UNITED STATES

DEPARTMENT OF THE INTERIOR

GEOLOGICAL SURVEY

WATER RESOURCES OF CLALLAM COUNTY, WASHINGTON:

PHASE 1 REPORT

By B. W. Drost

U.S. GEOLOGICAL SURVEY

Water-Resources Investigations Report 83-4227

Prepared in cooperation with the

CLAILAM COUNTY BOARD OF COMMISSIONERS and STA':E OF WASHINGTON DEPARTMENT OF ECOLOGY 


\section{UNITED STATES DEPARTMENT OF THE INTERIOR \\ WILLIAM P. CLARK, Secretary}

GEOLOGICAL SURVEY

Dallas L. Peck, Director

For additional information write to:

District Chief

U.S. Geological Survey

1201 Pacific Avenue - Suite 600

Tacoma, Washington 98402
Copies of this report

can be purchased from:

Open-File Services Section Western Distribution Branch

U.S. Geological Survey Box 25425, Federal Center Lakewood, Colorado 80225 (Telephone: (303) 234-5888) 


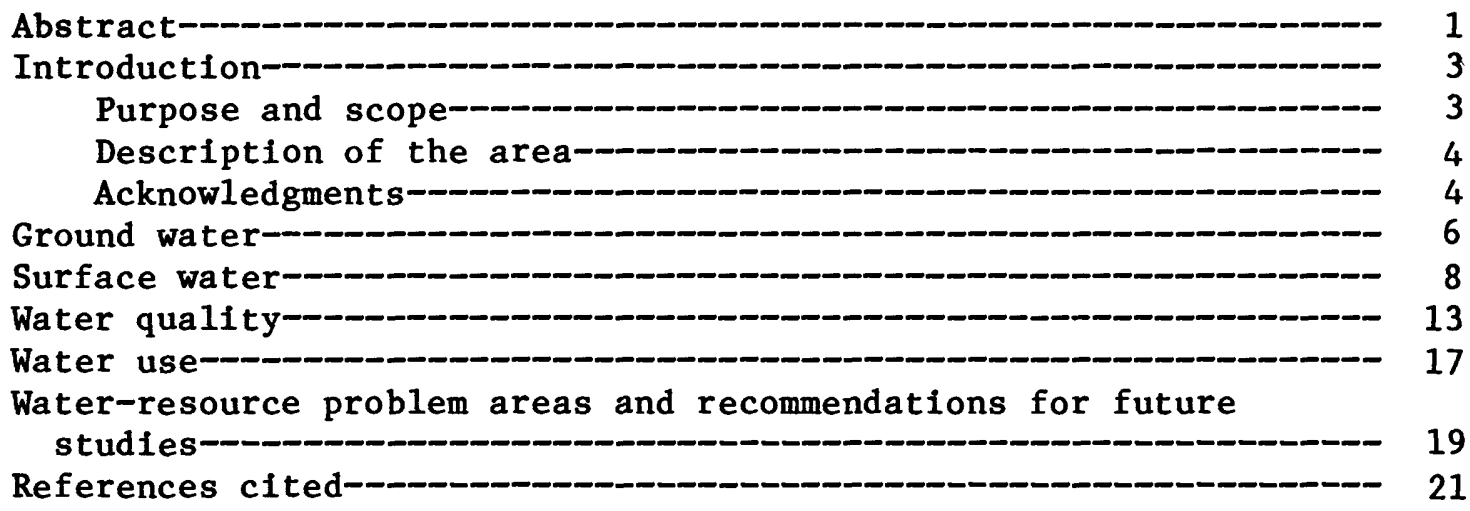

\section{ILLUSTRATIONS}

(P1ates 1-5 in pocket)

PLATES 1-5. Maps showing primary study areas:

1. Miller Peninsula and adjacent areas.

2. Sequim-Dungeness Peninsula.

3. Port Angeles and adjacent areas.

4. Soleduck-Calawah River basins from LaPush to Sappho and adjacent areas.

5. Clallam Bay-Sekiu and adjacent areas. 
TABLE 1. Summary of selected ground-water data in the primary study areas-_-_- 7

2. Summary of selected surface-water data-_- 9

3. Summary of selected ground-water-quality data-______-_ 15

4. Summary of selected surface-water-quality data -___-_-_ 16

5. Estimated annual water use -

6. Records of selected wells and springs- 24

7. Records of gaging stations- 78

8. Records of crest-stage partial record stations--_- 119

9. Records of miscellaneous streamflow measurement sites---- 130

10. Month-end reservoir contents of Lake Mills, 1927-78----- 161

11. Summary of selected data on lakes- 163

12. Chemical and physical quality of water from selected wells and springs-_-_- 168

13. Trace constituents in water from selected wells and springs----- 184

14. Chemical and physical quality of water from selected surface-water sites----- 185

15. Suspended sediment and related parameters in water from selected surface-water sites-_-_-_-_-

METRIC CONVERSION FACTORS

Multiply

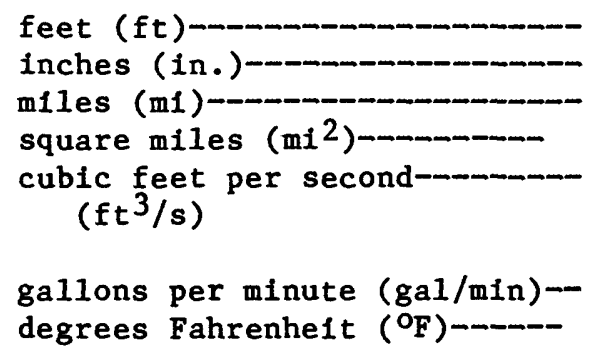

\section{By}

0.3048

2.540

1.609

2.59

28.32

0.02832

0.06309

0.5556 , after subtracting 32

\section{To obta1n}

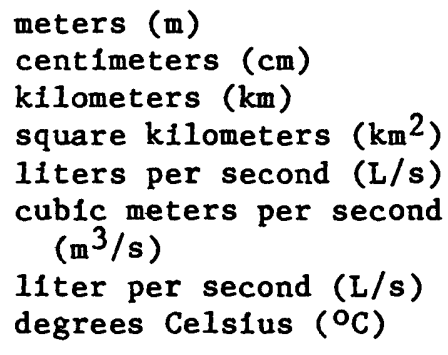

National Geodetic Vertical Datum of 1929 (NGVD of 1929): A geodetic datum derived from a general adjustment of the first-order level nets of both the United States and Canada, formerly called "mean sea level." NGVD of 1929 is referred to as sea level in this report. 


\section{WELL- AND AREA-LOCATION NUMBERING SYSTEM}

The well and area-location numbers used in this report give the location of wells and areas according to the official rectangular public-land survey. For example, in well number 28/13-34Bl, the part preceding the hyphen indicates successively the township and range (T. $28 \mathrm{~N}$., R. $13 \mathrm{~W}$.) north and west of the Willamette base line and meridian, respectively. The number following the hyphen indicates the section (sec. 34), and the letter (B) indicates the 40-acre subdivision of the section as shown in the sketch below. Last is a sequence number used to distinguish wells in the same 40-acre tract. Thus, well 28/1 3-34Bl is in the NW of the $\mathrm{NE}$ of sec. 34, T.28 N., R.13 W. An " $\mathrm{W}$ " following the sequence number indicates that the site is a spring.

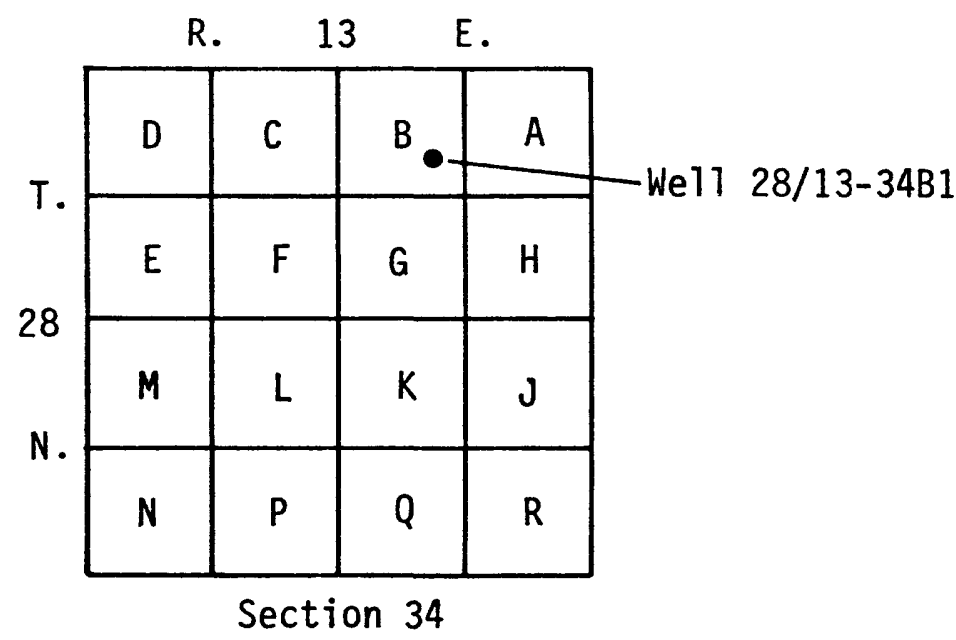

In computer-printout tables the same well is given the number $28 \mathrm{~N} / 13 \mathrm{~W}-34 \mathrm{Bl}$. On plates, which show numbered section, only the 40 -acre subdivision and sequence number are shown. Thus, well $28 / 13-34 \mathrm{Bl}$ is shown as $\mathrm{Bl}$. 


\title{
WATER RESOURCES OF CLALLAM COUNTY, WASHINGTON: PHASE I REPORT
}

\author{
By B. W. Drost
}

\begin{abstract}
This report concludes the first of two phases of investigation of the water resources of Clallam County. The study area includes all of the county, with emphasis on the more densely populated areas. The purpose of the first phase of study was to inventory the water resources of the county and identify any related problems.

Sufficient water is available to supply all present demands. Water supplies suitable for domestic use can be obtained in most of the populated parts of the county from wells drilled 100 feet or less into glacial and alluvial deposits. In populated areas underlain by bedrock, wells more than 100 feet deep are generally capable of supplying one home per well. The potential for obtaining domestic water supplies from wells in the unpopulated mountainous interior of the county is not known.

Surface water is abundant in the county and is the source of supply for most of the public water systems. The only streams that are sometimes dry or nearly so are in small drainage basins in bedrock in the mountainous interior and along parts of the southern coastline of the Strait of Juan de Fuca.

Annual water use (1978) is about 39 billion gallons. About 18 billion gallons is used for industrial purposes, 18 billion gallons for irrigation, and 3 billion gallons is used for domestic purposes. Most of the water is obtained from surface-water sources (38 billion gallons). The remaining 1 billion gallons is ground water and is obtained from about 4,000 wells.

Ground and surface waters are generally of excellent quality. The only serious ground-water-quality problem occurs in coastal areas where wells may yield water with large concentrations of chloride and dissolved-solids. Excessive concentrations of iron and (or) manganese were measured in about 24 percent of the wells tested. Minor taste, color, or odor problems were reported by about 34 percent of the well owners surveyed.

High values of turbidity, color, and coliform bacteria are widespread surface-water problems. However, standard filtering and chlorination treatment makes the water suitable for public supplies. The large concentrations of coliform bacteria apparently originate naturally in soils, and are generally associated with relatively low fecal-coliform concentrations. High ammonia concentration was observed at one site on the Bogachiel River near Forks, and is probably caused by sewage-disposal practices in the area.
\end{abstract}


Several monitoring activities would provide a continuing data base and alert system for managing the ground water resources. To detect saltwater encroachment in aquifers, chloride concentrations could be monitored in wells along several coastal areas. Water levels in wells tapping bedrock aquifers could be measured periodically to determine effects of increased aquifer development. Ammonia, nitrate, and fecal coliform bacteria concentrations could be monitored in ground and surface waters in the Forks area to observe any increases due to sewage disposal practices. 


\section{INTRODUCTION}

\section{Purpose and Scope}

Some of the oldest developed areas in western Washington are in Clallam County. In recent years, the county has experienced rapid growth in population and changes in the patterns of land use. Much of the land, especially in the northeastern part of the county, was previously used for irrigated agriculture, but in recent years the trend has been toward subdivision for residential uses. The changes in land use have caused changes in the patterns of water use, placing new stresses on the ground- and surface-water systems.

To evaluate the changes in water use, which will be considered in formulating a plan for recommended land-use practices for the future, the Clallam County Planning Commission and the State of Washington Department of Ecology requested that the U.S. Geological Survey conduct a study of the County's water resources. A two-phase study was agreed upon, and work began in late 1978.

The objectives of the study are to: (1) inventory the existing surface- and ground-water supplies of the county, and assess their quantity and quality; (2) determine the effects of irrigation on ground-water recharge in a northeastern part of the county (Sequim area); and (3) identify present and potential sources of contamination of the county's water resources and to determine if these present or potential contaminants will affect the quality or availability of water.

The study was undertaken in two phases. Phase I goals were to: (1) inventory and tabulate the existing water-resources data; (2) evaluate current water-resources conditions; (3) identify areas where water-quality problems and (or) water shortages exist; and (4) establish a network of monitoring sites in problem areas. This report completes Phase I of the study and provides a framework for completion of the more interpretive Phase II goals.

Phase II goals are (1) evaluation of data collected in the problem areas; (2) determination of the effects of changing land-use patterns in the problem areas (particularly the change in use from irrigated agriculture to domestic); and (3) preparation of water budgets for the problem areas; the budget will be used to test with a simple computerized numerical model various management alternatives.

From the start of the project, the Sequim area was treated as a "problem area" because irrigation and development is heaviest in that area. Therefore, in the Sequim area a greater concentration of monitoring sites was established during Phase I of the study. The Phase II report will present a thorough tabulation discussion of all data collected in the Sequim area. Included in this report are all of the available basic data for the county, except for the Sequim area where only selected representative data are included. 
Clallam County is the northernmost part of the Olympic Peninsula in western Washington (fig. 1). The county is bounded on the west by the Pacific Ocean, on the north by the Strait of Juan de Fuca, and on the east and south by Jefferson County.

The total area of the county is about $1,750 \mathrm{mi}^{2}$. Most of the county has rugged mountains with elevations reaching nearly $7,000 \mathrm{ft}$. The mountains are composed mainly of sandstone, conglomerate, siltstone, slate, and phyllite, and some basalt and mudflow breccias (Tabor and Cady, 1978). The coast east of Port Angeles is relatively flat and is composed of glacial and alluvial deposits. A large area in the western part of the county, from Forks to the Pacific Ocean and fron the Jefferson County line to areas north of Ozette Lake, is also made up predominantly of glacial and alluvial deposits, but it has a more rugged topography than the coastal area east of Port Angeles.

The primary study areas are the most densely populated portions of the county, and include the coastal strip 2 to $6 \mathrm{mi}$ wide along the Strait of Juan de Fuca between the east county line urid the Lyre River (pls. 1-3), the Soleduck-Calawah River basins from LaPush to Sappho (pl. 4), and a small coastal area including Sekiu and Clallam Bay (pl. 5). The total area is about $200 \mathrm{mi}^{2}$.

The county has a maritime climate characterized by cool, dry summers and mild, wet winters. About 75 percent of the annual precipitation falls during the period October-March. The Olympic Mountains cause a wide range in rainfall. Forks, on the western, windward side of the mountains, has an average annual precipitation of 116 in. At Sequim, on the northeast side of the mountains about $60 \mathrm{mi}$ from Forks, the annual precipitation is 16 in. Average annual temperature in the county is about $50^{\circ} \mathrm{F}$; the average temperature in July, the warmest month, is $63^{\circ} \mathrm{F}$ and the average temperature in December, the coldest month, is $36^{\circ} \mathrm{F}$.

The population of the county was about 44,200 in 1978 (State of Washington, 1978). In recent years the county has experienced an increase in its population growth rate. From 1940 through 1970, the average annual increase in population was 1.8 percent. From 1970 through 1978, the annual rate increased to 3.4 percent.

The county is largely undeveloped (ENCON, 1974), and is 80 to 85 percent forest-covered. About $\mathbf{5 0}$ percent of the county is National Park and or National Forest land. Between 5 and 10 percent of the county is agricultural land, about 5 percent is residential, and between 1 and 5 percent is used for other purposes, including industrial, commercial, and recreational.

\section{Acknowledgments}

This study was made in cooperation with the Clallam County Board of Commissioners and the State of Washington Department of Ecology. Unpublished data were obtained from the State of Washington Department of Social and Health Services. Many individual residents of the county aided the investigation by supplying information and granting access to their wells for water-quality sampling. 


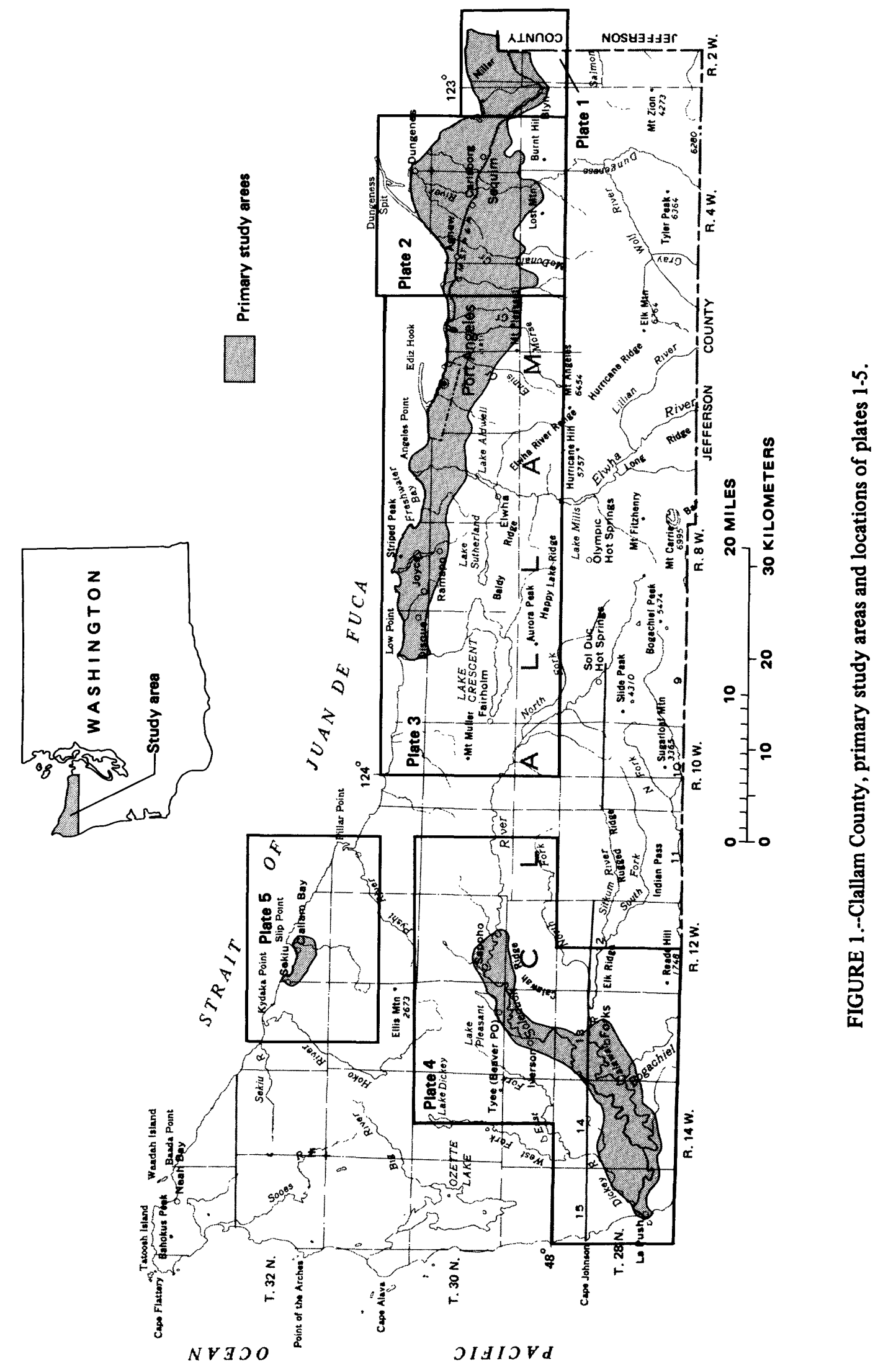




\section{GROUND WATER}

Ground-water data sources were: (1) U.S. Geological Survey well records, which include data on all wells and springs visited during previous USGS studies in the area; (2) Washington State Department of Ecology Water Well Reports; (3) field visits during the summer of 1979 to about 440 wells in the primary study areas; and (4) records of oil and gas exploration wells drilled during 1900-1978 (McFarland, 1979).

Records on about 2,100 wells and springs are available (table 6,p.24). Well and spring locations are shown on plates $1-5$.

Most of the well data in table 6 are as reported by the drillers who completed the wells. Well ownerships are listed as reported by drillers or as determined from field visits. Local numbers were assigned by the U.S. Geological Survey. Wells that are listed as having unchecked locations were assigned local well numbers on the basis of locations reported by drillers. Approximately 40 percent of the recorded wells have been field-checked. Altitudes were determined from topographic maps by U.S. Geological Survey personnel. In most cases, they are probably accurate to

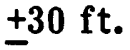

Wells reported as "dry" may actually yield some water. The term "dry" as used by some drillers refers to a well with insufficient yield to supply a household.

Specific-capacity values (the rate of discharge of water from a well divided by the drawdown of water level within the well) are determined mainly from short-term (less than 4 hours) bailer tests, where discharge rates and water-level measurements were not precisely determined.

A summary of selected ground-water data in the primary study areas is shown in table 1. An increase in drilling activity in recent years may be inferred from the available data. About $\mathbf{8 0}$ percent of the well records on file are for wells drilled in 1970 or after.

Sufficient water for most uses is obtained at relatively shallow depths in most parts of the primary study areas. Most wells are less than $100 \mathrm{ft}$ deep, except on the Miller Peninsula where the median well depth is $148 \mathrm{ft}$. Approximately 250 well owners were asked about the supply capacities of their wells during field visits in 1979. About 9 percent of the wells were reported as dry or yielding insufficient supplies at least once during their period of use.

Water levels are generally less than $50 \mathrm{ft}$ below land surface, but may exceed $300 \mathrm{ft}$ in places. A few flowing wells are found primarily along the shorelines of the Port Angeles area, the Sequim-Dungeness Peninsula, and the Miller Peninsula.

Most wells completed in unconsolidated materials have 6-in. diameter open-ended casings. Those completed in bedrock are uncased. Screens have been installed in some wells in unconsolidated deposits, particularly in wells on the Miller Peninsula and in the Sequim area. Generally the screens are $5 \mathrm{ft}$ in length.

Specific-capacity values vary widely. Generally, the alluvial materials of the Soleduck-Calawah area have the greatest values. The Port Angeles area has the lowest median value, primarily due to the low specific capacities observed in the large number of wells drilled into the bedrock along the southern margin of the area. 
TABLE 1.--Sumary of se lected ground-water data in Clallam County

\begin{tabular}{|c|c|c|c|c|c|}
\hline $\begin{array}{l}\text { Primary study areas (see pls.1-5 } \\
\text { for full descriptions) }\end{array}$ & $\begin{array}{l}\text { Miller } \\
\text { Peninsula }\end{array}$ & $\begin{array}{l}\text { Sequim- } \\
\text { Dungeness } \\
\text { Peninsula }\end{array}$ & $\begin{array}{l}\text { Port } \\
\text { Angeles }\end{array}$ & $\begin{array}{c}\text { Soleduck- } \\
\text { Calawah }\end{array}$ & $\begin{array}{l}\text { CTallam } \\
\text { Bay } \\
\text { Sekiu }\end{array}$ \\
\hline Number of well records on file & 84 & 983 & 219 & 57 & 5 \\
\hline $\begin{array}{l}\text { Date well completed (percentage of we } 11 \mathrm{~s} \text { ) } 1 \\
\text { First quarter of } 1980 \\
1975-79 \\
1970-74 \\
\text { pre-1970 } \\
\text { 01dest on file }\end{array}$ & $\begin{array}{r}4 \\
58 \\
17 \\
21 \\
1914\end{array}$ & $\begin{array}{r}1 \\
71 \\
20 \\
8 \\
1918\end{array}$ & $\begin{array}{r}0 \\
59 \\
7 \\
34 \\
1892\end{array}$ & $\begin{array}{r}7 \\
64 \\
9 \\
20 \\
1928\end{array}$ & $\begin{array}{r}0 \\
80 \\
0 \\
20 \\
1958\end{array}$ \\
\hline $\begin{array}{l}\text { Water use (percentage of wells) } \\
\text { Domestic } \\
\text { Public } \\
\text { Stock } \\
\text { Irrigation } \\
\text { Commercial-industrial } \\
\text { Aquiculture } \\
\text { Unused }\end{array}$ & $\begin{array}{r}82 \\
7 \\
1 \\
0 \\
0 \\
0 \\
10\end{array}$ & $\begin{array}{r}86 \\
4 \\
3 \\
4 \\
0 \\
0 \\
3\end{array}$ & $\begin{array}{r}90 \\
2 \\
1 \\
2 \\
1 \\
1 \\
3\end{array}$ & $\begin{array}{r}59 \\
14 \\
0 \\
0 \\
0 \\
0 \\
27\end{array}$ & $\begin{array}{r}40 \\
0 \\
0 \\
0 \\
0 \\
0 \\
60\end{array}$ \\
\hline $\begin{array}{l}\text { Maximum depth drilled }(\mathrm{ft})^{2} \\
\text { Well depth }(\mathrm{ft})\end{array}$ & 1,015 & 970 & 500 & 196 & 72 \\
\hline $\begin{array}{l}\text { Maximum } \\
\text { Minimum } \\
\text { Median }\end{array}$ & $\begin{array}{r}492 \\
10 \\
140\end{array}$ & $\begin{array}{r}842 \\
4 \\
78\end{array}$ & $\begin{array}{r}500 \\
6 \\
87\end{array}$ & $\begin{array}{r}196 \\
25 \\
81\end{array}$ & $\begin{array}{l}51 \\
22 \\
33\end{array}$ \\
\hline $\begin{array}{l}\text { Water level }(\mathrm{ft}) \\
\text { Maximum } \\
\text { Minimum } \\
\text { Median } \\
\text { Number of flowing wells } \\
\text { Number of "dry" holes } 3\end{array}$ & $\begin{array}{c}312 \\
\text { Flowing } \\
55 \\
2 \\
0\end{array}$ & $\begin{array}{l}275 \\
\text { Flowing } \\
26 \\
18 \\
22\end{array}$ & $\begin{array}{c}222 \\
\text { Flowing } \\
20 \\
7 \\
10\end{array}$ & $\begin{array}{r}106 \\
8 \\
48 \\
0 \\
8\end{array}$ & $\begin{array}{l}8 \\
7 \\
8 \\
0 \\
1\end{array}$ \\
\hline $\begin{array}{l}\text { Well finish (percentage of wells) } \\
\text { Open end or open hole } \\
\text { Screen or sand point } \\
\text { Perforations } \\
\text { Horizontal gallery } \\
\text { Walled }\end{array}$ & $\begin{array}{r}41 \\
45 \\
14 \\
0 \\
0\end{array}$ & $\begin{array}{r}50 \\
40 \\
10 \\
0 \\
0\end{array}$ & $\begin{array}{r}65 \\
18 \\
15 \\
1 \\
1\end{array}$ & $\begin{array}{r}47 \\
21 \\
32 \\
0 \\
0\end{array}$ & $\begin{array}{r}50 \\
0 \\
50 \\
0 \\
0\end{array}$ \\
\hline $\begin{array}{l}\text { Specific capacity }(\mathrm{gal} / \mathrm{min}) / \mathrm{ft} \\
\text { Maximum } \\
\text { Minimum } \\
\text { Median }\end{array}$ & $\begin{array}{r}61 \\
1.0\end{array}$ & $\begin{array}{r}460 \\
.0 \\
2.0\end{array}$ & $\begin{array}{r}396 \\
.0 \\
.5\end{array}$ & $\begin{array}{r}169 \\
.0 \\
4.8\end{array}$ & $\begin{array}{l}12 \\
.0 \\
2.0\end{array}$ \\
\hline
\end{tabular}

1 Mandatory filing of well records by drillers was not required by Washington State Department of Ecology prior to 1971. Therefore, the percentage of wells completed prior to 1970 is probably greater than indicated by the existing records.

2 These figures do not include oil test wells.

3 Wells reported as "dry" by drillers.

40.0 , less than 0.05 


\section{SURFACE WATER}

Surface-water data sources were: (1) U.S. Geological Survey computer files (WATSTORE) of continuous gaging station and crest-stage station records; (2) a compilation of miscellaneous streamflow measurements made during 1890-January 1961 (State of Washington Department of Conservation, 1964); (3) miscellaneous streamflow measurements made during January 1961-September 1978 (U.S. Geological Survey, 1961-79); (4) reservoir-content records for Lake Mills (near Port Angeles) during April 1927-September 1978 (U.S. Geological Survey, 1955, 1964, and 1961-79); and (5) lake data from two earlier reports (Wolcott, 1973 and Bortleson and others, 1976). Discharge data are contained in tables 7-9 (p.78). Surface-water sites are shown on plates 1-4.

A summary of selected surface-water discharge data is presented in table 2. The data in the table must be used with caution, particularly when comparing sites. Mean values have been calculated on the basis of the available data for each site. Because of the cyclic nature of weather patterns, the value is influenced greatly by the period and the length of record at any particular site and the type of data collected at that site. Generally, the longer the period of record, the more representative are the values obtained. When comparing sites, it is best to compare sites with similar periods of record.

The 7-day low-flow values in table 2 are as determined by Haushild and LaFrance (1978). A 7-day low-flow value at the 50-percent probability level means that during any year the minimum of the mean flow for 7-day periods has a 50-percent probability (chance of 1 in 2) of being less than or equal to that value. This is sometimes referred to as the 7-day 2-year low flow. The 5-percent probability (chance of 1 in 20) is sometimes referred to as the 7-day 20-year low flow.

The floodflow values in table 2 were determined by Cummans and others (1975) or were calculated from a regression equation presented in that report. A floodflow value at the 50-percent probability level means that during any year the maximum flow has 50-percent probability of exceeding that value. This is sometimes referred to as the 2-year floodflow. The 1-percent probability (chance of 1 in 100) is sometimes referred to as the 100-year floodflow. Three studies have been completed that show the flood inundation areas for most of Clallam County (Federal Insurance Administration, 1979 and 1980). 
TABLE 2.--Sumary of selected surface-water discharge data in Clallam County

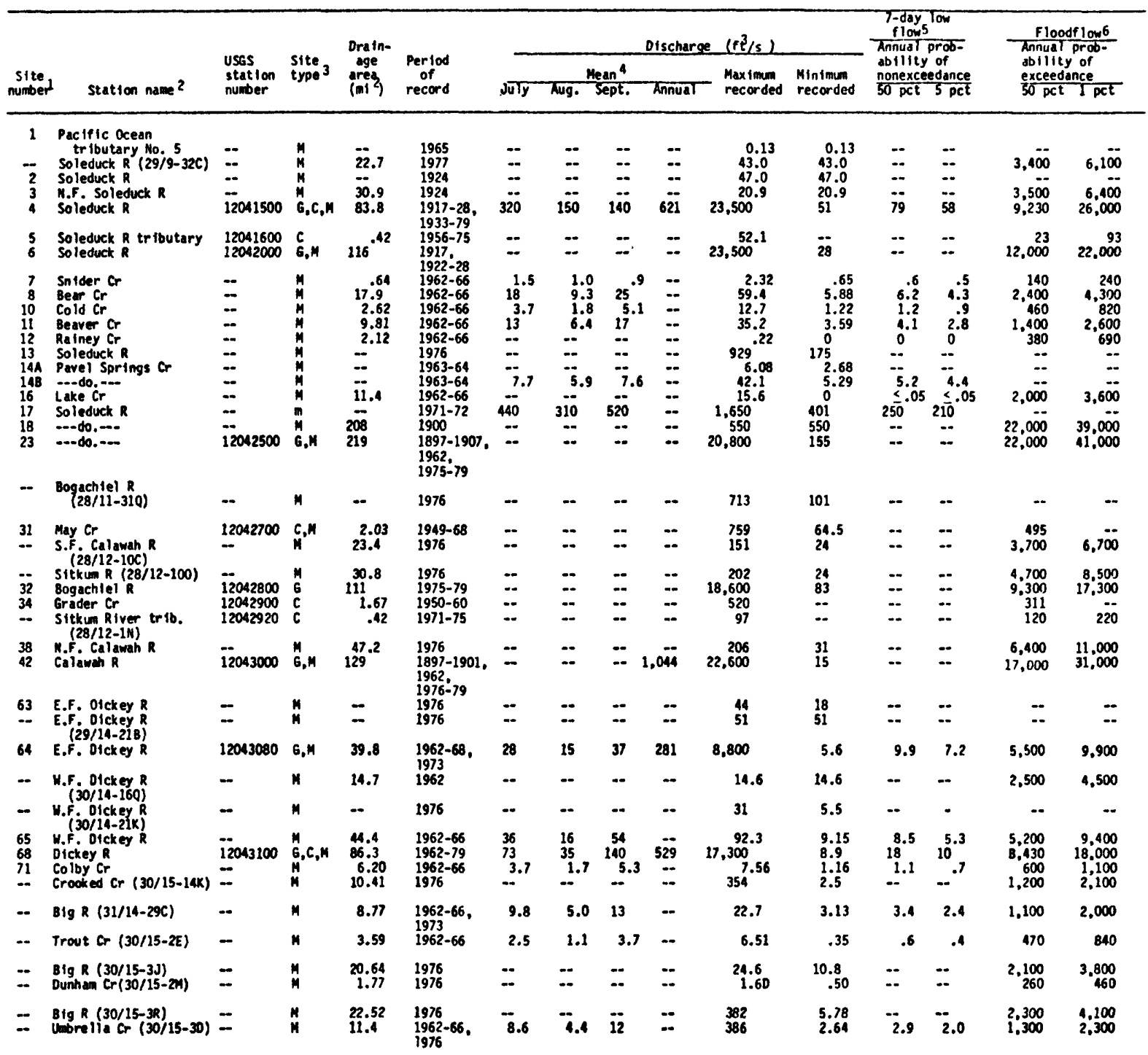


TABLE 2.--Sumary of selected surface-water discharge data in Clallam County--cont inued

\begin{tabular}{|c|c|c|c|c|c|c|c|c|c|c|c|c|c|c|c|}
\hline \multirow[b]{2}{*}{$\begin{array}{l}\text { Site } \\
\text { numb-1 } \\
\text { ber }\end{array}$} & \multirow[b]{2}{*}{ Station name? } & \multirow[b]{2}{*}{$\begin{array}{l}\text { USGS } \\
\text { station } \\
\text { number }\end{array}$} & \multirow[b]{2}{*}{$\begin{array}{l}\text { Site } \\
\text { type }\end{array}$} & \multirow{2}{*}{$\begin{array}{l}\text { Dra 1n- } \\
\text { age } \\
\text { area } \\
\text { (nit) }\end{array}$} & \multirow[b]{2}{*}{$\begin{array}{l}\text { Period } \\
\text { of } \\
\text { record }\end{array}$} & \multicolumn{6}{|c|}{ Discharge $\left(\mathrm{ft}^{3} / \mathrm{s}\right)$} & \multirow{2}{*}{\multicolumn{2}{|c|}{$\begin{array}{l}\text { 7-day low } \\
\text { flow } \\
\text { Annualprob- } \\
\text { abllity of } \\
\text { nonexceedance } \\
50 \text { pet } 5 \text { pct }\end{array}$}} & \multirow{2}{*}{\multicolumn{2}{|c|}{$\begin{array}{l}\text { Floodf low } \\
\text { Arnual prob- } \\
\text { abillty of } \\
\text { exceedance } \\
50 \text { pct I pct }\end{array}$}} \\
\hline & & & & & & Juty & $\frac{\text { Me }}{\text { Kug. }}$ & $\frac{n^{4}}{\text { sept. }}$ & Annuat & - Maximum & $\begin{array}{l}\text { Minimum } \\
\text { recorded }\end{array}$ & & & & \\
\hline$\cdots$ & Ozette $R(31 / 15-30 R)$ & - & M & - & $1962-66$, & 100 & so & 140 & -- & 261 & 25.1 & 32 & 22 & -- & -- \\
\hline$\ddot{\ddot{z}}$ & $\begin{array}{l}\text { Cosi } C r \quad(31 / 15-29 N) \\
\text { orette } R(31 / 15-31 A) \\
\text { Thirty Cent } C r\end{array}$ & $\overline{12043150}$ & $\begin{array}{l}M \\
G \\
M\end{array}$ & $\begin{array}{l}5.82 \\
77.5 \\
.57\end{array}$ & $\begin{array}{l}1962-66 \\
1977-79 \\
1962-66\end{array}$ & $\begin{aligned} 2.3 \\
-. .6\end{aligned}$ & $\begin{array}{r}1.0 \\
-.3\end{array}$ & $\begin{aligned} 3.3 \\
-.9\end{aligned}$ & $\ddot{\ddot{\prime}}$ & $\begin{array}{r}7.24 \\
1.500 \\
1.51\end{array}$ & 20.14 &. .6 &. .4 & $\begin{array}{r}670 \\
6,600 \\
120\end{array}$ & $\begin{array}{r}1,200 \\
12,000 \\
220\end{array}$ \\
\hline$\ddot{*}$ & $\begin{array}{l}(32 / 15-22 \times) \\
\text { Milier Cr }(32,15-22 G) \\
\text { Sooes R }(32 / 15-21 A)\end{array}$ & $\overline{12043163}$ & $G_{G, M}$ & 32.0 & $\begin{array}{l}1962-66 \\
1971\end{array}$ &. .9 &.-4 & $\therefore .3$ & 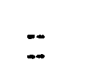 & $3,270^{2.78}$ & 9.11 &.$^{.3}$ & $\ldots .2$ & 3,900 & 7,390 \\
\hline -- & $\begin{array}{l}\text { Grimes } \operatorname{Cr}(32 / 15-15 \mathrm{M}) \\
\text { Gr imes } \operatorname{Cr}(32 / 15-8 A)\end{array}$ & $=$ & $\stackrel{M}{M}$ & 1.38 & $\begin{array}{l}1962-66 \\
1971\end{array}$ & 1.1 & $-\cdots$ & $\therefore .6$ & $=$ & 1400 & $27 \cdot \frac{.12}{3}$ & .3 & .2 & 260 & 470 \\
\hline$\because$ & $\begin{array}{l}\text { Waatch R (33/15-15K) } \\
\text { Wadtch } C_{r}\end{array}$ & 12043173 & $\underline{G}$ & 9.96 & $\begin{array}{l}1976-79 \\
1962-66\end{array}$ & $=$ & $\ddot{z}$ & $\because$ & $=$ & $\begin{array}{l}1,180 \\
3.40\end{array}$ & $\begin{array}{l}27.3 \\
.12 \\
.21\end{array}$ & 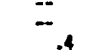 & $\ddot{z}_{3}$ & $1,6 \overrightarrow{600}$ & 2.800 \\
\hline .. & $\begin{array}{l}\text { (33/15-20MA- }) \\
\text { village } \mathrm{Cr}(33 / 15-10 \mathrm{P})\end{array}$ & - & $m$ & $\begin{array}{l}.03 \\
.65\end{array}$ & $1962-66$ & - & $\ldots$ & .. & - & 21.2 & 31 & $\stackrel{\bullet}{-}$ & & 20 & 220 \\
\hline$\because$ & 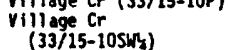 & $=$ & $m$ & .68 & - $1962-66$ & $=$ & 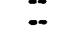 & 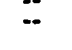 & $=$ & 1.97 & .06 & .1 & .1 & 130 & 230 \\
\hline-- & $S A 11 R(33 / 14-18 K)$ & 12043190 & $M$ & 5.42 & $\begin{array}{l}\text { 1962-66, } \\
1971 .-79 \\
1976-79\end{array}$ & 3.7 & 1.2 & 6.2 & -- & 762 & .41 & .6 & .3 & 790 & 1.400 \\
\hline $\begin{array}{l}\ddot{z} \\
\ddot{-}\end{array}$ & $\begin{array}{l}\text { Snow } C r\left(33 / 14-17 \mathrm{SH}_{4}\right) \\
\text { Bulliman } \mathrm{Cr}(33 / 14-20 \mathrm{~A}) \\
\text { Rasmussan } \mathrm{Cr} \\
(33 / 14-27 \mathrm{H})\end{array}$ & $=$ & $M_{M}^{M}$ & $\begin{array}{l}1.45 \\
3.69 \\
2.32\end{array}$ & $\begin{array}{l}1962-66 \\
1962-66 \\
1962-66\end{array}$ & $\begin{array}{l}.7 \\
1.7 \\
1.0\end{array}$ & $\begin{array}{l}.2 \\
.5 \\
.3\end{array}$ & $\begin{array}{l}1.2 \\
3.0 \\
1.7\end{array}$ & $\ddot{z}$ & $\begin{array}{l}3.01 \\
7.91 \\
4.75\end{array}$ & $\begin{array}{r}\leq .05 \\
. .20 \\
.13\end{array}$ & $\begin{array}{l}.1 \\
.3 \\
.1\end{array}$ & $\begin{array}{l}\leq .05 \\
.1 \\
.1\end{array}$ & $\begin{array}{l}250 \\
570 \\
410\end{array}$ & $\begin{array}{r}460 \\
1,000 \\
740\end{array}$ \\
\hline$\ddot{\ddot{z}}$ & $\begin{array}{l}\text { Jansen } C_{r}(33 / 14-26 R) \\
\text { O1sen } C r(32 / 14-1 A) \\
\text { N.F. Sek Iu R } \\
\text { (32/14-15NE4) }\end{array}$ & $\ddot{-}$ & $M_{M}^{M}$ & $\begin{array}{r}2.02 \\
1.14 \\
11.1\end{array}$ & $\begin{array}{l}1962-66 \\
1962-66 \\
1962-66\end{array}$ & $12^{1.2}$ & $\begin{array}{r}.4 .4 \\
5.8\end{array}$ & $\begin{array}{r}2.8 \\
1.7\end{array}$ & $\begin{array}{l}\because \\
\because-\end{array}$ & $\begin{array}{l}4.37 \\
2.30 \\
33.4\end{array}$ & $\begin{array}{r}.24 \\
.12 \\
3.29\end{array}$ & .2 & $\frac{.1}{2.05}$ & $\begin{array}{r}370 \\
200 \\
1,900\end{array}$ & $\begin{array}{r}660 \\
350 \\
3,400\end{array}$ \\
\hline-- & 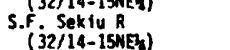 & -- & $M$ & 8.86 & $1962-66$ & 9.0 & 4.6 & 12 & - & 21.3 & 2.48 & 3.0 & 2.1 & 1,500 & 2,800 \\
\hline $\begin{array}{c}89 \\
89 \\
--\end{array}$ & $\begin{array}{l}\text { Carpenter or } \\
\text { Sek iu R } \\
\text { Hoko } R \text { tr ib } \\
\text { (31/14-23A) }\end{array}$ & $=$ & $\stackrel{M}{M}$ & $\begin{array}{r}2.90 \\
31.6 \\
.88\end{array}$ & $\begin{array}{l}1962-66 \\
1962-66 \\
1962-66\end{array}$ & $\ddot{29}_{.3}$ & 13 & $\ddot{42} .4$ & $\ddot{z}$ & $\begin{array}{r}2.79 \\
84.9 \\
.44\end{array}$ & 9.17 & $\overline{8.1}$ & .7 .2 & $\begin{array}{r}440 \\
3.900 \\
250\end{array}$ & $\begin{array}{r}790 \\
7.000 \\
450\end{array}$ \\
\hline -- & $\begin{array}{l}\text { Moko R tr tr. Mo. } 2 \\
(31 / 14-240)\end{array}$ & -- & $M$ & .94 & $1962-66$ & .6 & .3 & .9 & -. & 1.58 & .17 & .2 & .1 & 270 & 480 \\
\hline-- & $\begin{array}{l}\text { Hoko R trib } \\
\text { (31/13-76) }\end{array}$ & 12043270 & c & .67 & $1971-75$ & -- & -- & -- & -- & 149 & -- & -. & -. & 200 & 360 \\
\hline $\begin{array}{l}90 \\
91\end{array}$ & $\begin{array}{l}\text { Hoko R } \\
\text { Little Hoko R }\end{array}$ & 12043300 & $\mathrm{G}^{\mathrm{G}, \mathrm{C}}$ & $\begin{array}{l}51.2 \\
11.5\end{array}$ & $\begin{array}{l}1962-78 \\
1962-66 \\
1971-73\end{array}$ & ${ }_{8.0}^{62}$ & 31 & $\begin{array}{l}85 \\
12\end{array}$ & $\begin{array}{l}408 \\
--\end{array}$ & $\begin{array}{r}14,100 \\
122\end{array}$ & $\begin{array}{l}12 \\
2.04\end{array}$ & ${ }_{2.2}^{20}$ & 14.4 & $\begin{array}{l}6,270 \\
1,500\end{array}$ & $\begin{array}{r}14,000 \\
2,600\end{array}$ \\
\hline $\begin{array}{r}92 \\
93 \\
94 \\
95 \\
96 \\
97 \\
98 \\
99 \\
100 \\
101\end{array}$ & 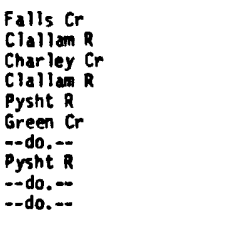 & 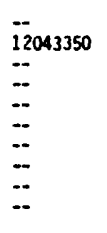 & $\begin{array}{l}M \\
G, M \\
M \\
M \\
M \\
M \\
M \\
M \\
M \\
M\end{array}$ & \begin{tabular}{l}
137.43 \\
5.23 \\
$\ddot{10.2}$ \\
1.96 \\
5.28 \\
\hdashline.- \\
37.6
\end{tabular} & $\begin{array}{l}1971-13 \\
1962-13 \\
1962-66 \\
1962-66 \\
1901 \\
1962-66 \\
1962-66 \\
1962-66 \\
1901 \\
1901 \\
1962-66 . \\
1971-73\end{array}$ & $\begin{array}{l}\ddot{9.8} \\
1.8 \\
\ddot{8.8} \\
.9 \\
1.6 \\
\ddot{17}\end{array}$ & $\begin{array}{r}4.1 \\
.3 \\
.6 \\
\because .6 \\
6.8\end{array}$ & $\begin{array}{l}\ddot{14} \\
2.8 \\
\ddot{12} \\
1.3 \\
2.6 \\
\ddot{20}\end{array}$ & 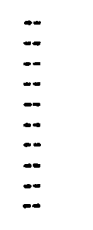 & $\begin{array}{c}127.26 \\
7.48 \\
100 \\
33.5 \\
4.29 \\
8.67 \\
114 \\
19 \\
560\end{array}$ & $\begin{array}{l}.10 \\
2.2 \\
.40 \\
6.71 \\
2.17 \\
.25 \\
.25 \\
19 \\
3.31\end{array}$ & \begin{tabular}{c}
$\because 3.0$ \\
.4 \\
-.4 .6 \\
.2 \\
.3 \\
$\because .3$ \\
\hdashline .9
\end{tabular} & $\begin{array}{r}\ddot{2.0} \\
.2 \\
\ddot{1.8} \\
.1 \\
.2 \\
\ddot{2.4}\end{array}$ & $\begin{array}{r}220 \\
12,000 \\
600 \\
\ddot{1,300} \\
250 \\
650 \\
\because \\
3,900\end{array}$ & $\begin{array}{r}390 \\
22.000 \\
1.100 \\
2.300 \\
4460 \\
1,200 \\
\ddot{70} \\
6,900\end{array}$ \\
\hline $\begin{array}{l}\ddot{-} \\
\ddot{102} \\
103 \\
104 \\
105 \\
105 \\
107 \\
108 \\
109\end{array}$ & 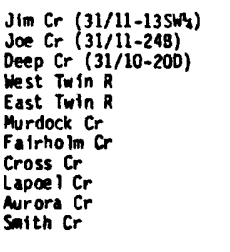 & $\begin{array}{l}- \\
\because \\
\ddot{12043430} \\
\ddot{\ddot{12043450}} \\
\ddot{-}\end{array}$ & $\begin{array}{l}M \\
M \\
M \\
M \\
G, C, M \\
M \\
M \\
C \\
M \\
M \\
M\end{array}$ & $\begin{array}{c}3.70 \\
1.51 \\
17.3 \\
12.8 \\
14.0 \\
2.26 \\
3.81 \\
.92 \\
1.15 \\
.61 \\
1.38\end{array}$ & $\begin{array}{l}1971-1 / 3 \\
1962-66 \\
1962-66 \\
1962-66 \\
1962-66 \\
1962-78 \\
1962-66 \\
1962-66 \\
1949-56 \\
1962-66 \\
1962-66 \\
1962-63 . \\
1965-66\end{array}$ & $\begin{array}{r}.2 \\
-.8 .3 \\
7.6 \\
8.0 \\
.1 \\
. .5 \\
-3.2 \\
. .\end{array}$ & $\begin{array}{l}.1 \\
\because 4.6 \\
4.5 \\
4.8 \\
\leq .05 \\
.3 \\
. .4 \\
. .4\end{array}$ & $\begin{array}{r}.2 \\
-8.0 \\
7.3 \\
7.8 \\
.1 \\
.2 \\
\because .0 \\
\therefore . .\end{array}$ & $\begin{array}{l}\because \\
\because \\
\ddot{64.7} \\
\because \\
\because \\
\because \\
\because\end{array}$ & $\begin{array}{r}2.56 \\
10.48 \\
20.3 \\
17.5 \\
1,220 \\
1.37 \\
11.10 \\
208 \\
6.42 \\
0.42 \\
7.64\end{array}$ & $\begin{array}{c}.04 \\
.05 \\
3.33 \\
3.00 \\
2.3 \\
.01 \\
.20 \\
. .03 \\
.03 \\
0\end{array}$ & $\begin{array}{l}\leq .05 \\
\leq .05 \\
3.5 \\
3.5 \\
3.8 \\
\leq .05 \\
. .2 \\
-. .6 \\
. .6 \\
-.-\end{array}$ & $\begin{array}{l}5.05 \\
\frac{x}{2.05} \\
2.5 \\
2.5 \\
2.8 \\
\leq .05 \\
.1 \\
\because \\
\therefore \\
\because\end{array}$ & $\begin{array}{l}360 \\
170 \\
900 \\
510 \\
859 \\
96 \\
330 \\
97 \\
120 \\
66 \\
130\end{array}$ & $\begin{array}{r}650 \\
3300 \\
1.700 \\
9.550 \\
1.200 \\
180 \\
612 \\
180 \\
220 \\
1120 \\
250\end{array}$ \\
\hline 110 & Lake Crescent trib. & 12043470 & c & .79 & $\begin{array}{l}1955-58 \\
1953-60\end{array}$ & .- & .- & .. & .. & 175 & .- & .. & .. & 82 & 150 \\
\hline
\end{tabular}


TABLE 2.--Sumary of selected surface-water discharge data in Clallan County--continued

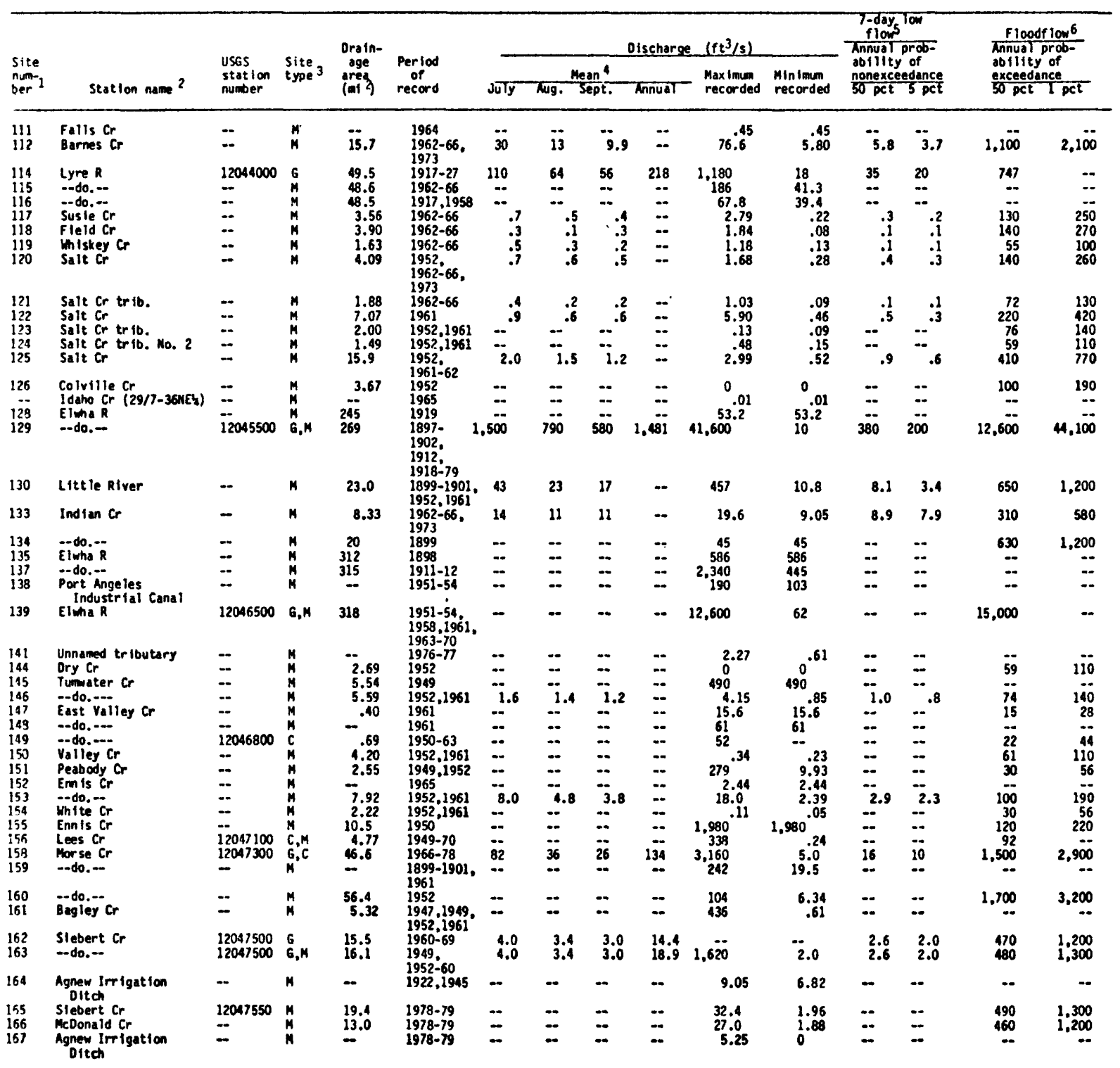


TABLE 2.--Sumary of selected surface-water discharge data in Clallam County--continued

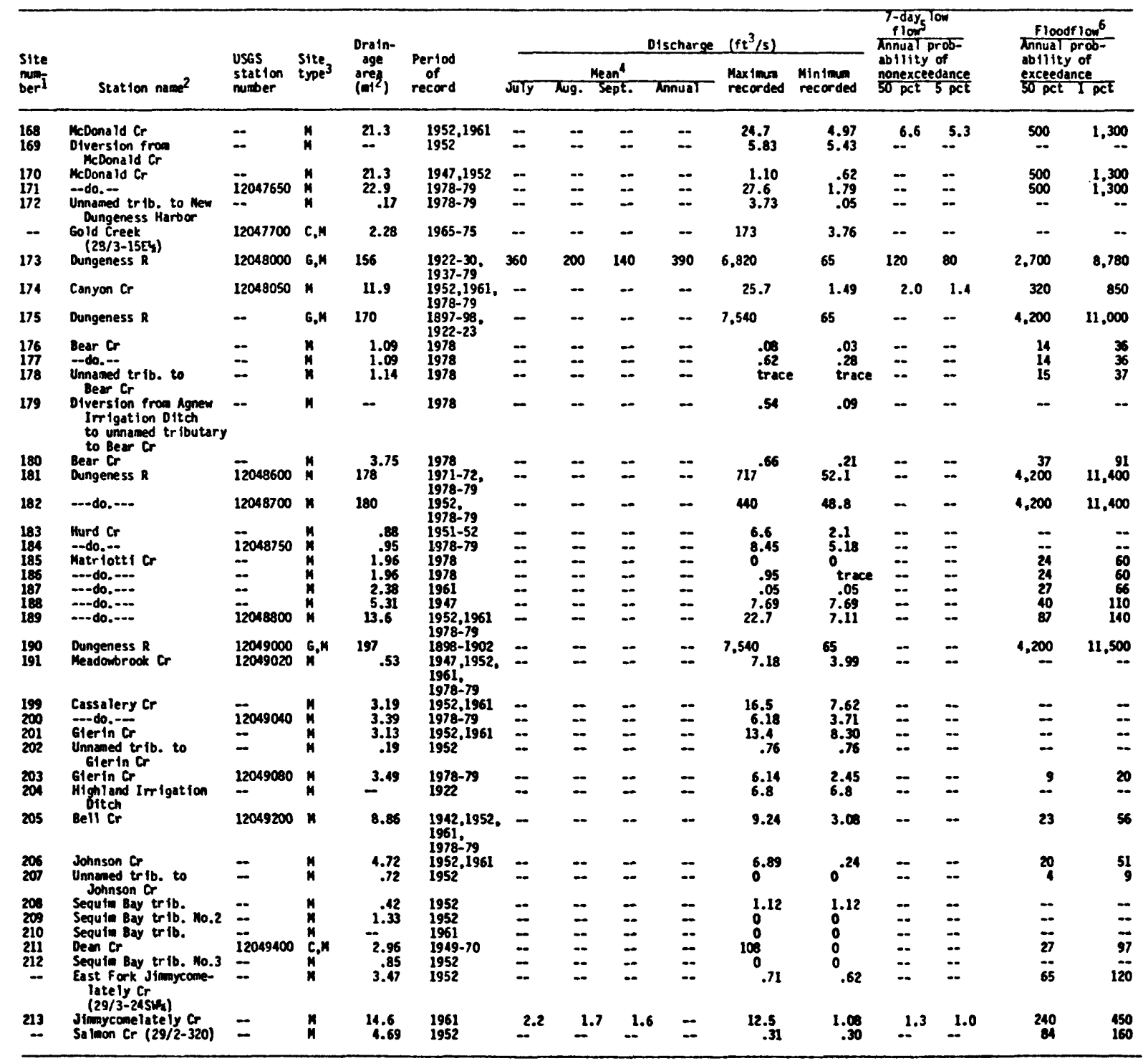

Numbered sites are shom on plates 1-5.

2abbreviated names are used in this table. Full names can be found in tables 7-9.

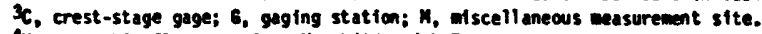

Hean monthly nows are trom Haush1ld and Lafrace, 1978.

Slow flows are from Mawshild and Lafrance, 1978.

6Flood flows are from Cumans and others. 1975, or as calculated from regression analyses presented in the same report. 


\section{WATER QUALITY}

Water-quality-data sources were: (1) U.S. Geological Survey computer files (WATSTORE); (2) State of Washington Department of Social and Health Services water-quality files as of March 1980; (3) a compilation of ground-water-quality records through 1961 (Van Denburgh and Santos, 1965); (4) USGS well records as of November 1981; (5) USGS reports on seawater intrusion along the coastal areas (Walters, 1971, and Dion and Sumioka 1981); (6) USGS reports on Indian Reservations in the county (USGS, 1961, Walters and others 1979 and 1980, and Fretwell, written commun., 1980) (7) USGS reports on studies in Olympic National Park (Walters, 1967 and 1970; Dion, 1979; Bortleson and Dion, 1979); (8) reports prepared by consultants (ENCON, 1974 and $\mathrm{CH}_{2} \mathrm{M}, 1967$ ), and (9) oral information from well owners and users during field visits. Tables 12-15 (p.168) contain ground- and surface-water-quality data for the county.

Ground and surface water within the county are generally of good quality. The available data were compared to the standards established by the U.S. Environmental Protection Agency (1975 and 1977) for safe drinking water. Tables 3 and 4 show the results of the comparisons.

The maximum contaminant levels (MCL's) listed are of primary importance because they refer to concentrations of constituents which, if exceeded, may affect the health of consumers. The proposed secondary recommended limits do not refer to health hazards but to concentrations that may affect the esthetic quality of the water.

In ground water, only turbidity and mercury levels have been observed to exceed their respective MCL's. Water with high turbidity values is hazardous primarily because it may affect chlorination processes. Most wells in the study area do not require chlorination, therefore turbidity is rarely a serious problem. A high mercury value was observed in one of two samples collected from the same water system on the same day. The second sample had no detectable mercury. This high mercury value may represent a pollution source within the distribution system or, more likely, an error in analysis; it is not considered to be a true concentration in the ground water.

Chloride, dissolved solids, and $\mathrm{pH}$, have exceeded their respective recommended limits in some ground-water samples. These samples generally come from wells in shoreline areas that penetrate the freshwater-saltwater zone of diffusion. Therefore, these high values generally do not indicate the presence of any pollutant, only naturally occurring salty water. Some of the $\mathrm{pH}$ values that exceeded the recommended limit were measured in waters obtained from several hot springs in the county and do not represent widespread conditions. 
Excessive concentrations of iron and (or) manganese in ground water are a common problem. About 24 percent of the wells tested had concentrations of either or both constituents that exceeded the recommended limits. Excessive concentrations of either of these constituents often create a bad taste, stain plumbing fixtures and laundry, and clog pumps and pipes. The high levels of color and turbidity observed are generally associated with samples containing excessive concentrations of iron and (or) manganese.

During field visits in 1979, about 250 well owners were asked about the quality of water from their wells. Problems were reported in 34 percent of the wells: 17 percent had staining or color problems, 16 percent had water with unpleasant taste, and 11 percent had bad odors. Some wells were reported as having two, or all three of the problems mentioned.

In surface water, only turbidity and coliform bacteria have been observed to exceed their respective MCL's. In samples where fecal-coliform data are also available, the fecal coliform concentrations are generally a small fraction of the total coliform. This suggests that most of the coliform present are not associated with a fecal source, but probably originated naturally in soils.

The only constituents or properties in surface water that exceed the recommended limits are iron, manganese, $\mathrm{pH}$, and color. However, none of these constituents is known to produce any serious problem in the county. 
TABLE 3.--Summary of selected ground-water-quality data in Clallam County

\begin{tabular}{|c|c|c|c|c|c|c|c|}
\hline Constituent 1 & $\begin{array}{l}\text { Number } \\
\text { of } \\
\text { sites } \\
\text { tested }\end{array}$ & $\begin{array}{l}\text { Number } \\
\text { of } \\
\text { samples } \\
\text { tested } \\
\end{array}$ & $\begin{array}{l}\text { Chemical stanc } \\
\text { Maximum con- } \\
\text { taminant leve }{ }^{2} \\
(E P A, 1975)\end{array}$ & $\begin{array}{ll}\text { dards } & \text { Nu } \\
\text { Proposed } & \text { of } \\
\text { secondary } & \text { ex } \\
\text { leve13 } & \text { ch } \\
\text { (EPA, 1977) } & \text { st } \\
\end{array}$ & $\begin{array}{l}\text { Uumber } \\
\text { of sites } \\
\text { exceeding } \\
\text { chemical } \\
\text { tandard }\end{array}$ & $\begin{array}{l}\text { Number of } \\
\text { samples } \\
\text { exceeding } \\
\text { chemical } \\
\text { standard } \\
\end{array}$ & $\begin{array}{l}\text { Maximum } \\
\text { value } \\
\text { observed }\end{array}$ \\
\hline $\begin{array}{l}\text { Iron } \\
\text { Manganese } \\
\text { Sulfate } \\
\text { Chloride } \\
\text { Fluoride } \\
\text { Nitrate } \\
\text { Dissolved solids } 5 \\
\text { pH } \\
\text { Color }\end{array}$ & $\begin{array}{r}98 \\
62 \\
72 \\
147 \\
70 \\
85 \\
57 \\
75 \\
68\end{array}$ & $\begin{array}{r}145 \\
103 \\
104 \\
230 \\
106 \\
125 \\
89 \\
116 \\
107\end{array}$ & $\begin{array}{l}-- \\
-- \\
-- \\
-- \\
2.0 \mathrm{mg} / \mathrm{L} \\
10 \mathrm{mg} / \mathrm{L} \\
-- \\
-- \\
--\end{array}$ & $\begin{array}{l}0.3 \mathrm{mg} / \mathrm{L} \\
0.05 \mathrm{mg} / \mathrm{L} \\
250 \mathrm{mg} / \mathrm{L} \\
250 \mathrm{mg} / \mathrm{L} \\
-- \\
-- \\
500 \mathrm{mg} / \mathrm{L} \\
<6.5 \text { or }>8.5 \\
15 \text { platinum- } \\
\text { cobalt units }\end{array}$ & $\begin{array}{r}24 \\
14 \\
0 \\
10 \\
0 \\
0 \\
16 \\
15 \\
5\end{array}$ & $\begin{array}{r}29 \\
21 \\
0 \\
16 \\
0 \\
0 \\
23 \\
22 \\
5\end{array}$ & $\begin{array}{l}17 \mathrm{mg} / \mathrm{L} \\
0.55 \mathrm{mg} / \mathrm{L} \\
81 \mathrm{mg} / \mathrm{L} \\
3,300 \mathrm{mg} / \mathrm{L} \\
1.6 \mathrm{mg} / \mathrm{L} \\
3.9 \mathrm{mg} / \mathrm{L} \\
1,570 \mathrm{mg} / \mathrm{L} \\
6.0 \text { to } 9.6 \\
90 \text { units }\end{array}$ \\
\hline $\begin{array}{l}\text { Turbidity } \\
\text { Arsenic } \\
\text { Barium } \\
\text { Cadmium } \\
\text { Chromium } \\
\text { Copper } \\
\text { Lead } \\
\text { Mercury } \\
\text { Selenium } \\
\text { Silver } \\
\text { Zinc } \\
\text { Endrin9 } \\
\text { Lindane } 9 \\
\text { Methoxychlor } 9 \\
\text { Toxaphene } \\
\text { 2,4-D9 } \\
2,4,5-\text { TP silvex } 9\end{array}$ & $\begin{array}{r}51 \\
18 \\
18 \\
18 \\
22 \\
7 \\
24 \\
18 \\
21 \\
21 \\
6 \\
3 \\
3 \\
3 \\
3 \\
3 \\
3\end{array}$ & $\begin{array}{r}83 \\
24 \\
24 \\
24 \\
28 \\
7 \\
27 \\
22 \\
24 \\
24 \\
6 \\
3 \\
3 \\
3 \\
3 \\
3 \\
3\end{array}$ & $\begin{array}{l}1 \text { to } 5 \mathrm{JTU} \\
0.05 \mathrm{mg} / \mathrm{L} \\
1 \mathrm{mg} / \mathrm{L} \\
0.010 \mathrm{mg} / \mathrm{L} \\
0.05 \mathrm{mg} / \mathrm{L} \\
0.05 \mathrm{mg} / \mathrm{L} \\
0.05 \mathrm{mg} / \mathrm{L} \\
0.002 \mathrm{mg} / \mathrm{L} \\
0.01 \mathrm{mg} / \mathrm{m} \\
0.05 \mathrm{mg} / \mathrm{L} \\
-0 \\
0.0002 \mathrm{mg} / \mathrm{L} \\
0.004 \mathrm{mg} / \mathrm{L} \\
0.1 \mathrm{mg} / \mathrm{L} \\
0.005 \mathrm{mg} / \mathrm{L} \\
0.1 \mathrm{mg} / \mathrm{L} \\
0.01 \mathrm{mg} / \mathrm{L}\end{array}$ & $\begin{array}{l}-- \\
-- \\
-- \\
-- \\
-- \\
1 \mathrm{mg} / \mathrm{L} \\
-- \\
-- \\
-- \\
-- \\
5 \mathrm{mg} / \mathrm{L} \\
-- \\
-- \\
-- \\
-- \\
--\end{array}$ & $\begin{array}{l}12 \\
0 \\
0 \\
0 \\
0 \\
0 \\
07 \\
18 \\
0 \\
0 \\
0 \\
0 \\
0 \\
0 \\
0 \\
0 \\
0\end{array}$ & $\begin{array}{l}15 \\
0 \\
0 \\
0 \\
0 \\
0 \\
07 \\
18 \\
0 \\
0 \\
0 \\
0 \\
0 \\
0 \\
0 \\
0 \\
0\end{array}$ & $\begin{array}{l}45 \mathrm{JTU} \\
0.01 \mathrm{mg} / \mathrm{L} \\
<1 \mathrm{mg} / \mathrm{L} \\
<0.005 \mathrm{mg} / \mathrm{L} \\
<0.05 \mathrm{mg} / \mathrm{L} \\
<0.4 \mathrm{mg} / \mathrm{L} \\
<0.1 \mathrm{mg} / \mathrm{L} \\
<0.005 \mathrm{mg} / \mathrm{L} \\
<0.01 \mathrm{mg} / \mathrm{L} \\
<0.04 \mathrm{mg} / \mathrm{L} \\
<0.5 \mathrm{mg} / \mathrm{L} \\
<0.00005 \mathrm{mg} / \mathrm{L} \\
<0.00005 \mathrm{mg} / \mathrm{L} \\
<0.05 \mathrm{mg} / \mathrm{L} \\
<0.005 \mathrm{mg} / \mathrm{L} \\
<0.001 \mathrm{mg} / \mathrm{L} \\
<0.0001 \mathrm{mg} / \mathrm{L}\end{array}$ \\
\hline
\end{tabular}

1 The method of reporting each constituent is according to standard U.S. Geological Survey practice and is shown in the headings of table 12. Endrin, lindane, metoxychlor, toxaphene, 2,4-D, and 2,4,5-TP silvex are not included in table 12. These constituents are reported as concentration in "whole sample."

2 National Interm Primary Drinking Water Regulations (U.S. Environmental Protection Agency, 1975). Primary regulations are those which deal with constituents that may affect the health of consumers.

3 National Proposed Secondary Water Regulations (U.S. Environmental Protection Agency, 1977). Secondary regulations are those which deal with the esthetic qualities of drinking water. These are guidelines only.

4 The maximum contaminant level (MCL) for fluoride is dependent upon the annual average of the maximum daily air temperatures for the location in which the water-supply system is situated; from $1.4 \mathrm{mg} / \mathrm{L}$ $\left(26.3\right.$ to $\left.32.5^{\circ} \mathrm{C}\right)$ to $2.4 \mathrm{mg} / \mathrm{L}\left(\leq 12.0^{\circ} \mathrm{C}\right)$. A figure of $2.0 \mathrm{mg} / \mathrm{L}$ was selected for use in this table.

5 Sixteen samples from 12 sites had specific conductances greater than 1,000 micromhos, but were not tested for dissolved solids. All of these samples probably had concentrations exceeding $500 \mathrm{mg} / \mathrm{L}$.

6 Although the MCL for turbidity applies only to surface water, the relatively high turbidities in some ground water in the area warrant inclusion of turbidity in this summary. The MCL is 1 JTU, but a maximum of 5 JTU's may be allowed. A total of 13 samples had turbidities from 1 to 5 JTU's. Only those samples which exceeded $5 \mathrm{JTU}$ 's are included in this summary as exceeding the MCL.

7 Two samples were tested only to an accuracy of $0.1 \mathrm{mg} / \mathrm{L}$. It is therefore uncertain if these samples exceeded the MCL or not.

8 One sample was tested only to an accuracy of $0.005 \mathrm{mg} / \mathrm{L}$. It is therefore uncertain if this sample exceeded the MCL or not.

9 Wells $30 / 9-3001(6 / 22 / 78)$ and $29 / 9-3201$ and $F 1(6 / 26 / 768)$ were the only wells sampled. 
TABLE 4.--Summary of selected surface-water-quality data in Clallam County

\begin{tabular}{|c|c|c|c|c|c|c|c|}
\hline Const ituent $^{1}$ & $\begin{array}{l}\text { Number } \\
\text { of } \\
\text { sites } \\
\text { tested }\end{array}$ & $\begin{array}{l}\text { Number } \\
\text { of } \\
\text { samples } \\
\text { tested } \\
\end{array}$ & $\begin{array}{l}\text { Chemical stand } \\
\text { Maximum con- }{ }^{2} \\
\text { taminant level } \\
\text { (EPA, 1975) }\end{array}$ & $\begin{array}{ll}\text { dards } & \text { N } \\
\text { Proposed } & 0 \\
\text { secondary } & \text { e } \\
\text { level }{ }^{3} & \\
(E P A, 1977) & 5 \\
\end{array}$ & $\begin{array}{l}\text { Number } \\
\text { of sites } \\
\text { exceeding } \\
\text { chemical } \\
\text { standard }\end{array}$ & $\begin{array}{l}\text { Number of } \\
\text { samples } \\
\text { exceeding } \\
\text { chemical } \\
\text { standard }\end{array}$ & $\begin{array}{l}\text { Maximum } \\
\text { value } \\
\text { observed }\end{array}$ \\
\hline $\begin{array}{l}\text { Iron } \\
\text { Manganese } \\
\text { Sulfate } \\
\text { Chloride } \\
\text { Fluoride } 5 \\
\text { Nitrate } \\
\text { Dissolved solids } \\
\text { pH } \\
\text { Color }\end{array}$ & $\begin{array}{r}11 \\
9 \\
28 \\
28 \\
17 \\
103 \\
9 \\
26 \\
79\end{array}$ & $\begin{array}{r}104 \\
50 \\
246 \\
269 \\
201 \\
495 \\
157 \\
353 \\
304\end{array}$ & $\begin{array}{l}-- \\
-- \\
-- \\
-- \\
2.0 \mathrm{mg} / \mathrm{L} \\
10 \mathrm{mg} / \mathrm{L} \\
-- \\
-- \\
--\end{array}$ & $\begin{array}{l}0.3 \mathrm{mg} / \mathrm{L} \\
0.05 \mathrm{mg} / \mathrm{L} \\
250 \mathrm{mg} / \mathrm{L} \\
250 \mathrm{mg} / \mathrm{L} \\
-- \\
-- \\
500 \mathrm{mg} / \mathrm{L} \\
<6.5 \text { or }>8.5 \\
15 \text { plat inum- } \\
\text { cobalt un its }\end{array}$ & $\begin{array}{r}4 \\
2 \\
0 \\
0 \\
0 \\
0 \\
0 \\
8 \\
31\end{array}$ & $\begin{array}{r}15 \\
3 \\
0 \\
0 \\
0 \\
0 \\
0 \\
10 \\
86\end{array}$ & $\begin{array}{l}2.2 \mathrm{mg} / \mathrm{L} \\
0.21 \mathrm{mg} / \mathrm{L} \\
56 \mathrm{mg} / \mathrm{L} \\
97 \mathrm{mg} / \mathrm{L} \\
0.6 \mathrm{mg} / \mathrm{L} \\
2.2 \mathrm{mg} / \mathrm{L} \\
170 \mathrm{mg} / \mathrm{L} \\
6.1 \text { to } 8.4 \\
280 \text { units }\end{array}$ \\
\hline $\begin{array}{l}\text { Turbidity } 6 \\
\text { Colif orm bacteria } 7\end{array}$ & $\begin{array}{l}93 \\
93\end{array}$ & $\begin{array}{l}674 \\
598\end{array}$ & $\begin{array}{l}1 \text { to } 5 \mathrm{JTU} \\
1 \text { to } 4 \\
\text { col } / 100 \mathrm{~mL}\end{array}$ & -- & $\begin{array}{l}16 \\
91\end{array}$ & $\begin{array}{r}79 \\
524\end{array}$ & $\begin{array}{l}90 \mathrm{JTU} \\
5,400 \mathrm{col} / 10 \mathrm{dmL}\end{array}$ \\
\hline $\begin{array}{l}\text { Arsenic } \\
\text { Barium } \\
\text { Cadmi um } \\
\text { Chromium } \\
\text { Copper } \\
\text { Lead } \\
\text { Mercury } \\
\text { Selenium } \\
\text { Silver } \\
\text { Zinc } \\
\text { Endrin } \\
\text { Lindane } \\
\text { Methoxych lor } \\
\text { Toxaphene } \\
\text { 2,4-0 } \\
\text { 2,4,5-TP silvex }\end{array}$ & $\begin{array}{r}7 \\
4 \\
4 \\
14 \\
12 \\
10 \\
7 \\
4 \\
4 \\
12 \\
1 \\
1 \\
1 \\
1 \\
1 \\
1\end{array}$ & $\begin{array}{l}65 \\
14 \\
26 \\
77 \\
78 \\
27 \\
29 \\
26 \\
14 \\
68 \\
16 \\
16 \\
16 \\
16 \\
11 \\
11\end{array}$ & $\begin{array}{l}0.05 \mathrm{mg} / \mathrm{L} \\
1 \mathrm{mg} / \mathrm{L} \\
0.010 \mathrm{mg} / \mathrm{L} \\
0.05 \mathrm{mg} / \mathrm{L} \\
-- \\
0.05 \mathrm{mg} / \mathrm{L} \\
0.002 \mathrm{mg} / \mathrm{L} \\
0.01 \mathrm{mg} / \mathrm{L} \\
0.05 \mathrm{mg} / \mathrm{L} \\
-- \\
0.0002 \mathrm{mg} / \mathrm{L} \\
0.004 \mathrm{mg} / \mathrm{L} \\
0.1 \mathrm{mg} / \mathrm{L} \\
0.005 \mathrm{mg} / \mathrm{L} \\
0.1 \mathrm{mg} / \mathrm{L} \\
0.01 \mathrm{mg} / \mathrm{L}\end{array}$ & $\begin{array}{l}-- \\
-- \\
-- \\
-- \\
1 \mathrm{mg} / \mathrm{L} \\
-- \\
-- \\
-- \\
-- \\
5 \mathrm{mg} / \mathrm{L} \\
-- \\
-- \\
-- \\
-- \\
-- \\
--\end{array}$ & $\begin{array}{l}0 \\
0 \\
0 \\
0 \\
0 \\
0 \\
0 \\
0 \\
0 \\
0 \\
0 \\
0 \\
0 \\
0 \\
0 \\
0\end{array}$ & $\begin{array}{l}0 \\
0 \\
0 \\
0 \\
0 \\
0 \\
0 \\
0 \\
0 \\
0 \\
0 \\
0 \\
0 \\
0 \\
0 \\
0\end{array}$ & $\begin{array}{l}0.01 \mathrm{mg} / \mathrm{L} \\
<0.25 \mathrm{mg} / \mathrm{L} \\
<0.003 \mathrm{mg} / \mathrm{L} \\
0.04 \mathrm{mg} / \mathrm{L} \\
0.09 \mathrm{mg} / \mathrm{L} \\
0.011 \mathrm{mg} / \mathrm{L} \\
<0.001 \mathrm{mg} / \mathrm{L} \\
<0.003 \mathrm{mg} / \mathrm{L} \\
<0.01 \mathrm{mg} / \mathrm{L} \\
0.05 \mathrm{mg} / \mathrm{L} \\
N D \\
N D \\
N D \\
N D \\
N D \\
N D\end{array}$ \\
\hline
\end{tabular}

1The method of reporting each constituent is according to standard U.S. Geological Survey practice and is shown in the headings of table

2National Interm Primary Drinking Water Regulations (U.S. Environmental Protection Agency, 1975). Primary regulations are those which deal with constituents that may affect the health of consumers.

3National Proposed Secondary Water Regulations (U.S. Environmental Protection Agency, 1977). Secondary regulations are those which deal with the esthetic qualities of drinking water. These are guidelines on ly.

4ND = none detected.

5The maximum contaminant level (MCL) for fluoride is dependent upon the annual average of the maximum daily air temperatures for the location in which the water-supply system is situated; from $1.4 \mathrm{mg} / \mathrm{L}\left(26.3\right.$ to $\left.32.5^{\circ} \mathrm{C}\right)$ to $2.4 \mathrm{mg} / \mathrm{L}\left(\leq 12.0^{\circ} \mathrm{C}\right)$. A figure of $2.0 \mathrm{mg} / \mathrm{L}$ was selected for use in this table.

6The MCL for turbidity is 1 JT, but a maximum of 5 JTU's may be allowed. A total of 71 samples had turbidities from 2 to 5 JTU's. Only those samples which exceeded 5 JTU's are included in this summary as exceeding the MCL.

7The MCL for coliform bacteria ranges from 1 to 4 col/100 $\mathrm{mL}$ depending on the number and type of tests performed. A total of 11 samples had coliform counts from 1 to 4 col/100 mL. Only those samples which exceeded 4 col/100 $\mathrm{mL}$ are included in this sumary as exceeding the $\mathrm{MCL}$. 


\section{WATER USE}

Water-use data sources were: (1) U.S. Geological Survey records collected for an earlier report (Dion and Lum, 1977), and (2) data collected from managers of water systems during field visits in 1979.

Water use in the county is dominated by industrial users in the Port Angeles area and irrigators in the Sequim area. Total estimated use in 1978 was about 39 billion gallons per year. About 18 billion was used by industries, 18 billion was used for irrigation, and 3 billion was used for domestic purposes (drinking and cleaning water and watering of lawns and gardens). Other uses, including recreation, commercial, and stock use, are relatively insignificant. Most of the water used, 38 billion gallons, was obtained from surface-water sources (about one-half was from collector wells along major rivers). Table 5 shows the distribution of water use in the county in 1978.

The water-use data in table 5 show greater usage in the winter of 1978 than may be typical in most years. The monthly figures were strongly influenced by industrial water use in the Port Angeles Water System and may not be representative of long-term conditions. Total domestic use displays a more reasonable pattern of seasonal use, with average monthly use of 200 million gallons during the period October-March and 310 million gallons during the period April-September.

The available well records (about 2,100 wells) indicate that about 87 percent of the wells in the county are used as domestic supplies (serving less than five homes per well), about 6 percent are unused (this includes wells that have been destroyed) and about 4 percent are public-supply wells. The remaining 3 percent are used for irrigation, recreation, industry, stock supply, commerce, and aquiculture. Some of the domestic wells (5-1 0 percent) are also used for stock supply and irrigation. The total number of wells in the county is probably about 4,000 . 


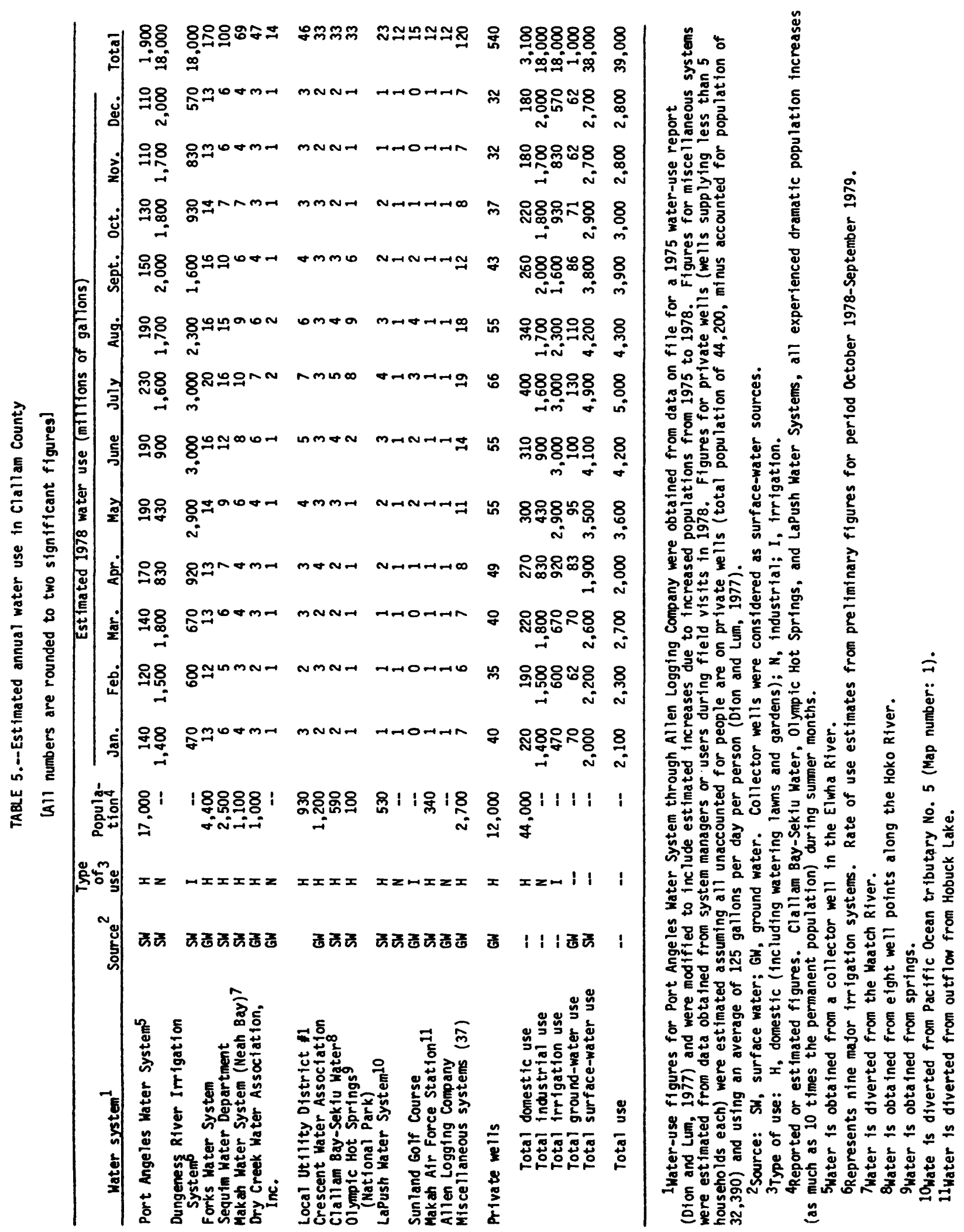




\section{WATER-RESOURCE PROBLEM AREAS AND \\ RECOMMENDATIONS FOR FUTURE STUDIES}

The water resources of the county have undergone (as of 1980) relatively little development. For this reason, few problems can be identified in the county; however, potential problems have been observed that may warrant further study.

Adequate ground water for individual domestic use is available in almost all of the developed areas. In some locations, where thin unconsolidated deposits overlie bedrock (primarily shales), attempts to install individual domestic wells have been unsuccessful. In most of these cases, two or three wells were drilled before an adequate yield was obtained. The successful wells occur when saturated fractures in the bedrock are intercepted. Areas where unsuccessful wells are relatively common include: (1) the foothills southeast of Port Angeles and 3-4 mi. inland (pl. 2); (2) the shoreline of Lake Crescent (pl. 2); (3) the Soleduck River valley from Forks to areas east of Sappho; and (4) the southwest shoreline of Sequim Bay (pl. 1). Very few wells have been drilled in the mountainous interior of the study area, but the available data suggest that there may be large areas of inadequate ground-water to supply even single domestic needs. A network of observation wells in the areas of relatively common unsuccessful wells could reveal any potential water-shortage problems before the occurrence of serious shortages.

Although most of the water presently (1978) used is from surface-water sources, the use of surface water is restricted to a few locations in the study area. About six major systems (table 5) supply essentially all the surface water used. The huge amount of precipitation that falls on most of the study area results in substantial surface-water flows. Although there are no supply problems, there are many areas where streamflows (table 2) sometimes are very small or dry up completely and therefore are less suitable for use. Identified areas with extremely low flows include tributaries in (1) the upper Soleduck River basin (pl. 3); (2) the Crescent Lake area (pl. 2); (3) the southwest side of Sequim Bay (pl. 1); (4) the Strait of Juan de Fuca area from Neah Bay to Olsen Creek and from the Lyre River to Port Angeles (pl. 2); and (5) the Hoko River area (pl. 4). Most of the streams in the areas of extreme low flows have small drainage basins, high relief, and are underlain by low permeable bedrock. Most of the small basins in the mountainous interior have not been gaged and the majority of them probably have periods of very little or no flow.

A few problems of poor ground-water quality are known to exist in the county. The most serious problem is salty (chloride concentrations in excess of $250 \mathrm{mg} / \mathrm{L}$ ) ground water in a few locations: (1) The Neah Bay area; (2) the shoreline from the southwest side of Sequim Bay to the northeast part of the Miller Peninsula (particularly the Diamond Point Area) (pl. 1); and (3) the lower end of the Hoko River valley (pl. 4). The salty ground water is encountered in wells that have been drilled into the freshwater-saltwater zone of diffusion. Saltwater intrusion produced by pumping has apparently not been a problem. Pumping of ground water from the freshwater-saltwater zone of diffusion may result in saltwater encroachment. This could lead to increased deterioration of water quality in existing wells and enlargment of the problem areas. Chloride concentrations should 
be monitored in a network of wells along the coastline of the Miller Peninsula to observe any water-quality changes that otherwise might go undetected until a serious saltwater encroachment problem arose. A detailed ground-water study would be needed to assess the potential impact of increased stresses on the ground-water system of the Miller Peninsula.

Large concentrations of iron and (or) manganese are a widespread ground-water problem in the county. The problem is greatest near LaPush, Forks, and the lower end of the Hoko River valley, where associated problems of excessive color and turbidity are also found.

Very few problems of poor surface-water quality have been observed. Excessive color, turbidity, and coliform bacteria are the only widespread problems, and each of these can be treated by standard filter and chlorination processes used in most public-supply systems.

One area of possible surface-water contamination has been observed (Fretwell, written commun., 1980). Samples taken from the Bogachiel River near Forks (site 35) in 1976 and 1977 had ammonia concentrations several times greater than those from samples taken at upstream or downstream sites. Fretwell concluded that the higher concentrations were due to inflow from Mill Creek, which drains a major part of the town of Forks. Much of the sewage from the town is discharged into gravel shafts drilled 50-70 ft deep. Ground water contaminated by this effluent, discharges into Mill Creek and combines with surface runoff. The available data indicate that no serious problem presently exists. Nevertheless, increased effluent discharge could very likely cause increases of ammonia, nitrate, and fecal-coliform bacteria concentrations. Such water-quality changes could be detected early by systematically collecting and analyzing ground- and surface-water samples. 


\section{REFERENCES CITED}

Bortleson, G. C., and Dion, N. P., 1979, Preferred and observed conditions for sockeye salmon in Ozette Lake and its tributaries, Clallam County, Washington: U.S. Geological Survey Water-Resources Investigations Open-File Report 78-64, 61 p.

Bortleson, G. C., Dion, N. P., McConne11, J. B., and Nelson, L. M., 1976, Reconnaissance data on lakes in Washington, volume 1, Clallam, Island, Jefferson, San Juan, Skagit, and Whatcom Counties: State of Washington Department of Ecology Water-Supply Bulletin 43, v. 1, 248 p.

$\mathrm{CH}_{2} \mathrm{M}$ (Cornell, Howland, Hayes, and Merryfield), 1967, A report on the water system at LaPush, Washington: $\mathrm{CH}_{2} \mathrm{M}$, in cooperation with U.S. Department of the Interior, Bureau of Indian Affairs, Seattle, Washington, $38 \mathrm{p}$.

Cummans, J. E., Collings, M. R., and Nassar, E. G., 1975, Magnitude and frequency of floods in Washington: U.S. Geological Survey Open-File Report 74-336, 40 p.

Dion, N. P., 1979, Reconnaissance of hydraulic conditions of thermal springs at Sol Duc Hot Springs Resort, 0lympic National Park, Washington: U.S. Geological Survey Administrative Report, 20 p.

Dion, N. P., and Lum, W. E. II, 1977, Municipal, industrial, and irrigation water use in Washington, 1975: U.S. Geological Survey Open-File Report 77-308, $34 \mathrm{p}$.

Dion, N. P., Walters, K. L., and Nelson, L. M., 1980, Water resources of the Makah Indian Reservation, Washington: U.S. Geological Survey Water-Resources Investigations 80-15, $54 \mathrm{p}$.

Dion, N. P., and Sumioka, S. S., 1981, Seawater intrusion along coastal Washington, 1978: Washington Department of Ecology Water-Supply Bulletin p. (in review).

ENCON, 1974, North Olympic Coastal Basin, Water quality management plan and 303(e) addendum, Basin No. 13-11-09, WRIA 17, 18, 19, 20, 226 p.

Federal Insurance Administration, 1979, Flood insurance study, Clallam County, Washington, unincorporated areas: U.S. Department of Housing and Urban Development, $45 \mathrm{p}$.

- - 1979, Flood insurance study, town of Forks, Clallam County, Washington: U.S. Department of Housing and Urban Development, $15 \mathrm{p}$.

---1980 , Flood insurance study, city of Port Angeles, Clallam County, Washington: U.S. Federal Emergency Management Agency, 12 p. 
Haushild, W. L., and LaFrance, D. E., 1978, Low-flow characteristics of streams on the Olympic Peninsula, Washington: U.S. Geological Survey Open-File Report 77-812, 25 p.

McFarland, C. R., 1979, $0 i 1$ and gas exploration in Washington, 1900-1978: State of Washington Department of Natural Resources, Division of Geology and Earth Resources, Information Circular 67, 119 p.

State of Washington Department of Conservation, 1964, Miscellaneous streamflow measurements in the State of Washington, 1890 to January 1961: Water-Supply Bulletin 23, 292 p.

State of Washington, Office of Financial Management, 1978, State of Washington pocket data book, 1978: $274 \mathrm{p}$.

State of Washington, Office of Program Planning and Fiscal Management, 1972: State of Washington 1970 census data book: $496 \mathrm{p}$.

Tabor, R. W., and Cady, W. M., 1978, Geologic map of the Olympic Peninsula, Washington: U.S. Geological Survey, Miscellaneous Investigations Serles, Map I-994.

U.S. Environmental Protection Agency, 1975, National interim primary drinking water regulations: Federal Register, v. 40, no. 248, p. 59566-59588.

-- - 1977, National secondary drinking water regulations: Proposed regulations: Federal Register, v. 42, no. 62, p. 17143-17146.

U.S. Geological Survey, 1955, Compllation of records of surface waters of the United States through September 1950, Part 12: U.S. Geological Survey Water-Supply Paper 1316, 592 p.

- -1961, Ground-water conditions near Neah Bay, Washington; Administrative Report, $12 \mathrm{p}$.

---1961-79, Water resources data for Washington, Part 1, Surface water records, 1960-78: Tacoma, Washington, reports published annually for years indicated.

- - 1964, Compilation of records of surface water of the United States, October 1950 to September 1960, Part 12: U.S. Geological Survey Water-Supply Paper 1736, 415 p.

Van Denburgh, A. S., and Santos, J. F., 1965, Ground water in Washington, its chemical and physical quality: State of Washington Department of Conservation Water-Supply Bulletin 24, 93 p. 
Walters, K. L., 1967, Reconnaissance of Sol Duc Hot Springs, 01ympic National Park, Washington: U.S. Geological Survey, Administrative Report, 4 p.

---1970, Water supplies for selected sites in 0lympic National Park, Washington: U.S. Geological Survey Open-File Report, 72 p.

---1971, Reconnaissance of sea-water intrusion along coastal Washington, 1966-68: State of Washington Department of Ecology Water-Supply Bulletin 32, 208 p.

Walters, K. L., Haushild, W. L., and Nelson, L. M., 1979, Water resources of the lower Elwha Indian Reservation, Washington: U.S. Geological Survey Water-Resources Investigations Open-File Report 79-82, 55 p.

Wolcott, E. E., 1973, Lakes of Washington, Volume 1, Western Washington (3d ed.): State of Washington Department of Ecology Water-Supply Bulletin 14, 619 p. 
TABLE 6.--Records of selected wells and springs in Clallam County.

Use of water: C, commercial; $\mathrm{H}$, domestic; I, irrigation; $\mathrm{N}$, industrial ;

$P$, public; $Q$, agricul ture; $R$, recreation; $S$, stock; $U$, unused.

Depth of well: wells which have been destroyed are recorded at their last known depth.

Water level: Below land surface unless accompanied by a "+," which indicates above land surface. D, dry; F, flowing.

Finish: F, perforated and gravel packed; $H$, horizontal gallery; 0 , open end; $P$, perforated or slotted; $S$, screen; $T$, sand point; $W$, walled; $X$, open nole.

Specific capacity: 0.0 , less than 0.05 .

Data available (LG): G, driller's log on file with U.S. Geological Survey in Tacoma, Washington.

Data available (CK): C, location has been field checked; U, location not field checked. 


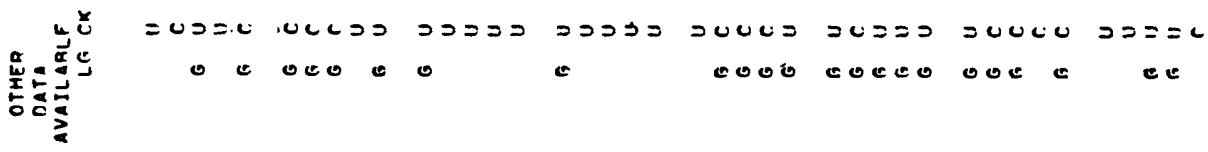

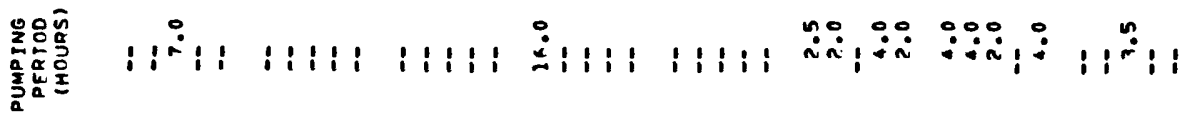

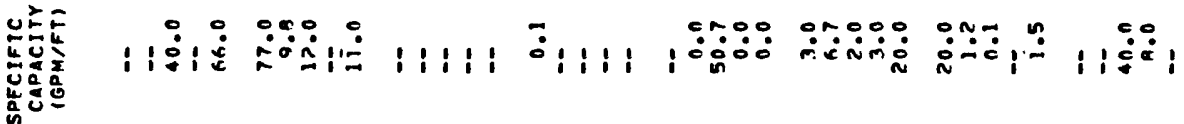

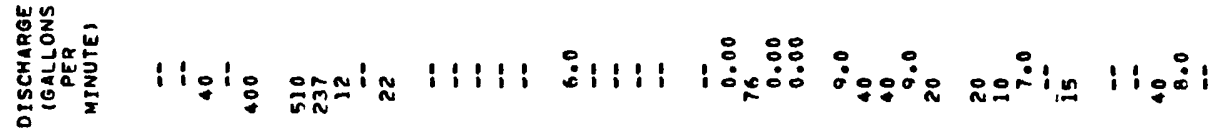

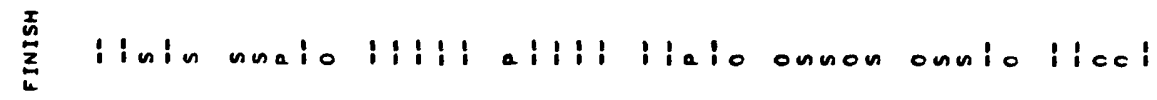

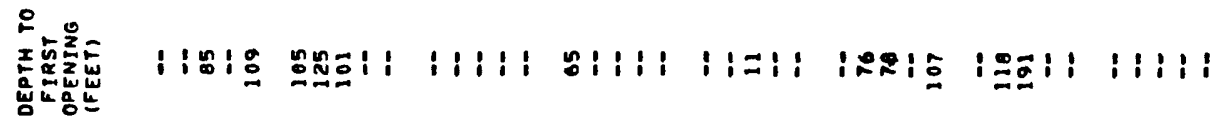
䉂

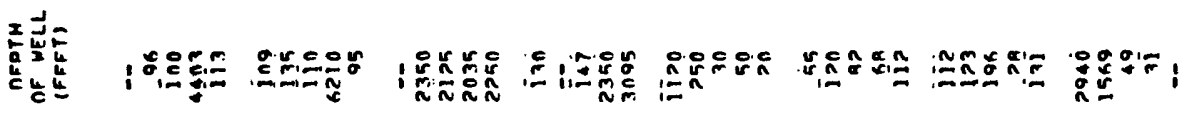

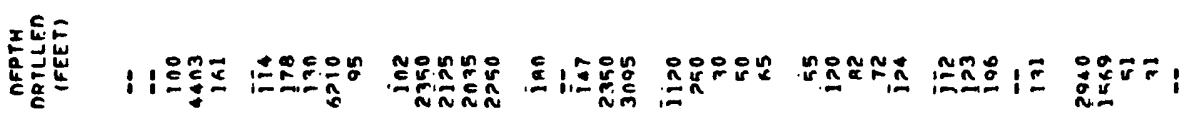

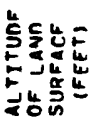

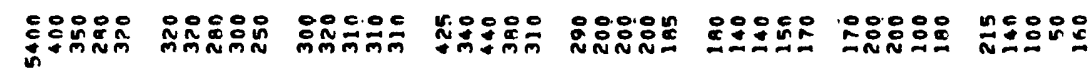

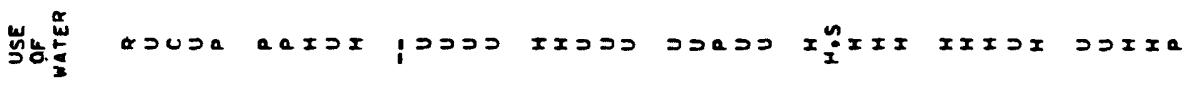
I
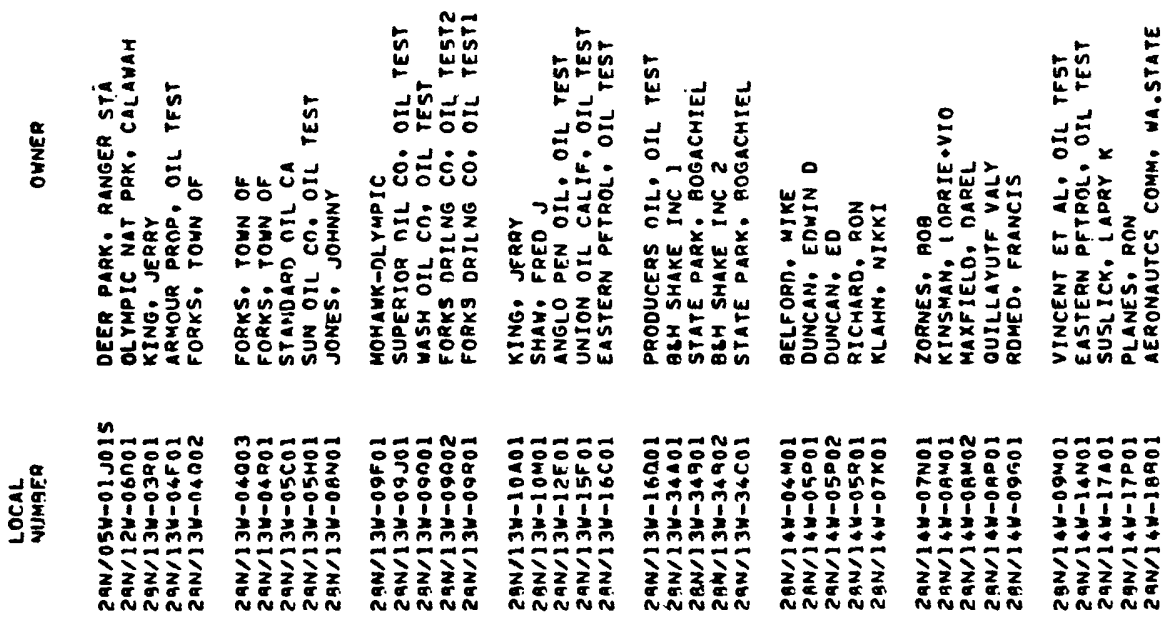


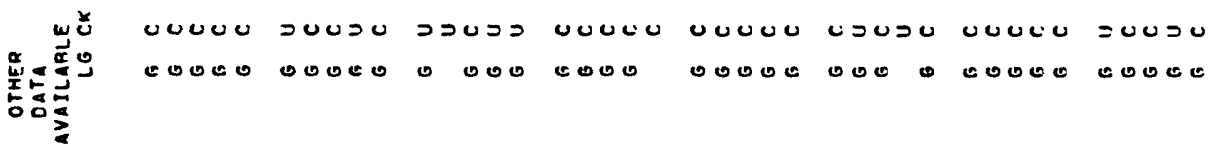

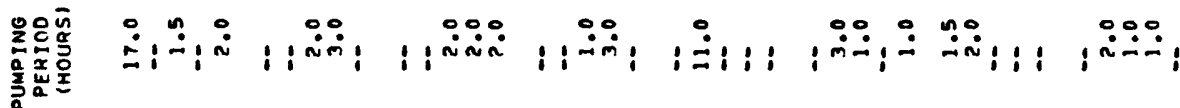

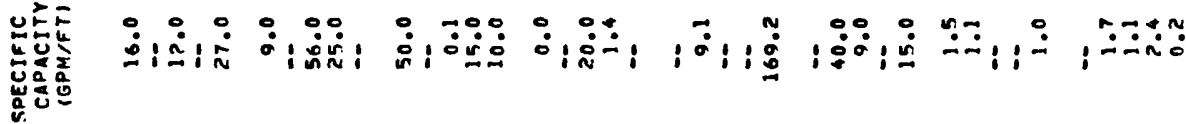

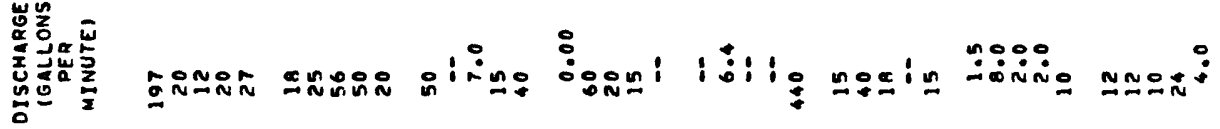

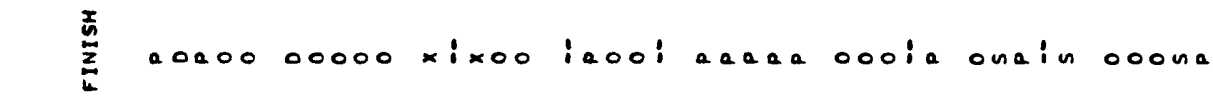
웅

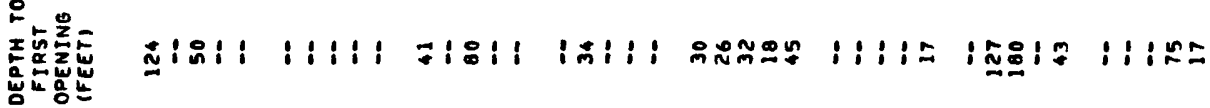

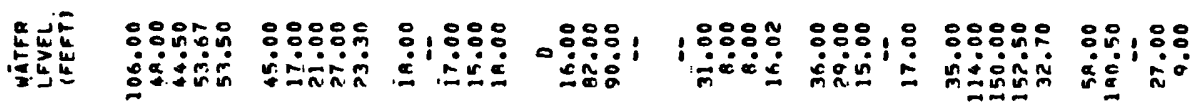

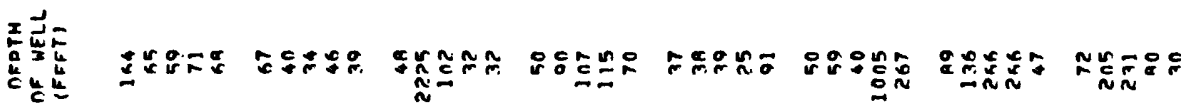

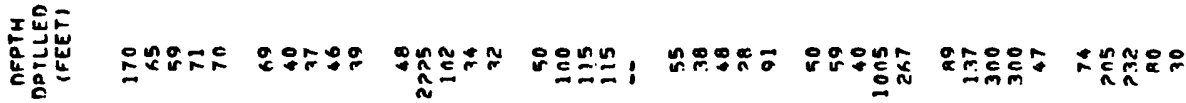

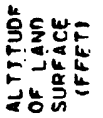

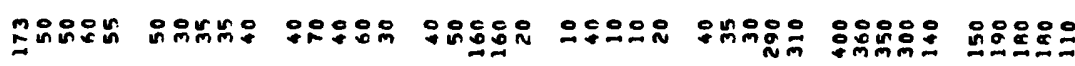
乌ัy

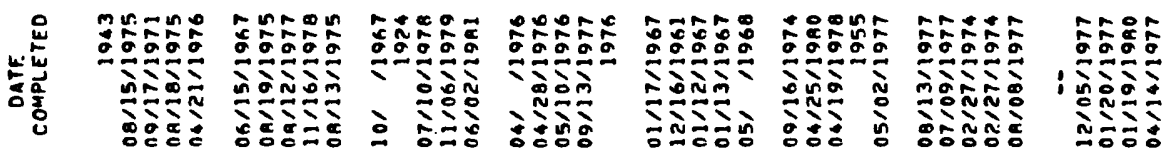

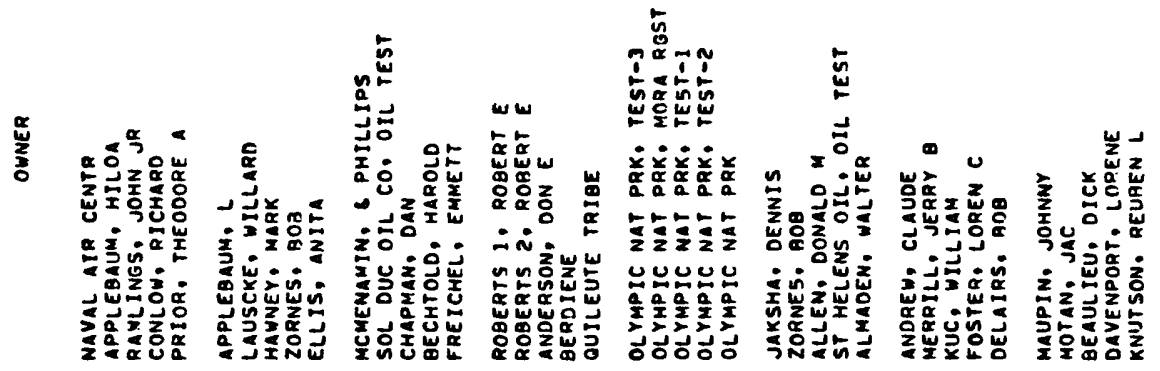

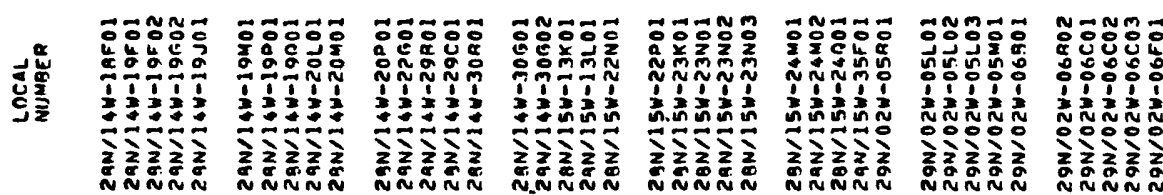




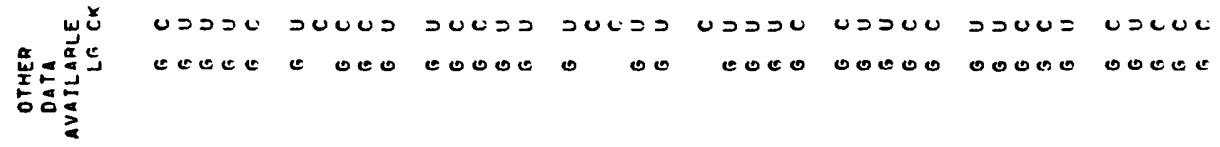
美最

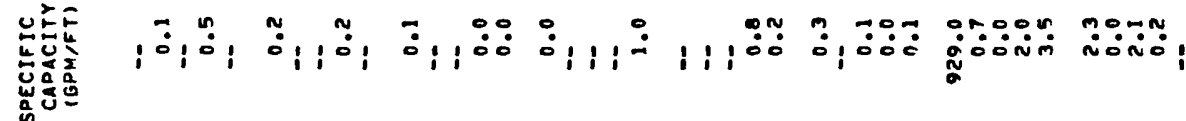

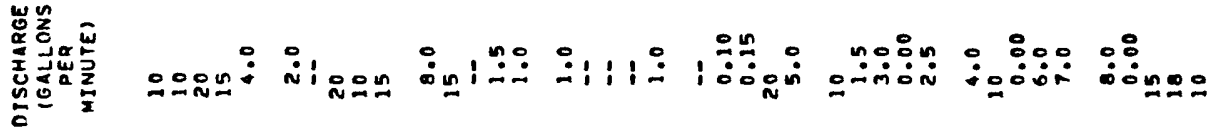

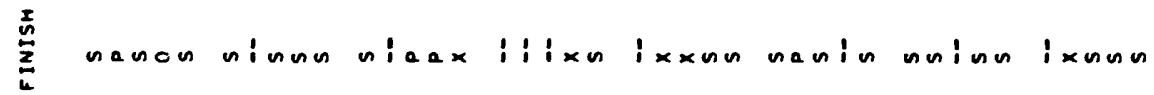

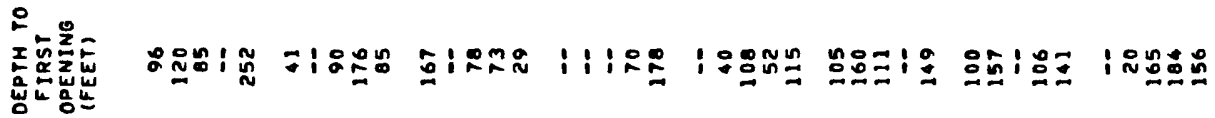

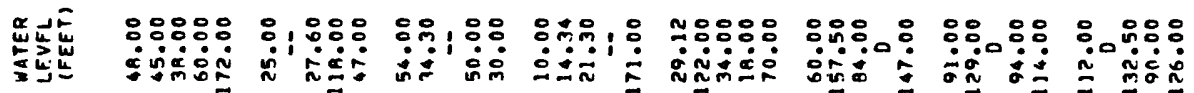

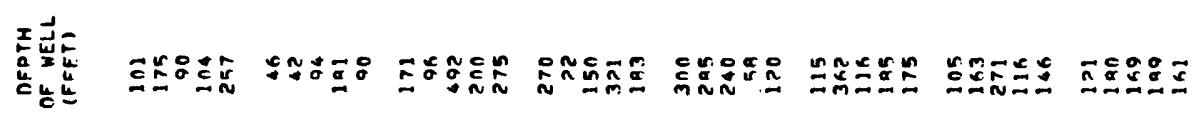

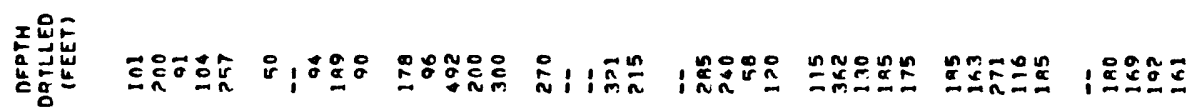

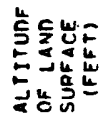

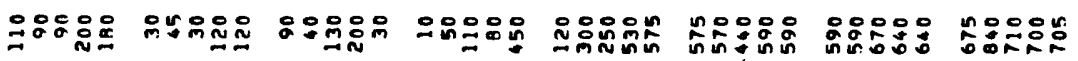

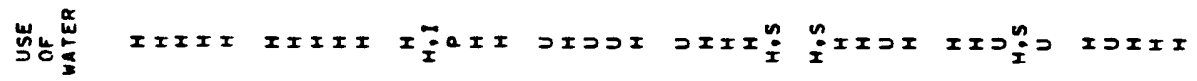

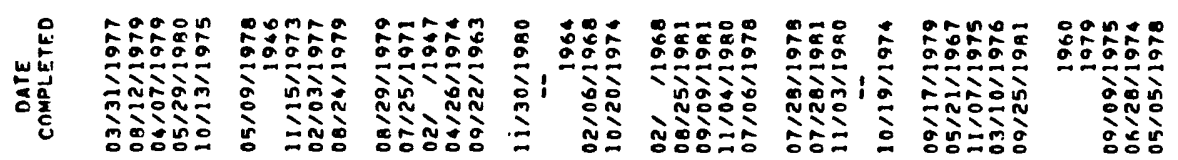
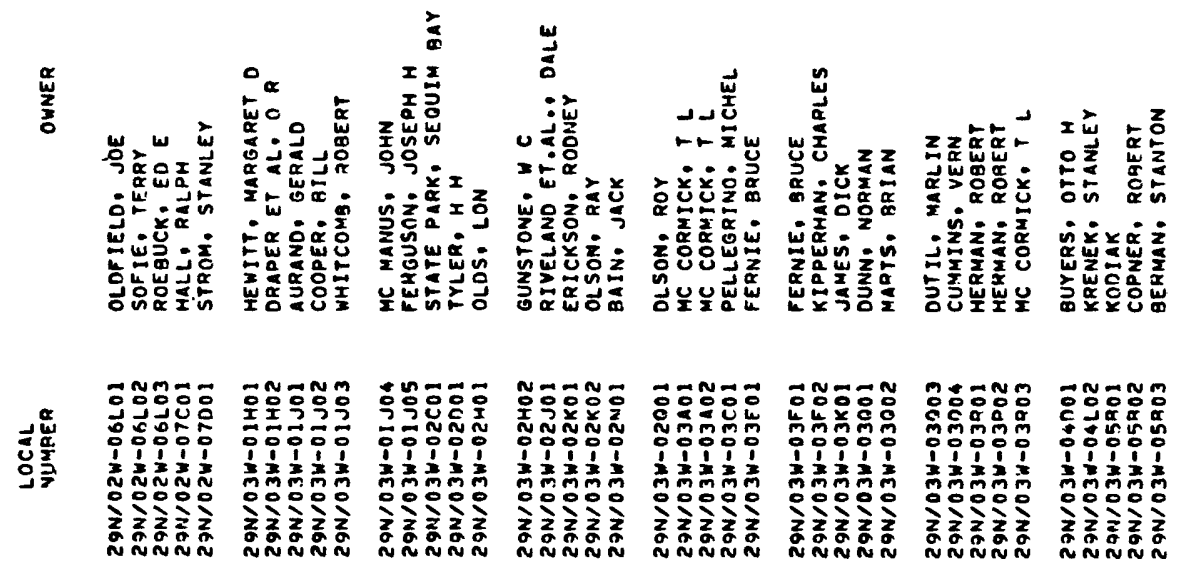


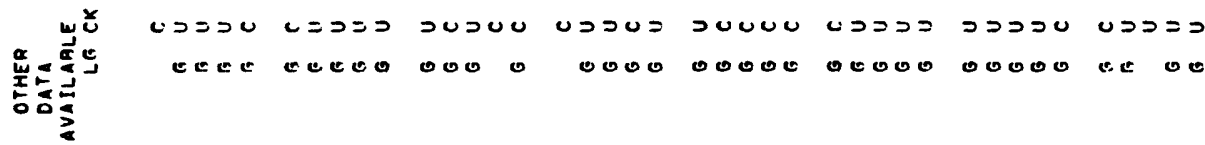

送最

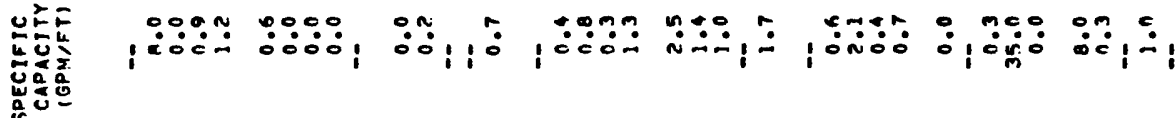

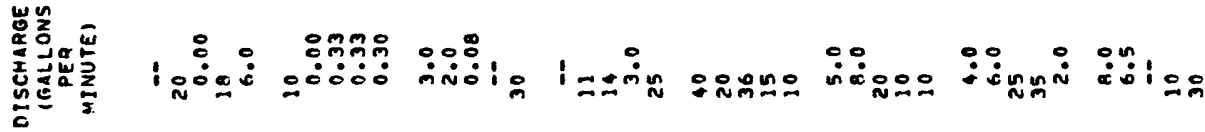

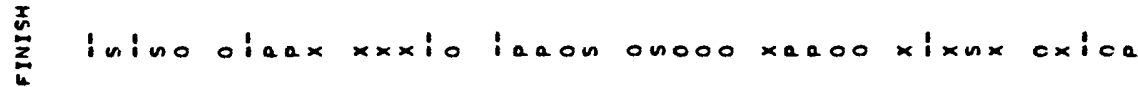

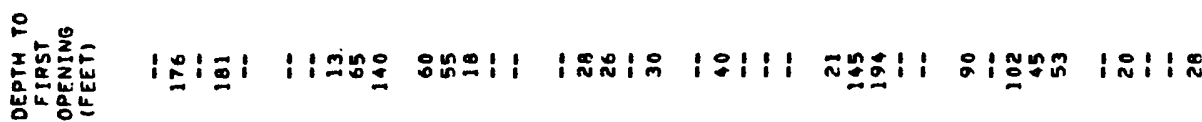
造

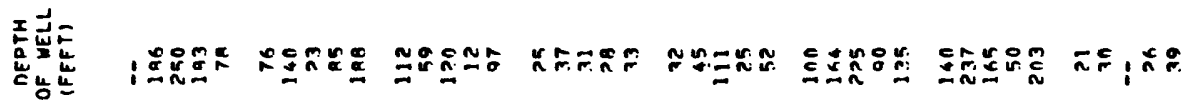

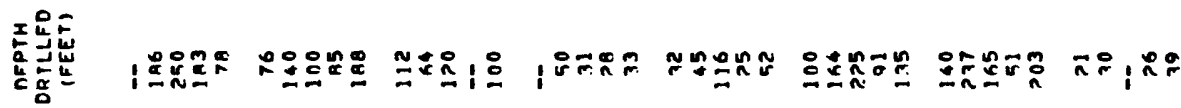

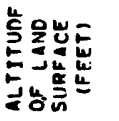

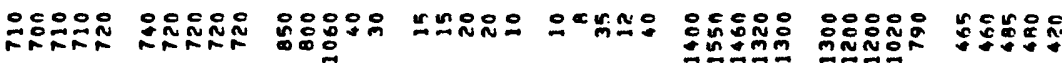

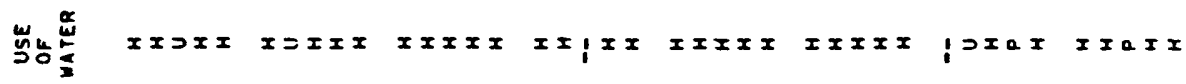
先
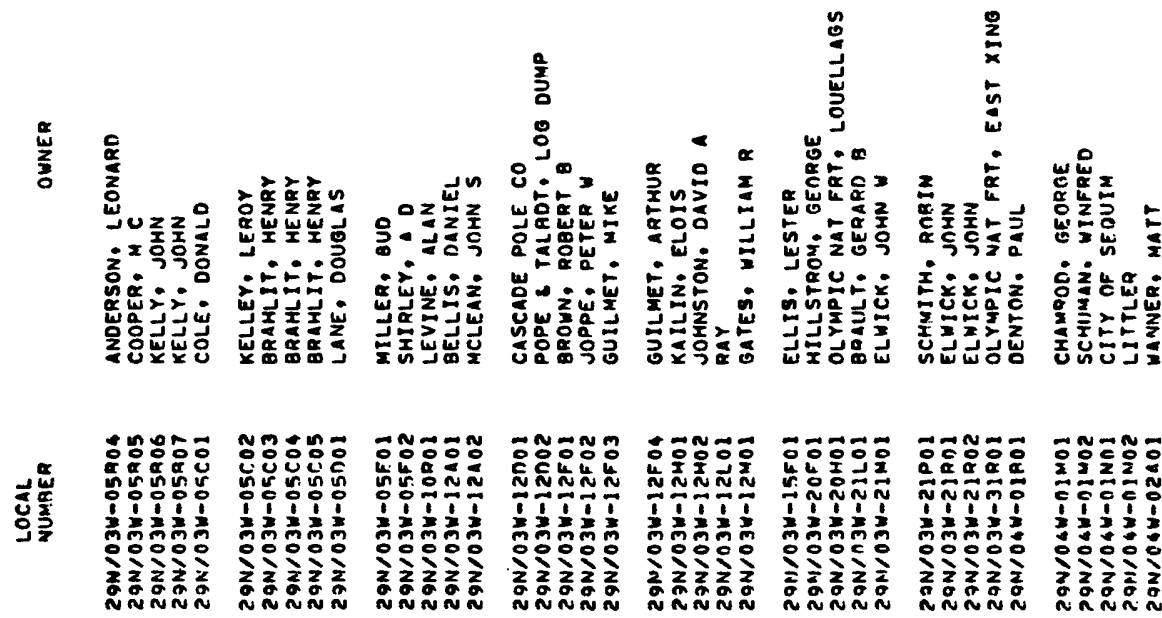


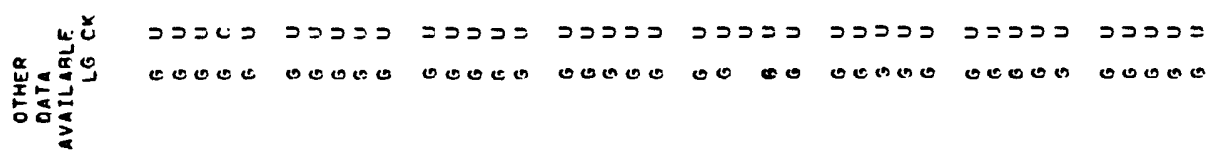

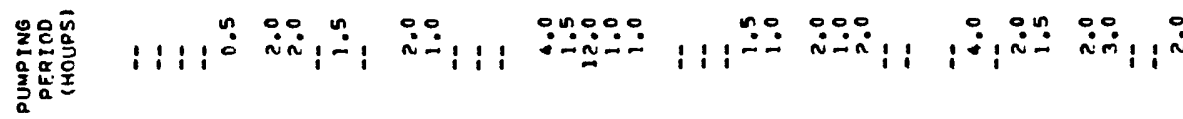

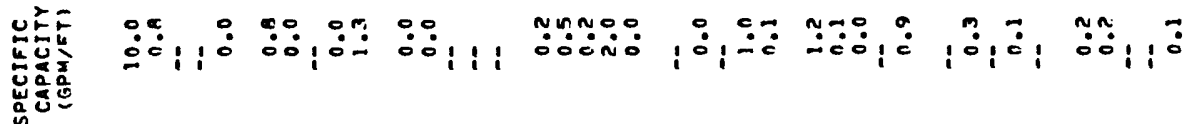

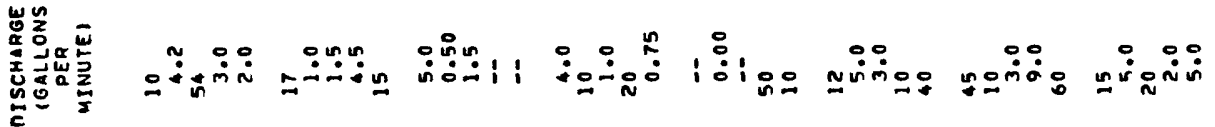
$\sum_{0}^{T}$ Daaa nxxaa xxaxx nuaxx $1: 1$ ixx nxxoo naoan anuxn 운

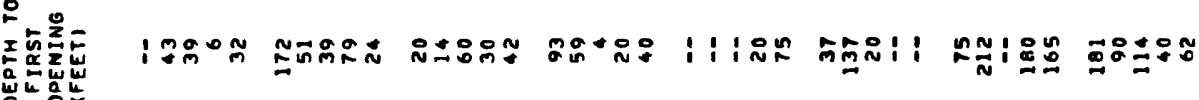
热

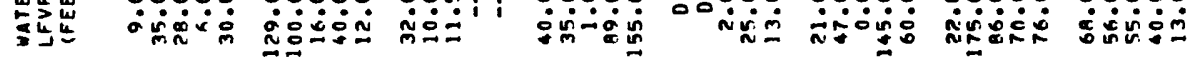

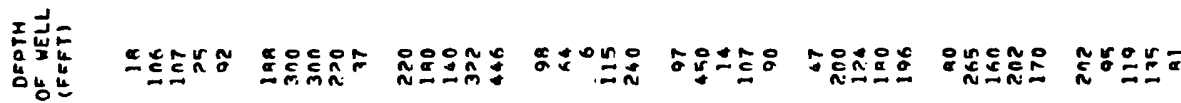

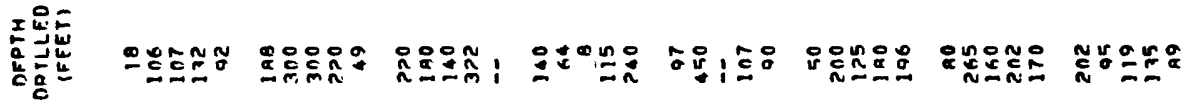

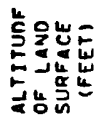

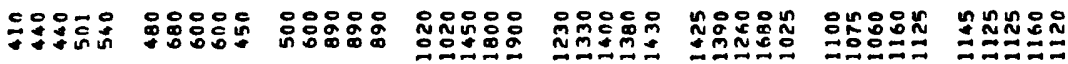

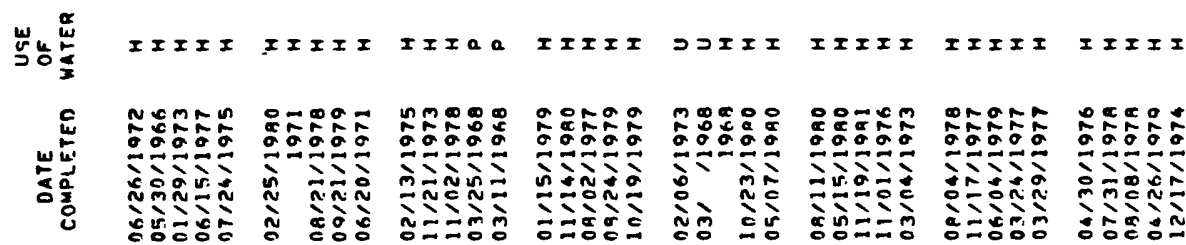
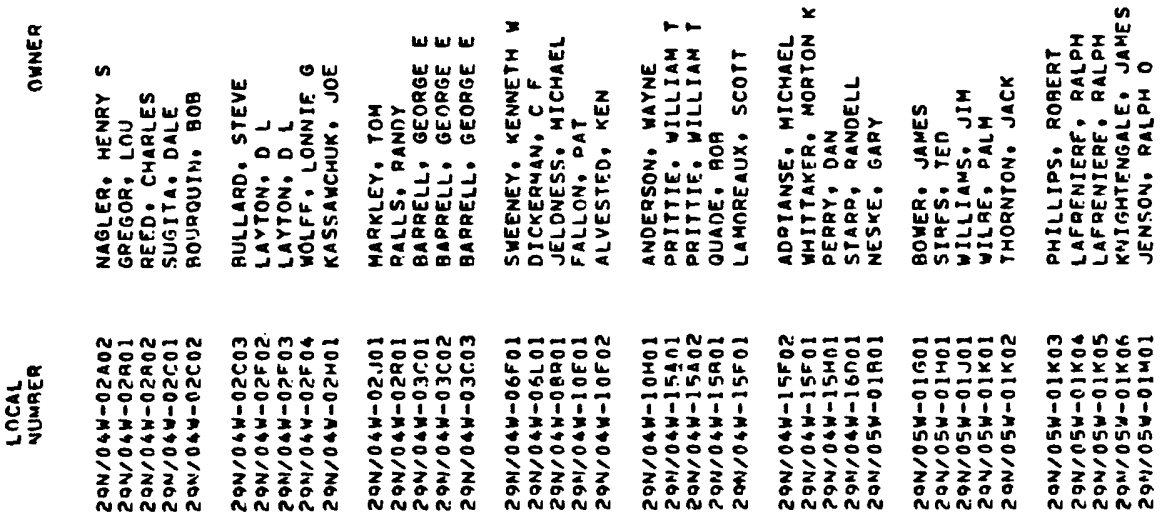


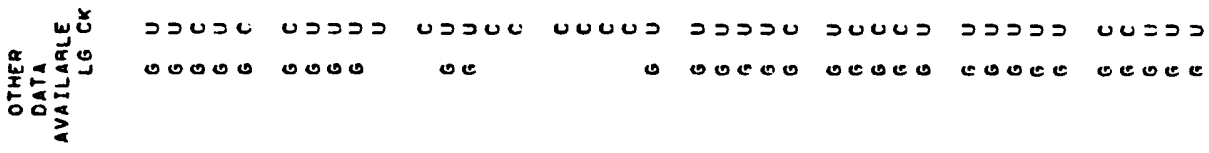

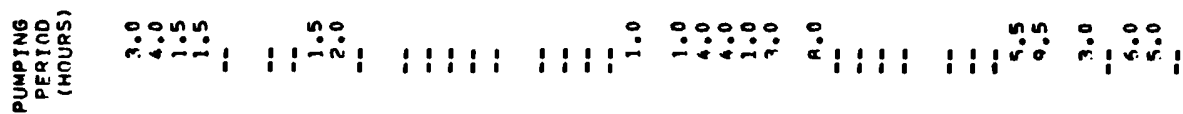

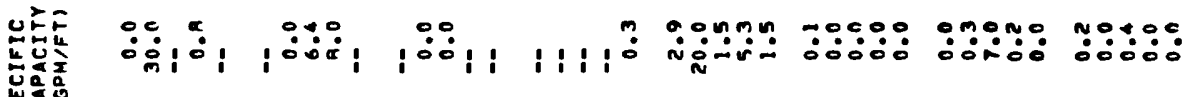

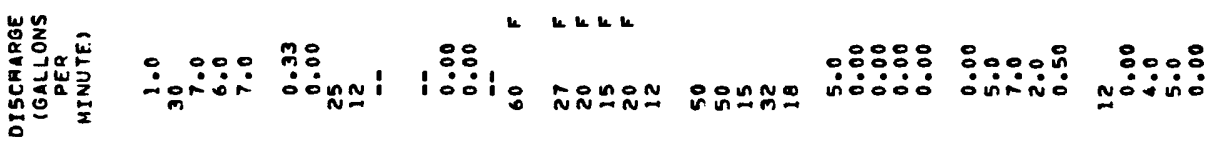

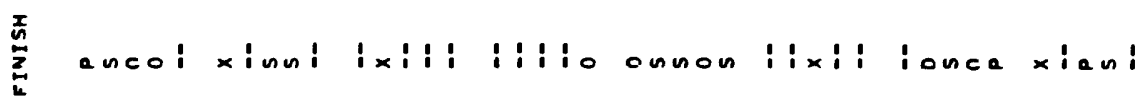

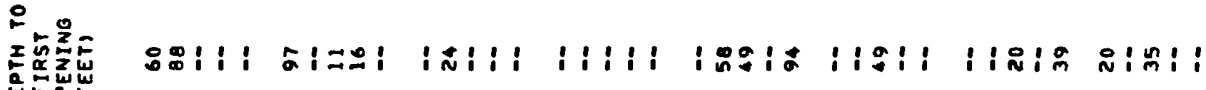
造

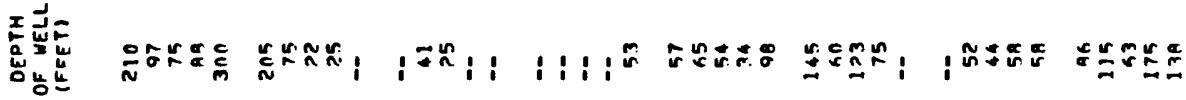

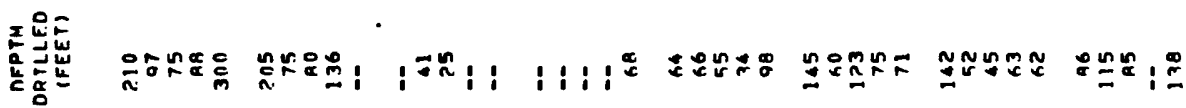

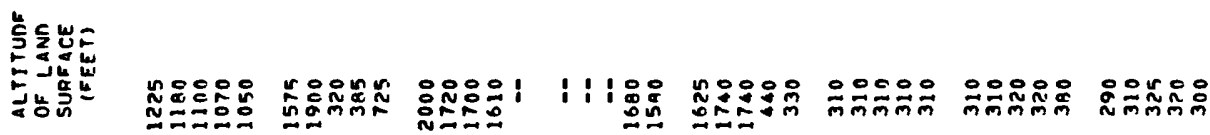

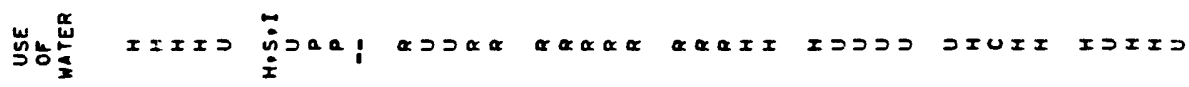

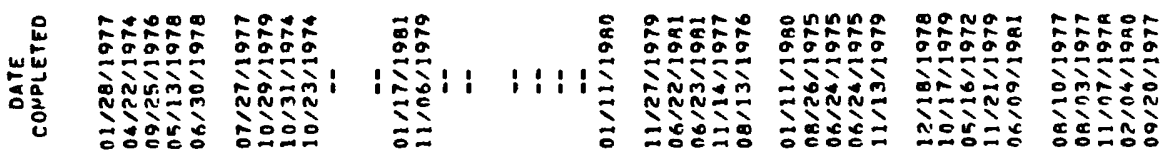




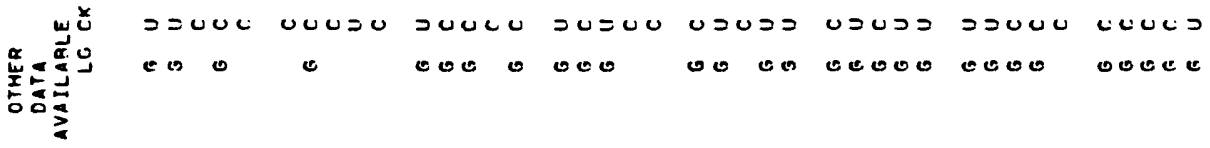
这高

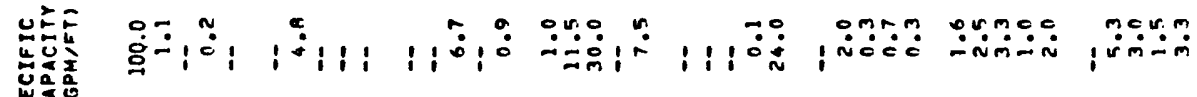

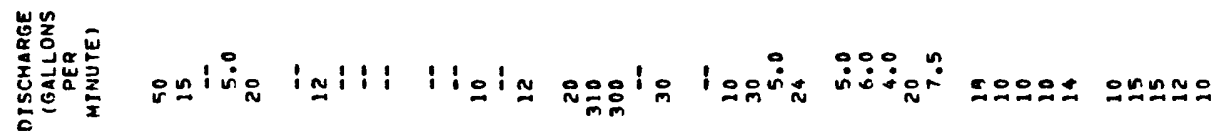

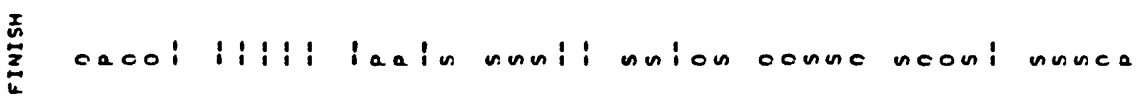

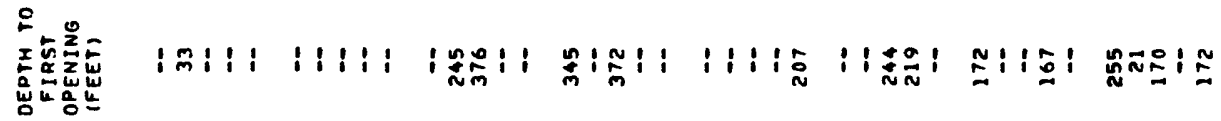

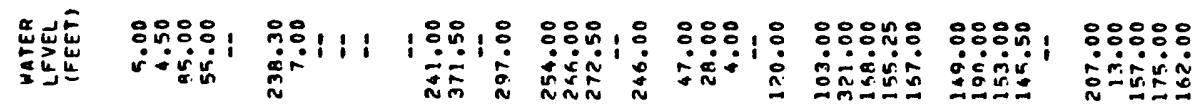

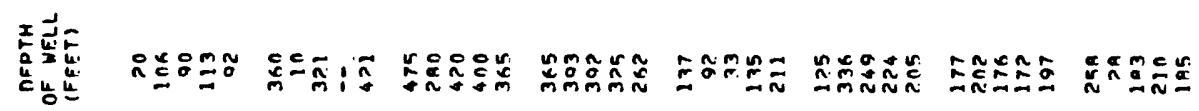

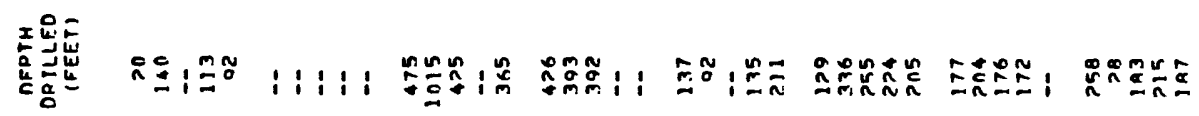

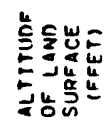

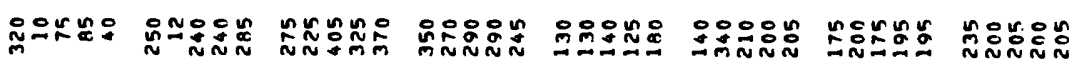

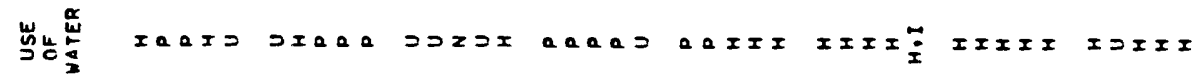

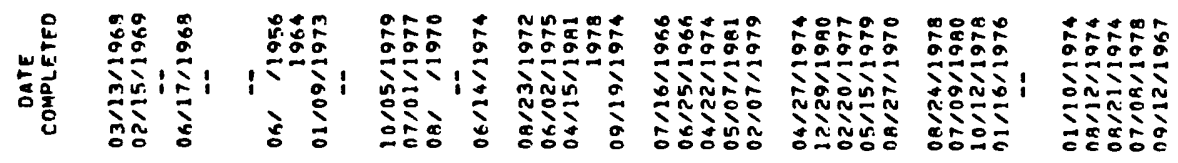
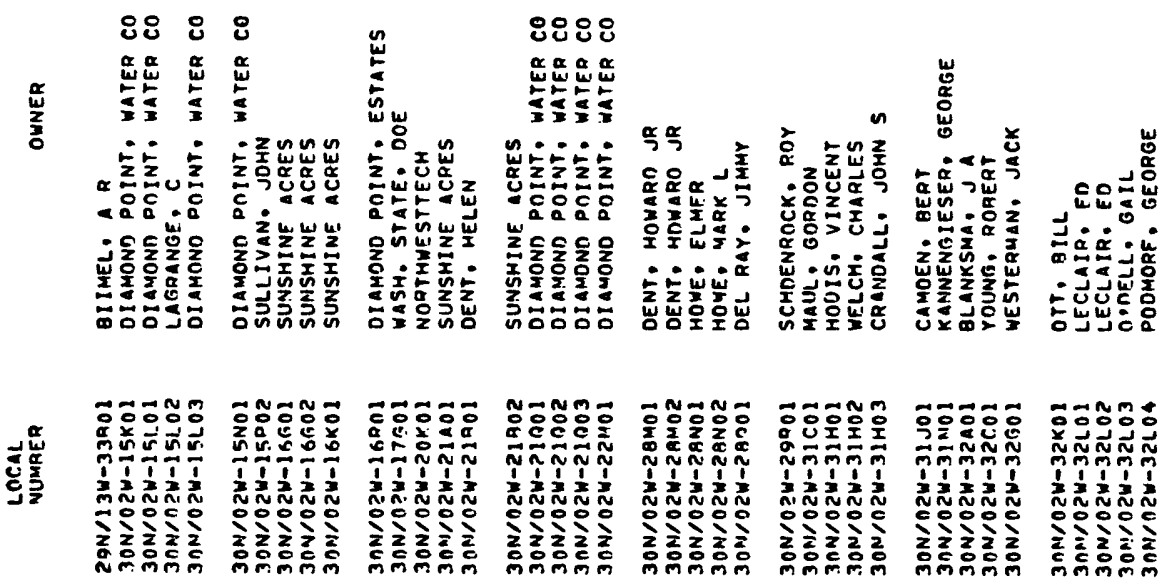


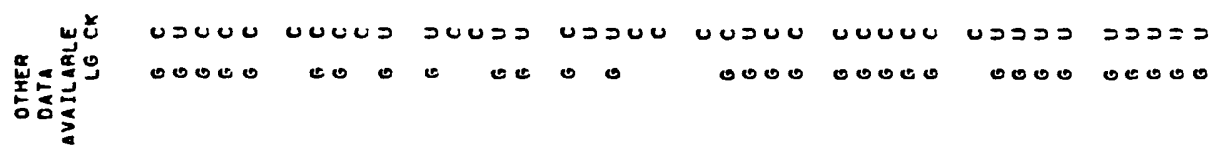

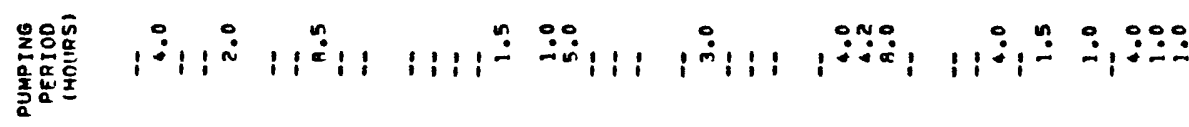

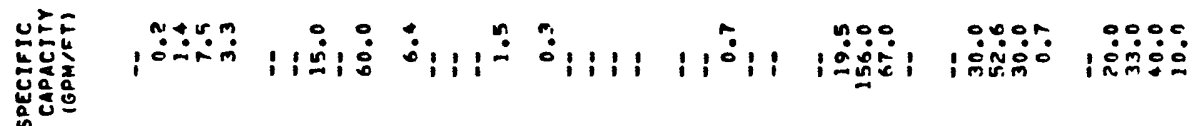

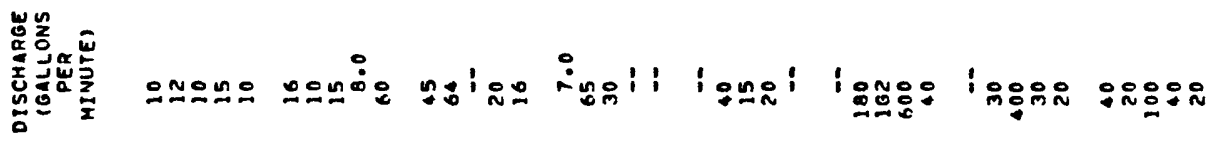

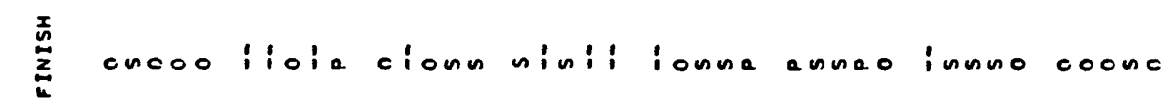

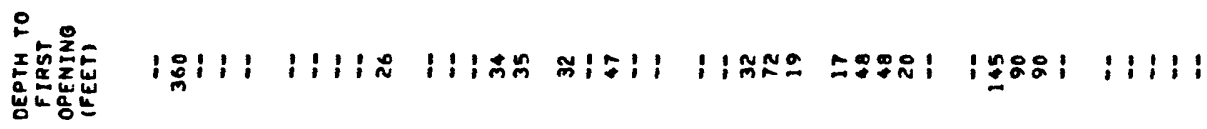

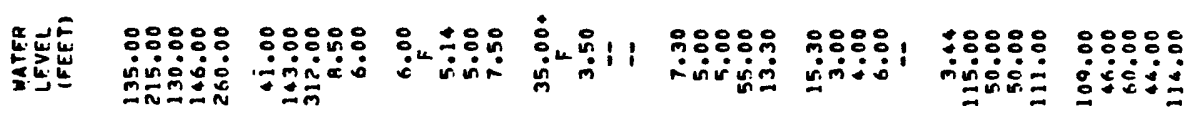

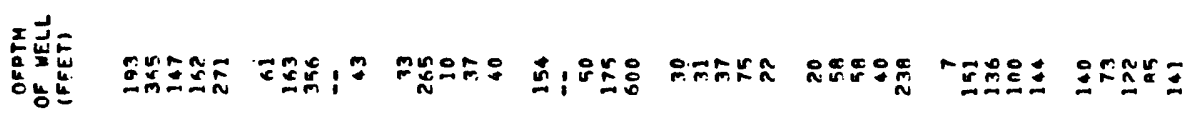

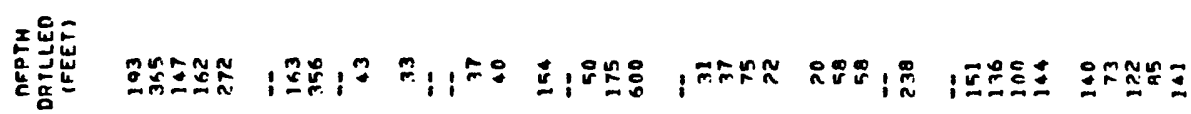

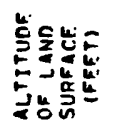

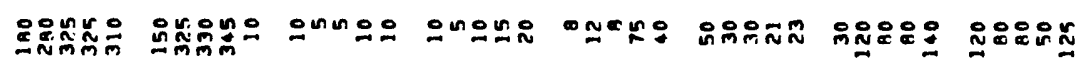

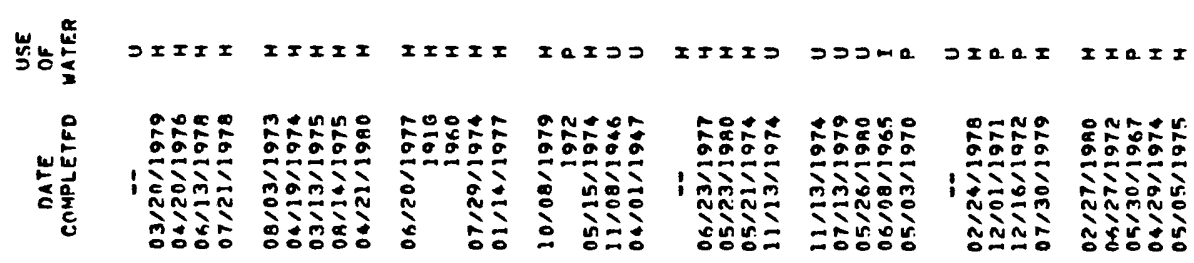

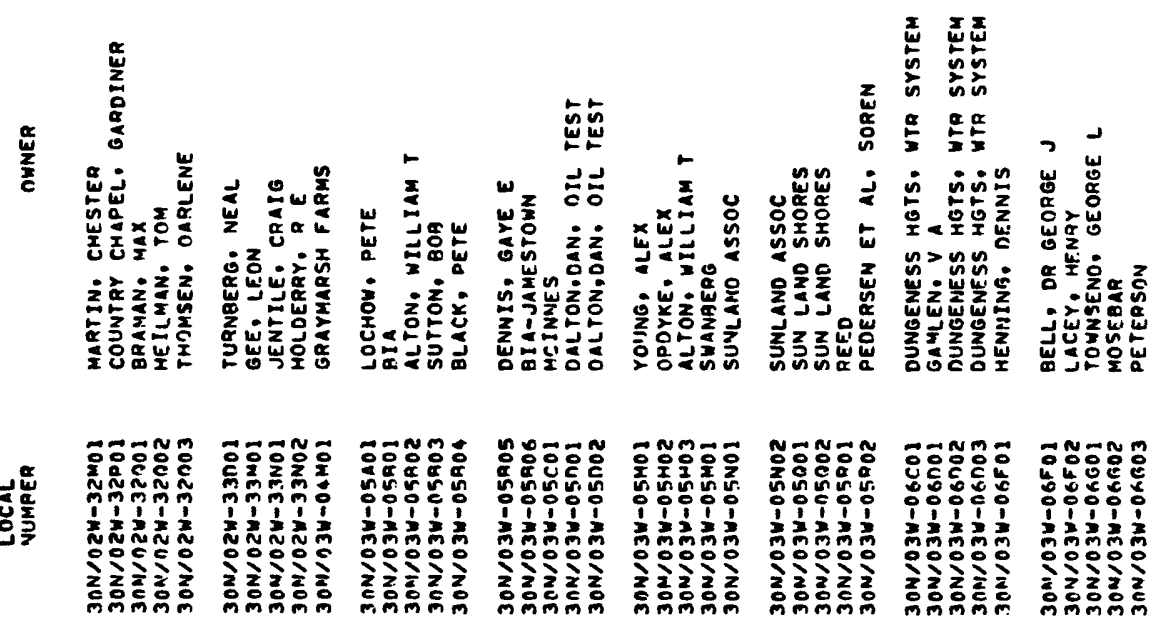




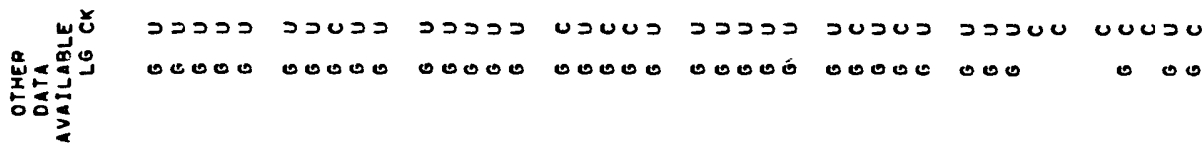

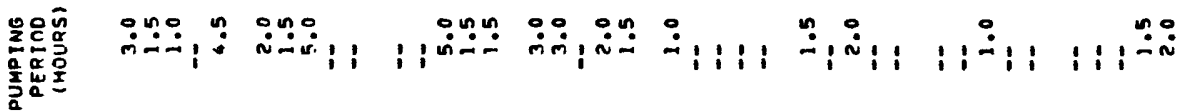

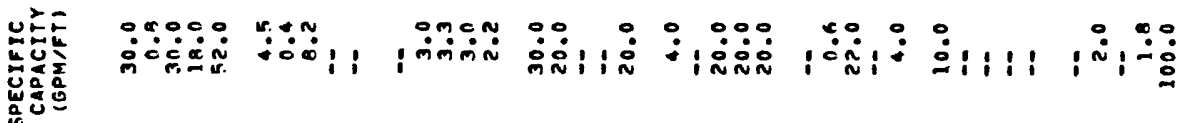

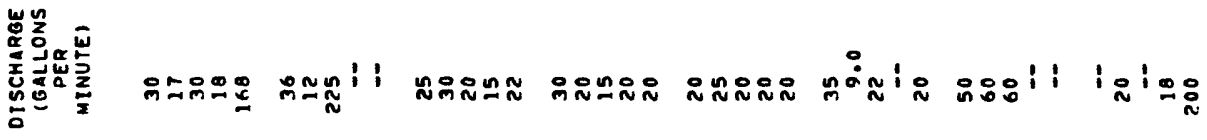

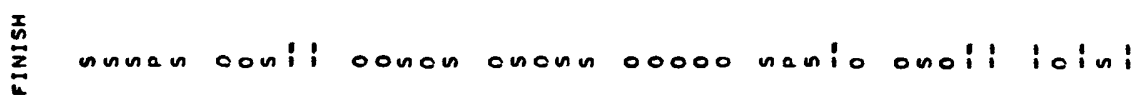

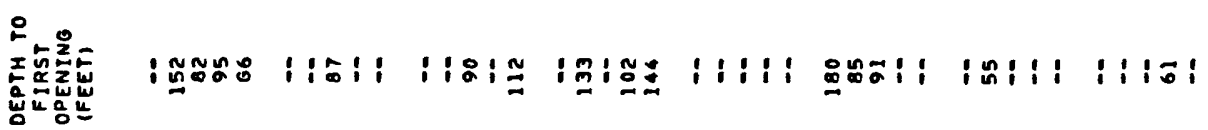

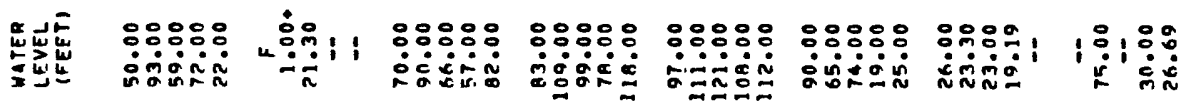

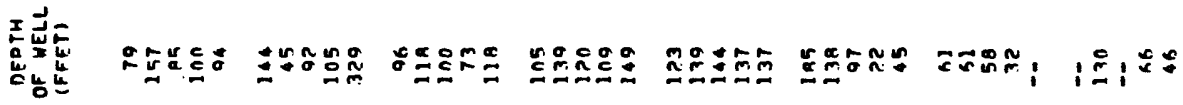

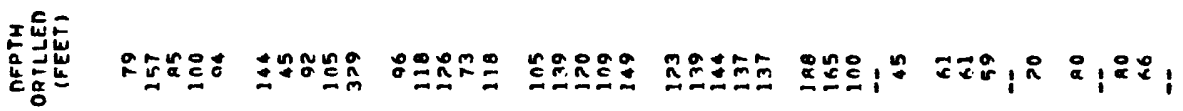

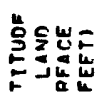

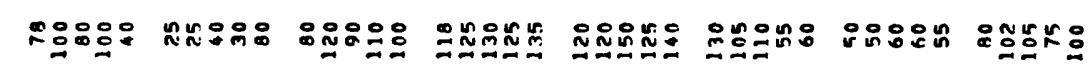

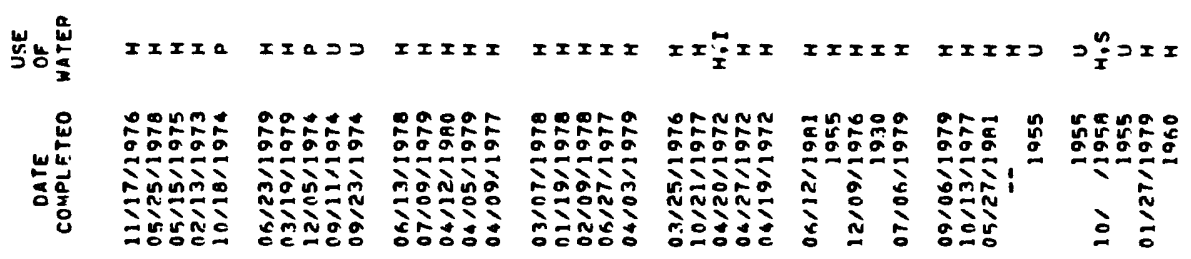
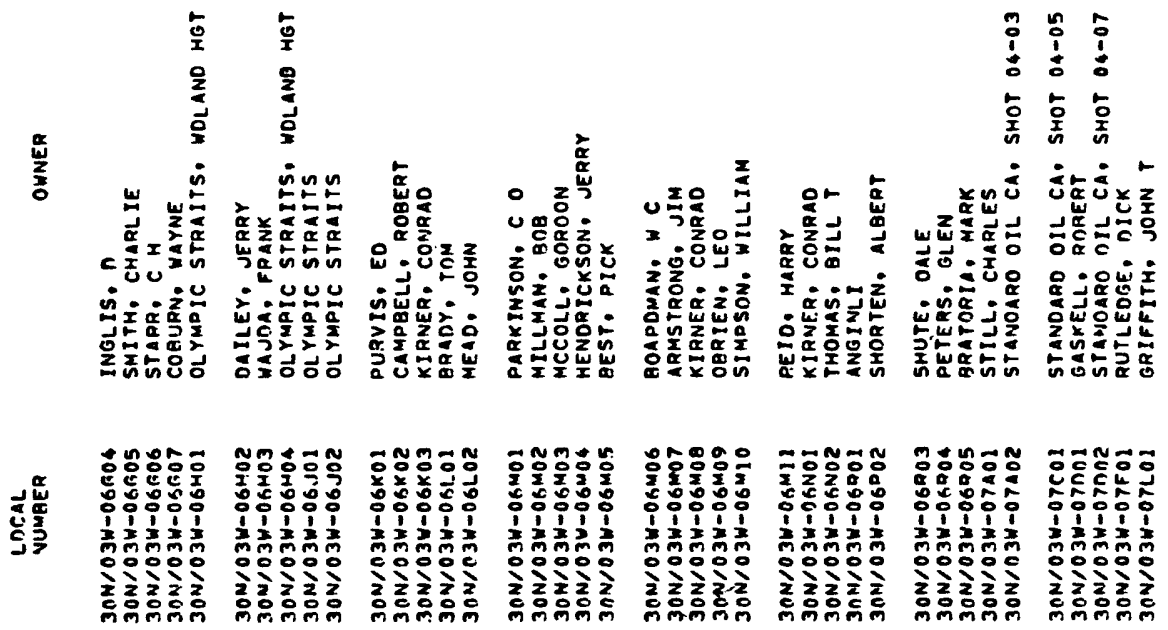


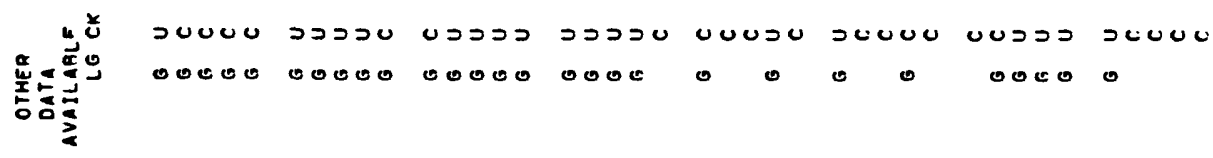
造育

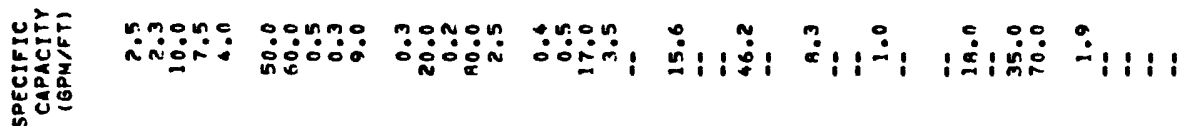

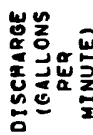

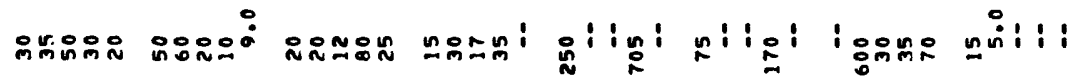

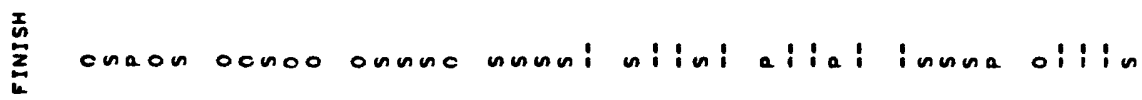

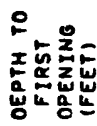

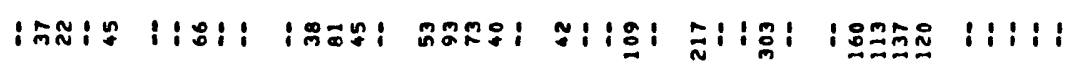
造

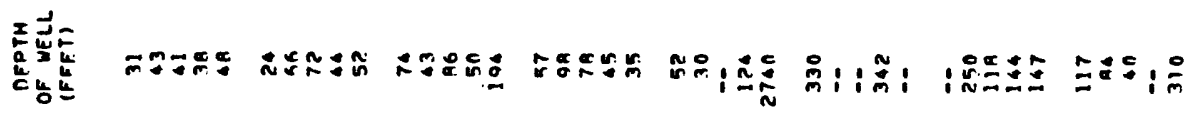

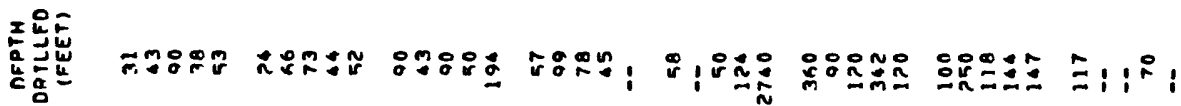

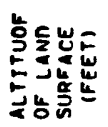

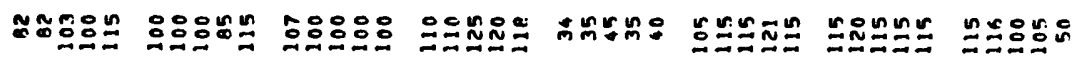

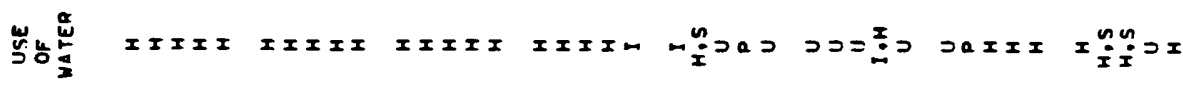
至

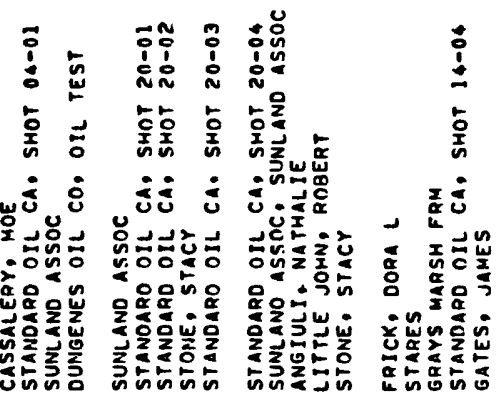

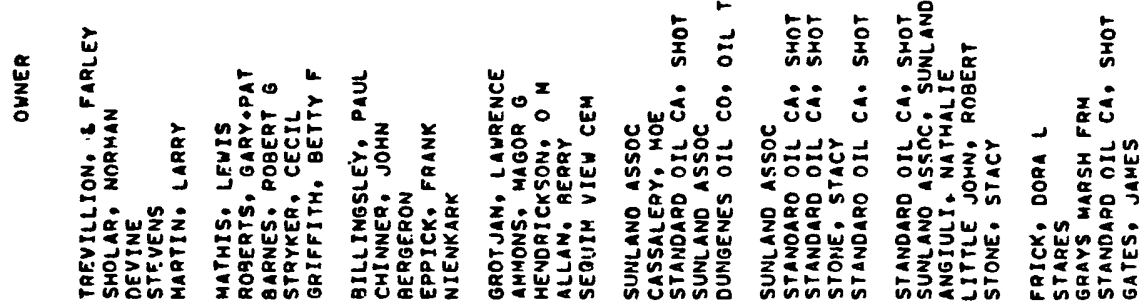

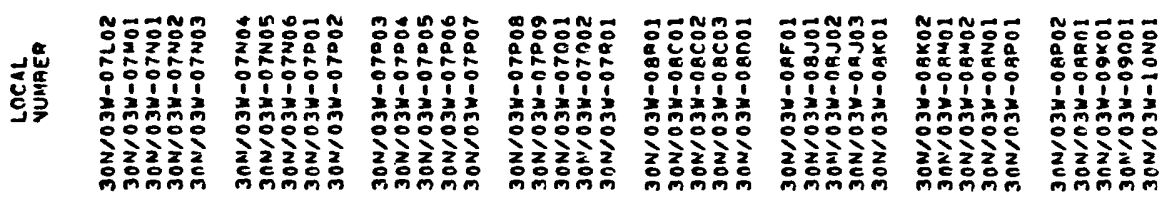




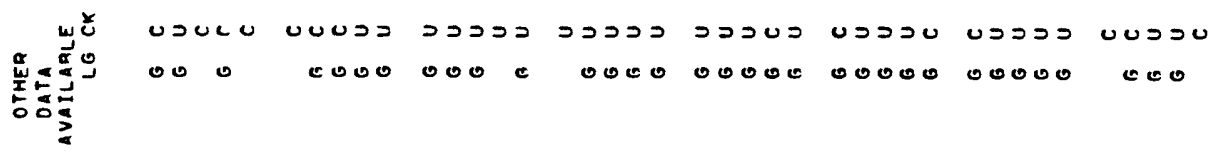

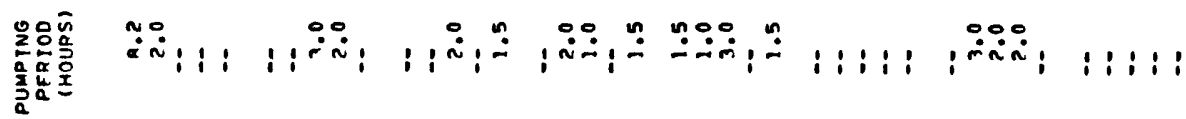

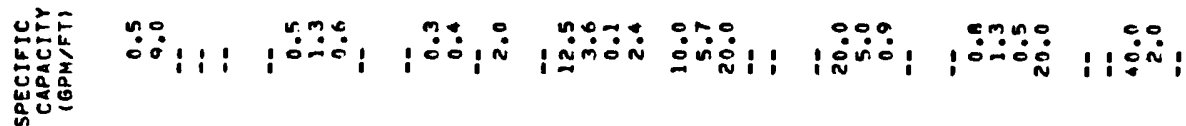

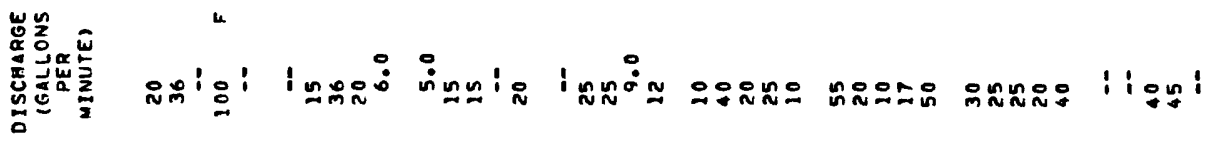

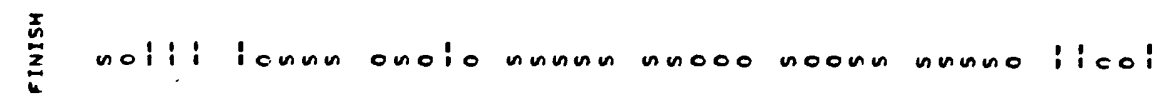
돈

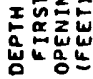

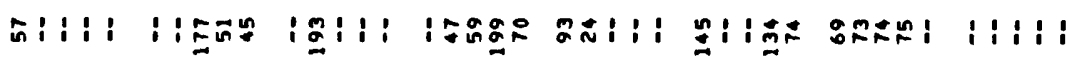
先

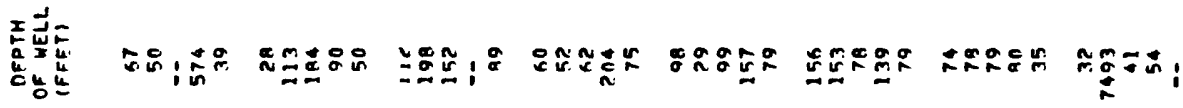

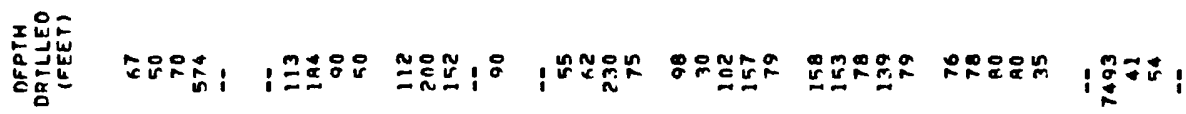

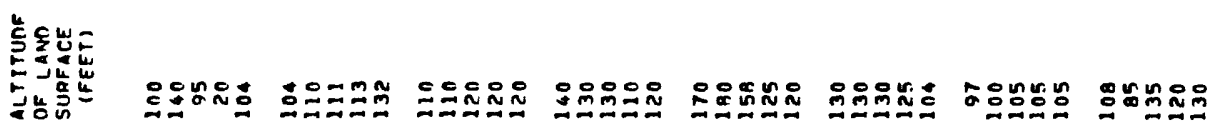

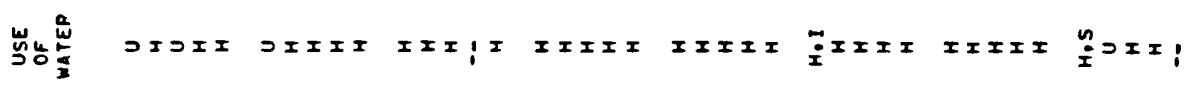
先
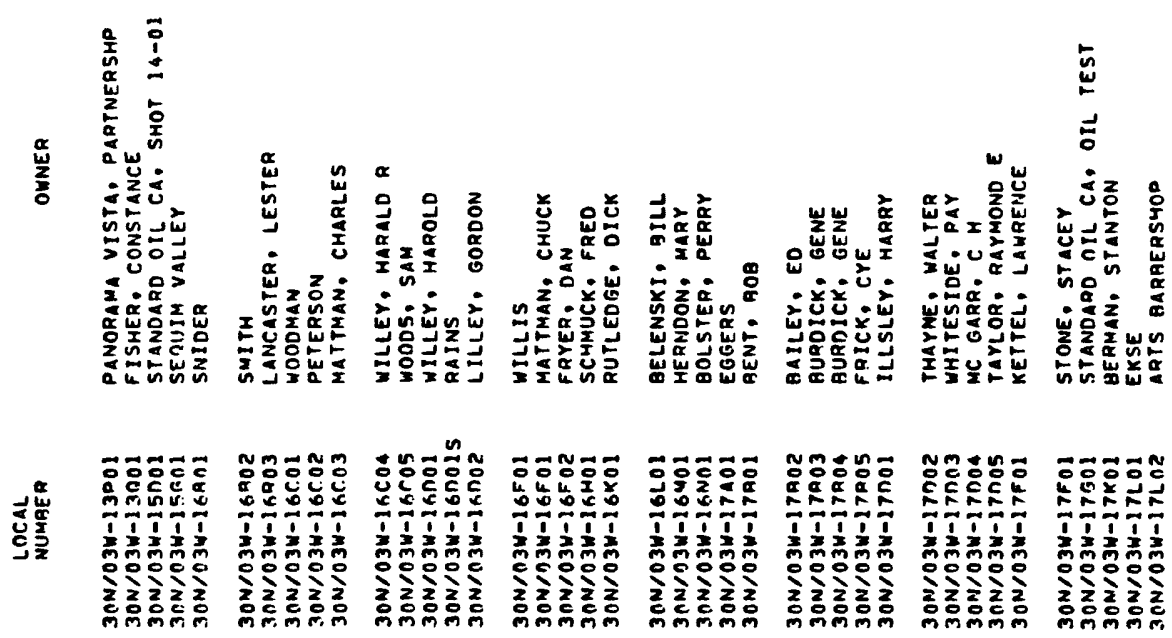


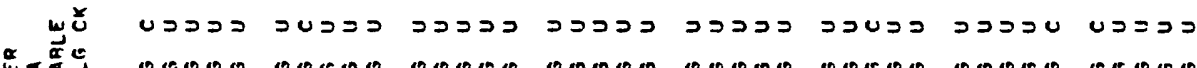

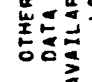

00000000000000000000000000000000000000

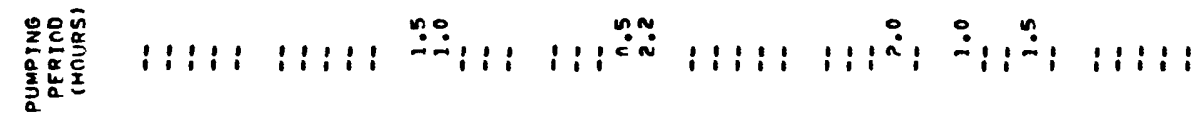

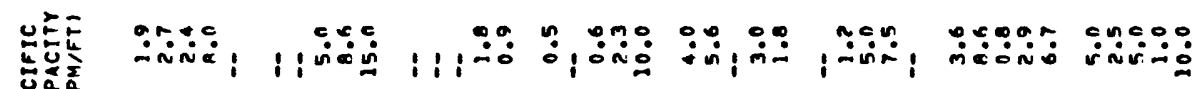

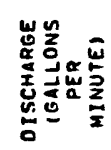

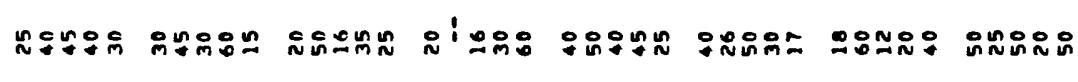
$\sum_{3}^{\mathbf{x}}$

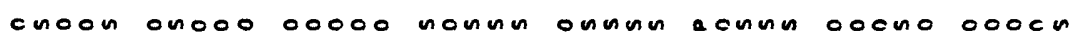

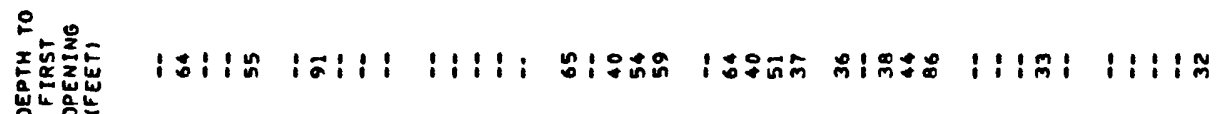

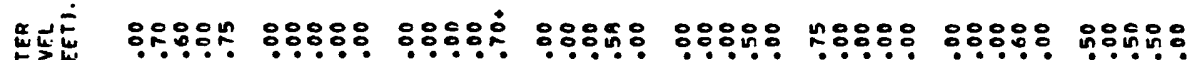

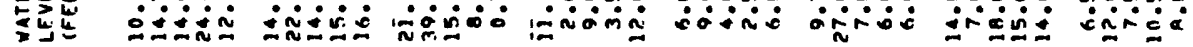

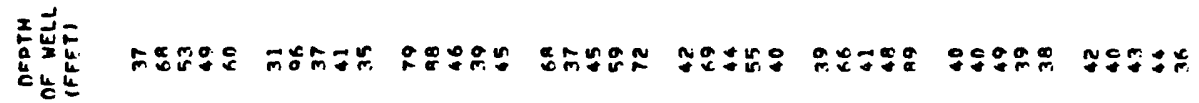

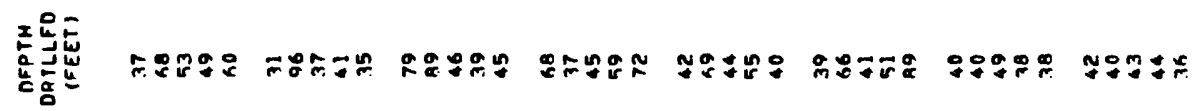

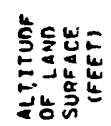

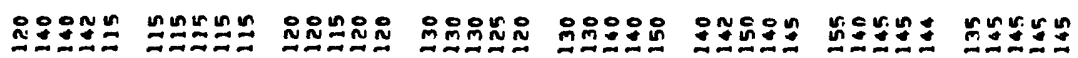

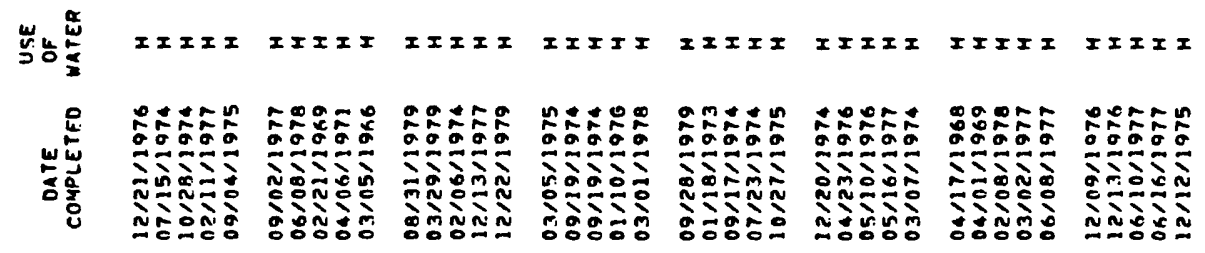
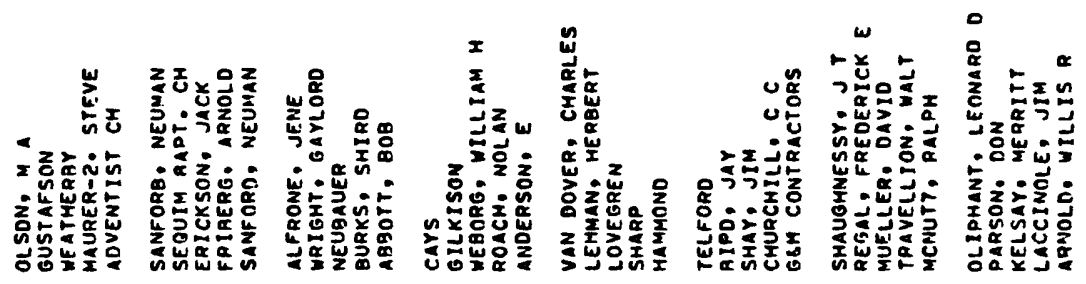


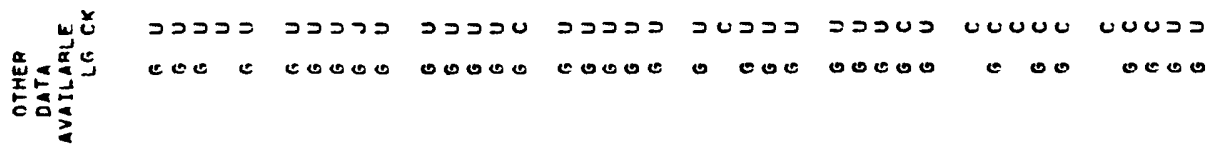

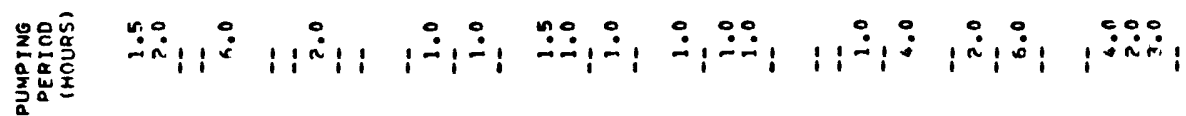

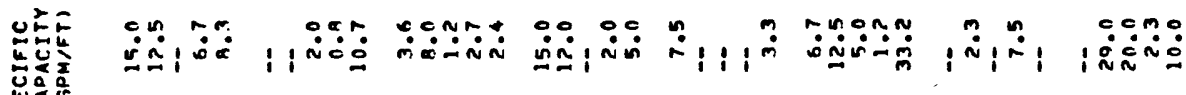

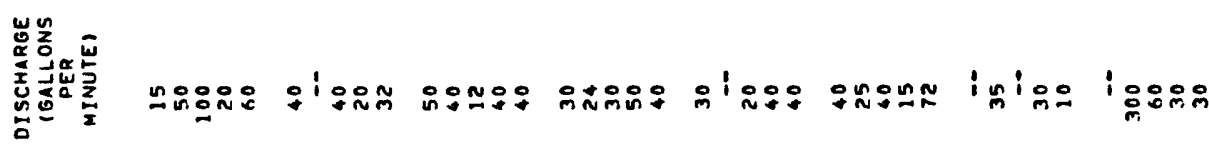

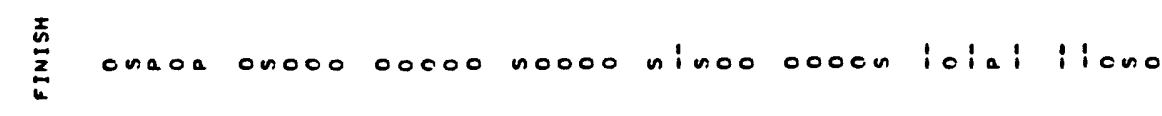

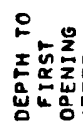

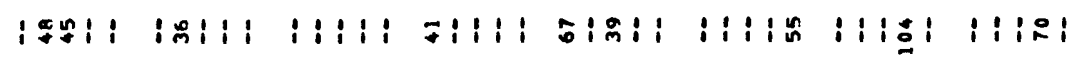

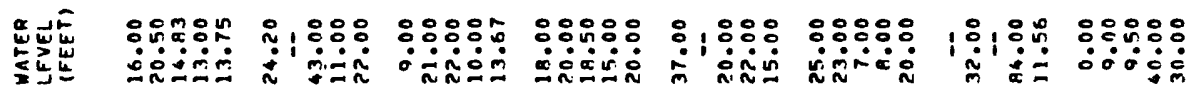

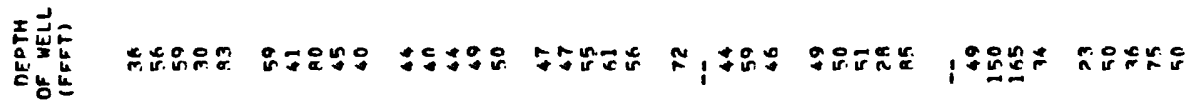

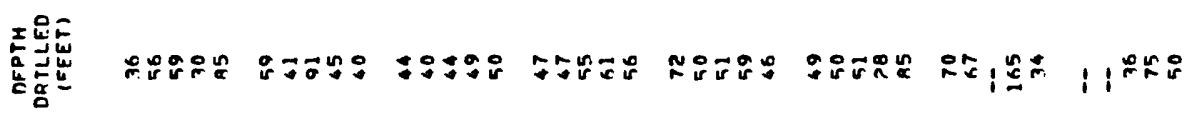

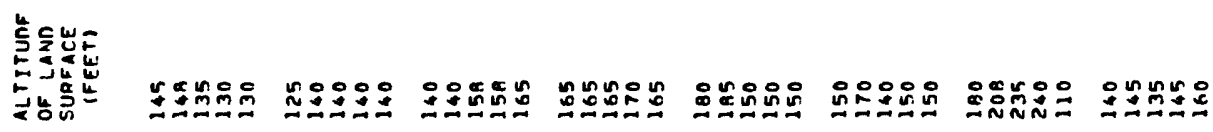

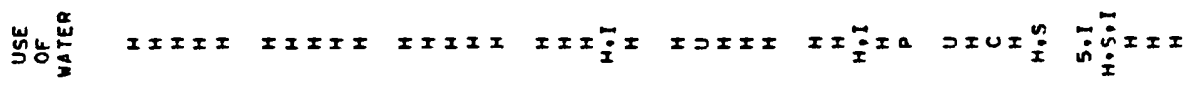

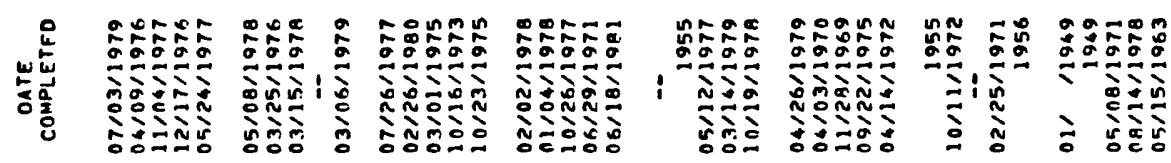

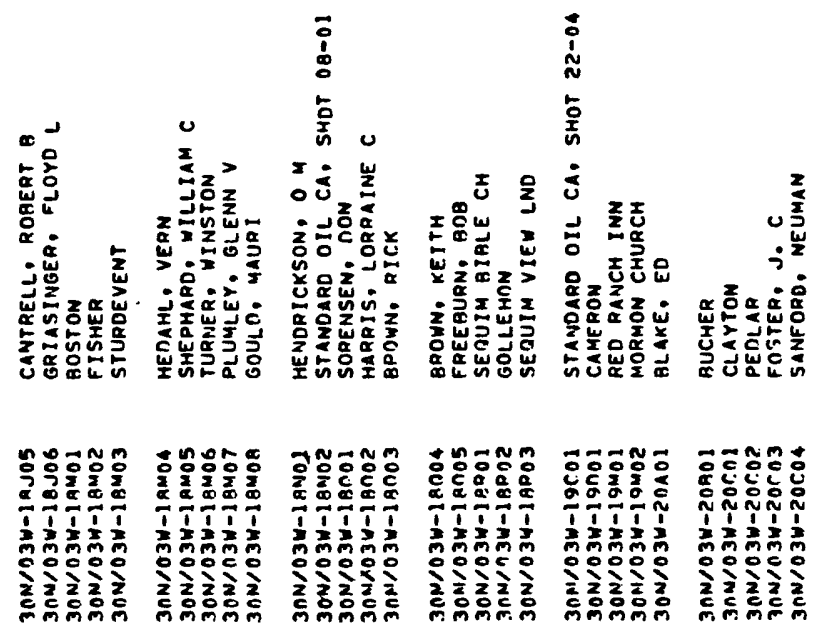




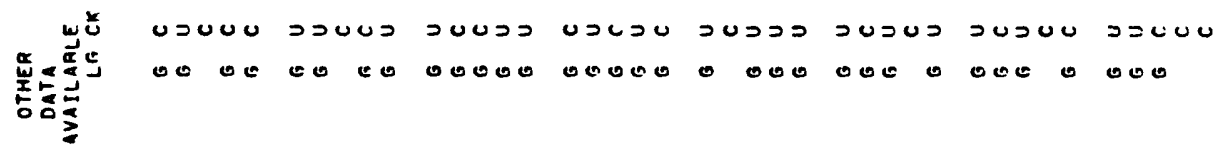

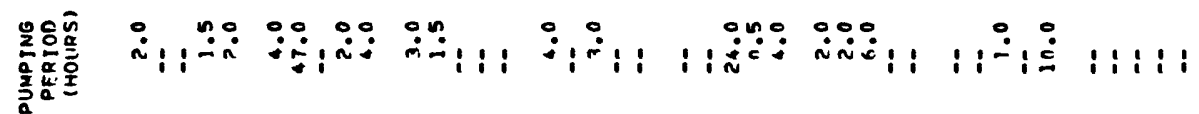

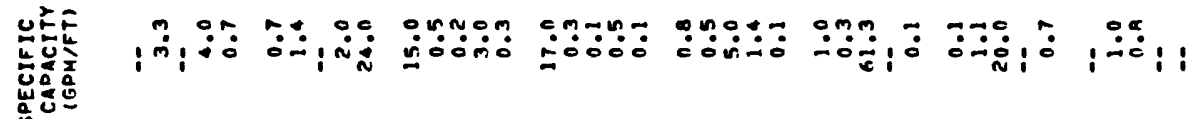

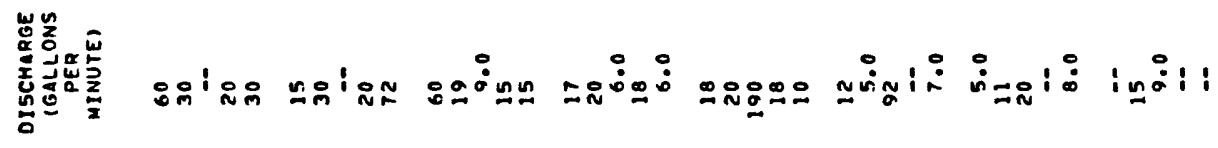

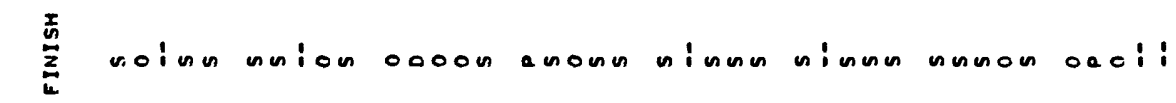

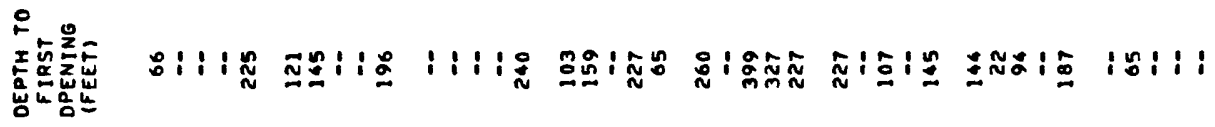

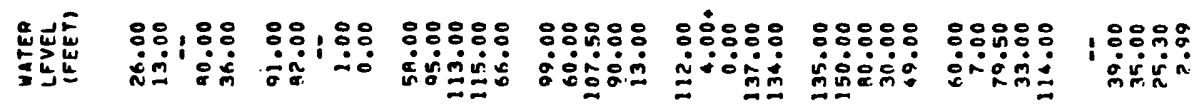

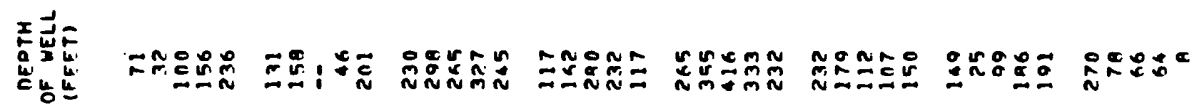

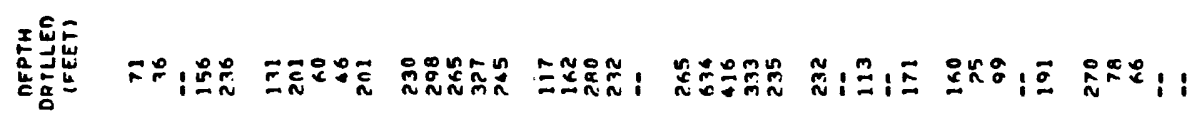

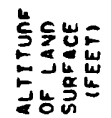

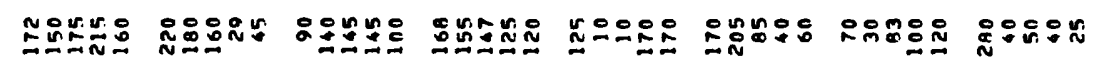

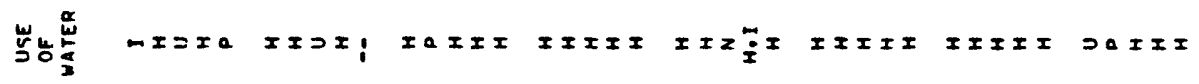

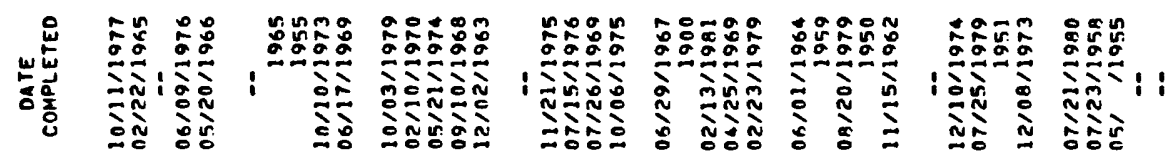
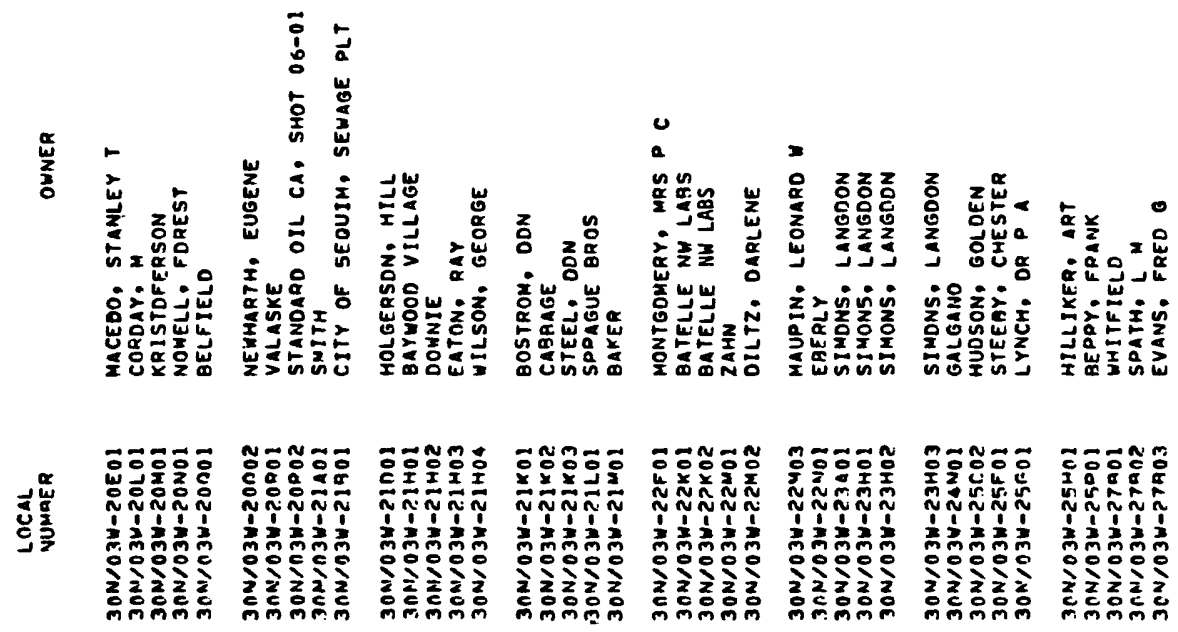


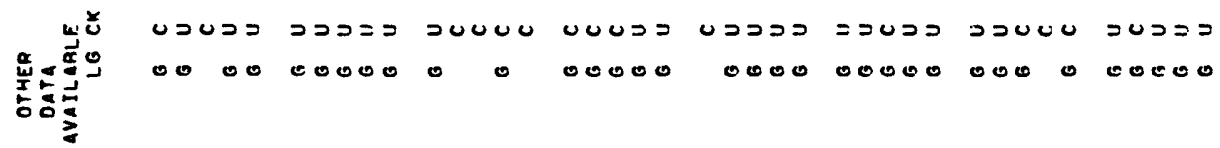

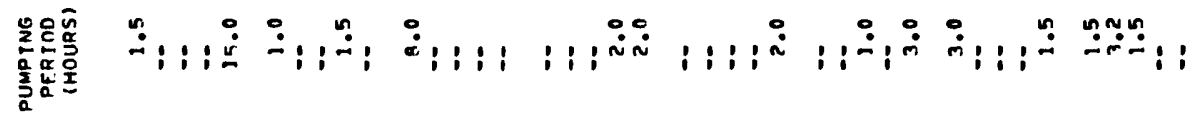

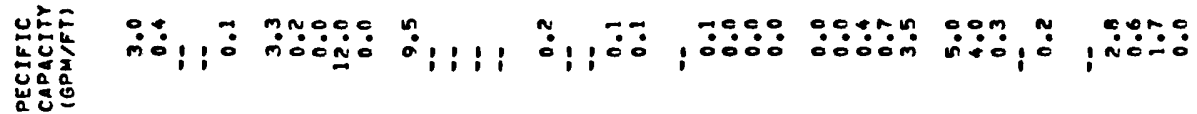

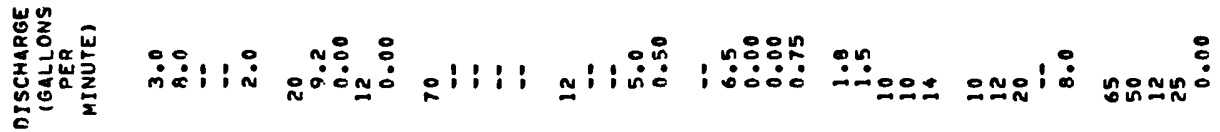

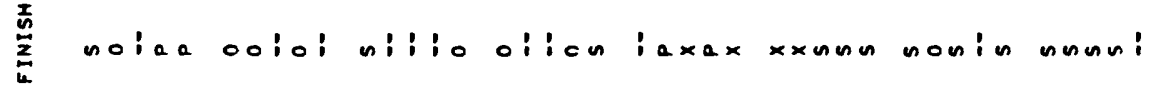

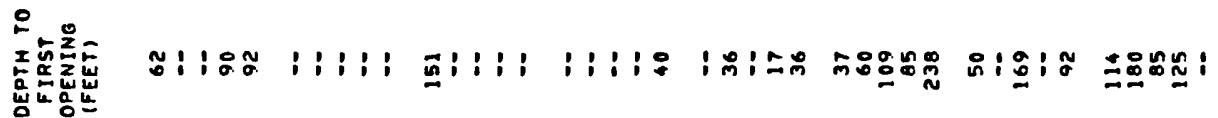

热

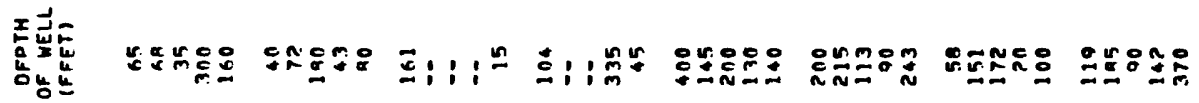

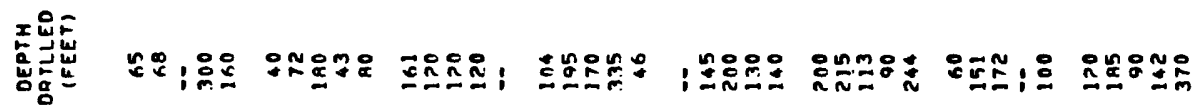

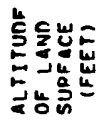
모ำกิ

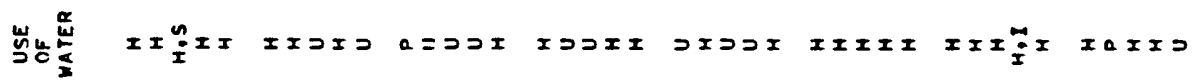

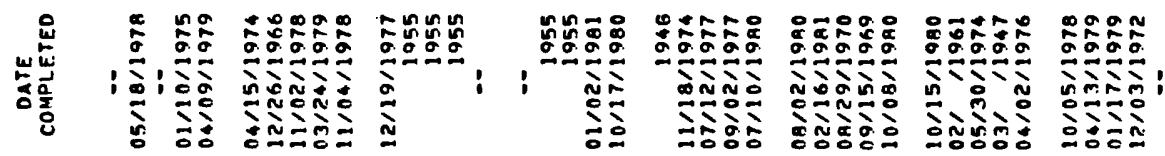

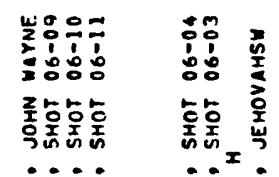

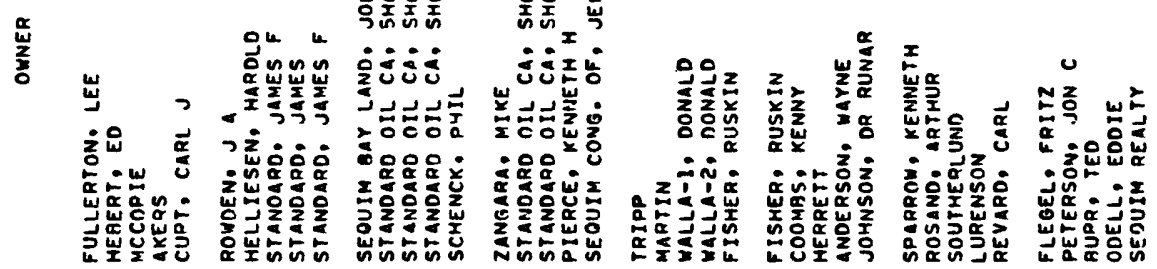

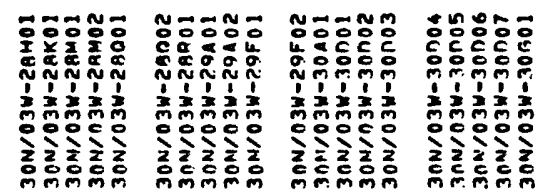




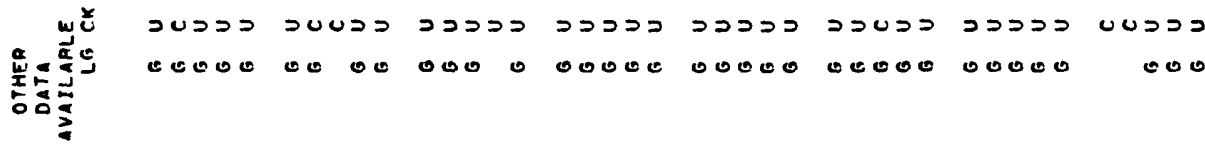

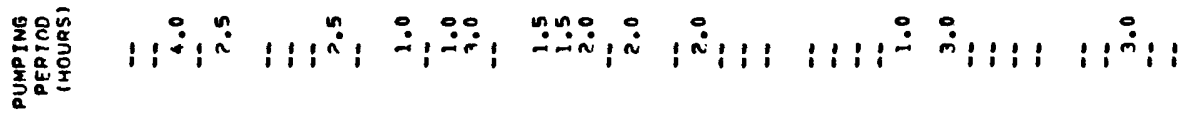
造

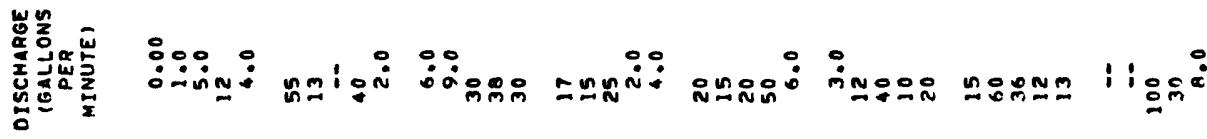

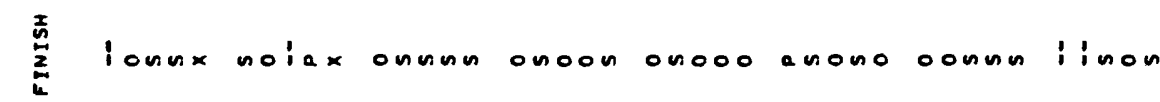

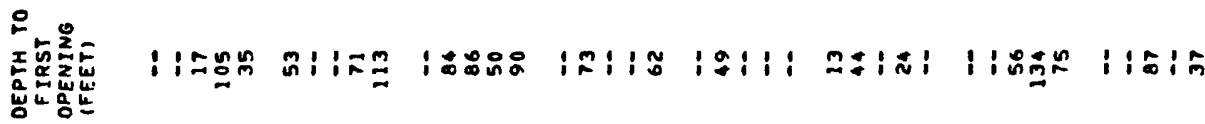

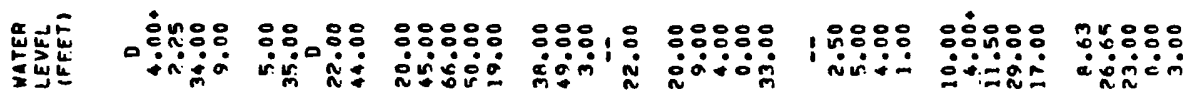

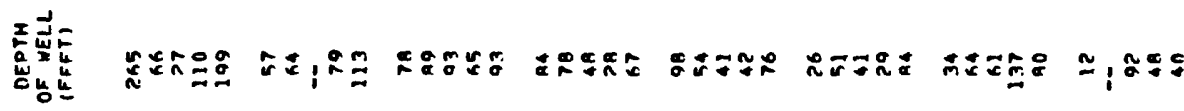

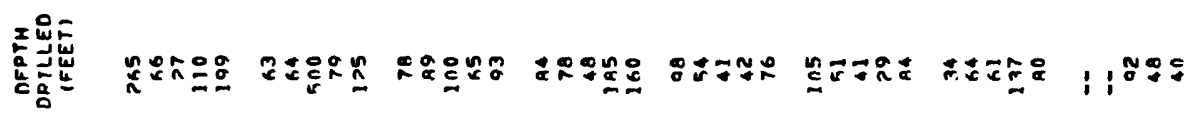

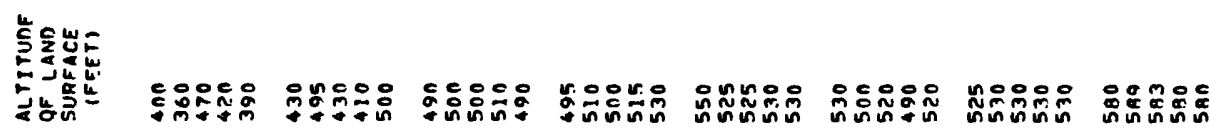

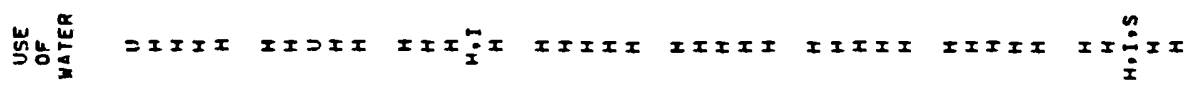

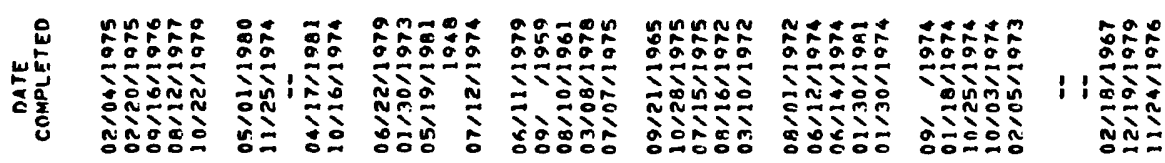




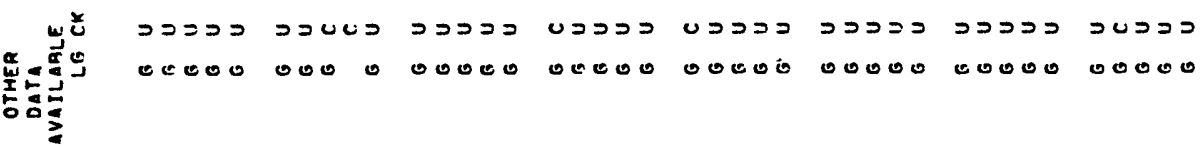

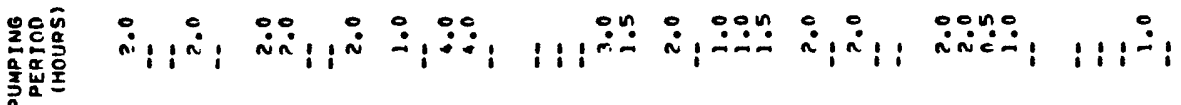

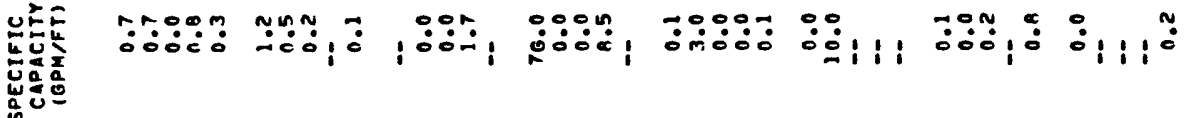

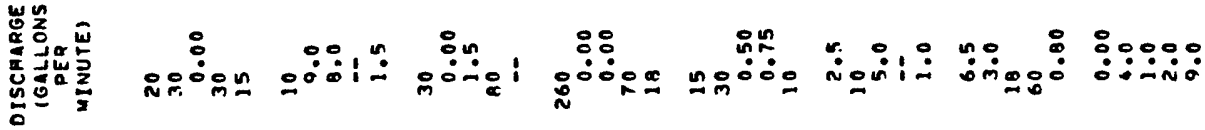

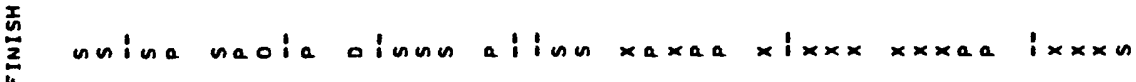
안.

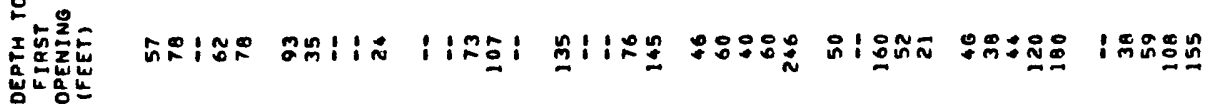
凯

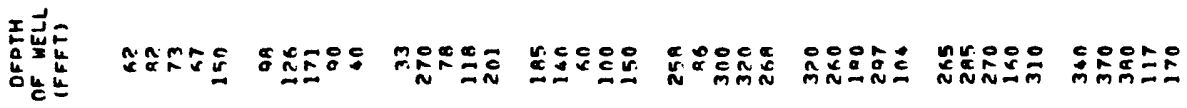

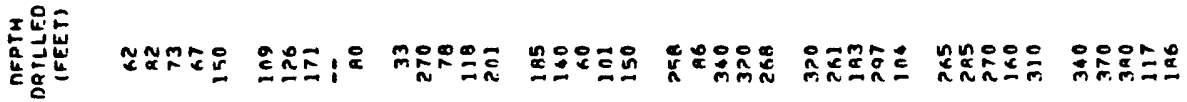

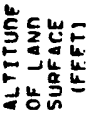

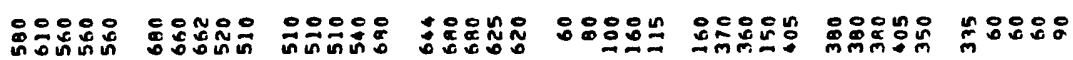

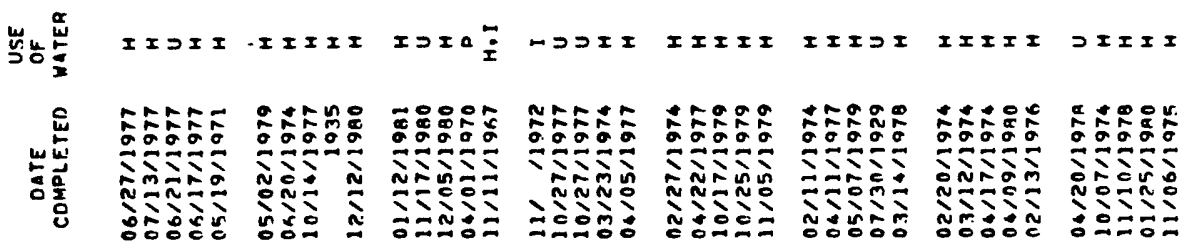




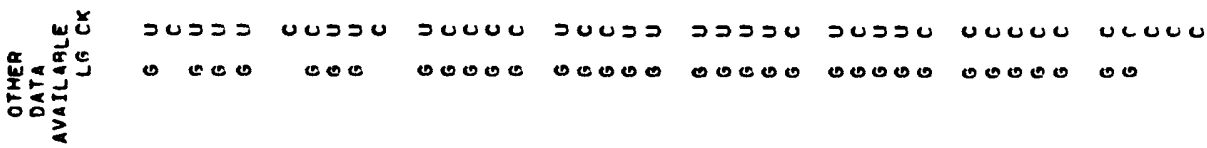

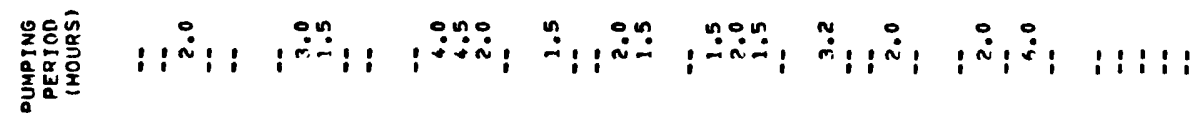

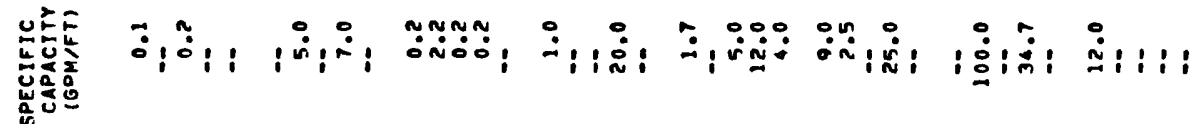

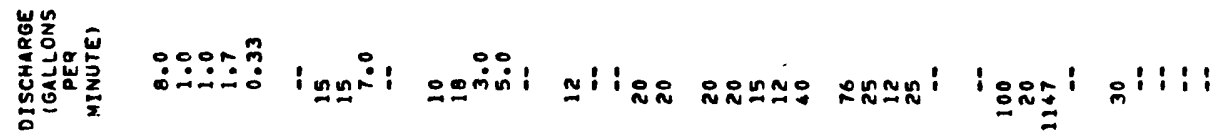

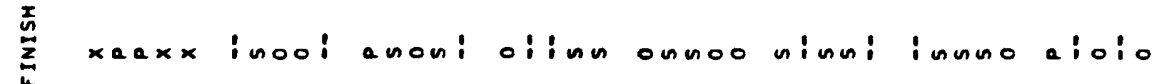
웅

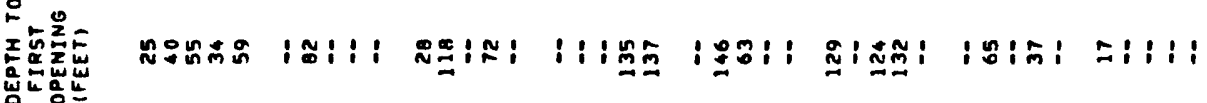

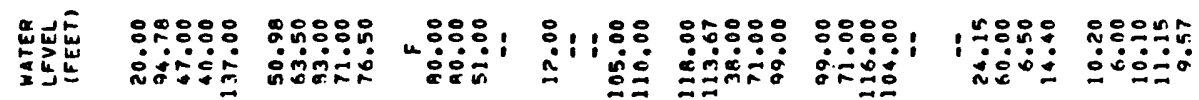

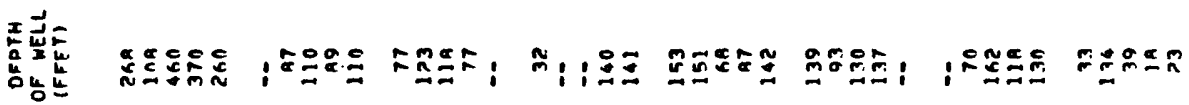

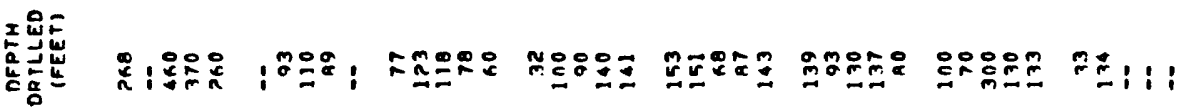

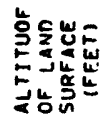

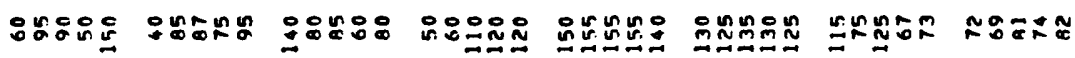

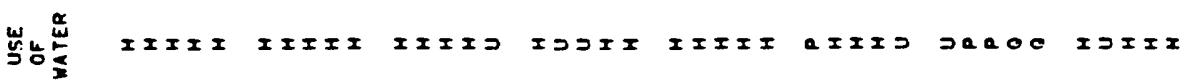

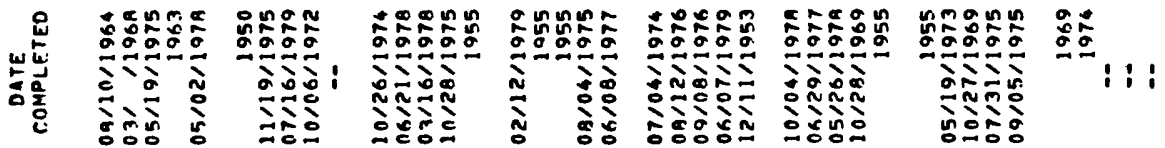
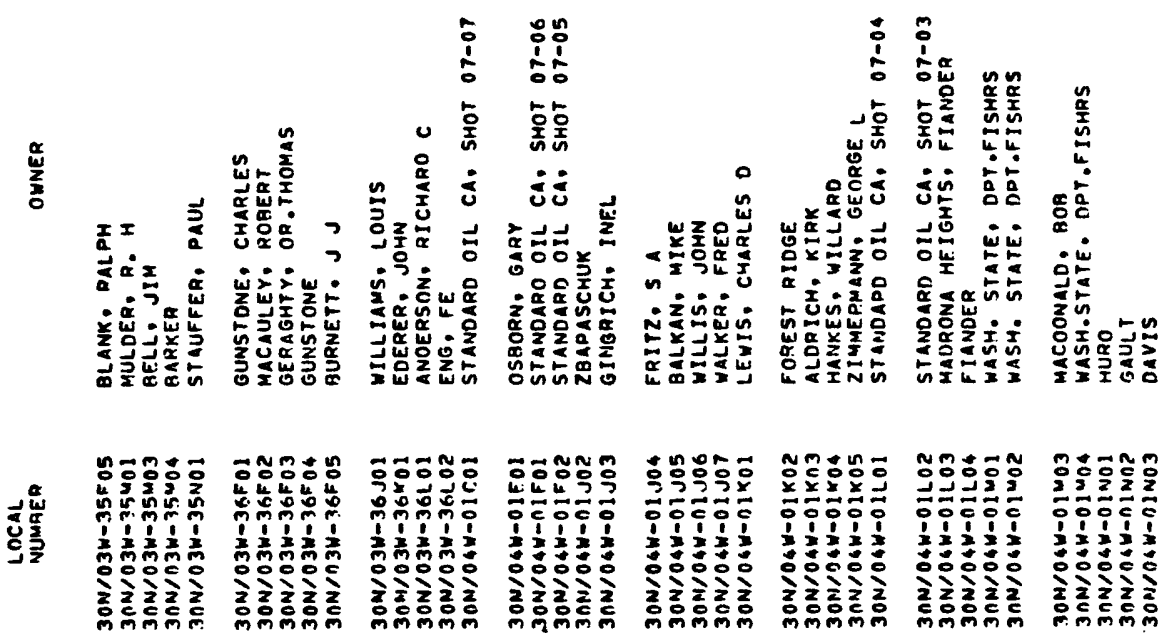


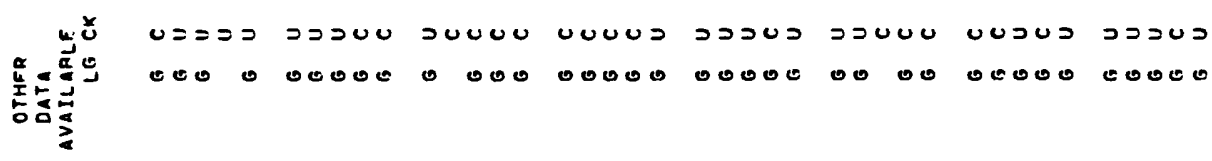

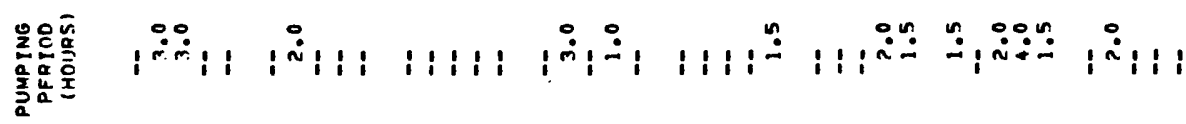

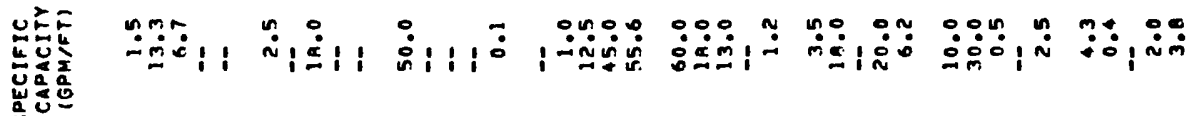

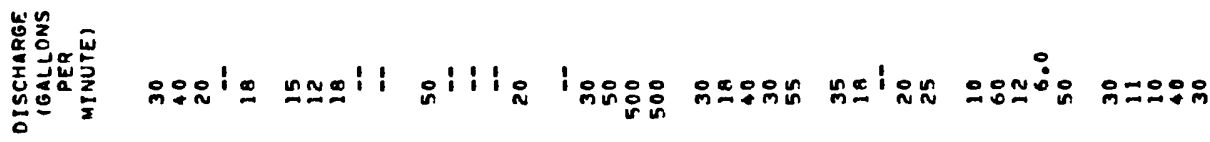

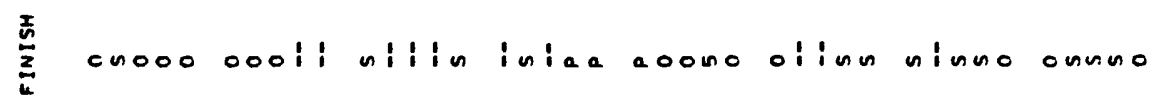

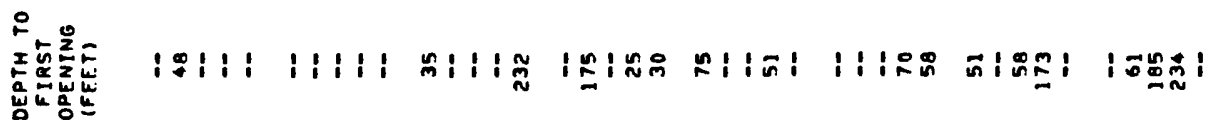
热

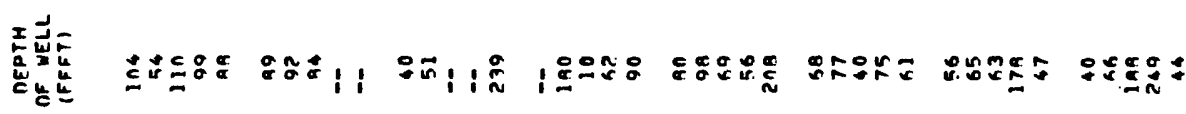

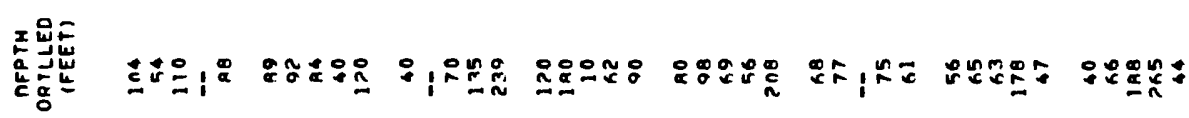

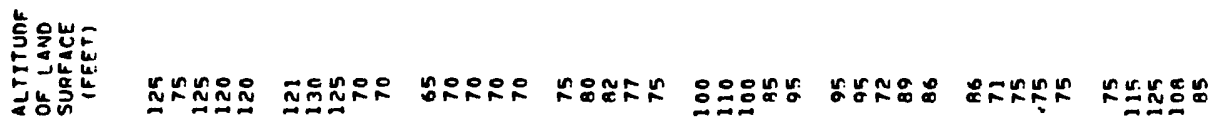

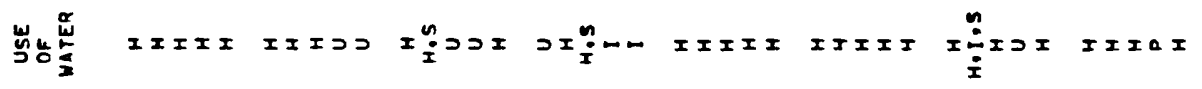
先

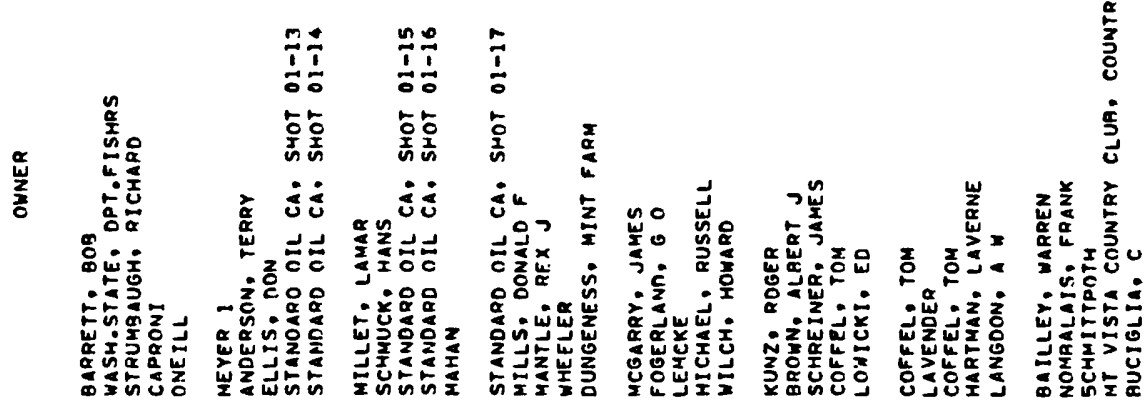




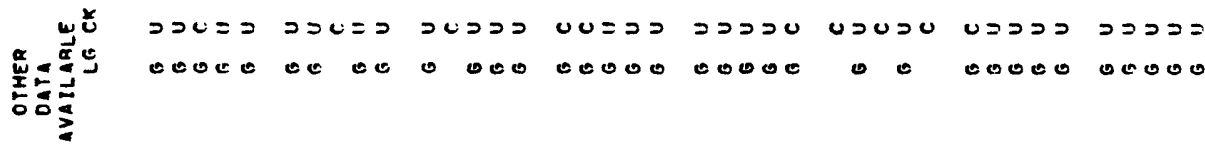

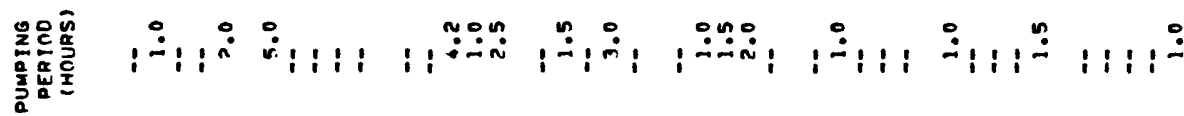

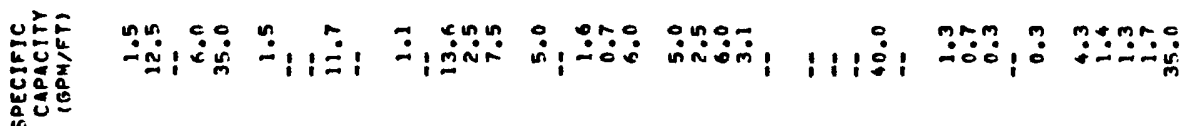

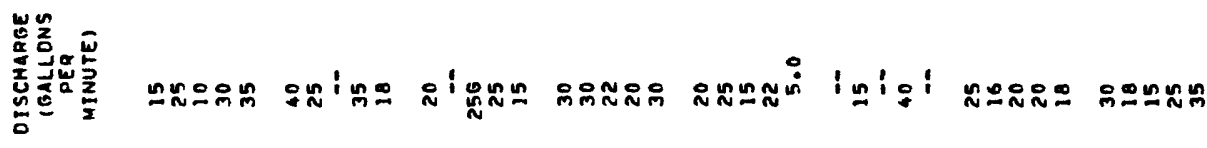

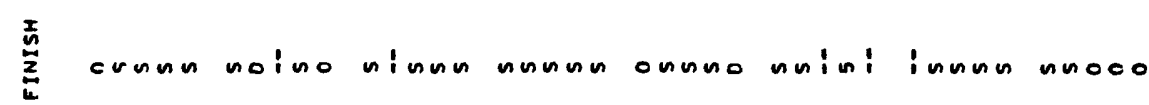

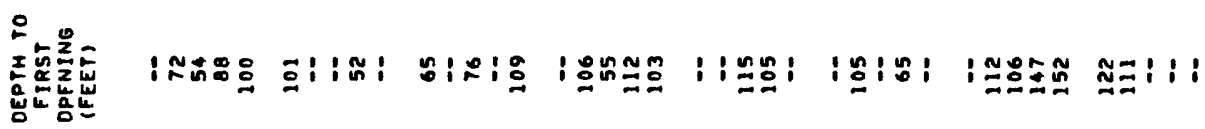
造

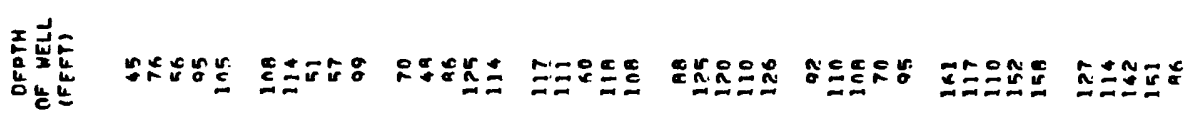

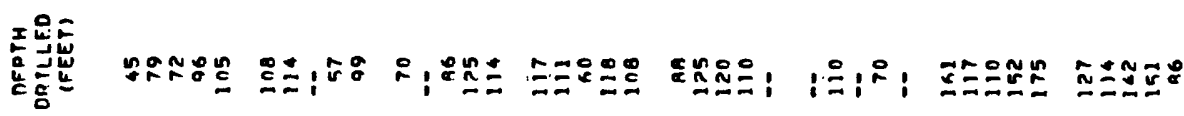

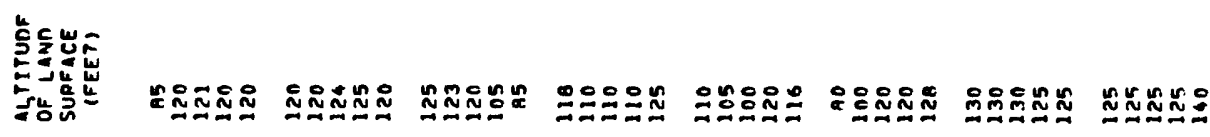

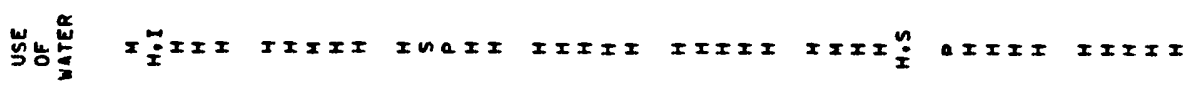

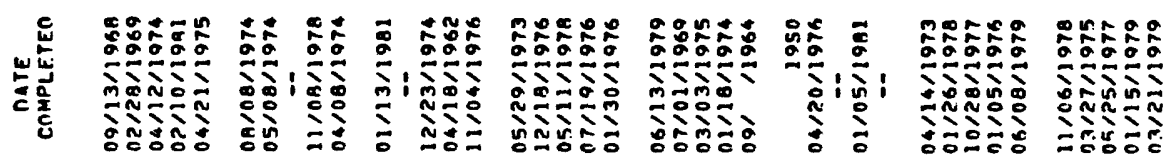




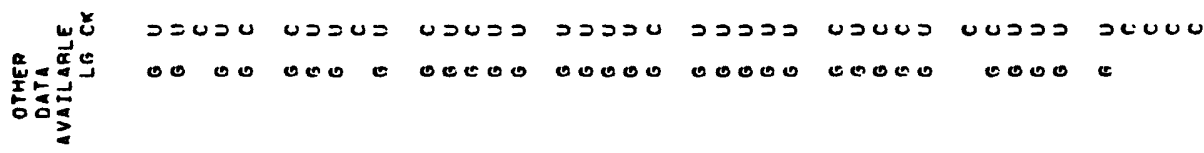

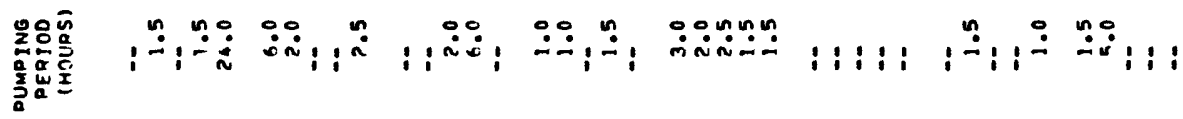

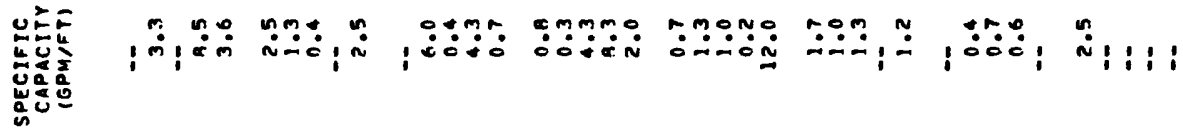

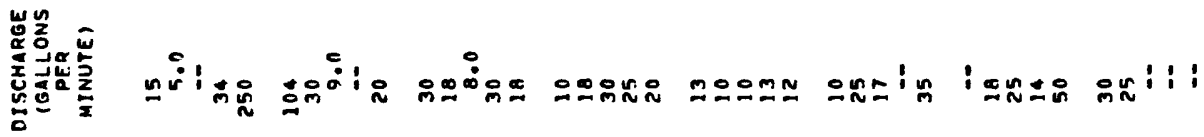

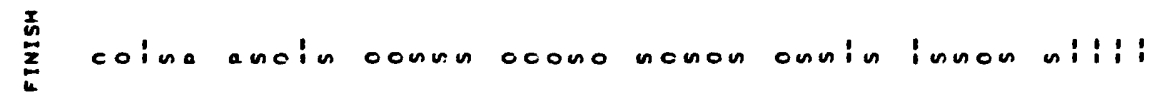

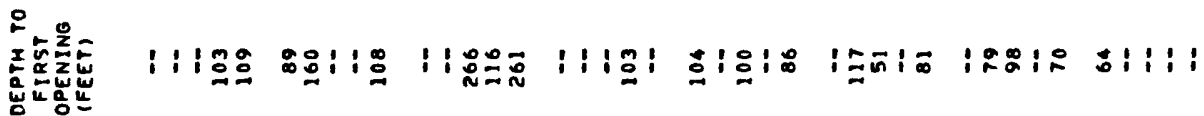

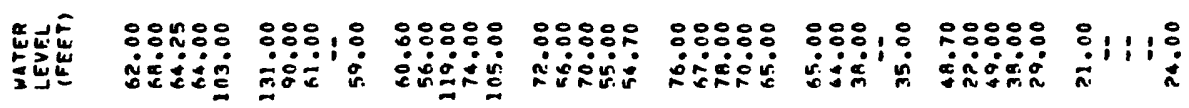

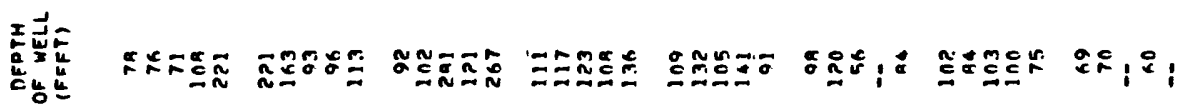

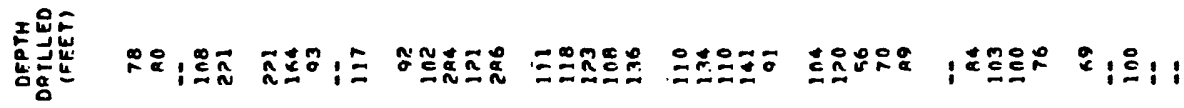

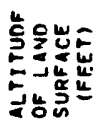

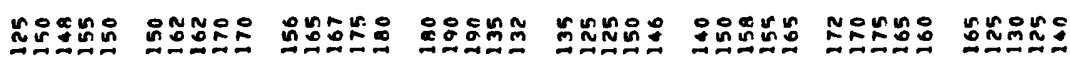

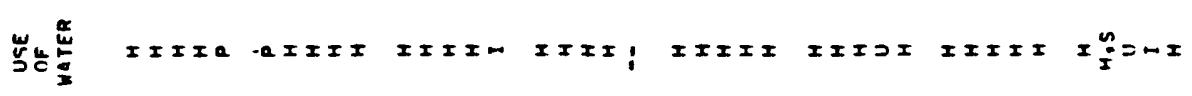
先
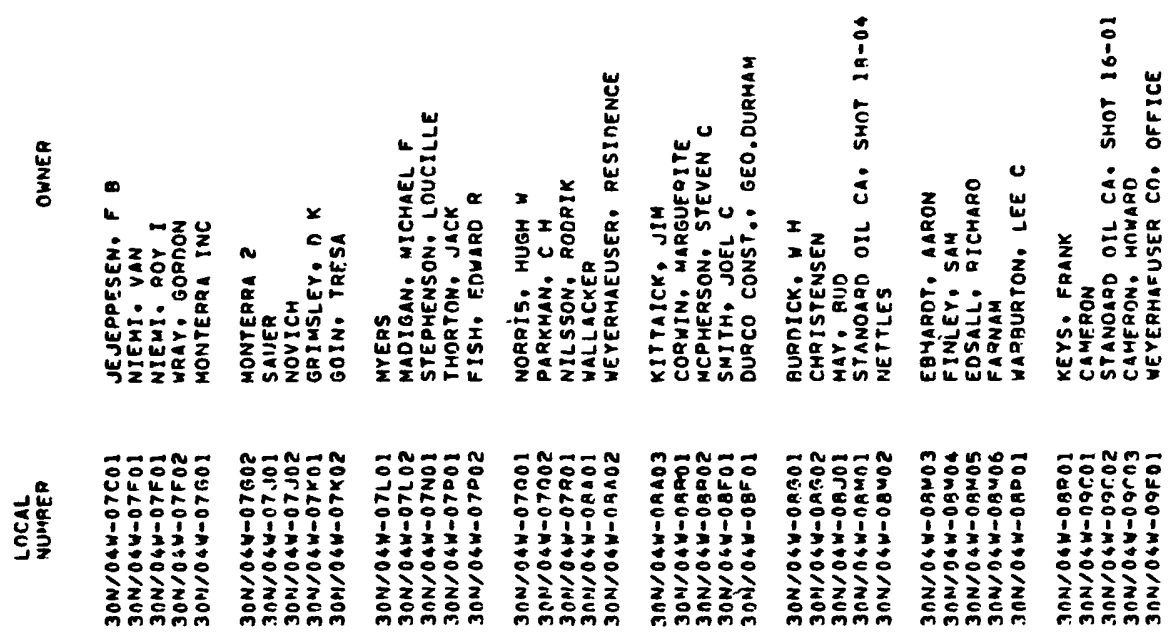


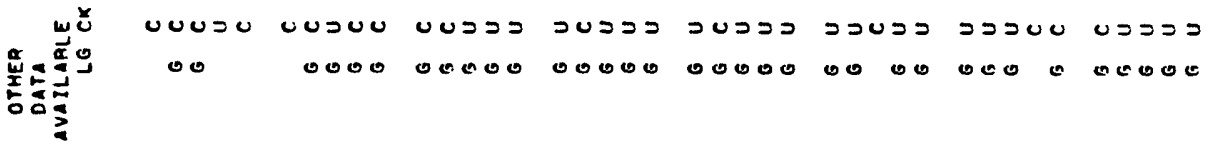
送最

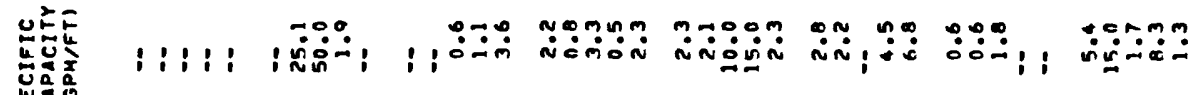

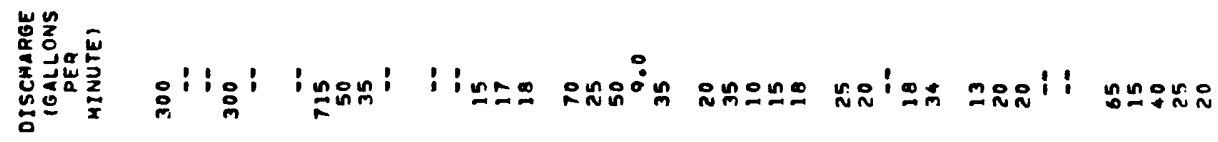

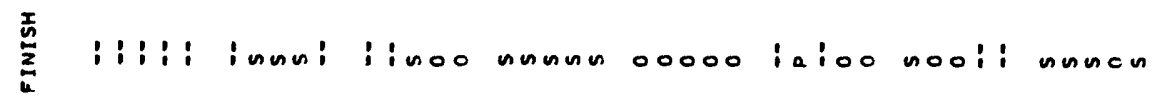

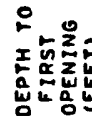

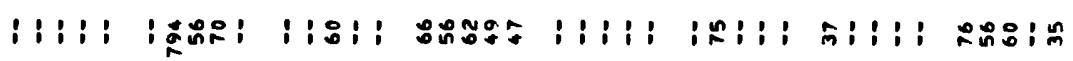

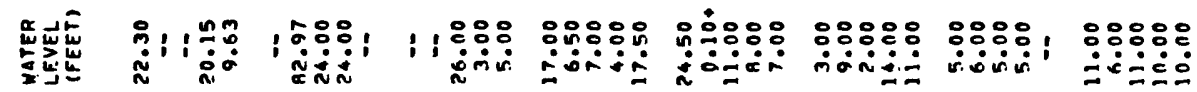

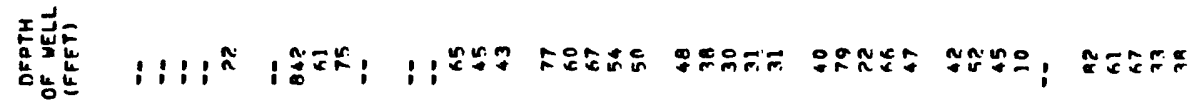

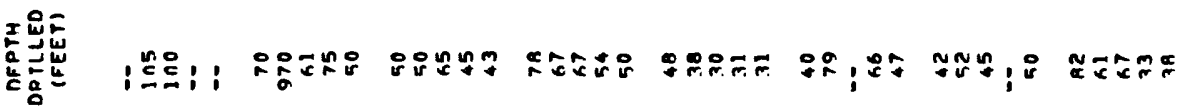

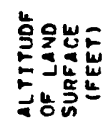

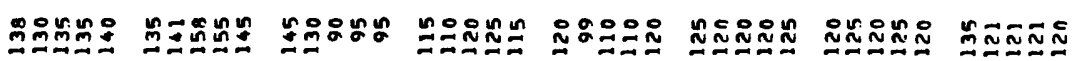

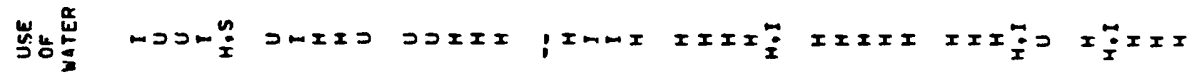

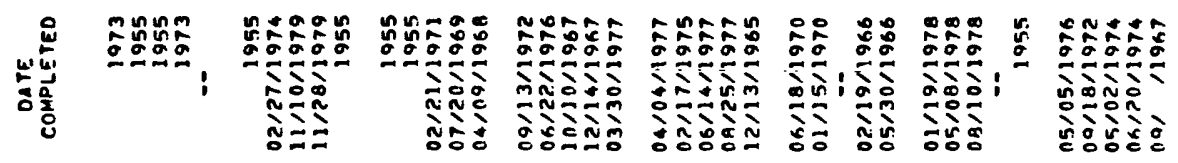

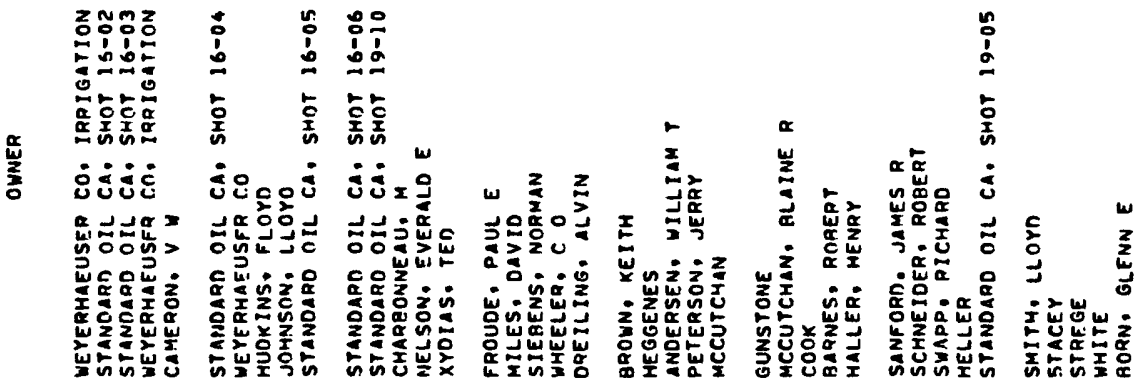

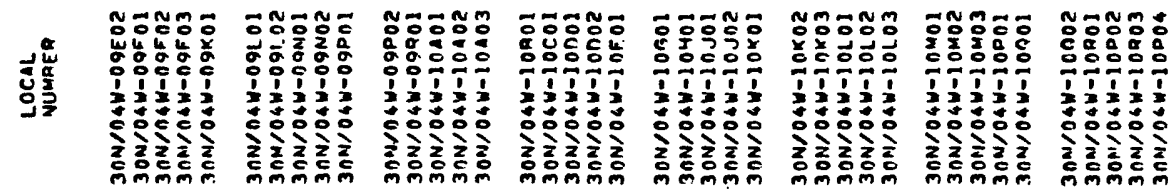




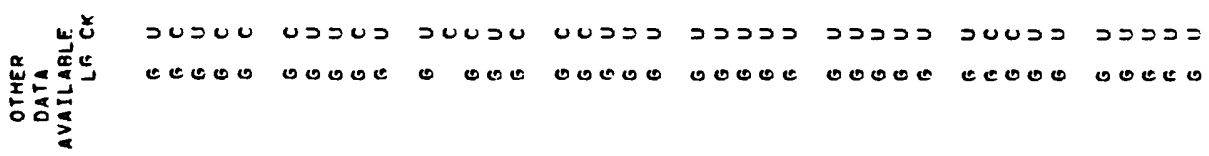

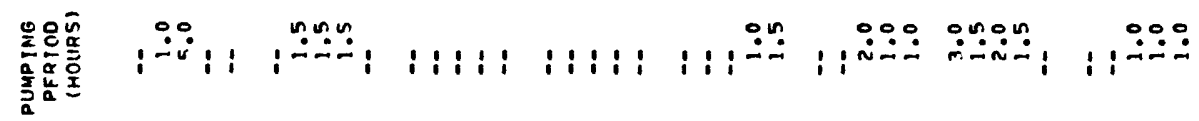

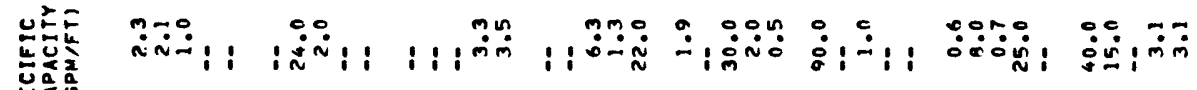

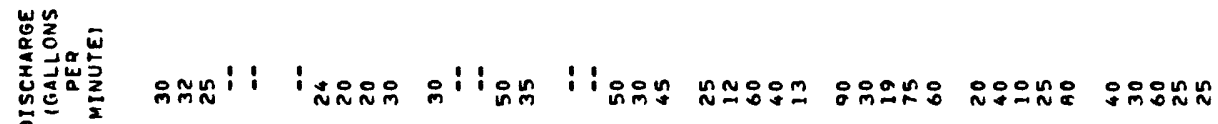
恶 $00011: 10000011001100000000000000000000000$

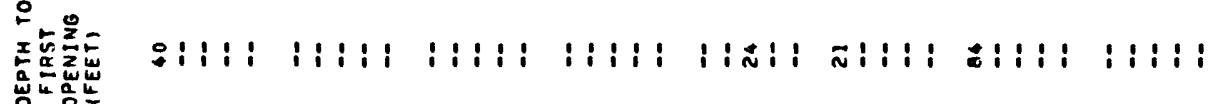
采点

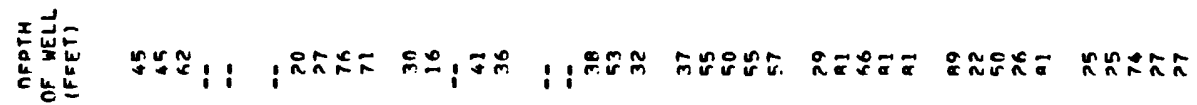

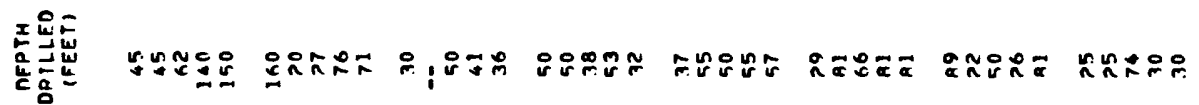

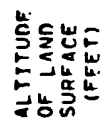

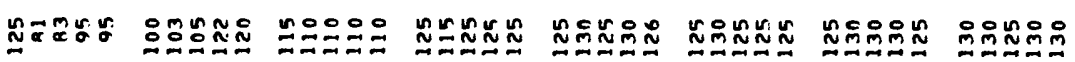

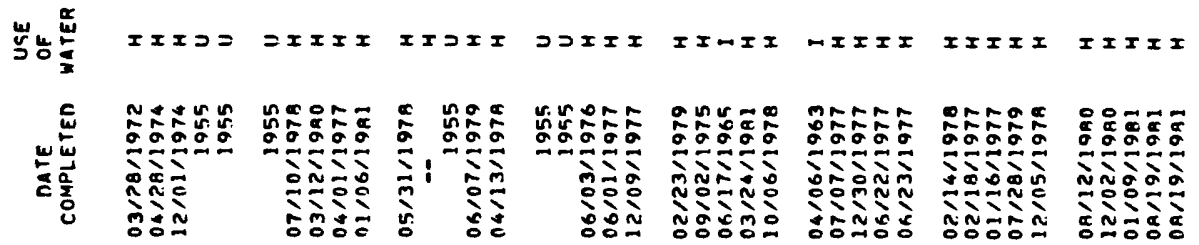

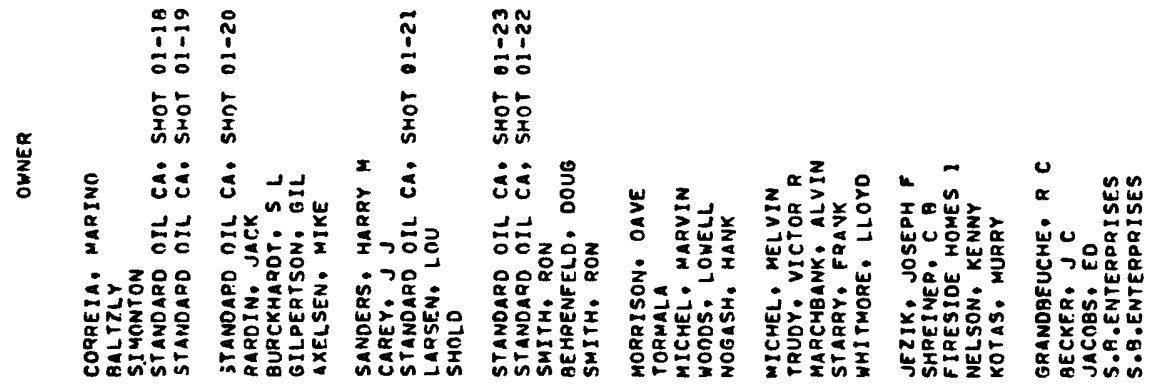

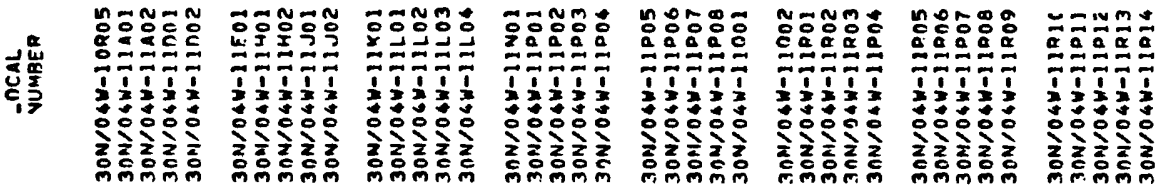




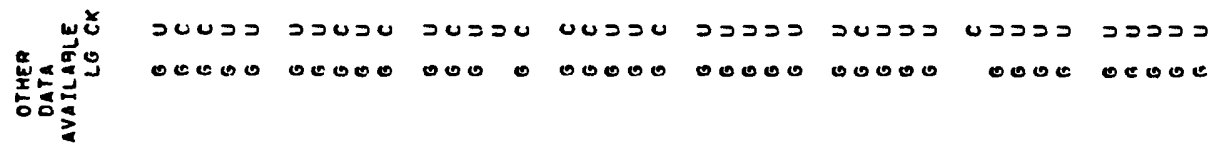
率最

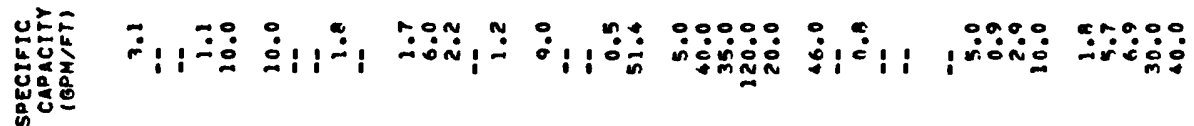

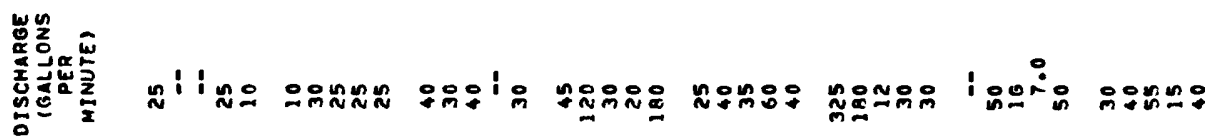

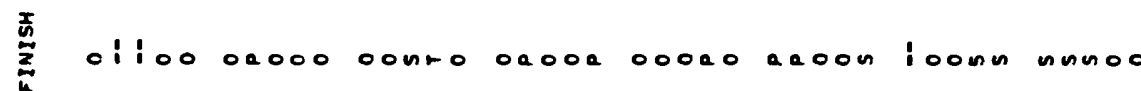

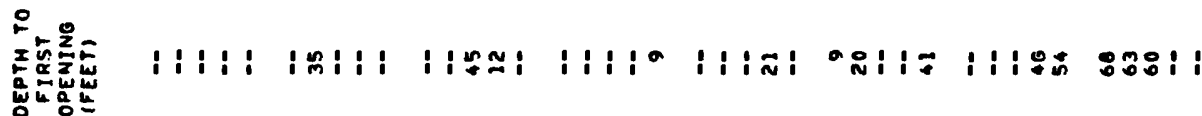
题

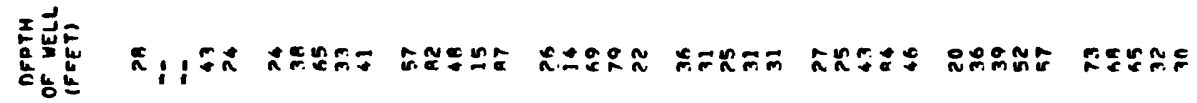

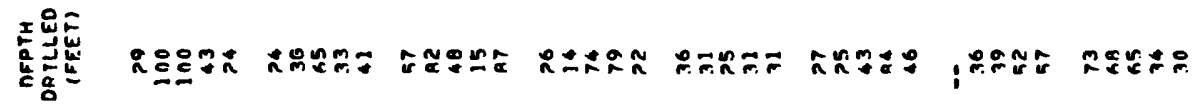

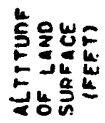

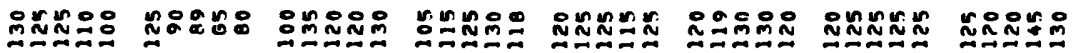

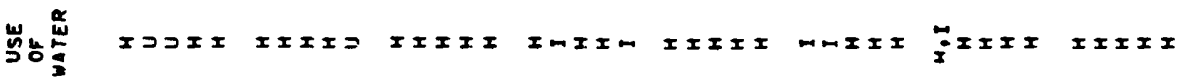
先

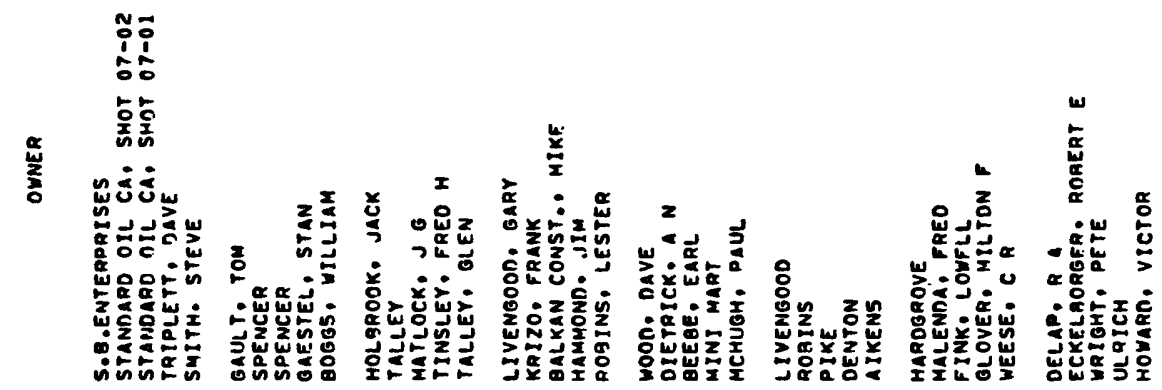




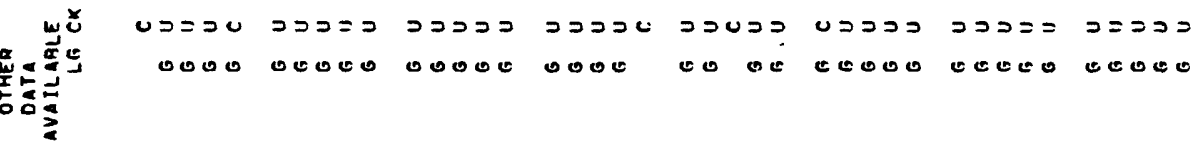

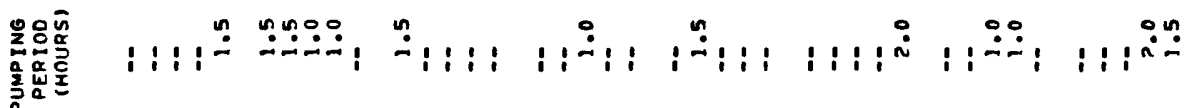

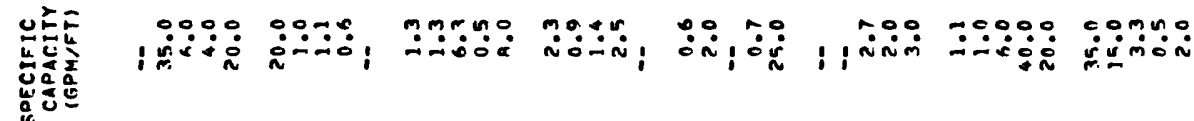

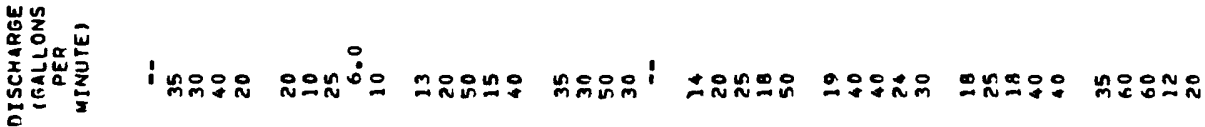

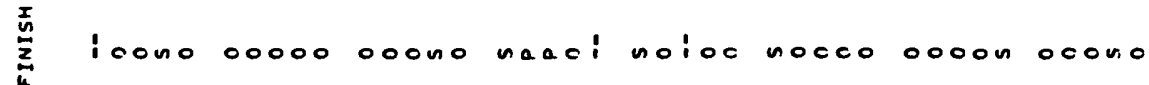
웅

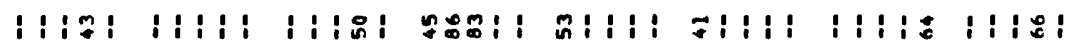
造

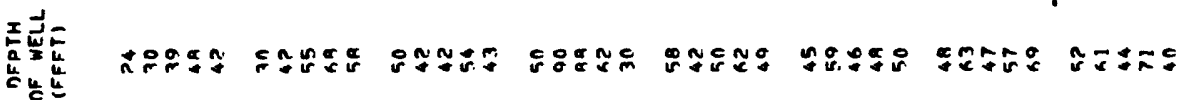

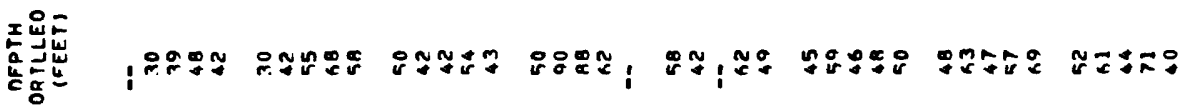

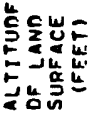

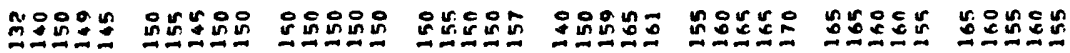

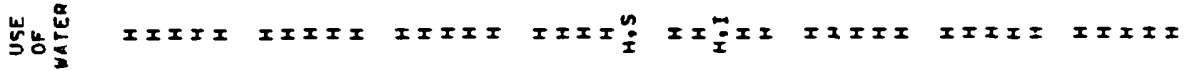

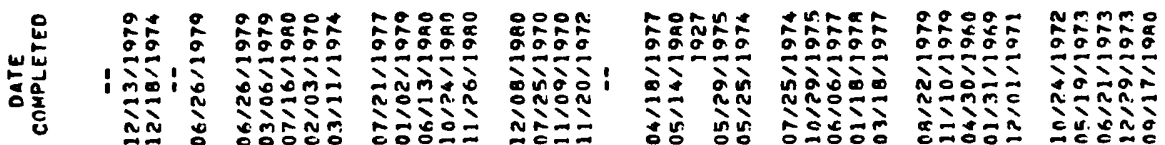




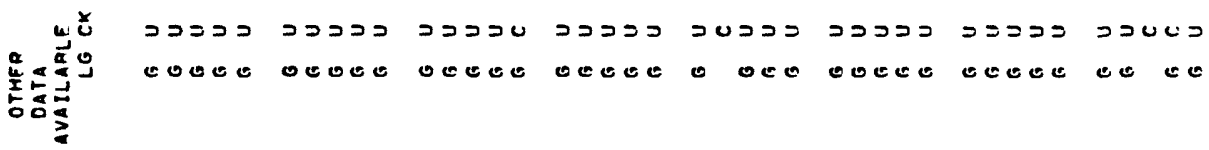

还最产

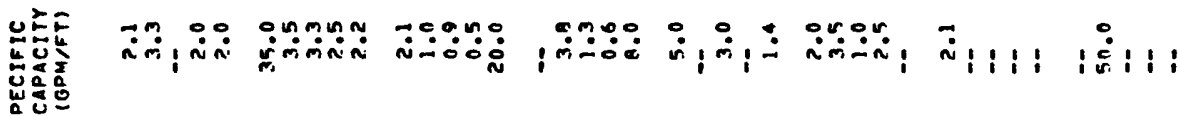

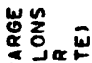

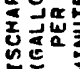

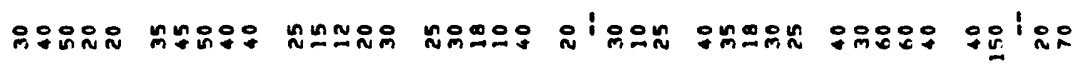

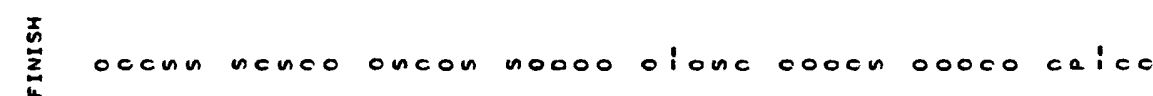

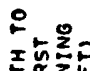

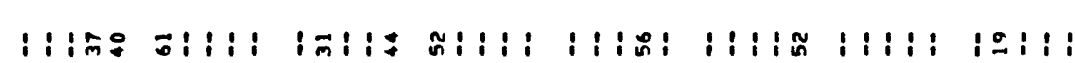

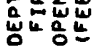
v

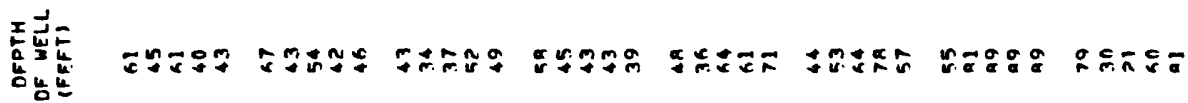

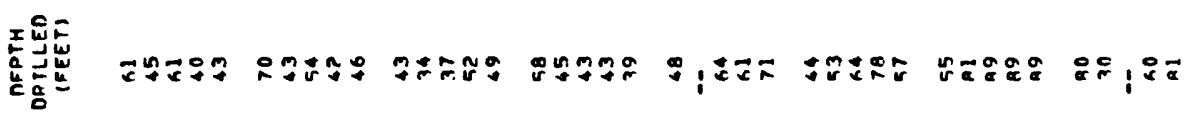

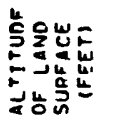

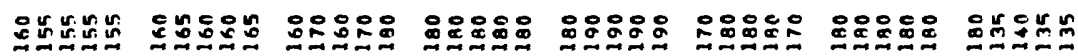

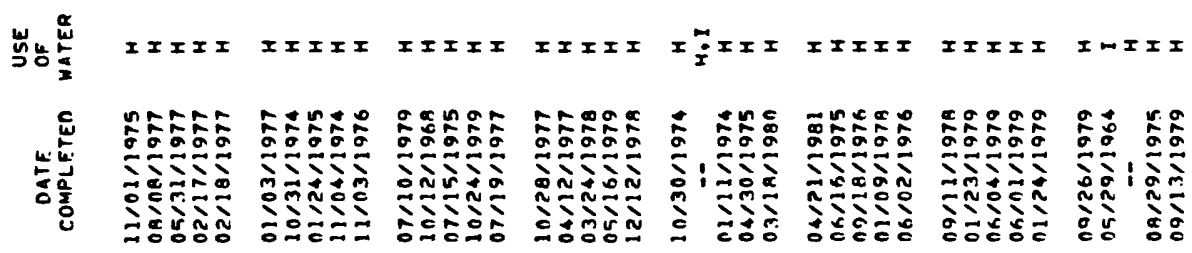

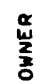
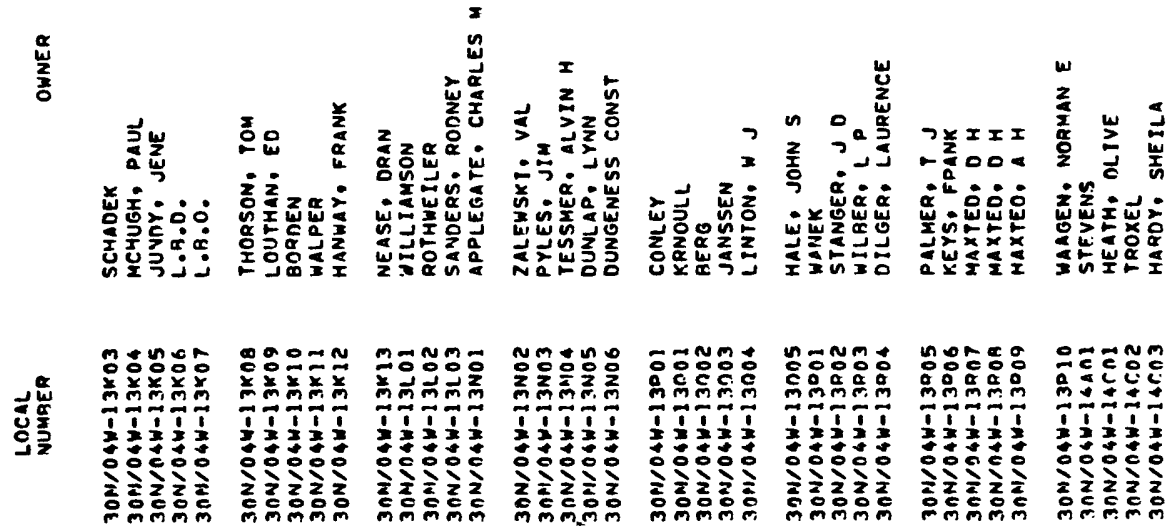


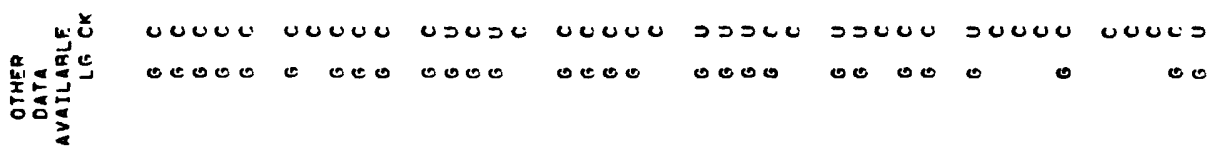

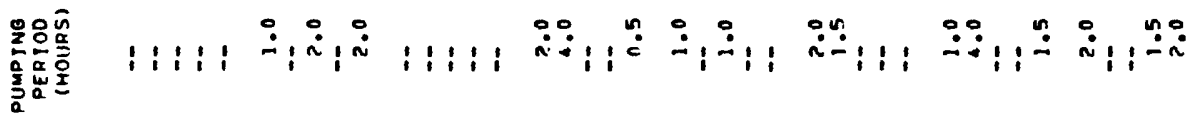

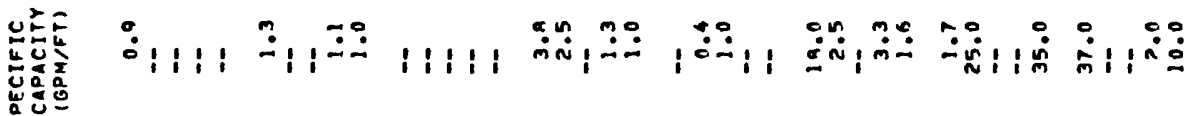

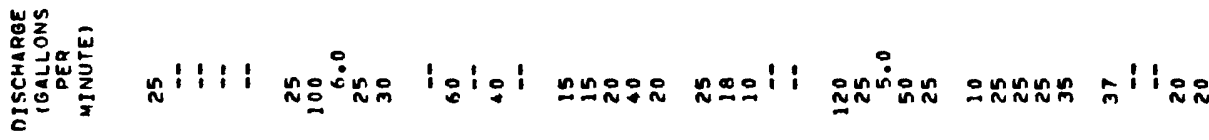

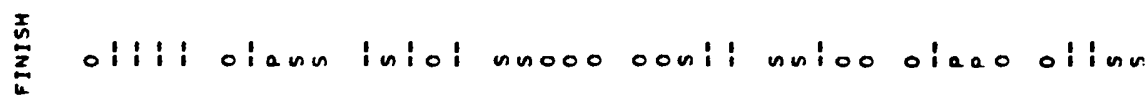

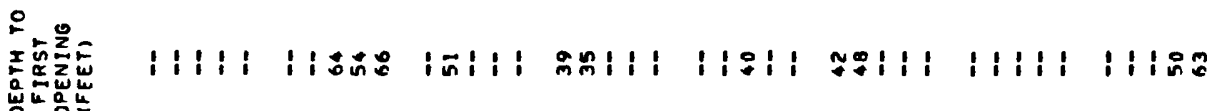

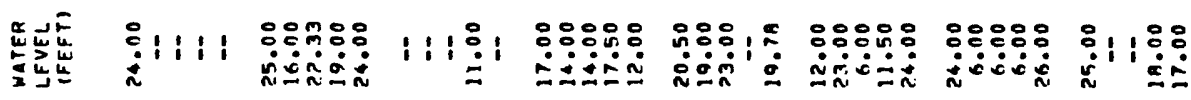

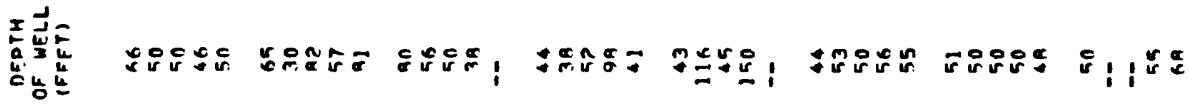

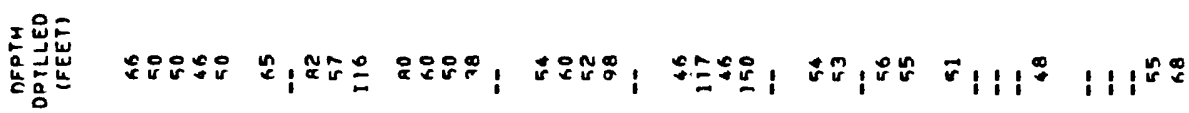

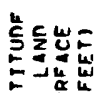

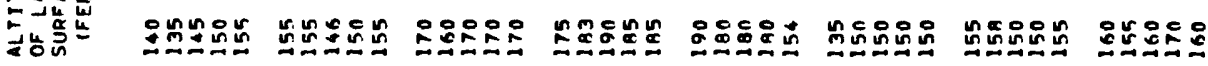

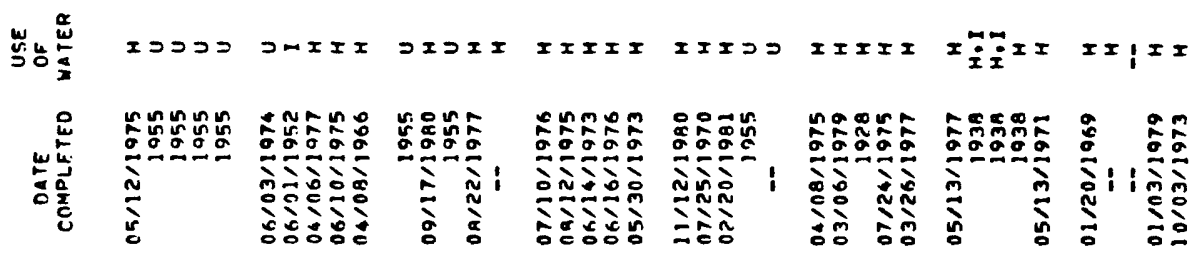

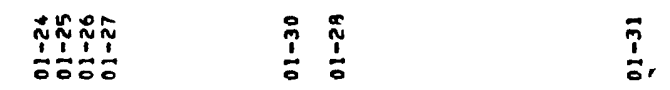

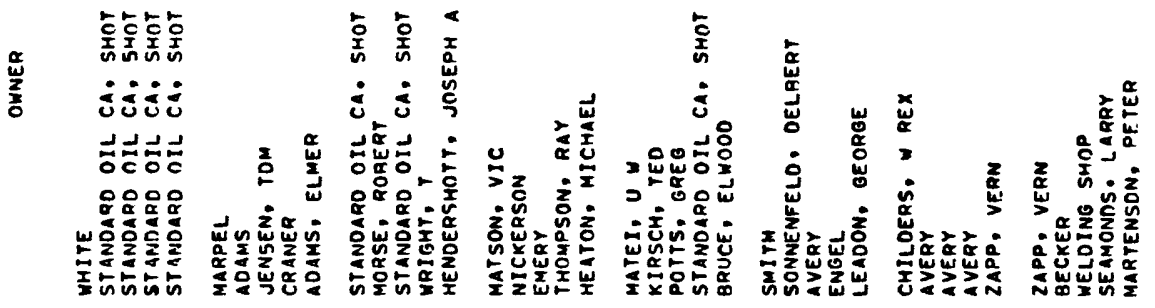

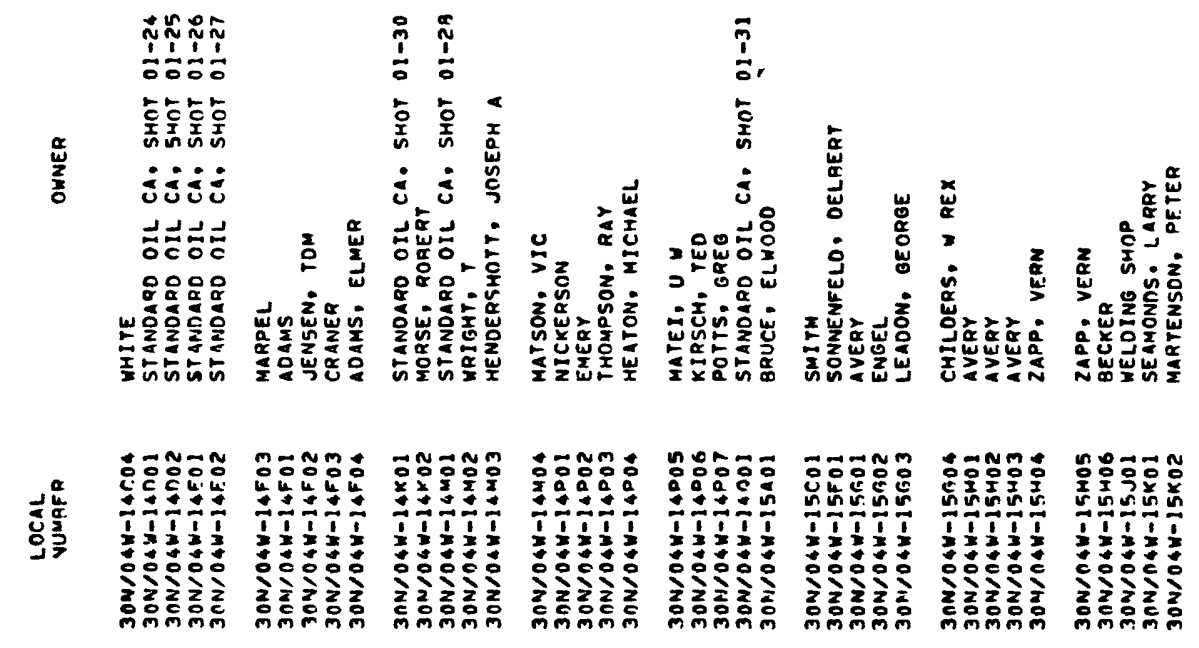




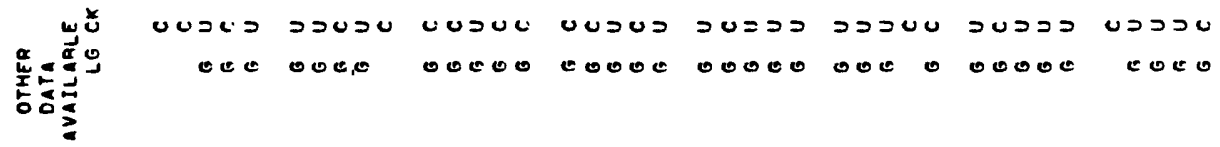

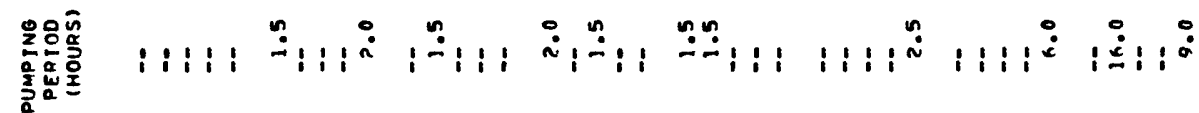

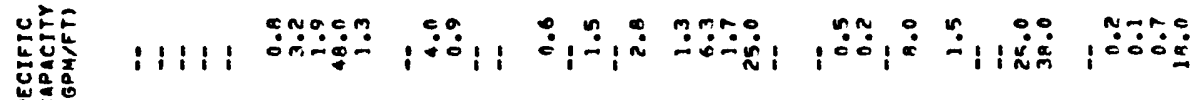

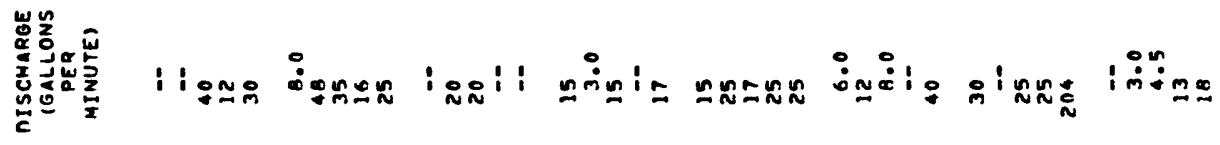

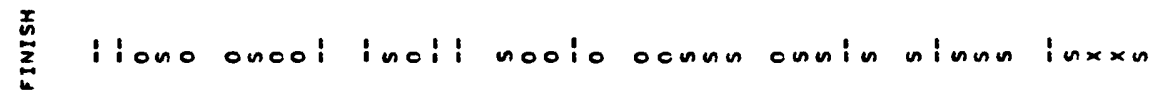
2

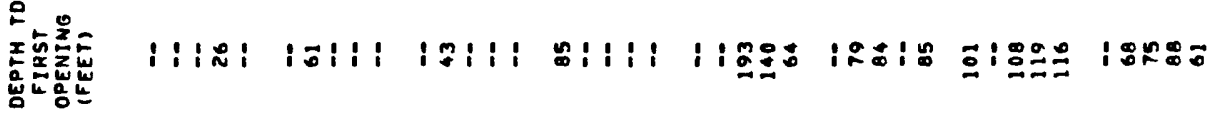

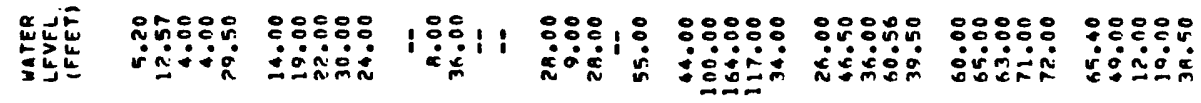

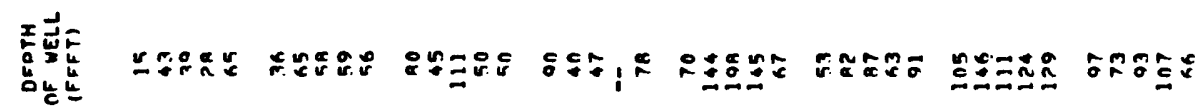

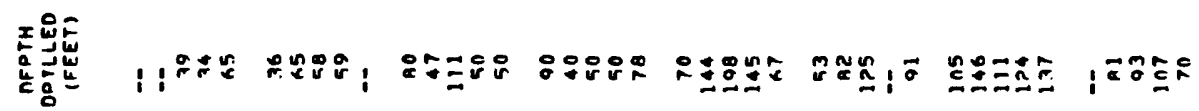

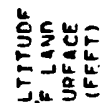

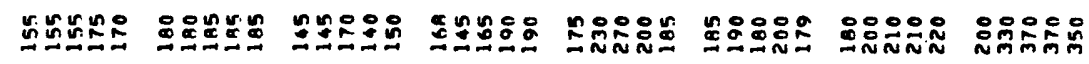

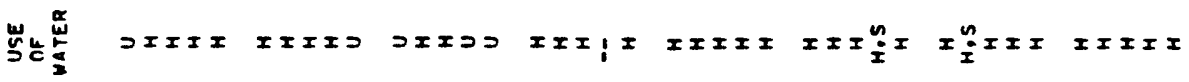

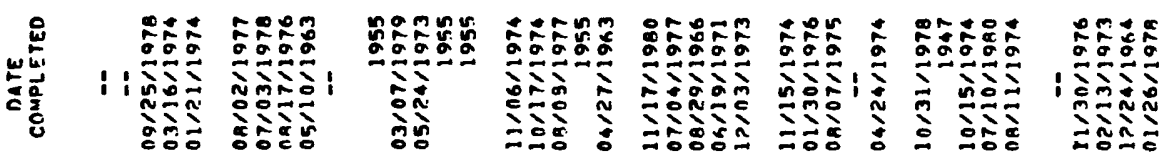

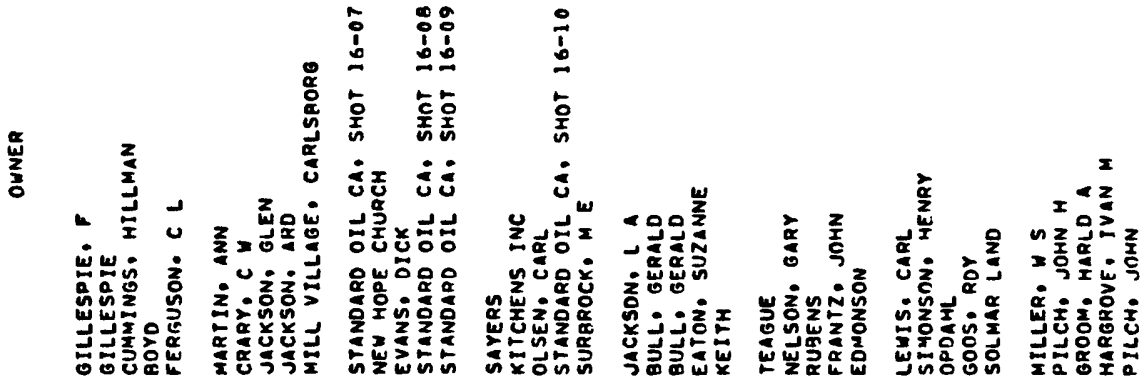

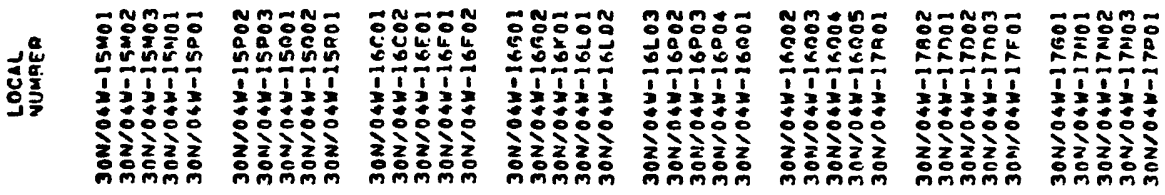




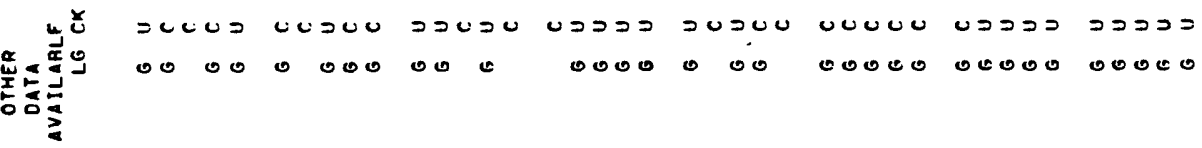
送亮

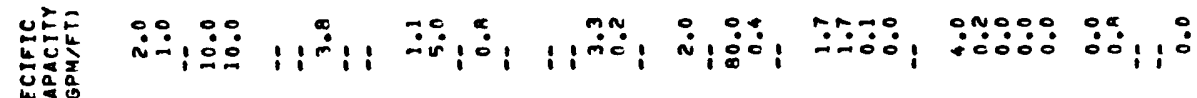

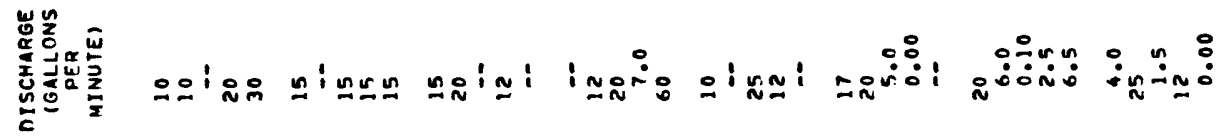

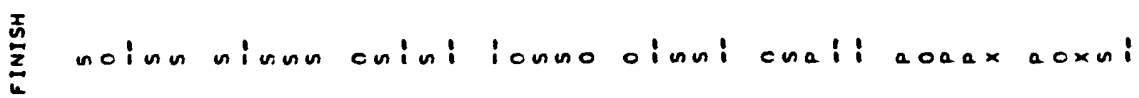

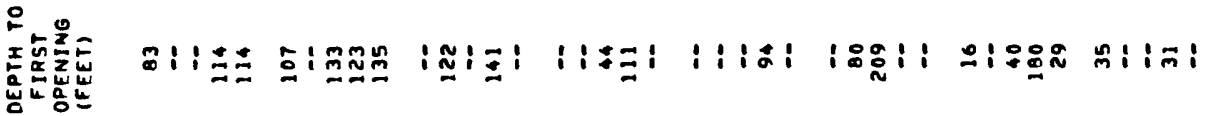

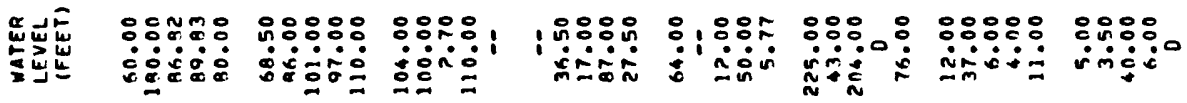

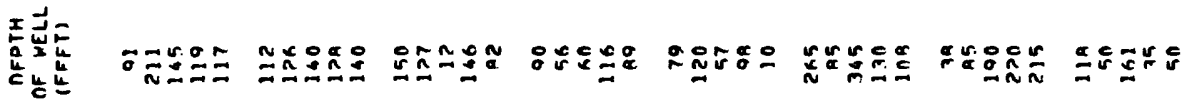

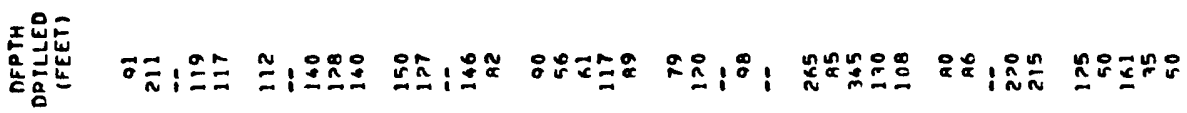

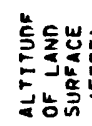

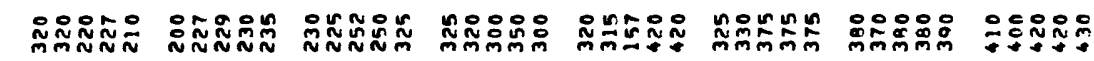

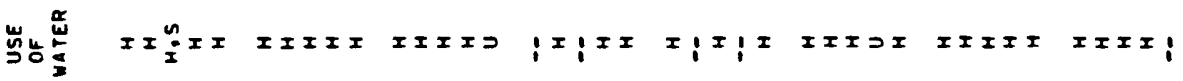
至

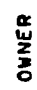

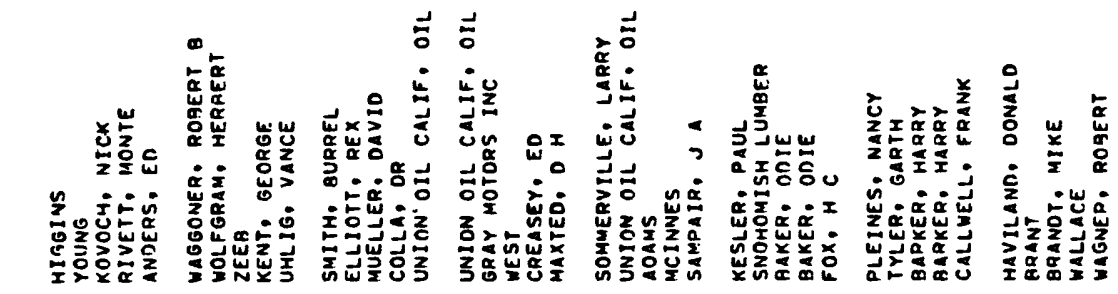

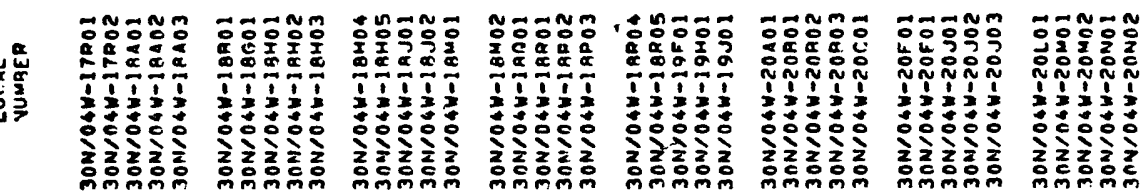




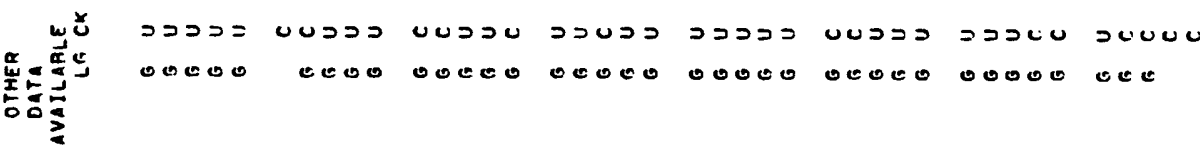

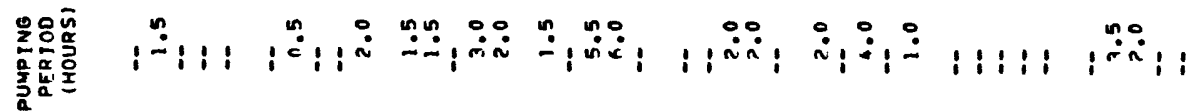

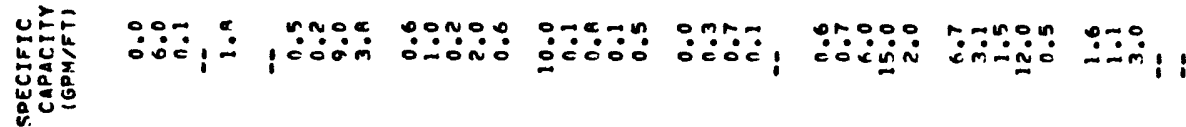

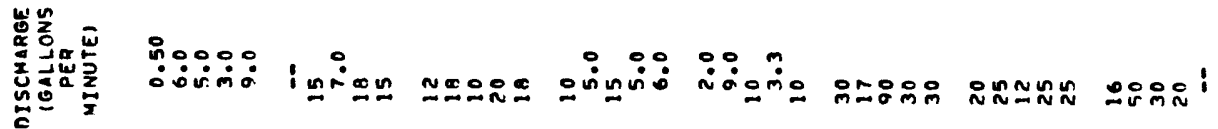

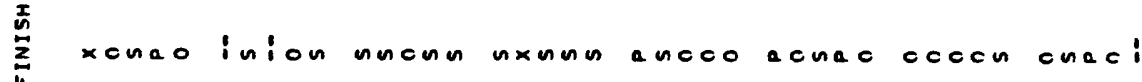
$\therefore$

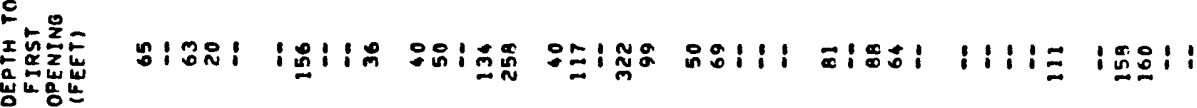

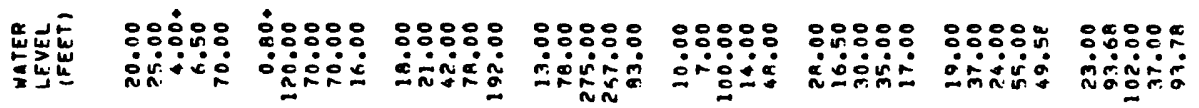

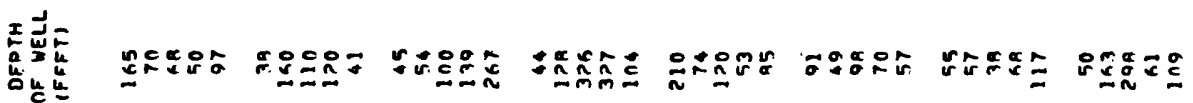

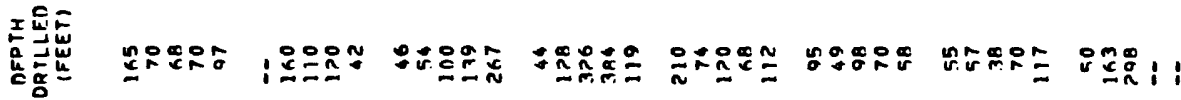

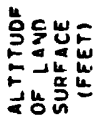

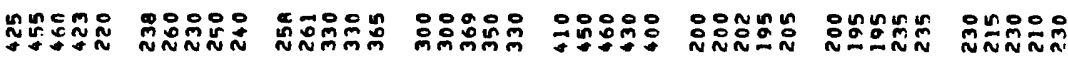

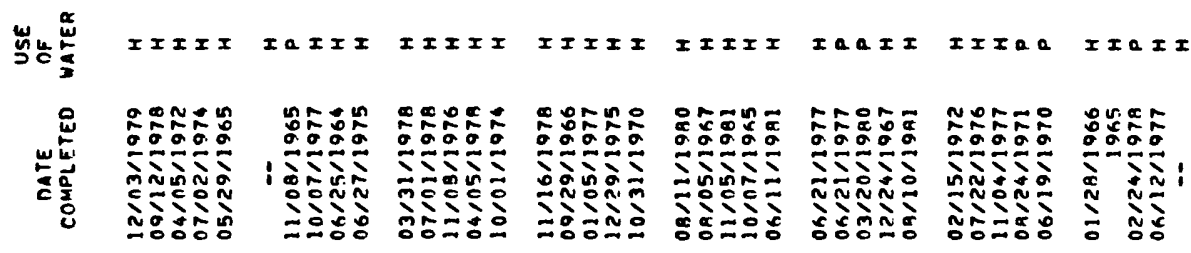




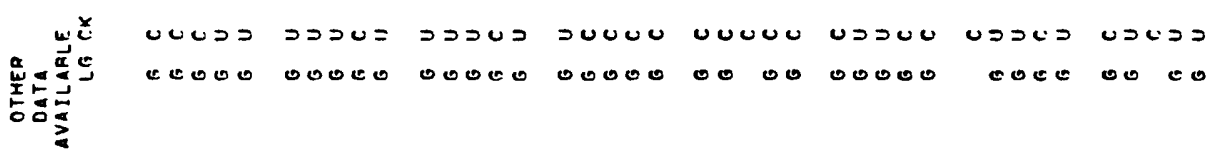
登最

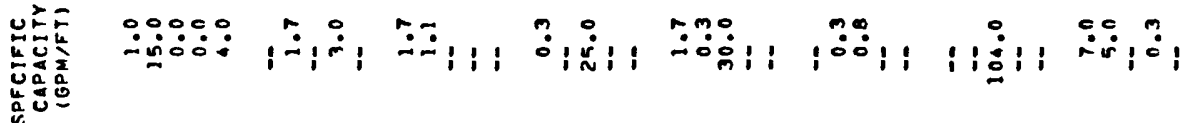

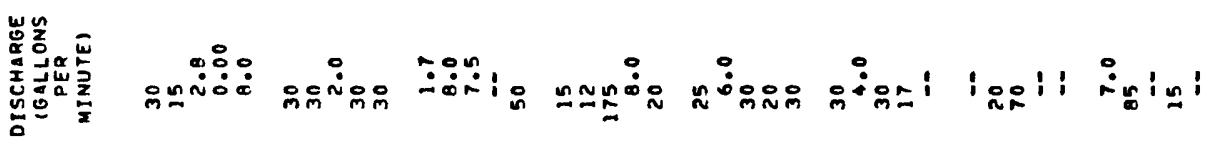

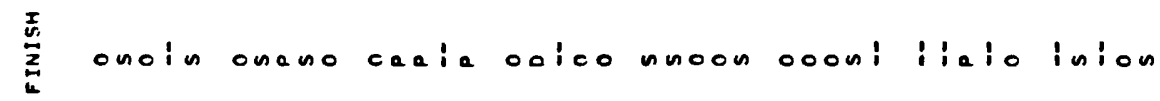

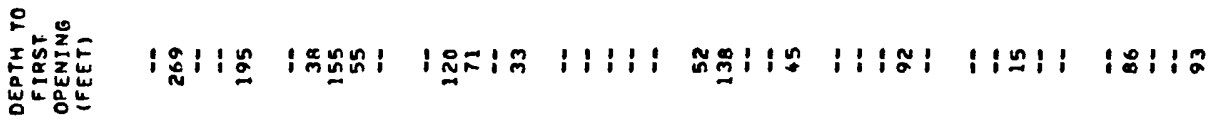

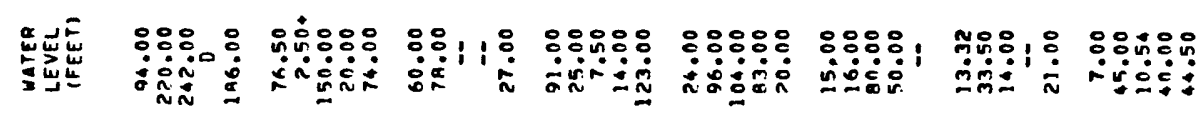

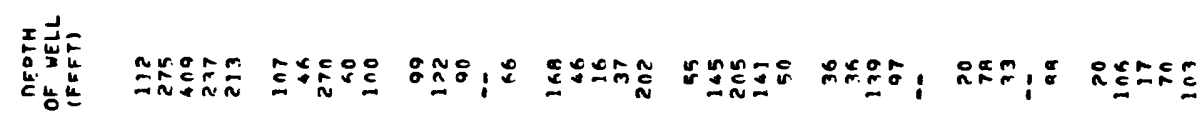

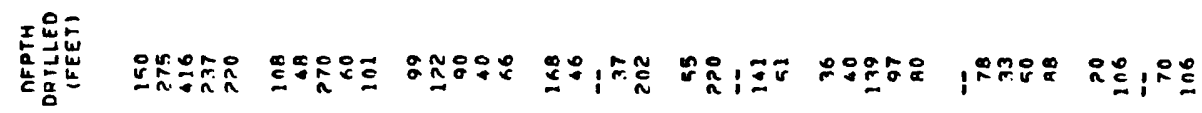

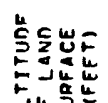

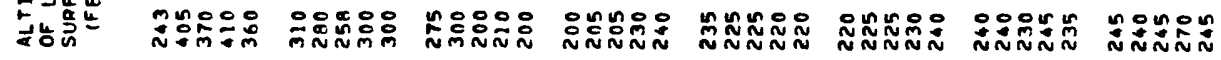

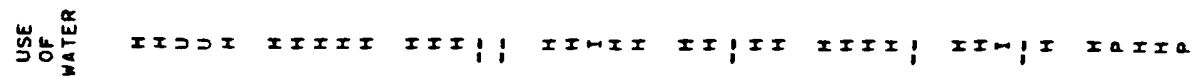
先
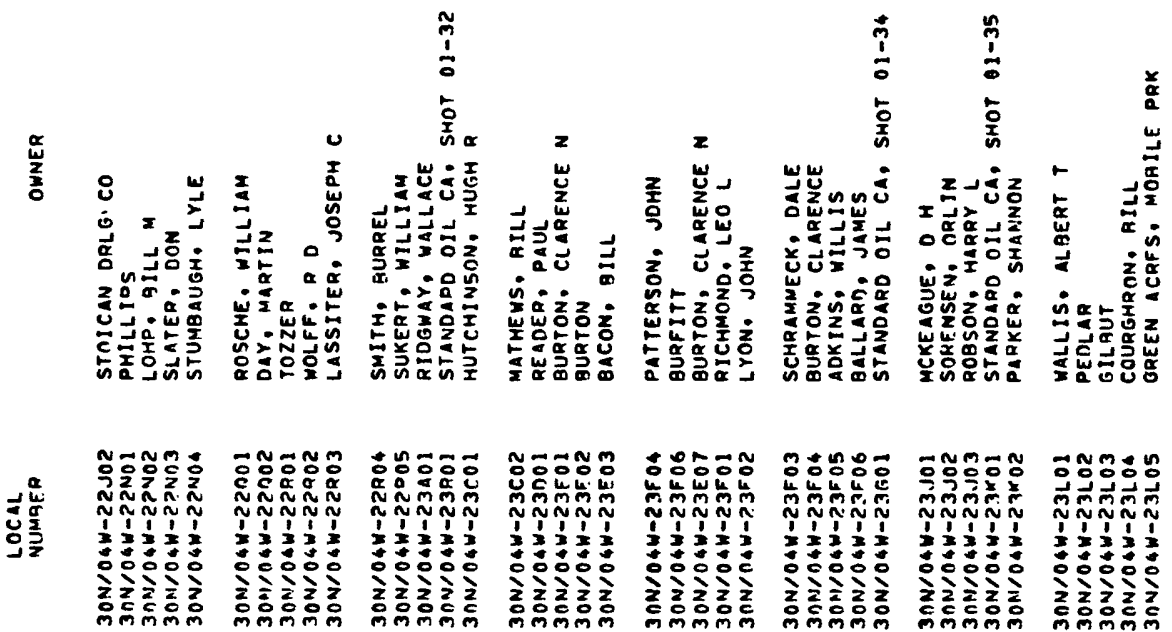


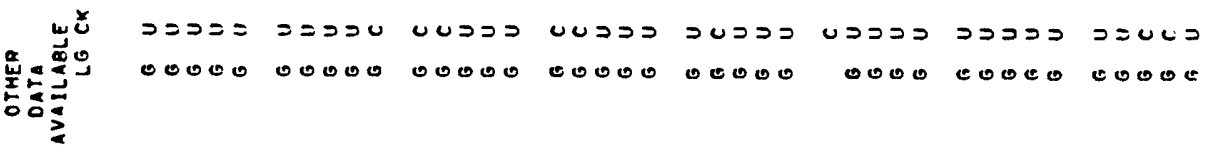

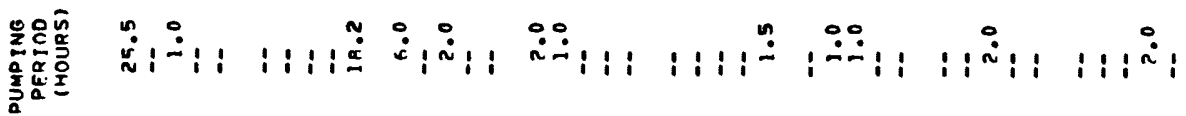

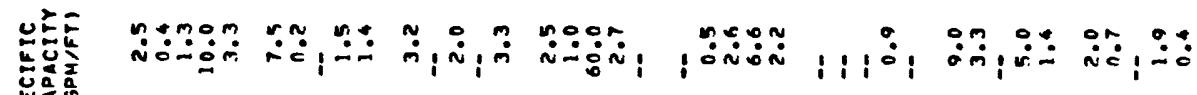

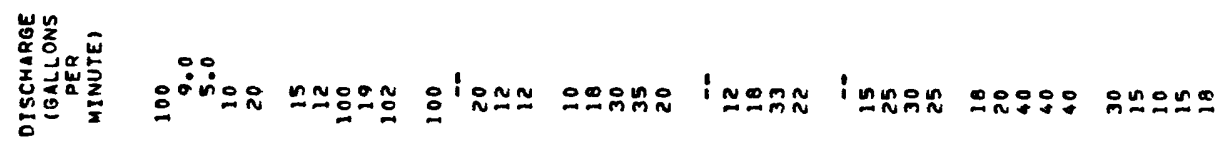

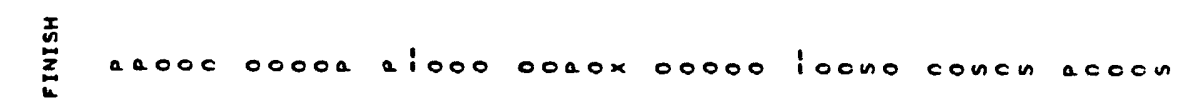

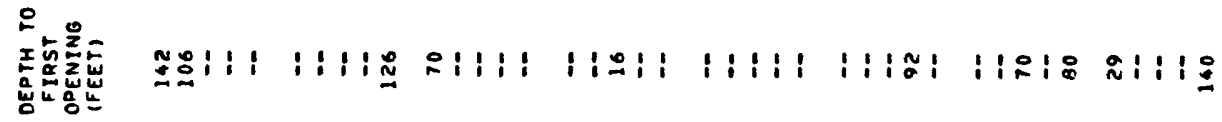

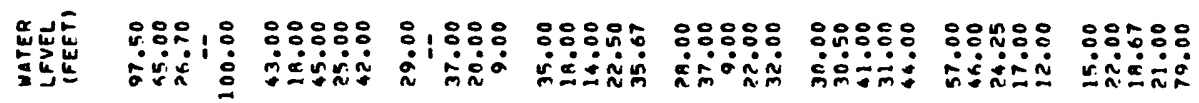

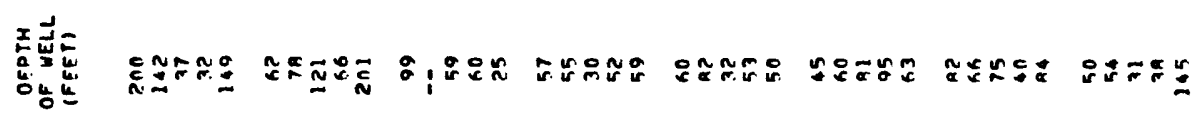

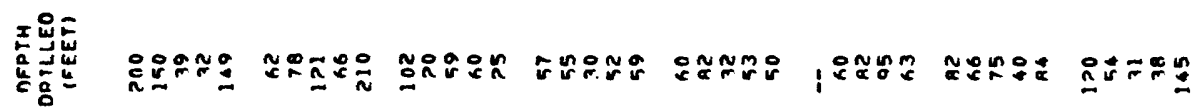

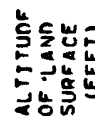

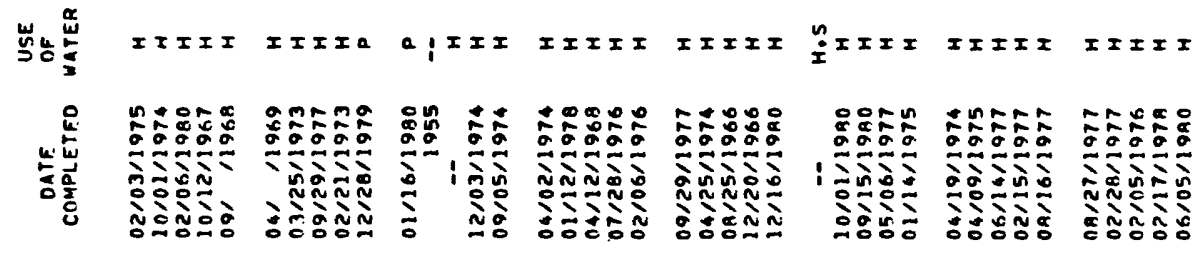
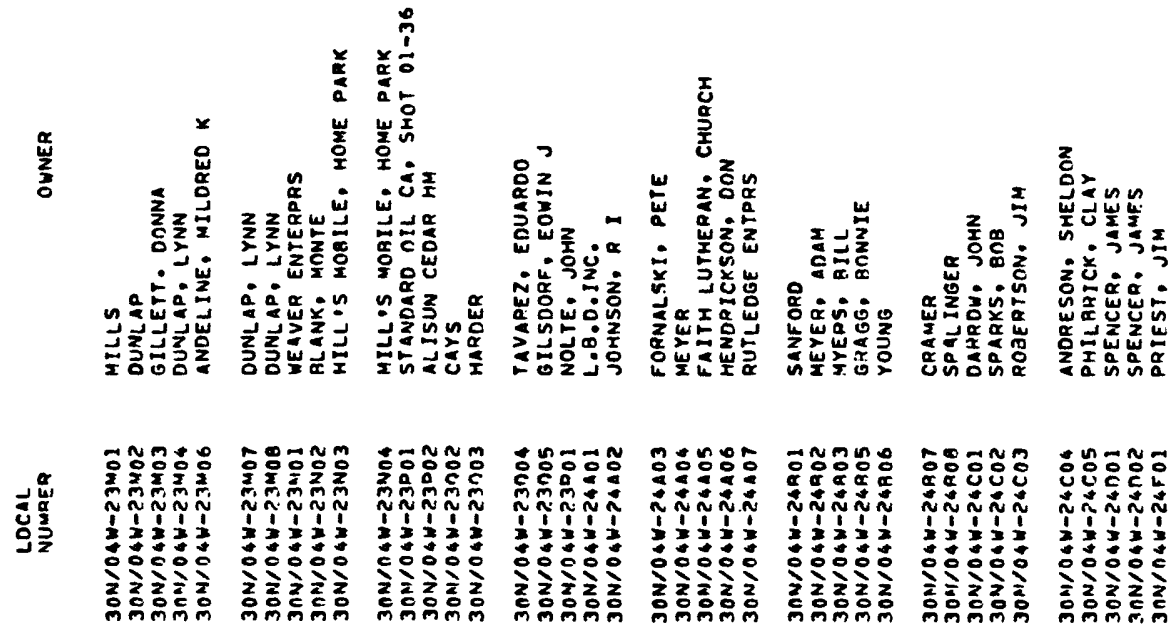


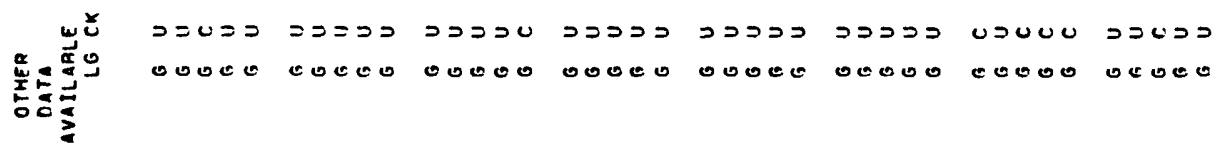
退亮

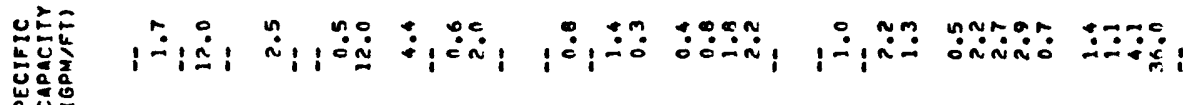
要

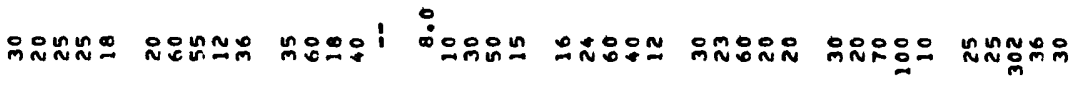

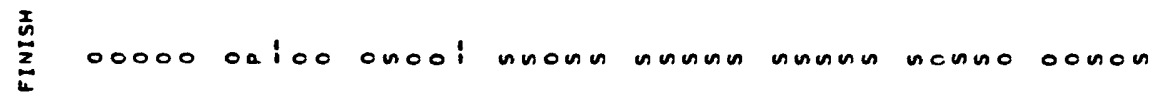

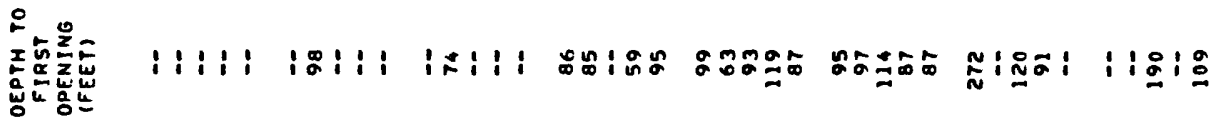
x

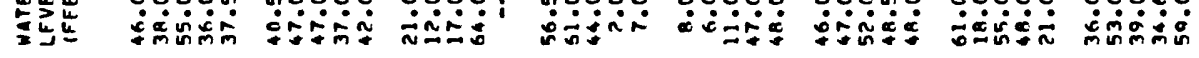

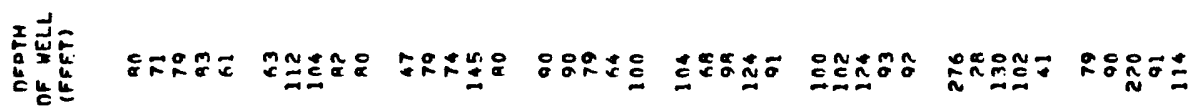

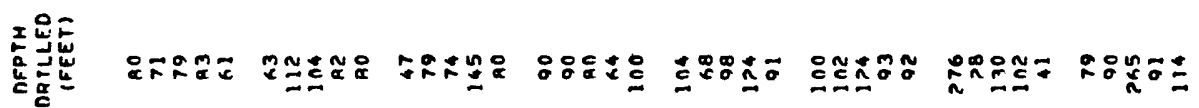

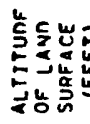

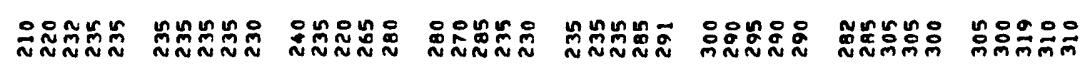

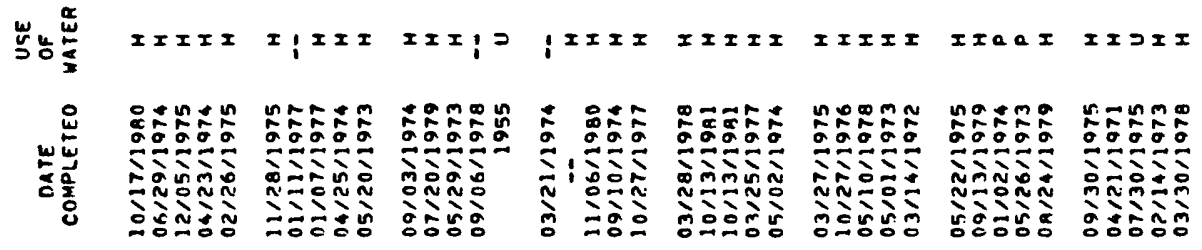

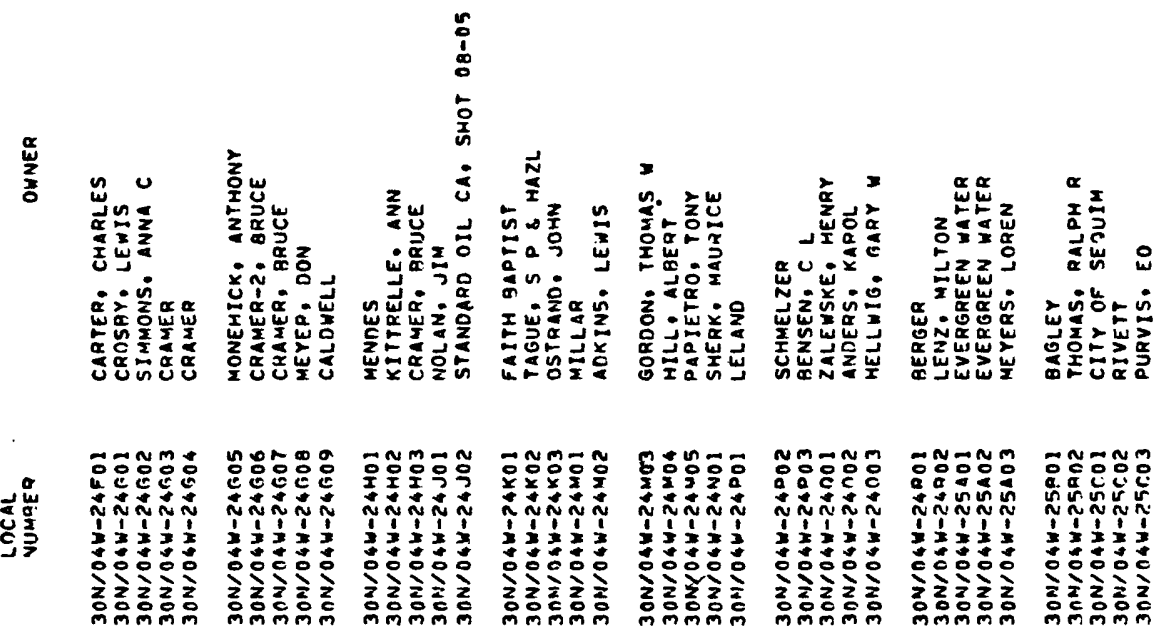




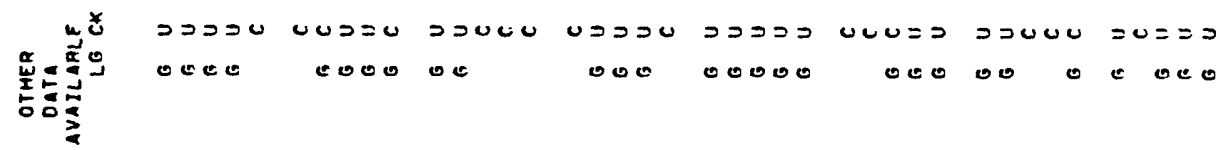

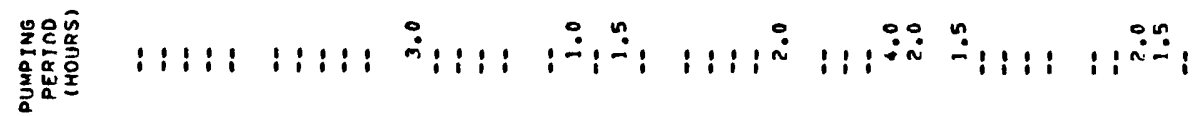

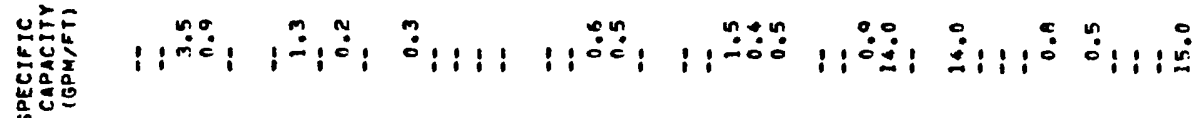
窟

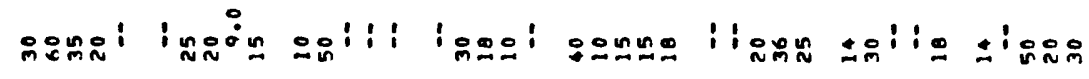

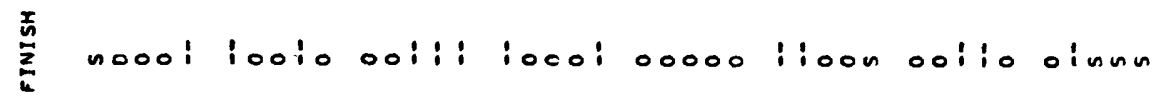

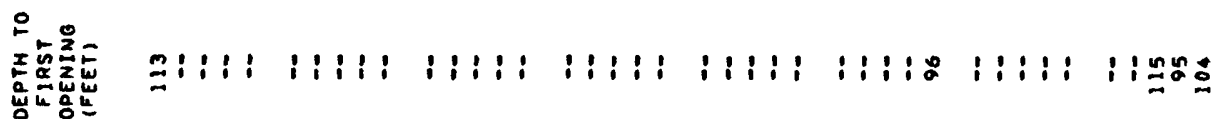

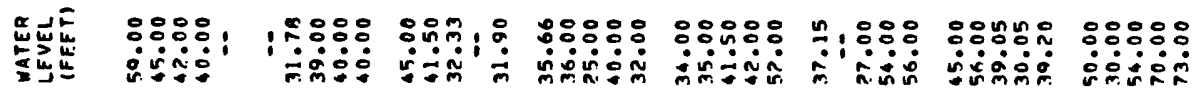

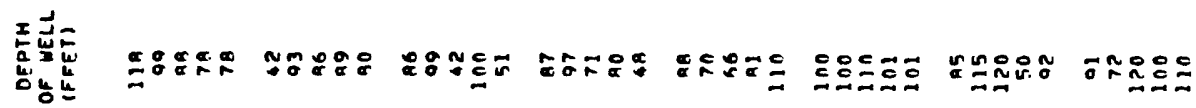

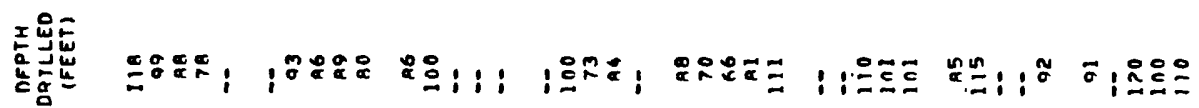

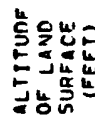

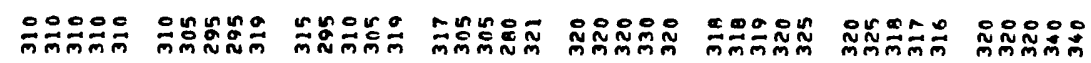

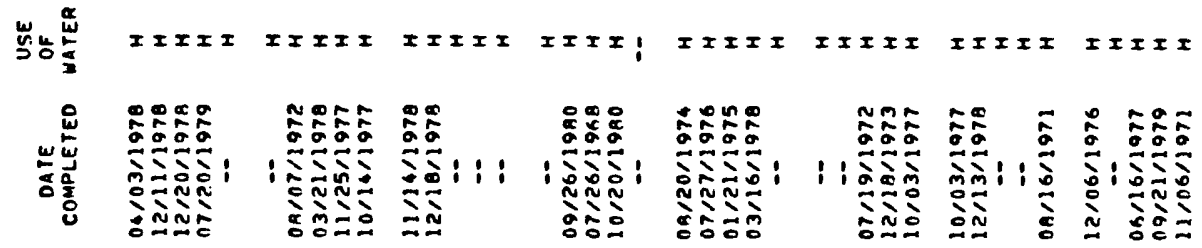

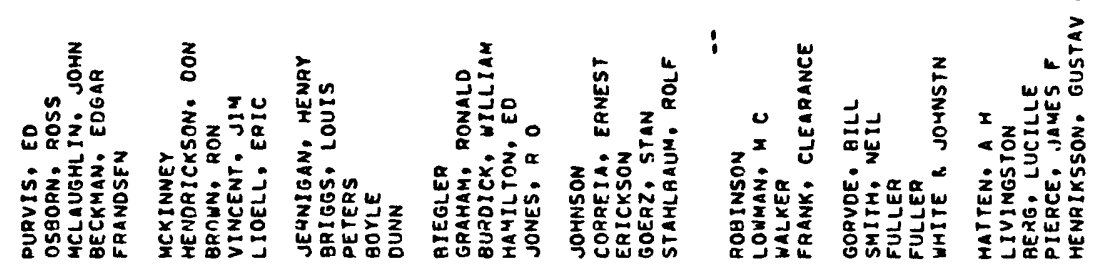

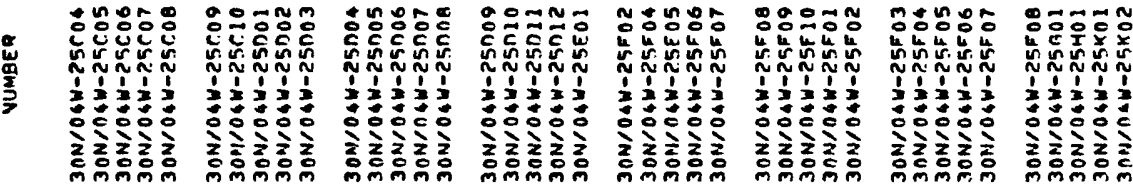




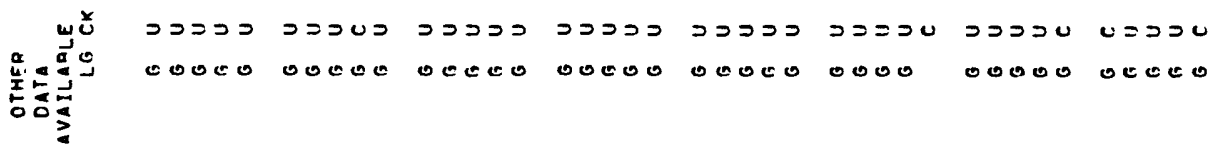

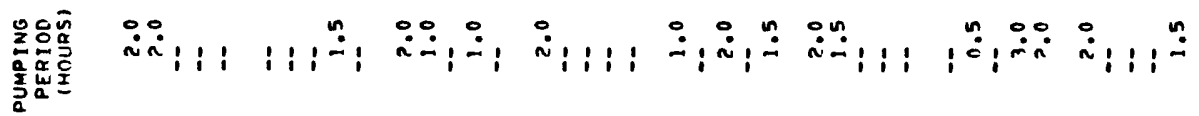

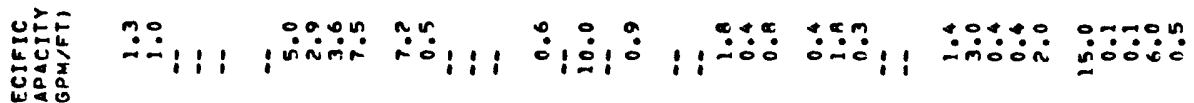

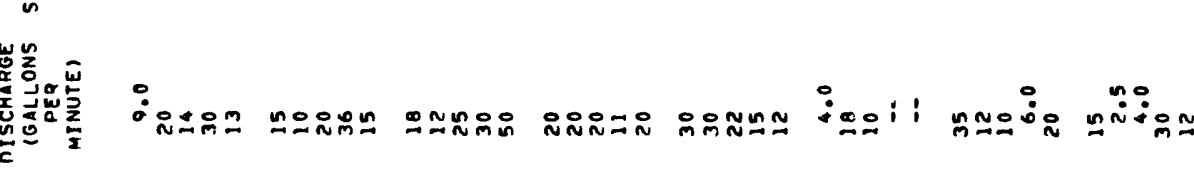

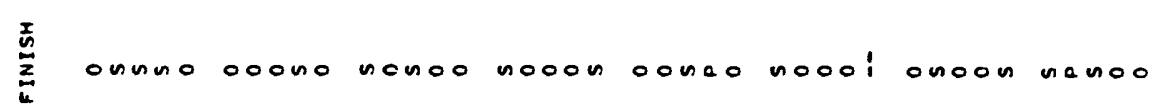

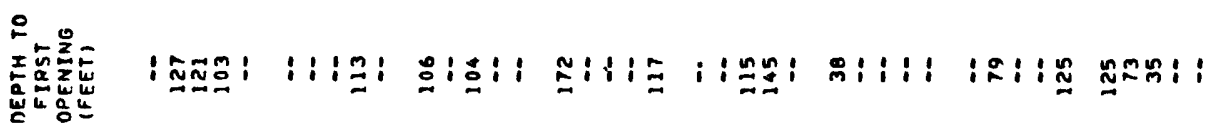
造

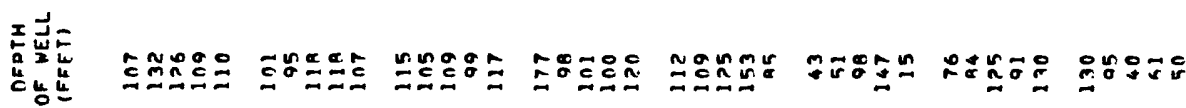

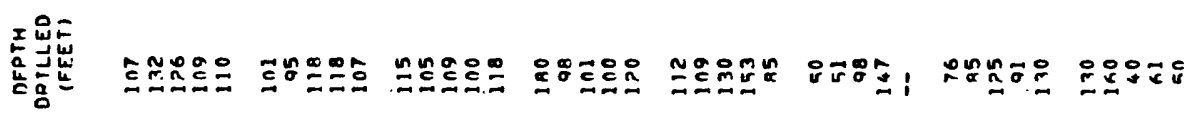
等

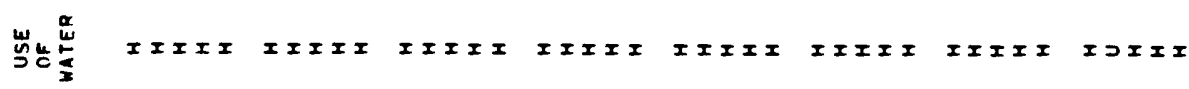
E
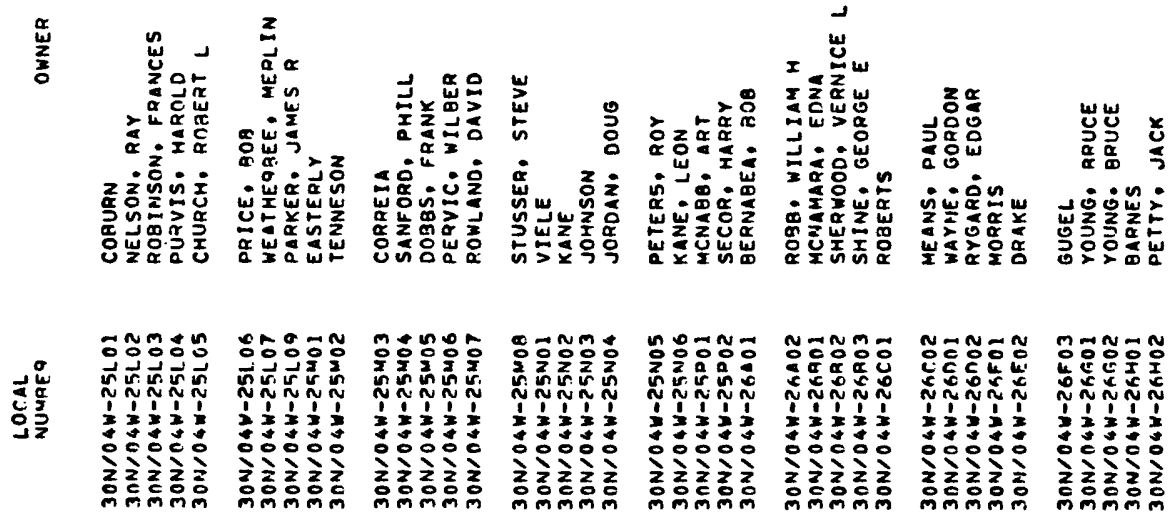


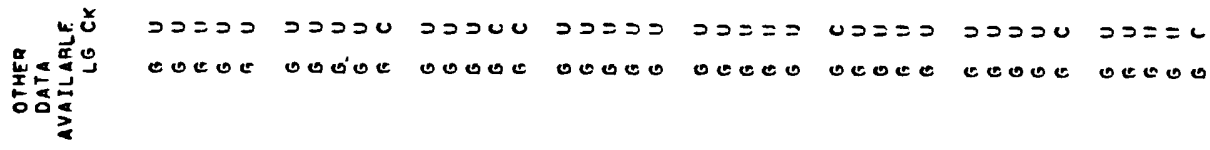

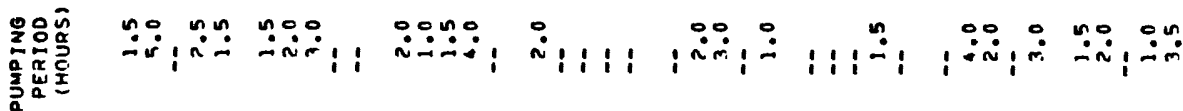

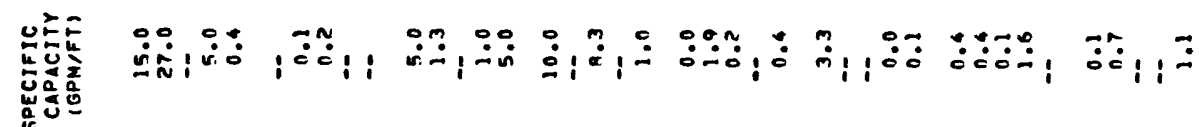

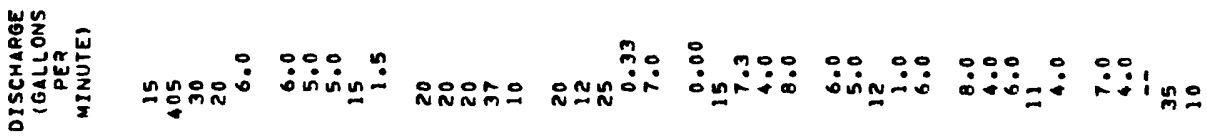

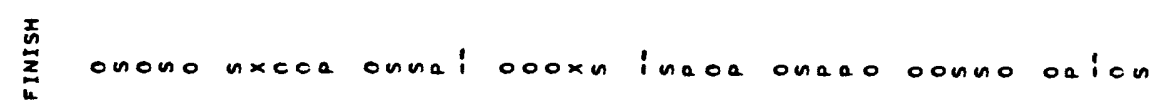

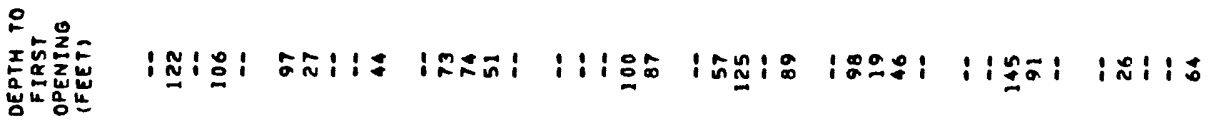

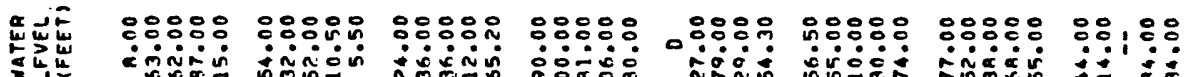

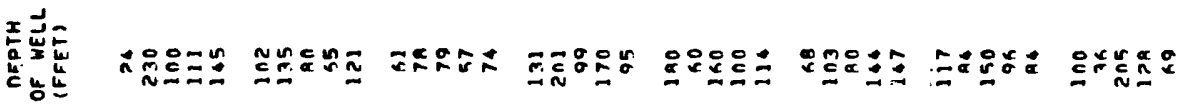

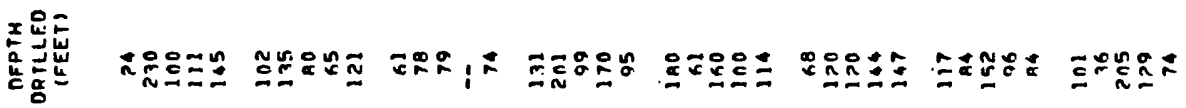
等

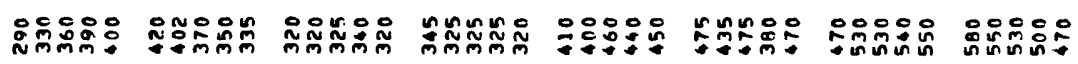

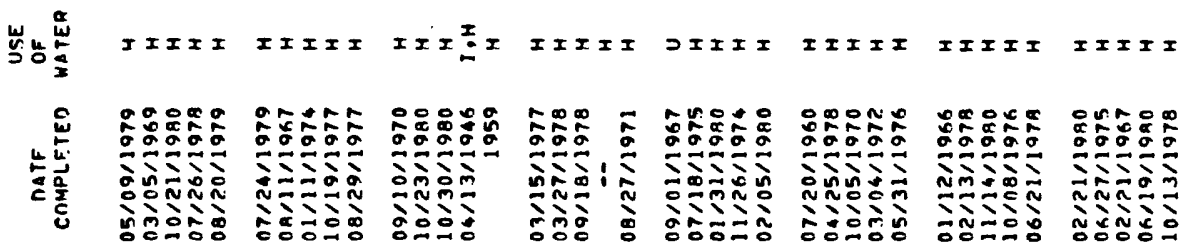

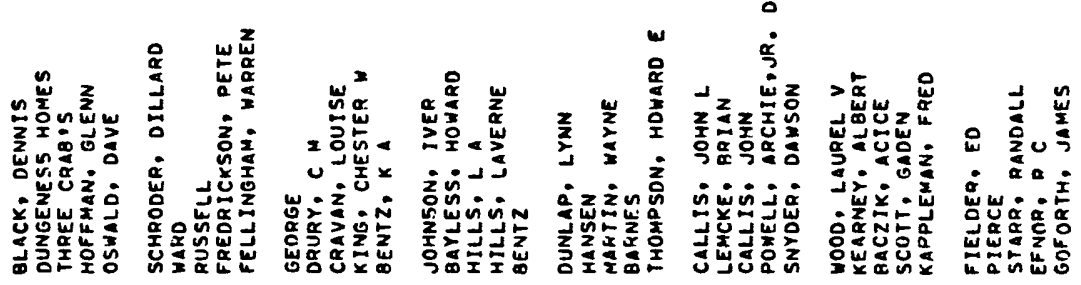




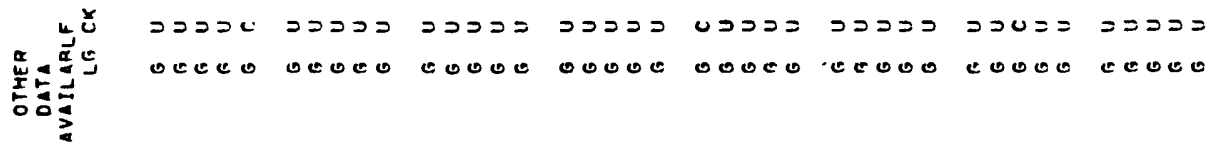

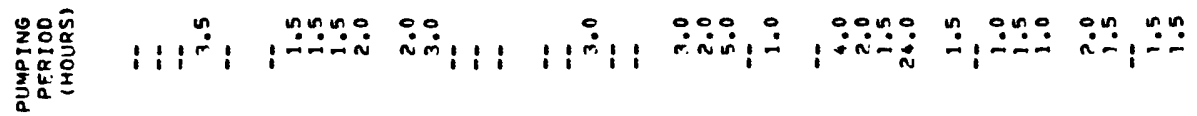

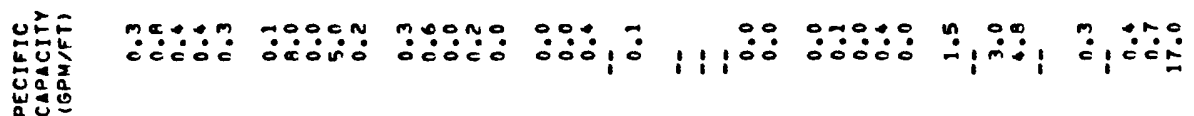

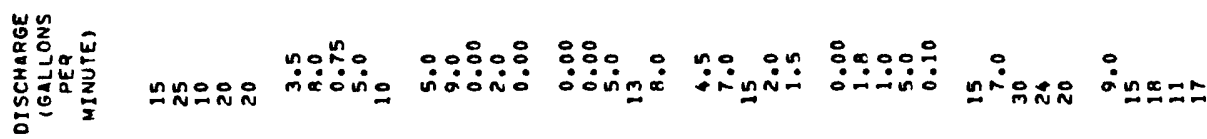

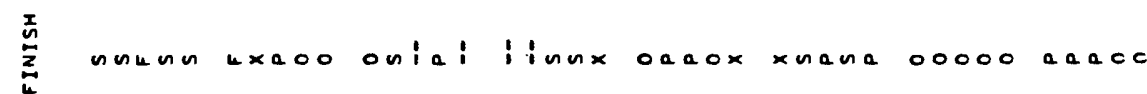

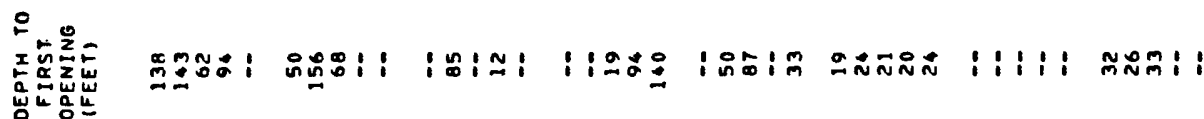

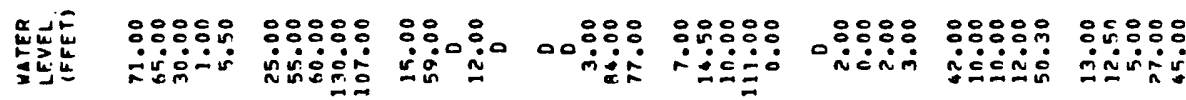

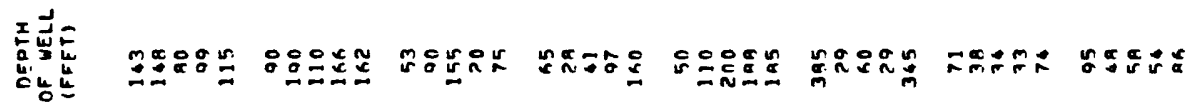

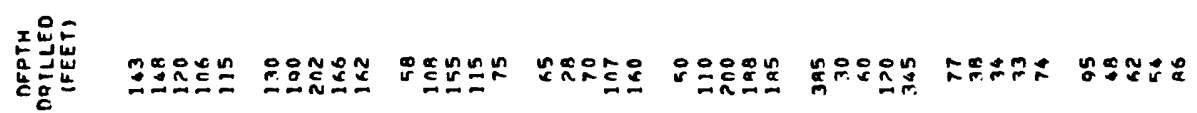

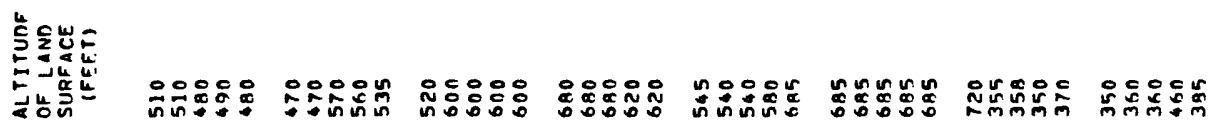

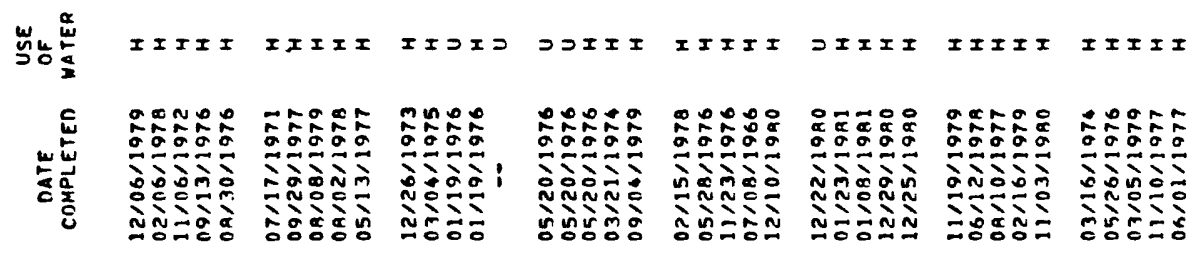
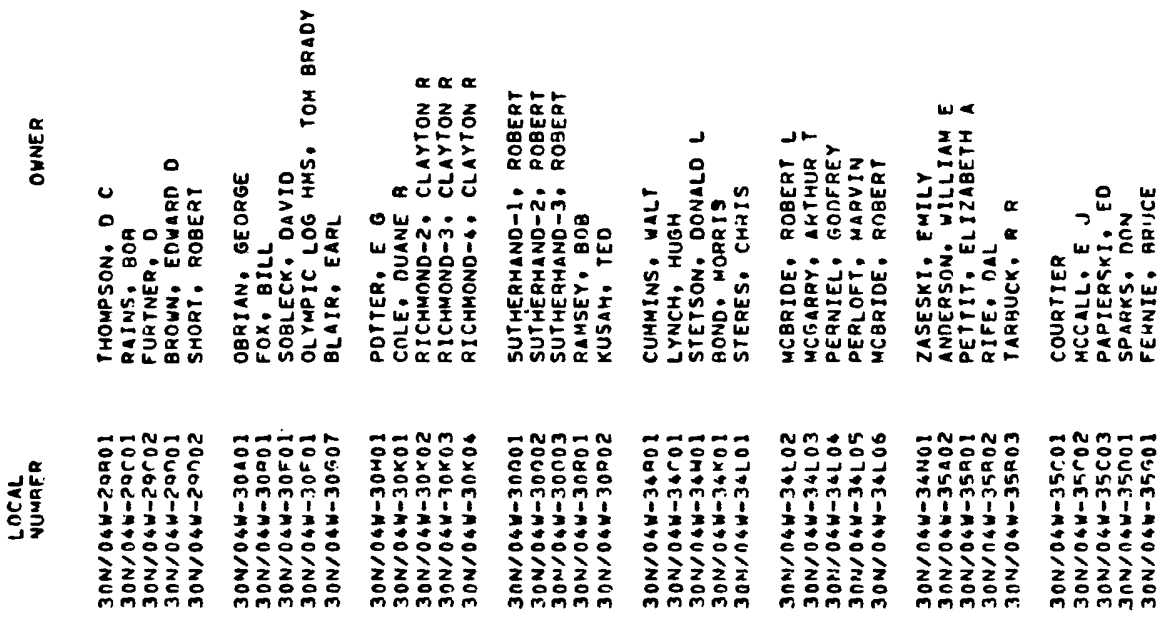


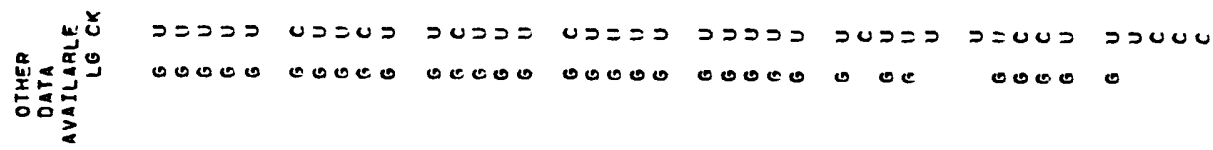

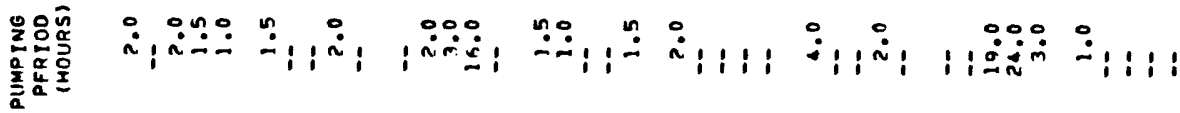

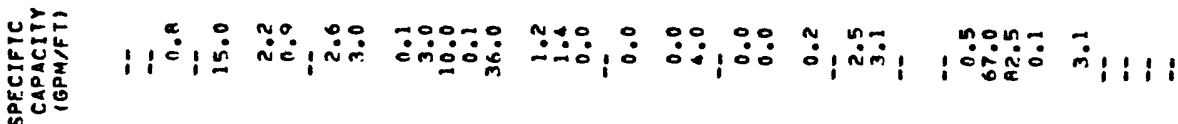

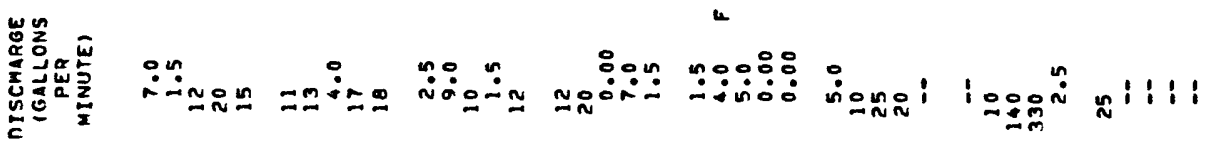

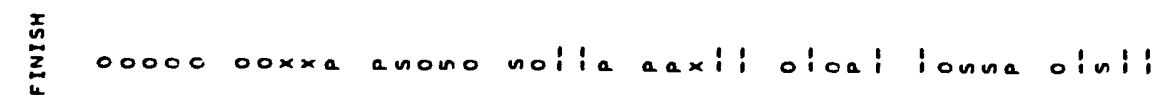

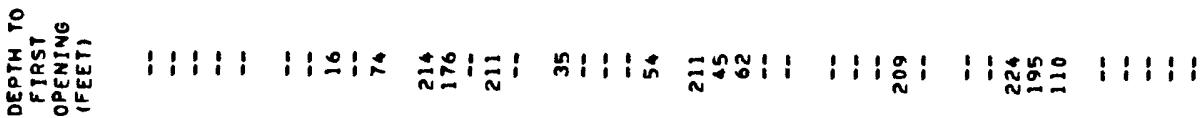

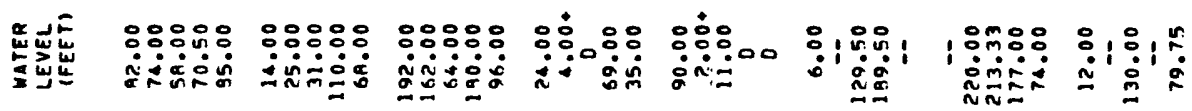

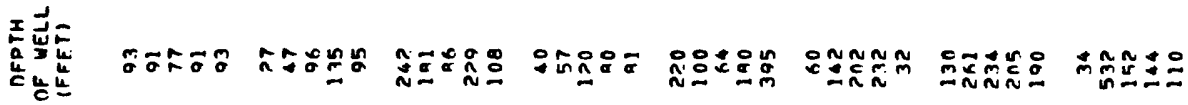

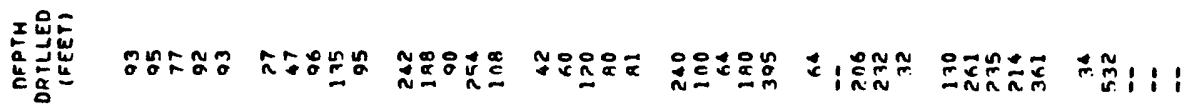
等

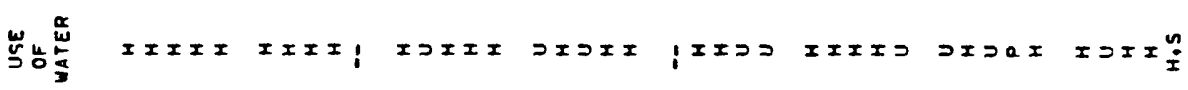

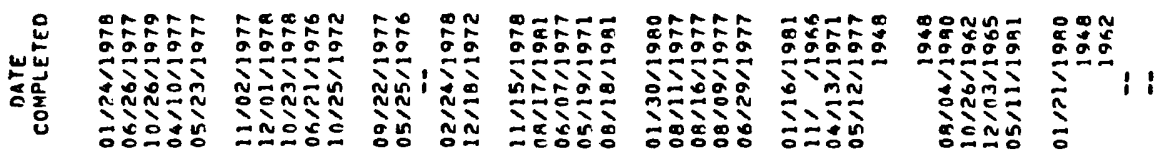

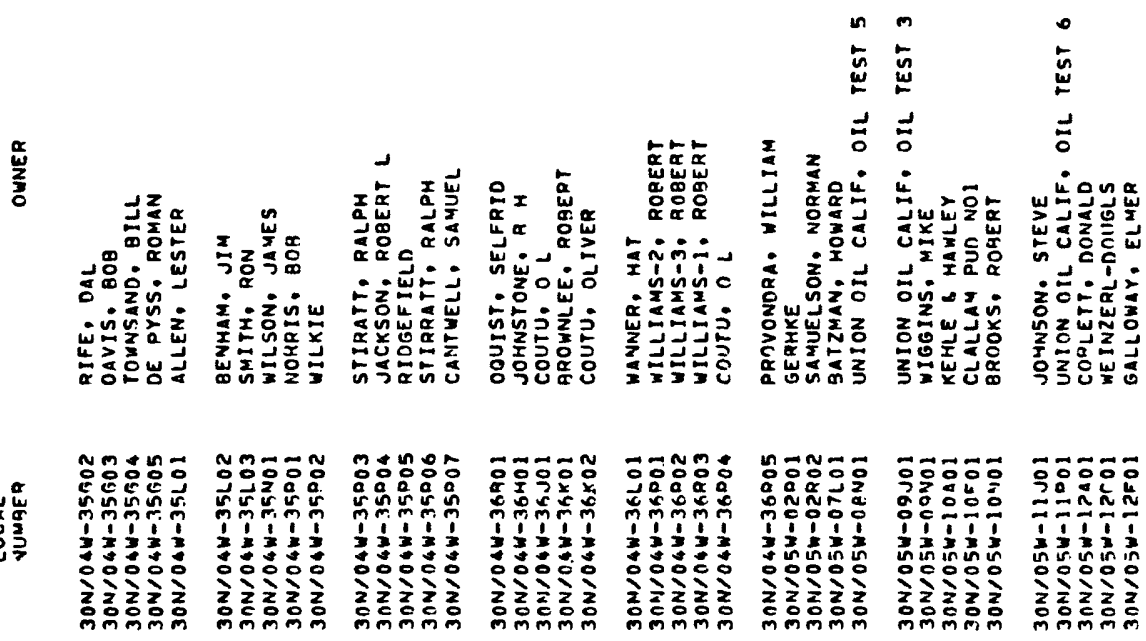




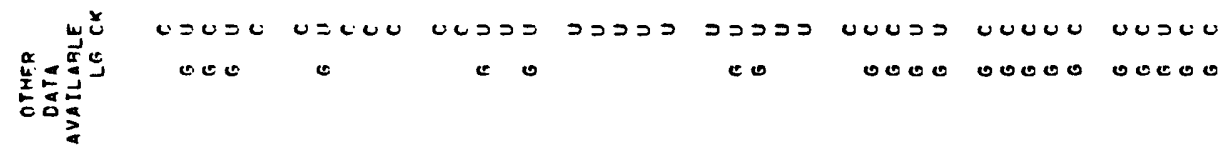

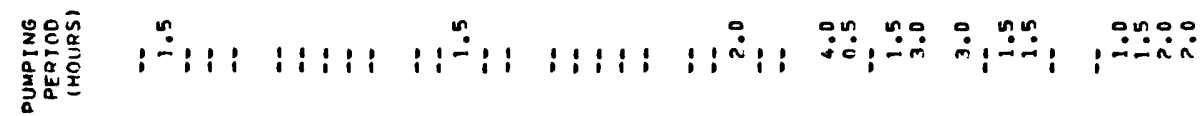

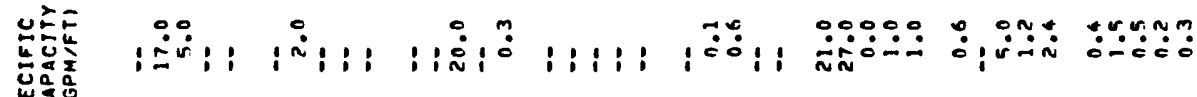

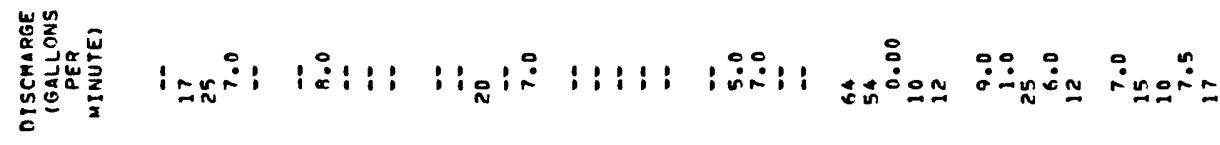

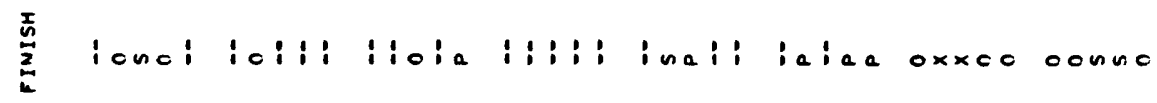

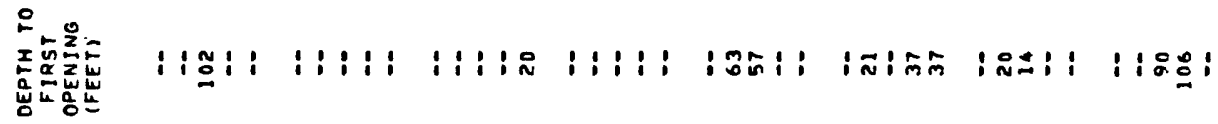

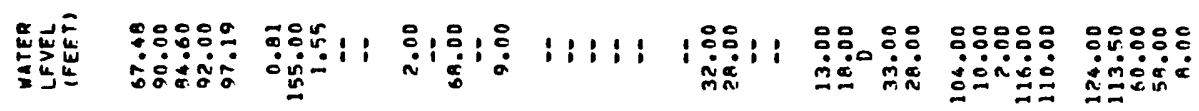

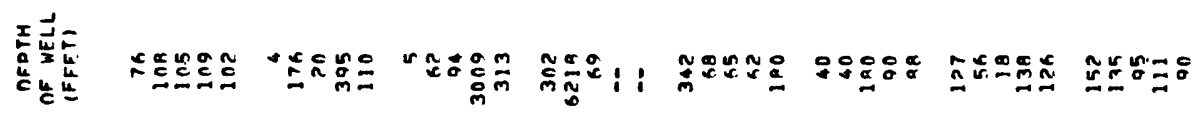

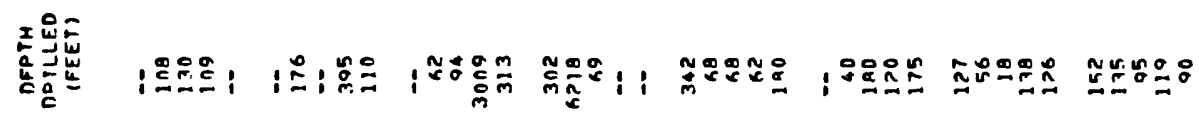

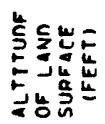

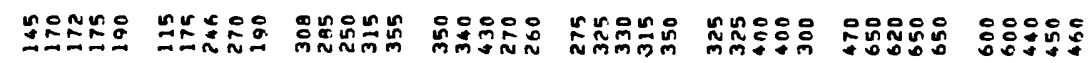

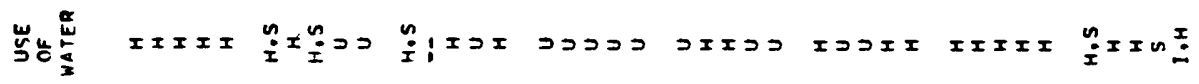
势

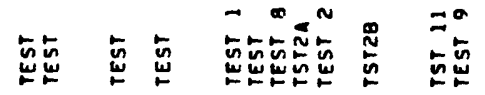

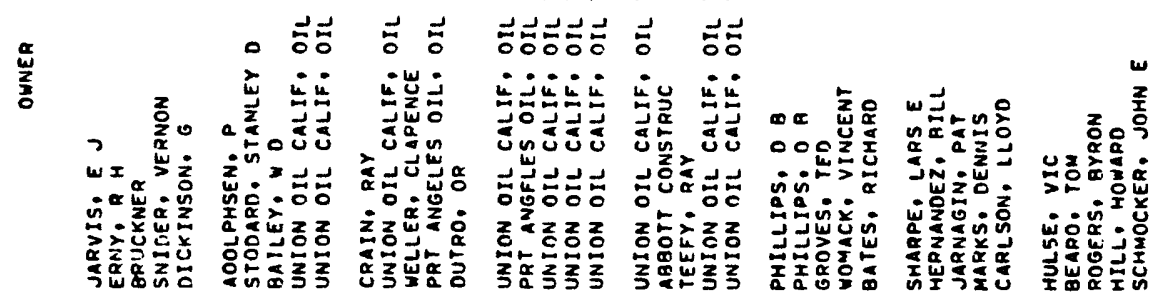

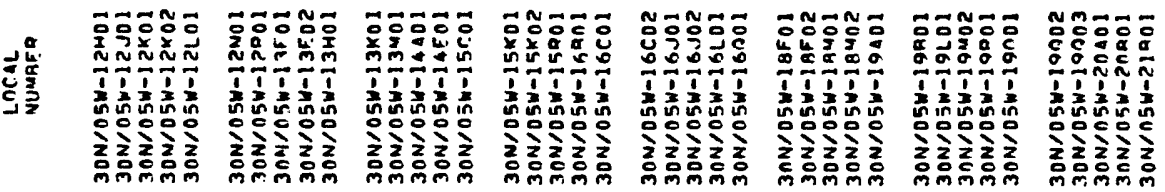




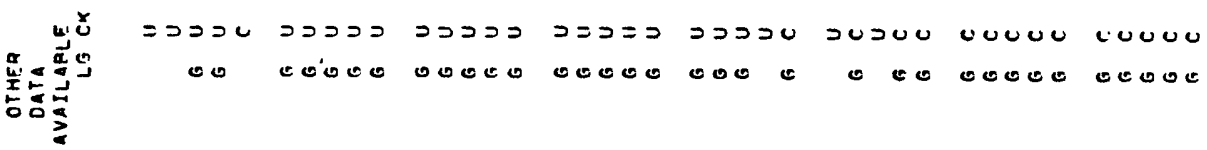

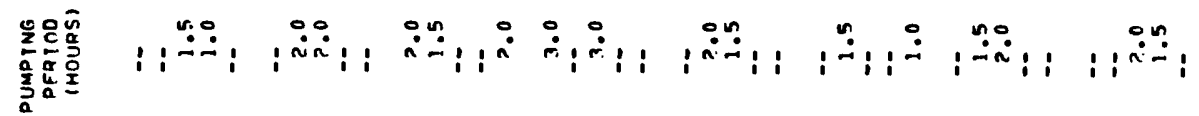

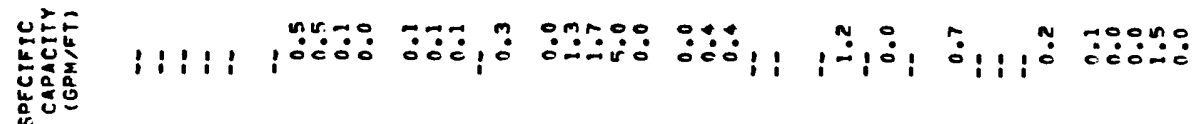

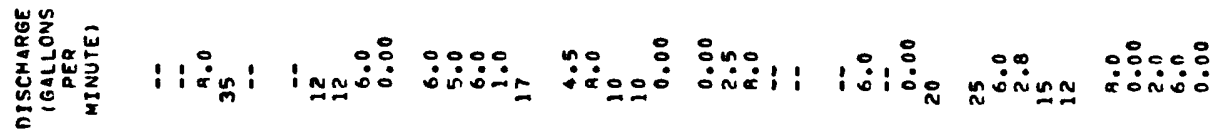

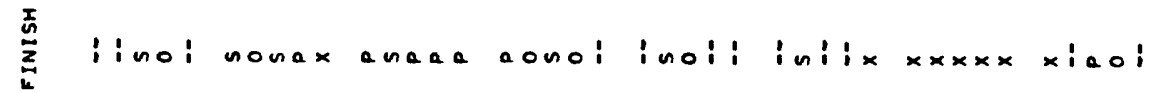
$\therefore$

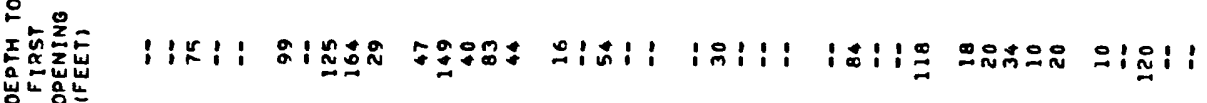

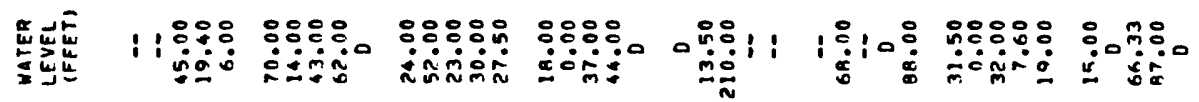

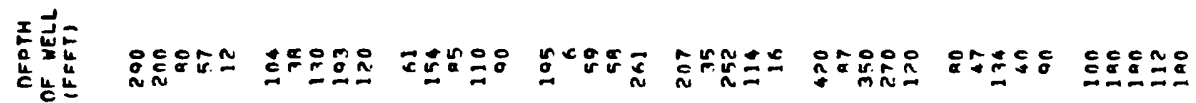
究影

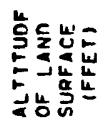

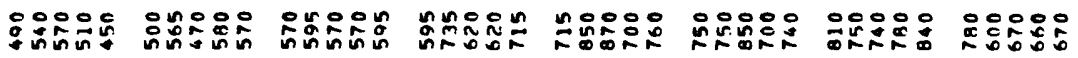

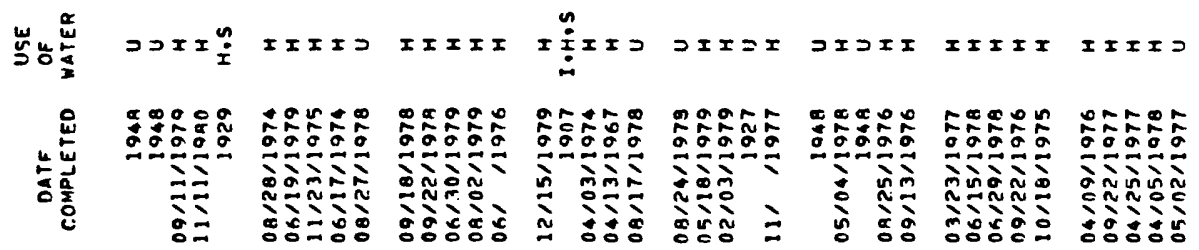

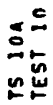

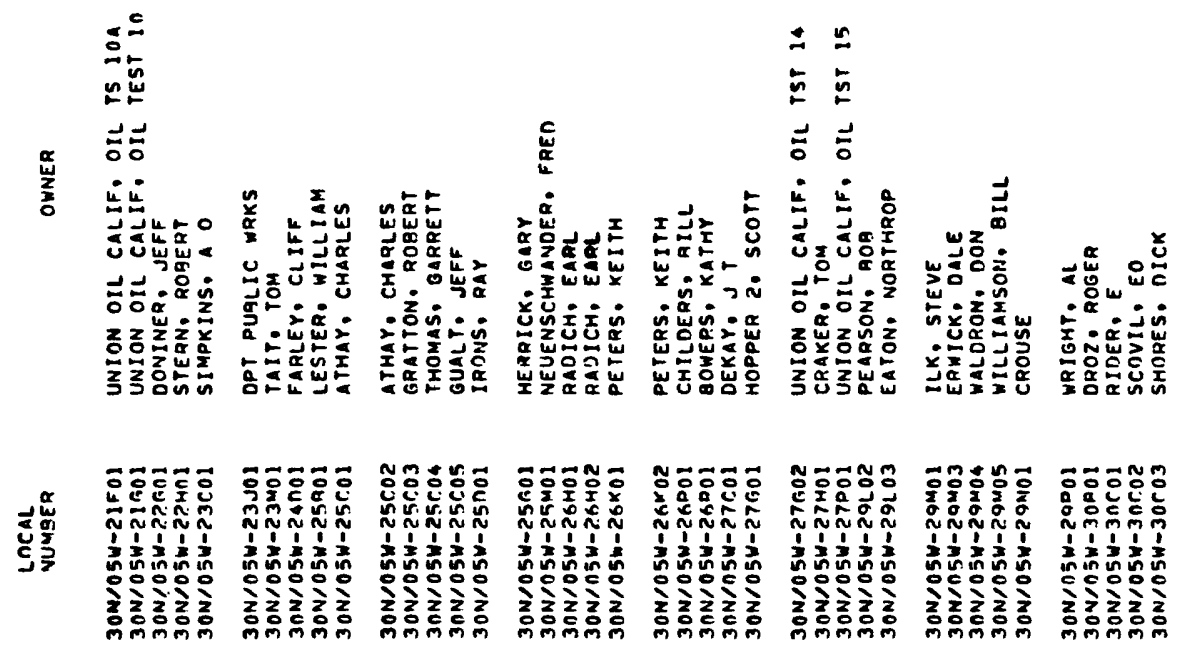




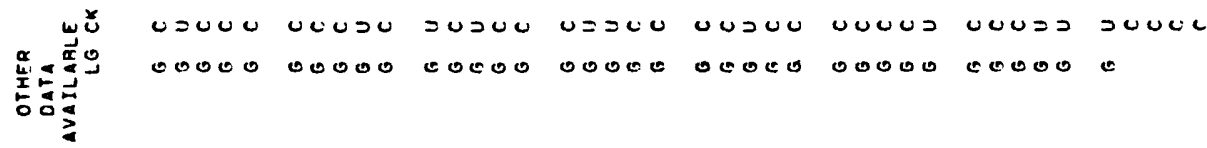

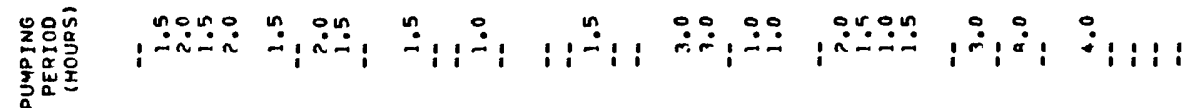

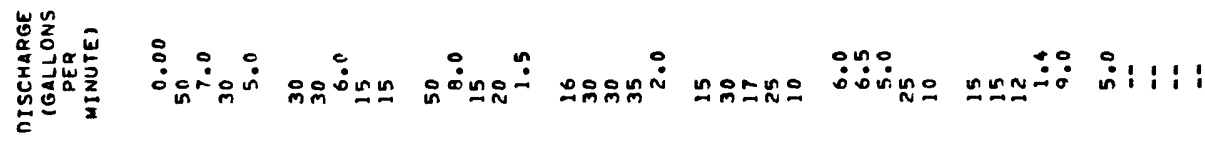

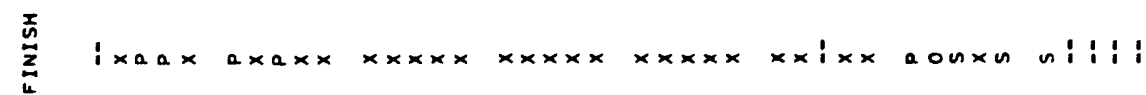

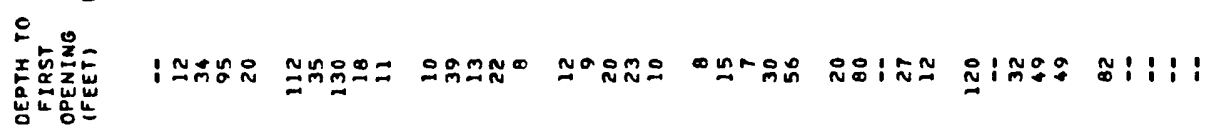
起点

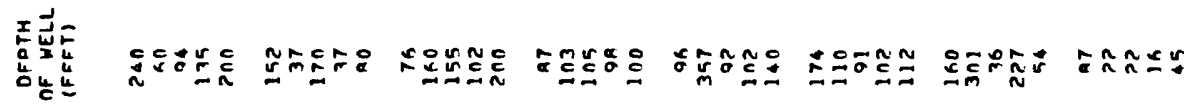

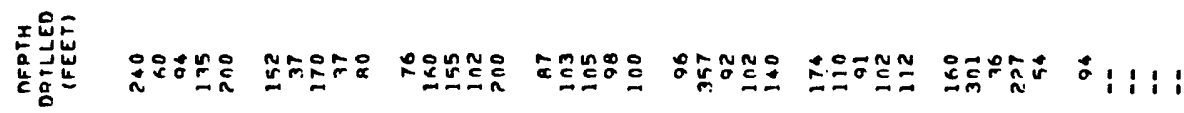

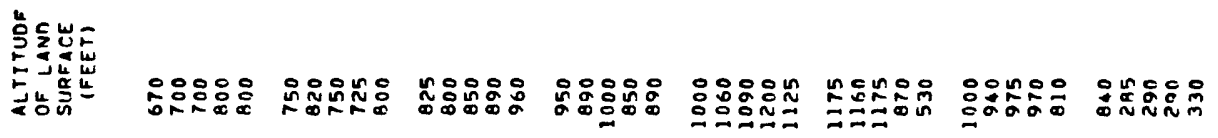
药范

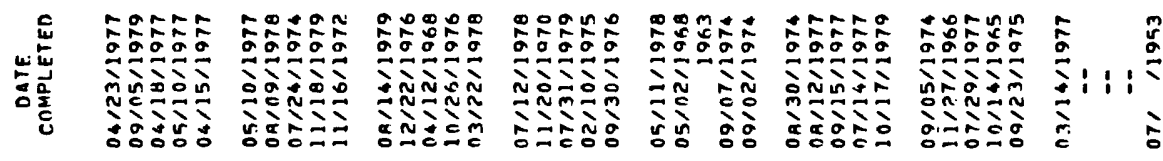

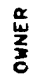

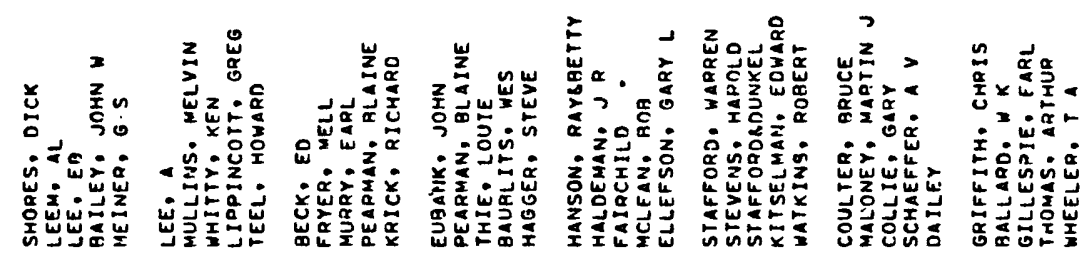




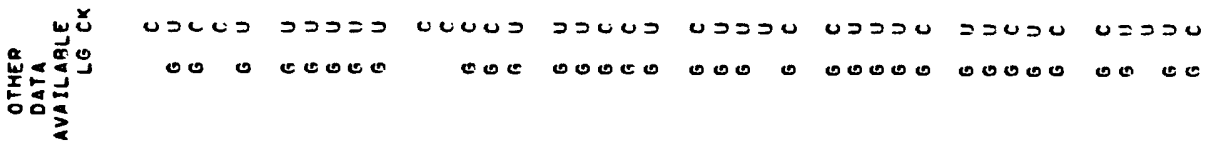

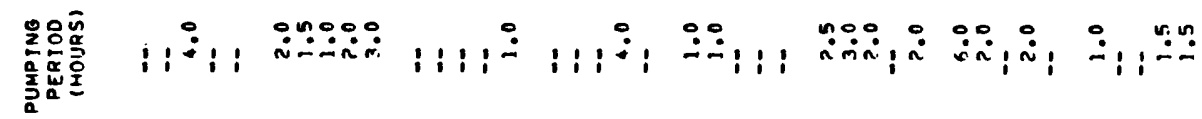

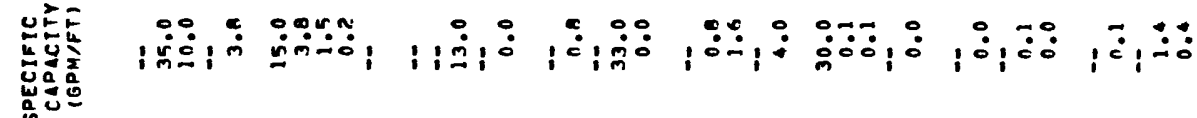

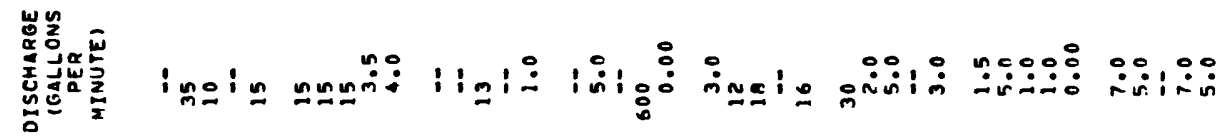

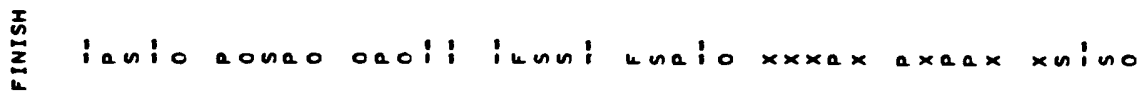
$\circ$

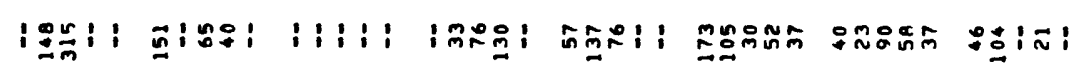
选

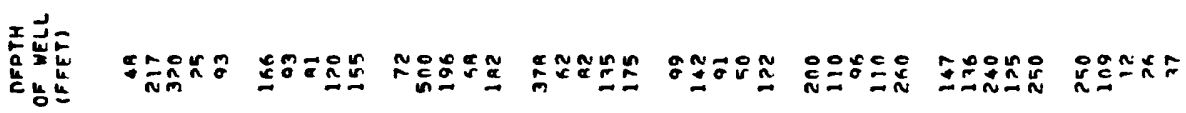

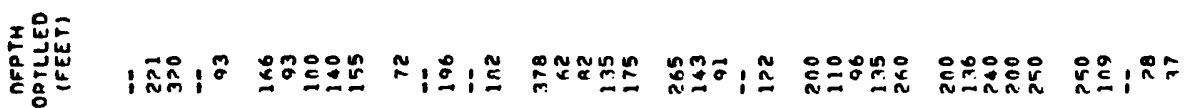

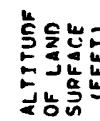

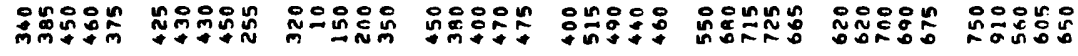

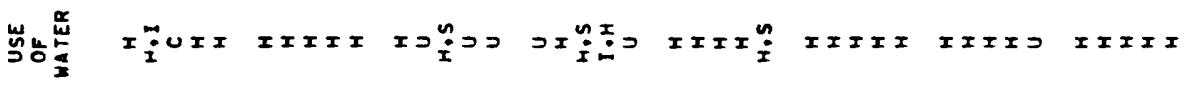

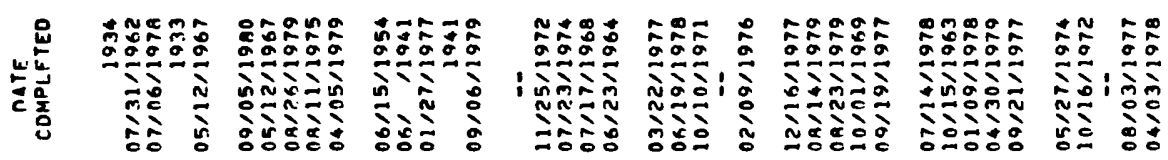
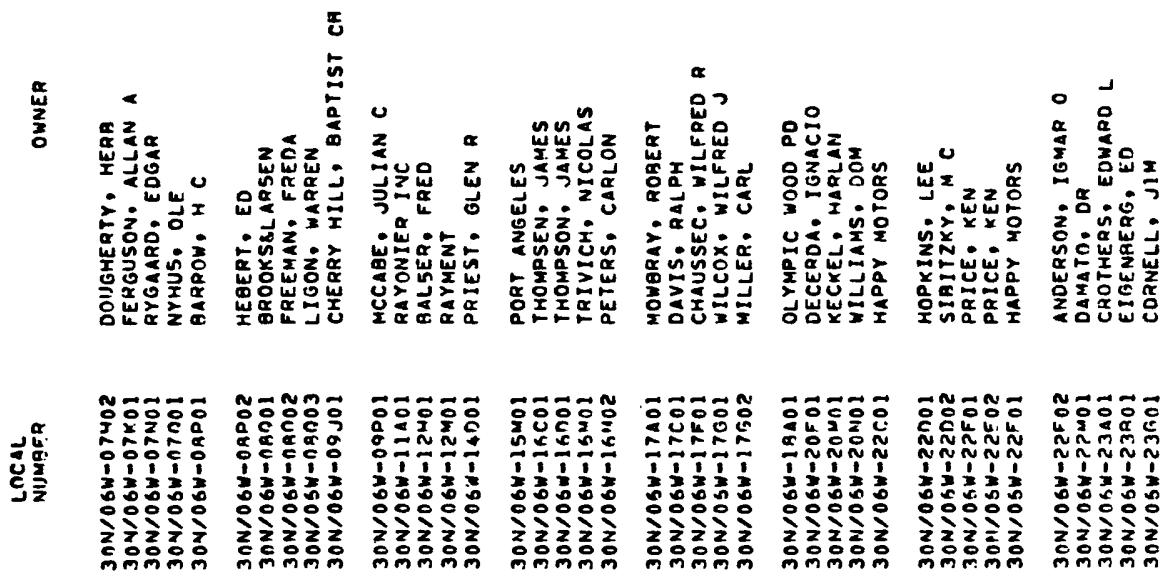


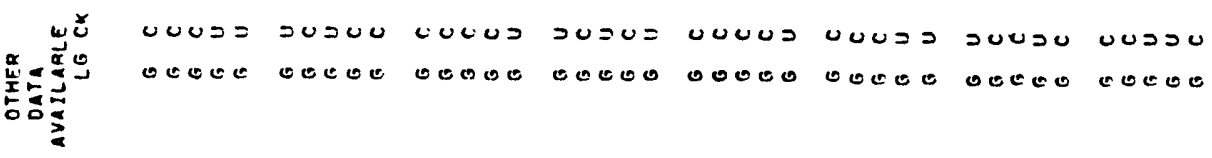

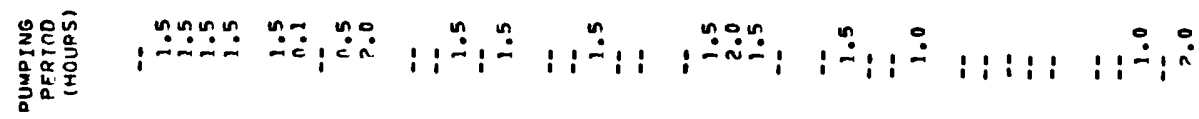

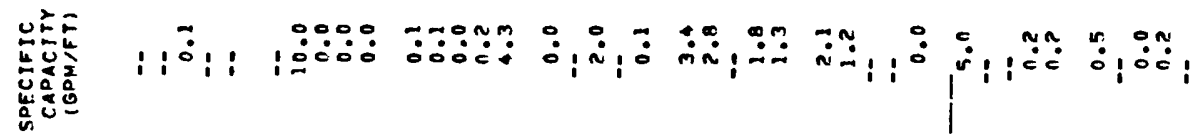

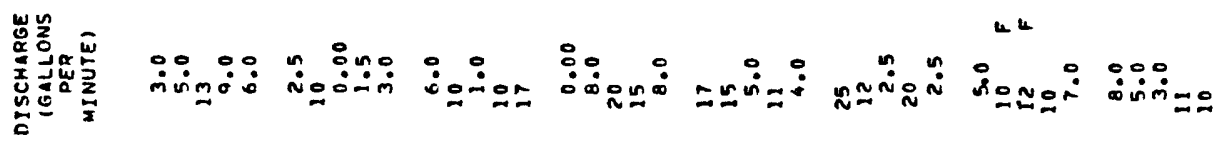
-

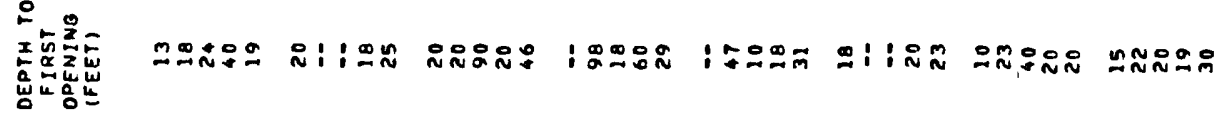

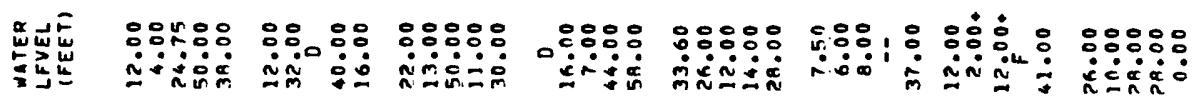

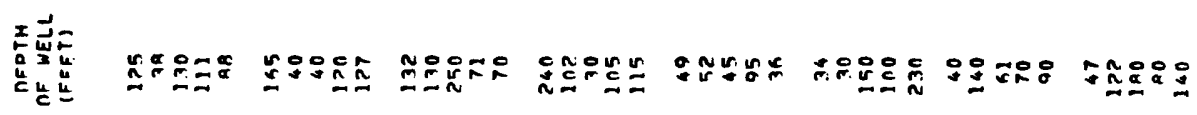

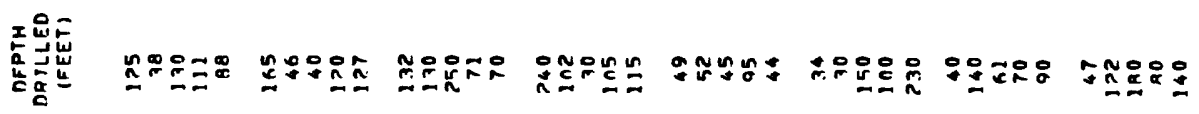

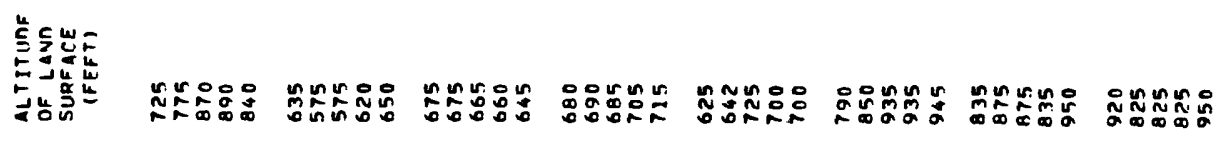

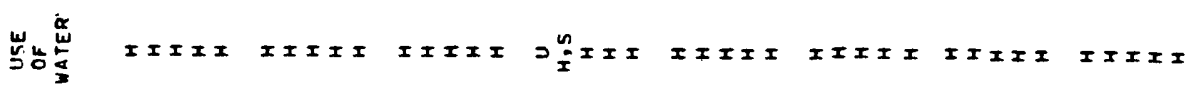

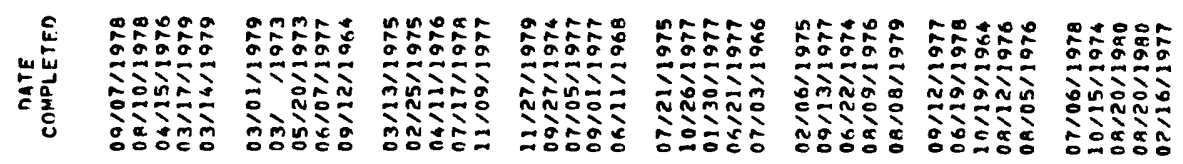

竎

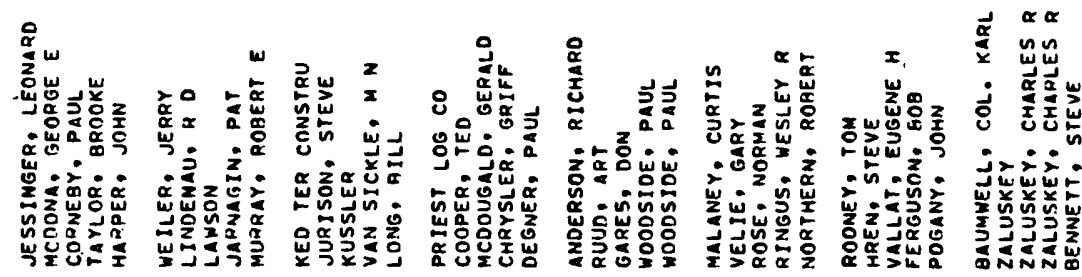

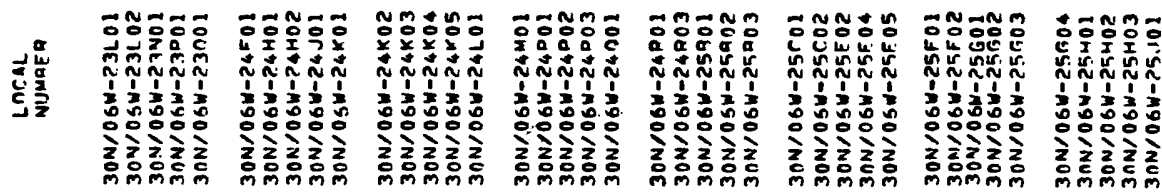




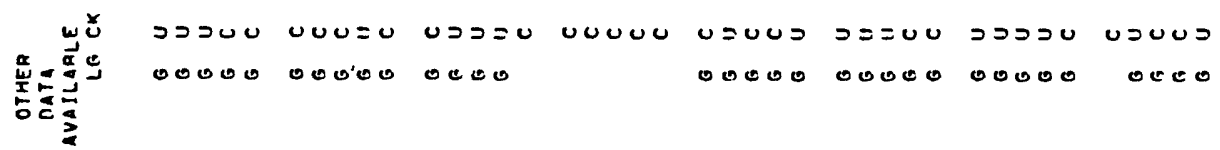

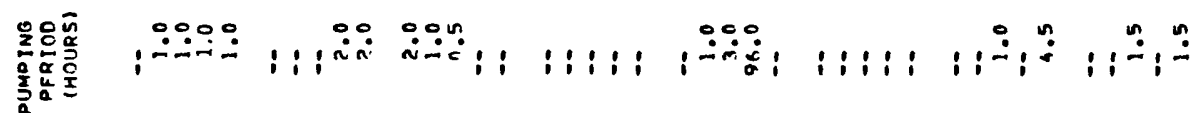

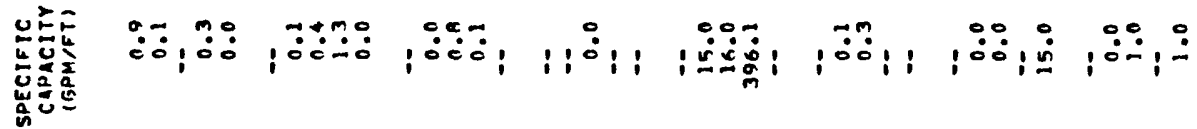

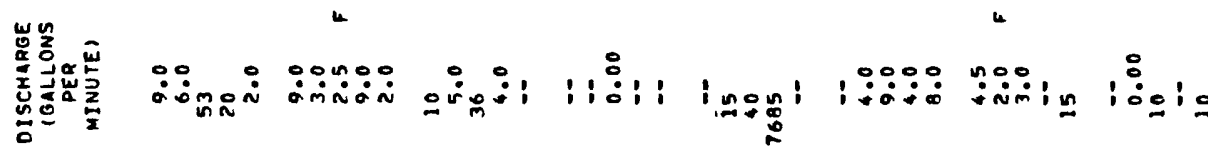

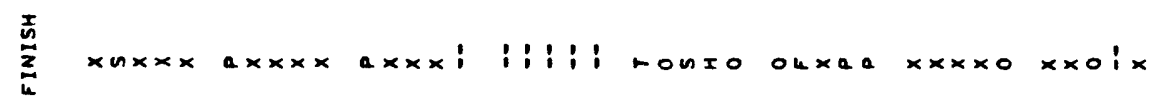
은

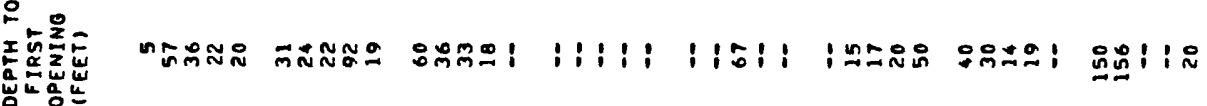

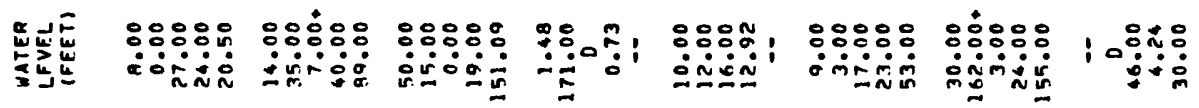

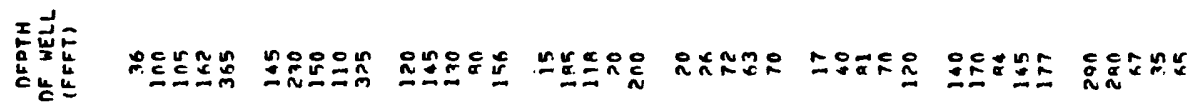

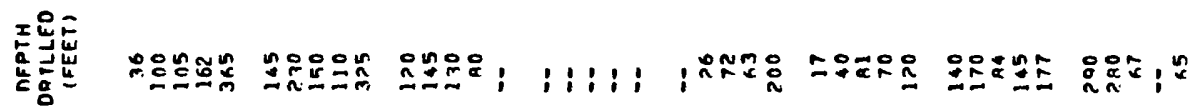

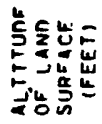

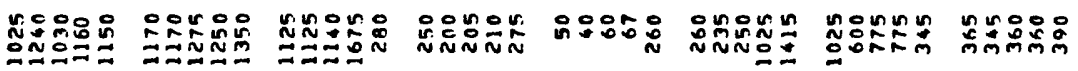
荡此

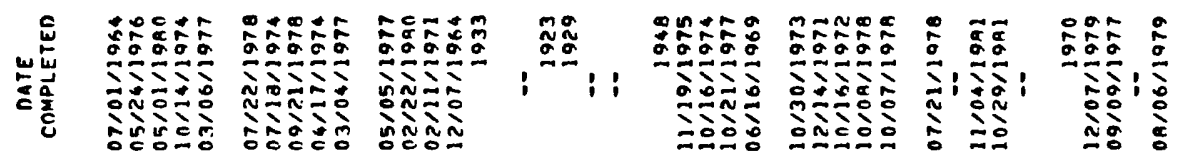
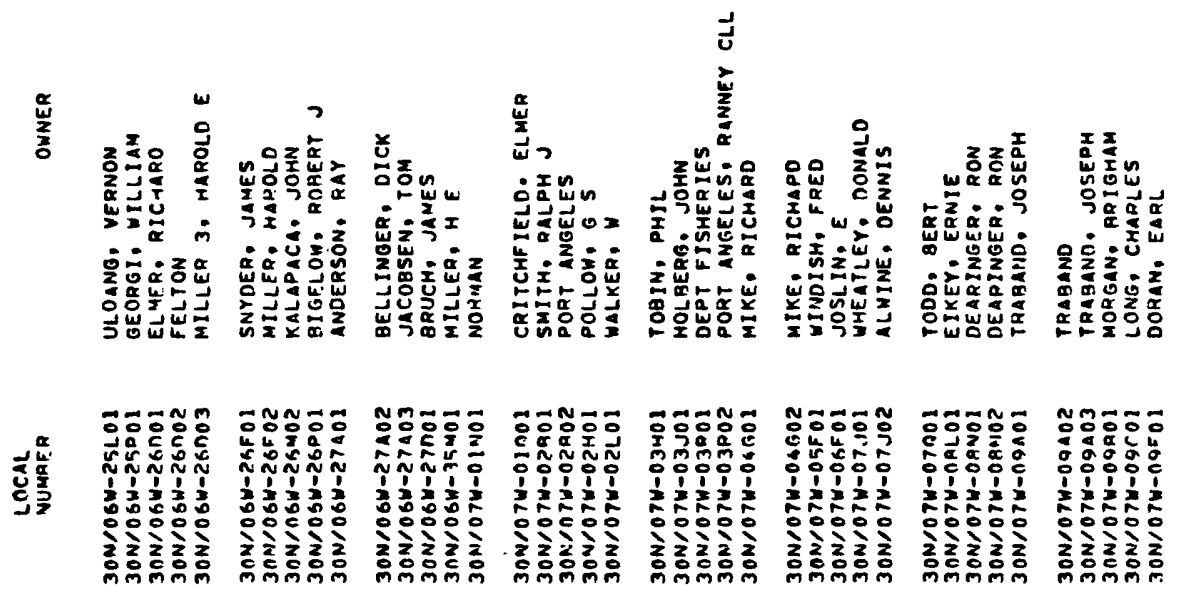


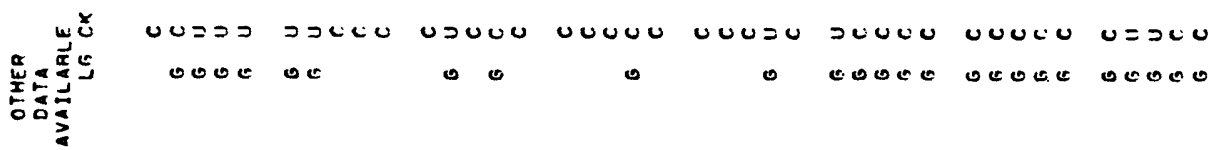

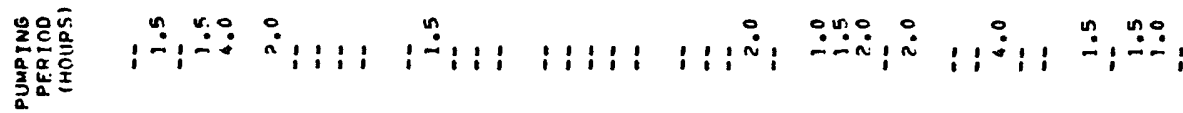

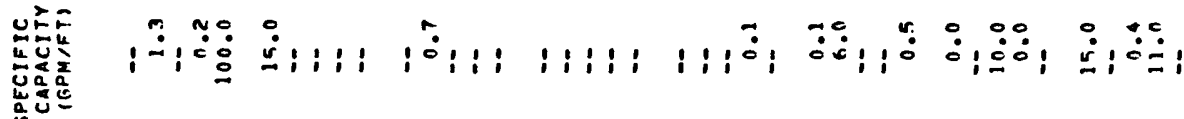

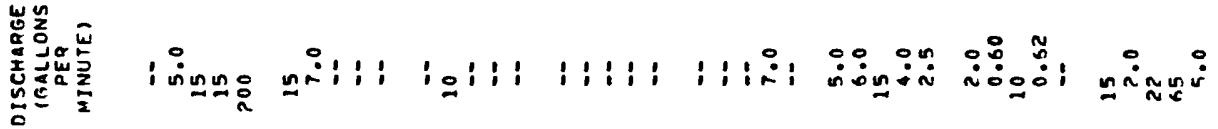

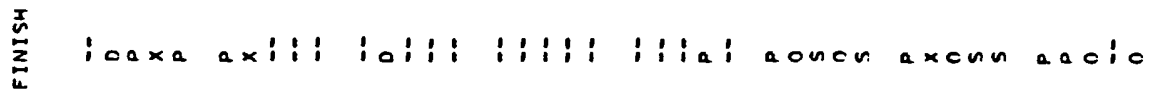

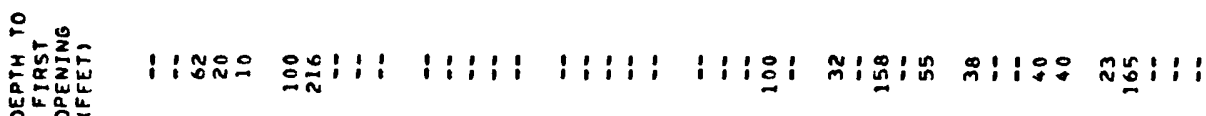

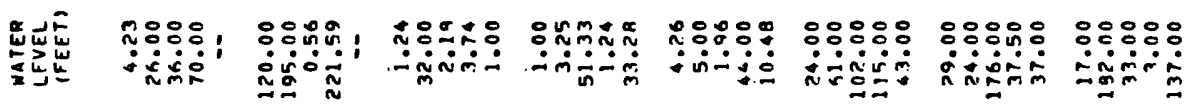

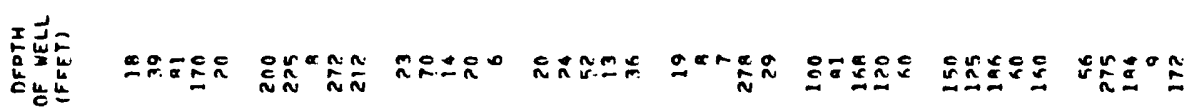

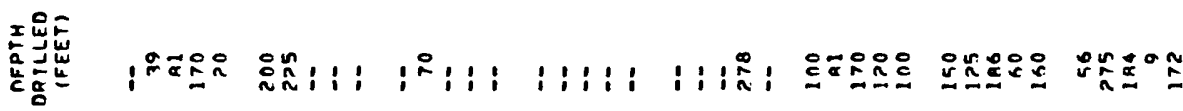
等

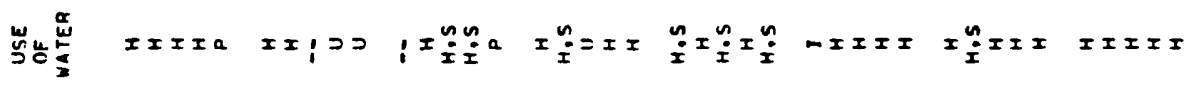

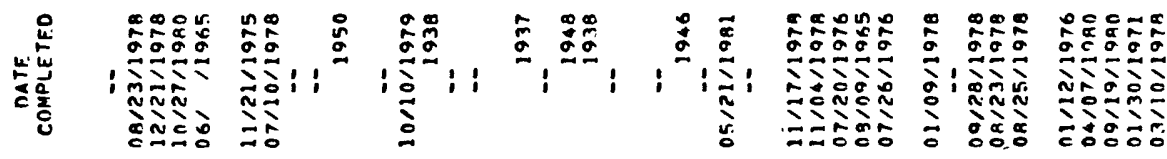

产

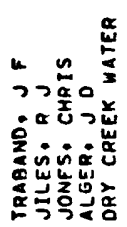

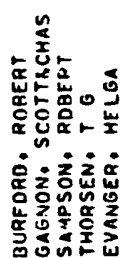

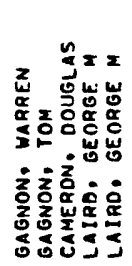

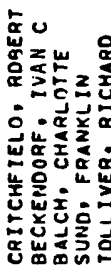
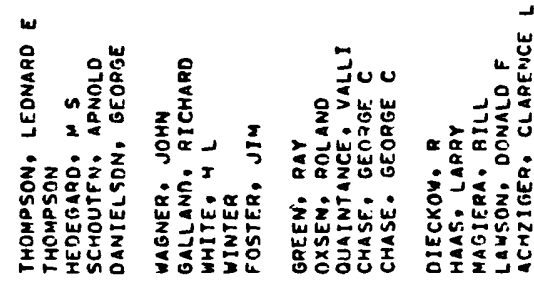
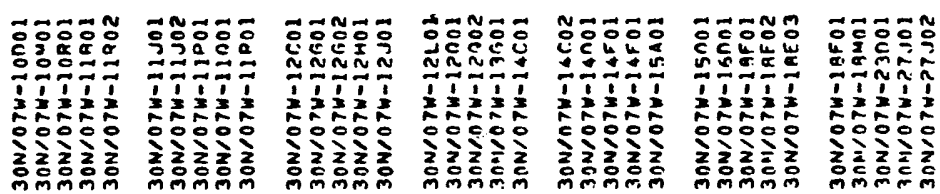


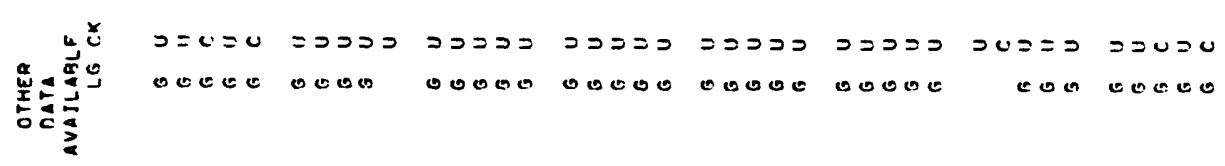

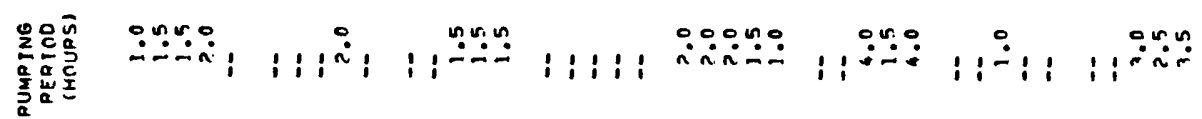

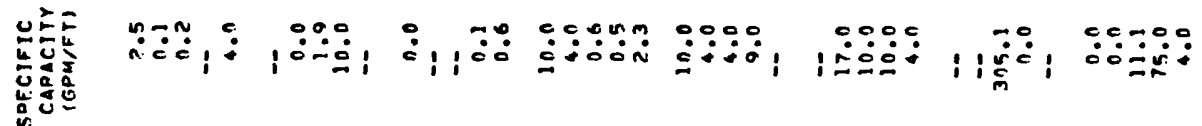

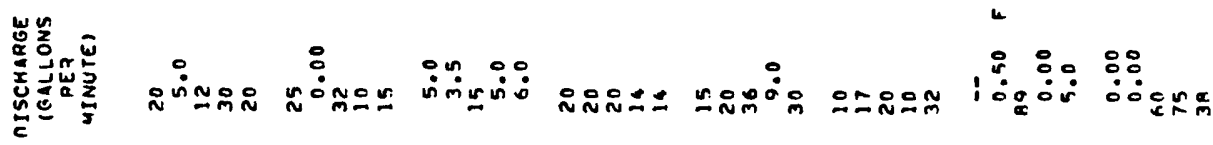

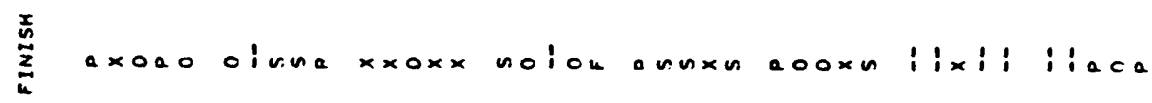

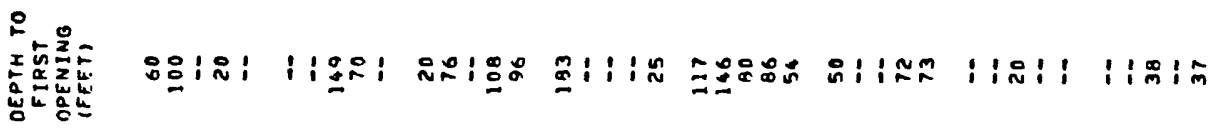

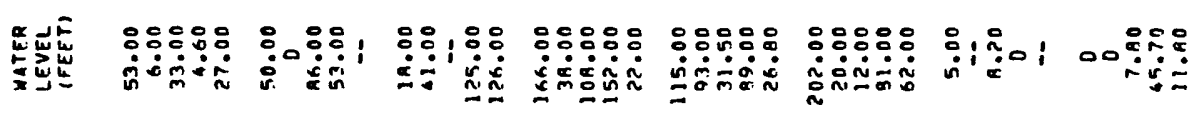

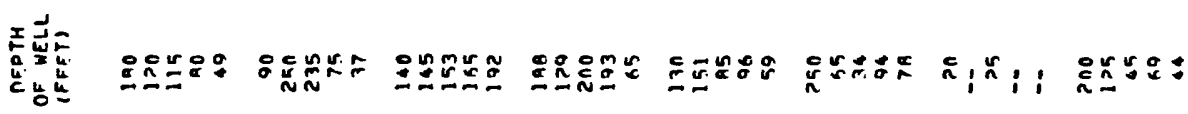

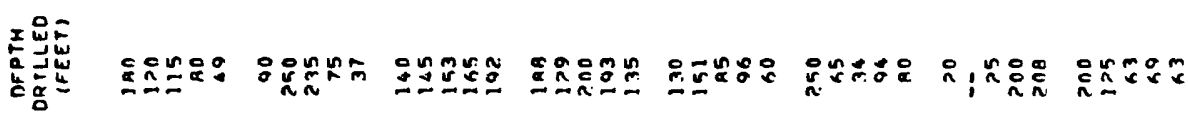

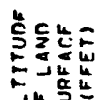

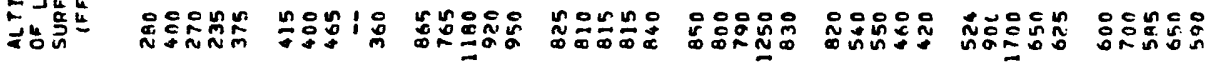

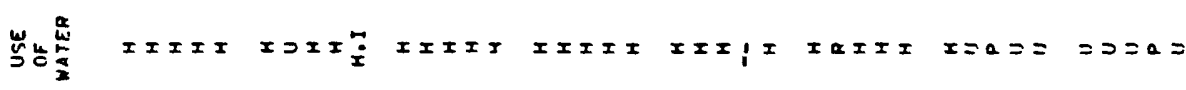

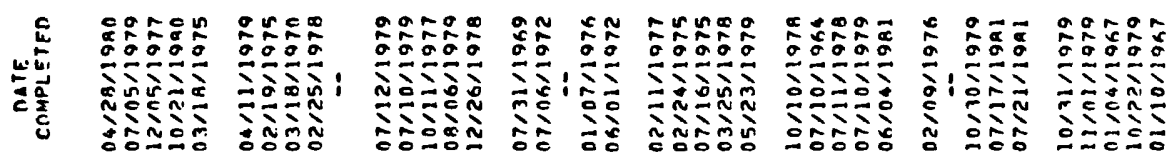




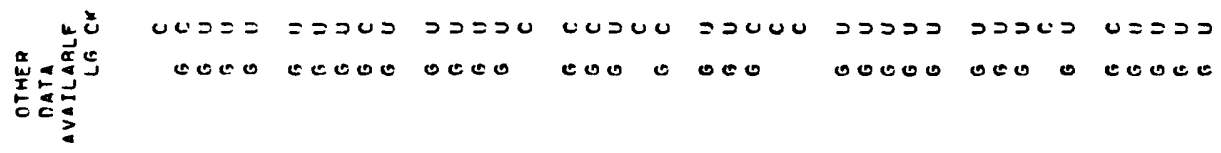
率商高

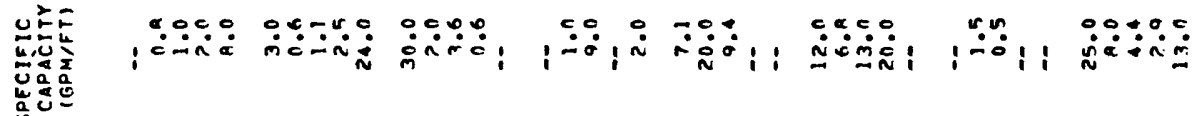

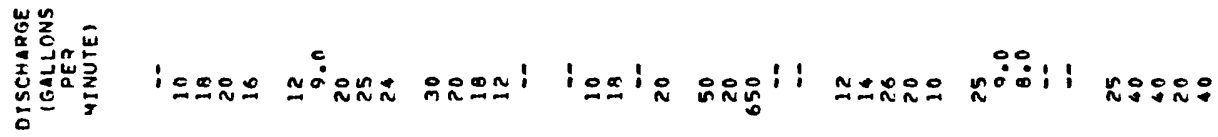

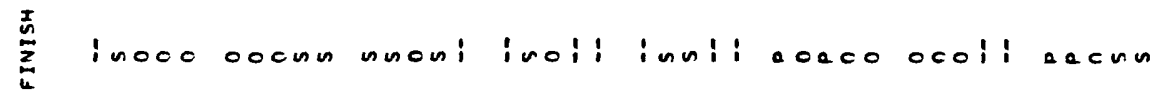
琶题

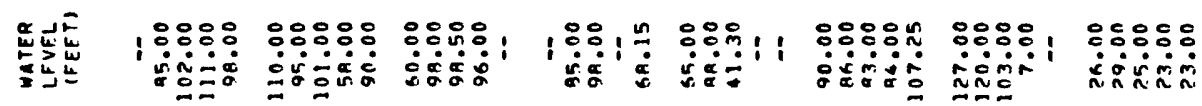

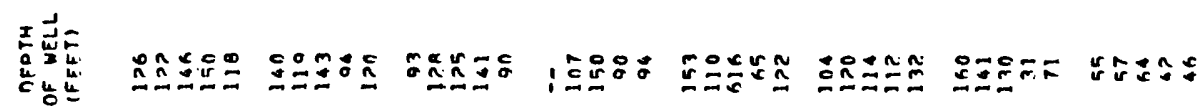

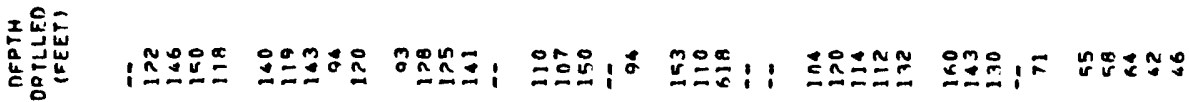

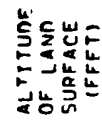

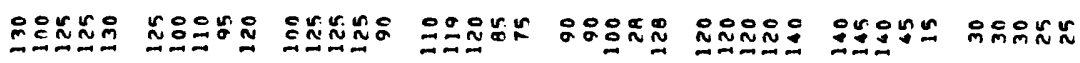

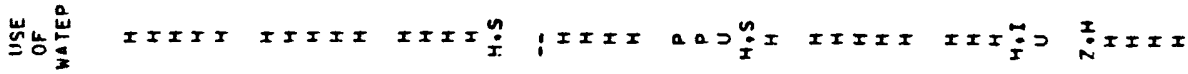

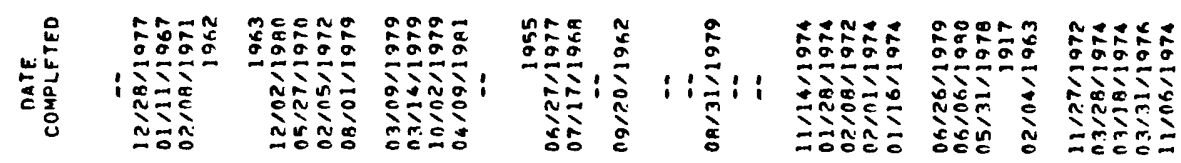

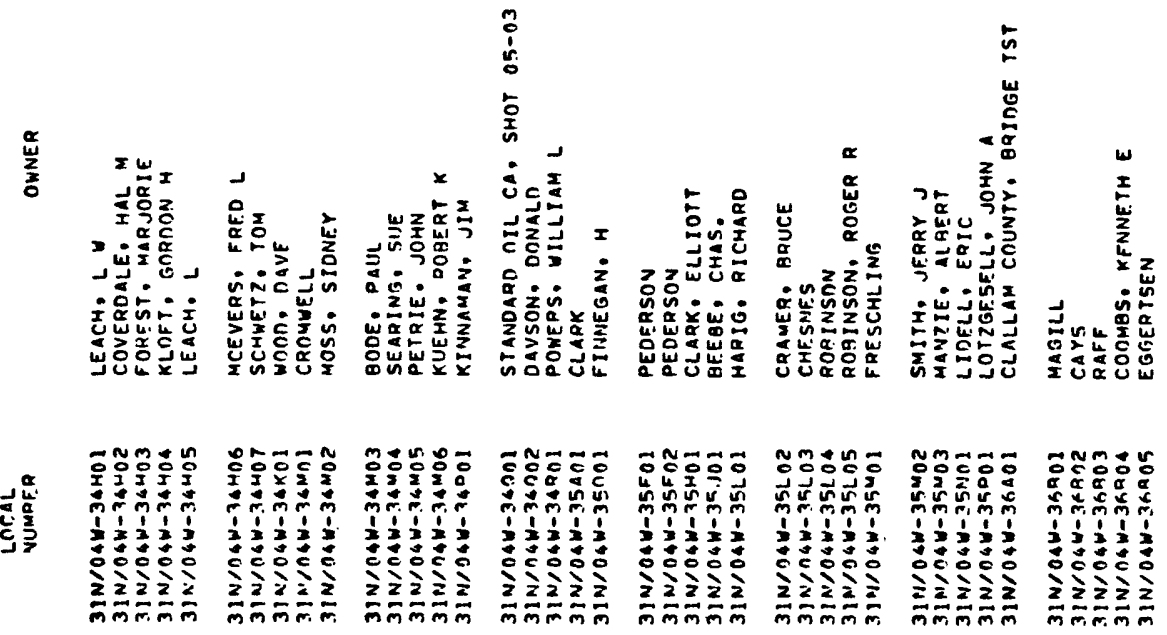




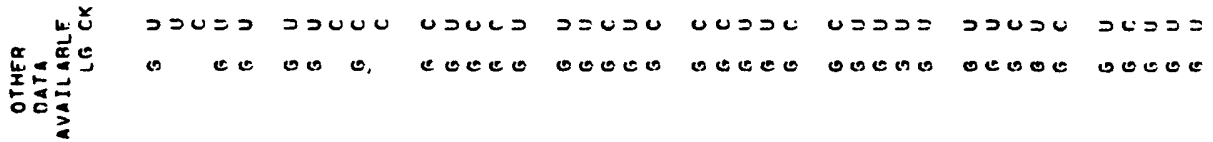

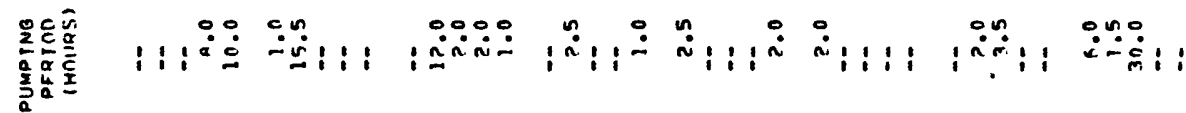

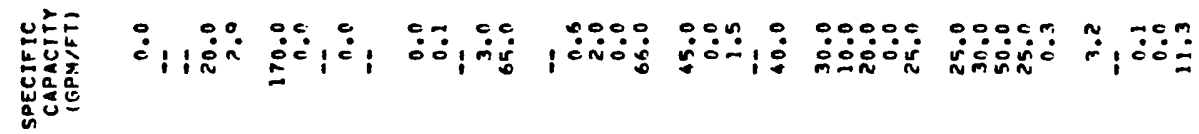

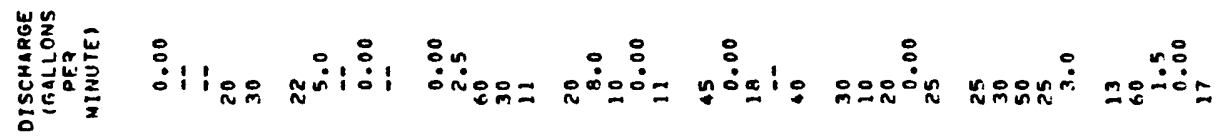

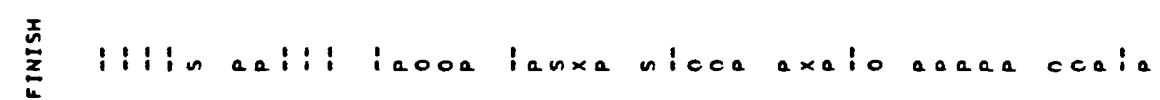

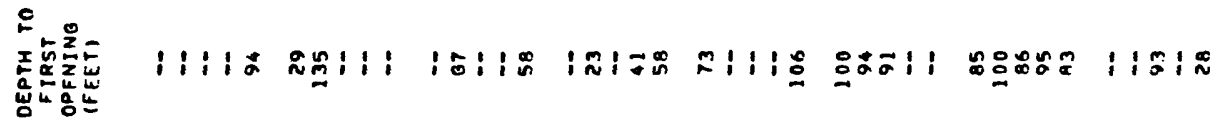

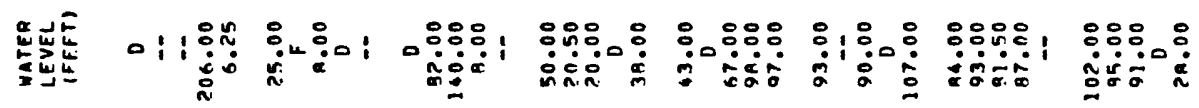

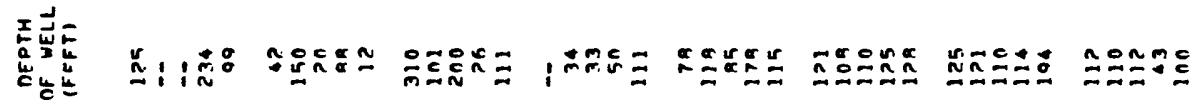

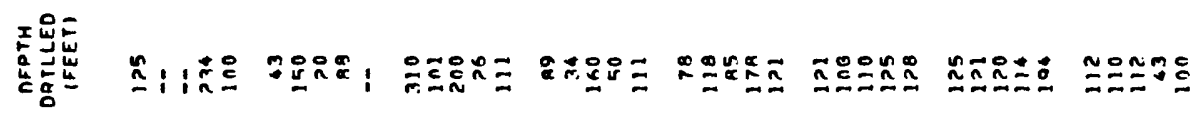

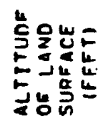

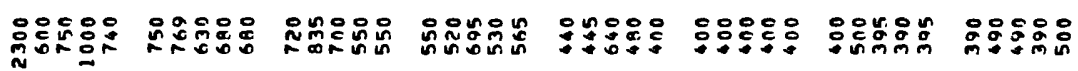

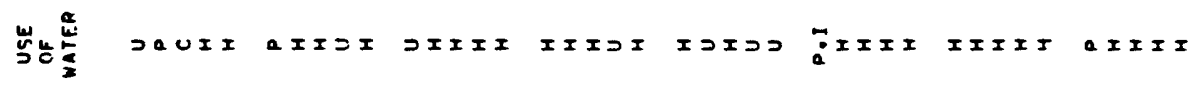

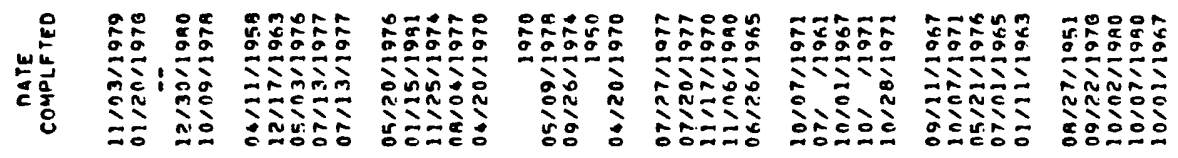

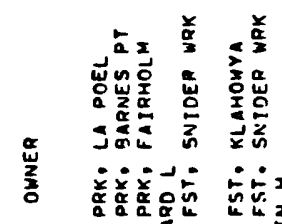

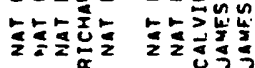

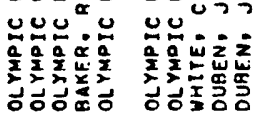
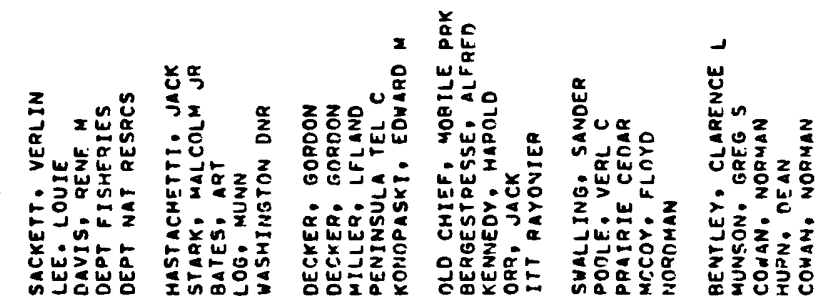
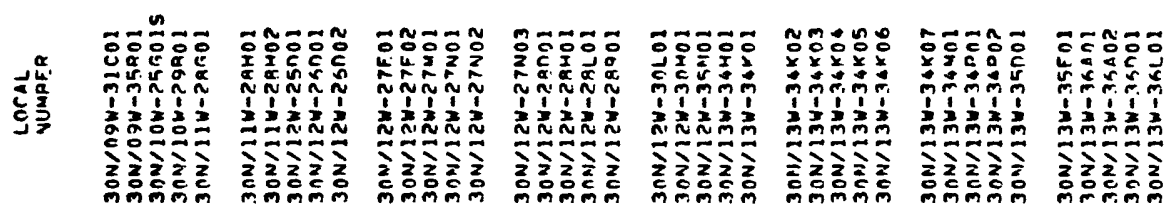


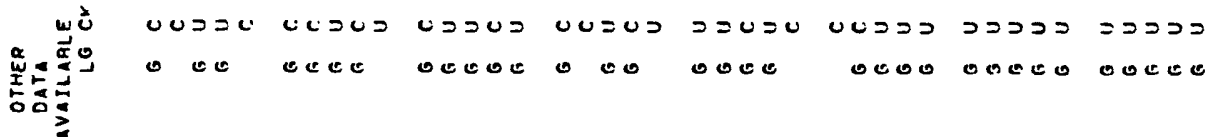

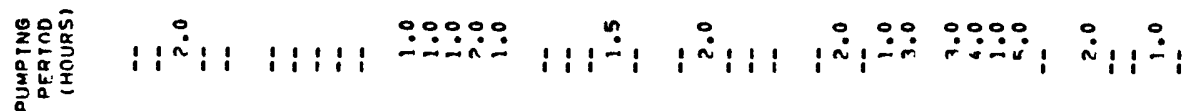

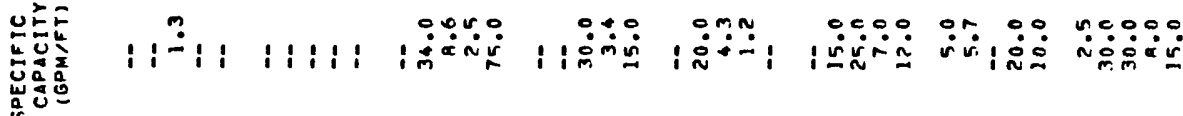

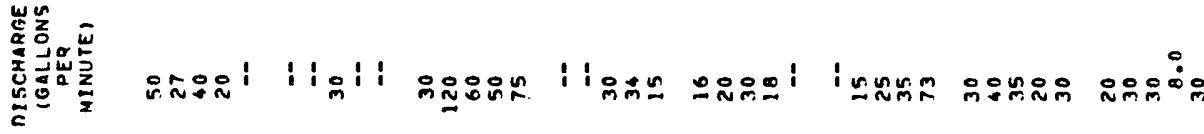

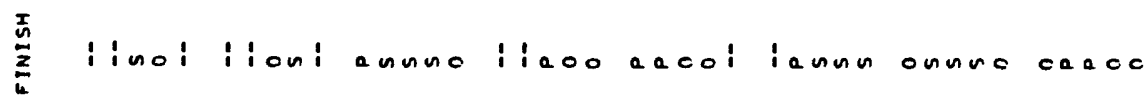

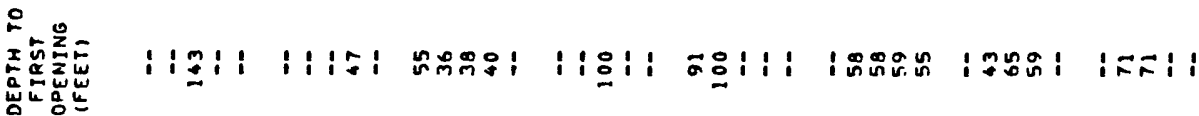

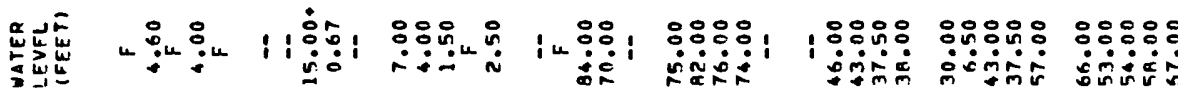

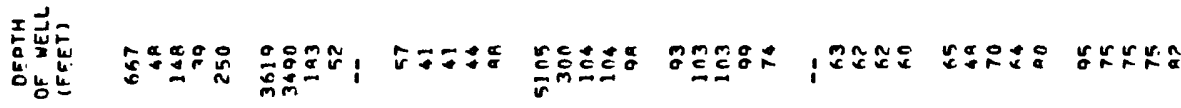

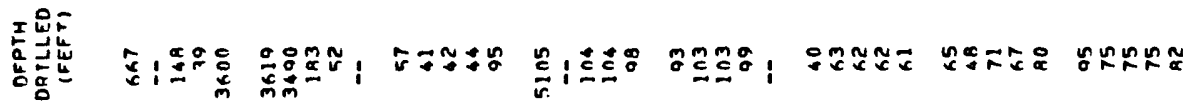

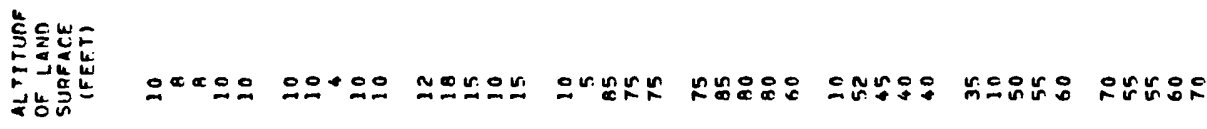

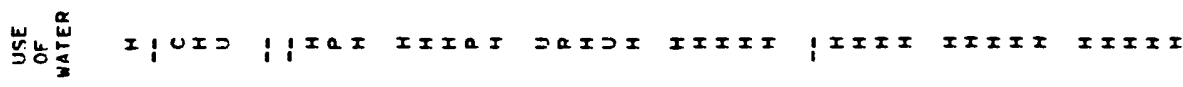

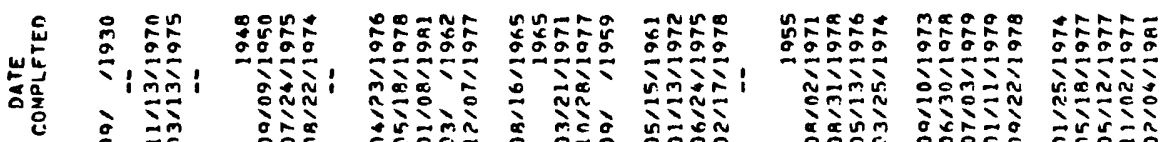
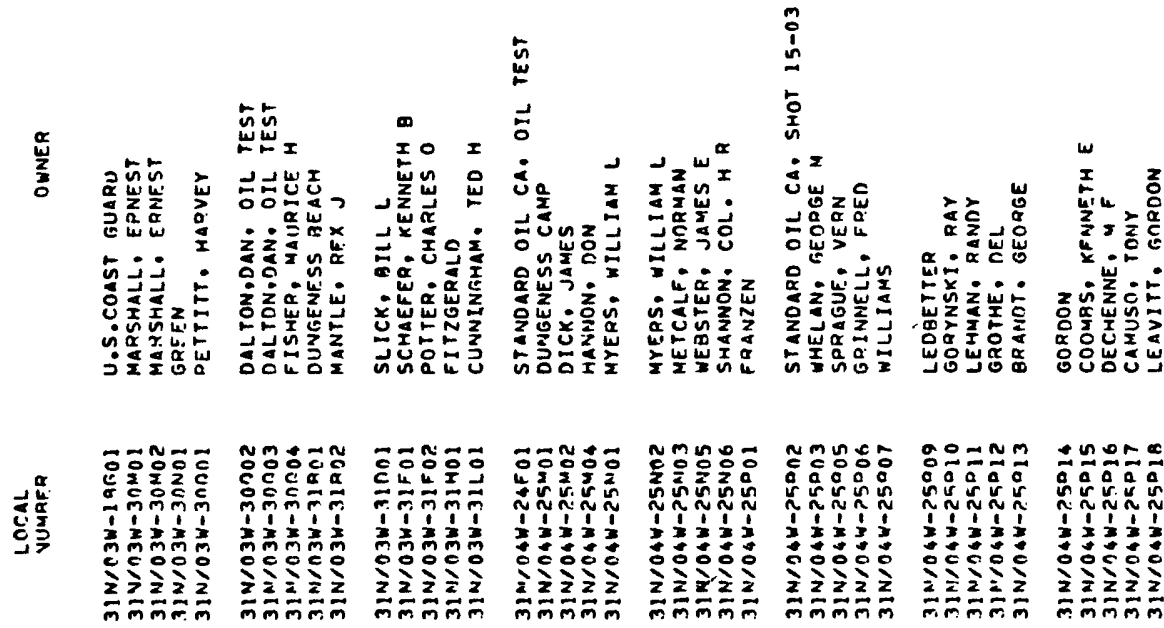


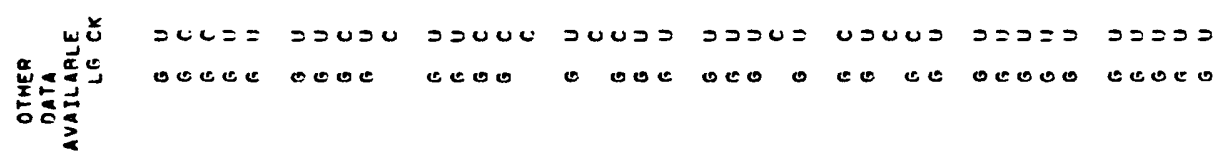

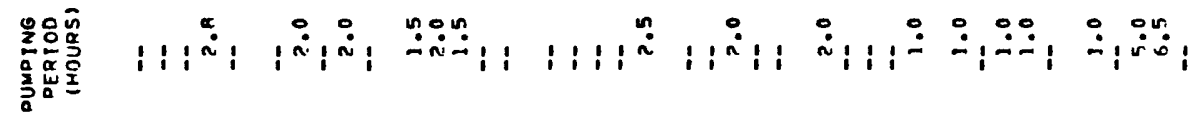

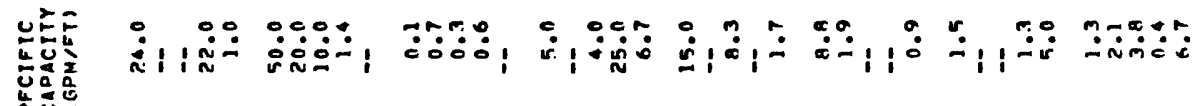

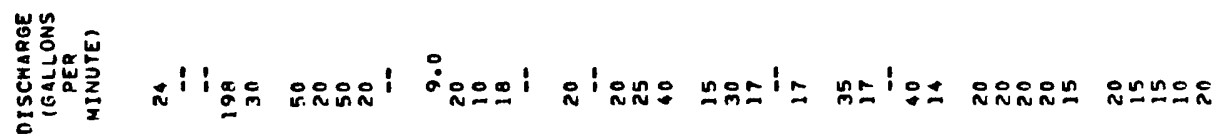
ellino nunol ocont olonn nocla nolico cooco ooone

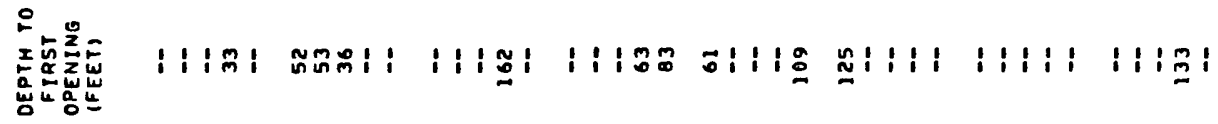

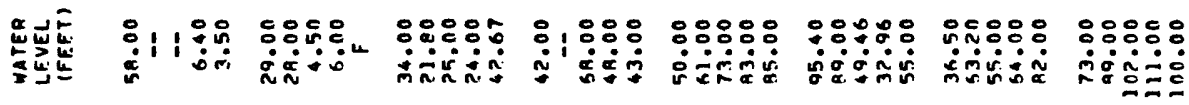

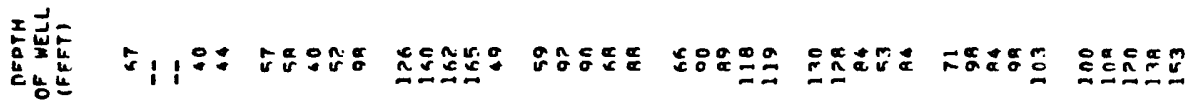

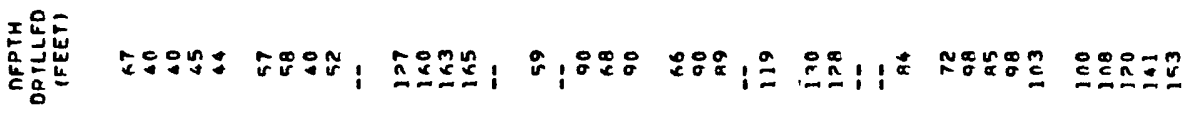
作

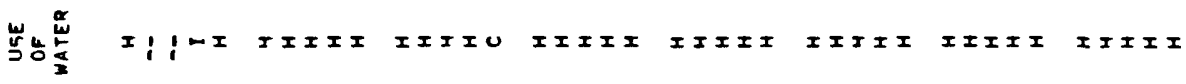

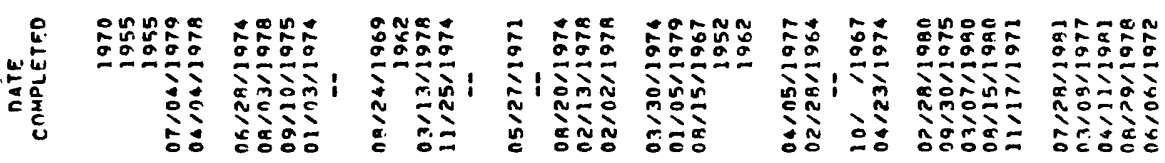
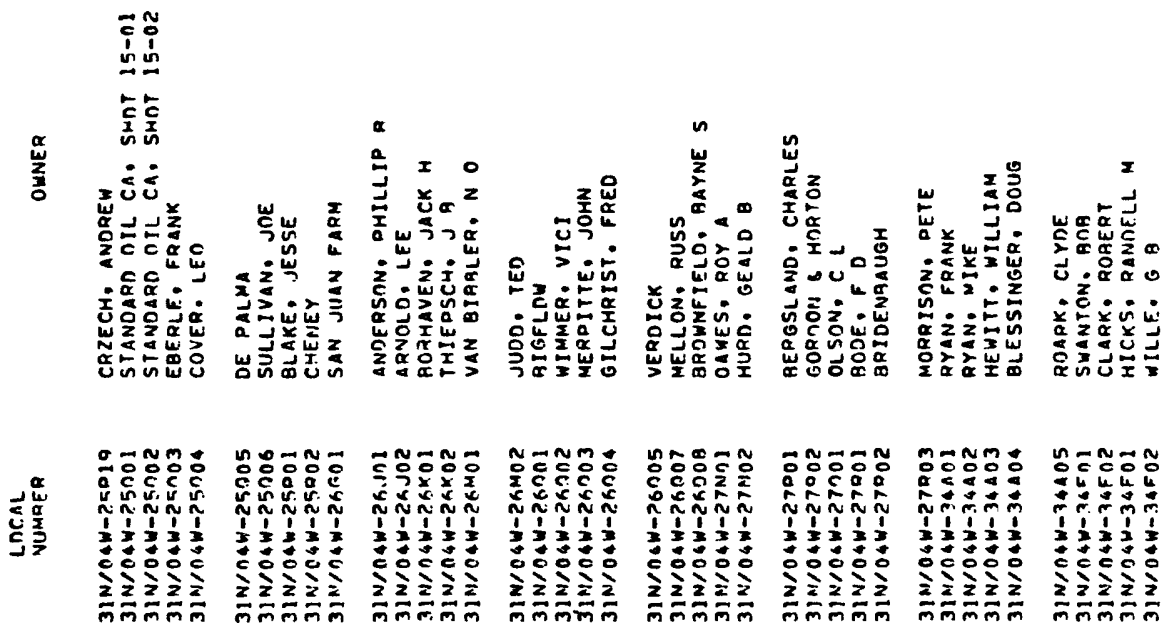


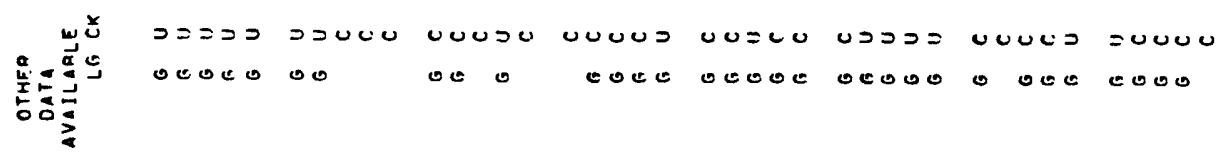

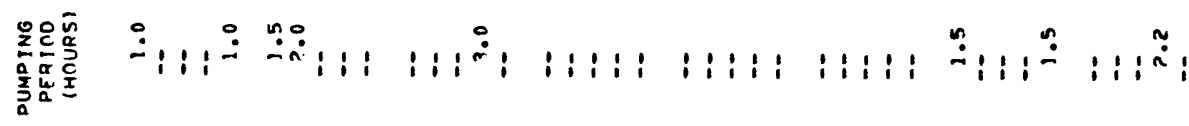

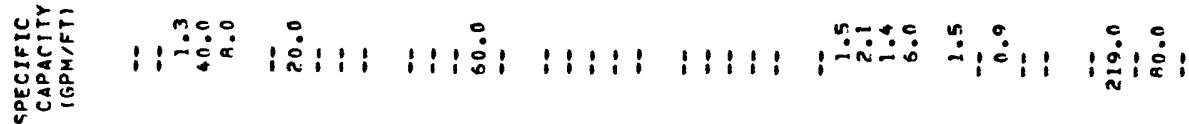

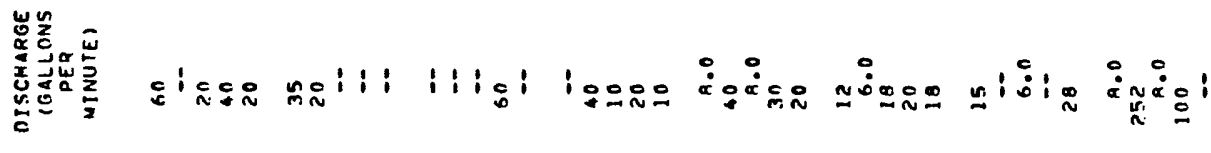

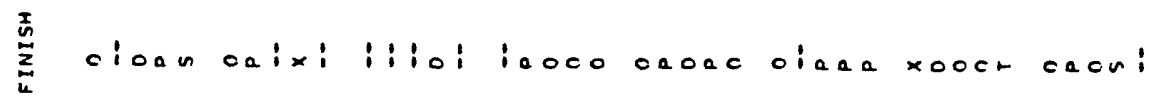

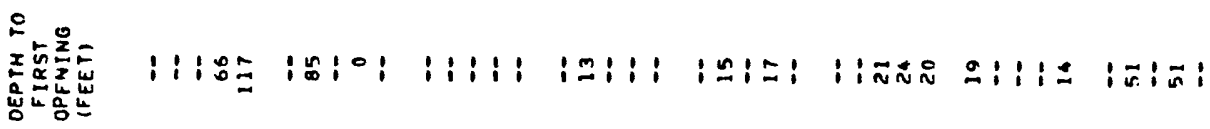

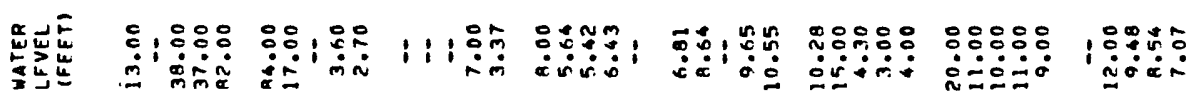

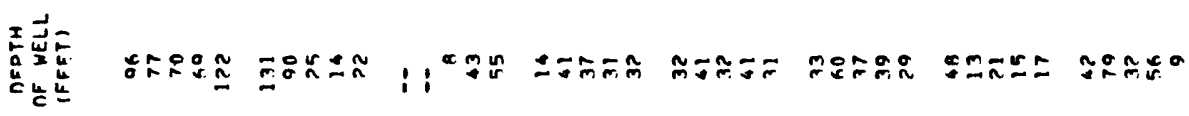

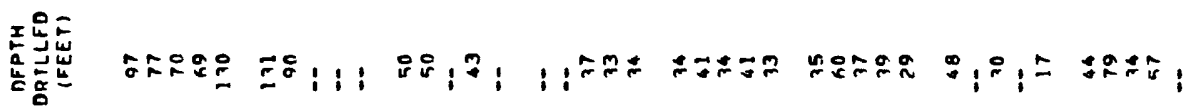
等

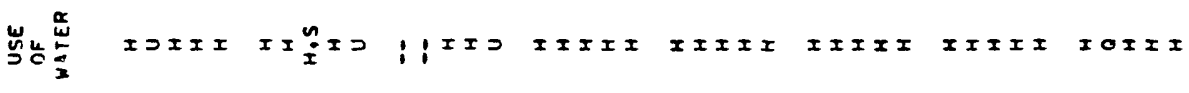

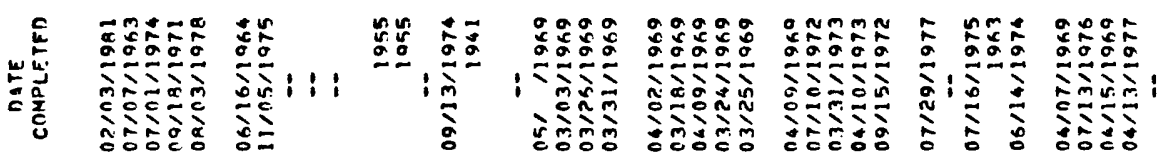
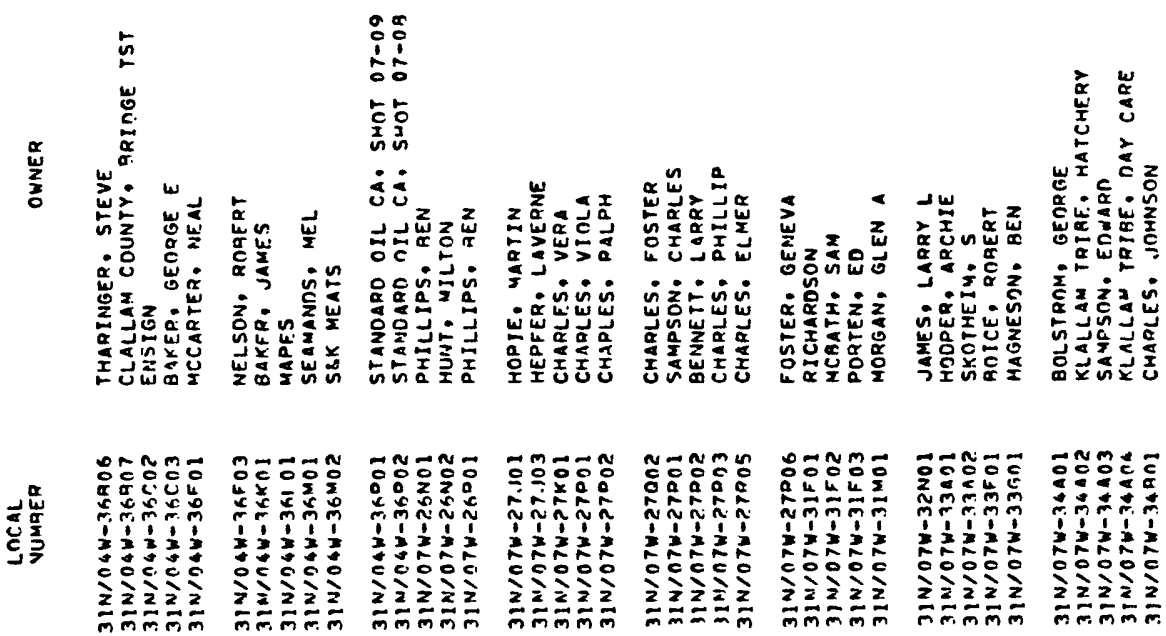


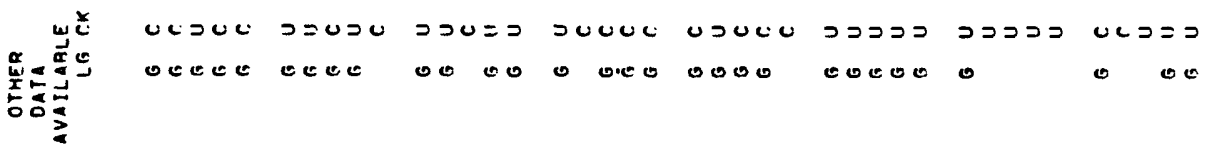

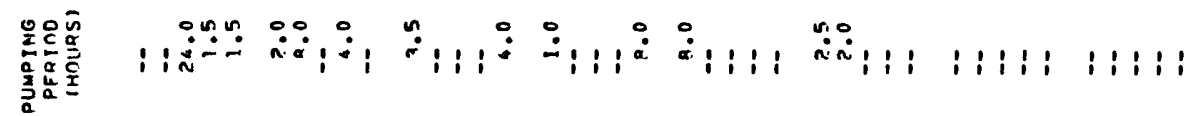

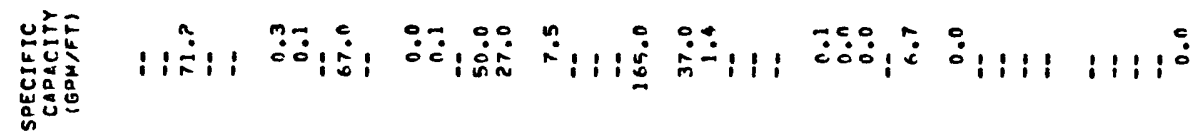

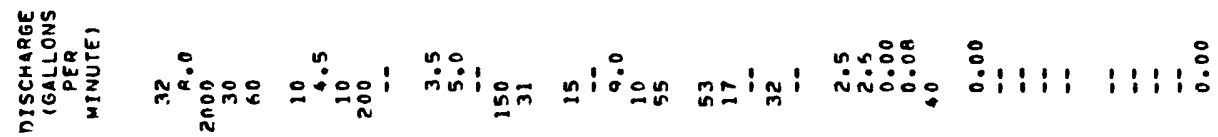

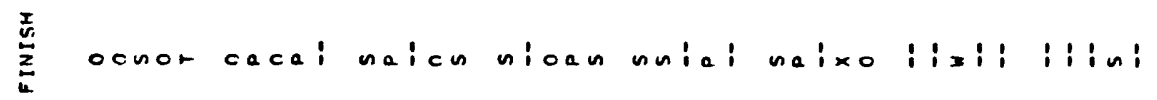

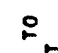

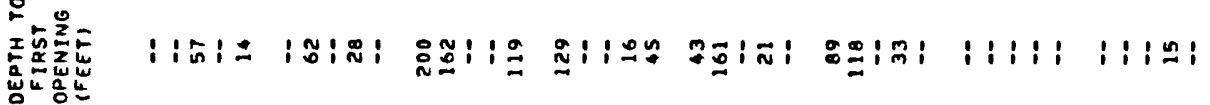

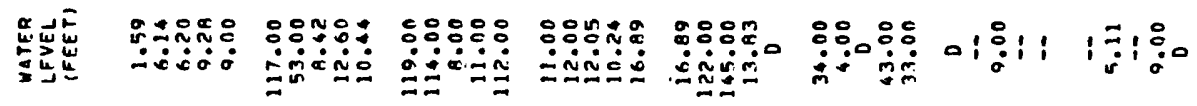

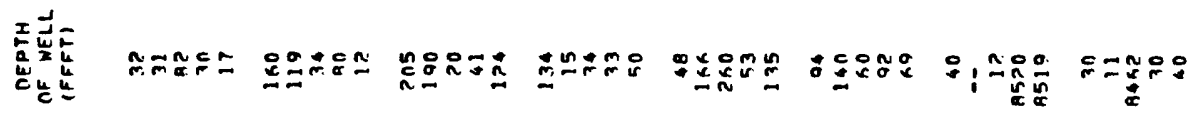

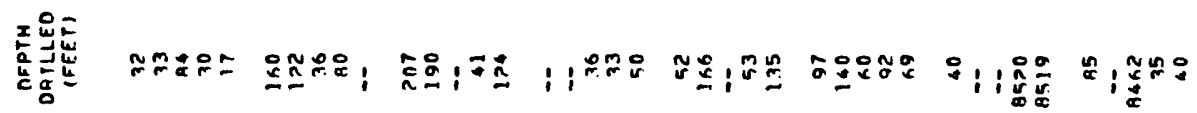
势

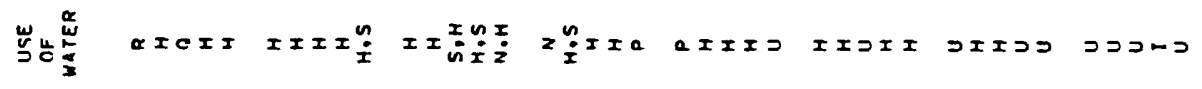

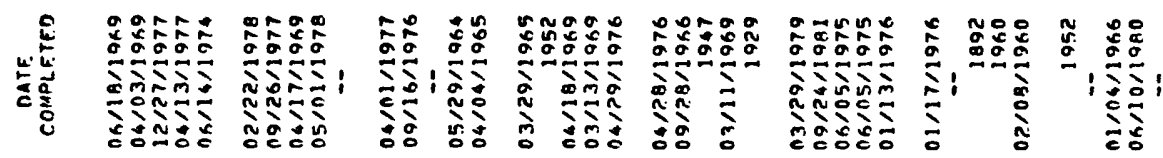
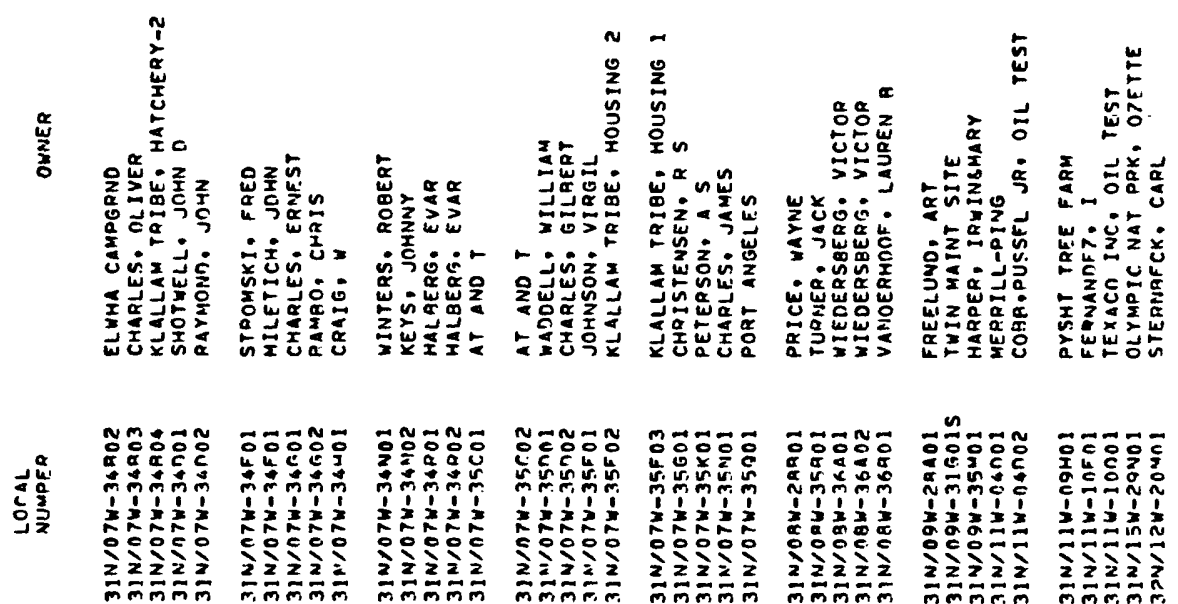


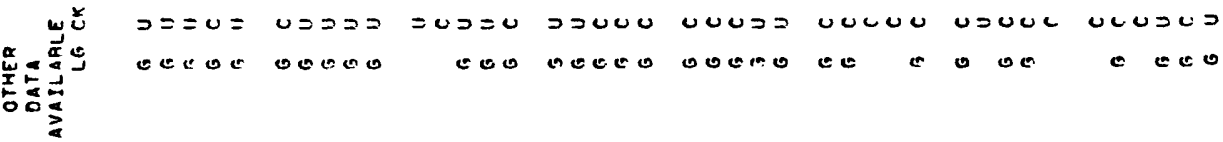
总象高

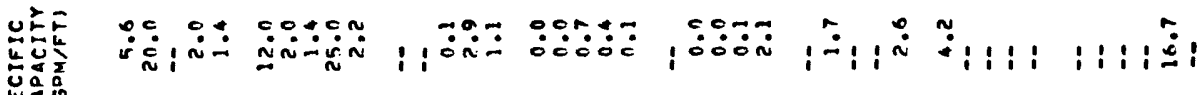

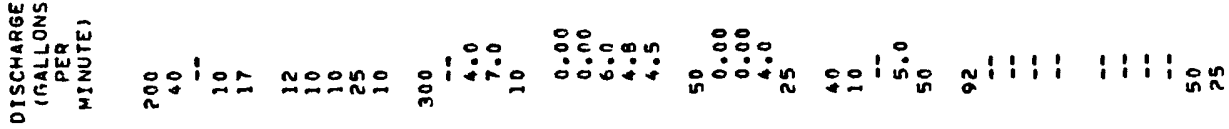

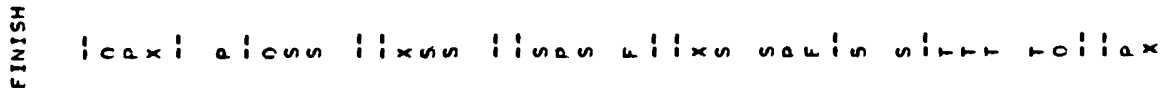

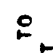

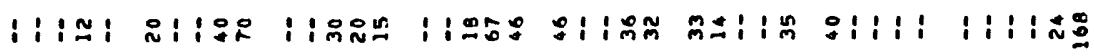

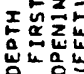

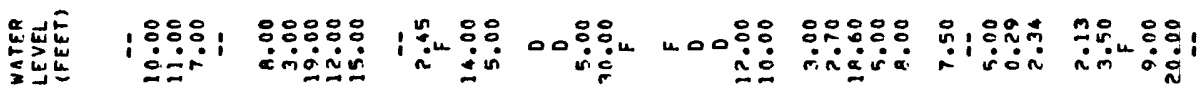

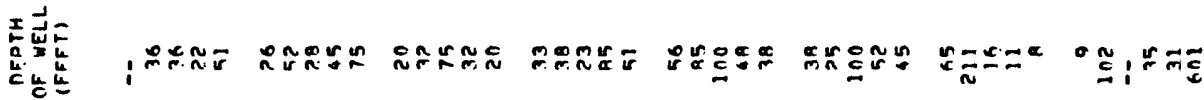

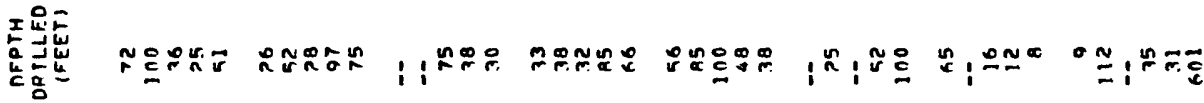

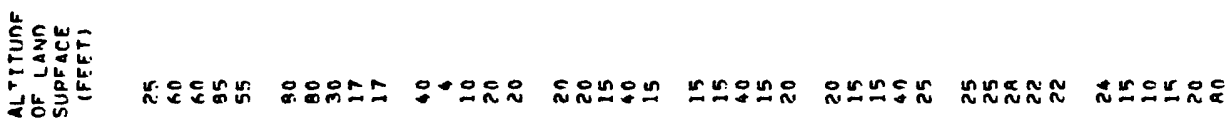

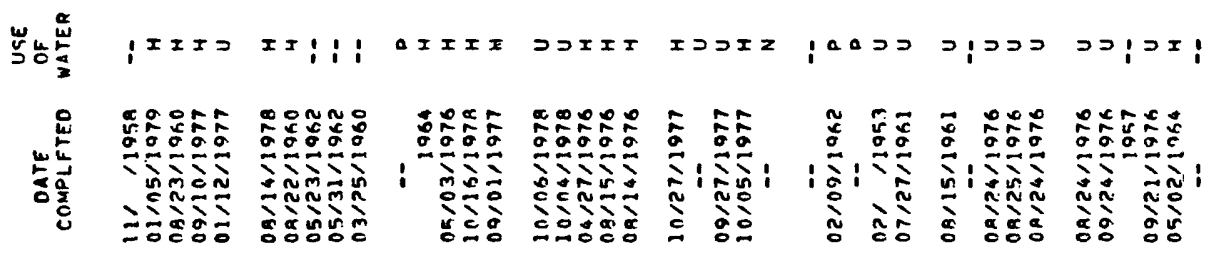
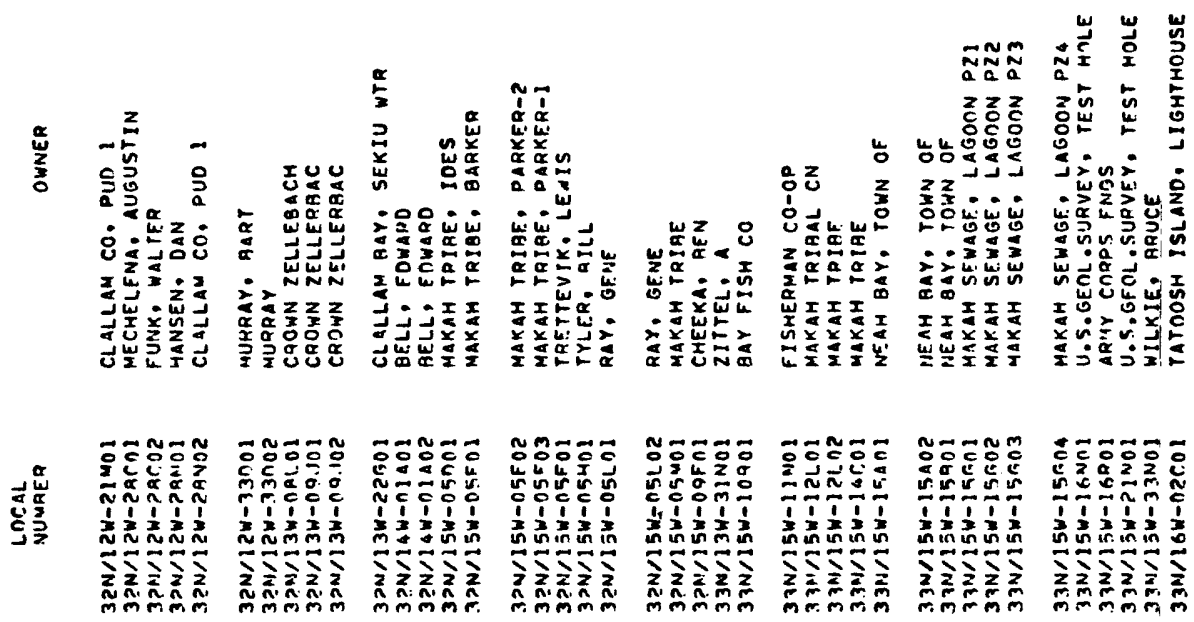
LOCATION--Lat 48002 '40", long 123057'28", in lot 4 SE⿺辶SW/ sec. 35, T.20 N., R. 10 H., Clallan County, 01ympic Mational Park, on right bank 0.1 mi downstream from south Fo $\mathbf{1 . 6}$ mi southwest of Fairholm, and at mile 54.9 .

DRAINAGE AREA--83.8 mi 2 .

PERIOD OF RECORD--October 1917 to September 1921, October 1933 to September 1971, October 1976 to current year.

GAGE-- Hater-stage recorder. Alt1tude of gage is 1,060 ft (from topographic map). Oct 1917 to Sept 1921 , water-stage recorder and Oct 4 to Nov 4, 1933, nonrecording gage, at same site at datum $1.2 \mathrm{ft}(0.37$ m) higher.

AVERAGE DISCHARGE--45 years (water years 1918-21, 1934-71, 1977-79), 621 ft3/s, 100,53 in/yr, 449,300 acre-ft/yr.

EXTREMES FOR PERIOD OF RECORD--Maxinum discharge, $23,500 \mathrm{ft} 3 / \mathrm{s}$ Nov 26,1949 , gage height, $16.42 \mathrm{ft} f \mathrm{from} \mathrm{high-water} \mathrm{mark} \mathrm{in} \mathrm{well,}$

from rating curve extended above $13,000 \mathrm{ft} 3 / \mathrm{s}$ on basis of slope-area measurement of peak flow; minimum, $51 \mathrm{ft} 3 / \mathrm{s}$ Sept 11 , 12 ,

1944; minimum gage height, $0.70 \mathrm{ft}$ kug 9, 10,. 1978.

REMARKS--Records excellent. No regulation or diversion above station.

DISCHARGF, IN CUAIC FEET PER SECOND, WATER YEAR OCTOAER 1917 TO SFPTEMAER 1918 MEAN VALUES

\begin{tabular}{|c|c|c|c|c|c|c|c|c|c|c|c|c|}
\hline 1919 & OCT & Nov & DEC & JAN & FEB & MAR & APR & MAY & JUN & JUL & AUG & SFP \\
\hline $\begin{array}{l}\text { TOTAL } \\
\text { MFAN } \\
\text { MAX } \\
\text { MIN }\end{array}$ & $\begin{array}{r}4422 \\
143 \\
250 \\
92\end{array}$ & $\begin{array}{r}7551 \\
252 \\
605 \\
101\end{array}$ & $\begin{array}{r}86177 \\
2780 \\
11500 \\
295\end{array}$ & $\begin{array}{r}44330 \\
1430 \\
4730 \\
580\end{array}$ & $\begin{array}{r}30672 \\
1095 \\
4410 \\
3 i 2\end{array}$ & $\begin{array}{r}26933 \\
869 \\
3730 \\
210\end{array}$ & $\begin{array}{r}19076 \\
636 \\
1430 \\
+38\end{array}$ & $\begin{array}{r}14180 \\
457 \\
842 \\
279\end{array}$ & $\begin{array}{r}12228 \\
408 \\
1070 \\
231\end{array}$ & $\begin{array}{r}5128 \\
165 \\
231 \\
111\end{array}$ & $\begin{array}{r}3869 \\
125 \\
207 \\
91\end{array}$ & $\begin{array}{r}2157 \\
71.9 \\
84 \\
60\end{array}$ \\
\hline WTR YR & 1918 TOTAL & 256723 & MEAN & 703 & $\max$ & 11500 & 460 & & & & & \\
\hline 1919 & OCT & Nov & DEC & JAN & FEB & MAR & APR & MaY & JUN & JUL & AUG & SEP \\
\hline $\begin{array}{l}\text { TOTAL } \\
\text { MFAN } \\
\text { MAX } \\
\text { MIN }\end{array}$ & $\begin{array}{r}15484 \\
499 \\
6100 \\
58\end{array}$ & $\begin{array}{r}25143 \\
838 \\
3850 \\
379\end{array}$ & $\begin{array}{r}54483 \\
1758 \\
8910 \\
508\end{array}$ & $\begin{array}{r}42742 \\
1379 \\
5050 \\
753\end{array}$ & $\begin{array}{r}24923 \\
890 \\
4040 \\
445\end{array}$ & $\begin{array}{r}17473 \\
564 \\
1080 \\
365\end{array}$ & $\begin{array}{r}26793 \\
893 \\
1440 \\
539\end{array}$ & $\begin{array}{r}23281 \\
751 \\
1470 \\
498\end{array}$ & $\begin{array}{r}19899 \\
663 \\
1040 \\
553\end{array}$ & $\begin{array}{r}17348 \\
560 \\
742 \\
361\end{array}$ & $\begin{array}{r}7572 \\
244 \\
334 \\
147\end{array}$ & $\begin{array}{r}36 A_{1}^{-} \\
123 \\
210 \\
90\end{array}$ \\
\hline WTR YR & 1919 TOTAL & 27882? & MFAN & 764 & MAX & B910 & 58 & & & & & \\
\hline $192 n$ & OCT & NOV & nFC & JAN & FEB & MAR & APR & mar & JUN & JUL & AUG & SEP \\
\hline $\begin{array}{l}\text { TOTAL } \\
\text { MFAN } \\
\text { MAX } \\
\text { MIN }\end{array}$ & $\begin{array}{r}2787 \\
89.9 \\
153 \\
75\end{array}$ & $\begin{array}{r}24653 \\
822 \\
6960 \\
141\end{array}$ & $\begin{array}{r}28787 \\
979 \\
41: 0 \\
150\end{array}$ & $\begin{array}{r}31074 \\
1002 \\
4040 \\
293\end{array}$ & $\begin{array}{r}13017 \\
449 \\
921 \\
190\end{array}$ & $\begin{array}{r}14305 \\
461 \\
2400 \\
175\end{array}$ & $\begin{array}{r}9361 \\
312 \\
428 \\
252\end{array}$ & $\begin{array}{r}11257 \\
363 \\
854 \\
259\end{array}$ & $\begin{array}{r}15371 \\
512 \\
848 \\
326\end{array}$ & $\begin{array}{r}6712 \\
217 \\
390 \\
125\end{array}$ & $\begin{array}{r}3487 \\
112 \\
310 \\
84\end{array}$ & $\begin{array}{r}18509 \\
617 \\
2010 \\
84\end{array}$ \\
\hline WTR YR & 1920 TDTAL & 179320 & MEAN & 490 & $\operatorname{MAX}$ & 6960 & 75 & & & & & \\
\hline 1921 & OCT & NOV & DFC & JARi & FEB & MAR & APR & MAY & JUN & JUL & AUG & SEP \\
\hline $\begin{array}{l}\text { TOTAL } \\
\text { MFAN } \\
\text { MAX } \\
\text { MTN }\end{array}$ & $\begin{array}{r}35108 \\
1133 \\
4830 \\
4.34\end{array}$ & $\begin{array}{r}21875 \\
729 \\
1860 \\
230\end{array}$ & $\begin{array}{r}30033 \\
969 \\
3220 \\
468\end{array}$ & $\begin{array}{r}35404 \\
1142 \\
4530 \\
527\end{array}$ & $\begin{array}{r}36214 \\
1293 \\
7840 \\
472\end{array}$ & $\begin{array}{r}20310 \\
655 \\
1260 \\
392\end{array}$ & $\begin{array}{r}14850 \\
495 \\
772 \\
309\end{array}$ & $\begin{array}{r}22891 \\
738 \\
1070 \\
436\end{array}$ & $\begin{array}{r}26750 \\
892 \\
1260 \\
645\end{array}$ & $\begin{array}{r}14866 \\
480 \\
742 \\
350\end{array}$ & $\begin{array}{r}7569 \\
244 \\
334 \\
159\end{array}$ & $\begin{array}{r}15854 \\
528 \\
3630 \\
142\end{array}$ \\
\hline WTO YR & TOTAL & 281724 & NEAN & $77 ?$ & $\max$ & 7840 & 142 & & & & & \\
\hline 1934 & OCT & NnV & DEC & $J A N$ & FEA & MAR & APR & MAY & JUN & JUL & AUG & SEP \\
\hline $\begin{array}{l}\text { TOTAL } \\
\text { MFAN } \\
\text { MAX } \\
\text { MIN }\end{array}$ & $\begin{array}{r}22605 \\
732 \\
2430 \\
208\end{array}$ & $\begin{array}{r}21060 \\
702 \\
3200 \\
296\end{array}$ & $\begin{array}{r}77342 \\
2495 \\
10700 \\
336\end{array}$ & $\begin{array}{r}54155 \\
1747 \\
4700 \\
915\end{array}$ & $\begin{array}{r}18243 \\
652 \\
1190 \\
308\end{array}$ & $\begin{array}{r}27352 \\
882 \\
3170 \\
276\end{array}$ & $\begin{array}{r}15587 \\
520 \\
1050 \\
338\end{array}$ & $\begin{array}{r}1765 A \\
570 \\
1700 \\
315\end{array}$ & $\begin{array}{r}7608 \\
254 \\
381 \\
168\end{array}$ & $\begin{array}{r}5288 \\
171 \\
714 \\
112\end{array}$ & $\begin{array}{r}3363 \\
108 \\
203 \\
78\end{array}$ & $\begin{array}{r}3223 \\
107 \\
342 \\
75 \\
95\end{array}$ \\
\hline WTR YR & 1934 TOTAL & 273575 & MFAN & 750 & $\max$ & 10700 & 475 & & & & & \\
\hline 1935 & OCT & Nov & DEC & JAN & FEB & MAR & APR & MAY & JUN & JUL & AUG & SEP \\
\hline $\begin{array}{l}\text { TOTAL } \\
\text { MFANI } \\
\text { MAX } \\
\text { MIN }\end{array}$ & $\begin{array}{r}14344 \\
463 \\
2520 \\
72\end{array}$ & $\begin{array}{r}44659 \\
1489 \\
10500 \\
625\end{array}$ & $\begin{array}{r}31589 \\
1119 \\
3930 \\
501\end{array}$ & $\begin{array}{r}79952 \\
2579 \\
11900 \\
375\end{array}$ & $\begin{array}{r}31604 \\
1129 \\
2780 \\
600\end{array}$ & $\begin{array}{r}19742 \\
637 \\
2460 \\
338\end{array}$ & $\begin{array}{r}12414 \\
414 \\
605 \\
256\end{array}$ & $\begin{array}{r}1598 ? \\
516 \\
726 \\
412\end{array}$ & $\begin{array}{r}16831 \\
561 \\
878 \\
372\end{array}$ & $\begin{array}{r}10801 \\
34 \mathrm{~B} \\
515 \\
218\end{array}$ & $\begin{array}{r}4493 \\
145 \\
212 \\
104\end{array}$ & $\begin{array}{r}6325 \\
211 \\
1310 \\
71\end{array}$ \\
\hline WTP YP & 19.35 TOTAL & 298736 & MEAN & 791 & $\max$ & 11900 & 71 & & & & & \\
\hline 1936 & nc: & Nov & OEC & JAN & FEB & MAR & APR & $\operatorname{mar}$ & JUN & JUL & AUG & SFP \\
\hline $\begin{array}{l}\text { TOTAI } \\
\text { MFAN } \\
\text { NAX } \\
\text { MIN }\end{array}$ & $\begin{array}{r}7030 \\
227 \\
676 \\
95\end{array}$ & $\begin{array}{r}9543 \\
318 \\
1000 \\
129\end{array}$ & $\begin{array}{r}17567 \\
567 \\
1060 \\
214\end{array}$ & $\begin{array}{r}33181 \\
1070 \\
3160 \\
402\end{array}$ & $\begin{array}{r}11088 \\
382 \\
1460 \\
200\end{array}$ & $\begin{array}{r}72234 \\
717 \\
1780 \\
286\end{array}$ & $\begin{array}{r}17087 \\
570 \\
1000 \\
228\end{array}$ & $\begin{array}{r}24661 \\
796 \\
1300 \\
586\end{array}$ & $\begin{array}{r}21514 \\
717 \\
1760 \\
402\end{array}$ & $\begin{array}{r}10619 \\
343 \\
686 \\
182\end{array}$ & $\begin{array}{r}3902 \\
126 \\
182 \\
93\end{array}$ & $\begin{array}{r}3879 \\
129 \\
566 \\
84\end{array}$ \\
\hline WTP YR & 1936 TOTAL & 182305 & MEAN & 498 & $\max$ & MIN & 84 & & & & & \\
\hline 1937 & OCT & Nov & DEC & JAN & FEB & MAR & APR & MAY & JUN & JUL & AUG & SFI \\
\hline $\begin{array}{l}\text { TOTAL } \\
\text { MFAN } \\
\text { MAX } \\
\text { MIN }\end{array}$ & $\begin{array}{r}2715 \\
87.5 \\
204 \\
70\end{array}$ & $\begin{array}{r}2481 \\
82.7 \\
150 \\
60\end{array}$ & $\begin{array}{r}32846 \\
1060 \\
4890 \\
69\end{array}$ & $\begin{array}{r}6595 \\
213 \\
366 \\
120\end{array}$ & $\begin{array}{r}9707 \\
347 \\
1310 \\
120\end{array}$ & $\begin{array}{r}18203 \\
587 \\
1200 \\
286\end{array}$ & $\begin{array}{r}24570 \\
819 \\
3570 \\
399\end{array}$ & $\begin{array}{r}25606 \\
826 \\
1840 \\
421\end{array}$ & $\begin{array}{r}26958 \\
899 \\
1420 \\
615\end{array}$ & $\begin{array}{r}11207 \\
362 \\
630 \\
195\end{array}$ & $\begin{array}{r}4696 \\
151 \\
206 \\
113\end{array}$ & $\begin{array}{r}29.5 ? \\
95.1 \\
796 \\
72\end{array}$ \\
\hline WTR YR & TITAL & 168436 & MFAN & 461 & $\max$ & MIN & 60 & & & & & \\
\hline
\end{tabular}


TABLE 7.--Records of gaging stations in Clallaw County--continued

12041500 SOLEDUCK RIVER MEAR FAIRHOLM, WA (Site 4) -- continued

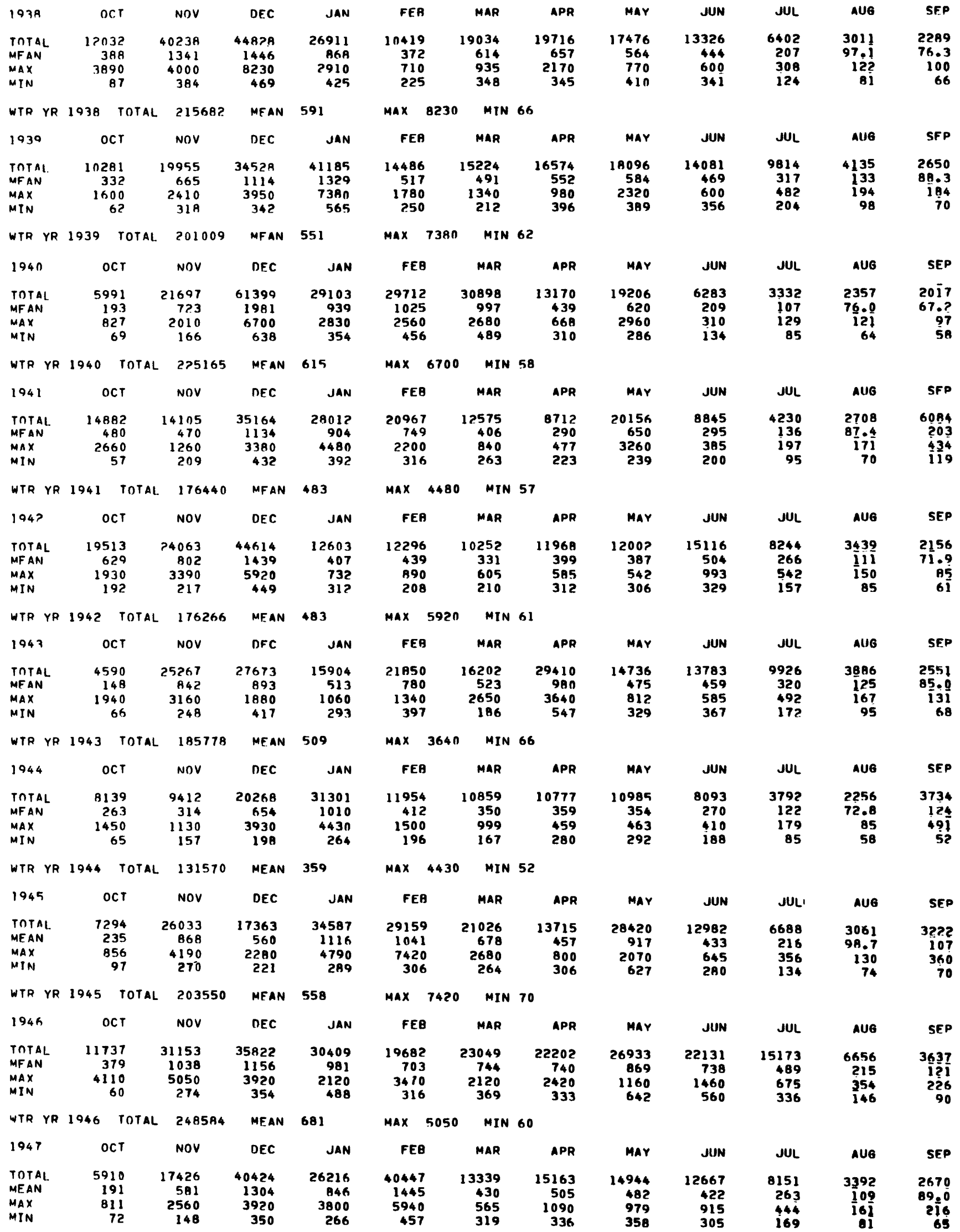

WTR YR 1947 TOTAL 200749 MFAN 550 MAX 5940 MIN 65 
TABLE 7.--Records of gaging stations in Clallan County--continued

12041500 SOLEDUCK RIVER MEAR FAIRHOLM, WA (SIte 4) -- continued

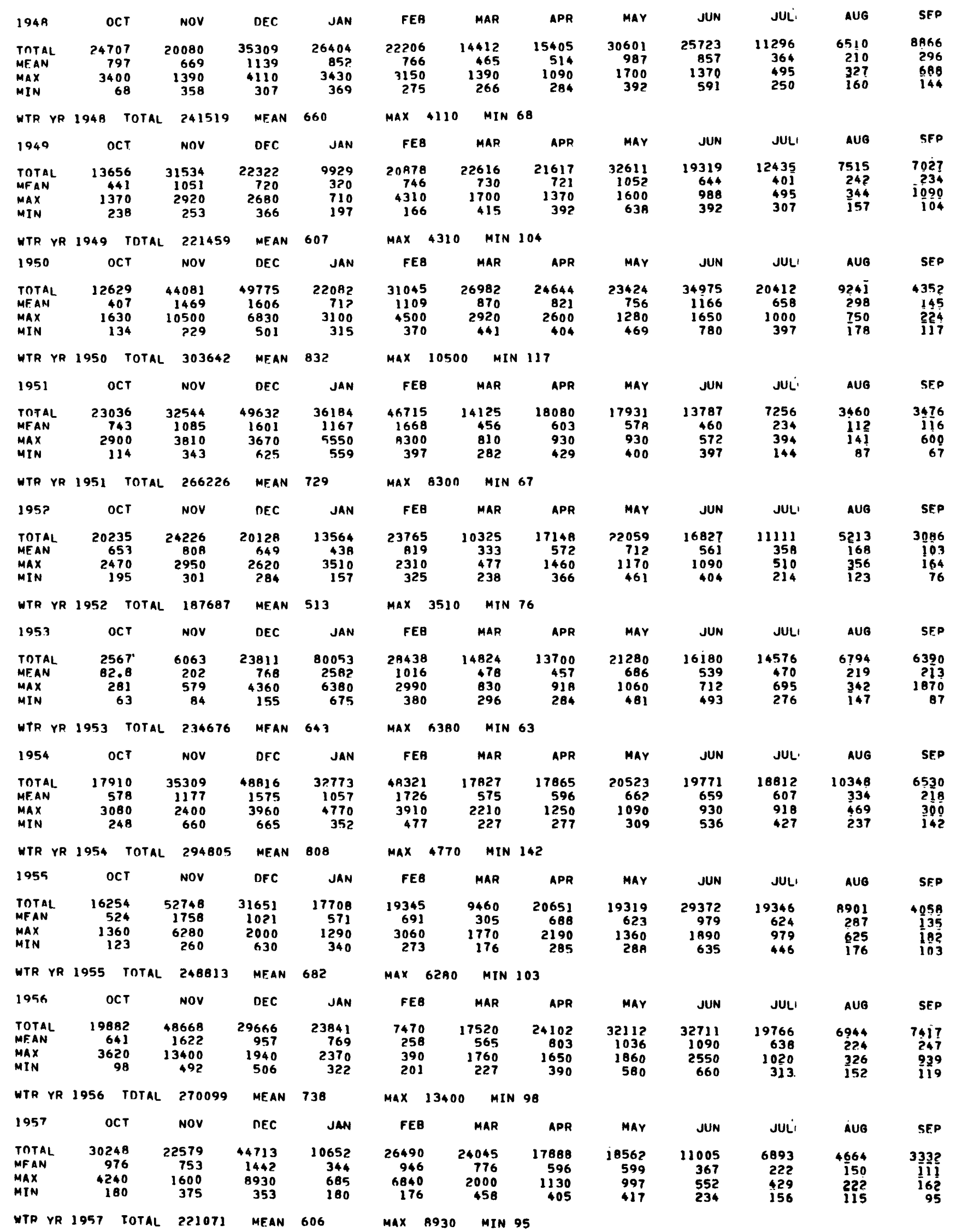


TABLE 7,--Records of gaging stations in Clallam County--continued 12041500 SOLEDUCK RIVER NEAR FAIRHOLM, WA (S1 te 4) -- continued

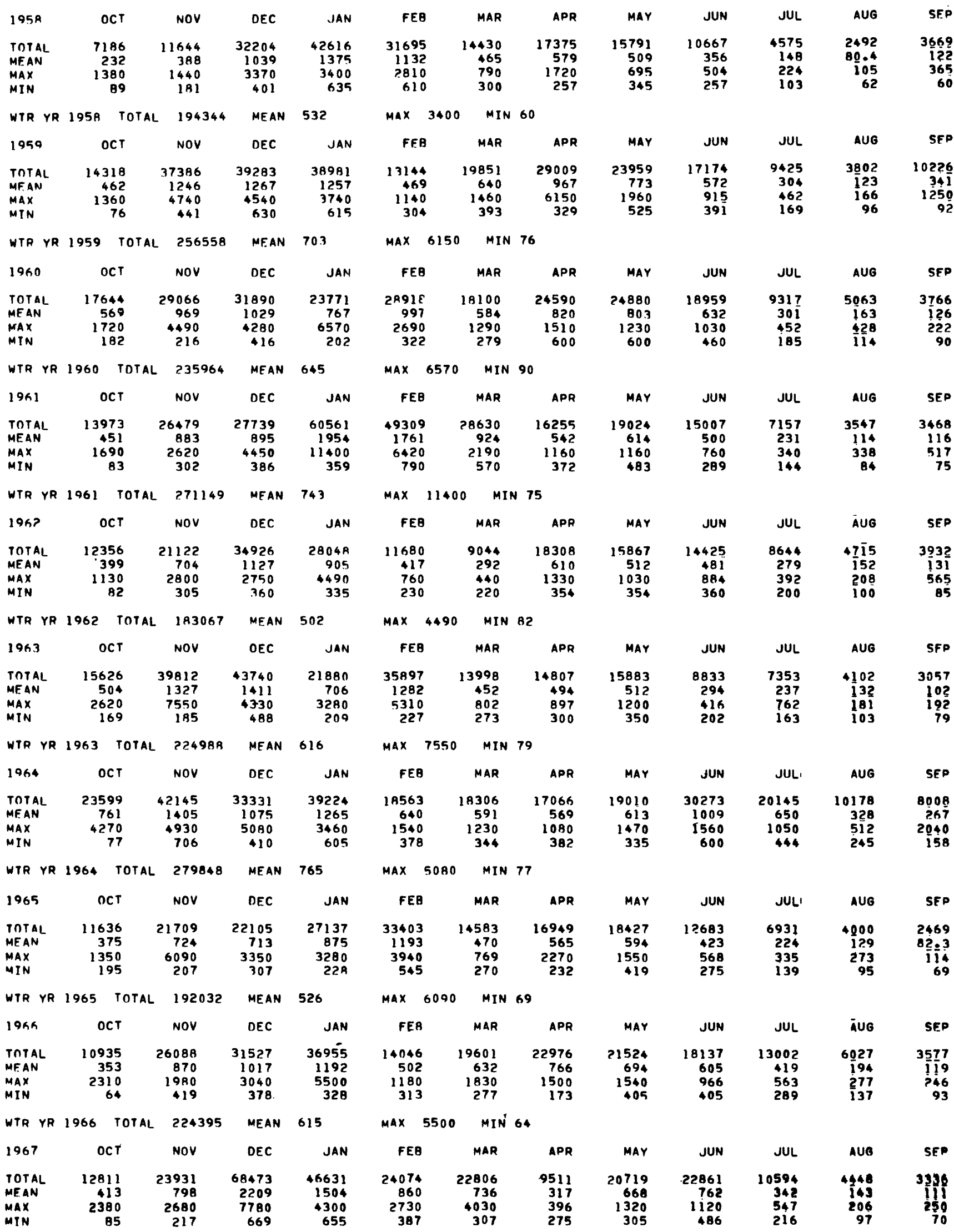


TABLE 7.--Records of gaging stations in Clallam County--continued 12041500 SOLEDUCK RIVER NEAR FAIRHOLM, HA (Site 4) -- continuea

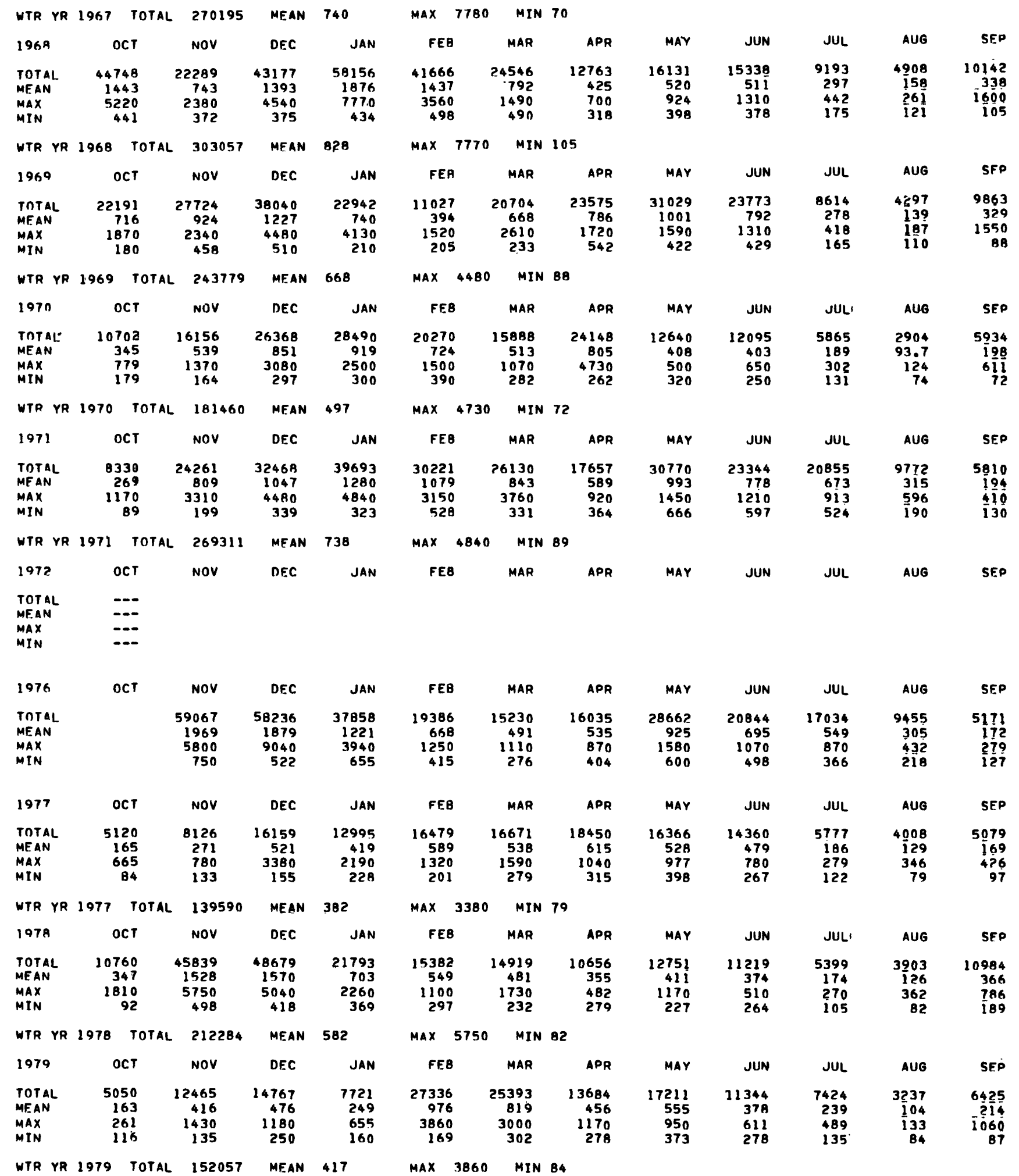


TABLE 7.--Records of gaging stations in Clallam County--continued

12042000 SOLEDUCK RIVER NEAR BEAVER, HA (S1 te 6)

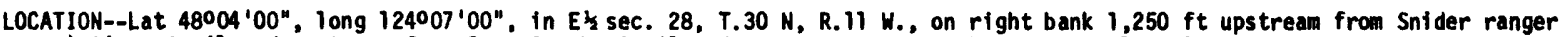
station, 4 miles downstream from Camp Creek. 9 miles downstream from South Fork, and il miles east of Beaver.

PERIOD OF RECORD--Oct 1921 to Sept 1922, Dec 1922 to Oct 1925, May 1926 to Sept 1926, Apr 1927 to Sept 1928

DRAINAGE AREA--116 sq $\mathrm{mi}$.

GAGE--Staff gage. Altitude of gage is $730 \mathrm{ft}$ (from topographic map). Nov 13 to Dec 16, 1921, staff gage $250 \mathrm{ft}$ upstrean at same datum.

EXTREMES--1922-28. Maximum discharge, $23,500 \mathrm{ft}^{3} / \mathrm{s}$ Dec 12,1921 (gage height, $14.7 \mathrm{ft}$ from graph based on gage readings); minimum observed, $28 \mathrm{ft} 3 / \mathrm{s}$ Sept 14,1926 (gage height, $1.10 \mathrm{ft}$ ).

REMARKS--No diversion or regulation above station.

OISCHARGE, IN CUBIC FEET PER SECOND, WATER YEAR OCTOAER I921 TO SFPTEMBER İT2 MEAN VALUES

\begin{tabular}{|c|c|c|c|c|c|c|c|c|c|c|c|c|}
\hline $192 ?$ & OCT & NOV & DEC & JAN & FEB & MAR & APR & MAY & JUN & JUL' & AUG & SEP \\
\hline $\begin{array}{l}\text { TOTAL } \\
\text { MFAN } \\
\text { MAX } \\
\text { MIN }\end{array}$ & $\begin{array}{r}41540 \\
1340 \\
1340 \\
1340\end{array}$ & $\begin{array}{r}41420 \\
1381 \\
5870 \\
340\end{array}$ & $\begin{array}{r}66180 \\
2135 \\
18200 \\
780\end{array}$ & $\begin{array}{r}10230 \\
330 \\
330 \\
330\end{array}$ & $\begin{array}{r}10133 \\
362 \\
840 \\
198\end{array}$ & $\begin{array}{r}6513 \\
210 \\
253 \\
186\end{array}$ & $\begin{array}{r}11272 \\
376 \\
570 \\
238\end{array}$ & $\begin{array}{r}33540 \\
108 ? \\
2180 \\
520\end{array}$ & $\begin{array}{r}26280 \\
876 \\
1860 \\
520\end{array}$ & $\begin{array}{r}9065 \\
292 \\
545 \\
153\end{array}$ & $\begin{array}{r}3717 \\
120 \\
162 \\
83\end{array}$ & $\begin{array}{r}4145 \\
138 \\
425 \\
72\end{array}$ \\
\hline WTR YR & 1922 TOTAL & 264035 & MEAN & 723 & $\max :$ & 18200 & 172 & & & & & \\
\hline 1927 & OCT & NOV & DEC & JAN & FEB & MAR & APR & MAY & JUN & JUL & AUG & SFP \\
\hline $\begin{array}{l}\text { TOTAL } \\
\text { MFAN } \\
\text { MAX } \\
\text { MIN }\end{array}$ & & & $\begin{array}{r}48170 \\
1554 \\
9840 \\
200\end{array}$ & $\begin{array}{r}66298 \\
2139 \\
7230 \\
360\end{array}$ & $\begin{array}{r}15314 \\
547 \\
1960 \\
238\end{array}$ & $\begin{array}{r}13763 \\
444 \\
840 \\
285\end{array}$ & $\begin{array}{r}16613 \\
554 \\
780 \\
448\end{array}$ & $\begin{array}{r}18653 \\
602 \\
960 \\
40 ?\end{array}$ & $\begin{array}{r}15700 \\
523 \\
840 \\
380\end{array}$ & $\begin{array}{r}9883 \\
319 \\
545 \\
162\end{array}$ & $\begin{array}{r}3520 \\
114 \\
157 \\
75\end{array}$ & $\begin{array}{r}2549 \\
85.0 \\
238 \\
54\end{array}$ \\
\hline 1924 & OCT & NOV & DEC & JAN & FEB & MAR & APR & MAY & JUN & JUi & AUG & SEP. \\
\hline $\begin{array}{l}\text { TOTAL } \\
\text { MFAN } \\
\text { MAX } \\
\text { MIN }\end{array}$ & $\begin{array}{r}6090 \\
196 \\
1100 \\
60\end{array}$ & $\begin{array}{r}15399 \\
513 \\
3470 \\
117\end{array}$ & $\begin{array}{r}57412 \\
1852 \\
12800 \\
425\end{array}$ & $\begin{array}{r}4068 A \\
1313 \\
15300 \\
400\end{array}$ & $\begin{array}{r}61330 \\
2115 \\
11600 \\
780\end{array}$ & $\begin{array}{r}13032 \\
420 \\
1100 \\
205\end{array}$ & $\begin{array}{r}9562 \\
319 \\
695 \\
220\end{array}$ & $\begin{array}{r}10535 \\
340 \\
470 \\
235\end{array}$ & $\begin{array}{r}6319 \\
211 \\
300 \\
146\end{array}$ & $\begin{array}{r}3322 \\
107 \\
151 \\
72\end{array}$ & $\begin{array}{r}1863 \\
60.1 \\
114 \\
43\end{array}$ & $\begin{array}{r}8996 \\
300 \\
2620 \\
34\end{array}$ \\
\hline WTR YR & 1924 TOTAL & $23454 \mathrm{~A}$ & MFAN & 641 & $\max$ & 15300 & 34 & & & & & \\
\hline 1925 & OCT & NoV & DEC & JAN & FEB & MAR & APR & MAY & JUN & JUL & AUB & SEP \\
\hline $\begin{array}{l}\text { TOTAL } \\
\text { MFAN } \\
\text { MAX } \\
\text { MIN }\end{array}$ & $\begin{array}{r}33577 \\
1083 \\
2620 \\
333\end{array}$ & $\begin{array}{r}41222 \\
1374 \\
4480 \\
633\end{array}$ & $\begin{array}{r}42999 \\
1387 \\
4170 \\
439\end{array}$ & $\begin{array}{r}39718 \\
1281 \\
4170 \\
462\end{array}$ & $\begin{array}{r}60100 \\
2146 \\
6440 \\
608\end{array}$ & $\begin{array}{r}17978 \\
580 \\
1580 \\
333\end{array}$ & $\begin{array}{r}16323 \\
544 \\
1110 \\
294\end{array}$ & $\begin{array}{r}20731 \\
669 \\
1410 \\
485\end{array}$ & $\begin{array}{r}14873 \\
496 \\
583 \\
417\end{array}$ & $\begin{array}{r}8249 \\
266 \\
395 \\
144\end{array}$ & $\begin{array}{r}3363 \\
108 \\
140 \\
77\end{array}$ & $\begin{array}{r}1678 \\
55.9 \\
77 \\
44\end{array}$ \\
\hline WTR YR & 1925 TOTAL & 300811 & MEAN & 824 & $\max$ & MIN & 44 & & & & & \\
\hline 1926 & OCT & NOV & DEC & JAN & FEB & MAR & APR & MAY & JUN & JULI & AUE & SEP \\
\hline $\begin{array}{l}\text { TOTAL } \\
\text { MEAN } \\
\text { MAX } \\
\text { MIN }\end{array}$ & $\begin{array}{r}1382 \\
44.6 \\
50 \\
38\end{array}$ & & & & & & & $\begin{array}{r}12957 \\
418 \\
633 \\
313\end{array}$ & $\begin{array}{r}6115 \\
204 \\
353 \\
121\end{array}$ & $\begin{array}{r}2585 \\
83.4 \\
117 \\
59\end{array}$ & $\begin{array}{r}1574 \\
50.8 \\
67 \\
40\end{array}$ & $\begin{array}{r}1933 \\
64.4 \\
353 \\
28\end{array}$ \\
\hline 1927 & OCT & NOV & DEC & JAN & FEB & MAR & APR & MAY & JUN & JUL' & AUE & SEB⿱ \\
\hline $\begin{array}{l}\text { TOTAL } \\
\text { MEAN } \\
\text { MAX } \\
\text { MIN }\end{array}$ & & & & & & & $\begin{array}{r}15705 \\
524 \\
1250 \\
333\end{array}$ & $\begin{array}{r}26350 \\
850 \\
1410 \\
509\end{array}$ & $\begin{array}{r}22345 \\
745 \\
1330 \\
509\end{array}$ & $\begin{array}{r}10995 \\
355 \\
485 \\
209\end{array}$ & $\begin{array}{r}4230 \\
1.36 \\
353 \\
94\end{array}$ & $\begin{array}{r}9378 \\
313 \\
1330 \\
130\end{array}$ \\
\hline J92A & OCT & NOV & DEC & JAN & FEB & MAR & APR & MaY & JUN & Jui & AUG & SEP \\
\hline $\begin{array}{l}\text { TOTAL } \\
\text { MFAN } \\
\text { MAX } \\
\text { MIN }\end{array}$ & $\begin{array}{r}22786 \\
735 \\
2850 \\
276\end{array}$ & $\begin{array}{r}41265 \\
1376 \\
3600 \\
417\end{array}$ & $\begin{array}{r}21629 \\
698 \\
2100 \\
353\end{array}$ & $\begin{array}{r}52536 \\
1695 \\
5500 \\
445\end{array}$ & $\begin{array}{r}13173 \\
454 \\
740 \\
260\end{array}$ & $\begin{array}{r}30357 \\
979 \\
3880 \\
228\end{array}$ & $\begin{array}{r}22691 \\
756 \\
1960 \\
489\end{array}$ & $\begin{array}{r}23042 \\
743 \\
1250 \\
583\end{array}$ & $\begin{array}{r}12339 \\
411 \\
633 \\
293\end{array}$ & $\begin{array}{r}5840 \\
188 \\
276 \\
115\end{array}$ & $\begin{array}{r}2465 \\
79.5 \\
113 \\
55\end{array}$ & $\begin{array}{r}18 \overline{16} 6 \\
60.5 \\
200 \\
45\end{array}$ \\
\hline
\end{tabular}

WTR YR 1928 TOTAL 249939 MEAN 683 MAX 5500 MIN 45 
TABLE 7.--Records of gaging stations in Clallan County--continued

12042500 SOLEDUCK RIVER MEAR QUILLAYUTE, WA (SIte 23)

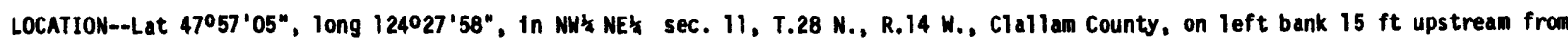
county bridge, 3.9 ifi west of Forks and at mile 13.9.

DRAINAGE AREA--219 mi2.

PERIOD OF RECORD--Nov 1897 to Mar 1900, Jan 1901 to Dec 1901, Oct to current year.

GAGE-- Water-stage recorder. Altitude of gage is $150 \mathrm{ft}$ (from topographic map). Prior to Dec 31 , 1901, nonrecording gage at different datum.

EXTREMES FOR PERIOD OF RECORD--Maximum discharge, $20,800 \mathrm{ft}^{3} / \mathrm{s}$, Nov 1,1977 , gage height, $15.77 \mathrm{ft}$ from rating curve extended above $6,700 \mathrm{ft}^{3} / \mathrm{s}$; minimum discharge observed, $155 \mathrm{ft} 3 / \mathrm{s}$ 0ct $16,17,1901$.

REMARKS--Water-discharge records excellent except those above $10,000 \mathrm{ft} 3 / \mathrm{s}$, which are fair. No regulation or diversion above station. OISCHARGF, IN CUAIC FEET PER SECOND, WATER YEAR OCTOAER 1 A97 TO SEPTEMBER 1898

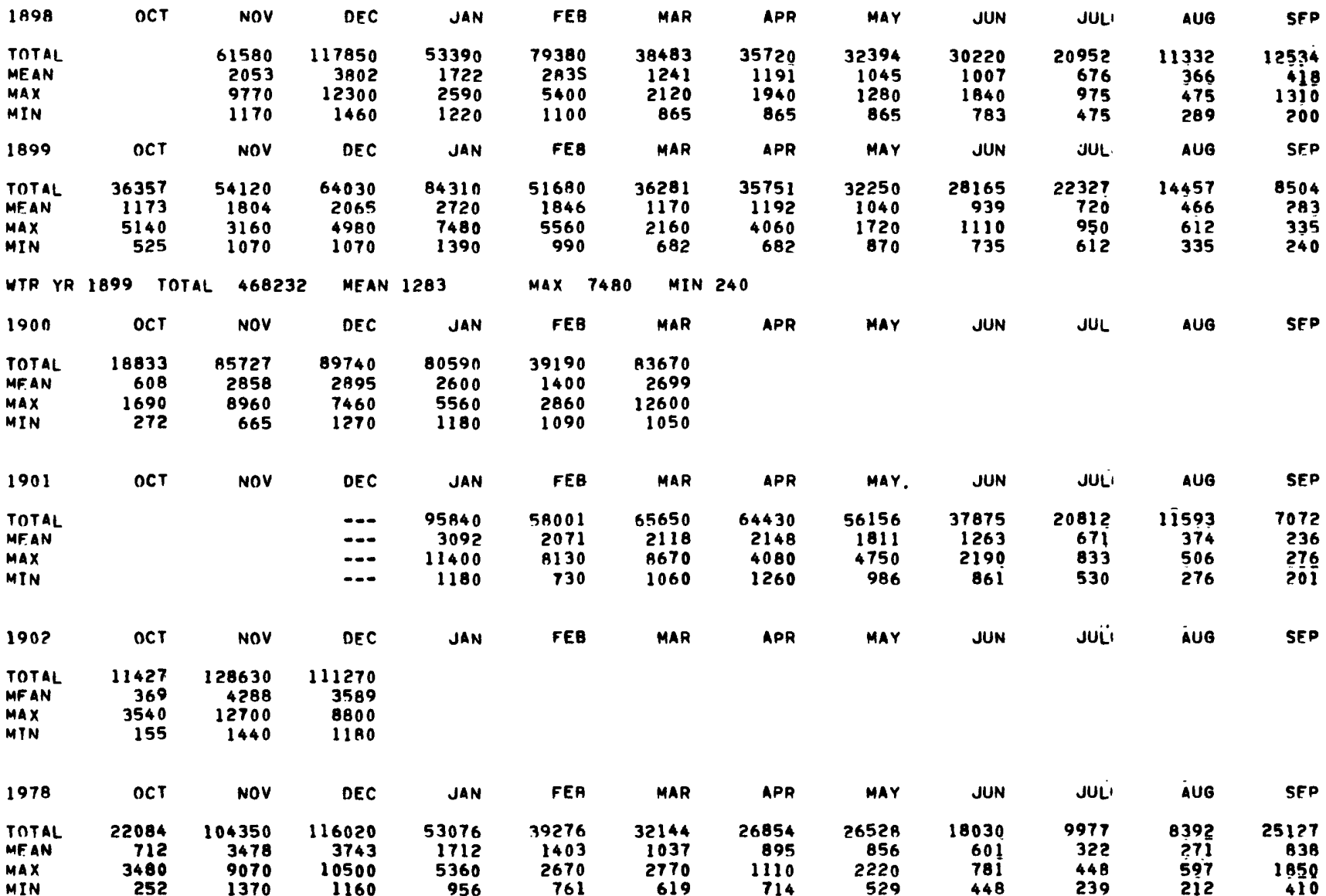

WTR YR 1978 TOTAL 481858 MEAN 1320 MAX 10500 MIN 212

PROVISIONAL DATA

\begin{tabular}{|c|c|c|c|c|c|c|c|c|c|c|c|c|}
\hline 1979 & DCT & NOV & DEC & JAN & FEB & MAR & APR & MAY & JUN & JULi & Áug & SEP \\
\hline $\begin{array}{l}\text { TOTAL } \\
\text { MEAN } \\
\text { MAX } \\
\text { MIN }\end{array}$ & $\begin{array}{r}14249 \\
460 \\
760 \\
340\end{array}$ & $\begin{array}{r}29270 \\
976 \\
2700 \\
400\end{array}$ & $\begin{array}{r}41436 \\
1337 \\
2130 \\
805\end{array}$ & $\begin{array}{r}21371 \\
689 \\
1590 \\
510\end{array}$ & $\begin{array}{r}78266 \\
2795 \\
7360 \\
508\end{array}$ & $\begin{array}{r}61684 \\
1990 \\
7080 \\
720\end{array}$ & $\begin{array}{r}30892 \\
1030 \\
2280 \\
681\end{array}$ & $\begin{array}{r}27954 \\
902 \\
1660 \\
598\end{array}$ & $\begin{array}{r}16344 \\
545 \\
801 \\
426\end{array}$ & $\begin{array}{r}12282 \\
396 \\
691 \\
260\end{array}$ & $\begin{array}{r}6660 \\
215 \\
253 \\
180\end{array}$ & $\begin{array}{r}12035 \\
401 \\
1500 \\
180\end{array}$ \\
\hline
\end{tabular}

WTR YR 1979 TOTAL 352443 MEAN 966 MAX 7360 MIN 180 
TABLE 7.--Records of gaging stations in Clallam County--continued

12042800 SOLEDUCK RIVER NEAR FORKS, WA (SIte 32)

LOCATION--Lat 47053'40", Iong 124021 '19", In NEK NEl sec. 34, T.28 N., R.13 W., Clallan County on right bank 50 ft downstrean from U.S. Highway 101, 0.6 mi downstream from Bear Creek, 4.2 mi southeast of Forks and at mile 15.3.

DRAINAGE AREA--111 m $^{2}$.

PERIOO OF RECORD--Apri1 1975 to current year.

GAEE--Hater stage recorder. Al titude of gage is $170 \mathrm{ft}$ (from topographic map).

EXTREMES FOR PERIOD OF RECORD--Maximum discharge, 23,800 ft3/s Dec 2, 1975, gage height, 15.81 ft from rating curve extended above $6,000 \mathrm{ft} 3 / \mathrm{s}$ on basis of step-backwater anaiysis; minimum, $83 \mathrm{ft} 3 / \mathrm{s}$ Aug $10,1978,7.61 \mathrm{ft}$.

REMARKS--Records good except those above $8,000 \mathrm{ft} 3 / \mathrm{s}$ and those for periods Oct 1-19, Dec 10 to Jan 17 , Mar 25 to Apr 4, which are fair. No regulation or tiversion above station.

$$
\text { DISCHARGF, IN CUAIC FEET PER SECOND, WATER YEAR OCTORER } 1974 \text { TO SEPTEMBER I975 }
$$

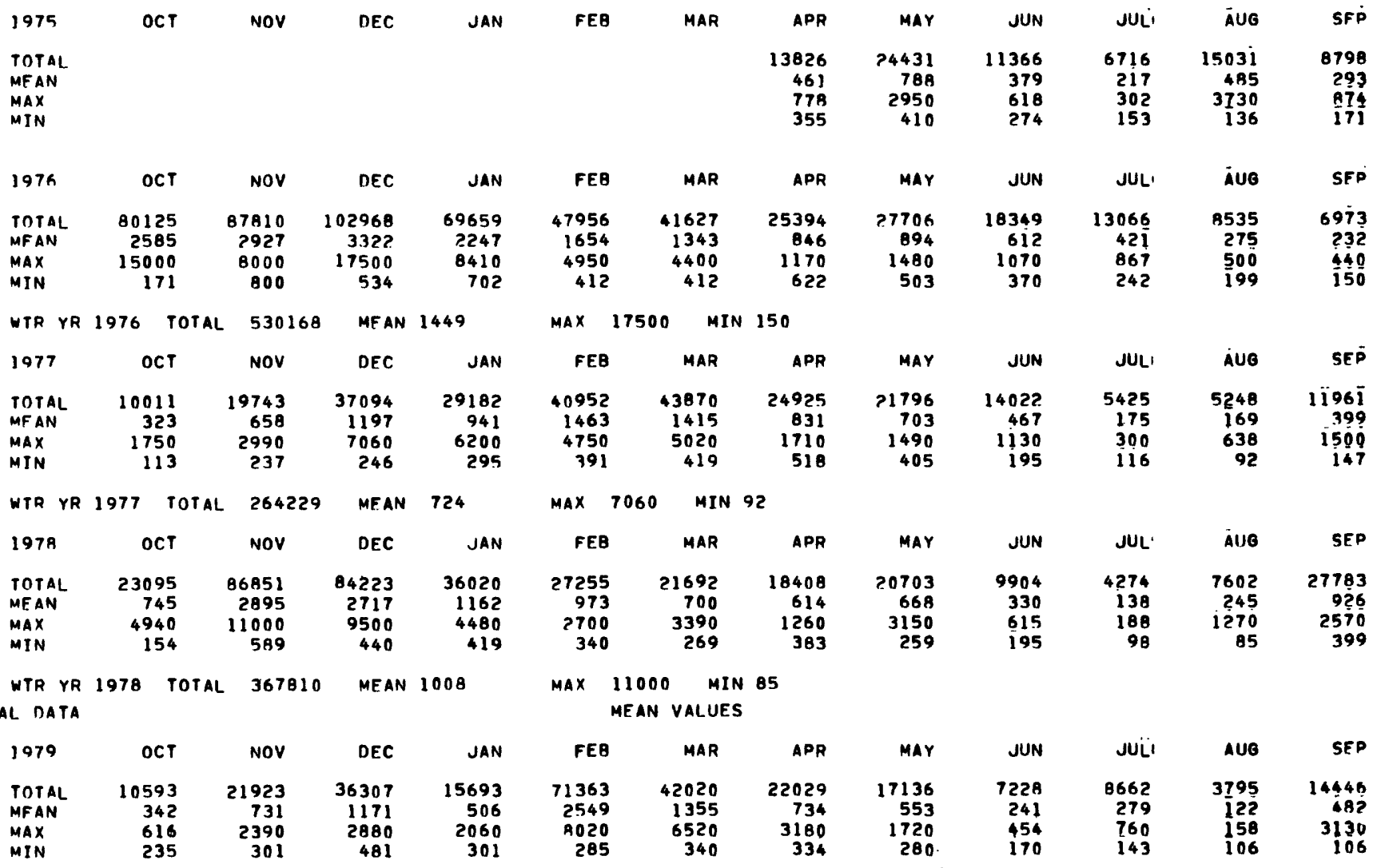

WTR YR 1979 TOTAL 271195 MEAN 743 MAX 8020 MIN 106 
TABLE 7.--Records of gaging stations in Clallam County--continued

12043000 CALAWAH RIVER NEAR FORKS, WA (SI te 42)

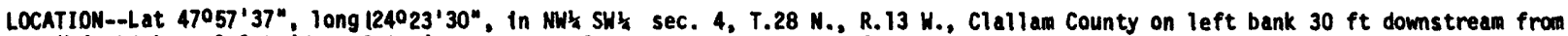
U.S. Highway 101 bridge, $0.8 \mathrm{ml}$ northwest of Forks, and at mile 6.6 .

DRAINAGE AREA--129 mi2.

PERIOD OF RECORD--Nov 1897 to Dec 1901, Jan 1976 to current year.

GACE--Water-stage recorder. Datum of gage is $201.58 \mathrm{ft}$, National Geodetic Vertical Datum of 1929. Nov 1897 to Dec 1901 , nonrecording gage at sane site but at dfferent datum; Oct to Dec 1975, nonrecording gage and crest-stage gage at sane site and datum.

AVERAGE DISCHARGE--6 years (water years 1899-1901, 1977-79) 1,044 ft3/s, $109.91 \mathrm{in} / \mathrm{yr}, 756,200 \mathrm{acre-ft/yr}$.

EXTREMES FOR PERIOD OF RECORD--Maximum dicharge, $22,600 \mathrm{ft}^{3} / \mathrm{s}$, Dec 2, 1975 , gage height, $16.99 \mathrm{ft}$, from rating curve extended above $6,000 \mathrm{ft}^{3} / \mathrm{s}$ on basis of step-backwater analysis; maximum gage height, $17.2 \mathrm{ft}$, from graph based on gage readings, Nov 18 , 1897 ; mintmum, $15 \mathrm{ft}^{3} / \mathrm{s}$ Sept 28, 1899.

REMARKS--Hater-discharge records excellent. No regulation or diversion above station.

DISCMARGE, IN CUBIC FEET PER SECOND, WATER YEAR OCTOBER 1897 TO SEPTEMBER 1898 MEAN VALUES

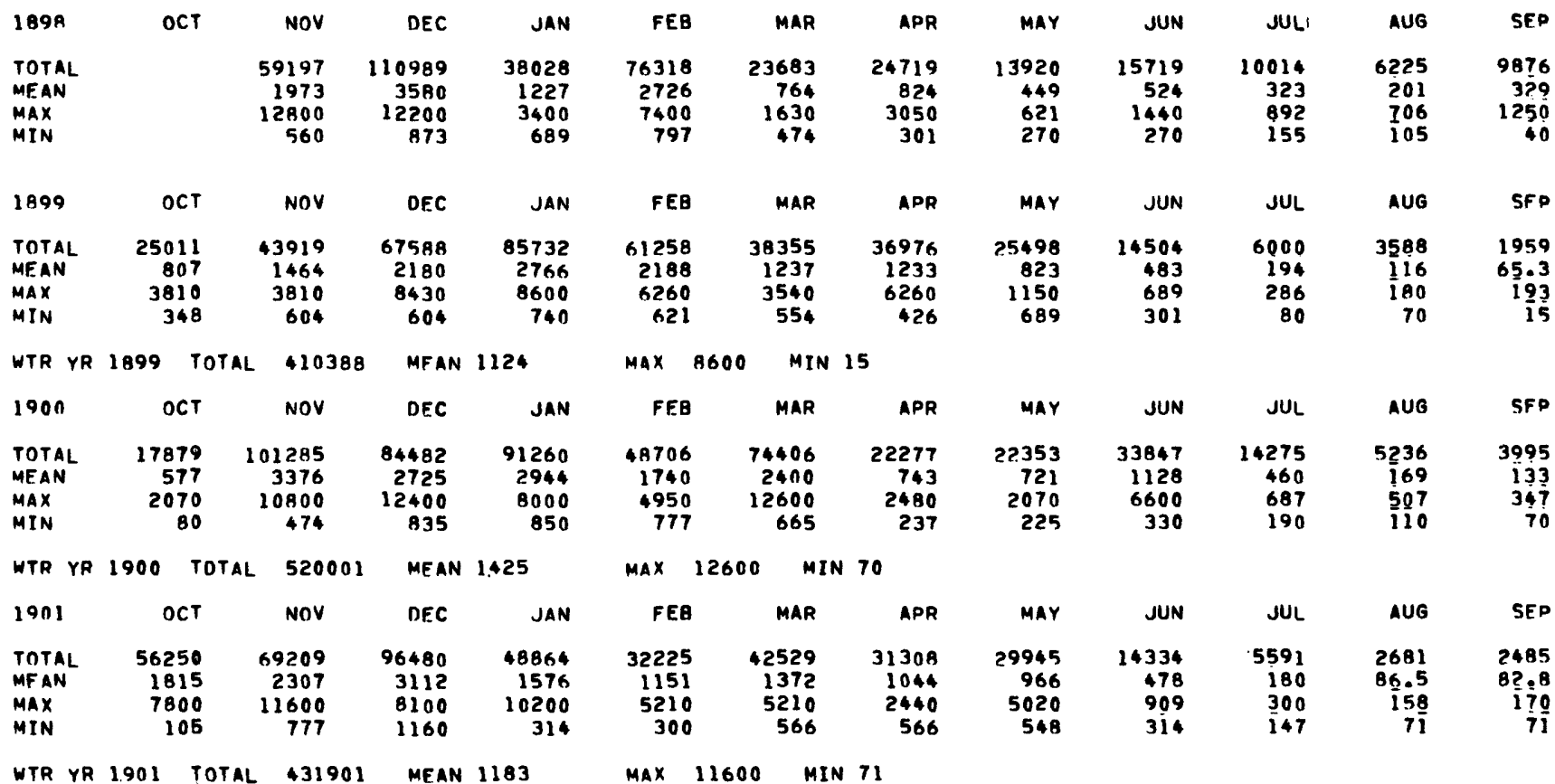


TABLE 7.--Records of gaging stations in Clallam Counity--continued

12043000 CALAKAH RIVER MEAR FORKS, WA (Site 42) -- continued

\begin{tabular}{|c|c|c|c|c|c|c|c|c|c|c|c|c|}
\hline $190 ?$ & OCT & Nov & DEC & JAN & FEB & MAR & APR & MAY & JUN & JUL & AUG & SEP \\
\hline $\begin{array}{l}\text { TOTAL } \\
\text { MEAN } \\
\text { MAX } \\
\text { MIN }\end{array}$ & $\begin{array}{r}7096 \\
229 \\
2700 \\
71\end{array}$ & $\begin{array}{r}73506 \\
2450 \\
8710 \\
762\end{array}$ & $\begin{array}{r}64691 \\
2087 \\
6540 \\
566\end{array}$ & & & & & & & & & \\
\hline $197 \mathrm{~h}$ & OCT & NOV & DEC & JAN & FEB & MAR & APR & MAY & JUN & JUL & AUG & SFP \\
\hline $\begin{array}{l}\text { TOTAL } \\
\text { MEAN } \\
\text { MAX } \\
\text { MIN }\end{array}$ & & & & $\begin{array}{r}69686 \\
2248 \\
8760 \\
750\end{array}$ & $\begin{array}{r}52845 \\
1822 \\
5180 \\
468\end{array}$ & $\begin{array}{r}50214 \\
1620 \\
5000 \\
590\end{array}$ & $\begin{array}{r}27260 \\
909 \\
1500 \\
655\end{array}$ & $\begin{array}{r}19318 \\
623 \\
1170 \\
312\end{array}$ & $\begin{array}{r}12280 \\
409 \\
906 \\
228\end{array}$ & $\begin{array}{r}7560 \\
244 \\
448 \\
144\end{array}$ & $\begin{array}{r}5323 \\
172 \\
336 \\
114\end{array}$ & $\begin{array}{r}505 ? \\
168 \\
267 \\
122\end{array}$ \\
\hline 1977 & OCT & NOV & DEC & JAN & FEB & MAR & $A P R$ & MAY & JUN & JULI & Áug & SFP \\
\hline $\begin{array}{l}\text { TOTAL } \\
\text { MEAN } \\
\text { MAX } \\
\text { MIN }\end{array}$ & $\begin{array}{r}8242 \\
266 \\
1350 \\
103\end{array}$ & $\begin{array}{r}17267 \\
576 \\
2340 \\
202\end{array}$ & $\begin{array}{r}33550 \\
1082 \\
5930 \\
250\end{array}$ & $\begin{array}{r}24525 \\
791 \\
5630 \\
320\end{array}$ & $\begin{array}{r}37907 \\
1354 \\
5520 \\
356\end{array}$ & $\begin{array}{r}50041 \\
1614 \\
5680 \\
600\end{array}$ & $\begin{array}{r}23406 \\
780 \\
1460 \\
440\end{array}$ & $\begin{array}{r}17587 \\
567 \\
1220 \\
348\end{array}$ & $\begin{array}{r}11339 \\
378 \\
898 \\
170\end{array}$ & $\begin{array}{r}4950 \\
160 \\
285 \\
114\end{array}$ & $\begin{array}{r}4265 \\
138 \\
368 \\
92\end{array}$ & $\begin{array}{r}9624 \\
321 \\
1200 \\
128\end{array}$ \\
\hline WTR YR & 1978 TOTAL & L $\quad 354318$ & MFAN & 971 & $\max$ & 10100 & 91 & & & & & \\
\hline 1979 & OCT & Nov & DEC & JAN & FEB & MAR & $A P R$ & MAY & JUN & JUL & AUG & SEP \\
\hline $\begin{array}{l}\text { TOTAL } \\
\text { ME:AN } \\
\text { MAX } \\
\text { MIN }\end{array}$ & $\begin{array}{r}9469 \\
305 \\
452 \\
205\end{array}$ & $\begin{array}{r}20171 \\
672 \\
2280 \\
287\end{array}$ & $\begin{array}{r}36127 \\
1165 \\
2690 \\
486\end{array}$ & $\begin{array}{r}14765 \\
476 \\
1460 \\
290\end{array}$ & $\begin{array}{r}78755 \\
2813 \\
11600 \\
302\end{array}$ & $\begin{array}{r}42517 \\
1372 \\
6250 \\
355\end{array}$ & $\begin{array}{r}25429 \\
848 \\
3720 \\
350\end{array}$ & $\begin{array}{r}13753 \\
444 \\
1250 \\
214\end{array}$ & $\begin{array}{r}4946 \\
165 \\
278 \\
114\end{array}$ & $\begin{array}{r}6930 \\
224 \\
695 \\
114\end{array}$ & $\begin{array}{r}3051 \\
98.4 \\
111 \\
90\end{array}$ & $\begin{array}{r}13545 \\
452 \\
3100 \\
95\end{array}$ \\
\hline WTR YR & 1979 TO & 269458 & MEAN & 738 & $\max$ & 11600 & 90 & & & & & \\
\hline
\end{tabular}


TABLE 7,--Records of gaging stations in Clallaw County---continued

\section{EAST FORK DICKEY RIVER NEAR LAPUSH, WA (SI te 64)}

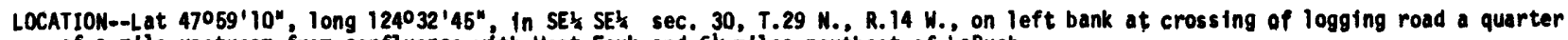
of a mile upstrean from confluence with West Fork and $6 \frac{1}{2}$ iniles northest of Lapush.

DRAINAGE AREA--39.8 $\mathrm{mt} 2$.

RECORDS AVALLABLE--AUg to OCt 1962, Apr 1963 to Sept 1968.

GAGE--Water-stage recorder. altitude of gage is $70 \mathrm{ft}$ (from topographic map).

AVERAGE DISCHARGE--5 years $(1964-68), 281 \mathrm{ft}^{3} / \mathrm{s}(203,400$ acre- $\mathrm{ft} / \mathrm{yr})$.

EXTREMES--1962-68. Maximum disharge 8,800 ft3/s Jan 18, 1968 (gage height, $16.32 \mathrm{ft} 3 / \mathrm{s}$ ), fron rating curve extended above $3,700 \mathrm{ft}^{3} / \mathrm{s}$ on basis of slope-area measurements at gage heights 10.19 and $16.32 \mathrm{ft}$; ininimum, $5.6 \mathrm{ft} 3 / \mathrm{s}$ Aug $11-20$, 1967 (gage height, $1.18 \mathrm{ft}$ ).

REMARKS--Records excellent. No regulation or diversion above station.

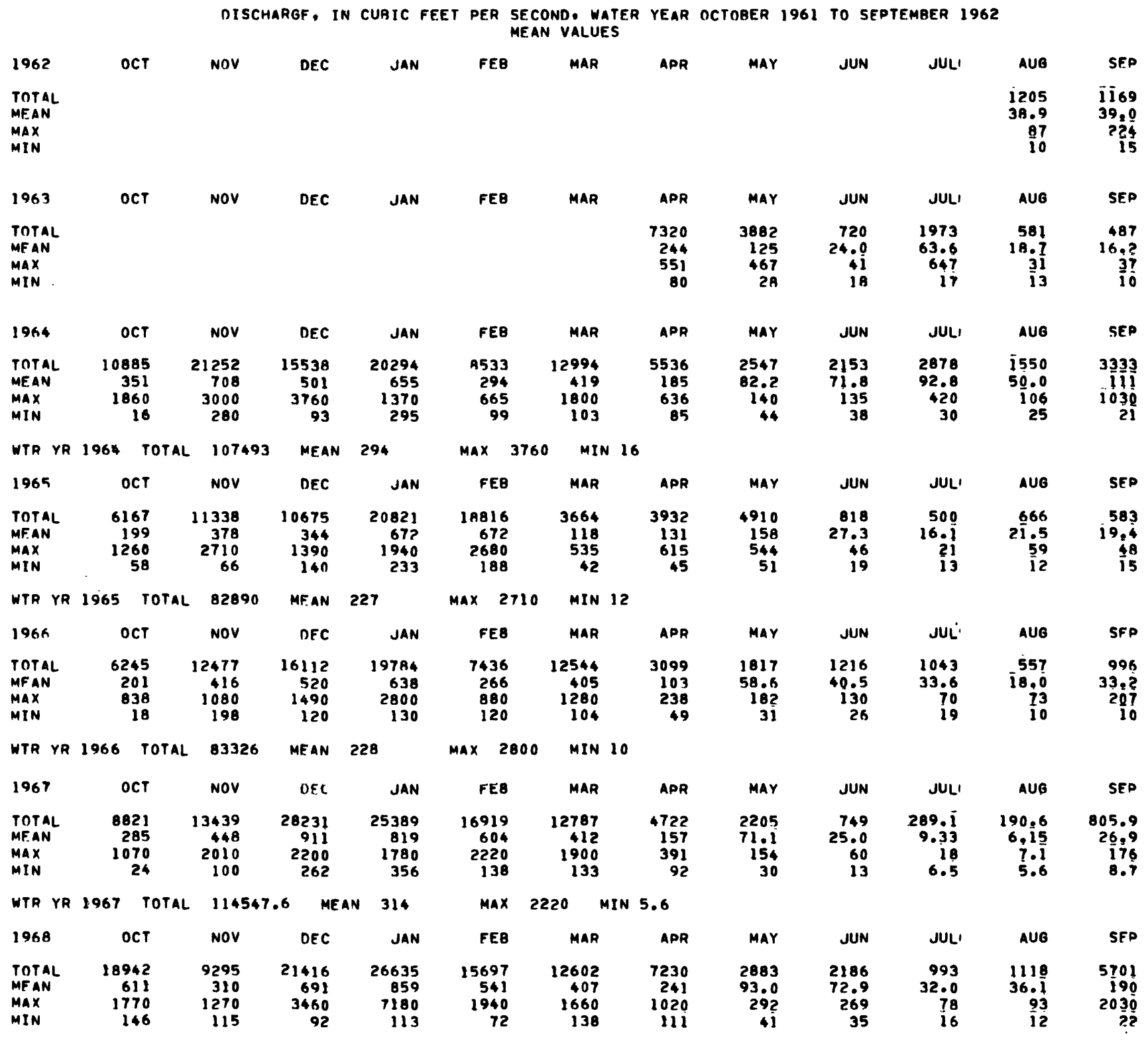


TABLE 7.--Records of gaging stations in Clalian County--continued

12043100 DICKEY RIVER NEAR LA PUSH, WA (SI te 68)

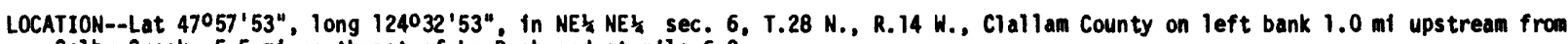
Colby Creek, $5.5 \mathrm{mi}$ northeast of La Push and at mile 6.0.

DRAINAGE AREA--86.3 $\mathrm{mi}^{2}$.

PERIOD OF RECORD--Sept 1962 to Sept 1973, Aug 1976 to current year.

GAGE--Water-stage recorder. Al titude of gage is $50 \mathrm{ft}$ (from topographic map).

AVERAGE DISCHARGE--14 years (water years 1963-73, 1977-79), $529 \mathrm{ft} 3 / \mathrm{s}, 83.18 \mathrm{in} / \mathrm{yr}, 382,900 \mathrm{acre}-\mathrm{ft} / \mathrm{yr}$.

EXTREMES FOR PERIOD OF RECORD--Maximum discharge, 17, $300 \mathrm{ft}^{3} / \mathrm{s}$ Jan 19, 1968, gage height, $22.15 \mathrm{ft}$ from high-water mark in well,

from rating curve extended above $4,900 \mathrm{ft} 3 / \mathrm{s}$ on basis of slope-area measurement of peak flow; minimum, $8.9 \mathrm{ft} 3 / \mathrm{s} \mathrm{Aug.} 29,30$,

31, 1967, gage height, $0.79 \mathrm{ft}$.

$$
\begin{aligned}
& \text { DISCHARGE, IN CUAIC FEET PER SECOND, WATER YEAR OCTOBER } 1961 \text { TO SEPTEMBER I962 } \\
& \text { MEAN VALUES }
\end{aligned}
$$

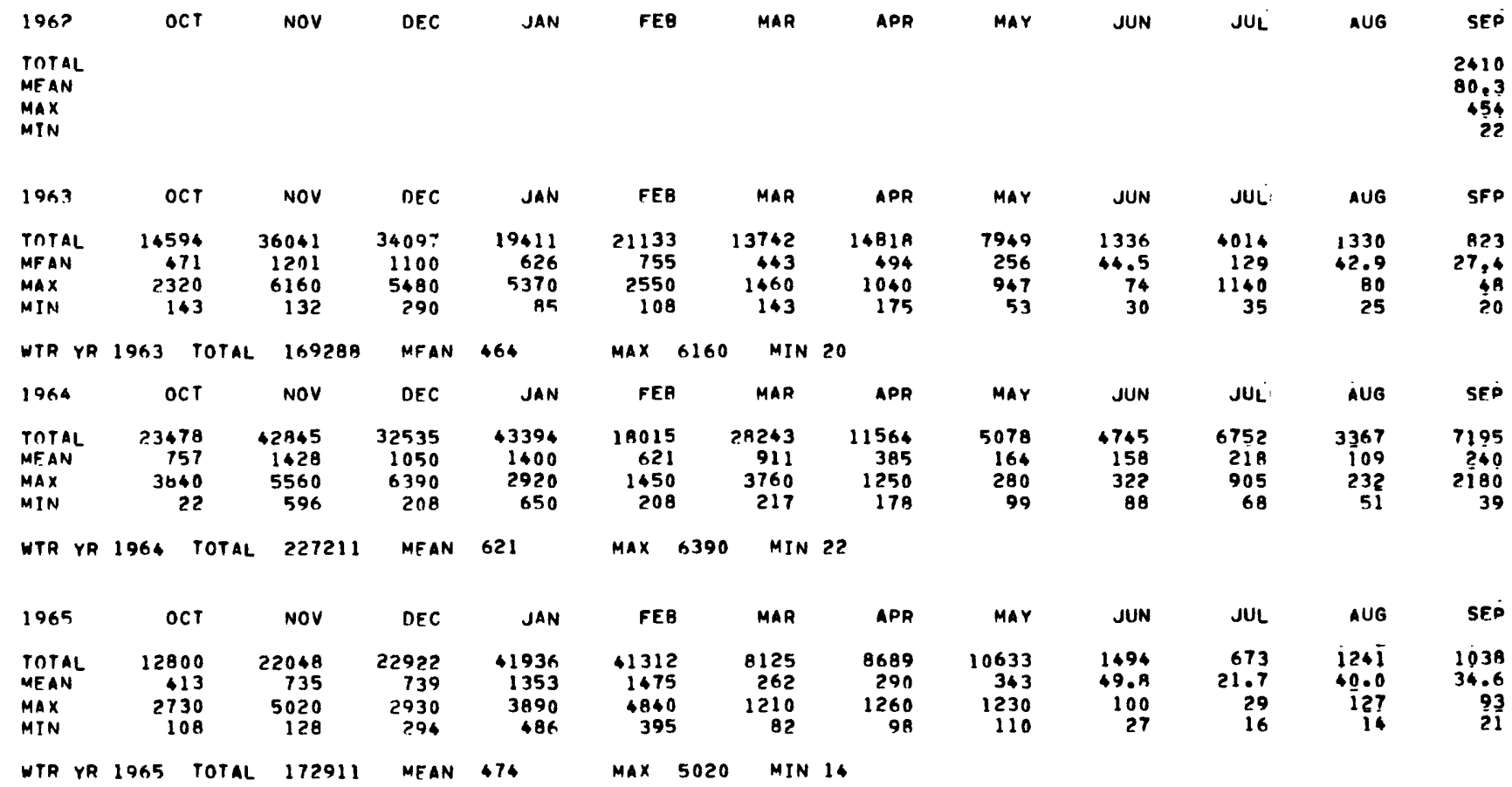


TABLE 7.--Records of gaging stations in Clallam County--continued

12043100 DICKEY RIVER NEAR LA PUSH, WA (Site 68) -- continued

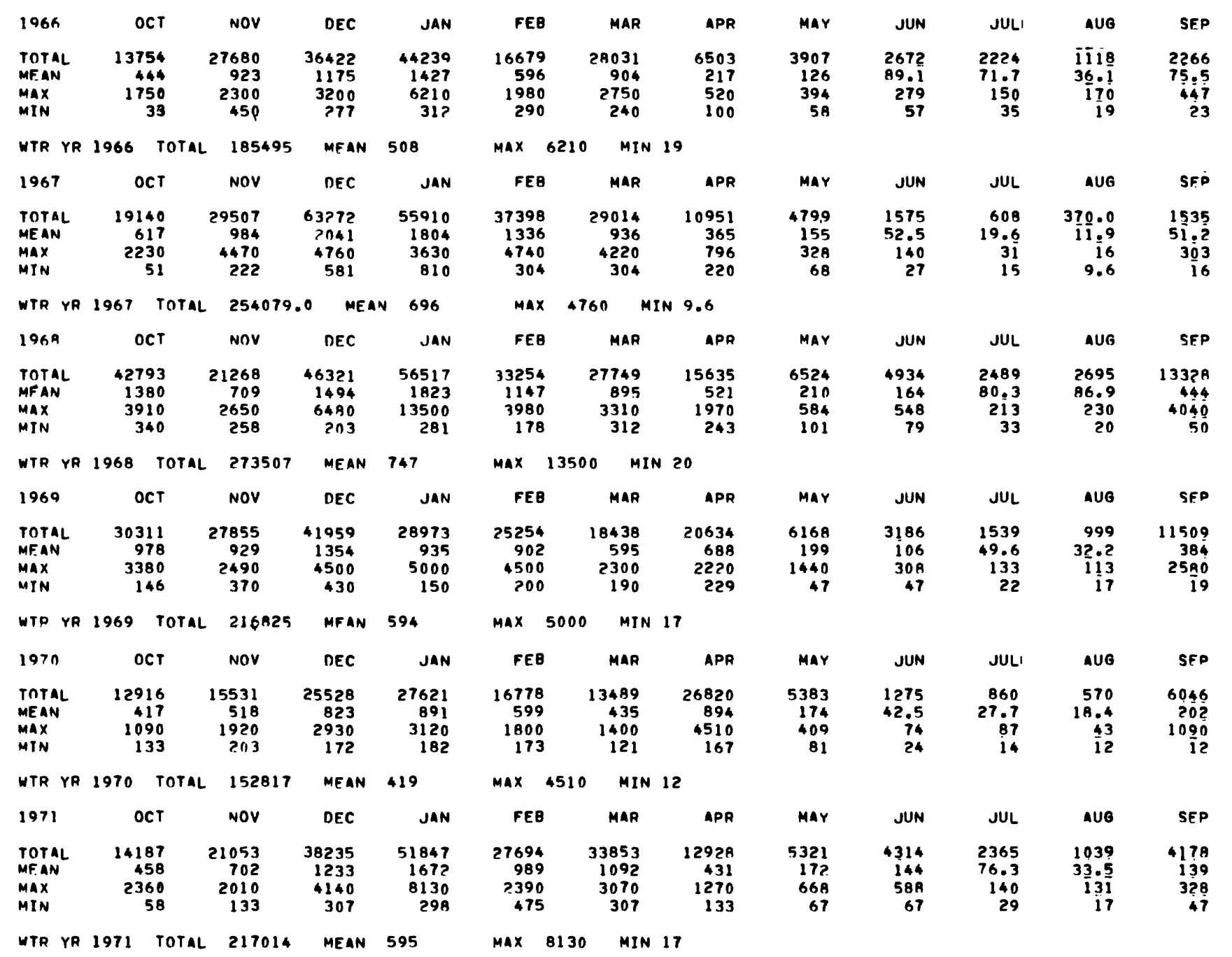


TABLE 7.--Records of gaging stations in Clallan County--continued

12043100 DICKEY RIVER MEAR LA PUSH, HA (Site 68) -- continued

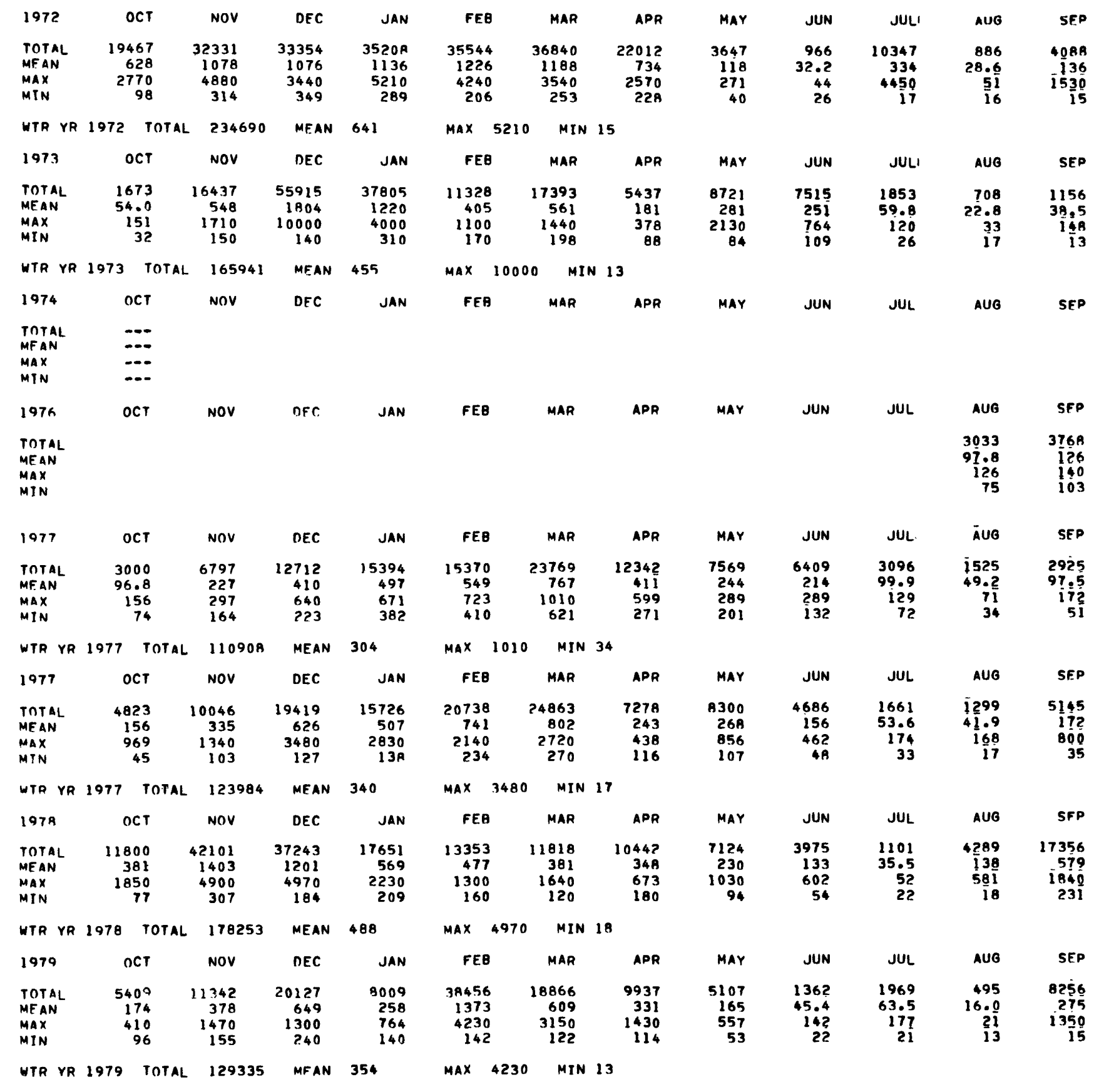


TABLE 7.--Records of gaging stations in Clallam County--continued

12043150 OZETTE RIVER AT OZETTE, HA

LOCATION--Lat 48009'13", long 124040'04", in NEL NEL sec. 31, T.31 N., R.15 W., Clallam County, on right bank, 50 ft west of

Ozette Ranger station, $75 \mathrm{ft}$ upstream from foot bridge, $250 \mathrm{ft}$ downstream from Ozette Lake and 3 mi southeast of Cap Alava.

DRAIMAGE AREA--77.5 $\mathrm{mi} 2$.

PERIOD OF RECORD--OCt 1977 to current year.

GAEE-Water-stage recorder. Altitude of gage is $30 \mathrm{ft}$ from topographic map.

EXTREMES FOR PERIO0 OF RECORD--Maximum discharge, $1,500 \mathrm{ft}^{3} / \mathrm{s}\left(42.5 \mathrm{~m}^{3} / \mathrm{s}\right) \mathrm{Dec} .3,1977$, gage height, $38.50 \mathrm{ft} ; \mathrm{minimum}, 32 \mathrm{ft} / \mathrm{s}$ Aug 22, 1977, gage height, $31.40 \mathrm{ft}$.

REMARKS--Records good. Flow affected by natural regulation from Ozette Lake. No diversion above station.

DISCHAKGE, IN CUBIC FEET PER SECOND, WATER YEAR OCTOAER 1975 TO SEPTEMBER 1976

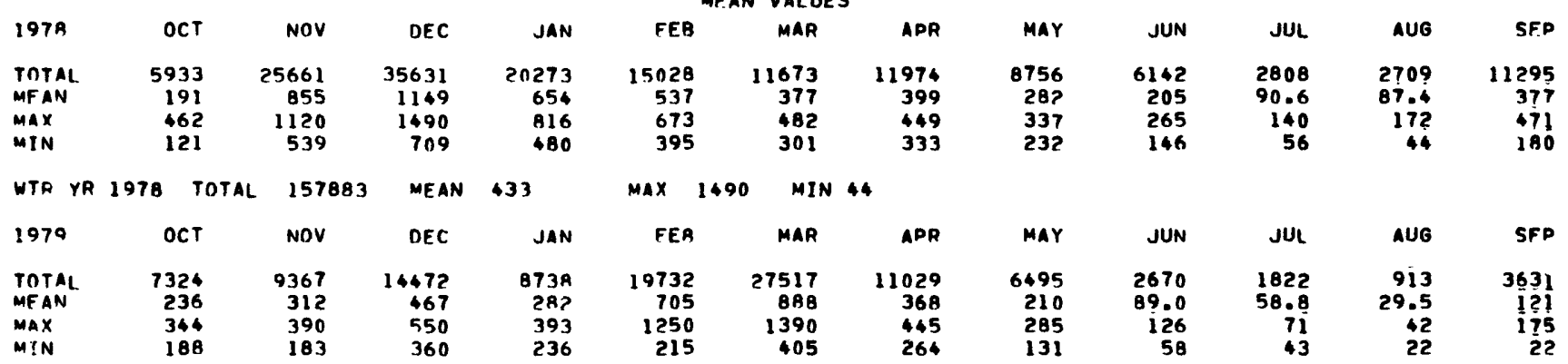

WTR YR 1979 TOTAL 113710 MEAN 312 MAX 1390 MIN 22 
TABLE 7.--Records of gaging stations in Clallan County--continued

12043163 SOOES RIVER BELOW MILLER CREEK MEAR OZETTE, WA

LOCATION--Lat 48015'58", long 124037'30", in NEK NE⿺ sec. 21 , T.32 N., R.15 W., Clallan County, on right bank at downstream side of Crown Zellerback' logging road, $0.6 \mathrm{ml}$ downstream from Milier Creek and 8 mi north of $0 z$ zette.

DRAINAGE AREA- $-32.0 \mathrm{mi} 2$.

PERIOD OF RECORD--Mar 1976 to current year.

GAGE--Water-stage recorder. Altitude of gage is $70 \mathrm{ft}$ (from topographic map).

EXTREMES FOR PERIOD OF RECORD--Maximum discharge 3,270 $\mathrm{ft}^{3} / \mathrm{s}$ Nov 25, 1977, gage height, $13.39 \mathrm{ft} ; \mathrm{minimum} 9.9 \mathrm{ft} 3 / \mathrm{s} \mathrm{Aug} \mathrm{8,} \mathrm{9,} 1978$, gage height, $5.54 \mathrm{ft}$.

REMARKS--Water-discharge records good. No regulation or diversion above station.

DISCHARGE. IN CUBIC FEET PER SECOND. WATER YEAR OCTORER 1975 TO SEPTEMBER 1976 MEAN VALUES

\begin{tabular}{|c|c|c|c|c|c|c|c|c|c|c|c|c|}
\hline 1976 & OCT & NOV & DEC & JAN & FEB & MAR & APR & MAY & JUN & JUL & AUG & SFP \\
\hline $\begin{array}{l}\text { TOTAL } \\
\text { MEAN } \\
\text { MAX } \\
\text { MIN }\end{array}$ & & & & & & $\ldots$ & $\begin{array}{r}4337 \\
145 \\
330 \\
68\end{array}$ & $\begin{array}{r}2378 \\
76.7 \\
278 \\
31\end{array}$ & $\begin{array}{r}2886 \\
96.2 \\
373 \\
37\end{array}$ & $\begin{array}{r}1517 \\
48.9 \\
241 \\
24\end{array}$ & $\begin{array}{r}1684 \\
54.3 \\
297 \\
18\end{array}$ & $\begin{array}{r}1977 \\
65.9 \\
327 \\
26\end{array}$ \\
\hline 1977 & OCT & Nov & DEC & JAN & FEB & MAR & $A P P$ & MAY & JUN & JUL & AUG & SEP \\
\hline $\begin{array}{l}\text { TOTAL } \\
\text { MFAN } \\
\text { MAX } \\
\text { MIN }\end{array}$ & $\begin{array}{r}2697 \\
87.0 \\
466 \\
23\end{array}$ & $\begin{array}{r}4787 \\
160 \\
870 \\
46\end{array}$ & $\begin{array}{r}8165 \\
263 \\
1260 \\
51\end{array}$ & $\begin{array}{r}5222 \\
201 \\
1430 \\
55\end{array}$ & $\begin{array}{r}9396 \\
336 \\
1200 \\
75\end{array}$ & $\begin{array}{r}9077 \\
293 \\
950 \\
100\end{array}$ & $\begin{array}{r}3117 \\
104 \\
272 \\
55\end{array}$ & $\begin{array}{r}2947 \\
95.1 \\
250 \\
52\end{array}$ & $\begin{array}{r}2099 \\
7000 \\
217 \\
30\end{array}$ & $\begin{array}{r}1338 \\
43.2 \\
169 \\
24\end{array}$ & $\begin{array}{r}922 \\
29.7 \\
175 \\
13\end{array}$ & $\begin{array}{r}269 ? \\
89 . ? \\
434 \\
24\end{array}$ \\
\hline WTR YR & 1977 TOTAL & 53459 & MFAN & 146 & $\operatorname{MAx} 1430$ & MIN & 13 & & & & & \\
\hline 1.978 & OCT & Nov & ner & JAN & FEB & MAR & APR & MAY & JUN & JUL & AUG & SEP \\
\hline $\begin{array}{l}\text { TOTAL } \\
\text { MEAN } \\
\text { MAX } \\
\text { MTN }\end{array}$ & $\begin{array}{r}4130 \\
133 \\
732 \\
37\end{array}$ & $\begin{array}{r}18447 \\
615 \\
1590 \\
195\end{array}$ & $\begin{array}{r}16808 \\
542 \\
2070 \\
85\end{array}$ & $\begin{array}{r}7730 \\
249 \\
936 \\
85\end{array}$ & $\begin{array}{r}5828 \\
208 \\
582 \\
68\end{array}$ & $\begin{array}{r}4994 \\
161 \\
696 \\
49\end{array}$ & $\begin{array}{r}4309 \\
144 \\
320 \\
64\end{array}$ & $\begin{array}{r}3437 \\
111 \\
543 \\
40\end{array}$ & $\begin{array}{r}1630 \\
54.3 \\
247 \\
26\end{array}$ & $\begin{array}{r}562 \\
18.1 \\
26 \\
12\end{array}$ & $\begin{array}{r}2792 \\
90.1 \\
477 \\
10\end{array}$ & $\begin{array}{r}5899 \\
197 \\
832 \\
72\end{array}$ \\
\hline $\begin{array}{l}\text { WTR YR } \\
\text { IL DATA }\end{array}$ & 1978 TOTAL & 76566 & MEAN & 210 & $\operatorname{MAX} 2070$ & MIN & 10 & & & & & \\
\hline 1979 & DCT & NOV & DEC & JAN & FEB & MAR & APR & MAY & JUN & JULI & AUG & SEP \\
\hline $\begin{array}{l}\text { TOTAL } \\
\text { MEAN } \\
\text { MAX } \\
\text { MIN }\end{array}$ & $\begin{array}{r}1838 \\
59.3 \\
172 \\
35\end{array}$ & $\begin{array}{r}5210 \\
174 \\
724 \\
45\end{array}$ & $\begin{array}{r}8133 \\
262 \\
610 \\
80\end{array}$ & $\begin{array}{r}2929 \\
94.5 \\
370 \\
50\end{array}$ & $\begin{array}{r}12823 \\
458 \\
1360 \\
47\end{array}$ & $\begin{array}{r}7972 \\
257 \\
1210 \\
72\end{array}$ & $\begin{array}{r}4361 \\
145 \\
666 \\
53\end{array}$ & $\begin{array}{r}1966 \\
63.4 \\
204 \\
33\end{array}$ & $\begin{array}{r}820 \\
27.3 \\
70 \\
18\end{array}$ & $\begin{array}{r}930 \\
30.0 \\
84 \\
14\end{array}$ & $\begin{array}{r}357 \\
11.5 \\
14 \\
10\end{array}$ & $\begin{array}{r}2219 \\
7400 \\
335 \\
11\end{array}$ \\
\hline
\end{tabular}

PROVISIONAL DATA

WTR YR 1979 TOTAL 49558 MFAN 136 MAX 1360 MIN 10 
12043173 WAMTCH RIVER BELON EDUCKET CREEK AT NEAH BAY, HA

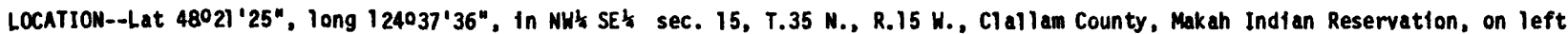
bank at Neah Bay pumping plant, $80 \mathrm{ft}$ downstream from Educket Creek, and $0.7 \mathrm{mi}$ south of Neah Bay.

DRAINAGE AREA--9.96 $\mathrm{ml}^{2}$.

PERIOD OF RECORD--May 1976 to current year.

GAGE--Water-stage recorder. Altitude of gage $1 \mathrm{~s} 20 \mathrm{ft}$ (from topographic map).

EXTREMES FOR PERIOD OF RECORD--Maximum discharge, 1,180 ft3/s Mov 25, 1977, gage height, 8.76 ft; minimum, $0.12 \mathrm{ft} 3 / \mathrm{s} \mathrm{Aug} \mathrm{9,} 1978$, gage height, $0.62 \mathrm{ft}$.

REMARKS--Hater-discharge records good except those below $2.0 \mathrm{ft} 3 / \mathrm{s}$, which are poor. Makah Water Utility diverts approximately 2l acre-ft annually at the gage, for muntcidal use. No regulation.

DISCHARGE, IN CUBIC FEET PER SECOND, WATER YEAR OCTOAER 1975 TO SEPTEMBER 1976

\begin{tabular}{|c|c|c|c|c|c|c|c|c|c|c|c|c|}
\hline 1976 & OCT & NOV & DEC & JAN & FEB & MAR & APR & MAY & JUN & JUL & AUG & SEP \\
\hline $\begin{array}{l}\text { TOTAL } \\
\text { MFAN } \\
\text { MAX } \\
\text { MIN }\end{array}$ & & & & & & & & $\begin{array}{r}638.7 \\
20.6 \\
104 \\
5.4\end{array}$ & $\begin{array}{r}778.3 \\
25.9 \\
123 \\
8.8\end{array}$ & $\begin{array}{r}279.8 \\
9.03 \\
59 \\
3.2\end{array}$ & $\begin{array}{r}12.6 \\
13.3 \\
69 \\
2.1\end{array}$ & $\begin{array}{r}553.3 \\
1.9 .4 \\
12 ? \\
5.2\end{array}$ \\
\hline 1977 & OCT & NOV & DEC & JAN & FEB & MAR & APR & MAY & JUN & JUL & AUG & SEP \\
\hline $\begin{array}{l}\text { TOTAL } \\
\text { MEAN } \\
\text { MAX } \\
\text { MIN }\end{array}$ & $\begin{array}{r}906.5 \\
29.2 \\
152 \\
4.9\end{array}$ & $\begin{array}{r}1266 \\
42.2 \\
224 \\
14\end{array}$ & $\begin{array}{r}2095 \\
67.6 \\
320 \\
14\end{array}$ & $\begin{array}{r}1973 \\
63.6 \\
450 \\
12\end{array}$ & $\begin{array}{r}1926 \\
68.8 \\
290 \\
18\end{array}$ & $\begin{array}{r}2639 \\
85.1 \\
340 \\
20\end{array}$ & $\begin{array}{r}624 \\
20.8 \\
50 \\
10\end{array}$ & $\begin{array}{r}651.0 \\
21.0 \\
69 \\
8.8\end{array}$ & $\begin{array}{r}420.6 \\
14.0 \\
48 \\
5.7\end{array}$ & $\begin{array}{r}425.1 \\
13.7 \\
64 \\
4.5\end{array}$ & $\begin{array}{r}202.73 \\
6.54 \\
65 \\
.44\end{array}$ & $\begin{array}{r}880.5 \\
29.4 \\
177 \\
6.6\end{array}$ \\
\hline WTR YR & 1977 TOTAL & 14009.43 & MEAN & 38.4 & $\operatorname{MAX} 450$ & MIN & .44 & & & & & \\
\hline 1978 & OCT & NoV & DEC & IAN & FEB & MAR & APR & MAY & JUN & JUL & AUG & SFP \\
\hline $\begin{array}{l}\text { TOTAL } \\
\text { MFAN } \\
\text { MAX } \\
\text { MIN }\end{array}$ & $\begin{array}{r}1539.8 \\
49.7 \\
320 \\
6.7\end{array}$ & $\begin{array}{r}4890 \\
163 \\
447 \\
23\end{array}$ & $\begin{array}{r}4158 \\
134 \\
574 \\
14\end{array}$ & $\begin{array}{r}2+10 \\
77.7 \\
345 \\
18\end{array}$ & $\begin{array}{r}1873 \\
66.9 \\
219 \\
17\end{array}$ & $\begin{array}{r}1527 \\
49.3 \\
248 \\
11\end{array}$ & $\begin{array}{r}1305 \\
43.5 \\
119 \\
13\end{array}$ & $\begin{array}{r}843.5 \\
27.2 \\
141 \\
7.0\end{array}$ & $\begin{array}{r}294.0 \\
9.80 \\
57 \\
3.4\end{array}$ & $\begin{array}{r}73.5 \\
2.37 \\
5.2 \\
i .1\end{array}$ & $\begin{array}{r}845.30 \\
27.3 \\
160 \\
.60\end{array}$ & $\begin{array}{r}176 ? \\
58,7 \\
246 \\
15\end{array}$ \\
\hline WTR YR & 1978 TOTAL & 21521.10 & MEAN & 59.0 & $\operatorname{MAX} 574$ & MIN & .60 & & & & & \\
\hline 1979 & OCT & Nov & DEC & JAN & FEB & MAR & APR & MAY & JUN & JUL & ÁUG & SEP \\
\hline $\begin{array}{l}\text { TOTAL } \\
\text { MEAN } \\
\text { MAX } \\
\text { MIN }\end{array}$ & $\begin{array}{r}539.8 \\
17.4 \\
43 \\
8.7\end{array}$ & $\begin{array}{r}1606 \\
53.5 \\
283 \\
11\end{array}$ & $\begin{array}{r}2571 \\
82.9 \\
257 \\
18\end{array}$ & $\begin{array}{r}820 \\
26.5 \\
129 \\
12\end{array}$ & $\begin{array}{r}4378 \\
156 \\
423 \\
11\end{array}$ & $\begin{array}{r}2064.9 \\
66.6 \\
402 \\
9.9\end{array}$ & $\begin{array}{r}1378.7 \\
46.0 \\
246 \\
9.1\end{array}$ & $\begin{array}{r}480.5 \\
15.5 \\
45 \\
5.5\end{array}$ & $\begin{array}{r}142.5 \\
4.75 \\
9.9 \\
2.0\end{array}$ & $\begin{array}{r}366.6 \\
11.8 \\
50 \\
2.7\end{array}$ & $\begin{array}{r}47.58 \\
1.53 \\
2.9 \\
.76\end{array}$ & $\begin{array}{r}641.3 \\
21.4 \\
90 \\
1.2\end{array}$ \\
\hline
\end{tabular}

WTR YR 1979 TOTAL 15036.88 MEAN 41.2 MAX 423 MIN .76 
LOCATION--Lat 48027'27", long 124033'37", in NW SE sec. 18, T.33 N., R.14 W., Clallam County, Makah Indian Reservation, on right bank $100 \mathrm{ft}$ upstream from State Highway $112,0.2 \mathrm{mi}$ upstrean from mouth, and $3.0 \mathrm{mi}$ east of Neah Bay.

DRAINAGE AREA- $-5.42 \mathrm{~m} \mathrm{t}^{2}$.

PERIOD OF RECORD--June 1976 to current year.

GAGE--Water-stage recorder. Altitude of gage is $20 \mathrm{ft}$ (from topographic map).

EXTRMES FOR PERIOD OF RECORD--Maximum discharge, $762 \mathrm{ft} 3 / \mathrm{s}$ Nov 25,1977 , gage height, $6.08 \mathrm{ft}$; minimim, $0.41 \mathrm{ft} 3 / \mathrm{s}$ Aug 8, 9,1978 , gage helght, $2.94 \mathrm{ft}$.

REMARKS-- Hater discharge records good. No regulation. Minor diversion above station for domestic use.

$$
\text { DISCHARGE, IN CUAIC FEET PER SECOND, WATER YEAR OCTOBER } 1975 \text { TO SEPTEMBER } 1976
$$
MEAN VALUES

\begin{tabular}{|c|c|c|c|c|c|c|c|c|c|c|c|c|}
\hline 1976 & OCT & NOV & DEC & JAN & FEB & MAR & APR & MaY & JUN & JUL. & AUG & SEP \\
\hline $\begin{array}{l}\text { TOTAL } \\
\text { MEAN } \\
\text { MAX } \\
\text { MIN }\end{array}$ & & & & & & & & & $\begin{array}{r}380.4 \\
12.7 \\
40 \\
4.6\end{array}$ & $\begin{array}{r}150.3 \\
4.85 \\
21 \\
2.0\end{array}$ & $\begin{array}{r}140.4 \\
4.53 \\
15 \\
1.4\end{array}$ & $\begin{array}{r}210.8 \\
7.03 \\
37 \\
2.6\end{array}$ \\
\hline 1977 & OCT & NOV & DEC & JAN & FEA & MAR & $A P R$ & MaY & JUN & JUL & ÃUG & \\
\hline $\begin{array}{l}\text { TOTAL } \\
\text { MEAN } \\
\text { MAX } \\
\text { MIN }\end{array}$ & $\begin{array}{r}506.6 \\
16.3 \\
96 \\
2.4\end{array}$ & $\begin{array}{r}801.7 \\
26.7 \\
133 \\
6.2\end{array}$ & $\begin{array}{r}369.4 \\
44.2 \\
196 \\
5.4\end{array}$ & $\begin{array}{r}1160.0 \\
37.4 \\
292 \\
5.1\end{array}$ & $\begin{array}{r}1713 \\
61.2 \\
205 \\
13\end{array}$ & $\begin{array}{r}2138 \\
69.0 \\
254 \\
18\end{array}$ & $\begin{array}{r}690.4 \\
23.0 \\
41 \\
9.4\end{array}$ & $\begin{array}{r}568.2 \\
18.3 \\
4.4 \\
8.4\end{array}$ & $\begin{array}{r}417.7 \\
13.9 \\
4.4 \\
3.1\end{array}$ & $\begin{array}{r}143.3 \\
4.62 \\
15 \\
2.3\end{array}$ & $\begin{array}{r}148.4 \\
4.79 \\
42 \\
1.3\end{array}$ & $\begin{array}{r}622.7 \\
20.8 \\
90 \\
3.3\end{array}$ \\
\hline WTR YR & 1977 TOTAL & -10279.4 & MEAN & 28.2 & MAX 292 & MIN I & .3 & & & & & \\
\hline 1978 & OCT & NOV & DEC & JAN & FEB & MAR & APR & MaY & JUN & $J U L$ & AUG & \\
\hline $\begin{array}{l}\text { TOTAL } \\
\text { MEAN } \\
\text { MAX } \\
\text { MIN }\end{array}$ & $\begin{array}{r}1035.6 \\
33.4 \\
205 \\
3.5\end{array}$ & $\begin{array}{r}32.90 \\
110 \\
316 \\
17\end{array}$ & $\begin{array}{r}2689.5 \\
86.8 \\
369 \\
9.5\end{array}$ & $\begin{array}{r}1710 \\
55.2 \\
232 \\
16\end{array}$ & $\begin{array}{r}1215 \\
43.4 \\
119 \\
12\end{array}$ & $\begin{array}{r}1017.6 \\
32.8 \\
129 \\
7.4\end{array}$ & $\begin{array}{r}902 \\
30.1 \\
63 \\
11\end{array}$ & $\begin{array}{r}638.2 \\
20.6 \\
90 \\
4.9\end{array}$ & $\begin{array}{r}265.6 \\
8.85 \\
29 \\
3.6\end{array}$ & $\begin{array}{r}59.95 \\
1.93 \\
3.4 \\
.85\end{array}$ & $\begin{array}{r}415.76 \\
13.4 \\
74 \\
.49\end{array}$ & $\begin{array}{r}1312 \\
43.7 \\
16 \frac{7}{1}\end{array}$ \\
\hline WTR YR & 1978 TOTAL & .14551 .2 & MEAN & 19.9 & $\max 369$ & MIN & .49 & & & & & \\
\hline 1979 & OCT & NOV & DEC & JAN & FEB & MAR & APR & MAY & JUN & JUL & AUG & \\
\hline $\begin{array}{l}\text { TOTAL } \\
\text { MEAN } \\
\text { MAX } \\
\text { MIN }\end{array}$ & $\begin{array}{r}372.8 \\
12.0 \\
29 \\
5.3\end{array}$ & $\begin{array}{r}929.3 \\
31.0 \\
162 \\
5.3\end{array}$ & $\begin{array}{r}1646 \\
53.1 \\
129 \\
11\end{array}$ & $\begin{array}{r}429.8 \\
13.9 \\
53 \\
4.9\end{array}$ & $\begin{array}{r}2838.8 \\
101 \\
322 \\
7.6\end{array}$ & $\begin{array}{r}1236.9 \\
39.9 \\
253 \\
4.3\end{array}$ & $\begin{array}{r}643.2 \\
21.4 \\
1.7 \\
4.0\end{array}$ & $\begin{array}{r}250.0 \\
8.06 \\
22 \\
2.6\end{array}$ & $\begin{array}{r}86.6 \\
2.89 \\
9.2 \\
1.7\end{array}$ & $\begin{array}{r}156.9 \\
5.06 \\
18 \\
1.6\end{array}$ & $\begin{array}{r}33.06 \\
1.07 \\
1.6 \\
.78\end{array}$ & $\begin{array}{r}350.6 \\
11.7 \\
5 ? \\
1.4\end{array}$ \\
\hline
\end{tabular}

WTR YR 1979 TOTAL A973.96 MEAN 24.6 MAX 322 MIN .78 
TABLE 7,--Records of gaging stations in Clallam County--continued

12043300 HOKO RIVER NEAR SEKIU, WA (SIte 90)

LOCATION--Lat $48^{\circ} 14^{\prime} 30^{\prime \prime}$, long $124022^{\prime} 57^{\prime \prime}$, In NE⿺ SWl sec. 28, T.32 N., R. 13 H., Clallam County, on right bank 2.2 mi upstream from Little Hoko River and $4.0 \mathrm{mi}$ southwest of Sekiu.

DRAINAGE AREA- $-51.2 \mathrm{mt} 2$.

PERIOD OF RECORD--July 1962 to Sep 1974.

GACE--Hater-stage recorder. Altitude of gage $1550 \mathrm{ft}$ (from topographic map).

AVERAGE DISCHARGE--12 years (water years 1963-74) $408 \mathrm{ft} 3 / \mathrm{s}, 108.22 \mathrm{in} / \mathrm{yr}, 295,600 \mathrm{acre-ft/yr}$.

EXTREMES FOR PERIOD OF RECORD--Maximum discharge, 14,100 ft3/s Dec 25, 1972, gage helght, $14.17 \mathrm{ft}$, from rating curve extended above

$2,100 \mathrm{ft} 3 / \mathrm{s}$ on basis of slope-area measurement at gage helght, $12.49 \mathrm{ft}$; minimum, $12 \mathrm{ft} 3 / \mathrm{s}$ Aug 30 , 1967 ; minimum gage height,

$0.97 \mathrm{ft}$ Sept. 15, 16, 1973 .

REMARKS--Records good. No regulation or diversion above station.

DISCHARGE, IN CUBIC FEET PER SECOND, WATER YEAR OCTOAER 1961 TO SFPTEMBER 1962 MEAN VALUES

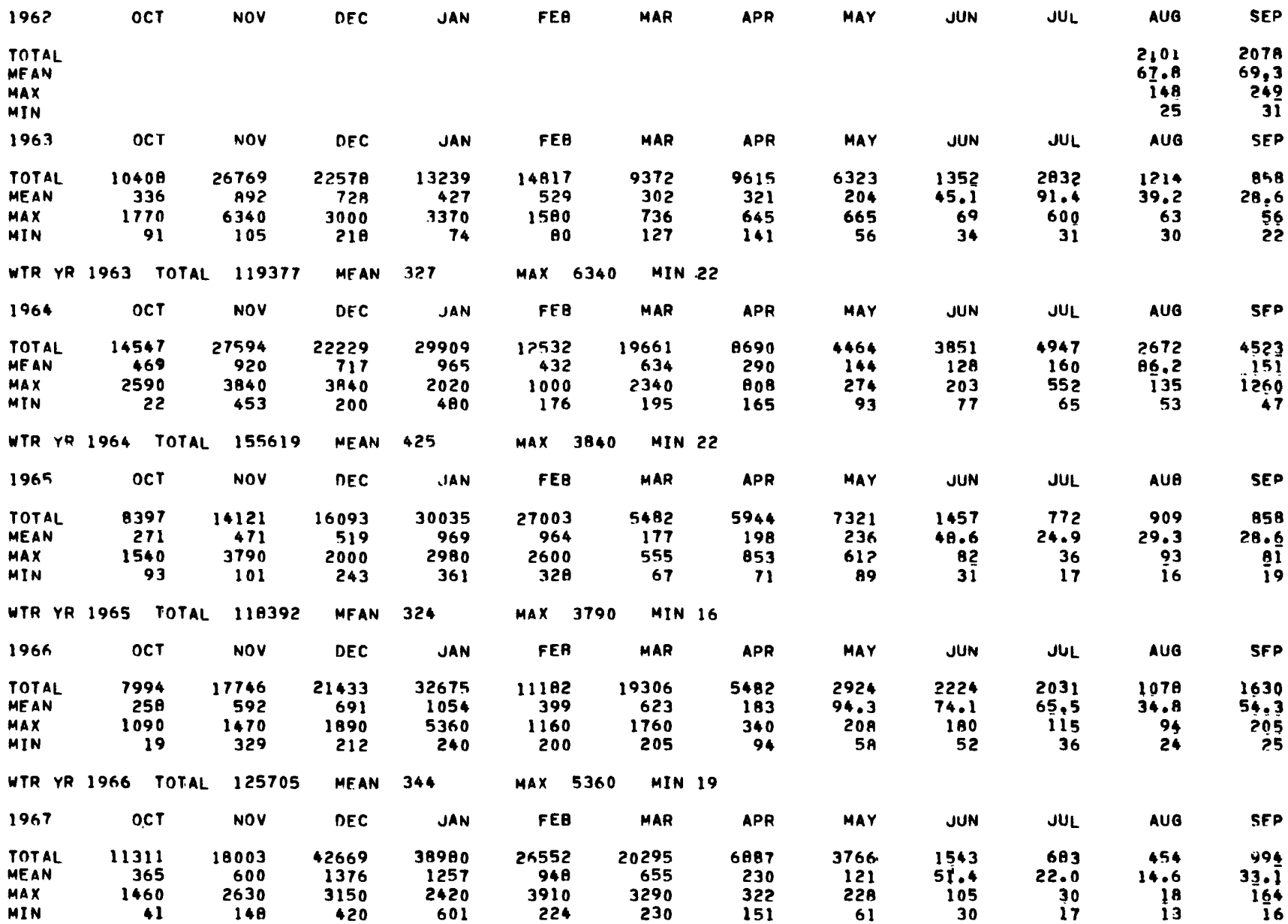

WTR YR 1967 TOTAL 172137 MEAN 472 MAX 3910 MIN 13 
TABLE 7.--Records of gaging stations in Clallaln County--continued

12043300 HOKO RIVER MEAR SEIKU, WA (Site 90) -. continued

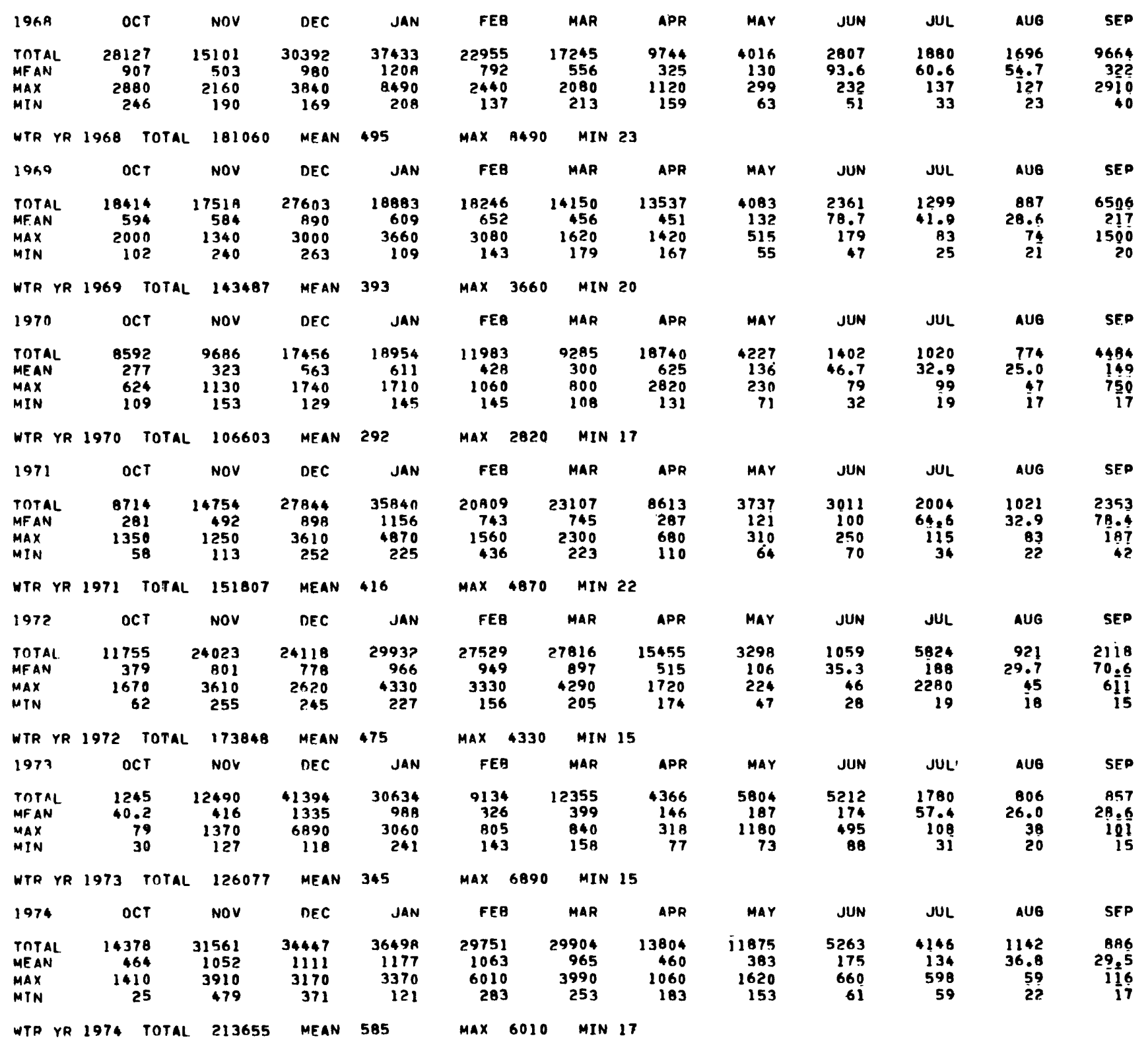




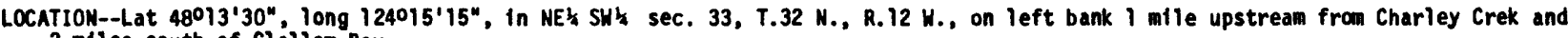
2 miles south of Clailian Bay.

DRAINAGE AREA--137 m12.

RECORDS AYAILABLE--Aug to Oct 1962

GAGE--Hater-stage recorder. Altttude of gage is $50 \mathrm{ft}$ (from topographic map).

EXTREMES--Maximum dafly discharge during period, $127 \mathrm{ft}^{3} / \mathrm{s}$ Oct 9 ; minimur discharge, $2.2 \mathrm{ft} 3 / \mathrm{s}$ July 29 to Aug 2 (gage height, $0.92 \mathrm{ft}$ ).

REMARKS--Records excellent. Some diversion for domestic use. Ho regulation.

DISCHARGF, IN CUAIC FEET PER SECOND, WATER YEAR OCTOAER 1961 TO SFPTEMBER I962

\begin{tabular}{|c|c|c|c|c|c|c|c|c|c|c|c|c|}
\hline 196? & OCT & NOV & DEC & JAN & FEA & MAR & APR & MAY & JUN & JUL & AUG & SFP \\
\hline $\begin{array}{l}\text { TOTAL } \\
\text { MEAN } \\
\text { MAX } \\
\text { MIN }\end{array}$ & & & & & & & & & & & $\begin{array}{r}320.2 \\
10.3 \\
23 \\
2.2\end{array}$ & $\begin{array}{r}433.7 \\
14.5 \\
70 \\
3.2\end{array}$ \\
\hline
\end{tabular}

12043430 EAST TWIN RIVER NEAR PYSHT, WA. (SIte 103)

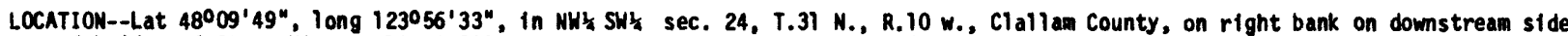
of bridge of State Highway 12, 1,300 ft upstream from mouth, and 8.4 miles southeast of Pysht.

DRAIMAGE AREA. $--14.0 \mathrm{~m}^{2}$.

PERIOO OF RECORD.--Aug 1962 to Sept 1972.

AVERAGE DISCHARGE.--10 years (water years 1963-72), $64.7 \mathrm{ft} 3 / \mathrm{s}, 62.76 \mathrm{ln} / \mathrm{yr}, 46,880 \mathrm{acre}-\mathrm{ft} / \mathrm{yr}$.

EXTREMES FOR PERIOD OF RECORD--Maximum discharge, $1,220 \mathrm{ft}^{3} / \mathrm{s}$ Nov 18, 1962 (gage height, $6.62 \mathrm{ft}$, site then in use), from rating

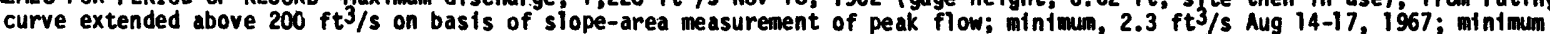
gage helght, $0.68 \mathrm{ft}$ Aug 25, 26, 27, 1967.

REMARKS--Records good except those for period of no gage-helght record, which are fair. No regulation or diversion above station.

DISCHARGF, IN CUBIC FEET PER SFCONO, WATEN YEAR OCTOBER ISOI TO SEPTEMBER 1962 MF. AN VALUES

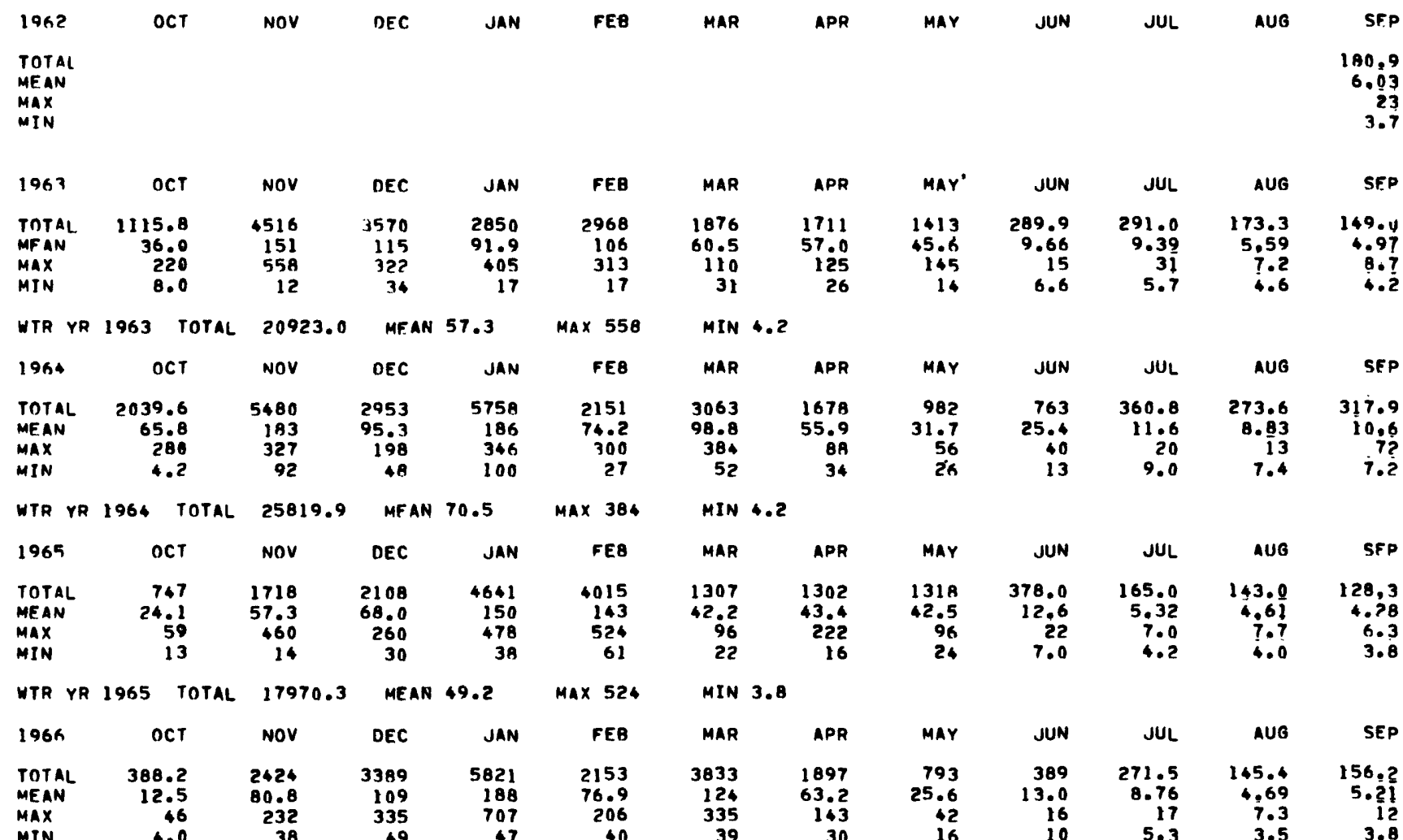

WTR YR 1966 TOTAL 21660.3 MEAN 59.3 MAX 707 MIN 3.5 
TABLE 7.--Records of gaging stations in Clallam County--continued

12043430 EAST TWIN RIVER MEAR PYSHT, WA. (Site 103) -- continued

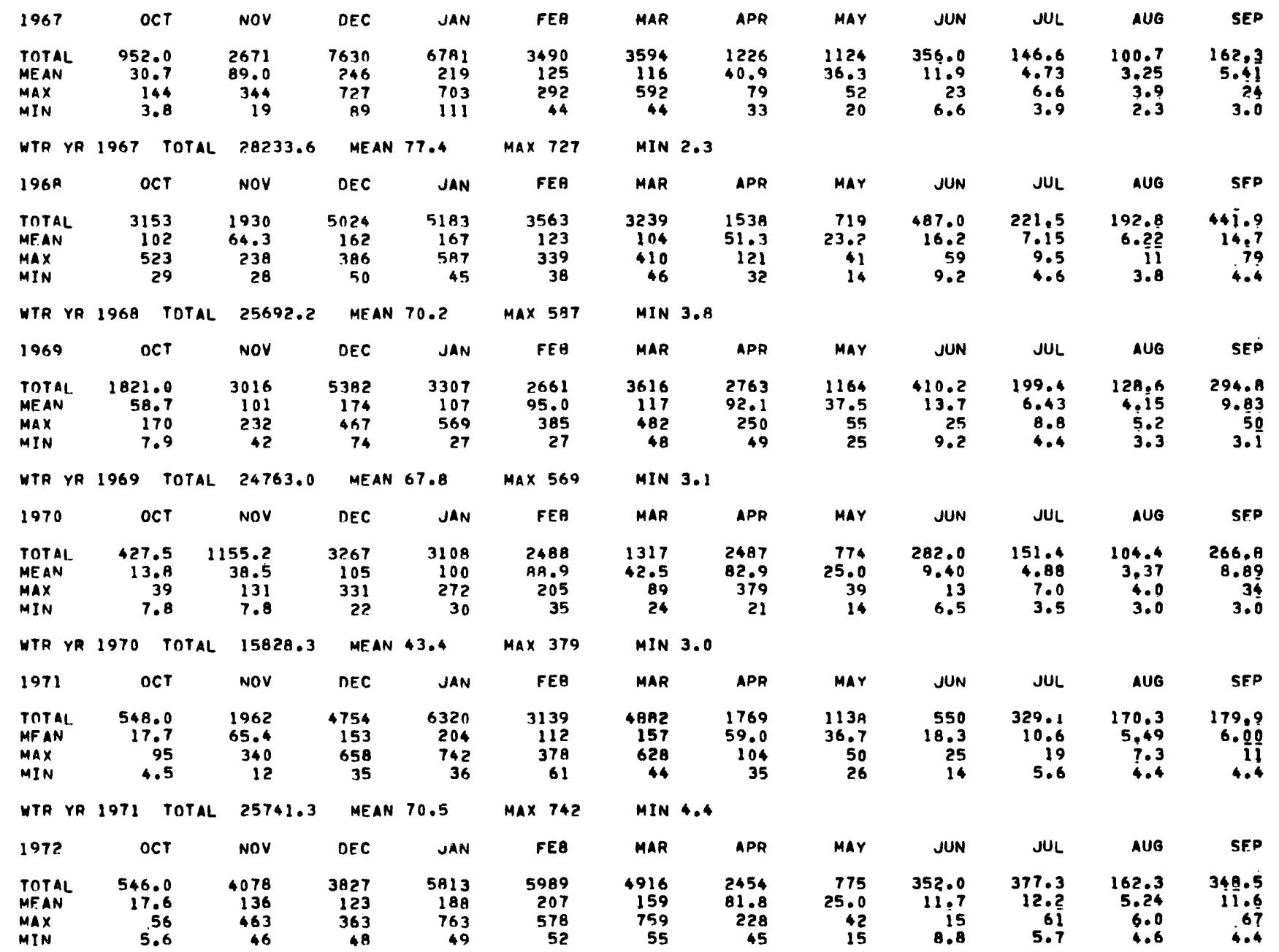

WTR YR 1972 TOTAL 29638.1 MEAN 81.0 MAX 763 MIN 4.4 
TABLE 7.--Records of gaging stations in Clallam County--continued

12044000 LYRE RIVER AT PIEDMONT, WA. (S1 te 114)

LOCATION--Lat 48005'35", Iong 123047'30", in NEk sec. 14, T.30 N., R.9 W., on north shore of Crescent Lake, on dock at Log Cabin Hotel at Pledmont, and half a mlle upstream from iake outlet.

DRAIMAGE AREA- $-49.5 \mathrm{mi}^{2}$.

PERIOO OF RECORD--Oct 1917 to Sept 1927.

GAGE--Staff gage. Alti tude of gage is $580 \mathrm{ft}$ (from topographic map). Oct 15, 1917, to 0ct 16, 1922, and Oct 1, 1923, to Dec 16, 1925, water-stage recorder three-quarters of a mile downstrean at different datums.

AVERAGE DISCHARGE--10 years (water years 1917-27), $218 \mathrm{ft} 3 / \mathrm{s}, 59.64 \mathrm{in} / \mathrm{yr}, 157,500 \mathrm{acre}-\mathrm{ft} / \mathrm{yr}$.

EXTREMES--9117-27: Maximum discharge observed, 1,180 ft3/s Jan. 10, 11, 1923; minimum discharge, $18 \mathrm{ft} 3 / \mathrm{s}$ Sept $19,1924$.

REMARKS--No diversion or regulation above station.

DISCHARGE, IN CUAIC FEET PFR SECDND, WATER YEAR OCTORER 1917 TO SEPTEMAER 1918 MEAN VALUES

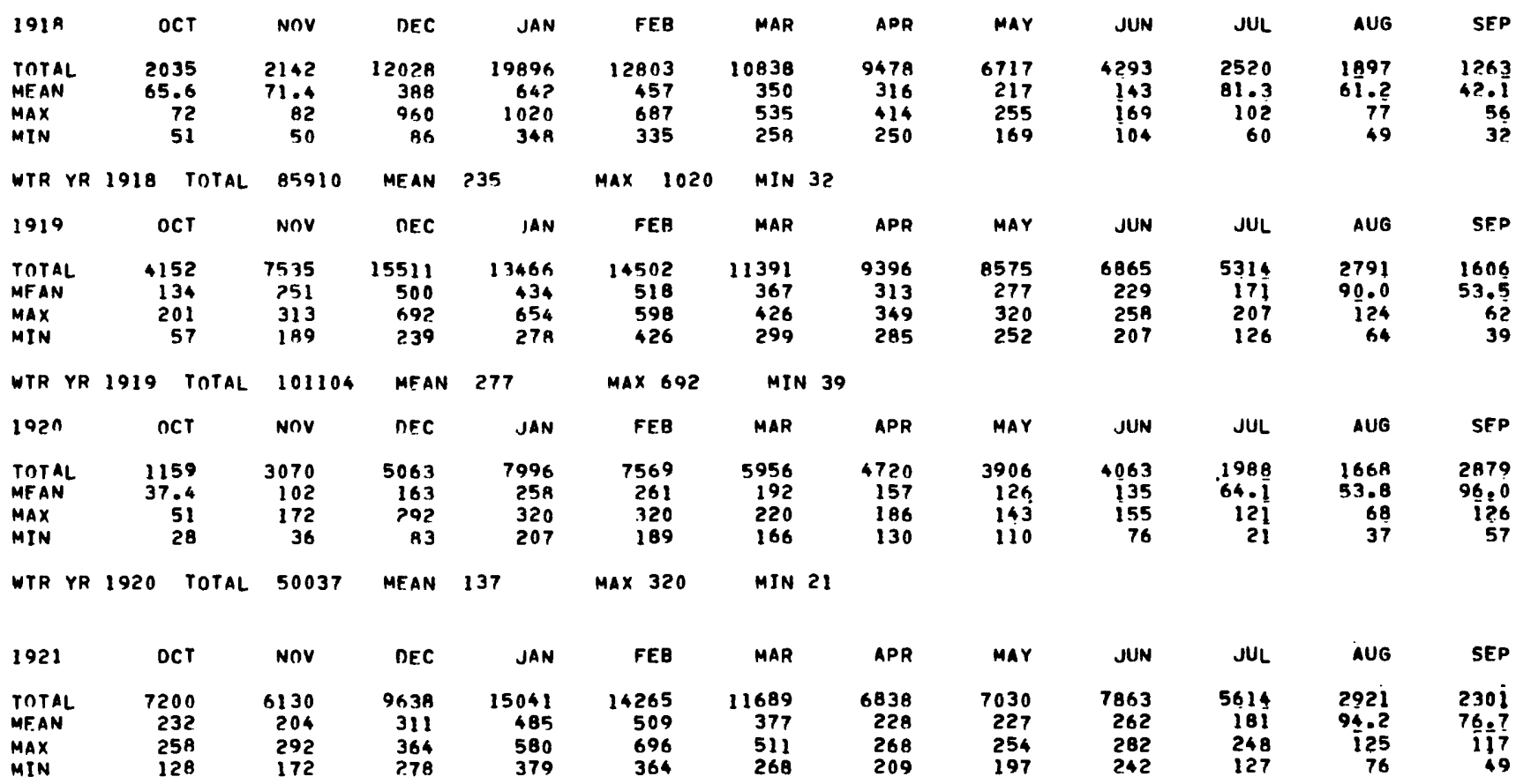

WTR YR 1921 TOTAL 96530 MEAN 264 MAX 696 MIN 49 
TABLE 7.--Records of gaging stations in Clallam County--continued

12044000 LYRE RIVER AT PIEDMONT, WA. (Site 114) - continued

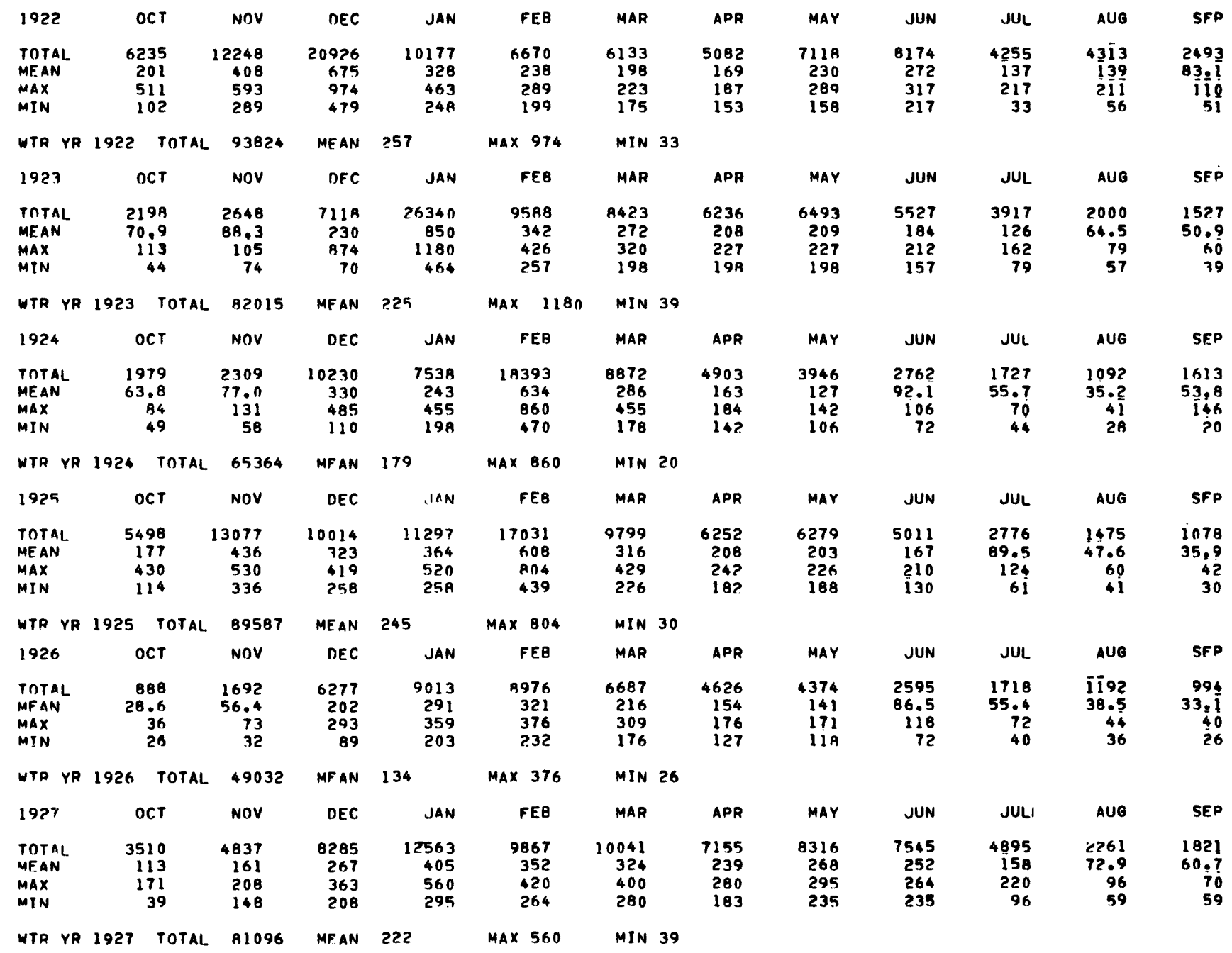


LOCATION--Lat 48003'18", long $123034^{\prime} 55^{\prime \prime}$, in NE' NWh sec. 33, T.30 N., R.7 W., Clall am County, Olympic National Forest, on right bank $300 \mathrm{ft}$ upstream from site of McDonald Bridge (now removed), 0.7 mi upstream from Littie River, 8 mi southwest of Port Angeles, and at mile 8.6.

DRAIMAGE AREA--269 mi $^{2}$

PERIOD OF RECORD--OCt 1897 to DeC 1901, Nov-Dec 1912, Oct 191 B to current year.

GAGE-- Hater-stage recorder. Datum of gage is $200.00 \mathrm{ft}$ (National Geodetic Vertical Datum of 1929. Oct 1, 1897, to Dec 31,1901, nonrecording gage at Mcdonal Bridge at different datum. Dec 9, 1918, to May 1, 1936, water-stage recorder under McDonald bridge at datum $7.4 \mathrm{ft}$ higher.

AVERAGE DISCHARGE--65 years (water years 1890-1901, 1919-1979) 1,481 ft3/s, $74.75 \mathrm{in} / \mathrm{yr}, 1,072,000$ acre-ft/yr adjusted for storage since Apri1 1927.

EXTREMES FOR PERIOD OF RECORD--Maximum discharge, $41,600 \mathrm{ft}^{3} / \mathrm{s}$ Nov 18, 1897, gage height, $14.5 \mathrm{ft}$, from graph based on gage readings, site and datum then in use, from rating curve extended above $3,300 \mathrm{ft}^{3} / \mathrm{s}$ on basis of two determination of flow over dam at discharge $26,700 \mathrm{ft}^{3} / \mathrm{s}$ and $30,100 \mathrm{ft}^{3} / \mathrm{s}$, referred to 1897 datum; minimum dally, $10 \mathrm{ft} 3 / \mathrm{s}$ oct 3,1938 .

REMARKS-- Hater-discharge records excellent. Water is diverted through Gilnes Canyon powerhouse and returned to river above gage. Flow partly regulated by Lake Mills $4.9 \mathrm{mi}$ upstream.

DISCHARGe, IN CUBIC, FEET PER SECOND, WATER YEAR OCTOAER 1897 TO SEPTEMBER 1898 MEAN VALUES

\begin{tabular}{|c|c|c|c|c|c|c|c|c|c|c|c|c|}
\hline 1898 & OCT & NOV & DEC & JAN & FEQ & MAR & $A P R$ & MAY & JUN & JUL & AUG & SEP \\
\hline $\begin{array}{l}\text { TOTAL } \\
\text { MFAN } \\
\text { MAX } \\
\text { MIN }\end{array}$ & $\begin{array}{r}19694 \\
635 \\
1690 \\
300\end{array}$ & $\begin{array}{r}71930 \\
2398 \\
24400 \\
562\end{array}$ & $\begin{array}{r}91800 \\
2961 \\
11800 \\
1150\end{array}$ & $\begin{array}{r}49327 \\
1591 \\
3580 \\
855\end{array}$ & $\begin{array}{r}60447 \\
2159 \\
3260 \\
967\end{array}$ & $\begin{array}{r}38732 \\
1249 \\
1860 \\
687\end{array}$ & $\begin{array}{r}36846 \\
1228 \\
2150 \\
687\end{array}$ & $\begin{array}{r}62240 \\
2008 \\
2600 \\
1240\end{array}$ & $\begin{array}{r}66990 \\
2233 \\
3280 \\
1640\end{array}$ & $\begin{array}{r}54420 \\
17.55 \\
2380 \\
1290\end{array}$ & $\begin{array}{r}37224 \\
1201 \\
1609 \\
631\end{array}$ & $\begin{array}{r}20844 \\
695 \\
2170 \\
470\end{array}$ \\
\hline WTR YR & 1898 TOT & AL $\quad 610494$ & MEAN & 1673 & $\max 24$ & MIN & 300 & & & & & \\
\hline 1899 & OCT & NoV & DEC & JAN & FEB & MAR & APR & MAY & JUN & JUL & ÃUG & SFP \\
\hline $\begin{array}{l}\text { TOTAL } \\
\text { MEAN } \\
\text { MAX } \\
\text { MIN }\end{array}$ & $\begin{array}{r}27578 \\
890 \\
2920 \\
330\end{array}$ & $\begin{array}{r}31973 \\
1066 \\
1680 \\
687\end{array}$ & $\begin{array}{r}37784 \\
1219 \\
3310 \\
520\end{array}$ & $\begin{array}{r}45545 \\
1469 \\
3320 \\
660\end{array}$ & $\begin{array}{r}38395 \\
1371 \\
3320 \\
770\end{array}$ & $\begin{array}{r}21191 \\
684 \\
1210 \\
510\end{array}$ & $\begin{array}{r}22894 \\
763 \\
1920 \\
520\end{array}$ & $\begin{array}{r}38754 \\
1250 \\
2020 \\
575\end{array}$ & $\begin{array}{r}63740 \\
2125 \\
2820 \\
1630\end{array}$ & $\begin{array}{r}63190 \\
2038 \\
2470 \\
1630\end{array}$ & $\begin{array}{r}30987 \\
1000 \\
1630 \\
575\end{array}$ & $\begin{array}{r}17085 \\
570 \\
805 \\
510\end{array}$ \\
\hline WTR YR & 1899 TOT & AL $\quad 439116$ & MEAN & 1203 & $\max 33$ & MIN 3 & 330 & & & & & \\
\hline 1900 & OCT & NOV & DEC & JAN & FEB & MAR & $A P R$ & MAY & JUN & JUL & AUG & SEP \\
\hline $\begin{array}{l}\text { TOTAL } \\
\text { MEAN } \\
\text { MAX } \\
\text { MIN }\end{array}$ & $\begin{array}{r}23029 \\
743 \\
1680 \\
475\end{array}$ & $\begin{array}{r}140012 \\
4667 \\
17700 \\
595\end{array}$ & $\begin{array}{r}90140 \\
2908 \\
12400 \\
1000\end{array}$ & $\begin{array}{r}78070 \\
2518 \\
6950 \\
1030\end{array}$ & $\begin{array}{r}25767 \\
920 \\
1570 \\
690\end{array}$ & $\begin{array}{r}98065 \\
3163 \\
22400 \\
710\end{array}$ & $\begin{array}{r}52314 \\
1744 \\
6950 \\
930\end{array}$ & $\begin{array}{r}59480 \\
1919 \\
2950 \\
1100\end{array}$ & $\begin{array}{r}83710 \\
2790 \\
8740 \\
1260\end{array}$ & $\begin{array}{r}38902 \\
1255 \\
1890 \\
810\end{array}$ & $\begin{array}{r}29181 \\
941 \\
1310 \\
650\end{array}$ & $\begin{array}{r}20148 \\
672 \\
1140 \\
562\end{array}$ \\
\hline NTR YR & 1900 ТОТ & AL $\quad 738818$ & MEAN & 2024 & $\operatorname{MAX} 22$ & MIN & 475 & & & & & \\
\hline 1901 & OCT & NOV & DEC & JAN & FEB & MAR & APR & MAY & JUN & JUL & AUG & SEP \\
\hline $\begin{array}{l}\text { TOTALL } \\
\text { MEAN } \\
\text { MAX } \\
\text { MIN }\end{array}$ & $\begin{array}{r}41137 \\
1327 \\
5320 \\
495\end{array}$ & $\begin{array}{r}49482 \\
1649 \\
4910 \\
930\end{array}$ & $\begin{array}{r}136750 \\
4411 \\
17300 \\
1570\end{array}$ & $\begin{array}{r}56370 \\
1818 \\
10300 \\
985\end{array}$ & $\begin{array}{r}46446 \\
1659 \\
8960 \\
645\end{array}$ & $\begin{array}{r}44870 \\
1447 \\
5600 \\
685\end{array}$ & $\begin{array}{r}28703 \\
957 \\
1300 \\
625\end{array}$ & $\begin{array}{r}67680 \\
2183 \\
6720 \\
1070\end{array}$ & $\begin{array}{r}64780 \\
2159 \\
4710 \\
1230\end{array}$ & $\begin{array}{r}50890 \\
1642 \\
2100 \\
1340\end{array}$ & $\begin{array}{r}36349 \\
1173 \\
1420 \\
873\end{array}$ & $\begin{array}{r}21338 \\
711 \\
979 \\
605\end{array}$ \\
\hline MTR YR & 1901 TOT & AL $\quad 644795$ & MEAN & 1767 & $\max 17$ & MIN & 495 & & & & & \\
\hline 1902 & OCT & NOV & DEC & JAN & FEB & MAR & APR & MAY & JUN & JULI & AUG & SEP \\
\hline $\begin{array}{l}\text { TOTALL } \\
\text { MEAN } \\
\text { MAX } \\
\text { MIN }\end{array}$ & $\begin{array}{r}20570 \\
664 \\
1900 \\
460\end{array}$ & $\begin{array}{r}96227 \\
3208 \\
20200 \\
795\end{array}$ & $\begin{array}{r}64850 \\
2092 \\
6040 \\
1070\end{array}$ & & & & & & & & & \\
\hline $191 ?$ & OCT & NoV & DEC & JAN & FEB & MAR & APR & MAY & JUN & JUL & AUG & SFP \\
\hline $\begin{array}{l}\text { TOTAL } \\
\text { MEAN } \\
\text { MAX } \\
\text { MIN }\end{array}$ & & $\ddot{-\infty}$ & $\begin{array}{r}44611 \\
1439 \\
3200 \\
28 B\end{array}$ & & & & & & & & & \\
\hline 1919 & OCT & Nov & DEC & JAN & FEB & MAR & APR & MAXYY & JUN & JUL & AUG & SEP \\
\hline $\begin{array}{l}\text { TOTAL } \\
\text { MEAN } \\
\text { MAX } \\
\text { MIN }\end{array}$ & $\begin{array}{r}40300 \\
1300 \\
1300 \\
1300\end{array}$ & $\begin{array}{l}48000 \\
1600 \\
1600 \\
1600\end{array}$ & $\begin{array}{r}76504 \\
2468 \\
6660 \\
800\end{array}$ & $\begin{array}{r}64329 \\
2075 \\
6070 \\
54 B\end{array}$ & $\begin{array}{r}51500 \\
1839 \\
5430 \\
1040\end{array}$ & $\begin{array}{r}30681 \\
990 \\
1480 \\
784\end{array}$ & $\begin{array}{r}52390 \\
1746 \\
2880 \\
1050\end{array}$ & $\begin{array}{r}65170 \\
2102 \\
3550 \\
1470\end{array}$ & $\begin{array}{r}69380 \\
2313 \\
2820 \\
1990\end{array}$ & $\begin{array}{r}71370 \\
2302 \\
2820 \\
1800\end{array}$ & $\begin{array}{r}40338 \\
1301 \\
1600 \\
935\end{array}$ & $\begin{array}{r}1970 \mathrm{~B} \\
657 \\
871 \\
440\end{array}$ \\
\hline
\end{tabular}

WTR YR 1919 TOTAL 629670 MFAN 1725 MAX 6660 MIN 440 
TABLE 7.---Records of gaging stations in Clallan County--continued

12045500 ELHHA RIVER AT MCDONALD BRIDGE, MEAR PORT ANGELES, WA (S1 te 129) -- continued

\begin{tabular}{|c|c|c|c|c|c|c|c|c|c|c|c|}
\hline 1925 & OCT & NOV & DEC & JAN & FEB & MAR & APR & MAY & JUN & JULI & AUB \\
\hline $\begin{array}{l}\text { TOTAL } \\
\text { MFAN } \\
\text { MAX } \\
\text { MIN }\end{array}$ & $\begin{array}{r}64128 \\
2069 \\
5520 \\
696\end{array}$ & $\begin{array}{r}78200 \\
2607 \\
6810 \\
1420\end{array}$ & $\begin{array}{r}62580 \\
2019 \\
4480 \\
1080\end{array}$ & $\begin{array}{r}45520 \\
146 A \\
3120 \\
763\end{array}$ & $\begin{array}{r}65900 \\
2354 \\
6070 \\
1090\end{array}$ & $\begin{array}{r}31079 \\
1003 \\
1670 \\
731\end{array}$ & $\begin{array}{r}47075 \\
1569 \\
3350 \\
715\end{array}$ & $\begin{array}{r}80470 \\
2596 \\
4220 \\
1410\end{array}$ & $\begin{array}{r}65250 \\
2175 \\
2980 \\
1670\end{array}$ & $\begin{array}{r}47910 \\
1545 \\
2100 \\
1020\end{array}$ & $\begin{array}{r}23267 \\
751 \\
1040 \\
510\end{array}$ \\
\hline
\end{tabular}

WTR YR 1925 TOTAL 625008 MEAN 1712 MAX 6810 MIN 350

\begin{tabular}{|c|c|c|c|c|c|c|c|c|c|c|c|c|}
\hline 926 & OCT & NOV & DFC & JAN & FEB & MAR & APR & MAY & JUN & JUL & AUG & SEP \\
\hline $\begin{array}{l}\text { TOTAL } \\
\text { MF.AN } \\
\text { MAX }\end{array}$ & $\begin{array}{r}9714 \\
313 \\
517 \\
365\end{array}$ & $\begin{array}{r}12261 \\
409 \\
715 \\
754\end{array}$ & $\begin{array}{r}57563 \\
1857 \\
4700\end{array}$ & $\begin{array}{r}44740 \\
1443 \\
4160\end{array}$ & $\begin{array}{r}46926 \\
1676 \\
3200 \\
926\end{array}$ & $\begin{array}{r}32821 \\
1059 \\
1500 \\
850\end{array}$ & $\begin{array}{r}35098 \\
1170 \\
1660 \\
836\end{array}$ & $\begin{array}{r}35286 \\
1138 \\
1660 \\
890\end{array}$ & $\begin{array}{r}27653 \\
922 \\
1300 \\
746\end{array}$ & $\begin{array}{r}18446 \\
595 \\
826 \\
430\end{array}$ & $\begin{array}{r}13749 \\
444 \\
632 \\
360\end{array}$ & $\begin{array}{r}9910 \\
330 \\
55 ? \\
258\end{array}$ \\
\hline
\end{tabular}

$\begin{array}{lllllll}\text { MIN } & 265 & 254 & 715 & 917 & 926 & 850\end{array}$

WTR YR 1926 TOTAL 344167 MEAN 943 MAX 4700 MIN 254

\begin{tabular}{|c|c|c|c|c|c|c|c|c|c|c|c|c|c|}
\hline 1927 & OCT & Nov & DEC & JAN & FEE & & MAR & APR & MAY & JUN & JUL & AUG & SEP \\
\hline $\begin{array}{l}\text { TOTAL } \\
\text { MFAN } \\
\text { MAX } \\
\text { MIN }\end{array}$ & $\begin{array}{r}36059 \\
1163 \\
4620 \\
355\end{array}$ & $\begin{array}{r}40286 \\
1343 \\
3350 \\
559\end{array}$ & $\begin{array}{r}59402 \\
1916 \\
5890 \\
926\end{array}$ & $\begin{array}{r}56177 \\
181 ? \\
4990 \\
989\end{array}$ & $\begin{array}{r}40978 \\
1464 \\
2840 \\
762\end{array}$ & & $\begin{array}{r}35425 \\
1143 \\
1720 \\
890\end{array}$ & $\begin{array}{r}21809 \\
727 \\
1320 \\
203\end{array}$ & $\begin{array}{r}51330 \\
1656 \\
2420 \\
750\end{array}$ & $\begin{array}{r}89140 \\
2971 \\
4990 \\
1670\end{array}$ & $\begin{array}{r}58880 \\
1899 \\
2700 \\
1340\end{array}$ & $\begin{array}{r}2 A 878 \\
932 \\
1490 \\
648\end{array}$ & $\begin{array}{r}24844 \\
828 \\
2040 \\
566\end{array}$ \\
\hline WTR YR & 1927 TOTAL & L $\quad 543208$ & MEAN & 1488 & $\operatorname{MAX}$ & 5890 & MIN & 203 & & & & & \\
\hline $192 \mathrm{~A}$ & oct & NOV & DEC & JAN & FE & & MAR & APR & MAY & JUN & JUL & AUG & SEP \\
\hline $\begin{array}{l}\text { TOTAL } \\
\text { MEAN } \\
\text { MAX } \\
\text { MIN }\end{array}$ & $\begin{array}{r}40288 \\
1300 \\
3930 \\
548\end{array}$ & $\begin{array}{r}63460 \\
2115 \\
4650 \\
1040\end{array}$ & $\begin{array}{r}40227 \\
1298 \\
3250 \\
373\end{array}$ & $\begin{array}{r}85112 \\
2746 \\
7100 \\
762\end{array}$ & $\begin{array}{r}33163 \\
1144 \\
1720 \\
484\end{array}$ & & $\begin{array}{r}46522 \\
1501 \\
3940 \\
426\end{array}$ & $\begin{array}{r}4089 A \\
1363 \\
1890 \\
938\end{array}$ & $\begin{array}{r}72160 \\
2328 \\
3350 \\
1380\end{array}$ & $\begin{array}{r}49990 \\
1666 \\
2060 \\
1250\end{array}$ & $\begin{array}{r}32454 \\
1047 \\
1470 \\
673\end{array}$ & $\begin{array}{r}19207 \\
620 \\
751 \\
522\end{array}$ & $\begin{array}{r}13076 \\
434 \\
660 \\
38\end{array}$ \\
\hline WTR YR & 1928 TOTAL & L $\quad 536507$ & MEAN & 1466 & MAX & 7100 & MIN & 38 & & & & & \\
\hline 1929 & ocr & Nov & DEC & JAN & FEB & & MAR & APR & MAY & JUN & JUL & AUG & SFD \\
\hline $\begin{array}{l}\text { TOTAL } \\
\text { MEAN } \\
\text { MAX } \\
\text { MIN }\end{array}$ & $\begin{array}{r}25717 \\
830 \\
4010 \\
36\end{array}$ & $\begin{array}{r}32402 \\
1080 \\
3320 \\
478\end{array}$ & $\begin{array}{r}31028 \\
1001 \\
2270 \\
272\end{array}$ & $\begin{array}{r}25199 \\
813 \\
1340 \\
600\end{array}$ & $\begin{array}{r}13325 \\
476 \\
600 \\
322\end{array}$ & & $\begin{array}{r}15833 \\
511 \\
1260 \\
204\end{array}$ & $\begin{array}{r}26999 \\
900 \\
1340 \\
653\end{array}$ & $\begin{array}{r}55580 \\
1793 \\
3040 \\
1070\end{array}$ & $\begin{array}{r}64150 \\
2138 \\
2860 \\
1550\end{array}$ & $\begin{array}{r}43072 \\
1389 \\
2180 \\
918\end{array}$ & $\begin{array}{r}23336 \\
753 \\
1000 \\
560\end{array}$ & $\begin{array}{r}127 ? ? \\
424 \\
566 \\
33\end{array}$ \\
\hline
\end{tabular}

WTR YR 1929 TOTAL 369368 MEAN 1012 MAX 4010 MIN 33

\begin{tabular}{|c|c|c|c|c|c|c|c|c|c|c|c|c|}
\hline 920 & OCT & NOV & DEC & JAN & FEB & MAR & APR & MAY & JUN & JUL & AUG & SEP \\
\hline $\begin{array}{l}\text { TOTAL } \\
\text { MEAN } \\
\text { MAX }\end{array}$ & $\begin{array}{r}11818 \\
381 \\
558\end{array}$ & $\begin{array}{r}34148 \\
1138 \\
2400\end{array}$ & $\begin{array}{r}44611 \\
1439 \\
3200\end{array}$ & $\begin{array}{r}52488 \\
1693 \\
5660\end{array}$ & $\begin{array}{r}35777 \\
1234 \\
2340\end{array}$ & $\begin{array}{r}26786 \\
864 \\
2540\end{array}$ & $\begin{array}{r}20670 \\
689 \\
922\end{array}$ & $\begin{array}{r}29807 \\
962 \\
1910\end{array}$ & $\begin{array}{r}46114 \\
1537 \\
2230\end{array}$ & $\begin{array}{r}37957 \\
1224 \\
1880\end{array}$ & $\begin{array}{r}22306 \\
720 \\
939\end{array}$ & $\begin{array}{r}39916 \\
1331 \\
2920\end{array}$ \\
\hline
\end{tabular}

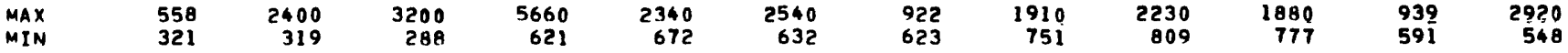

WTR YR 1920 TOTAL 102398 MEAN 1099 MAX 5660 MIN 280

1921 OCT NOV DEC JAN

$\begin{array}{lrrrr}\text { TOTAL } & 85980 & 56355 & 57680 & 67060 \\ \text { MEAN } & 2774 & 1879 & 1861 & 2163\end{array}$

MAX $\quad 6550 \quad 4000 \quad 3590 \quad 4300$

$\begin{array}{lllll}M I N & 1450 & 843 & 1080 & 1300\end{array}$

WTR YR 1921 TOTAL 728673 MEAN 1996

1922 OCT NOV DEC JAN

$\begin{array}{rrrrrrrr}\text { FEB } & \text { MAR } & \text { APR } & \text { MAY } & \text { JUN } & \text { JUL } & \text { AUG } & \text { SFP } \\ 65070 & 46072 & 33553 & 62280 & 110070 & 70960 & 40259 & 33334 \\ 2324 & 1486 & 118 & 2009 & 3669 & 2289 & 1299 & 1111 \\ 7580 & 2550 & 1500 & 3290 & 5480 & 3290 & 1790 & 3450 \\ 1020 & 992 & 829 & 952 & 2690 & 1760 & 829 & 646\end{array}$

MAX 7580 MIN 646

\begin{tabular}{|c|c|c|c|c|c|c|c|c|c|c|c|c|}
\hline 1922 & OCT & Nov & DEC & JAN & FEB & MAR & APR & MAY & JUN & JUL & AUG & SFP \\
\hline $\begin{array}{l}\text { TOTAL } \\
\text { ME:AN } \\
\text { MAX } \\
\text { MIN }\end{array}$ & $\begin{array}{r}84049 \\
2711 \\
10500 \\
802\end{array}$ & $\begin{array}{r}78060 \\
2602 \\
6680 \\
1040\end{array}$ & $\begin{array}{r}109910 \\
3545 \\
13300 \\
1210\end{array}$ & $\begin{array}{r}28501 \\
919 \\
1340 \\
744\end{array}$ & $\begin{array}{r}21429 \\
765 \\
1080 \\
702\end{array}$ & $\begin{array}{r}17322 \\
559 \\
708 \\
458\end{array}$ & $\begin{array}{r}20723 \\
691 \\
968 \\
499\end{array}$ & $\begin{array}{r}62074 \\
2002 \\
4180 \\
952\end{array}$ & $\begin{array}{r}89800 \\
2993 \\
4770 \\
2100\end{array}$ & $\begin{array}{r}43314 \\
1397 \\
2280 \\
952\end{array}$ & $\begin{array}{r}22493 \\
726 \\
984 \\
600\end{array}$ & $\begin{array}{r}17834 \\
594 \\
864 \\
486\end{array}$ \\
\hline WTR YR & 1922 TOTAL & -595509 & MEAN & 1632 & $\operatorname{MAX} 1330$ & MIN & 458 & & & & & \\
\hline 1923 & OCT & NOV & DEC & JAN & FEB & MAR & APR & MAY & JUN & JUL & ÁUG & SEP \\
\hline $\begin{array}{l}\text { TOTAL } \\
\text { MFAN } \\
\text { MAX } \\
\text { MIN }\end{array}$ & $\begin{array}{r}21551 \\
695 \\
3280 \\
401\end{array}$ & $\begin{array}{r}17879 \\
596 \\
1010 \\
478\end{array}$ & $\begin{array}{r}51790 \\
1671 \\
7330 \\
374\end{array}$ & $\begin{array}{r}87304 \\
2816 \\
6200 \\
900\end{array}$ & $\begin{array}{r}30910 \\
1104 \\
2340 \\
652\end{array}$ & $\begin{array}{r}27407 \\
884 \\
1500 \\
690\end{array}$ & $\begin{array}{r}36420 \\
1214 \\
1660 \\
1000\end{array}$ & $\begin{array}{r}64060 \\
2066 \\
3140 \\
1040\end{array}$ & $\begin{array}{r}68340 \\
2278 \\
3520 \\
1660\end{array}$ & $\begin{array}{r}50010 \\
1613 \\
2760 \\
970\end{array}$ & $\begin{array}{r}23303 \\
752 \\
898 \\
62 ?\end{array}$ & $\begin{array}{r}15148 \\
505 \\
933 \\
390\end{array}$ \\
\hline WTR YR & 1923 TOTAL & 494122 & ME.AN & 1354 & $\operatorname{mAX} 7330$ & MIN & 374 & & & & & \\
\hline 1924 & OCr & Nov & DEC & JAN & FEB & MAR & APR & MAY & JUN & JUL & AUG & SFD \\
\hline $\begin{array}{l}\text { TOTAL } \\
\text { MEAN } \\
\text { MAX } \\
\text { MIN }\end{array}$ & $\begin{array}{r}15326 \\
494 \\
1120 \\
344\end{array}$ & $\begin{array}{r}20179 \\
673 \\
2460 \\
376\end{array}$ & $\begin{array}{r}71634 \\
2311 \\
6710 \\
680\end{array}$ & $\begin{array}{r}51469 \\
1660 \\
12000 \\
786\end{array}$ & $\begin{array}{r}120950 \\
3826 \\
10200 \\
1640\end{array}$ & $\begin{array}{r}30739 \\
992 \\
1960 \\
654\end{array}$ & $\begin{array}{r}22530 \\
751 \\
1300 \\
568\end{array}$ & $\begin{array}{r}54902 \\
1771 \\
2810 \\
752\end{array}$ & $\begin{array}{r}40714 \\
1357 \\
2270 \\
986\end{array}$ & $\begin{array}{r}26045 \\
840 \\
1370 \\
627\end{array}$ & $\begin{array}{r}17411 \\
562 \\
668 \\
380\end{array}$ & $\begin{array}{r}22176 \\
739 \\
3680 \\
310\end{array}$ \\
\hline
\end{tabular}

WTR YR 1924 TOTAL 484075 MEAN 1323 MAX 12000 MIN 310 


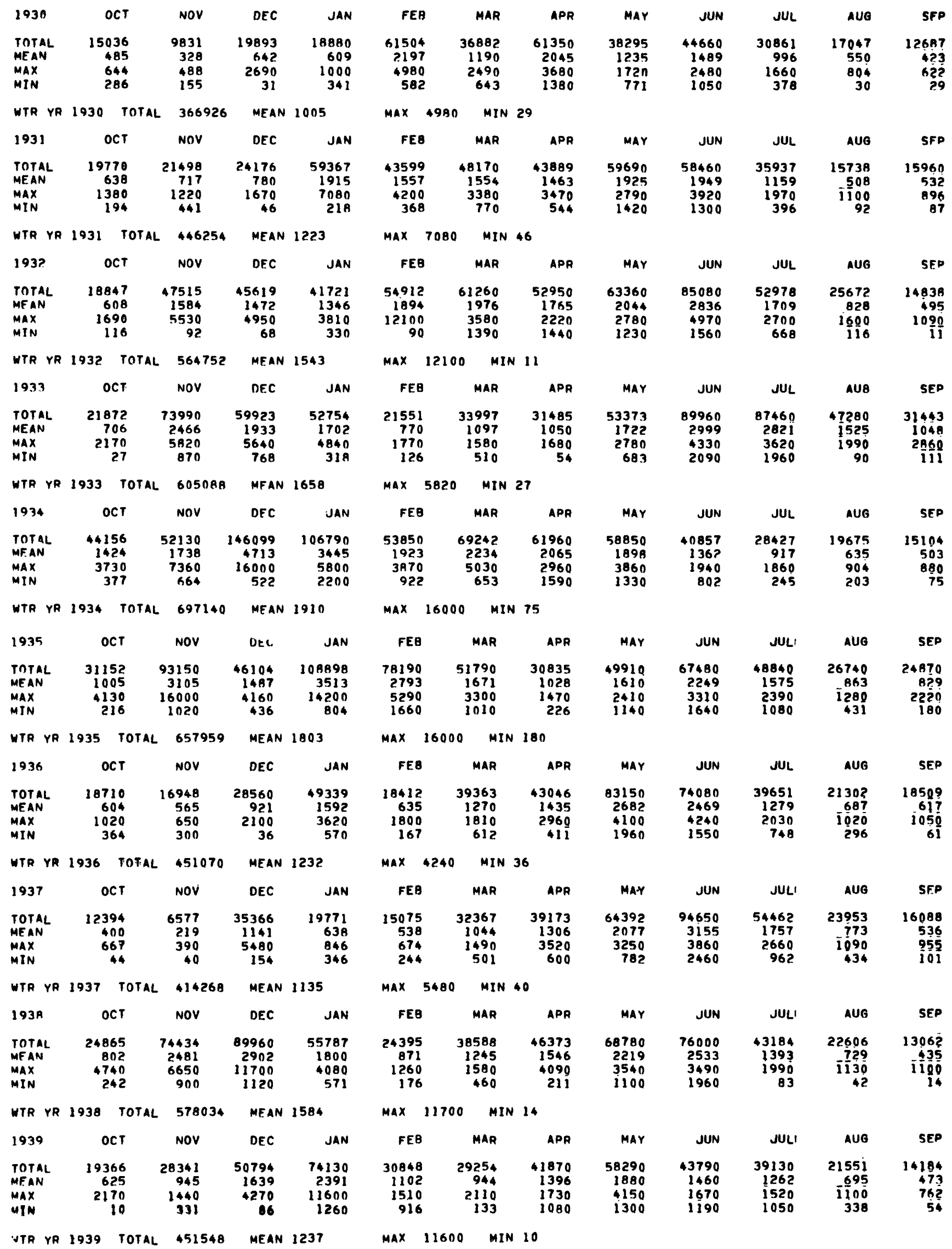


TABLE 7.--Records of gaging stations in Clallam County--continued

12045500 ELWHA RIVER AT MCDONALD BRIDGE, MEAR PORT ANGELES, WA (SIte 129) -- continued

\begin{tabular}{|c|c|c|c|c|c|c|c|c|c|c|c|}
\hline 1940 & OCT & NOV & DEC & JAN & FEB & MAR & APR & MAY & JUN & JUL' & AUE \\
\hline $\begin{array}{l}\text { TOTAL } \\
\text { MEAN } \\
\text { MAX } \\
\text { MIN }\end{array}$ & $\begin{array}{r}15825 \\
510 \\
759 \\
194\end{array}$ & $\begin{array}{r}30883 \\
1029 \\
1540 \\
272\end{array}$ & $\begin{array}{r}112200 \\
3619 \\
8900 \\
1880\end{array}$ & $\begin{array}{r}70120 \\
2262 \\
6750 \\
960\end{array}$ & $\begin{array}{r}58840 \\
2029 \\
3460 \\
1230\end{array}$ & $\begin{array}{r}64920 \\
2094 \\
3610 \\
1260\end{array}$ & $\begin{array}{r}41130 \\
1371 \\
1670 \\
1200\end{array}$ & $\begin{array}{r}61020 \\
1968 \\
4090 \\
1420\end{array}$ & $\begin{array}{r}38553 \\
1285 \\
1600 \\
917\end{array}$ & $\begin{array}{r}20923 \\
675 \\
1000 \\
82\end{array}$ & $\begin{array}{r}16713 \\
539 \\
704 \\
177\end{array}$ \\
\hline
\end{tabular}

WTR YR 1940 TOTAL 541967 MEAN 1481 MAX 8900 MIN 22

\begin{tabular}{|c|c|c|c|c|c|c|c|c|c|c|c|c|c|}
\hline 1941 & OCT & NOV & DEC & JAN & FEB & & MAR & APR & MaY & JUN & JUL & AUG & SEP \\
\hline $\begin{array}{l}\text { TOTAL } \\
\text { MEAN } \\
\text { MAX } \\
\text { MIN }\end{array}$ & $\begin{array}{r}41135 \\
1327 \\
4910 \\
228\end{array}$ & $\begin{array}{r}32918 \\
1097 \\
1660 \\
658\end{array}$ & $\begin{array}{r}62267 \\
2009 \\
3910 \\
919\end{array}$ & $\begin{array}{r}54828 \\
1769 \\
5660 \\
860\end{array}$ & $\begin{array}{r}46246 \\
1652 \\
4230 \\
986\end{array}$ & & $\begin{array}{r}32763 \\
1057 \\
1560 \\
809\end{array}$ & $\begin{array}{r}29413 \\
980 \\
1220 \\
746\end{array}$ & $\begin{array}{r}47138 \\
1521 \\
3330 \\
968\end{array}$ & $\begin{array}{r}38537 \\
1285 \\
1690 \\
978\end{array}$ & $\begin{array}{r}24075 \\
777 \\
1130 \\
76\end{array}$ & $\begin{array}{r}14969 \\
483 \\
612 \\
378\end{array}$ & $\begin{array}{r}17416 \\
581 \\
1220 \\
156\end{array}$ \\
\hline WTR YR & 1941 TOTAL & 441705 & MEAN & 1210 & MAX & 5660 & MIN & 76 & & & & & \\
\hline $194 ?$ & OCT & NOV & DEC & JAN & FEB & & MAR & APR & MAY & JUN & JUL & AUG & SFP \\
\hline $\begin{array}{l}\text { TOTAL } \\
\text { MEAN } \\
\text { MAX } \\
\text { MIN }\end{array}$ & $\begin{array}{r}32860 \\
1060 \\
2760 \\
445\end{array}$ & $\begin{array}{r}41406 \\
1380 \\
4160 \\
443\end{array}$ & $\begin{array}{r}87790 \\
2832 \\
11100 \\
1150\end{array}$ & $\begin{array}{r}30190 \\
974 \\
1150 \\
602\end{array}$ & $\begin{array}{r}27460 \\
981 \\
1420 \\
559\end{array}$ & & $\begin{array}{r}20333 \\
656 \\
798 \\
422\end{array}$ & $\begin{array}{r}24950 \\
832 \\
1080 \\
532\end{array}$ & $\begin{array}{r}33819 \\
1091 \\
1960 \\
499\end{array}$ & $\begin{array}{r}47350 \\
1578 \\
2340 \\
1320\end{array}$ & $\begin{array}{r}32838 \\
1059 \\
1560 \\
783\end{array}$ & $\begin{array}{r}16280 \\
525 \\
699 \\
163\end{array}$ & $\begin{array}{r}11284 \\
376 \\
776 \\
12\end{array}$ \\
\hline WTR YR & 1942 TOTAL & 406560 & MEAN & 1114 & $\operatorname{MAX}$ & 11100 & MIN & 12 & & & & & \\
\hline 1943 & OCT & NOV & DEC & JAN & FEB & & MAR & APR & MaY & JUN & JUL & AUG & SEP \\
\hline $\begin{array}{l}\text { TOTAL } \\
\text { MEAN } \\
\text { MAX } \\
\text { MIN }\end{array}$ & $\begin{array}{r}9946 \\
321 \\
644 \\
115\end{array}$ & $\begin{array}{r}32289 \\
1076 \\
3560 \\
355\end{array}$ & $\begin{array}{r}44409 \\
1433 \\
2160 \\
828\end{array}$ & $\begin{array}{r}33070 \\
1067 \\
1750 \\
480\end{array}$ & $\begin{array}{r}35705 \\
1275 \\
1870 \\
670\end{array}$ & & $\begin{array}{r}30676 \\
990 \\
3210 \\
254\end{array}$ & $\begin{array}{r}66290 \\
2210 \\
4930 \\
1300\end{array}$ & $\begin{array}{r}47435 \\
1530 \\
2740 \\
873\end{array}$ & $\begin{array}{r}54820 \\
1827 \\
2600 \\
1350\end{array}$ & $\begin{array}{r}47498 \\
1532 \\
2290 \\
972\end{array}$ & $\begin{array}{r}21583 \\
696 \\
980 \\
320\end{array}$ & $\begin{array}{r}14701 \\
490 \\
755 \\
34\end{array}$ \\
\hline
\end{tabular}

WTR YR 1943 TOTAL 438422 MFAN 1201 MAX 4930 MIN 34

\begin{tabular}{|c|c|c|c|c|c|c|c|c|c|c|c|c|c|c|}
\hline 1944 & \multicolumn{2}{|c|}{ OCT } & NOV & DEC & JAN & \multicolumn{2}{|c|}{ FEB } & MAR & APR & MAY & JUN & JUL & AUG & SEP \\
\hline $\begin{array}{l}\text { TOTAL } \\
\text { MEAN } \\
\text { MAX } \\
\text { MIN }\end{array}$ & \multicolumn{2}{|c|}{$\begin{array}{r}16553 \\
534 \\
1880 \\
192\end{array}$} & $\begin{array}{r}18996 \\
633 \\
1100 \\
413\end{array}$ & $\begin{array}{r}37026 \\
1194 \\
6340 \\
470\end{array}$ & $\begin{array}{r}48016 \\
1549 \\
5320 \\
386\end{array}$ & \multicolumn{2}{|c|}{$\begin{array}{r}25610 \\
883 \\
1480 \\
427\end{array}$} & $\begin{array}{r}21869 \\
705 \\
1110 \\
443\end{array}$ & $\begin{array}{r}22305 \\
744 \\
1030 \\
396\end{array}$ & $\begin{array}{r}35847 \\
1156 \\
1670 \\
712\end{array}$ & $\begin{array}{r}41095 \\
1370 \\
1700 \\
902\end{array}$ & $\begin{array}{r}21584 \\
696 \\
1280 \\
95\end{array}$ & $\begin{array}{r}13444 \\
434 \\
724 \\
250\end{array}$ & $\begin{array}{r}12184 \\
406 \\
566 \\
44\end{array}$ \\
\hline WTR YR & 1944 & TOTAL & 314529 & MEAN & 859 & $\operatorname{MAX}$ & 6340 & MIN & 44 & & & & & \\
\hline 1945 & \multicolumn{2}{|c|}{ OCT } & NOV & DEC & JAN & \multicolumn{2}{|c|}{ FEB } & MAR & APR & MAY & JUN & JUL & AUG & SFP \\
\hline $\begin{array}{l}\text { TOTAL } \\
\text { MEAN } \\
\text { MAX } \\
\text { MIN }\end{array}$ & \multicolumn{2}{|c|}{$\begin{array}{r}14789 \\
477 \\
1140 \\
263\end{array}$} & $\begin{array}{r}43555 \\
1452 \\
3930 \\
600\end{array}$ & $\begin{array}{r}39417 \\
1272 \\
4210 \\
282\end{array}$ & $\begin{array}{r}58775 \\
1896 \\
5780 \\
569\end{array}$ & \multicolumn{2}{|c|}{$\begin{array}{r}52575 \\
1878 \\
8020 \\
772\end{array}$} & $\begin{array}{r}35606 \\
1149 \\
3070 \\
602\end{array}$ & $\begin{array}{r}27121 \\
904 \\
1100 \\
640\end{array}$ & $\begin{array}{r}70200 \\
2265 \\
3310 \\
1390\end{array}$ & $\begin{array}{r}55220 \\
1841 \\
2940 \\
1170\end{array}$ & $\begin{array}{r}34146 \\
1101 \\
1520 \\
443\end{array}$ & $\begin{array}{r}19836 \\
640 \\
816 \\
334\end{array}$ & $\begin{array}{r}15710 \\
524 \\
823 \\
224\end{array}$ \\
\hline WTR YR & 1945 & TOTAL & 466950 & MFAN & 1279 & $\operatorname{MAX}$ & 8020 & MIN & 224 & & & & & \\
\hline 1946 & & 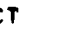 & NOV & DEC & JAN & FE & & MAR & APR & MAY & JUN & JUL & AUG & SEP \\
\hline $\begin{array}{l}\text { TOTAL } \\
\text { MEAN } \\
\text { MAX } \\
\text { MIN }\end{array}$ & $\begin{array}{r}204 \\
65 \\
278 \\
3\end{array}$ & & $\begin{array}{r}42861 \\
1429 \\
3070 \\
592\end{array}$ & $\begin{array}{r}56580 \\
1825 \\
4490 \\
614\end{array}$ & $\begin{array}{r}52060 \\
1679 \\
2930 \\
1040\end{array}$ & $\begin{array}{r}3023 \\
108 \\
262 \\
47\end{array}$ & & $\begin{array}{r}35291 \\
1138 \\
1980 \\
734\end{array}$ & $\begin{array}{r}44120 \\
1471 \\
2480 \\
680\end{array}$ & $\begin{array}{r}91440 \\
2950 \\
3790 \\
1540\end{array}$ & $\begin{array}{r}80070 \\
2669 \\
3880 \\
1810\end{array}$ & $\begin{array}{r}65120 \\
2101 \\
2820 \\
1570\end{array}$ & $\begin{array}{r}33117 \\
1068 \\
1650 \\
750\end{array}$ & $\begin{array}{r}17114 \\
570 \\
926 \\
217\end{array}$ \\
\hline
\end{tabular}

WTR YR 1946 TOTAL 568437 MEAN 1557 MAX 4490 MIN 217

1947 OCT NOV DEC JAN

$\begin{array}{lrrrr}\text { TOTAL } & 16820 & 23546 & 69863 & 41868 \\ \text { MEAN } & 543 & 785 & 2254 & 1351 \\ \text { MAX } & 1070 & 2780 & 5480 & 4480\end{array}$

$\begin{array}{rrr}\text { FEB } & \text { MAR } & \text { APR } \\ & & \\ \text { A5890 } & 35467 & 35934 \\ 3068 & 1144 & 1198 \\ 11200 & 1590 & 2010\end{array}$

$\begin{array}{rrr}11200 & 1590 & 2010 \\ 1210 & 866 & 689\end{array}$

MAX $11200 \quad$ MIN 98

$\begin{array}{rrr}\text { FEB } & \text { MAR } & \text { APR } \\ 35633 & 27876 & 27104 \\ 1229 & 899 & 903 \\ 2 \text { A60 } & 1650 & 1580 \\ 674 & 538 & 494\end{array}$

$\begin{array}{rr}\text { MAY } & \text { JUN } \\ & \\ 79259 & 105350 \\ 2557 & 3512 \\ 5990 & 5830 \\ 999 & 2400\end{array}$

$\begin{array}{rrr}\text { JUL } & \text { AUG } & \text { SED } \\ 33016 & 16863 & 10338 \\ 1065 & 544 & 345 \\ 1530 & 818 & 563 \\ 625 & 298 & 98\end{array}$

WTR YR 1947 TOTAL 474589 MEAN 1300

$\begin{array}{lrrrr}\text { 194B } & \text { OCT } & \text { NOV } & \text { DEC } & \text { JAN } \\ \text { TRTAL } & 47327 & 43549 & 63555 & 54510 \\ \text { MEAN } & 1527 & 1452 & 2050 & 1758 \\ \text { MAX } & 6960 & 2770 & 5680 & 4430 \\ \text { MIN } & 361 & 643 & 592 & 1110\end{array}$

WTR YR 1948 TOTAL 586386 MEAN 1602

1949 OCT NOV DEC JAN

MAX 6960 MIN 168

$\begin{array}{lrrrr}\text { TOTAL } & 31063 & 44216 & 40234 & 21445 \\ \text { MEAN } & 1002 & 1474 & 1298 & 697 \\ \text { MAX } & 2040 & 4030 & 3360 & 1030 \\ \text { MIN } & 609 & 666 & 410 & 440\end{array}$

$\begin{array}{rrr}\text { FEB } & \text { MAR } & \text { APR } \\ 34587 & 45780 & 46906 \\ 1235 & 1477 & 1564 \\ 4770 & 2460 & 2580 \\ 270 & 1130 & 710\end{array}$

MAY

JUN

98070

3164
4920

4920
1670

76870

2562

3790

1510

MAX 4920 MIN 171

SEP

61

22

WTR YR 1949 TOTAL 547759 MEAN 1501 


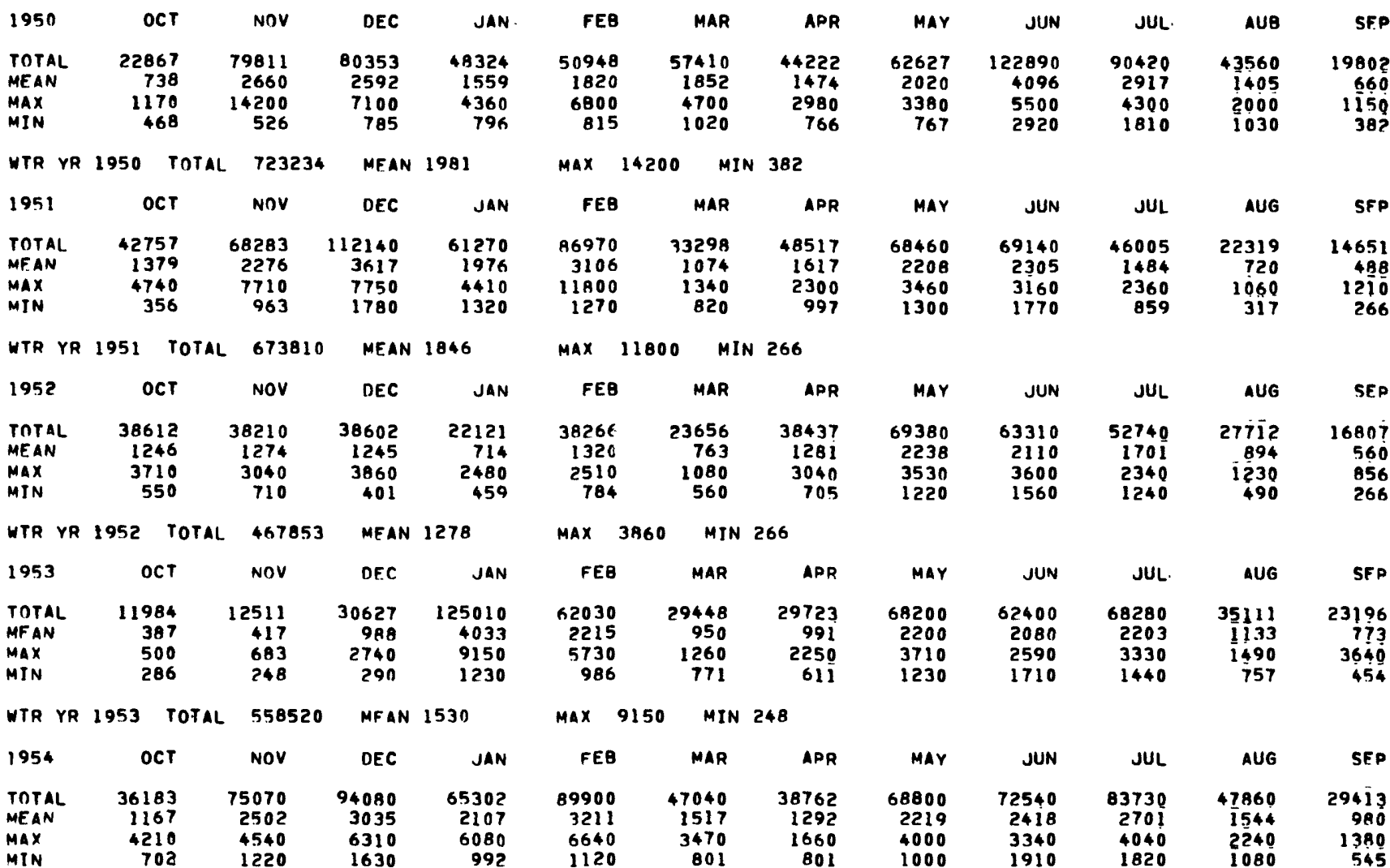

WTR YR 1954 TOTAL 748680 MEAN 2051 MAX 6640 MIN 545

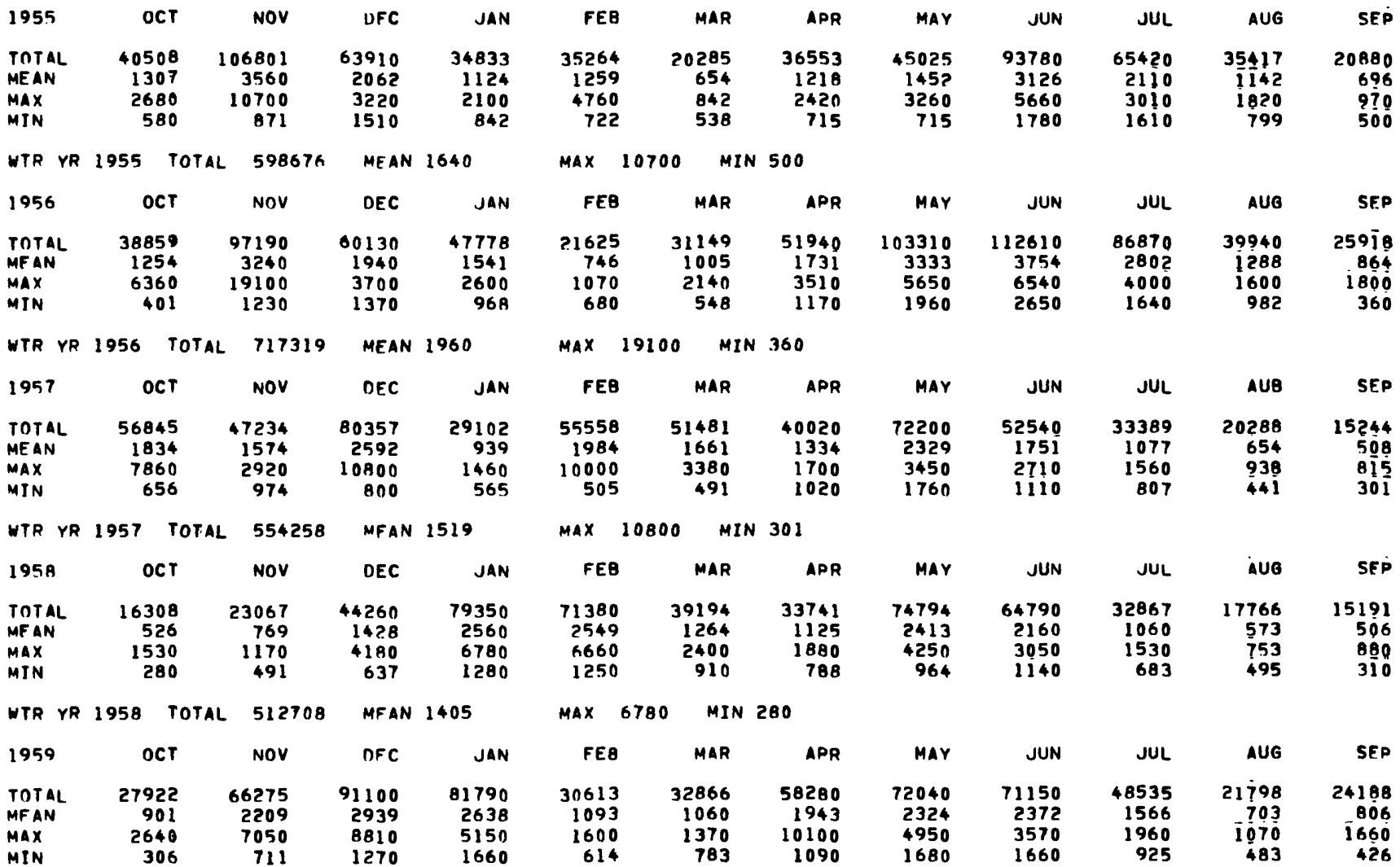

WTR YR 1959 TOTAL 626557 MEAN 1717 MAX 10100 MIN 306 
TABLE 7.--Records of gaging stations in Clallam County--continued

12045500 ELHHA RIVER AT MCDONALD BRIDGE, NEAR PORT ANGELES, WA (Site 129)

\begin{tabular}{|c|c|c|c|c|c|c|c|c|c|c|c|c|}
\hline 1960 & $\mathrm{OCT}$ & NOV & DEC & JAN & FEB & MAR & APR & MAY & JUN & JUL & AUO & SFO \\
\hline $\begin{array}{l}\text { TOTAL } \\
\text { MEAN }\end{array}$ & $\begin{array}{r}29854 \\
963\end{array}$ & $\begin{array}{r}64073 \\
2136\end{array}$ & $\begin{array}{r}65460 \\
2112\end{array}$ & $\begin{array}{r}47439 \\
1530\end{array}$ & $\begin{array}{r}64582 \\
2227\end{array}$ & $\begin{array}{r}37495 \\
1210\end{array}$ & $\begin{array}{r}52370 \\
1746\end{array}$ & $\begin{array}{r}60420 \\
1949\end{array}$ & $\begin{array}{r}73070 \\
2436\end{array}$ & $\begin{array}{r}46079 \\
1486\end{array}$ & $\begin{array}{r}23543 \\
-759\end{array}$ & $\begin{array}{r}13956 \\
465\end{array}$ \\
\hline $\begin{array}{l}\text { MAX } \\
\text { MIN }\end{array}$ & $\begin{array}{r}2230 \\
454\end{array}$ & $\begin{array}{r}10400 \\
513\end{array}$ & $\begin{array}{l}9410 \\
1010\end{array}$ & $\begin{array}{r}11300 \\
581\end{array}$ & $\begin{array}{r}5900 \\
917\end{array}$ & $\begin{array}{r}2070 \\
781\end{array}$ & $\begin{array}{l}2630 \\
1230\end{array}$ & $\begin{array}{r}3230 \\
1420\end{array}$ & $\begin{array}{l}4090 \\
1620\end{array}$ & $\begin{array}{r}2120 \\
949\end{array}$ & $\begin{array}{r}1180 \\
477\end{array}$ & $\begin{array}{l}683 \\
304\end{array}$ \\
\hline
\end{tabular}

WTR YR 1960 TOTAL 578341 MEAN 1580

MAX $11300 \quad$ MIN 304

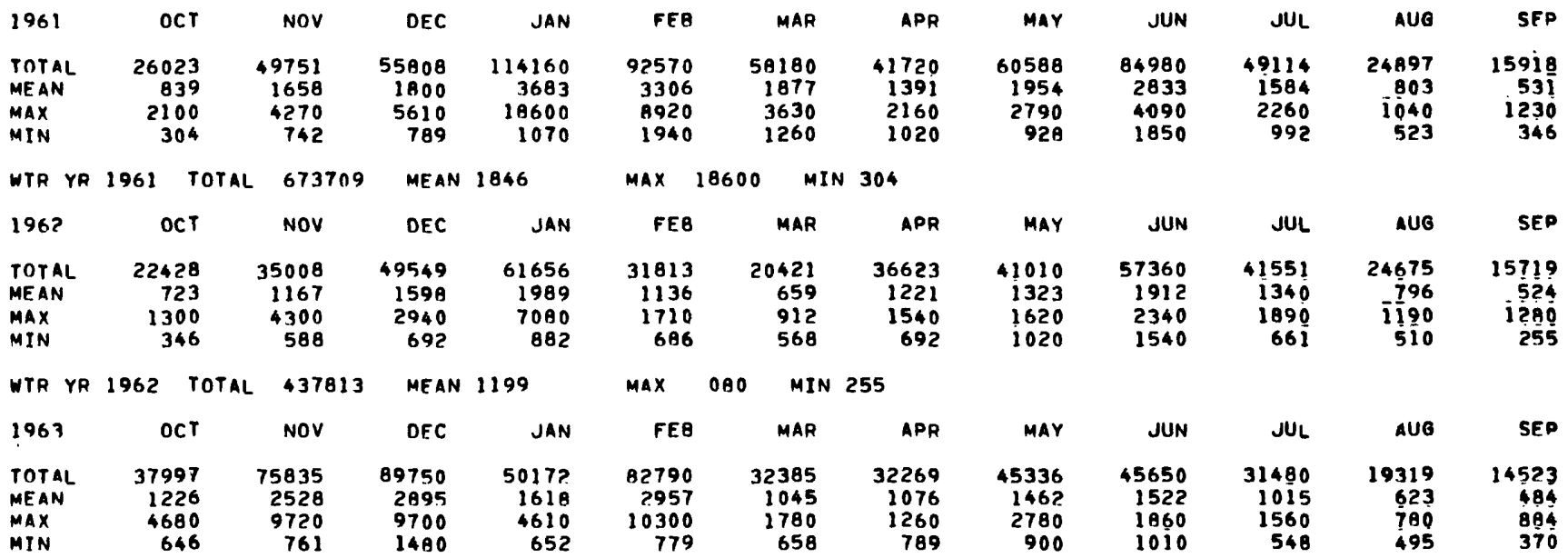

WTR YR 1963 TOTAL 557506 MFAN 1527 MAX 10300 MIN 370

$\begin{array}{lrrrr}\text { TOTAL } & 39303 & 71130 & 62510 & 70580 \\ \text { MEAN } & 1268 & 2371 & 2016 & 2277 \\ \text { MAX } & 5110 & 6410 & 5200 & 6100 \\ \text { MIN } & 345 & 1400 & 1020 & 1470\end{array}$

$\begin{array}{rrr}\text { FEB } & \text { MAR } & \text { APR } \\ 37143 & 31206 & 31383 \\ 1281 & 1007 & 1046 \\ 2460 & 1240 & 1260 \\ 863 & 779 & 700\end{array}$

$\begin{array}{rrrrr}\text { MAY } & \text { JUN } & \text { JUL } & \text { AUG } & \text { SFP } \\ 44280 & 92100 & 69500 & 34189 & 20217 \\ 1429 & 3070 & 2242 & 1103 & 674 \\ 3640 & 4400 & 3280 & 1500 & 2630 \\ 767 & 1840 & 1490 & 730 & 394\end{array}$

WTR YR 1964 TOTAL 603549 MFAN 1649

MAX $6410 \quad$ MIN 345

1965 OCT NOV DEC JAN

$\begin{array}{lrrrr}\text { TOTAL } & 22157 & 30389 & 39214 & 43175 \\ \text { MEAN } & 715 & 1013 & 1265 & 1393\end{array}$

$\begin{array}{lrrrr}\text { MEAN } & 715 & 1013 & 1265 & 1393 \\ \text { MAX } & 1700 & 7120 & 4630 & 4700\end{array}$

$\begin{array}{lrrr}\text { MIN } & 401 & 242 & 359\end{array}$

WTR YR 1965 TOTAL 447118 MFAN 1225

$196 \mathrm{~K}$ OCT NOV DEC JAN

$\begin{array}{lllll}\text { TOTAL } & 23637 & 46633 & 51320 & 53830\end{array}$

$\begin{array}{lrrrr}\text { TOTAL } & 23637 & 46633 & 51320 & 53830 \\ \text { MEAN } & 762 & 1554 & 1655 & 1736 \\ \text { MAX } & 3460 & 2970 & 3800 & 5400\end{array}$

FEB MAR APR

MAY JUN

JULI

AUG

SEP

$\begin{array}{lll}57560 & 33950 & 38200\end{array}$

$2056 \quad 2095 \quad 1273$

53420

59520

$3621 \mathrm{i}$

21165

12157

1984
2610

1168

683

1130

1300

758

1010

405

MAX 7120 MIN 242

$\begin{array}{rrrrrrrr}\text { FEB } & \text { MAR } & \text { APR } & \text { MAY } & \text { JUN } & \text { JUL } & \text { AUG } & \text { SEP } \\ 27556 & 34611 & 53160 & 70080 & 69710 & 55850 & 31110 & 16874 \\ 984 & 1116 & 1772 & 2261 & 2324 & 1802 & 1004 & 562 \\ 1490 & 2910 & 2460 & 4190 & 3680 & 2280 & 1550 & \text { B53 } \\ 700 & 664 & 1130 & 1280 & 1540 & 14,20 & 578 & 315\end{array}$

WTR YR 1966 TOTAL 534371 MEAN 1464

$\operatorname{MAX} 5400 \quad$ MIN 306

1967 OCT NOV DEC JAN

$\begin{array}{lllll}\text { TOTAL } & 25540 & 40794 & 129390 & 75820\end{array}$

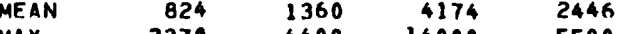

$\begin{array}{rrrrr}\text { MAX } & 3370 & 4400 & 14000 & 5500 \\ \text { MIN } & 296 & 556 & 1660 & 1590\end{array}$

WTR YR 1967 TOTAL 655541 MEAN 1796

1968 OCT NOV DEC JAN

$\begin{array}{lrrrr}\text { TOTAL } & 86800 & 58116 & 75280 & 117380 \\ \text { MEAN } & 2800 & 1937 & 2428 & 3786\end{array}$

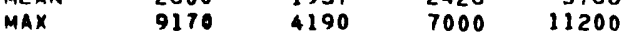

MIN $\quad 1170 \quad 916 \quad 1030 \quad 1300$

WTR YR $\$ 968$ TOTAL 719665 MEAN 1966

1969 OCT NOV DEC JAN

$\begin{array}{rrr}\text { FEB } & \text { MAR } & \text { APR } \\ 44925 & 41687 & 24578 \\ 1604 & 1345 & 819 \\ 3140 & 5320 & 1190 \\ 905 & 814 & 556\end{array}$

$\begin{array}{rrrrr}\text { MAY } & \text { JUN } & \text { JUL } & \text { AUE } & \text { SEP } \\ & & & & \\ 60252 & 103180 & 60530 & 30926 & 17919 \\ 1944 & 3439 & 1953 & 998 & 597 \\ 3870 & 4440 & 3190 & 1320 & 980 \\ 592 & 2220 & 1270 & 527 & 428\end{array}$

MAX $14000 \quad$ MIN 196

$\begin{array}{rrrrrrrr}\text { FEB } & \text { MAR } & \text { APR } & \text { MAY } & \text { JUN } & \text { JULI } & \text { AUE } & \text { SEP } \\ 82450 & 59920 & 32477 & 47780 & 63190 & 46608 & 25434 & 24230 \\ 2843 & 1933 & 1083 & 1541 & 2106 & 1503 & 820 & 808 \\ 5580 & 3720 & 1450 & 2470 & 4000 & 2190 & 1090 & 2050 \\ 1360 & 1170 & 821 & 1130 & 1560 & 958 & 408 & 271\end{array}$

MAX 11200 MIN 271

$\begin{array}{lrrrr}\text { TOTAL } & 34999 & 52712 & 64280 & 48625 \\ \text { MEAN } & 1129 & 1757 & 2074 & 1569 \\ \text { MAX } & 3360 & 3520 & 4560 & 5190 \\ \text { MIN } & 438 & 952 & 1160 & 644\end{array}$

WTR YR 1969 TOTAL 606589 MEAN 1662 


\begin{tabular}{|c|c|c|c|c|c|c|c|c|c|c|c|c|}
\hline 1970 & $O C T$ & nuV & DEC & JAN & FEE & MAR & APR & MAY & JUN & JUL' & AUG & SEP \\
\hline $\begin{array}{l}\text { TOTAL } \\
\text { MEAN } \\
\text { MAX } \\
\text { MIN }\end{array}$ & $\begin{array}{r}26917 \\
868 \\
1550 \\
534\end{array}$ & $\begin{array}{r}33181 \\
1106 \\
1940 \\
544\end{array}$ & $\begin{array}{r}46808 \\
1510 \\
3770 \\
578\end{array}$ & $\begin{array}{r}51125 \\
1649 \\
4590 \\
503\end{array}$ & $\begin{array}{r}4423 \\
158 \\
268 \\
1100\end{array}$ & $\begin{array}{r}36084 \\
1164 \\
2070 \\
736\end{array}$ & $\begin{array}{r}40256 \\
1342 \\
4830 \\
483\end{array}$ & $\begin{array}{r}41941 \\
1353 \\
2580 \\
661\end{array}$ & $\begin{array}{r}63430 \\
2114 \\
3600 \\
1200\end{array}$ & $\begin{array}{r}35922 \\
1159 \\
1610 \\
752\end{array}$ & $\begin{array}{r}18373 \\
593 \\
760 \\
384\end{array}$ & $\begin{array}{r}16959 \\
565 \\
989 \\
301\end{array}$ \\
\hline WTR YR & 1970 TOTAL & $\quad 455226$ & MEAN & 1247 & $\max$ & 4830 & 301 & & & & & \\
\hline 1971 & OCT & Nov & DEC & JAN & FEE & MAR & APR & MAY & JUN & Juili & AUG & SFP \\
\hline $\begin{array}{l}\text { TOTAL } \\
\text { MEAN } \\
\text { MAX } \\
\text { MIN }\end{array}$ & $\begin{array}{r}14900 \\
481 \\
1220 \\
270\end{array}$ & $\begin{array}{r}37147 \\
1238 \\
4610 \\
370\end{array}$ & $\begin{array}{r}46578 \\
1503 \\
4190 \\
610\end{array}$ & $\begin{array}{r}62083 \\
2003 \\
5490 \\
622\end{array}$ & $\begin{array}{r}64230 \\
229 \\
4800 \\
1280\end{array}$ & $\begin{array}{r}47244 \\
1524 \\
4330 \\
928\end{array}$ & $\begin{array}{r}41931 \\
1398 \\
2150 \\
731\end{array}$ & $\begin{array}{r}87350 \\
2818 \\
3880 \\
1760\end{array}$ & $\begin{array}{r}84550 \\
2818 \\
4050 \\
2070\end{array}$ & $\begin{array}{r}89150 \\
2876 \\
4250 \\
1820\end{array}$ & $\begin{array}{r}50910 \\
1642 \\
3320 \\
1030\end{array}$ & $\begin{array}{r}23460 \\
782 \\
1260 \\
502\end{array}$ \\
\hline WTR YR & 1971 TOTAL & 649533 & MEAN & 1780 & $\max$ & 5490 & 270 & & & & & \\
\hline 1972 & OCT & NOV & DEC & JAN & FEE & MAR & APR & MAY & JUN & JUL' & AUG & SFP \\
\hline $\begin{array}{l}\text { TOTAL } \\
\text { MEAN } \\
\text { MAX } \\
\text { MIN }\end{array}$ & $\begin{array}{r}20365 \\
657 \\
899 \\
466\end{array}$ & $\begin{array}{r}48329 \\
1611 \\
4240 \\
906\end{array}$ & $\begin{array}{r}35987 \\
1161 \\
2700 \\
108\end{array}$ & $\begin{array}{r}42518 \\
1372 \\
5570 \\
616\end{array}$ & $\begin{array}{r}5492 \\
189 \\
625 \\
85\end{array}$ & $\begin{array}{r}102510 \\
3307 \\
6420 \\
1460\end{array}$ & $\begin{array}{r}52600 \\
1753 \\
4570 \\
1150\end{array}$ & $\begin{array}{r}80620 \\
2601 \\
5330 \\
1290\end{array}$ & $\begin{array}{r}90660 \\
3022 \\
4120 \\
2320\end{array}$ & $\begin{array}{r}76580 \\
2470 \\
4540 \\
1540\end{array}$ & $\begin{array}{r}37271 \\
1202 \\
1720 \\
801\end{array}$ & $\begin{array}{r}23628 \\
788 \\
2350 \\
532\end{array}$ \\
\hline WTR YR & 1972 TOTAL & 665989 & MEAN & 1820 & $\max$ & 6420 & 466 & & & & & \\
\hline 1973 & OCT & NoV & DEC & JAN & FEE & MAR & APR & MAY & JUN & JUL' & AUG & SEF \\
\hline $\begin{array}{l}\text { TOTAL } \\
\text { MEAN } \\
\text { MAX } \\
\text { MIN }\end{array}$ & $\begin{array}{r}16677 \\
538 \\
759 \\
396\end{array}$ & $\begin{array}{r}27898 \\
930 \\
1780 \\
421 .\end{array}$ & $\begin{array}{r}73408 \\
2368 \\
10300 \\
385\end{array}$ & $\begin{array}{r}63990 \\
2064 \\
6030 \\
1060\end{array}$ & $\begin{array}{r}26817 \\
958 \\
2090 \\
682\end{array}$ & $\begin{array}{r}31883 \\
1028 \\
1890 \\
700\end{array}$ & $\begin{array}{r}20836 \\
695 \\
943 \\
459\end{array}$ & $\begin{array}{r}51768 \\
1670 \\
4640 \\
873\end{array}$ & $\begin{array}{r}52700 \\
1757 \\
2250 \\
1410\end{array}$ & $\begin{array}{r}36616 \\
1181 \\
1470 \\
842\end{array}$ & $\begin{array}{r}20481 \\
661 \\
991 \\
448\end{array}$ & $\begin{array}{r}13869 \\
462 \\
888 \\
345\end{array}$ \\
\hline
\end{tabular}

WTR YR 1973 TOTAL 436943 MEAN 1197 MAX 10300 MIN 345

\begin{tabular}{|c|c|c|c|c|c|c|c|c|c|c|c|c|}
\hline 1974 & OCT & NoV & DEC & JAN & FEB & MAR & APR & MAY & JUN & JUL! & AUG & SEP \\
\hline $\begin{array}{l}\text { TOTAL } \\
\text { MEAN } \\
\text { MAX } \\
\text { MIN }\end{array}$ & $\begin{array}{r}22162 \\
715 \\
2090 \\
330\end{array}$ & $\begin{array}{r}44702 \\
1490 \\
5310 \\
537\end{array}$ & $\begin{array}{r}84230 \\
2717 \\
8040 \\
1390\end{array}$ & $\begin{array}{r}115222 \\
3717 \\
18100 \\
836\end{array}$ & $\begin{array}{r}43690 \\
1560 \\
2990 \\
1000\end{array}$ & $\begin{array}{r}65792 \\
2122 \\
6250 \\
772\end{array}$ & $\begin{array}{r}46100 \\
1537 \\
2190 \\
1200\end{array}$ & $\begin{array}{r}60930 \\
1965 \\
3890 \\
1270\end{array}$ & $\begin{array}{r}112560 \\
3752 \\
5400 \\
2220\end{array}$ & $\begin{array}{r}85540 \\
2759 \\
3890 \\
2100\end{array}$ & $\begin{array}{r}49370 \\
1593 \\
2600 \\
1210\end{array}$ & $\begin{array}{r}24805 \\
827 \\
1230 \\
523\end{array}$ \\
\hline
\end{tabular}

WTR YR 1974 TOTAL 755103 MEAN 2069 MAX 18100 MIN 330

\begin{tabular}{|c|c|c|c|c|c|c|c|c|c|c|c|c|c|c|}
\hline 1975 & $O C$ & & Nov & DEC & JAN & FE & & MAR & APR & MAY & JUN & JULI & AUG & SEP \\
\hline $\begin{array}{l}\text { TOTAL } \\
\text { MEAN } \\
\text { MAX } \\
\text { MIN }\end{array}$ & $\begin{array}{r}1449 \\
46 \\
64 \\
40\end{array}$ & & $\begin{array}{r}32501 \\
1083 \\
3620 \\
352\end{array}$ & $\begin{array}{r}59950 \\
1934 \\
8240 \\
1030\end{array}$ & $\begin{array}{r}55460 \\
1789 \\
4340 \\
1010\end{array}$ & $\begin{array}{r}3183 \\
113 \\
211 \\
90\end{array}$ & & $\begin{array}{r}6814 \\
1188 \\
3010 \\
703\end{array}$ & $\begin{array}{r}23451 \\
782 \\
968 \\
640\end{array}$ & $\begin{array}{r}53502 \\
1726 \\
3340 \\
689\end{array}$ & $\begin{array}{r}79220 \\
2641 \\
4030 \\
1560\end{array}$ & $\begin{array}{r}62560 \\
2018 \\
3440 \\
1230\end{array}$ & $\begin{array}{r}34 \pm 47 \\
1111 \\
2960 \\
860\end{array}$ & $\begin{array}{r}21214 \\
707 \\
1150 \\
479\end{array}$ \\
\hline WTR YR & 1975 & TOTAL & 505448 & MEAN & 1385 & $\operatorname{mAX}$ & 8240 & MIN & 352 & & & & & \\
\hline 1976 & $\mathrm{OC}$ & & NOV & OEC & JAN & FE & & MAR & APR & MAY & JUN & JUL & AUG & SEP \\
\hline $\begin{array}{l}\text { TOTAL } \\
\text { MEAN } \\
\text { MAX } \\
\text { MIN }\end{array}$ & $\begin{array}{r}6848 \\
220 \\
967 \\
45\end{array}$ & & $\begin{array}{r}107190 \\
3573 \\
10600 \\
1910\end{array}$ & $\begin{array}{r}124420 \\
4014 \\
14200 \\
1330\end{array}$ & $\begin{array}{r}81950 \\
2644 \\
6750 \\
1600\end{array}$ & $\begin{array}{r}4294 \\
148 \\
205 \\
120\end{array}$ & & $\begin{array}{r}0126 \\
972 \\
1260 \\
728\end{array}$ & $\begin{array}{r}33358 \\
1112 \\
1900 \\
860\end{array}$ & $\begin{array}{r}72000 \\
2323 \\
3880 \\
1700\end{array}$ & $\begin{array}{r}68030 \\
2268 \\
3550 \\
1570\end{array}$ & $\begin{array}{r}73080 \\
2357 \\
3690 \\
1700\end{array}$ & $\begin{array}{r}39768 \\
1283 \\
1640 \\
920\end{array}$ & $\begin{array}{r}21618 \\
721 \\
1010 \\
602\end{array}$ \\
\hline WTR YR & 1976 & TOTAL & 762961 & MEAN & 2085 & $\max$ & 14200 & MIN & $\sqrt{451}$ & & & & & \\
\hline 1977 & $o c$ & & NOV & DEC & JAN & FE & & MAR & APR & MAY & JUN & JUL & äus & SEP \\
\hline $\begin{array}{l}\text { TOTAL } \\
\text { MEAN } \\
\text { MAX } \\
\text { MIN }\end{array}$ & $\begin{array}{r}1561 \\
50 \\
69 \\
36\end{array}$ & & $\begin{array}{r}17210 \\
574 \\
884 \\
385\end{array}$ & $\begin{array}{r}21107 \\
681 \\
2980 \\
393\end{array}$ & $\begin{array}{r}24205 \\
781 \\
3870 \\
438\end{array}$ & $\begin{array}{r}24998 \\
89 \\
1700 \\
45\end{array}$ & & $\begin{array}{r}8793 \\
929 \\
2130 \\
608\end{array}$ & $\begin{array}{r}35496 \\
1183 \\
2180 \\
614\end{array}$ & $\begin{array}{r}40330 \\
1301 \\
2250 \\
914\end{array}$ & $\begin{array}{r}47660 \\
1589 \\
2880 \\
1150\end{array}$ & $\begin{array}{r}24154 \\
779 \\
1250 \\
650\end{array}$ & $\begin{array}{r}21074 \\
680 \\
1250 \\
545\end{array}$ & $\begin{array}{r}17867 \\
596 \\
1090 \\
620\end{array}$ \\
\hline WTR YR & 1977 & TOTAL & 318508 & MEAN & 873 & $\max$ & 3870 & MIN & 365 & & & & & \\
\hline 1978 & OC & & NOV & DEC & JAN & FE & & MAR & APR & MAY & JUN & JUL & AUG & SEP \\
\hline $\begin{array}{l}\text { TOTAL } \\
\text { MEAN } \\
\text { MAX } \\
\text { MIN }\end{array}$ & $\begin{array}{r}2191 \\
70 \\
242 \\
34\end{array}$ & & $\begin{array}{r}82390 \\
2746 \\
7260 \\
1180\end{array}$ & $\begin{array}{r}98922 \\
3191 \\
8160 \\
992\end{array}$ & $\begin{array}{r}49458 \\
1595 \\
3080 \\
968\end{array}$ & $\begin{array}{r}3715 \\
132 \\
248 \\
848\end{array}$ & & $\begin{array}{r}7826 \\
1220 \\
3300 \\
776\end{array}$ & $\begin{array}{r}31998 \\
1067 \\
1560 \\
710\end{array}$ & $\begin{array}{r}42518 \\
1372 \\
1960 \\
928\end{array}$ & $\begin{array}{r}61220 \\
2041 \\
3150 \\
1340\end{array}$ & $\begin{array}{r}41390 \\
1335 \\
1860 \\
890\end{array}$ & $\begin{array}{r}23022 \\
743 \\
1380 \\
525\end{array}$ & $\begin{array}{r}31036 \\
1035 \\
2530 \\
644\end{array}$ \\
\hline WTR YR & 1978 & TDTAL & 558847 & MEAN & 1581 & $\max$ & 8160 & MIN & 342 & & & & & \\
\hline 1979 & OC & $C T$ & NOV & DEC & JAN & FEE & & MAR & $A P R$ & MAY & JUN & JUL & AUB & SEP \\
\hline $\begin{array}{l}\text { TOTAL } \\
\text { MEAN } \\
\text { MAX } \\
\text { MIN }\end{array}$ & $\begin{array}{r}1625 \\
52 \\
77 \\
41\end{array}$ & $\begin{array}{l}50 \\
24 \\
70 \\
10\end{array}$ & $\begin{array}{r}26330 \\
878 \\
2900 \\
.385\end{array}$ & $\begin{array}{r}23669 \\
764 \\
1260 \\
465\end{array}$ & $\begin{array}{r}13156 \\
424 \\
515 \\
346\end{array}$ & $\begin{array}{r}34491 \\
1232 \\
4040 \\
365\end{array}$ & & $\begin{array}{r}6402 \\
1819 \\
5340 \\
944\end{array}$ & $\begin{array}{r}26294 \\
876 \\
1370 \\
662\end{array}$ & $\begin{array}{r}57120 \\
1843 \\
2610 \\
1290\end{array}$ & $\begin{array}{r}48080 \\
1603 \\
2320 \\
1140\end{array}$ & $\begin{array}{r}33992 \\
1097 \\
1700 \\
710\end{array}$ & $\begin{array}{r}16794 \\
542 \\
770 \\
450\end{array}$ & $\begin{array}{r}20458 \\
682 \\
1860 \\
435\end{array}$ \\
\hline
\end{tabular}

WTR YA 1979 TOTAL 373036 MFAN 1022 MAX 5340 MIN 346 
TABLE 7.--Records of gaging stations in Clallam County--continued

12046500 EL WHA RIVER BELOW DIVERSION, MEAR PORT ANGELES, HA (SIte 139)

LOCATION--Lat $48^{\circ} 06^{\prime} 55^{\prime \prime}$, long $123^{\circ} 33^{\prime} 10^{\prime \prime}$, in NE- sec. 10, T.30 N., R.7 W., on right bank at upstream side of rall road bridge $21 / 2$ miles upstream from mouh and $31 / 2$ miles west of Port Angeles.

DRAINAGE AREA--318 $\mathrm{mi} 2$.

PERIOD OF RECORD--July 1951 to Sept 1954.

GACE--Water-stage recorder. Altitude of gage $1 \mathrm{~s} 60 \mathrm{ft}$ (from topographic map).

EXTREMES--1951-54: Maximum discharge recorded, $12,600 \mathrm{ft}^{3} / \mathrm{s}$ Jan 9, 1953 (gage height, $5.88 \mathrm{ft}$ ); minimum recorded, $62 \mathrm{ft} 3 / \mathrm{s}$ Nov 28, 1952 (gage height, $0.29 \mathrm{ft}$ ).

REMARKS--Flow affected by Lake MIIls and Lake Aldwell. Flow diverted through Gl ine Canyon powerhouse and returned to river above gage. Port Angeles industrial canal diverts water above gage. DISCHARGE, IN CUBIC FEET PER SECOND, WATER YEAR OCTOBER 1950 TO SFPTEMBER İ951
MEAN VALUES

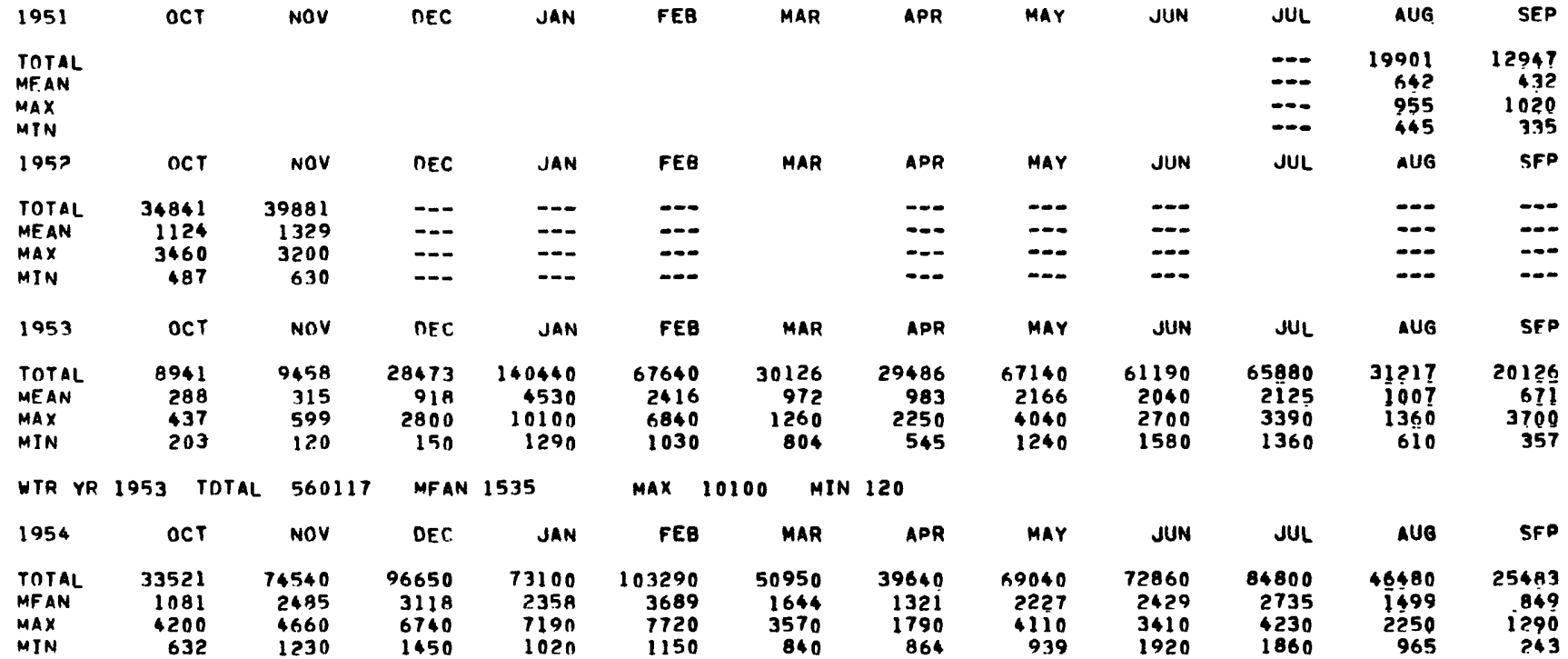

WTP YR 1954 TOTAL 770354 MEAN 2111 MAX 7720 MIN 243

12047300 MORSE CREEK NEAR PORT ANGELES, WA (Site 158)

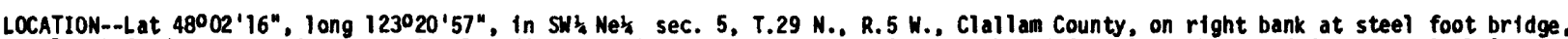
$1,000 \mathrm{ft}$ dounstream from Port Angeles diversion daw, $6.4 \mathrm{mt}$ upstream from mouth and $6.8 \mathrm{mi}$ southeast of ferry terminal in Port Angeles.

DRAINAGE AREA- $-46.6 \mathrm{mi} 2$.

PERIOD OF RECORD-JUI 1966 to Sept 1976.

GAGE--Water-stage recorder. Alt 1 tude of gage is $630 \mathrm{ft}$ (from topographic map).

AVERAGE DISCHARGE--10 years (water years 1967-76), $134 \mathrm{ft} 3 / \mathrm{s}, 97,080$ acre-ft/yr, adjusted for diversion.

EXTREMES FOR PERIOD OF RECORD--Maximum discharge, 3,160 $\mathrm{ft}^{3} / \mathrm{s}$ Dec 4, 1975, gage height, $7.19 \mathrm{ft}$ from rating curve extended above $1,300 \mathrm{ft}^{3} / \mathrm{s}$; mintmum, $5.0 \mathrm{ft}^{3} / \mathrm{s}$ Oct $8,13,1966$; minimum gage height, $1.18 \mathrm{ft}$ oct 17,1971 .

REMARKS--Records good. Some regulation by city of Port Angeles diversion dam 1,000 ft upstream.

Monthly and annual mean diversion, in $\mathrm{ft} 3 / \mathrm{s}$, for water year 1976 furnished by the city of Port Angeles are as follows:

OCT MOV DEC JAN FEB MAR APR MAY JUN JUL AUG SEPT YEAR

$\begin{array}{lllllllllllll}6.1 & 5.7 & 5.3 & 5.8 & 6.5 & 6.5 & 7.7 & 7.8 & 10.3 & 12.1 & 10.3 & 8.9 & 7.8\end{array}$

DISCHARGE, IN CURIC FEET PER SECONI. WATER YEAR OCTOAER 1965 TO SFPTEMBER 1966 MEAN VALUES

\begin{tabular}{|c|c|c|c|c|c|c|c|c|c|c|c|c|}
\hline 1966 & OCT & NOV & DEC & JAN & FEB & MAR & $A P R$ & MAY & JUN & JUL & AUG & SEP \\
\hline $\begin{array}{l}\text { TOTAL } \\
\text { MEAN } \\
\text { MAX } \\
\text { MIN }\end{array}$ & & & & & & & & & & $\ddot{-n}$ & $\begin{array}{r}1548 \\
49.9 \\
75 \\
33\end{array}$ & $\begin{array}{r}935 \\
31.8 \\
47 \\
23\end{array}$ \\
\hline 1967 & OCT & NOV & DEC & JAN & FEB & MAR & $A P R$ & MaY & JUN & JUL & AUG & SED \\
\hline $\begin{array}{l}\text { TOTAL } \\
\text { MFAN } \\
\text { MAX } \\
\text { MIN }\end{array}$ & $\begin{array}{r}1664 \\
53.7 \\
162 \\
16\end{array}$ & $\begin{array}{r}2949 \\
98.3 \\
338 \\
38\end{array}$ & $\begin{array}{r}9687 \\
312 \\
1240 \\
102\end{array}$ & $\begin{array}{r}7409 \\
239 \\
960 \\
126\end{array}$ & $\begin{array}{r}3772 \\
135 \\
232 \\
81\end{array}$ & $\begin{array}{r}3839 \\
124 \\
559 \\
65\end{array}$ & $\begin{array}{r}2427 \\
80.9 \\
98 \\
67\end{array}$ & $\begin{array}{r}6234 \\
201 \\
385 \\
84\end{array}$ & $\begin{array}{r}8171 \\
272 \\
331 \\
185\end{array}$ & $\begin{array}{r}3487 \\
112 \\
172 \\
71\end{array}$ & $\begin{array}{r}1480 \\
47.7 \\
69 \\
28\end{array}$ & $\begin{array}{r}839 \\
28,0 \\
48 \\
19\end{array}$ \\
\hline
\end{tabular}

WTR YR 1967 TOTAL 51958 MEAN 142 MAX 1240 MIN 16 
TABLE 7.--Records of gaging stations in Clallam County--continued

12047300 MORSE CREEK NEAR PORT ANGELES, WA (Site 158)--continued

\begin{tabular}{|c|c|c|c|c|c|c|c|c|c|c|c|c|}
\hline $196 A$ & эCT & NOV & DEC & JAN & FEB & MAR & APR & MAY & JUN & JUL & AUG & SEP \\
\hline $\begin{array}{l}\text { TOTAL } \\
\text { MFAN } \\
\text { MAX } \\
\text { MIN }\end{array}$ & $\begin{array}{r}4308 \\
139 \\
394 \\
61\end{array}$ & $\begin{array}{r}3342 \\
111 \\
202 \\
73\end{array}$ & $\begin{array}{r}6630 \\
214 \\
650 \\
73\end{array}$ & $\begin{array}{r}10379 \\
335 \\
1010 \\
111\end{array}$ & $\begin{array}{r}7791 \\
269 \\
602 \\
140\end{array}$ & $\begin{array}{r}4943 \\
159 \\
301 \\
110\end{array}$ & $\begin{array}{r}2355 \\
78.5 \\
110 \\
60\end{array}$ & $\begin{array}{r}3569 \\
115 \\
216 \\
79\end{array}$ & $\begin{array}{r}3965 \\
132 \\
238 \\
95\end{array}$ & $\begin{array}{r}2225 \\
711.8 \\
110 \\
40\end{array}$ & $\begin{array}{r}1277 \\
41.2 \\
58 \\
28\end{array}$ & $\begin{array}{r}1115 \\
37 . ? \\
64 \\
28\end{array}$ \\
\hline WTR YR & 1968 TOTAL & 51899 & ME AN & 142 & $\operatorname{MAX} 1010$ & MIN 28 & & & & & & \\
\hline 1969 & OCT & NOV & DEC & JAN & FEB & MAR & APR & MAY & JUN & UUL & AUG & SFP \\
\hline $\begin{array}{l}\text { TOTAL } \\
\text { MEAN } \\
\text { MAX } \\
\text { MIN }\end{array}$ & $\begin{array}{r}1624 \\
52.4 \\
166 \\
27\end{array}$ & $\begin{array}{r}3516 \\
117 \\
326 \\
50\end{array}$ & $\begin{array}{r}6247 \\
202 \\
502 \\
91\end{array}$ & $\begin{array}{r}424 A \\
137 \\
462 \\
66\end{array}$ & $\begin{array}{r}2631 \\
94.0 \\
300 \\
56\end{array}$ & $\begin{array}{r}4011 \\
129 \\
403 \\
62\end{array}$ & $\begin{array}{r}414 A \\
138 \\
250 \\
111\end{array}$ & $\begin{array}{r}7840 \\
253 \\
403 \\
100\end{array}$ & $\begin{array}{r}6754 \\
225 \\
351 \\
122\end{array}$ & $\begin{array}{r}2558 \\
82.5 \\
128 \\
49\end{array}$ & $\begin{array}{r}1282 \\
41.4 \\
51 \\
32\end{array}$ & $\begin{array}{r}1191 \\
39.7 \\
83 \\
23\end{array}$ \\
\hline WTR YR & 1969 TOTAL & 46050 & ME AN & 126 & $\operatorname{MAX} 502$ & MIN 23 & & & & & & \\
\hline 1970 & OCT & NOV & DEC & JAN & FEB & MAP & APR & MAY & JUN & JUL' & AUG & SEP \\
\hline $\begin{array}{l}\text { TOTAL } \\
\text { MEAN } \\
\text { MAX } \\
\text { MIN }\end{array}$ & $\begin{array}{r}1132 \\
36.5 \\
56 \\
27\end{array}$ & $\begin{array}{r}2764 \\
92.1 \\
300 \\
28\end{array}$ & $\begin{array}{r}4000 \\
129 \\
468 \\
36\end{array}$ & $\begin{array}{r}4319 \\
139 \\
405 \\
62\end{array}$ & $\begin{array}{r}3657 \\
131 \\
273 \\
87\end{array}$ & $\begin{array}{r}2855 \\
92.1 \\
196 \\
61\end{array}$ & $\begin{array}{r}3233 \\
108 \\
508 \\
56\end{array}$ & $\begin{array}{r}3892 \\
126 \\
206 \\
72\end{array}$ & $\begin{array}{r}4531 \\
151 \\
295 \\
90\end{array}$ & $\begin{array}{r}1949 \\
62.9 \\
88 \\
45\end{array}$ & $\begin{array}{r}1004 \\
32.4 \\
45 \\
24\end{array}$ & $\begin{array}{r}940 \\
28.0 \\
42 \\
16\end{array}$ \\
\hline WTE YR & 1970 TOTAL & 34176 & ME.AN & 93.6 & MAX $50 B$ & MIN 16 & & & & & & \\
\hline 1971 & OCT & NOV & DEC & JAN & FEB & MAR & APA & MAY & JUN & JUL & AUG & SFP \\
\hline $\begin{array}{l}\text { TOTAL } \\
\text { MEAN } \\
\text { MAX } \\
\text { MIN }\end{array}$ & $\begin{array}{r}630.6 \\
20.3 \\
70 \\
7.9\end{array}$ & $\begin{array}{r}1855 \\
61.8 \\
284 \\
23\end{array}$ & $\begin{array}{r}3836 \\
124 \\
511 \\
42\end{array}$ & $\begin{array}{r}5943 \\
192 \\
500 \\
64\end{array}$ & $\begin{array}{r}5580 \\
199 \\
460 \\
115\end{array}$ & $\begin{array}{r}4668 \\
151 \\
460 \\
85\end{array}$ & $\begin{array}{r}3567 \\
119 \\
186 \\
80\end{array}$ & $\begin{array}{r}6232 \\
201 \\
310 \\
125\end{array}$ & $\begin{array}{r}5877 \\
196 \\
284 \\
i 49\end{array}$ & $\begin{array}{r}4928 \\
159 \\
205 \\
138\end{array}$ & $\begin{array}{r}2292 \\
73.9 \\
139 \\
48\end{array}$ & $\begin{array}{r}1227 \\
40.9 \\
66\end{array}$ \\
\hline
\end{tabular}

WTR YR 1971 TOTAL 46635.8 MEAN 128 MAX 511 MIN 7.9

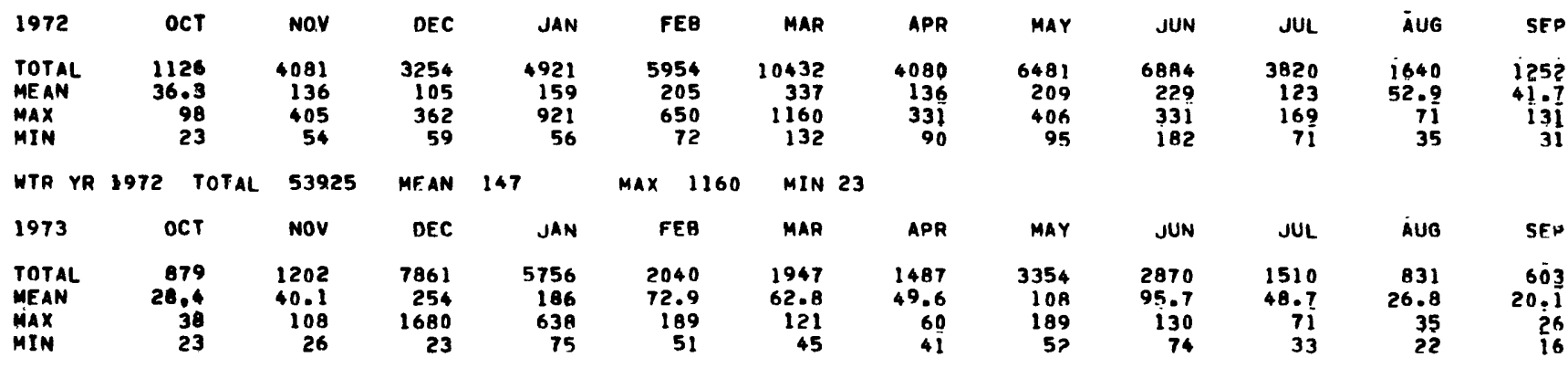

WTR YR 1973 TOTAL 30340 MEAN 83.1 MAX 1680 MIN 16

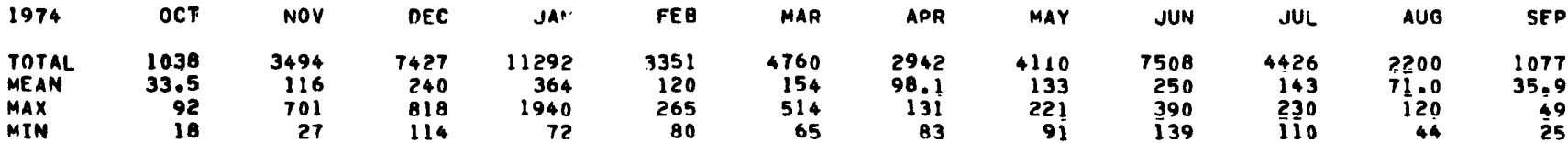

WTR YR 1974 TOTAL 53625 MEAN 147 MAX 1940 MIN 18

\begin{tabular}{|c|c|c|c|c|c|c|c|c|c|c|c|}
\hline 1975 & OCT & Nov & DEC & JAN & FEB & MAR & $A P R$ & MaY & JUN & JUL & AUG \\
\hline $\begin{array}{l}\text { TOTAL } \\
\text { MEAN } \\
\text { MAX } \\
\text { MIN }\end{array}$ & $\begin{array}{r}787 \\
25.4 \\
34 \\
22\end{array}$ & $\begin{array}{r}2122 \\
70.7 \\
300 \\
21\end{array}$ & $\begin{array}{r}3210 \\
104 \\
555 \\
47\end{array}$ & $\begin{array}{r}3354 \\
108 \\
300 \\
60\end{array}$ & $\begin{array}{r}2768 \\
98.9 \\
330 \\
55\end{array}$ & $\begin{array}{r}2506 \\
80.8 \\
167 \\
49\end{array}$ & $\begin{array}{r}1531 \\
51.0 \\
60 \\
43\end{array}$ & $\begin{array}{r}4223 \\
136 \\
236 \\
61\end{array}$ & $\begin{array}{r}5372 \\
179 \\
297 \\
104\end{array}$ & $\begin{array}{r}3063 \\
98.8 \\
159 \\
54\end{array}$ & $\begin{array}{r}1818 \\
58.6 \\
109 \\
34\end{array}$ \\
\hline
\end{tabular}

WTR YR 1975 TOTAL 31996 MEAN 87.7 MAX 555 MIN 21

$\begin{array}{lrrrrrrrrrrrr}1976 & \text { OCT } & \text { NOV } & \text { DEC } & \text { JAN } & \text { FEB } & \text { MAR } & \text { APR } & \text { MAY } & \text { JUN } & \text { JUL } & \text { AUG } & \text { SFD } \\ \text { TOTAL } & 4617 & 9384 & 11456 & 6987 & 4519 & 3079 & 3173 & 6414 & 5098 & 4186 & 2153 & 1126 \\ \text { MEAN } & 155 & 313 & 370 & 225 & 156 & 99.3 & 106 & 207 & 170 & 135 & 69.5 & 37.5 \\ \text { MAX } & 690 & 1300 & 1640 & 606 & 262 & 147 & 186 & 360 & 267 & 201 & 89 & 47 \\ \text { MIN } & 26 & 145 & 118 & 121 & 112 & 82 & 76 & 140 & 117 & B 8 & 48 & 30\end{array}$

WTR YR 1976 TOTAL 62394 MEAN 170 MAX 1640 MIN 26

1977 OCT NOV DEC JAN FEB MAR

$\begin{array}{ll}\text { TOTAL } & -\infty \\ \text { MEAN } & -\infty \\ \text { MAX } & -\infty\end{array}$ 
LOCATION--Lat 48004'58", Iong 123016'52", in SW' NE⿺ sec. 23, T.30 N., R.5 H. on right bank 300 ft downstrean from Emery Creek, 3.2 miles upstream from mouth, and $7.4^{\circ}$ miles southeast of courthouse in Port Angeles.

DRAINAGE AREA--15.5 mi2 (Site 1); $16.1 \mathrm{mit}^{2}$ (Site 2).

PERIOD OF RECORD--Jun 1952 to Sept 1969 (discontinued).

GAGE--Hater-stage recorder. Altitude of gage is $280 \mathrm{ft}$ (from topographic map). Prior to Apr 22, 1960, at site 2, within 0.7 mile downs tream at different datum.

AVERAGE DISCHARGE- -17 years, $17.1 \mathrm{ft}^{3} / \mathrm{s}(14.98 \mathrm{in} / \mathrm{yr}, 12,390 \mathrm{acre}-\mathrm{ft} / \mathrm{yr})$.

EXTREMES--Maximum discharge, $1.620 \mathrm{ft} 3 / \mathrm{s}$ Nov 3, 1955 (gage height, $9.50 \mathrm{ft}$, site and datum then in use), from rating curve extended abvoe $260 \mathrm{ft} 3 / \mathrm{s}$ on basis of computations of peak flow trough culvert at gage heights, $4.25 \mathrm{and} 9.12 \mathrm{ft}$; ninimum, $2.0 \mathrm{ft} 3 / \mathrm{s}$

Sept 3-5, 1952, Aug 19-26, 1958, Aug 25, 26, 1959, Feb. 25, 1962, probabiy Dec. 16, 1964, Aug. 1, 2, 1965.

REMARKS--Records excellent except those for January and February which are good. No reguiation or diversion above station. DISCHARGE, IN CUAIC FEET PER SECONO, WATER YEAR OCTOAER 1951 TO SEPTEMBER I952 MEAN VALUES

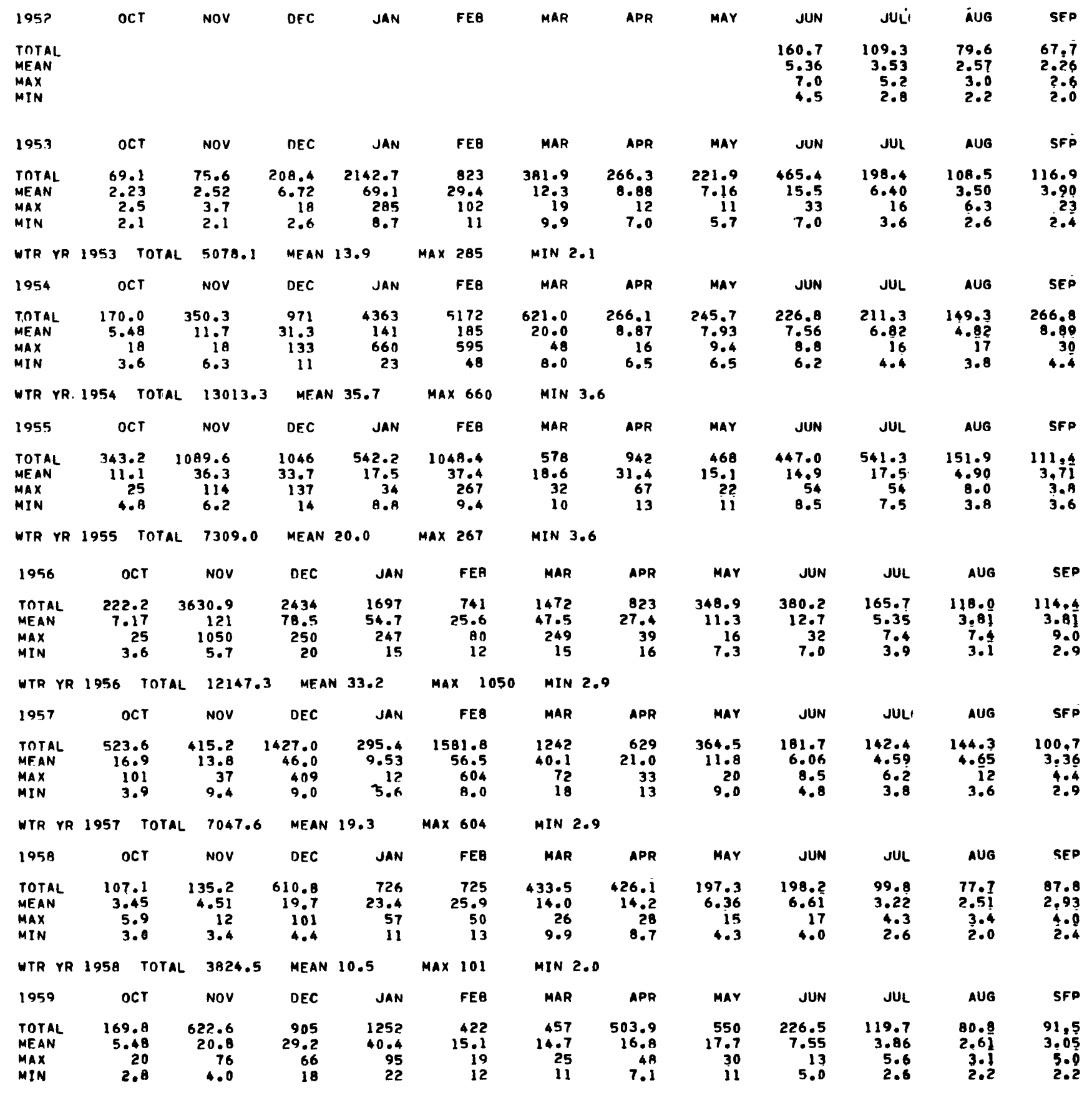

WTR YR 1959 TOTAL 5400.0 MEAN 14.8 MAX 95 MIN 2.2 
TABLE 7.--Records of gaging stations in Clallam County--continued

12047500 SIEBERT CREEK NEAR PORT NGELES, WA (Site 162 and 163)

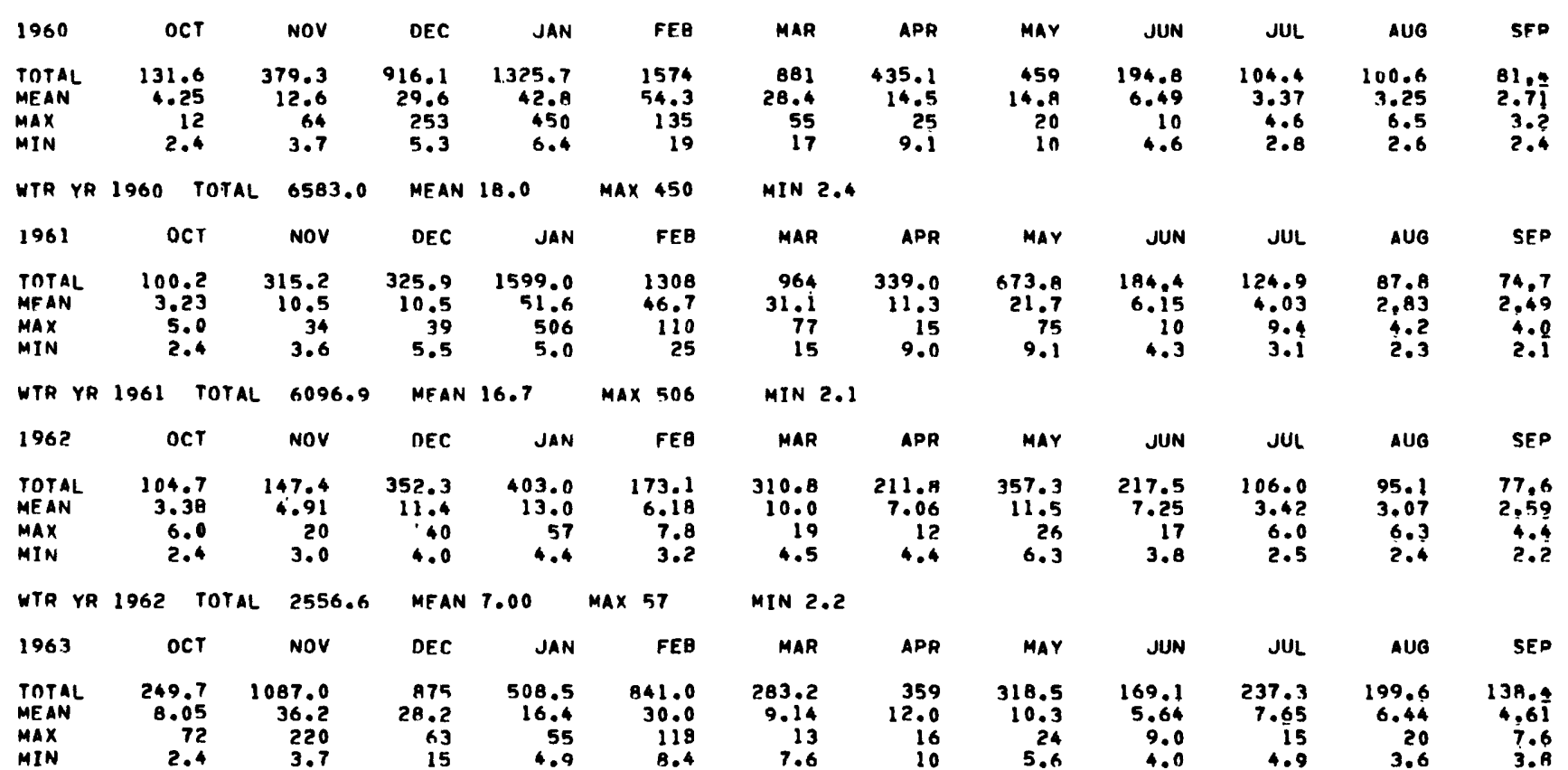

WTR YR 1963 TOTAL 5266.3 MEAN 14.4 MAX 220 MIN 2.4

\begin{tabular}{|c|c|c|c|c|c|c|c|c|c|c|c|c|}
\hline 1964 & OCT & NOV & DEC & JAN & FEB & MAR & APR & MAY & JUN & JUL & AUG & SFP \\
\hline $\begin{array}{l}\text { TOTAL } \\
\text { MEAN } \\
\text { MAX } \\
\text { MIN }\end{array}$ & $\begin{array}{r}566.5 \\
18.3 \\
113 \\
3.7\end{array}$ & $\begin{array}{r}901 \\
30.0 \\
80 \\
15\end{array}$ & $\begin{array}{r}759 \\
24.5 \\
41 \\
15\end{array}$ & $\begin{array}{r}1223 \\
39.5 \\
84 \\
22\end{array}$ & $\begin{array}{r}565.3 \\
19.5 \\
69 \\
8.6\end{array}$ & $\begin{array}{r}626 \\
20.2 \\
49 \\
12\end{array}$ & $\begin{array}{r}351.1 \\
11.7 \\
32 \\
6.9\end{array}$ & $\begin{array}{r}199.6 \\
6.44 \\
9.0 \\
5.8\end{array}$ & $\begin{array}{r}371.0 \\
12.4 \\
26 \\
6.5\end{array}$ & $\begin{array}{r}152.4 \\
4.92 \\
6.9 \\
3.5\end{array}$ & $\begin{array}{r}112.9 \\
3.64 \\
5.8 \\
3.0\end{array}$ & $\begin{array}{r}100.7 \\
3.36 \\
14 \\
2.8\end{array}$ \\
\hline WTR YR & 1964 TOTAL & 5928.5 & MEAN & 16.2 & $\max 113$ & MIN 2.8 & & & & & & \\
\hline 1965 & OCT & NOV & DEC & JAN & FEB & MAR & APR & MAY & JUN & JUL & AUG & SEP \\
\hline $\begin{array}{l}\text { TOTAL } \\
\text { MEAN } \\
\text { MAX } \\
\text { MIN }\end{array}$ & $\begin{array}{r}114.2 \\
3.68 \\
7.3 \\
3.0\end{array}$ & $\begin{array}{r}246.2 \\
8.21 \\
49 \\
4.2\end{array}$ & $\begin{array}{r}284.0 \\
9.16 \\
33 \\
3.0\end{array}$ & $\begin{array}{r}1109.8 \\
35.8 \\
188 \\
6.1\end{array}$ & $\begin{array}{r}1082 \\
38.6 \\
132 \\
19\end{array}$ & $\begin{array}{r}347.1 \\
11.2 \\
22 \\
6.0\end{array}$ & $\begin{array}{r}506.8 \\
16.9 \\
.84 \\
6.4\end{array}$ & $\begin{array}{r}241.7 \\
7.80 \\
1 ! \\
6.1\end{array}$ & $\begin{array}{r}132.9 \\
4.43 \\
6.1 \\
3.5\end{array}$ & $\begin{array}{r}89.6 \\
2.89 \\
4.0 \\
2.1\end{array}$ & $\begin{array}{r}88.4 \\
2.85 \\
6.1 \\
2.1\end{array}$ & $\begin{array}{r}83.4 \\
2.78 \\
4.2 \\
2.4\end{array}$ \\
\hline WTR YR & 1965 TOTAL & 4326.1 & MEAN & 11.9 & $\max 18 B$ & MIN 2.1 & & & & & & \\
\hline 1966 & OCT & NOV & DEC & JAN & FEB & MAR & APR & MAY & JUN & JUL & AUG & SFP \\
\hline $\begin{array}{l}\text { TOTAL } \\
\text { MEAN } \\
\text { MAX } \\
\text { MIN }\end{array}$ & $\begin{array}{r}91.2 \\
2.94 \\
4.5 \\
2.5\end{array}$ & $\begin{array}{r}160.3 \\
5.34 \\
16 \\
3.0\end{array}$ & $\begin{array}{r}335.1 \\
10.8 \\
36 \\
4.2\end{array}$ & $\begin{array}{r}1419.7 \\
45.8 \\
216 \\
7.9\end{array}$ & $\begin{array}{r}405.4 \\
14.5 \\
20 \\
9.4\end{array}$ & $\begin{array}{r}862.2 \\
27.8 \\
75 \\
8.9\end{array}$ & $\begin{array}{r}337.6 \\
11.3 \\
23 \\
6.2\end{array}$ & $\begin{array}{r}224.4 \\
7.24 \\
13 \\
5.8\end{array}$ & $\begin{array}{r}158.1 \\
5.27 \\
7.0 \\
4.2\end{array}$ & $\begin{array}{r}175.4 \\
5.66 \\
13 \\
3.2\end{array}$ & $\begin{array}{r}86.5 \\
2.79 \\
3.0 \\
2.5\end{array}$ & $\begin{array}{r}102.6 \\
3.42 \\
5.8 \\
2.5\end{array}$ \\
\hline
\end{tabular}

WTR YR 1966 TOTAL 4358.5 MEAN 11.9 MAX 216 MIN 2.5

$\begin{array}{lrrrrrrrrrrrr}1967 & \text { OCT } & \text { NOV } & \text { DEC } & \text { JAN } & \text { FEB } & \text { MAR } & \text { APR } & \text { MAY } & \text { JUN } & \text { JUL } & \text { AUG } & \text { SEP } \\ \text { TOTAL } & 171.9 & 287.9 & 1237 & 2080 & 584 & 543.1 & 585 & 414.3 & 200.2 & 111.1 & 81.4 & 78.0 \\ \text { MEAN } & 5.55 & 9.60 & 39.9 & 67.1 & 20.9 & 17.5 & 19.5 & 13.4 & 6.67 & 3.58 & 2.63 & 2.60 \\ \text { MAX } & 22 & 37 & 218 & 454 & 49 & 70 & 58 & 32 & 9.5 & 4.6 & 2.9 & 3.6 \\ \text { MIN } & 3.0 & 3.7 & 13 & 22 & 10 & 7.6 & 10 & 7.6 & 4.6 & 2.9 & 2.5 & 2.4\end{array}$

WTR YR 1967 TOTAL 6373.9 MEAN 17.5 MAX 454 MIN 2.4

\begin{tabular}{|c|c|c|c|c|c|c|c|c|c|c|c|c|}
\hline $196 A$ & $\mathrm{OCT}$ & NOV & DEC & JAN & FEB & MAR & APR & MAY & JUN & JUL & AUG & SED \\
\hline $\begin{array}{l}\text { TOTAL } \\
\text { MFAN } \\
\text { MAX } \\
\text { MIN }\end{array}$ & $\begin{array}{r}296.9 \\
9.58 \\
43 \\
3.3\end{array}$ & $\begin{array}{r}206.7 \\
6.89 \\
15 \\
4.8\end{array}$ & $\begin{array}{r}594.0 \\
19.2 \\
62 \\
4.6\end{array}$ & $\begin{array}{r}1560.1 \\
50.3 \\
232 \\
6.7\end{array}$ & $\begin{array}{r}1308 \\
45.1 \\
198 \\
14\end{array}$ & $\begin{array}{r}786 \\
25.4 \\
62 \\
14\end{array}$ & $\begin{array}{r}254.7 \\
8.49 \\
15 \\
5.6\end{array}$ & $\begin{array}{r}169.7 \\
5.47 \\
8.1 \\
4.2\end{array}$ & $\begin{array}{r}163.5 \\
5.45 \\
11 \\
3.6\end{array}$ & $\begin{array}{r}90.3 \\
2.91 \\
3.4 \\
2.4\end{array}$ & $\begin{array}{r}125.1 \\
4.04 \\
6.1 \\
2.4\end{array}$ & $\begin{array}{r}106, ? \\
3.54 \\
6.0 \\
2.8\end{array}$ \\
\hline WTR YR & 1968 TOTAL & 5661.2 & MFAN & 15.5 & $\operatorname{MAX} 232$ & MIN 2.4 & & & & & & \\
\hline 1969 & OCT & NOV & DEC & JAN & FEB & MAR & APR & MAY & JUN & JUL & ÄUG & SFP \\
\hline $\begin{array}{l}\text { TOTAL } \\
\text { MEAN } \\
\text { MAX } \\
\text { MIN }\end{array}$ & $\begin{array}{r}118.4 \\
3.62 \\
8.8 \\
2.8\end{array}$ & $\begin{array}{r}359.6 \\
12.0 \\
43 \\
3.9\end{array}$ & $\begin{array}{r}1150 \\
37.1 \\
121 \\
12\end{array}$ & $\begin{array}{r}722.0 \\
23.3 \\
95 \\
6.0\end{array}$ & $\begin{array}{r}627.5 \\
22.4 \\
76 \\
6.5\end{array}$ & $\begin{array}{r}941 \\
30.4 \\
92 \\
14\end{array}$ & $\begin{array}{r}672 \\
22.4 \\
50 \\
14\end{array}$ & $\begin{array}{r}348.4 \\
11.2 \\
17 \\
8.1\end{array}$ & $\begin{array}{r}225.3 \\
7.51 \\
25 \\
4.6\end{array}$ & $\begin{array}{r}135.1 \\
4.36 \\
7.4 \\
3.0\end{array}$ & $\begin{array}{r}84.8 \\
2.74 \\
3.2 \\
2.4\end{array}$ & $\begin{array}{r}94.3 \\
3.14 \\
8.1 \\
2.2\end{array}$ \\
\hline
\end{tabular}

WTR YR 1969 TOTAL 5478.4 MEAN 15.0 MAX IZI MIN 2.2 


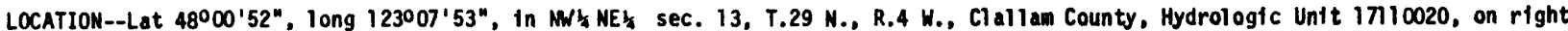
bank $1.0 \mathrm{mi}$ upstream from Canyon Creek, $4.8 \mathrm{mi}$ southwest of Sequim, and at mile 11.8 .

DRAIMAEE AREA--156 mi 2 .

PERIOD OF RECORD--June 1923 to Sept 1930, June 1937 to current year.

GAGE--Water-stage recorder. Datum of gage is $569.3 \mathrm{ft}$ National Geodetic Vertical Datum of 1929 (river profile survey). June 8 , 1923 , to Sept 30, 1930, nonrecording gage Just above fish-hatchery diversion $0.5 \mathrm{mf}$ downstream at different datum. June 19 to Aug 12 , 1937, nonrecording gage at present site and daturn.

AVERAGE DISCHARGE--48 years (water years 1924-30, 1938-78), $390 \mathrm{ft} 3 / \mathrm{s}, 33.95 \mathrm{in} / \mathrm{yr}, 282,600 \mathrm{acre}-\mathrm{ft} / \mathrm{yr}$.

EXTREMES--Maximum discharge, $6,820 \mathrm{ft} 3 / \mathrm{s}$ Nov 27,1949 , gage height, $8.58 \mathrm{ft}$ from floodnarks, from rating extended above $2,000 \mathrm{ft} 3 / \mathrm{s}$ on basis of slope-area measurement of peak flow; minimum discharge, $68 \mathrm{ft} 3 / \mathrm{s} \mathrm{Dec} 9,10$, 1972 , during perlod of 1 ce effect.

REMARKS. --Records good. No regulation or diversion above station.

DISCHARGE, IN CUBIC FEET PER SECONN, WATER YEAR OCTOBER 1922 TO SFPTEMBER 1923 MEAN VALUES

\begin{tabular}{|c|c|c|c|c|c|c|c|c|c|c|c|c|}
\hline 1923 & OCT & NOV & DEC & JAN & FEQ & MAR & APR & MAY & JUN & JUL & AUG & SEP \\
\hline $\begin{array}{l}\text { TOTAL } \\
\text { MEAN } \\
\text { MAX } \\
\text { MIN }\end{array}$ & & & & & & & & & $\begin{array}{r}21984 \\
733 \\
1130 \\
521\end{array}$ & $\begin{array}{r}17156 \\
553 \\
880 \\
333\end{array}$ & $\begin{array}{r}10586 \\
341 \\
670 \\
247\end{array}$ & $\begin{array}{r}6266 \\
219 \\
579 \\
152\end{array}$ \\
\hline 1924 & OCT & NOV & DEC & JAN & FEB & MAR & APR & Mar & JUN & JUL & AUG & SEP \\
\hline $\begin{array}{l}\text { TOTAL } \\
\text { MFAN } \\
\text { MAX } \\
\text { MIN }\end{array}$ & $\begin{array}{r}5509 \\
178 \\
344 \\
141\end{array}$ & $\begin{array}{r}5076 \\
169 \\
452 \\
125\end{array}$ & $\begin{array}{r}12139 \\
392 \\
2080 \\
158\end{array}$ & $\begin{array}{r}1141 A \\
36 A \\
4820 \\
15 B\end{array}$ & $\begin{array}{r}30213 \\
1042 \\
5140 \\
326\end{array}$ & $\begin{array}{r}7231 \\
233 \\
387 \\
165\end{array}$ & $\begin{array}{r}629 A \\
210 \\
298 \\
148\end{array}$ & $\begin{array}{r}15202 \\
490 \\
790 \\
248\end{array}$ & $\begin{array}{r}11710 \\
390 \\
556 \\
312\end{array}$ & $\begin{array}{r}9036 \\
291 \\
438 \\
216\end{array}$ & $\begin{array}{r}5 \$ 61 \\
176 \\
205 \\
119\end{array}$ & $\begin{array}{r}5017 \\
167 \\
413 \\
90\end{array}$ \\
\hline WTR YR & 1924 TOTAL & 124310 & MEAN & $34 n$ & $\operatorname{Max} 5140$ & MIN & 90 & & & & & \\
\hline 1925 & OCT & NOV & DEC & JAN & FEA & MAR & $A P R$ & MAY & JUN & JUL & AUG & SEP \\
\hline $\begin{array}{l}\text { TOTAL } \\
\text { MEAN } \\
\text { MAX } \\
\text { MIN }\end{array}$ & $\begin{array}{r}11756 \\
379 \\
1200 \\
154\end{array}$ & $\begin{array}{r}19067 \\
636 \\
2400 \\
295\end{array}$ & $\begin{array}{r}14570 \\
470 \\
1380 \\
295\end{array}$ & $\begin{array}{r}10665 \\
344 \\
675 \\
222\end{array}$ & $\begin{array}{r}13251 \\
473 \\
1470 \\
257\end{array}$ & $\begin{array}{r}7830 \\
253 \\
336 \\
211\end{array}$ & $\begin{array}{r}15578 \\
519 \\
1080 \\
258\end{array}$ & $\begin{array}{r}24793 \\
800 \\
1500 \\
425\end{array}$ & $\begin{array}{r}18776 \\
626 \\
1120 \\
425\end{array}$ & $\begin{array}{r}15099 \\
487 \\
650 \\
357\end{array}$ & $\begin{array}{r}\text { A253 } \\
266 \\
357 \\
164\end{array}$ & $\begin{array}{r}5165 \\
172 \\
2 ? 2 \\
140\end{array}$ \\
\hline WTR YR & 1925 TOTIAL & 164803 & MEAN & 452 & $\max 2400$ & MIN & 140 & & & & & \\
\hline 1926 & OCT & NOV & DEC & JAN & FEB & MAR & APR & MAY & JUN & JUL & AUG & SEP \\
\hline $\begin{array}{l}\text { TOTAL } \\
\text { MEAN } \\
\text { MAX } \\
\text { MIN }\end{array}$ & $\begin{array}{l}3686 \\
125 \\
147 \\
118\end{array}$ & $\begin{array}{r}4444 \\
146 \\
192 \\
112\end{array}$ & $\begin{array}{r}9084 \\
293 \\
740 \\
182\end{array}$ & $\begin{array}{r}6944 \\
224 \\
408 \\
173\end{array}$ & $\begin{array}{r}6804 \\
243 \\
425 \\
156\end{array}$ & $\begin{array}{r}6114 \\
197 \\
258 \\
156\end{array}$ & $\begin{array}{r}8365 \\
279 \\
425 \\
201\end{array}$ & $\begin{array}{r}9522 \\
307 \\
478 \\
212\end{array}$ & $\begin{array}{r}8658 \\
289 \\
425 \\
222\end{array}$ & $\begin{array}{r}5541 \\
179 \\
245 \\
132\end{array}$ & $\begin{array}{r}4140 \\
134 \\
201 \\
112\end{array}$ & $\begin{array}{r}3136 \\
105 \\
132 \\
82\end{array}$ \\
\hline WTR YR & 1926 TOTIAL & 76642 & MEAN & 210 & $\max 740$ & $M I N$ & 82 & & & & & \\
\hline $19 ? 7$ & OCT & NOV & DEC & JAN & FEB & MAR & APR & Mar & JUN & JUL & AUG & SFP \\
\hline $\begin{array}{l}\text { TOTAL } \\
\text { MFAN } \\
\text { MAX } \\
\text { MIN }\end{array}$ & $\begin{array}{r}8134 \\
262 \\
1080 \\
99\end{array}$ & $\begin{array}{r}9036 \\
301 \\
1040 \\
140\end{array}$ & $\begin{array}{r}15953 \\
515 \\
2150 \\
270\end{array}$ & $\begin{array}{r}13928 \\
449 \\
1710 \\
259\end{array}$ & $\begin{array}{r}11270 \\
403 \\
740 \\
258\end{array}$ & $\begin{array}{r}9475 \\
306 \\
408 \\
270\end{array}$ & $\begin{array}{r}9921 \\
331 \\
650 \\
234\end{array}$ & $\begin{array}{r}16035 \\
517 \\
1000 \\
357\end{array}$ & $\begin{array}{r}29355 \\
979 \\
1710 \\
550\end{array}$ & $\begin{array}{r}19342 \\
624 \\
710 \\
495\end{array}$ & $\begin{array}{r}10358 \\
334 \\
512 \\
201\end{array}$ & $\begin{array}{r}6676 \\
223 \\
442 \\
147\end{array}$ \\
\hline WTR YR & 1927 TOTAL & 159483 & MFAN & 437 & $\operatorname{MAX} 2150$ & MIN & 99 & & & & & \\
\hline 1928 & OCT & NOV & DEC & JAN & FEO & MAR & APR & MAY & JUN & JUL & AUG & SFP \\
\hline $\begin{array}{l}\text { TOTAL } \\
\text { MFAN } \\
\text { MAX } \\
\text { MIN }\end{array}$ & $\begin{array}{r}7525 \\
243 \\
595 \\
156\end{array}$ & $\begin{array}{r}9985 \\
333 \\
620 \\
182\end{array}$ & $\begin{array}{r}8189 \\
264 \\
650 \\
125\end{array}$ & $\begin{array}{r}18694 \\
609 \\
1400 \\
147\end{array}$ & $\begin{array}{r}7758 \\
268 \\
326 \\
212\end{array}$ & $\begin{array}{r}8797 \\
264 \\
650 \\
192\end{array}$ & $\begin{array}{r}9433 \\
314 \\
442 \\
245\end{array}$ & $\begin{array}{r}17395 \\
561 \\
920 \\
312\end{array}$ & $\begin{array}{r}13149 \\
436 \\
530 \\
326\end{array}$ & $\begin{array}{r}9055 \\
292 \\
374 \\
201\end{array}$ & $\begin{array}{r}4220 \\
143 \\
192 \\
99\end{array}$ & $\begin{array}{r}2814 \\
93,6 \\
164 \\
77\end{array}$ \\
\hline WTR YR & 1928 TOTAL & 117414 & MEAN & 321 & MAX 1400 & MIN & 77 & & & & & \\
\hline 1929 & OCT & NOV & DEC & JAN & FEB & MAR & APR & MAY & JUN & JULI & AUB & SEP \\
\hline $\begin{array}{l}\text { TOTAL } \\
\text { MEAN } \\
\text { MAX } \\
\text { MIN }\end{array}$ & $\begin{array}{r}5744 \\
185 \\
595 \\
82\end{array}$ & $\begin{array}{r}6180 \\
206 \\
390 \\
164\end{array}$ & $\begin{array}{l}5759 \\
186 \\
478 \\
132\end{array}$ & $\begin{array}{r}4134 \\
133 \\
192 \\
112\end{array}$ & $\begin{array}{r}2965 \\
106 \\
112 \\
99\end{array}$ & $\begin{array}{r}4149 \\
134 \\
164 \\
105\end{array}$ & $\begin{array}{r}6037 \\
201 \\
326 \\
118\end{array}$ & $\begin{array}{r}16266 \\
525 \\
645 \\
297\end{array}$ & $\begin{array}{r}19975 \\
666 \\
1000 \\
478\end{array}$ & $\begin{array}{r}13854 \\
447 \\
650 \\
312\end{array}$ & $\begin{array}{r}7063 \\
226 \\
326 \\
173\end{array}$ & $\begin{array}{r}3878 \\
129 \\
164 \\
93\end{array}$ \\
\hline WTR YR & 1929 TOTAL & 96004 & MEAN & 263 & $\operatorname{MAX} 1000$ & MIN $B$ & 32 & & & & & \\
\hline 1930 & OCT & NOV & DEC & JAN & FE. 8 & MÁR & $A P R$ & MAY & JUN & JUL & AUG & SEP \\
\hline $\begin{array}{l}\text { TOTAL } \\
\text { MEAN } \\
\text { MAX } \\
\text { MIN }\end{array}$ & $\begin{array}{r}2996 \\
96.7 \\
132 \\
12\end{array}$ & $\begin{array}{r}2597 \\
86.6 \\
132 \\
77\end{array}$ & $\begin{array}{r}5795 \\
187 \\
740 \\
77\end{array}$ & $\begin{array}{r}3259 \\
105 \\
147 \\
65\end{array}$ & $\begin{array}{r}8313 \\
297 \\
920 \\
125\end{array}$ & $\begin{array}{r}6547 \\
211 \\
374 \\
125\end{array}$ & $\begin{array}{r}11231 \\
374 \\
495 \\
297\end{array}$ & $\begin{array}{r}1120 ? \\
361 \\
460 \\
312\end{array}$ & $\begin{array}{r}14567 \\
486 \\
880 \\
390\end{array}$ & $\begin{array}{r}8810 \\
284 \\
390 \\
201\end{array}$ & $\begin{array}{r}5230 \\
169 \\
201 \\
132\end{array}$ & $\begin{array}{r}4014 \\
134 \\
1104\end{array}$ \\
\hline UTR YR & 1930 TOTAL & 84563 & MFAN & 232 & $\max 920$ & MIN 7 & 77 & & & & & \\
\hline
\end{tabular}




\begin{tabular}{|c|c|c|c|c|c|c|c|c|c|c|c|c|}
\hline 1937 & OCT & NOV & DEC & JAN & FEB & MAR & APR & MaY & JUN & JUL & AUG & SEP \\
\hline $\begin{array}{l}\text { TOTAL } \\
\text { MEAN } \\
\text { MAX } \\
\text { MIN }\end{array}$ & & & & & & & & & $\begin{array}{r}26402 \\
880 \\
1200 \\
692\end{array}$ & $\begin{array}{r}17.199 \\
555 \\
860 \\
300\end{array}$ & $\begin{array}{r}7080 \\
228 \\
320 \\
153\end{array}$ & $\begin{array}{r}4148 \\
138 \\
174 \\
107\end{array}$ \\
\hline $193 A$ & OCT & NOV & DEC & JAN & FEB & MAR & APR & MAY & JUN & JUL & AUO & SFP \\
\hline $\begin{array}{l}\text { TOTAL } \\
\text { MEAN } \\
\text { MAX } \\
\text { MIN }\end{array}$ & $\begin{array}{r}4836 \\
156 \\
898 \\
104\end{array}$ & $\begin{array}{r}11758 \\
392 \\
1020 \\
140\end{array}$ & $\begin{array}{r}17839 \\
575 \\
3240 \\
217\end{array}$ & $\begin{array}{r}12188 \\
393 \\
7.14 \\
240\end{array}$ & $\begin{array}{r}6093 \\
218 \\
408 \\
172\end{array}$ & $\begin{array}{r}8101 \\
261 \\
418 \\
194\end{array}$ & $\begin{array}{r}13145 \\
438 \\
1070 \\
188\end{array}$ & $\begin{array}{r}22095 \\
713 \\
1230 \\
402\end{array}$ & $\begin{array}{r}26357 \\
879 \\
1220 \\
655\end{array}$ & $\begin{array}{r}16639 \\
537 \\
690 \\
321\end{array}$ & $\begin{array}{r}7086 \\
229 \\
321 \\
162\end{array}$ & $\begin{array}{l}4548 \\
152 \\
183 \\
123\end{array}$ \\
\hline WTR YR & 1938 TOTAL & 150685 & MEAN & 413 & $\operatorname{MAX} 3240$ & MIN & 104 & & & & & \\
\hline 1939 & OCT & NOV & DEC & JAN & FEB & MAR & APR & MAY & JUN & JUL & AUG & SFP \\
\hline $\begin{array}{l}\text { TOTAL } \\
\text { MEAN } \\
\text { MAX } \\
\text { MIN }\end{array}$ & $\begin{array}{r}5266 \\
170 \\
525 \\
112\end{array}$ & $\begin{array}{r}4792 \\
160 \\
280 \\
125\end{array}$ & $\begin{array}{r}7924 \\
256 \\
607 \\
137\end{array}$ & $\begin{array}{r}14235 \\
459 \\
2560 \\
211\end{array}$ & $\begin{array}{r}5657 \\
202 \\
442 \\
149\end{array}$ & $\begin{array}{r}7108 \\
229 \\
486 \\
125\end{array}$ & $\begin{array}{r}9863 \\
329 \\
414 \\
259\end{array}$ & $\begin{array}{r}13895 \\
448 \\
903 \\
320\end{array}$ & $\begin{array}{r}13616 \\
454 \\
550 \\
370\end{array}$ & $\begin{array}{r}11636 \\
375 \\
464 \\
288\end{array}$ & $\begin{array}{r}5896 \\
190 \\
259 \\
141\end{array}$ & $\begin{array}{r}3754 \\
125 \\
156 \\
109\end{array}$ \\
\hline WTR YR & 1939 TOTIAL & 103642 & MEAN & 284 & $\max 2560$ & MIN & 109 & & & & & \\
\hline 1940 & OCI & NOV & DEC & JAN & FEB & MAR & APR & MAY & JUN & JUL & AUO & SFP \\
\hline $\begin{array}{l}\text { TOTAL } \\
\text { MFAN } \\
\text { MAX } \\
\text { MIN }\end{array}$ & $\begin{array}{r}3731 \\
120 \\
196 \\
98\end{array}$ & $\begin{array}{r}5055 \\
169 \\
419 \\
102\end{array}$ & $\begin{array}{r}22929 \\
740 \\
2320 \\
347\end{array}$ & $\begin{array}{r}18166 \\
586 \\
1840 \\
259\end{array}$ & $\begin{array}{r}11522 \\
397 \\
557 \\
280\end{array}$ & $\begin{array}{r}12411 \\
400 \\
570 \\
322\end{array}$ & $\begin{array}{r}10330 \\
344 \\
446 \\
276\end{array}$ & $\begin{array}{r}20643 \\
666 \\
1050 \\
499\end{array}$ & $\begin{array}{r}16063 \\
535 \\
710 \\
394\end{array}$ & $\begin{array}{r}9537 \\
308 \\
422 \\
232\end{array}$ & $\begin{array}{r}5813 \\
188 \\
220 \\
159\end{array}$ & $\begin{array}{r}4674 \\
156 \\
250 \\
129\end{array}$ \\
\hline WTR YR & 1940 TOTAL & 140874 & MEAN & 385 & $\operatorname{MAX} 2320$ & MIN & 98 & & & & & \\
\hline 1941 & OCT & NOV & DEC & JAN & FEB & MAR & APR & Mar & JUN & JUL & AUO & SEP \\
\hline $\begin{array}{l}\text { TOTAL } \\
\text { MEAN } \\
\text { MAX } \\
\text { MIN }\end{array}$ & $\begin{array}{r}10786 \\
348 \\
1210 \\
117\end{array}$ & $\begin{array}{r}7816 \\
261 \\
557 \\
187\end{array}$ & $\begin{array}{r}13462 \\
434 \\
795 \\
266\end{array}$ & $\begin{array}{r}12172 \\
393 \\
1420 \\
226\end{array}$ & $\begin{array}{r}10041 \\
359 \\
710 \\
206\end{array}$ & $\begin{array}{r}7425 \\
240 \\
361 \\
190\end{array}$ & $\begin{array}{r}8288 \\
276 \\
370 \\
212\end{array}$ & $\begin{array}{r}12904 \\
416 \\
641 \\
232\end{array}$ & $\begin{array}{r}15244 \\
508 \\
755 \\
398\end{array}$ & $\begin{array}{r}10667 \\
344 \\
462 \\
218\end{array}$ & $\begin{array}{r}5391 \\
174 \\
242 \\
141\end{array}$ & $\begin{array}{r}4561 \\
152 \\
218 \\
117\end{array}$ \\
\hline WTR YR & 1941 TOTAL & 118757 & MEAN & 325 & $\operatorname{MAX} 1420$ & MIN & 117 & & & & & \\
\hline 1942 & OCT & NOV & DEC & JAN & FEB & MAR & APR & MAY & JUN & JUL & AUO & SEP \\
\hline $\begin{array}{l}\text { TOTAL } \\
\text { MEAN } \\
\text { MAX } \\
\text { MIN }\end{array}$ & $\begin{array}{r}6473 \\
209 \\
526 \\
121\end{array}$ & $\begin{array}{r}10912 \\
364 \\
1300 \\
134\end{array}$ & $\begin{array}{r}22166 \\
715 \\
2860 \\
290\end{array}$ & $\begin{array}{r}7779 \\
251 \\
356 \\
208\end{array}$ & $\begin{array}{r}6070 \\
217 \\
338 \\
158\end{array}$ & $\begin{array}{r}5038 \\
163 \\
195 \\
135\end{array}$ & $\begin{array}{r}8115 \\
271 \\
446 \\
201\end{array}$ & $\begin{array}{r}14777 \\
477 \\
932 \\
241\end{array}$ & $\begin{array}{r}19135 \\
638 \\
869 \\
517\end{array}$ & $\begin{array}{r}14154 \\
457 \\
860 \\
270\end{array}$ & $\begin{array}{r}6216 \\
201 \\
259 \\
142\end{array}$ & $\begin{array}{r}3608 \\
120 \\
142 \\
100\end{array}$ \\
\hline WTR YR & 1942 TOTAL & 124443 & MEAN & 341 & $\operatorname{MAX} 2860$ & MIN & 100 & & & & & \\
\hline 1943 & OCT & NOV & DEC & JAN & FEB & MAR & APR & MAY & JUN & JUL & AUG & SEP \\
\hline $\begin{array}{l}\text { TOTAL } \\
\text { MEAN } \\
\text { MAX } \\
\text { MIN }\end{array}$ & $\begin{array}{r}3299 \\
106 \\
315 \\
87\end{array}$ & $\begin{array}{r}6738 \\
225 \\
531 \\
108\end{array}$ & $\begin{array}{r}8702 \\
281 \\
459 \\
152\end{array}$ & $\begin{array}{r}7297 \\
235 \\
423 \\
155\end{array}$ & $\begin{array}{r}7152 \\
255 \\
390 \\
175\end{array}$ & $\begin{array}{l}6000 \\
194 \\
604 \\
129\end{array}$ & $\begin{array}{r}13815 \\
461 \\
770 \\
327\end{array}$ & $\begin{array}{r}13916 \\
449 \\
920 \\
285\end{array}$ & $\begin{array}{r}17587 \\
586 \\
930 \\
414\end{array}$ & $\begin{array}{r}15338 \\
495 \\
770 \\
356\end{array}$ & $\begin{array}{r}7163 \\
231 \\
327 \\
174\end{array}$ & $\begin{array}{r}4124 \\
137 \\
168 \\
112\end{array}$ \\
\hline WTR YR & 1943 TOTAL & 111131 & MEAN & 304 & $\max 930$ & MIN & 87 & & & & & \\
\hline 1944 & OCT & NOV & DEC & JAN & FEB & MAR & APR & MAY & JUN & JUL & AUG & SEP \\
\hline $\begin{array}{l}\text { TOTAL } \\
\text { MEAN } \\
\text { MAX } \\
\text { MIN }\end{array}$ & $\begin{array}{r}4631 \\
149 \\
405 \\
106\end{array}$ & $\begin{array}{r}4123 \\
137 \\
200 \\
115\end{array}$ & $\begin{array}{r}6418 \\
207 \\
884 \\
115\end{array}$ & $\begin{array}{l}6050 \\
195 \\
508 \\
111\end{array}$ & $\begin{array}{r}3936 \\
136 \\
263 \\
108\end{array}$ & $\begin{array}{r}5006 \\
161 \\
386 \\
97\end{array}$ & $\begin{array}{r}5562 \\
185 \\
259 \\
141\end{array}$ & $\begin{array}{r}10622 \\
343 \\
582 \\
222\end{array}$ & $\begin{array}{r}12367 \\
412 \\
623 \\
304\end{array}$ & $\begin{array}{r}6818 \\
220 \\
292 \\
158\end{array}$ & $\begin{array}{r}4005 \\
129 \\
154 \\
106\end{array}$ & $\begin{array}{r}3250 \\
108 \\
180 \\
92\end{array}$ \\
\hline WTR YR & 1944 TOTIAL & 72788 & MEAN & 199 & $\operatorname{MAX} 884$ & MIN 9 & 92 & & & & & \\
\hline 1945 & OCT & NOV & DEC & JAN & FEB & MAR & APR & MAY & JUN & JUL & AUO & SEP \\
\hline $\begin{array}{l}\text { TOTAL } \\
\text { MEAN } \\
\text { MAX } \\
\text { MIN }\end{array}$ & $\begin{array}{r}3408 \\
110 \\
389 \\
80\end{array}$ & $\begin{array}{r}7115 \\
237 \\
489 \\
126\end{array}$ & $\begin{array}{r}6801 \\
219 \\
637 \\
112\end{array}$ & $\begin{array}{r}8553 \\
276 \\
604 \\
110\end{array}$ & $\begin{array}{r}12680 \\
417 \\
1600 \\
180\end{array}$ & $\begin{array}{r}7162 \\
231 \\
620 \\
155\end{array}$ & $\begin{array}{r}7196 \\
240 \\
402 \\
159\end{array}$ & $\begin{array}{r}18866 \\
609 \\
1020 \\
437\end{array}$ & $\begin{array}{r}17368 \\
579 \\
804 \\
426\end{array}$ & $\begin{array}{r}12685 \\
409 \\
544 \\
251\end{array}$ & $\begin{array}{r}6197 \\
200 \\
251 \\
146\end{array}$ & $\begin{array}{r}4497 \\
150 \\
333 \\
115\end{array}$ \\
\hline WTR YR & 1945 TOTAL & 111528 & MEAN & 306 & $\operatorname{MAX} 1600$ & MIN & 80 & & & & & \\
\hline 1946 & OCT & NOV & DEC & JAN & FEB & MAR & APR & MAY & JUN & JUL & AUB & SFP \\
\hline $\begin{array}{l}\text { TOTAL } \\
\text { MEAN } \\
\text { MAX } \\
\text { MIN }\end{array}$ & $\begin{array}{r}3934 \\
127 \\
412 \\
84\end{array}$ & $\begin{array}{r}6435 \\
215 \\
479 \\
119\end{array}$ & $\begin{array}{r}9613 \\
310 \\
697 \\
156\end{array}$ & $\begin{array}{r}8758 \\
283 \\
485 \\
198\end{array}$ & $\begin{array}{r}5419 \\
194 \\
427 \\
138\end{array}$ & $\begin{array}{r}6231 \\
201 \\
319 \\
151\end{array}$ & $\begin{array}{r}9539 \\
318 \\
658 \\
156\end{array}$ & $\begin{array}{r}21200 \\
684 \\
970 \\
357\end{array}$ & $\begin{array}{r}20894 \\
696 \\
1020 \\
550\end{array}$ & $\begin{array}{r}19554 \\
631 \\
848 \\
470\end{array}$ & $\begin{array}{r}10039 \\
324 \\
508 \\
240\end{array}$ & $\begin{array}{r}4953 \\
165 \\
237 \\
119\end{array}$ \\
\hline
\end{tabular}

WTR YR 1946 TOTAL 126569 MFAN 347 MAX 1020 MIN B4 
TABLE 7.--Records of gaging stations in Clallam County--continued

12048000 DUNGENESS RIVER MEAR SEQUIM, HA, (S1te 173) - continued

$\begin{array}{lrrrrrrrrrrrr}1947 & \text { OCT } & \text { NOV } & \text { DEC } & \text { JAN } & \text { FEB } & \text { MAR } & \text { APR } & \text { MAY } & \text { JUN } & \text { JUL } & \text { AUG } & \text { SFD } \\ \text { TOTAL } & 4133 & 5200 & 10426 & 8336 & 18244 & 9472 & 10415 & 19293 & 16544 & 10728 & 5759 & 3906 \\ \text { MEAN } & 133 & 173 & 336 & 269 & 652 & 306 & 347 & 622 & 551 & 346 & 186 & 130 \\ \text { MAX } & 295 & 510 & 993 & 966 & 2050 & 408 & 508 & 923 & \text { B96 } & 483 & 256 & 179 \\ \text { MIN } & 97 & 93 & 185 & 141 & 258 & 226 & 230 & 418 & 360 & 237 & 138 & 110\end{array}$

WTR YR 1947 TOTAL 122458 MEAN 336 MAX 2050 MIN 93

\begin{tabular}{|c|c|c|c|c|c|c|c|c|c|c|c|c|}
\hline $194 \mathrm{~A}$ & OCT & NOV & DEC & JAN & FEB & MAR & APR & MAY & JUN & JUL' & AUG & SFD \\
\hline $\begin{array}{l}\text { TOTAL } \\
\text { MEAN } \\
\text { MAX } \\
\text { MIN }\end{array}$ & $\begin{array}{r}10833 \\
349 \\
1630 \\
118\end{array}$ & $\begin{array}{r}7959 \\
265 \\
567 \\
179\end{array}$ & $\begin{array}{r}12820 \\
414 \\
1270 \\
162\end{array}$ & $\begin{array}{r}10323 \\
333 \\
567 \\
237\end{array}$ & $\begin{array}{r}8236 \\
284 \\
828 \\
176\end{array}$ & $\begin{array}{r}6642 \\
214 \\
508 \\
148\end{array}$ & $\begin{array}{r}7959 \\
265 \\
514 \\
162\end{array}$ & $\begin{array}{r}23926 \\
772 \\
2200 \\
222\end{array}$ & $\begin{array}{r}35892 \\
1196 \\
1830 \\
860\end{array}$ & $\begin{array}{r}18114 \\
584 \\
812 \\
424\end{array}$ & $\begin{array}{r}9557 \\
308 \\
471 \\
208\end{array}$ & $\begin{array}{r}6469 \\
216 \\
632 \\
134\end{array}$ \\
\hline
\end{tabular}

WTR YR 1948 TOTAL 158729 MFAN 434 MAX 2200 MIN 118

\begin{tabular}{|c|c|c|c|c|c|c|c|c|c|c|c|}
\hline 1949 & OCT & NOV & DEC & JAN & FER & MAR & APR & MAY & JUN & JUL & AUG \\
\hline $\begin{array}{l}\text { TOTAL } \\
\text { MEAN } \\
\text { MAX } \\
\text { MIN }\end{array}$ & $\begin{array}{r}7257 \\
234 \\
558 \\
150\end{array}$ & $\begin{array}{r}9149 \\
305 \\
1040 \\
140\end{array}$ & $\begin{array}{r}10318 \\
333 \\
1450 \\
170\end{array}$ & $\begin{array}{r}4369 \\
141 \\
191 \\
114\end{array}$ & $\begin{array}{r}10677 \\
381 \\
1910 \\
96\end{array}$ & $\begin{array}{r}9862 \\
318 \\
473 \\
213\end{array}$ & $\begin{array}{r}11550 \\
385 \\
571 \\
209\end{array}$ & $\begin{array}{r}23931 \\
772 \\
1210 \\
338\end{array}$ & $\begin{array}{r}20901 \\
697 \\
1050 \\
402\end{array}$ & $\begin{array}{r}1477 ? \\
477 \\
659 \\
348\end{array}$ & $\begin{array}{r}9125 \\
294 \\
448 \\
237\end{array}$ \\
\hline
\end{tabular}

WTR YR 1949 TOTAL 138651 MEAN 380

MAX 1910 MIN 96

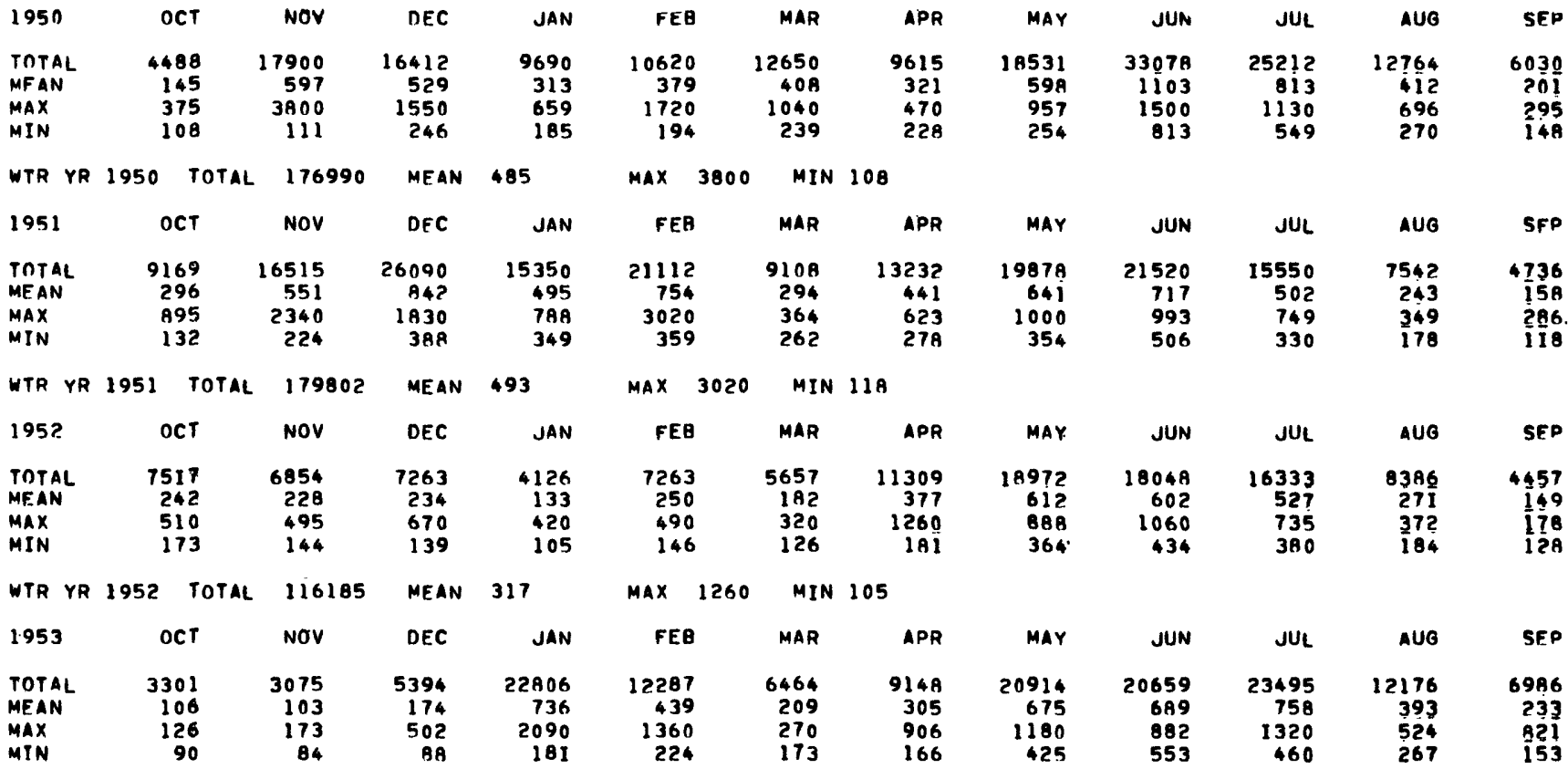

WTR YR 1953 TOTAL 146705 MFAN 402 MAX 2090 MIN A4

\begin{tabular}{|c|c|c|c|c|c|c|c|c|c|c|c|c|}
\hline 1954 & OCT & NOV & DEC & JAN & FEB & MAR & $A P R$ & MAY & JUN & JUL & AUO & SEP \\
\hline $\begin{array}{l}\text { TOTAL } \\
\text { MEAN } \\
\text { MAX } \\
\text { MIN }\end{array}$ & $\begin{array}{r}8989 \\
290 \\
648 \\
186\end{array}$ & $\begin{array}{r}17425 \\
581 \\
1450 \\
304\end{array}$ & $\begin{array}{r}17697 \\
571 \\
1440 \\
340\end{array}$ & $\begin{array}{r}15295 \\
493 \\
1930 \\
250\end{array}$ & $\begin{array}{r}22833 \\
815 \\
1790 \\
312\end{array}$ & $\begin{array}{r}11907 \\
384 \\
\text { B18 } \\
240\end{array}$ & $\begin{array}{r}9337 \\
311 \\
440 \\
230\end{array}$ & $\begin{array}{r}19788 \\
638 \\
1050 \\
270\end{array}$ & $\begin{array}{r}22415 \\
747 \\
1080 \\
535\end{array}$ & $\begin{array}{r}26041 \\
840 \\
1420 \\
589\end{array}$ & $\begin{array}{r}16165 \\
521 \\
750 \\
383\end{array}$ & $\begin{array}{r}10932 \\
364 \\
524 \\
240\end{array}$ \\
\hline
\end{tabular}

WTR YR 1954 TOTAL 198824 MEAN 545 MAX 1930 MIN 186

\begin{tabular}{|c|c|c|c|c|c|c|c|c|c|c|c|c|}
\hline 1955 & OCT & NOV & DEC & JAN & FEQ & MAR & APR & MAY & JUN & JUL' & AUG & SFD \\
\hline $\begin{array}{l}\text { TOTAL } \\
\text { MEAN } \\
\text { MAX } \\
\text { MIN }\end{array}$ & $\begin{array}{r}13294 \\
429 \\
906 \\
203\end{array}$ & $\begin{array}{r}25992 \\
866 \\
2440 \\
270\end{array}$ & $\begin{array}{r}14521 \\
468 \\
679 \\
383\end{array}$ & $\begin{array}{r}8021 \\
259 \\
397 \\
203\end{array}$ & $\begin{array}{r}7593 \\
271 \\
1010 \\
176\end{array}$ & $\begin{array}{r}5136 \\
166 \\
224 \\
146\end{array}$ & $\begin{array}{r}7945 \\
265 \\
553 \\
178\end{array}$ & $\begin{array}{r}14398 \\
464 \\
1130 \\
181\end{array}$ & $\begin{array}{r}27389 \\
913 \\
1560 \\
491\end{array}$ & $\begin{array}{r}20212 \\
652 \\
866 \\
486\end{array}$ & $\begin{array}{r}10873 \\
.351 \\
559 \\
256\end{array}$ & $\begin{array}{r}645 ? \\
215 \\
274 \\
153\end{array}$ \\
\hline
\end{tabular}

WTR YR 1955 TOTAL 161826 MEAN 443

MAX 2440 MTN 146

$\begin{array}{lrrrrrrrrrrrr}1956 & \text { OCT } & \text { NOV } & \text { DEC } & \text { JAN } & \text { FEB } & \text { MAR } & \text { APR } & \text { MAY } & \text { JUN } & \text { JUL } & \text { AUG } & \text { SEP } \\ & & & & & & & & & & \\ \text { TOTAL } & 8019 & 23176 & 14256 & 10871 & 5245 & 6614 & 15259 & 27695 & 34526 & 25955 & 12444 & 7043 \\ \text { MFAN } & 259 & 773 & 460 & 351 & 181 & 213 & 509 & 893 & 1151 & 837 & 401 & 235 \\ \text { MAX } & 934 & 5060 & 911 & 599 & 248 & 524 & 1100 & 1530 & 1760 & 1140 & 550 & 368 \\ \text { MIN } & 148 & 300 & 259 & 215 & 158 & 139 & 183 & 452 & 806 & 479 & 278 & 180\end{array}$

WTR YR 1956 TOTAL 191103 MEAN 522 MAX 5060 MIN 139 
TABLE 7.--Records of gaging stations in Clallam County--continued

12048000 DUNGENESS RIVER NEAR SEQUIM, HA, (Site 173) -- continued

\begin{tabular}{|c|c|c|c|c|c|c|c|c|c|c|c|c|c|c|}
\hline 1957 & \multicolumn{2}{|c|}{$\mathrm{OCT}$} & NOV & DEC & JAN & \multicolumn{2}{|c|}{ FEB } & MAR & APR & MAY & JUN & JUL & AUG & SFP \\
\hline $\begin{array}{l}\text { TOTAL } \\
\text { MEAN } \\
\text { MAX } \\
\text { MIN }\end{array}$ & \multicolumn{2}{|c|}{$\begin{array}{r}14003 \\
452 \\
1400 \\
161\end{array}$} & $\begin{array}{r}10325 \\
344 \\
600 \\
198\end{array}$ & $\begin{array}{r}16248 \\
524 \\
2310 \\
195\end{array}$ & $\begin{array}{r}6229 \\
201 \\
290 \\
145\end{array}$ & \multicolumn{2}{|c|}{$\begin{array}{r}12615 \\
451 \\
2560 \\
130\end{array}$} & $\begin{array}{r}12007 \\
387 \\
678 \\
239\end{array}$ & $\begin{array}{r}9374 \\
312 \\
866 \\
236\end{array}$ & $\begin{array}{r}25686 \\
829 \\
1610 \\
642\end{array}$ & $\begin{array}{r}19253 \\
642 \\
1020 \\
420\end{array}$ & $\begin{array}{r}11567 \\
373 \\
500 \\
277\end{array}$ & $\begin{array}{r}7293 \\
235 \\
285 \\
174\end{array}$ & $\begin{array}{r}5394 \\
180 \\
277 \\
146\end{array}$ \\
\hline WTR YR & 1957 & TOTAL & 149994 & MEAN & 411 & $\max$ & 2560 & MIN & 130 & & & & & \\
\hline 1958 & \multicolumn{2}{|c|}{$\mathrm{OCT}$} & NOV & DEC & JAN & \multicolumn{2}{|c|}{ FEB } & MAR & APR & MAY & JUN & JUL & AUG & SEP \\
\hline $\begin{array}{l}\text { TOTAL } \\
\text { MEAN } \\
\text { MAX } \\
\text { MIN }\end{array}$ & \multicolumn{2}{|c|}{$\begin{array}{r}5391 \\
174 \\
342 \\
118\end{array}$} & $\begin{array}{r}5219 \\
174 \\
280 \\
141\end{array}$ & $\begin{array}{r}7615 \\
246 \\
856 \\
136\end{array}$ & $\begin{array}{r}13513 \\
436 \\
1210 \\
215\end{array}$ & \multicolumn{2}{|c|}{$\begin{array}{r}16319 \\
583 \\
2030 \\
239\end{array}$} & $\begin{array}{r}9001 \\
290 \\
565 \\
208\end{array}$ & $\begin{array}{r}7610 \\
254 \\
374 \\
190\end{array}$ & $\begin{array}{r}26953 \\
869 \\
1540 \\
334\end{array}$ & $\begin{array}{r}26828 \\
894 \\
1240 \\
488\end{array}$ & $\begin{array}{r}13401 \\
432 \\
577 \\
308\end{array}$ & $\begin{array}{r}6902 \\
223 \\
300 \\
174\end{array}$ & $\begin{array}{r}4794 \\
160 \\
196 \\
128\end{array}$ \\
\hline WTR YR & 1958 & TOTAL & 143546 & MEAN & 393 & MAX & 2030 & MIN & 118 & & & & & \\
\hline 1959 & \multicolumn{2}{|c|}{ OCT } & NOV & DEC & JAN & \multicolumn{2}{|c|}{ FEB } & MAR & APR & MAY & JUN & JUL & AUG & SFP \\
\hline $\begin{array}{l}\text { TOTAL } \\
\text { MEAN } \\
\text { MAX } \\
\text { MIN }\end{array}$ & \multicolumn{2}{|c|}{$\begin{array}{r}6498 \\
210 \\
551 \\
110\end{array}$} & $\begin{array}{r}11781 \\
393 \\
1070 \\
183\end{array}$ & $\begin{array}{r}17390 \\
561 \\
1610 \\
330\end{array}$ & $\begin{array}{r}17560 \\
567 \\
1450 \\
350\end{array}$ & \multicolumn{2}{|c|}{$\begin{array}{r}6577 \\
235 \\
360 \\
183\end{array}$} & $\begin{array}{r}6349 \\
205 \\
340 \\
160\end{array}$ & $\begin{array}{r}12110 \\
404 \\
2100 \\
238\end{array}$ & $\begin{array}{r}20497 \\
661 \\
1070 \\
440\end{array}$ & $\begin{array}{r}21821 \\
727 \\
1030 \\
554\end{array}$ & $\begin{array}{r}158 \$ 9 \\
511 \\
642 \\
319\end{array}$ & $\begin{array}{r}7617 \\
246 \\
370 \\
175\end{array}$ & $\begin{array}{r}5936 \\
198 \\
365 \\
151\end{array}$ \\
\hline WTR YR & $\$ 959$ & TOTAL & -149994 & MEAN & 411 & $\operatorname{MAX}$ & 2100 & MIN & $11 n$ & & & & & \\
\hline 1960 & \multicolumn{2}{|c|}{ OCT } & NOV & DEC & JAN & \multicolumn{2}{|c|}{ FEB } & MAR & $A P R$ & MaY & JUN & JUL & AUG & SED \\
\hline $\begin{array}{l}\text { TOTAL } \\
\text { MEAN } \\
\text { MAX } \\
\text { MIN }\end{array}$ & \multicolumn{2}{|c|}{$\begin{array}{r}5834 \\
188 \\
331 \\
143\end{array}$} & $\begin{array}{r}11615 \\
387 \\
1820 \\
113\end{array}$ & $\begin{array}{r}15178 \\
490 \\
2200 \\
208\end{array}$ & $\begin{array}{r}12013 \\
388 \\
3300 \\
15 B\end{array}$ & \multicolumn{2}{|c|}{$\begin{array}{r}15448 \\
533 \\
1320 \\
235\end{array}$} & $\begin{array}{r}8757 \\
282 \\
510 \\
181\end{array}$ & $\begin{array}{r}10910 \\
364 \\
577 \\
278\end{array}$ & $\begin{array}{r}17531 \\
566 \\
956 \\
415\end{array}$ & $\begin{array}{r}23575 \\
786 \\
1270 \\
490\end{array}$ & $\begin{array}{r}14134 \\
456 \\
690 \\
310\end{array}$ & $\begin{array}{r}7132 \\
230 \\
305 \\
170\end{array}$ & $\begin{array}{r}4099 \\
137 \\
173 \\
106\end{array}$ \\
\hline WTR YR & 1960 & TOTAL & 146226 & MEAN & 400 & $\operatorname{Max}$ & 3300 & MTN & 106 & & & & & \\
\hline 1961 & \multicolumn{2}{|c|}{ OCT } & NOV & DEC & JAN & \multicolumn{2}{|c|}{ FEA } & MAR & APR & MAY & JUN & JUL & AUG & SFP \\
\hline $\begin{array}{l}\text { TOTAL } \\
\text { ME AN } \\
\text { MAX } \\
\text { MIN }\end{array}$ & \multicolumn{2}{|c|}{$\begin{array}{r}4486 \\
145 \\
278 \\
102\end{array}$} & $\begin{array}{r}7458 \\
249 \\
614 \\
128\end{array}$ & $\begin{array}{r}9713 \\
313 \\
1020 \\
164\end{array}$ & $\begin{array}{r}21790 \\
703 \\
3830 \\
178\end{array}$ & \multicolumn{2}{|c|}{$\begin{array}{r}17131 \\
612 \\
1170 \\
413\end{array}$} & $\begin{array}{r}14046 \\
453 \\
1030 \\
333\end{array}$ & $\begin{array}{r}10629 \\
354 \\
564 \\
284\end{array}$ & $\begin{array}{r}19911 \\
642 \\
940 \\
413\end{array}$ & $\begin{array}{r}32447 \\
1082 \\
1730 \\
630\end{array}$ & $\begin{array}{r}20574 \\
664 \\
956 \\
403\end{array}$ & $\begin{array}{r}10075 \\
325 \\
455 \\
212\end{array}$ & $\begin{array}{r}5 ? 55 \\
175 \\
351 \\
129\end{array}$ \\
\hline WTR YR & 1961 & TOTAL & $-\quad 173515$ & MEAN & 475 & $\max$ & 3830 & MIN & 102 & & & & & \\
\hline 1962 & \multicolumn{2}{|c|}{ OCT } & NOV & DEC & JAN & \multicolumn{2}{|c|}{ FEB } & MAR & APR & MAY & JUN & JUL & AUG & SEP \\
\hline $\begin{array}{l}\text { TOTAL } \\
\text { MEAN } \\
\text { MAX } \\
\text { MIN }\end{array}$ & \multicolumn{2}{|c|}{$\begin{array}{r}4567 \\
147 \\
225 \\
119\end{array}$} & $\begin{array}{r}5681 \\
189 \\
581 \\
125\end{array}$ & $\begin{array}{r}6461 \\
208 \\
360 \\
129\end{array}$ & $\begin{array}{r}12638 \\
408 \\
1190 \\
182\end{array}$ & \multicolumn{2}{|c|}{$\begin{array}{r}6117 \\
218 \\
346 \\
133\end{array}$} & $\begin{array}{r}4128 \\
133 \\
153 \\
121\end{array}$ & $\begin{array}{r}7816 \\
261 \\
370 \\
144\end{array}$ & $\begin{array}{r}10964 \\
354 \\
702 \\
203\end{array}$ & $\begin{array}{r}18800 \\
627 \\
747 \\
450\end{array}$ & $\begin{array}{r}12997 \\
419 \\
534 \\
315\end{array}$ & $\begin{array}{r}7530 \\
243 \\
355 \\
162\end{array}$ & $\begin{array}{r}4528 \\
151 \\
268 \\
121\end{array}$ \\
\hline WTR YR & 1962 & TOTAL & 102227 & MEAN & 280 & $\max$ & 1190 & MIN & 119 & & & & & \\
\hline 1963 & \multicolumn{2}{|c|}{$\mathrm{OCT}$} & NOV & DEC & JAN & \multicolumn{2}{|c|}{ FEB } & MAR & APR & MAY & JUN & JUL & AUG & SEP \\
\hline $\begin{array}{l}\text { TOTAL } \\
\text { MEAN } \\
\text { MAX } \\
\text { MIN }\end{array}$ & \multicolumn{2}{|c|}{$\begin{array}{r}11658 \\
376 \\
1420 \\
167\end{array}$} & $\begin{array}{r}16821 \\
561 \\
1840 \\
212\end{array}$ & $\begin{array}{r}20580 \\
664 \\
2140 \\
383\end{array}$ & $\begin{array}{r}11033 \\
356 \\
888 \\
184\end{array}$ & $\begin{array}{r}2053 \\
73 \\
218 \\
19\end{array}$ & & $\begin{array}{r}7719 \\
249 \\
378 \\
196\end{array}$ & $\begin{array}{r}7287 \\
243 \\
365 \\
181\end{array}$ & $\begin{array}{r}14630 \\
472 \\
929 \\
225\end{array}$ & $\begin{array}{r}17798 \\
593 \\
776 \\
410\end{array}$ & $\begin{array}{r}13822 \\
446 \\
589 \\
322\end{array}$ & $\begin{array}{r}8513 \\
275 \\
344 \\
214\end{array}$ & $\begin{array}{r}5716 \\
191 \\
267 \\
153\end{array}$ \\
\hline WTR YR & 1963 & TOTAL & . 156111 & MEAN & 428 & $\max$ & 2180 & MIN & 153 & & & & & \\
\hline 1964 & & :T & NOV & DEC & JAN & FE & & MAR & APR & MAY & JUN & JUL & AUG & SEP \\
\hline $\begin{array}{l}\text { TOTAL } \\
\text { MEAN } \\
\text { MAX } \\
\text { MIN }\end{array}$ & $\begin{array}{r}110 \\
35 \\
16 \\
1\end{array}$ & & $\begin{array}{r}16964 \\
565 \\
1440 \\
318\end{array}$ & $\begin{array}{r}15230 \\
491 \\
1340 \\
294\end{array}$ & $\begin{array}{r}16340 \\
527 \\
1620 \\
326\end{array}$ & $\begin{array}{r}804 \\
27 \\
43 \\
20\end{array}$ & & $\begin{array}{r}6546 \\
211 \\
322 \\
178\end{array}$ & $\begin{array}{r}7734 \\
258 \\
34 B \\
225\end{array}$ & $\begin{array}{r}12766 \\
412 \\
920 \\
218\end{array}$ & $\begin{array}{r}27215 \\
907 \\
1250 \\
534\end{array}$ & $\begin{array}{r}18725 \\
604 \\
882 \\
375\end{array}$ & $\begin{array}{r}8776 \\
283 \\
380 \\
193\end{array}$ & $\begin{array}{l}5131 \\
171 \\
456 \\
135\end{array}$ \\
\hline WTR YR & 1964 & TOTAL & 154539 & MEAN & 422 & $M: X$ & 1620 & MIN & 135 & & & & & \\
\hline 1965 & & $\mathrm{CT}$ & NOV & DEC & JAN & FE & & MAR & APR & MAY & JUN & JUL & AUG & SEP \\
\hline $\begin{array}{l}\text { TOTAL } \\
\text { MEAN } \\
\text { MAX } \\
\text { MIN }\end{array}$ & $\begin{array}{r}47 \\
1 \\
2 \\
1\end{array}$ & $\begin{array}{l}13 \\
53 \\
89 \\
15\end{array}$ & $\begin{array}{r}6201 \\
207 \\
1300 \\
117\end{array}$ & $\begin{array}{r}1326 \\
236 \\
922 \\
135\end{array}$ & $\begin{array}{r}11573 \\
373 \\
1210 \\
129\end{array}$ & $\begin{array}{r}1246 \\
44 \\
114 \\
27\end{array}$ & & $\begin{array}{r}6834 \\
220 \\
264 \\
166\end{array}$ & $\begin{array}{r}9961 \\
332 \\
834 \\
166\end{array}$ & $\begin{array}{r}14249 \\
460 \\
765 \\
278\end{array}$ & $\begin{array}{r}18330 \\
611 \\
946 \\
403\end{array}$ & $\begin{array}{r}12030 \\
388 \\
552 \\
275\end{array}$ & $\begin{array}{r}7056 \\
228 \\
286 \\
159\end{array}$ & $\begin{array}{r}3859 \\
129 \\
156 \\
104\end{array}$ \\
\hline WTR YR & 1965 & TOTAL & 114624 & MEAN & 314 & $\max$ & 1300 & MIN & 104 & & & & & \\
\hline 1966 & & $\mathrm{ET}$ & NOV & DEC & JAN & FE & & MAR & APR & MaY & JUN & JUL & AUG & SEP \\
\hline $\begin{array}{l}\text { TOTAL } \\
\text { MEAN } \\
\text { MAX } \\
\text { MIN }\end{array}$ & $\begin{array}{c}49 \\
1 \\
1\end{array}$ & & $\begin{array}{r}8909 \\
297 \\
564 \\
135\end{array}$ & $\begin{array}{r}8760 \\
283 \\
632 \\
166\end{array}$ & $\begin{array}{r}8982 \\
290 \\
768 \\
159\end{array}$ & $\begin{array}{r}482 \\
17 \\
26 \\
14\end{array}$ & & $\begin{array}{r}7400 \\
239 \\
652 \\
135\end{array}$ & $\begin{array}{r}12135 \\
405 \\
612 \\
307\end{array}$ & $\begin{array}{r}20660 \\
666 \\
1130 \\
312\end{array}$ & $\begin{array}{r}20243 \\
675 \\
1000 \\
436\end{array}$ & $\begin{array}{r}18606 \\
600 \\
758 \\
458\end{array}$ & $\begin{array}{r}9914 \\
320 \\
468 \\
200\end{array}$ & $\begin{array}{r}5670 \\
189 \\
242 \\
161\end{array}$ \\
\hline
\end{tabular}

WTR YR 1966 TOTAL 131063 MEAN 359 MAX 1130 MIN 103 
TABLE 7.--Records of gaging stations in Clall an County--continued

12048000 DUNGEMESS RIVER NEAR SEqUIM, WA, (Site 173) -- continued

\begin{tabular}{|c|c|c|c|c|c|c|c|c|c|c|c|c|}
\hline 1967 & OCT & Nov & DEC & JAN & FEB & MAR & $A P R$ & Mar & JUN & JUL & AUG & SEP \\
\hline $\begin{array}{l}\text { TOTAL } \\
\text { MEAN } \\
\text { MAX } \\
\text { MIN }\end{array}$ & $\begin{array}{r}6516 \\
210 \\
525 \\
119\end{array}$ & $\begin{array}{r}8849 \\
295 \\
772 \\
142\end{array}$ & $\begin{array}{r}27251 \\
879 \\
2630 \\
332\end{array}$ & $\begin{array}{r}15030 \\
485 \\
1270 \\
292\end{array}$ & $\begin{array}{r}8409 \\
300 \\
490 \\
196\end{array}$ & $\begin{array}{r}10679 \\
344 \\
1400 \\
183\end{array}$ & $\begin{array}{r}6671 \\
222 \\
296 \\
186\end{array}$ & $\begin{array}{r}20396 \\
65 A \\
1200 \\
253\end{array}$ & $\begin{array}{r}35435 \\
1181 \\
1540 \\
807\end{array}$ & $\begin{array}{r}22621 \\
730 \\
1120 \\
505\end{array}$ & $\begin{array}{r}11382 \\
367 \\
500 \\
249\end{array}$ & $\begin{array}{r}650 ? \\
217 \\
305 \\
158\end{array}$ \\
\hline WTR YR & 1967 TOTAL & -179741 & MEAN & 492 & MAX 2630 & MIN & 119 & & & & & \\
\hline 1968 & OCT & NOV & DEC & JAN & FEB & MAR & APR & MAY & JUN & JUL & AUG & SEP \\
\hline $\begin{array}{l}\text { TOTAL } \\
\text { MEAN } \\
\text { MAX } \\
\text { MIN }\end{array}$ & $\begin{array}{r}17388 \\
561 \\
1360 \\
280\end{array}$ & $\begin{array}{r}11680 \\
389 \\
674 \\
223\end{array}$ & $\begin{array}{r}15225 \\
491 \\
1960 \\
180\end{array}$ & $\begin{array}{r}33337 \\
1075 \\
3160 \\
323\end{array}$ & $\begin{array}{r}19576 \\
675 \\
1610 \\
352\end{array}$ & $\begin{array}{r}13554 \\
437 \\
1070 \\
288\end{array}$ & $\begin{array}{r}6806 \\
227 \\
316 \\
176\end{array}$ & $\begin{array}{r}13542 \\
437 \\
1240 \\
252\end{array}$ & $\begin{array}{r}19158 \\
639 \\
1210 \\
431\end{array}$ & $\begin{array}{r}14376 \\
464 \\
721 \\
330\end{array}$ & $\begin{array}{r}7974 \\
257 \\
334 \\
20 i\end{array}$ & $\begin{array}{r}\text { 5AA3 } \\
196 \\
307 \\
139\end{array}$ \\
\hline WTR YR & 1968 TOTAL & - 178499 & MEAN & 488 & $\max 3160$ & MIN & 139 & & & & & \\
\hline 1969 & OCT & NOV & DEC & JAN & FEB & MAR & APR & MAY & JUN & JUL & AUG & SFP \\
\hline $\begin{array}{l}\text { TOTAL } \\
\text { MEAN } \\
\text { MAX } \\
\text { MIN }\end{array}$ & $\begin{array}{r}6730 \\
217 \\
575 \\
131\end{array}$ & $\begin{array}{r}9257 \\
309 \\
598 \\
180\end{array}$ & $\begin{array}{r}13845 \\
447 \\
1060 \\
221\end{array}$ & $\begin{array}{r}10283 \\
332 \\
1100 \\
175\end{array}$ & $\begin{array}{r}6876 \\
246 \\
868 \\
145\end{array}$ & $\begin{array}{r}7896 \\
255 \\
610 \\
145\end{array}$ & $\begin{array}{r}11369 \\
379 \\
622 \\
280\end{array}$ & $\begin{array}{r}27647 \\
89 ? \\
1640 \\
343\end{array}$ & $\begin{array}{r}31002 \\
1033 \\
1500 \\
532\end{array}$ & $\begin{array}{r}13976 \\
451 \\
682 \\
311\end{array}$ & $\begin{array}{r}7321 \\
236 \\
315 \\
167\end{array}$ & $\begin{array}{r}6988 \\
233 \\
520 \\
140\end{array}$ \\
\hline WTR YR & 2969 TOTAL & $-\quad 153190$ & MEAN & 420 & $\max 1640$ & MIN & 131 & & & & & \\
\hline $197 n$ & OCT & NOV & DEC & JAN & FEB & MAR & $A P R$ & MaY & JUN & JULI & AUG & SEP \\
\hline $\begin{array}{l}\text { TOTAL } \\
\text { MEAN } \\
\text { MAX } \\
\text { MIN }\end{array}$ & $\begin{array}{r}5799 \\
187 \\
291 \\
137\end{array}$ & $\begin{array}{r}6963 \\
232 \\
515 \\
132\end{array}$ & $\begin{array}{r}10211 \\
326 \\
1060 \\
134\end{array}$ & $\begin{array}{r}10985 \\
354 \\
1100 \\
162\end{array}$ & $\begin{array}{r}8607 \\
307 \\
530 \\
227\end{array}$ & $\begin{array}{r}6916 \\
223 \\
356 \\
170\end{array}$ & $\begin{array}{r}7165 \\
239 \\
622 \\
165\end{array}$ & $\begin{array}{r}13801 \\
445 \\
873 \\
191\end{array}$ & $\begin{array}{r}23986 \\
800 \\
1410 \\
470\end{array}$ & $\begin{array}{r}13152 \\
424 \\
719 \\
239\end{array}$ & $\begin{array}{r}6196 \\
200 \\
259 \\
154\end{array}$ & $\begin{array}{r}4432 \\
147 \\
197 \\
119\end{array}$ \\
\hline WTR YR & 1970 TOTAL & 118103 & MEAN & 324 & $\max 1410$ & MIN & 129 & & & & & \\
\hline 1971 & OCT & NOV & DEC & JAN & FEB & MAR & APR & MAY & JUN & JUL & AUG & SEP \\
\hline $\begin{array}{l}\text { TOTAL } \\
\text { MEAN } \\
\text { MAX } \\
\text { MIN }\end{array}$ & $\begin{array}{r}3685 \\
119 \\
176 \\
93\end{array}$ & $\begin{array}{r}5793 \\
193 \\
717 \\
104\end{array}$ & $\begin{array}{r}7885 \\
254 \\
788 \\
135\end{array}$ & $\begin{array}{r}12849 \\
414 \\
1340 \\
144\end{array}$ & $\begin{array}{r}14193 \\
507 \\
1060 \\
282\end{array}$ & $\begin{array}{r}9306 \\
300 \\
638 \\
212\end{array}$ & $\begin{array}{r}8930 \\
298 \\
450 \\
220\end{array}$ & $\begin{array}{r}20659 \\
666 \\
988 \\
424\end{array}$ & $\begin{array}{r}23485 \\
783 \\
1340 \\
555\end{array}$ & $\begin{array}{r}24565 \\
792 \\
1140 \\
550\end{array}$ & $\begin{array}{r}14378 \\
464 \\
964 \\
282\end{array}$ & $\begin{array}{r}6608 \\
220 \\
380 \\
156\end{array}$ \\
\hline WTR YR & 1971 TOTAL & 152335 & MEAN & 417 & $\operatorname{MAX} 1340$ & MIN & 93 & & & & & \\
\hline $197 ?$ & OCT & Nov & DEC & JAN & FEB & MAR & APR & MAY & JUN & JUL & AUG & SEP \\
\hline $\begin{array}{l}\text { TOTAL } \\
\text { MEAN } \\
\text { MAX } \\
\text { MIN }\end{array}$ & $\begin{array}{r}4683 \\
151 \\
233 \\
126\end{array}$ & $\begin{array}{r}8576 \\
286 \\
748 \\
141\end{array}$ & $\begin{array}{r}\text { 6AOA } \\
220 \\
629 \\
148\end{array}$ & $\begin{array}{r}8829 \\
285 \\
1220 \\
139\end{array}$ & $\begin{array}{r}12439 \\
429 \\
1560 \\
170\end{array}$ & $\begin{array}{r}25396 \\
819 \\
2090 \\
386\end{array}$ & $\begin{array}{r}12353 \\
412 \\
840 \\
286\end{array}$ & $\begin{array}{r}23386 \\
754 \\
1610 \\
346\end{array}$ & $\begin{array}{r}28217 \\
941 \\
1260 \\
758\end{array}$ & $\begin{array}{r}22177 \\
715 \\
1000 \\
520\end{array}$ & $\begin{array}{r}11706 \\
378 \\
530 \\
260\end{array}$ & $\begin{array}{r}6511 \\
219 \\
500 \\
170\end{array}$ \\
\hline WTR YR & 2972 TOTAL & 171141 & MEAN & 468 & $\max 2090$ & MIN & 126 & & & & & \\
\hline 1973 & OCT & NoV & DEC & JAN & FEA & MAR & APR & MaY & JUN & $J U L$ & AUG & SEP \\
\hline $\begin{array}{l}\text { TOTAL } \\
\text { MFAN } \\
\text { MAX } \\
\text { MIN }\end{array}$ & $\begin{array}{r}4214 \\
136 \\
183 \\
105\end{array}$ & $\begin{array}{r}5590 \\
186 \\
550 \\
120\end{array}$ & $\begin{array}{r}25550 \\
502 \\
2590 \\
68\end{array}$ & $\begin{array}{r}13297 \\
429 \\
1090 \\
220\end{array}$ & $\begin{array}{r}5582 \\
199 \\
415 \\
153\end{array}$ & $\begin{array}{r}5649 \\
182 \\
299 \\
155\end{array}$ & $\begin{array}{r}5614 \\
187 \\
241 \\
145\end{array}$ & $\begin{array}{r}14783 \\
477 \\
988 \\
202\end{array}$ & $\begin{array}{r}16667 \\
556 \\
805 \\
358\end{array}$ & $\begin{array}{r}12112 \\
391 \\
519 \\
279\end{array}$ & $\begin{array}{r}6813 \\
220 \\
331 \\
153\end{array}$ & $\begin{array}{r}47 ? 1 \\
157 \\
210 \\
130\end{array}$ \\
\hline WTR YR & 1973 TOTAL & 110592 & MEAN & 303 & $\max 2590$ & MIN & 68 & & & & & \\
\hline 1974 & OCT & NOV & DEC & JAN & FEB & MAR & $\triangle P R$ & MaY & JUN & JUL & AUG & SEP \\
\hline $\begin{array}{l}\text { TOTAL } \\
\text { MEAN } \\
\text { MAX } \\
\text { MIN }\end{array}$ & $\begin{array}{r}5375 \\
173 \\
462 \\
90\end{array}$ & $\begin{array}{r}10409 \\
347 \\
1280 \\
154\end{array}$ & $\begin{array}{r}16655 \\
537 \\
2630 \\
271\end{array}$ & $\begin{array}{r}23946 \\
772 \\
3400 \\
190\end{array}$ & $\begin{array}{r}A 298 \\
296 \\
554 \\
207\end{array}$ & $\begin{array}{r}13141 \\
424 \\
1400 \\
174\end{array}$ & $\begin{array}{r}9018 \\
301 \\
410 \\
262\end{array}$ & $\begin{array}{r}16051 \\
51 A \\
944 \\
325\end{array}$ & $\begin{array}{r}30943 \\
1031 \\
1540 \\
604\end{array}$ & $\begin{array}{r}25895 \\
835 \\
1140 \\
674\end{array}$ & $\begin{array}{r}14414 \\
465 \\
754 \\
338\end{array}$ & $\begin{array}{r}7030 \\
234 \\
360 \\
155\end{array}$ \\
\hline WTR YR & 1974 TOTAL & 181175 & MEAN & 496 & $\operatorname{mAX} 3400$ & MIN & 90 & & & & & \\
\hline 1975 & OCT & Nov & DEC & JAN & FEB & MAR & $A P R$ & MAY & JUN & JUL & AUG & SEP \\
\hline $\begin{array}{l}\text { TOTAL } \\
\text { MEAN } \\
\text { MAX } \\
\text { MIN }\end{array}$ & $\begin{array}{l}448 y \\
145 \\
206 \\
119\end{array}$ & $\begin{array}{r}5760 \\
192 \\
485 \\
117\end{array}$ & $\begin{array}{r}10901 \\
352 \\
1700 \\
146\end{array}$ & $\begin{array}{r}9930 \\
320 \\
800 \\
160\end{array}$ & $\begin{array}{r}5863 \\
209 \\
365 \\
150\end{array}$ & $\begin{array}{r}6330 \\
204 \\
389 \\
139\end{array}$ & $\begin{array}{r}5135 \\
171 \\
209 \\
132\end{array}$ & $\begin{array}{r}14502 \\
468 \\
841 \\
209\end{array}$ & $\begin{array}{r}21571 \\
719 \\
1090 \\
393\end{array}$ & $\begin{array}{r}18380 \\
593 \\
1080 \\
321\end{array}$ & $\begin{array}{r}9777 \\
315 \\
517 \\
250\end{array}$ & $\begin{array}{r}5934 \\
198 \\
314 \\
146\end{array}$ \\
\hline WTR YR & 1975 TOTAL & -118564 & MEAN & 325 & $\max 1700$ & MIN & 117 & & & & & \\
\hline 1976 & $\mathrm{OCT}$ & Nov & DEC & JAN & FE 8 & MAR & APR & MAY & JUN & JUL & AUG & SFP \\
\hline $\begin{array}{l}\text { TOTAL } \\
\text { MEAN } \\
\operatorname{MAX} \\
\operatorname{MIN}\end{array}$ & $\begin{array}{r}13692 \\
442 \\
1860 \\
139\end{array}$ & $\begin{array}{r}21801 \\
727 \\
2750 \\
339\end{array}$ & $\begin{array}{r}28433 \\
917 \\
3900 \\
310\end{array}$ & $\begin{array}{r}1659 A \\
535 \\
1220 \\
330\end{array}$ & $\begin{array}{r}9831 \\
339 \\
465 \\
265\end{array}$ & $\begin{array}{r}7252 \\
234 \\
268 \\
211\end{array}$ & $\begin{array}{r}8910 \\
297 \\
505 \\
217\end{array}$ & $\begin{array}{r}19439 \\
627 \\
981 \\
450\end{array}$ & $\begin{array}{r}17658 \\
589 \\
939 \\
354\end{array}$ & $\begin{array}{r}18382 \\
593 \\
865 \\
427\end{array}$ & $\begin{array}{r}10449 \\
337 \\
427 \\
253\end{array}$ & $\begin{array}{r}6000 \\
200 \\
259 \\
177\end{array}$ \\
\hline
\end{tabular}

WTR YR 1976 TOTAL 178445 MEAN 488 MAX 3900 MIN 139 
TABLE 7.--Records of gaging stations in Clallan County--continued

12048000 DUNGENESS RIVER NEAR SEQUIM, WA. (S1 te 173) -. continued

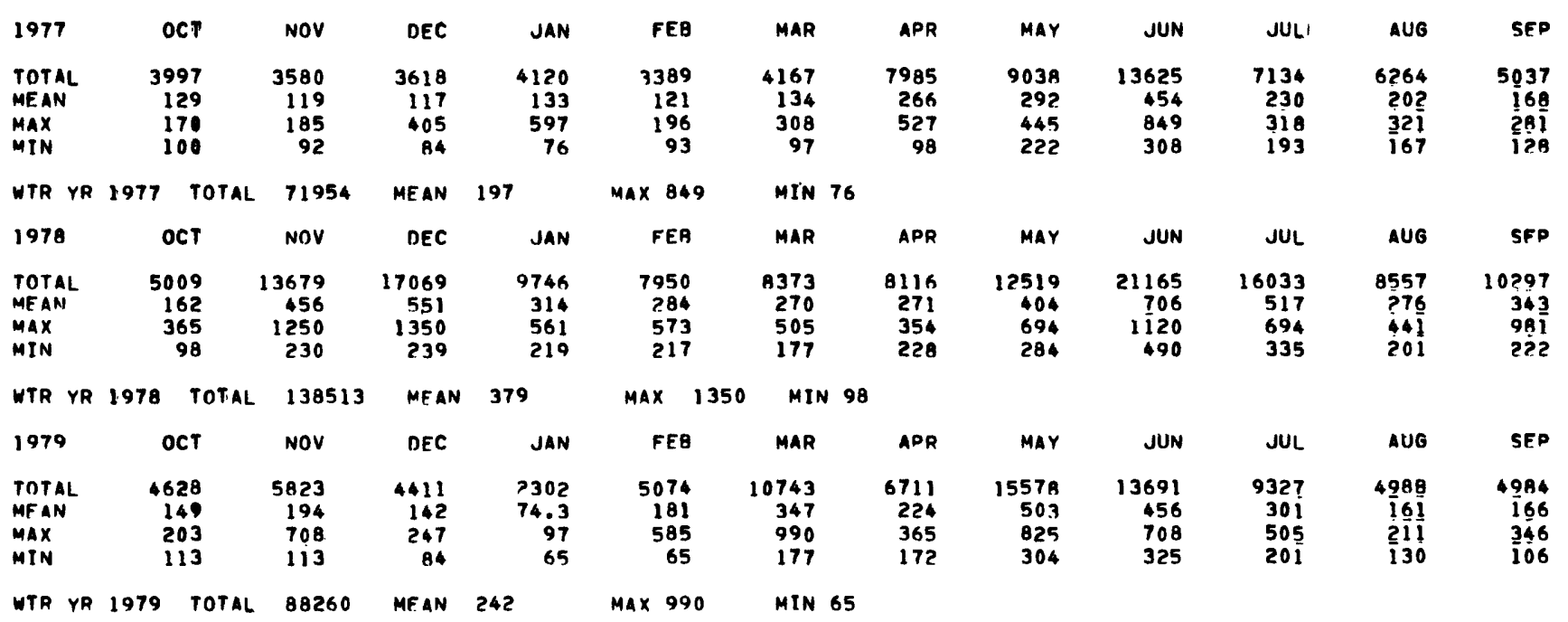


TABLE 8.-Records of crest-stage partial-record stations in Clallam County.

Crest-stage partial-record stations

The following table contains annual maximum discharges for crest-stage stations. A crest-stage gage is a device which will register the peak stage occurring between inspections of the gage. A stage-discharge relation for each gage is developed from discharge measurements made by indirect measurements of peak flow or by current meter. The date of the maximum discharge is not always certain, but is usually determined by comparison with nearby continuous-record stations, weather records, or local inquiry. Only the maximum discharge for each water year is given. Information on some lower floods may have been obtained, but is not published herein. 
Table 8.--continued

\section{Quillayute River Basin}

12041500 Soleduck River near Fairholm (site number 4) Location. --(see gaging station record)

Drainage area.--83.8 $\mathrm{mi}^{2}$.

\section{Annual peaks}

Water

year

1972

1973

1974

1975

1976
Date

Mar 5,1972
Dec 25, 1972
Jan 16, 1974
Dec 21, 1974
Dec 3, 1975

Gage height

$(\mathrm{ft})$

9.93
11.17
11.42
10.45
12.76
Discharge

$\left(\mathrm{ft}^{3} / \mathrm{s}\right)$

9,760
11,900
12,400
10,600
15,000

12041600 Soleduck River Tributary near Fairholm (site number 5)

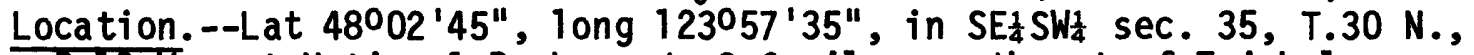
R.10W., at National Park road, 2.6 miles southwest of Fairholm. Drainage area. $--0.42 \mathrm{mi}^{2}$.

\section{Annual peaks}

Water

year

1956

1957

1958

1959

1960

1961

1962

1963

1964

1965

1966

1967

1968

1969

1970

1971

1972

1973

1974

1975
Date

Nov 3, 1955

Dec 9, 1956

Dec 25, 1957

Apr 29, 1959

Dec 14, 1959

Jan 15, 1961

Jan 3, 1962

Nov 19,1962

Oct 21, 1963

Nov 30,1964

Jan 12, 1966

Dec 13, 1966

Jan 19, 1968

Jan 4, 1969

Apr 9, 1970

Dec 6, 1970

Mar 5, 1972

Dec 26, 1972

Dec 15, 1973

Dec 21,1974
Gage height

$(\mathrm{ft})$

11.21

11.53

9.16

9.35

9.15

11.32

8.85

9.85

8.90

9.07

9.83

9.82

10.50

9.21

9.08

9.59

10.43

11.24

10.42

9.76
Discharge

$\left(\mathrm{ft}^{3} / \mathrm{s}\right)$

47.8

52.1

17.3

20

17

50

12

28

13

16

28

28

38

18

16

24

37

48

37 
Table 8.---continued

12042700 May Creek near Forks (site number 31)

Location.--Lat $47^{\circ} 52^{\prime} 55^{\prime \prime}$, long $124^{\circ} 21^{\prime} 00^{\prime \prime}$, in SWl sec. 35, T.28N., R.13W., at U.S. Highway 101, 5.0 miles south of Forks. Drainage area. $--2.03 \mathrm{mi}$.

\section{Annual peaks}

Water

year

1950

1951

1952

1953

1954

1955

1956

1957

1958

1959

1960

1961

1962

1963

1964

1965

1966

1967

1968
Date

Dec 28, 1949

Feb 10, 1951

Jan 30,1952

Jan 2, 1953

Feb 17-21, 1954

Nov 18, 1954

Nov 3, 1955

Dec 9, 1956

Dec 25, 1957

Apr 29, 1959

Dec 14, 1959

Feb 21, 1961

Jan 3, 1962

Nov 19,1962

Dec 23, 1963

Jan 4, 1965

Jan 12, 1966

Jan 19, 1967

Jan 19, 1968
Gage height

( $\mathrm{ft}$ )

Discharge

23.75

22.87

19.62

22.60

22.62

27.96

26.02

27.82

21.15

25.05

26.77

30.21

19.25

32.82

24.42

23.86

23.59

21.92

30.72 $\left(\mathrm{ft}^{3} / \mathrm{s}\right)$

475

439

278

427

428

624

554

617

303

522

582

694

238

759

499

478

465

395

711 
Table 8.--continued

12042900 Grader Creek near Forks (site number 34)

Location. --Lat $47^{\circ} 55^{\prime} 40^{\prime \prime}$, long $124^{\circ} 24^{\prime} 25^{\prime \prime}$, in SW sec. 17, T.28N., R.13W., at U.S. Highway 101, 2.0 miles southwest of Forks.

Drainage area. $--1.67 \mathrm{mi}^{2}$. Area of 1 akes and ponds, $0 . \mathrm{mi}^{2}$.

Annual peaks

Water

year

1950

1951

1952

1953

1954

1955

1956

1957

1958

1959

1960

1961

1962

1963

1964

1965

1966

1967

1968

1969

1970

1971

1972

1973

1974

1975

1976

1977

1978
Date

Nov 26, 1949

Feb 10, 1951

Nov 30, 1951

Jan 31,1953

Feb 19, 1954

Nov 18, 1954

Nov 3, 1955

Dec 9, 1956

Dec 25, 1957

Apr 29, 1959

Dec 14, 1959

Feb 21, 1961

Jan 3, 1962

Nov 19,1962

Nov 26, 1963

Jan 4, 1965

Jan 12, 1966

Jan 19, 1967

Jan 19, 1968

Jan 4, 1969

Apr 9, 1970

Jan 26, 1971

Jan 20, 1972

Jan 26, 1972

Dec 15, 1973

Dec 21,1974

Dec 26, 1975

Mar 7, 1977

Nov 1, 1977
Gage height

( ft)

Discharge

$\left(\mathrm{ft}^{3} / \mathrm{s}\right)$

20.94

235

265

210

335

288

21.04

20.58

407

21.98

503

520

199

334

20.50

415

21.70

455

22.05

18.92

163

485

22.32

312

20.89

328

20.30

20.14

264

250

22.68

20.40

521

244

20.69

20.42

274

246

20.98

303

23.68

20.78

597

283

21.04

336

22.46

502

19.28

147

21.11

355 
Table 8.--continued

12042920 Sitkum River tributary near Forks.

Location. --Lat $47^{0} 57^{\prime} 19^{\prime \prime}$, long 124012'11", on line between SW 1 sec. 1 and SE $\mathrm{SeC}^{\frac{1}{4}}$ 2, T.28 N., R.12 W., at Forest Service road 8 miles east of Forks. Drainage area. $-0.42 \mathrm{mi} 2$

\section{Annual peaks}

Water

year

Date

1971

1972

1973

1974

1975
Dec 6, 1970

$\operatorname{Mar} 5,1972$

Dec 26, 1972

Dec 15, 1973

Dec 21,1974
Gage height

$(\mathrm{ft})$

9.18

9.86

10.76

9.39

9.24
Discharge

$\left(\mathrm{ft}^{3} / \mathrm{s}\right)$

52

69

97

57

53

12043100 Dickey River near La Push (map number 71 ). Location. --(see gaging station record). Drainage area. $--86.3 \mathrm{mi}$.

\section{Annual peaks}

Water

year

1974

1975

1976
Date

Feb 2, 1974

Dec 21, 1974

Dec 2, 1975
Gage height

$(\mathrm{ft})$

13.29

10.31

13.97
Discharge

$\left(\mathrm{ft}^{3} / \mathrm{s}\right)$

7,810

5,170

8,490 
Table 8.--continued

\section{Hoko River Basin}

12043270 Hoko River tributary near Sekiu.

Location.--Lat 47012'14", long 124025'08", in SW 1 NEE $\frac{1}{4}$ sec.7, T.31 N.,

R.T3W., at county road 250 feet above mouth and 7 miles southwest of Sekiu. Drainage area. $--0.67 \mathrm{mi}^{2}$.

Annual peaks

Water

year

1971

1972

1973

1974

1975
Date

$$
\text { Jan 24, } 1971
$$

Dec 8, 1971

Dec 25, 1972

Feb 3, 1974

Dec 21, 1974
Gage height

$(\mathrm{ft})$

5.62

6.64

7.66

5.97

6.84
Discharge

( $\mathrm{ft}^{3 / \mathrm{s}}$ )

60

100

149

73

109

12043300 Hoko River near Seiku (site number 90)

Location. -- (see gaging station record)

Drainage area. $--51.2 \mathrm{mi} 2$.

\section{Annual peaks}

Water

year

1975

1976

1977

1978
Date

Dec 21, 1974

Dec 1,1975

Mar 8, 1977

Nov 25, 1977
Gage height

(ft)

$$
\begin{array}{r}
9.83 \\
11.62 \\
7.20 \\
9.11
\end{array}
$$

Discharge

$\left(\mathrm{ft}^{3} / \mathrm{s}\right)$

$$
\begin{aligned}
& 6,960 \\
& 9,690 \\
& 3,620 \\
& 5,950
\end{aligned}
$$


Table 8.--continued

\section{East Twin River Basin}

12043430 East Twin River near Pysht (site number 103)

Location.--( see gaging station record)

Drainage area. $--14.0 \mathrm{mi}^{2}$.

Annual peaks

Water

year

1973

1974

1975

1976

1977

1978
Date

Dec 26, 1972

Jan 16, 1974

Feb 12, 1975

Dec 2, 1975

Mar 8, 1977

Dec 2, 1977
Gage height

( ft)

Discharge

$\left(\mathrm{ft}^{3} / \mathrm{s}\right)$

5.40

5.32

4.59

5.48

4.71

5.39
1,140

1,110

877

1,170

913

\section{Lyre River Basin}

12043450 Cross Creek near Fairholm (site number 106)

Location. --Lat $48^{\circ} 03^{\prime} 20^{\prime \prime}$, long $123^{\circ} 52^{\prime} 35^{\prime \prime}$, in NE $\frac{1}{4}$ sec. 31 , T.30 N., R.9W., at U.S. Highway 101, 2.1 miles east of Fairholm. Drainage area. $--0.92 \mathrm{mi}^{2}$.

\section{Annual peaks}

Water

year

1950

1951

1952

1953

1954

1955

1956
Date

Nov 26, 1949

Feb 9, 1951

Nov 27, 1951

Jan 9, 1953

Jan 5, 1954

Nov 18, 1954

Nov 3, 1955
Gage height

$(\mathrm{ft})$

16.74

15.60

14.37

15.00

15.50

14.75

18.37
Discharge

( $\mathrm{ft}^{3} / \mathrm{s}$ )

112

59.7

15.8

36.0

55.0

27.1

208 
Table 8.--continued

12043470 Lake Crescent tributary near Piedmont (site number 110). Location. --Lat $48^{\circ} 03^{\prime} 00^{\prime \prime}$, long $123^{\circ} 48^{\prime} 05^{\prime \prime}$, in NW $\frac{1}{4} \mathrm{sec}$. 35, T.30 N., R.9W., at U.S. Highway 101, 3 miles south of Piedmont.

Drainage area. $--0.79 \mathrm{mi} 2$.

Annual peaks

Water

year

1956

1957

1958
Date

Nov 3, 1955

Dec 9, 1956

Dec 25, 1957
Gage height

$(\mathrm{ft})$

10.97

12.52

9.69
Discharge

$(\mathrm{ft} 3 / \mathrm{s})$

94.5

175

45

Valley Creek Basin

12046800 East Valley Creek at Port Angeles (site number 149). Location. --Lat $48^{\circ} 06^{\prime} 10^{\prime \prime}$, long $123^{\circ} 26^{\prime} 20^{\prime \prime}$, in SW $\frac{1}{4}$ sec. 15, T.30 N., R.6W., at county road, 1 mile south of Port Angeles city limits. (Prior to Oct 1961 gage was 0.2 mile downstream). Drainage area. $--0.69 \mathrm{mi}^{2}$.

\section{Annual peaks}

Water

year

1950

1951

1952

1953

1954

1955

1956

1957

1958

1959

1960

1961

1962

1963

\section{Date}

Feb 24, 1950

Feb 10, 1951

Dec 18, 1951

Jan 8,1953

Jan 5, 1954

Feb 7, 1955

Nov 3, 1955

Dec 9, 1956

Dec 25, 1957

Jan 24, 1959

Jan 29, 1960

Jan 15, 1961

Nov 25, 1962
Gage height

( ft)

28.52

26.21

25.48

26.28

30.04

27.70

26.96

28.26

25.95

26.41

30.26

$\star 33.12$
Discharge

$\left(\mathrm{ft}^{3} / \mathrm{s}\right)$

31.7

15.0

7.0

15.7

38.0

27.5

23.1

30.4

11.6

17

38.0

52

$<6$

20

* Gage-height is the result of culvert being plugged. Discharge may have been increased by failure of a dam located 0.8 mile upstream. 
Table 8.--continued

\section{Lees Creek Basin}

12047100 Lees Creek at Port Angeles (site number 156).

Location. --Lat $48^{\circ} 06^{\prime} 20^{\prime \prime}$, long $123^{\circ} 22^{\prime} 55^{\prime \prime}$, in SW $\frac{1}{4} \mathrm{Sec} .12, \mathrm{~T} .30 \mathrm{~N}$. , R.6W., at U.S. Highway 101, 1 mile east of Port Angeles city limits Drainage area. $--4.77 \mathrm{mi}^{2}$.

\section{Annual peaks}

$\begin{array}{ll}\begin{array}{l}\text { Water } \\ \text { year }\end{array} & \text { Date } \\ 1949 & \text { Feb 16, 1949 } \\ 1950 & \text { Feb 25, 1950 } \\ 1951 & \text { Feb 9, 1951 } \\ 1952 & \text { Dec 5, 1951 } \\ 1953 & \text { Jan 31, 1953 } \\ 1954 & \text { Jan 5, 1954 } \\ 1955 & \text { Feb 7, 1955 } \\ 1956 & \text { Nov 3, 1955 } \\ 1957 & \text { Dec 9, 1956 } \\ 1958 & \text { Jan 16, 1958 } \\ 1959 & \text { Jan 24, 1959 } \\ 1960 & \text { Jan 29, 1960 } \\ 1961 & \text { Jan 15, 1961 } \\ 1962 & \text { Jan 3, 1962 } \\ 1963 & \text { Nov 25, 1962 } \\ 1964 & \text { Jan 16, 1964 } \\ 1965 & \text { Jan 30, 1965 } \\ 1966 & \text { Jan 13, 1966 } \\ 1967 & \text { Jan 19, 1967 } \\ 1968 & \text { Feb 3, 1968 } \\ 1969 & \text { Jan 4, 1969 } \\ 1970 & \text { Dec 13, 1969 }\end{array}$

Gage height

$(\mathrm{ft})$

16.62

11.70

11.67

10.82

11.42

14.24

13.10

12.03

12.78

10.37

10.65

18.56

19.52

9.85

11.11

10.29

10.78

18.02

16.40

15.53

15.16
Discharge

( $\mathrm{ft}^{3} / \mathrm{s}$ )

316

98.8

99.0

70.0

90.8

205

157

114

141

57.5

66

321

338

43

81

56

70

$<20$

137

77

50

38 
Table 8.--continued

Morse Creek Basin

12047300 Morse Creek near Port Angeles (site number 158)

Location. --(see gaging station record).

Drainage area. $--46.6 \mathrm{mi}^{2}$.

Annual peaks

Water

year

1977

1978
Date

Mar 8, 1977

Nov 1,1977
Gage height

$(\mathrm{ft})$

3.96

4.99
Discharge

$\left(\mathrm{ft}^{3} / \mathrm{s}\right)$

441

914

\section{Dungeness River Basin}

12047700 Gold Creek near Blyn.

Location. --Lat $47^{\circ} 55^{\prime} 15^{\prime \prime}$, long $123^{\circ} 02^{\prime} 30^{\prime \prime}$, near center of east 1/2 sec. 15, T.28 N., R.3 W., at logging road, $71 / 2$ miles southwest of Blyn. Drainage area. $--2.28 \mathrm{mi}^{2}$.

\section{Annual peaks}

Water

year

1965

1966

1967

1968

1969

1970

1971

1972

1973

1974

1975
Date

Jan 30, 1965

Nov 19, 1965

Dec 13, 1966

Jan 14, 1968

Dec 3, 1968

Dec 13, 1969

Jan 31, 1971

Mar 5, 1972

Dec 19, 1972

Jan 16, 1974

Dec 21, 1974
Gage height ( ft)

5.32

4.97

6.40

7.64

6.03

6.16

5.16

5.95

5.88

6.77

6.09
Discharge

$\left(\mathrm{ft}^{3} / \mathrm{s}\right)$

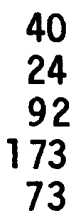

75

31

65

61

114

72 
Table 8.--continued

\section{Dean Creek Basin}

12049400 Dean Creek at Blyn (site number 211) Location. --Lat $48^{\circ} 01^{\prime} 30^{\prime \prime}$, long $123^{\circ} 00^{\prime} 35^{\prime}$ ', in NW1 sec. 12, T.29 N., R.3 W., at old highway, 50 feet east of U.S. Highway 101 and at the west edge of Blyn.

Drainage area. $--2.96 \mathrm{mi}^{2}$.

Annual peaks

$\begin{array}{ll}\begin{array}{l}\text { Water } \\ \text { year }\end{array} & \text { Date } \\ 1949 & \text { Feb 22, 1949 } \\ 1950 & \text { Feb 25, 1950 } \\ 1951 & \text { Dec 24, 1950 } \\ 1952 & \text { Dec 5, 1951 } \\ 1953 & \text { Jan 8, 1953 } \\ 1954 & \text { Feb 13, 1954 } \\ 1955 & \text { Feb 8, 1955 } \\ 1956 & \text { Mar 3, 1956 } \\ 1957 & \text { Feb 24, 1957 } \\ 1958 & \text { Dec 25, 1957 } \\ 1959 & \text { Nov 24, 1958 } \\ 1960 & \text { Jan 29, 1960 } \\ 1961 & \text { Mar 15 or May 4, 1961 } \\ 1962 & \text { Apr 27, 1962 } \\ 1963 & \text { Nov 25, 1962 } \\ & \\ 1964 & \text { Mar 21, 1964 } \\ 1965 & \text { Jan 30,1965 } \\ 1966 & \text { Dec 27, 1965 } \\ 1967 & \text { Jan 19, 1967 } \\ 1968 & \text { Jan 14, 1968 } \\ 1969 & \text { Feb 11, 1969 } \\ 1970 & \text { Jan 23, 1970 }\end{array}$

Gage height

$(\mathrm{ft})$

8.68

8.18

8.26

8.16

7.70

8.60

8.31

8.67

10.32

7.35

7.34

8.53

7.55

6.87

8.56

7.61

8.28

7.78

8.99

8.04

7.98

7.75
Discharge

( $\mathrm{ft}^{3 / \mathrm{s}}$ )

47.3

33.2

35.1

32.3

21.4

45.0

34.0

49.1

108

16.2

16

44

20

8

46

17

30

12

49

21

21

11 
TABLE 9.--Records of miscellaneous stream-flow measurement sites in $\mathrm{Clallam}$ County

\begin{tabular}{|c|c|c|c|c|c|c|}
\hline $\begin{array}{l}\text { Site } \\
\text { number }\end{array}$ & Stream & Iributary to & Location & $\begin{array}{l}\text { Drainage } \\
\text { area } \\
\text { (sq mi) } \\
\end{array}$ & $\begin{array}{l}\text { Meast } \\
\text { Date }\end{array}$ & $\begin{array}{l}\text { rements } \\
\text { Discharge } \\
\text { (cubic feet } \\
\text { per second) }\end{array}$ \\
\hline & \multicolumn{6}{|c|}{ Pacific Ocean Tributaries } \\
\hline 1 & $\begin{array}{l}\text { Pacific Ocean } \\
\text { tributary no. } 5\end{array}$ & ...do... & $\begin{array}{l}\text { SW \& sec. } 27, \text { T.28 N., R.15 W., just } \\
\text { above setting basin, } 0.9 \text { mile } \\
\text { southeast of La Push }\end{array}$ & -- & $9-16-65$ & .13 \\
\hline & \multicolumn{6}{|c|}{ Quill ayute River Basin } \\
\hline- & Soleduck River & Quillayute River & $\begin{array}{l}\text { Lat } 47058^{\prime} 10 " \text {, long } 123051^{\prime} 41^{\prime \prime} \text {, } \\
\text { in NEtNWt sec. } 32, \text { T.29 N., R.9 W.. } \\
\text { Clallam County, } 30 \text { ft downstrean } \\
\text { from Sol Duc Hot Springs drain. }\end{array}$ & 22.7 & $8-10-77$ & 43.0 \\
\hline 2 & ...do... & ...do... & 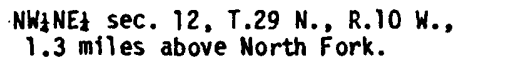 & -- & $9-18-24$ & 47.0 \\
\hline 3 & $\begin{array}{l}\text { North Fork } \\
\text { Soleduck River }\end{array}$ & Soleduck River & $\begin{array}{l}\text { NEt NEt sec. } 2, \text { T. } 29 \text { N., R.10 W., at } \\
\text { crossing of Sol Duc Hot Springs } \\
\text { road near mouth. }\end{array}$ & 30.9 & $9-18-24$ & 20.9 \\
\hline 4 & Soleduck River & Quill ayute River & 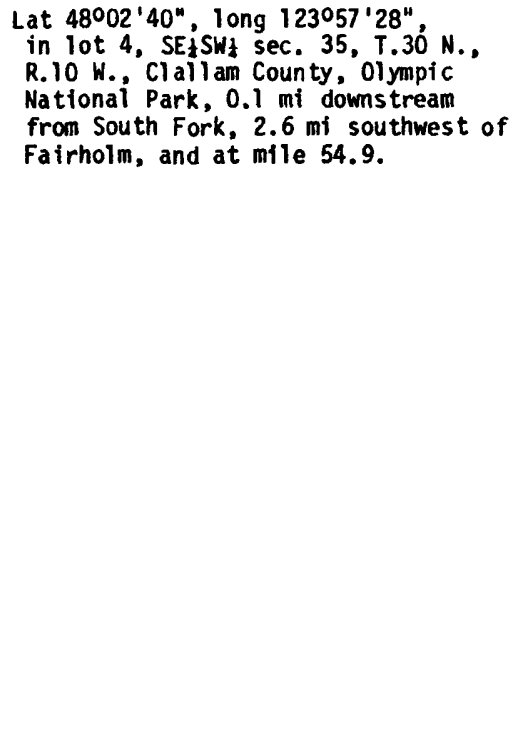 & 83.9 & $\begin{array}{l}8-29-17 \\
8-30-17 \\
11-13-21 \\
8-10-22 \\
9-14-22 \\
10-5-23 \\
2-2-24 \\
7-19-24 \\
9-14-24 \\
11-7-24 \\
4-14-25 \\
5-23-25 \\
7-13-25 \\
8-27-25 \\
5-29-26 \\
9-13-26 \\
4-28-27 \\
7-21-27 \\
8-25-27 \\
7-21-28 \\
8-29-73 \\
11-18-75 \\
12-19-75 \\
3-13-76 \\
4-9-76 \\
6-15-76 \\
8-10-76\end{array}$ & $\begin{array}{c}164 \\
163 \\
518 \\
159 \\
87 \\
80 \\
1,790 \\
114 \\
48.2 \\
898 \\
536 \\
540 \\
399 \\
120 \\
401 \\
52.0 \\
909 \\
321 \\
118 \\
157 \\
86.4 \\
1,050 \\
548 \\
869 \\
661 \\
1,350 \\
311\end{array}$ \\
\hline 6 & ...Do & ...Do & $\begin{array}{l}\text { SE dNE \&sec } 28 ., \text { T. } 30 \text { N., R. } 11 \text { W., at } \\
\text { gaging station "at Snider ranger } \\
\text { station, near Beaver," (operated } \\
\text { Oct. } 1921 \text { to Sept. 1928, fragmentary). }\end{array}$ & 116 & $\begin{array}{l}8-29-17 \\
8-30-17 \\
8-9-22 \\
9-14-22 \\
10-5-22 \\
10-6-22\end{array}$ & $\begin{array}{r}159 \\
134 \\
127 \\
78 \\
65 \\
102\end{array}$ \\
\hline
\end{tabular}


TABLE 9.--Records of miscellaneous stream-flow measurement sites in Clallam County--continued

\begin{tabular}{|c|c|c|c|c|c|c|}
\hline $\begin{array}{l}\text { S1 te } \\
\text { number }\end{array}$ & Stream & Tributary to & Location & $\begin{array}{l}\text { rainage } \\
\text { area } \\
\text { sq mil) }\end{array}$ & Meas & $\begin{array}{l}\text { rements } \\
\text { Discharge } \\
\text { (cubic feet } \\
\text { per second) }\end{array}$ \\
\hline & \multicolumn{6}{|c|}{ Quillayute River Basin } \\
\hline 7 & Snider Creek & Soleduck River & 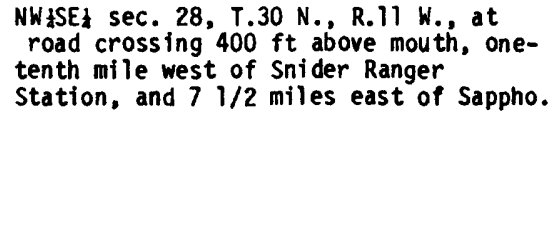 & 0.64 & $\begin{array}{l}7-17-62 \\
8-29-62 \\
5-22-63 \\
7-24-63 \\
9-30-63 \\
7-28-64 \\
9-8-65 \\
8-17-66\end{array}$ & $\begin{array}{r}1.37 \\
.84 \\
2.32 \\
1.99 \\
.68 \\
1.78 \\
.65 \\
.83\end{array}$ \\
\hline 8 & Bear Creek & ..Do & $\begin{array}{l}\text { SW \&SWA sec. } 27, T .30 \mathrm{~N} ., \mathrm{R} .12 \mathrm{~W} . \text {, at } \\
\text { U.S. Highway iol crossing, } 500 \mathrm{ft} \\
\text { above mouth, and } 2 \text { miles east of Sappho }\end{array}$ & $\begin{array}{l}17.9 \\
0 .\end{array}$ & $\begin{array}{l}7-17-62 \\
8-29-62 \\
5-22-63 \\
7-24-63 \\
10-2-63 \\
8-11-64 \\
9-8-65 \\
8-17-66\end{array}$ & $\begin{array}{c}13.8 \\
11.6 \\
31.5 \\
59.4 \\
7.43 \\
25.5 \\
5.88 \\
9.27\end{array}$ \\
\hline 10 & Cold Creek & Beaver Creek & $\begin{array}{l}\text { SW \& sec. 9, T. } 30 \text { N., R. } 12 \mathrm{~W} . \text {, at } \\
\text { Burnt Mountain Road crossing and } \\
3 \text { miles northeast of Sappho. }\end{array}$ & 2.62 & $\begin{array}{l}7-19-62 \\
8-29-62 \\
5-22-63 \\
7-24-63 \\
10-1-63 \\
8-11-64 \\
9-8-65 \\
8-17-66\end{array}$ & $\begin{array}{c}1.66 \\
3.48 \\
4.42 \\
12.7 \\
1.28 \\
5.22 \\
1.22 \\
1.96\end{array}$ \\
\hline 11 & Beaver Creek & Soleduck River & $\begin{array}{l}\text { NE } 1 \text { sec. } 20, T .30 \text { N., R. } 12 \text { W., at } \\
\text { Burnt Montain Road crossing and } \\
11 / 2 \text { miles northeast of Sappho. }\end{array}$ & 9.81 & $\begin{array}{l}7-19-62 \\
8-29-62 \\
5-22-63 \\
7-24-63 \\
10-1-63 \\
8-11-64 \\
9-8-65 \\
8-17-66\end{array}$ & $\begin{array}{c}7.96 \\
11.3 \\
19.0 \\
35.2 \\
5.03 \\
18.4 \\
3.59 \\
7.02\end{array}$ \\
\hline 12 & Rainey Creek & Beaver Creek & $\begin{array}{l}\text { SW 1SE \& sec. } 20, T .30 \text { N., R.12 W., at } \\
\text { Burnt Mountain Road crossing, i mile } \\
\text { nor theast of Sappho. }\end{array}$ & 2.12 & $\begin{array}{l}7-19-62 \\
8-29-62 \\
5-22-63 \\
7-24-63 \\
10-1-63 \\
8-11-64 \\
9-8-65 \\
8-17-66\end{array}$ & $\begin{array}{l}.02 \\
.005 \\
.22 \\
.03 \\
.001 \\
.14 \\
0 \\
.004\end{array}$ \\
\hline 13 & Soleduck River & Quillayute River & $\begin{array}{l}\text { Lat } 48004 \text { '07", long } 124017^{\prime} 39^{\prime \prime} \text {, in } \\
\text { in NE } 1 \mathrm{SW}^{\prime} \text { sec. } 30, \mathrm{~T} .30 \mathrm{~N} ., \mathrm{R} .12 \mathrm{~W} . \text {, } \\
\text { Clallam County, at U.S. Highway } 101 \\
\text { bridge, } 0.25 \text { mi downs tream from Beaver } \\
\text { Creek, } 0.8 \mathrm{mi} \text { west of Sappho, and at } \\
\text { mile } 32.0 \text {. }\end{array}$ & - & $\begin{array}{l}6-21-76 \\
7-19-76 \\
8-18-76 \\
10-7-76\end{array}$ & $\begin{array}{l}929 \\
574 \\
346 \\
175\end{array}$ \\
\hline
\end{tabular}


TABLE 9.--Records of miscellaneous stream-flow measurement sites in Clallam County--continued

\begin{tabular}{|c|c|c|c|c|c|c|}
\hline $\begin{array}{l}\text { Site } \\
\text { number }\end{array}$ & Stream & Tributary to & Location & $\begin{array}{l}\text { Drainage } \\
\text { area } \\
\text { (sq mi) } \\
\end{array}$ & $\begin{array}{l}\text { Measu } \\
\text { Date }\end{array}$ & $\begin{array}{l}\text { rements } \\
\text { Discharge } \\
\text { (cubic feet } \\
\text { per second) }\end{array}$ \\
\hline $14 A$ & $\begin{array}{l}\text { Pavel Springs } \\
\text { Creek }\end{array}$ & Soleduck River & $\begin{array}{l}\text { SW \& sec. } 31, T .30 \mathrm{~N}_{.}, R_{.} 12 \mathrm{~W}, 800 \mathrm{ft} \\
\text { above mouth and } \mathrm{i} 3 / 4 \mathrm{mi} \text { southwest } \\
\text { of Sappho. }\end{array}$ & -- & $\begin{array}{l}9-25-63 \\
6-3-64 \\
8-12-64\end{array}$ & $\begin{array}{l}2.68 \\
6.08 \\
4.68\end{array}$ \\
\hline 14B & ...Do & ...Do & 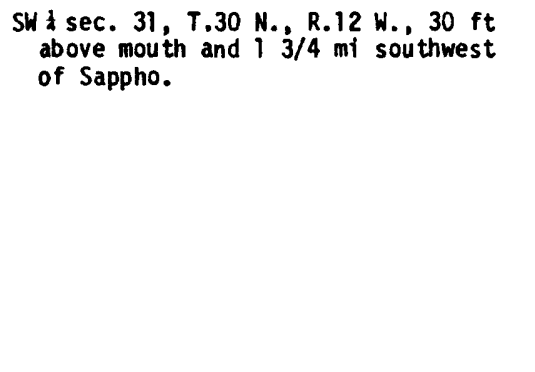 & - & $\begin{array}{l}9-25-63 \\
10-24-63 \\
10-29-63 \\
12-4-63 \\
1-7-64 \\
1-10-64 \\
2-13-64 \\
3-18-64 \\
4-22-64 \\
6-3-64 \\
7-9-64 \\
8-12-64 \\
9-16-64\end{array}$ & $\begin{array}{c}5.29 \\
7.92 \\
16.3 \\
41.0 \\
40.7 \\
42.1 \\
37.1 \\
40.9 \\
19.0 \\
14.2 \\
12.4 \\
11.6 \\
9.07\end{array}$ \\
\hline 16 & Lake Creek & ..Do & $\begin{array}{l}\text { SWISEd sec. 4, T.29 N., R.13. W., at } \\
\text { U.S. Highway loi crossing and } 2 \text { miles } \\
\text { southwest of Lake Pleasant. }\end{array}$ & 11.4 & $\begin{array}{l}7-19-62 \\
8-29-62 \\
5-22-63 \\
7-24-63 \\
9-10-63 \\
8-11-64 \\
9-8-65 \\
8-17-66\end{array}$ & $\begin{array}{l}.13 \\
15.08 \\
10.5 \\
0 \\
11.7 \\
0 \\
0\end{array}$ \\
\hline 17 & Soleduck River & Quillayute River & $\begin{array}{l}\text { Lat } 48001 \text { ' } 15^{\prime \prime} \text {, long } 124022 \text { '56" } \\
\text { SWINEI sec. } 16 \text {, T.29 N., R.I3 W., } \\
\text { Clallam County, at Maxfie1d Road } \\
\text { bridge over the Soleduck River, } \\
0.3 \text { mile east of Olympic Highway } \\
\text { U.S. I01, } 3.0 \text { miles southwest of } \\
\text { Lake Pleasant and } 4.8 \text { miles north } \\
\text { of Forks. }\end{array}$ & -- & $\begin{array}{l}12-16-71 \\
2-9-72 \\
4-12-72 \\
6-13-72 \\
8-8-72\end{array}$ & $\begin{array}{r}1,530 \\
1,610 \\
1,650 \\
863 \\
401\end{array}$ \\
\hline 18 & Soleduck River & Quillayute River & $\begin{array}{l}\text { SEtSE } 1 \text { sec. } 29, \text { T.29 N., R.13 } \mathrm{K}_{\text {., }} \\
\text { at crossing of U.S. Highway } 10 \mathrm{i} \text {. }\end{array}$ & 208 & $7-25-00$ & 550 \\
\hline 23 & ...Do & $\ldots$ Do & $\begin{array}{l}\text { NWINEt sec. 11, T.28 N., R.14 W., } \\
\text { at gaging station "near Quillayute," } \\
\text { (operated Nov. } 1897 \text { to Dec. 1901). }\end{array}$ & 219 & $\begin{array}{l}9-7-97 \\
8-9-62 \\
8-15-62 \\
8-29-62 \\
11-19-75 \\
12-18-75 \\
1-7-76 \\
2-12-76 \\
4-8-76 \\
6-17-76 \\
8-11-76 \\
10-15-76 \\
12-14-76 \\
2-9-77 \\
4-13-77 \\
6-24-77 \\
8-11-77\end{array}$ & $\begin{array}{r}858 \\
327 \\
251 \\
244 \\
2,640 \\
1,510 \\
2,200 \\
4,590 \\
1,360 \\
1,260 \\
439 \\
231 \\
826 \\
598 \\
220 \\
529 \\
225\end{array}$ \\
\hline
\end{tabular}


TABLE 9.--Records of miscellaneous stream-flow measurement sites in Clallam County--continued

\begin{tabular}{|c|c|c|c|c|c|c|}
\hline $\begin{array}{l}\text { Site } \\
\text { number }\end{array}$ & Stream & Tributary to & Location & $\begin{array}{l}\text { Drainage } \\
\text { area } \\
\text { (sq mi) } \\
\end{array}$ & Meas & $\begin{array}{l}\text { rements } \\
\text { Discharge } \\
\text { (cubic feet } \\
\text { per second) }\end{array}$ \\
\hline- & Bogachiel River & ...Do & $\begin{array}{l}\text { Lat } 47052^{\prime} 56^{\prime \prime}, \text { long } 124010^{\prime} 00^{\prime \prime} \text {, in } \\
\text { SWiSE t sec. 31., R.28 N., Rli W., } \\
\text { Clallam County, Oiympic National' } \\
\text { Park, at Bogachiel shel ter, } 11.2 \text { mile } \\
\text { southeast of Forks, at mile } 26.1 \text {. }\end{array}$ & es & $\begin{array}{l}5-7-76 \\
6-22-76 \\
7-20-76 \\
8-17-76 \\
10-5-76\end{array}$ & $\begin{array}{l}713 \\
391 \\
312 \\
204 \\
101\end{array}$ \\
\hline 31 & May Creek & Bogachiel River & $\begin{array}{l}\text { South line SWISW sec. } 35, T .28 \mathrm{~N} ., \\
\text { R. } 13 \text { W., at U.S. Highway } 101, \\
\text { (crest-s tage gage "near Fork"). }\end{array}$ & 2.03 & $1-7-54$ & 64.5 \\
\hline-- & $\begin{array}{l}\text { South Fork } \\
\text { Calawah River }\end{array}$ & Cal awah River & 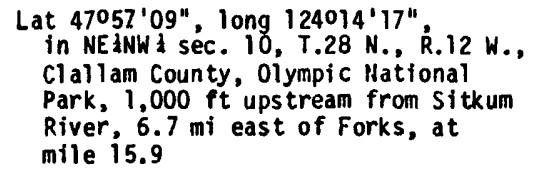 & 23.4 & $\begin{array}{l}5-6-76 \\
6-23-76 \\
7-21-76 \\
8-18-76 \\
10-6-76\end{array}$ & $\begin{array}{r}151 \\
61 \\
39 \\
32 \\
24\end{array}$ \\
\hline-- & Sitkum River & $\begin{array}{l}\text { South Fork } \\
\text { Calawah River }\end{array}$ & $\begin{array}{l}\text { Lat } 47057^{\prime} 12 " \text { " long } 124014 \text { ' } 30 " \text { " } \\
\text { in NWINWI sec. } 10, \mathrm{~T} .28 \mathrm{~N} . . \text { R. } 12 \text { W., } \\
\text { Clallam County, } 200 \mathrm{ft} \text { above mouth } \\
\text { and } 6.6 \text { mi east of Forks. }\end{array}$ & 30.8 & $\begin{array}{l}5-6-76 \\
6-23-76 \\
7-21-76 \\
8-18-76 \\
10-6-76\end{array}$ & $\begin{array}{r}202 \\
78 \\
43 \\
40 \\
24\end{array}$ \\
\hline 38 & $\begin{array}{l}\text { North Fork } \\
\text { Cal awah River }\end{array}$ & Cal awah River & $\begin{array}{l}\text { Lat } 47^{058^{\prime}} 16^{\prime \prime}, \text { long } 124019^{\prime} 53^{\prime \prime}, \\
\text { in SE \& SE sec. } 35, \mathrm{r} .29 \mathrm{~N} ., \mathrm{R} .13 \mathrm{~W} ., \\
\text { Clallam County, } 200 \mathrm{ft} \text { above mouth, } \\
2.8 \text { mi northeast of Forks }\end{array}$ & 47.2 & $\begin{array}{l}5-6-76 \\
6-23-76 \\
7-21-76 \\
8-18-76 \\
10-6-76\end{array}$ & $\begin{array}{r}206 \\
105 \\
71 \\
47 \\
31\end{array}$ \\
\hline 42 & Cal awah River & Bogachiel River & $\begin{array}{l}\text { NWASWA Sec. 4, T.28 N., R.13 W., } \\
\text { at U.S. Highway } 101 \text { crossing and } \\
\text { I/2 mile north of Forks. }\end{array}$ & 129 & $\begin{array}{l}9-6-97 \\
8-29-62\end{array}$ & $\begin{array}{l}467 \\
144\end{array}$ \\
\hline 63 & $\begin{array}{l}\text { East Fork Dickey } \\
\text { River }\end{array}$ & Quillayute River & 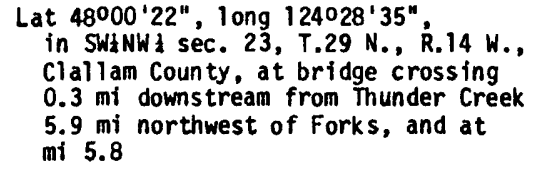 & -- & $\begin{array}{l}6-23-76 \\
7-21-76 \\
8-18-76 \\
10-6-76\end{array}$ & $\begin{array}{l}44 \\
23 \\
20 \\
18\end{array}$ \\
\hline-- & ...Do & ...Do & $\begin{array}{l}\text { Lat } 48000 \text { ' } 31 \text { ", long } 124030 \text { ' } 36 \text { " } \\
\text { in NWiNE } \$ \text { sec. } 21, T .28 \text { N., R.14 W., } \\
\text { Clallam County, } 7.2 \text { mi northwest of } \\
\text { Forks, and at mile } 3.6 \text {. }\end{array}$ & -- & $5-3-76$ & 51 \\
\hline 64 & $\begin{array}{l}\text { East Fork } \\
\text { Dickey River }\end{array}$ & Dickey River & $\begin{array}{l}\text { Lat } 47059 \text { '09", long } 124032 \text { ' } 44 \text { ", } \\
\text { in SEISEA sec. } 30, \mathrm{~T} .29 \mathrm{~N} \text {., R. } 14 \mathrm{~W} . \text {, } \\
\text { Clallam County, } 6.7 \mathrm{mi} \text { nor theast of } \\
\text { La Push and at mile } 0.2 \text {. }\end{array}$ & 39.8 & $\begin{array}{l}10-1-68 \\
8-29-73\end{array}$ & $\begin{array}{c}60.7 \\
9.77\end{array}$ \\
\hline-- & $\begin{array}{l}\text { West Fork } \\
\text { Oickey River }\end{array}$ & ...Do & $\begin{array}{l}\text { SWASEA sec. } 16, T .30 \mathrm{~N}, \text { R.14 } \mathrm{W} \text {, at } \\
\text { old logging road crossing, } 1 / 4 \mathrm{mi} \\
\text { downstream from Lake Oickey and } \\
11 \mathrm{mi} \text { northwest of Forks. }\end{array}$ & 14.7 & $8-28-62$ & 14.6 \\
\hline
\end{tabular}


TABLE 9.--Records of miscellaneous stream-flow measurement sites in Clallam County--continued

\begin{tabular}{|c|c|c|c|c|c|c|}
\hline $\begin{array}{l}\text { Site } \\
\text { number }\end{array}$ & Stream & Tributary to & Location & $\begin{array}{l}\text { Drainage } \\
\text { area } \\
\text { (sq } \mathrm{mi}) \\
\end{array}$ & Measu & $\begin{array}{l}\text { ements } \\
\text { Discharge } \\
\text { (cubic feet } \\
\text { per second) } \\
\end{array}$ \\
\hline-- & ...Do & ...Do & $\begin{array}{l}\text { Lat } 48005^{\prime} 17^{\prime \prime}, \text { long } 124030 \text { '08", } \\
\text { in NW } 1 \text { SEt sec. } 21, \mathrm{~T} .30 \mathrm{~N} ., \text { R. } 14 \mathrm{~W} . \text {, } \\
\text { Clall am County, } 0.8 \text { ni downstream fro } \\
\text { Lake Dickey, 1i mi nor thwest of Fork s } \\
\text { and at mile } 19.6 \text {. }\end{array}$ & $\begin{array}{l}-- \\
\text { om } \\
\text {, }\end{array}$ & $\begin{array}{l}5-6-76 \\
6-23-76 \\
7-21-76 \\
8-18-76 \\
10-6-76\end{array}$ & $\begin{array}{l}19 \\
31 \\
6.9 \\
5.5 \\
5.8\end{array}$ \\
\hline 65 & $\begin{array}{l}\text { West Fork } \\
\text { Dickey River }\end{array}$ & Dickey River & $\begin{array}{l}\text { SE \&SE } 1 \text { sec. } 30, T .29 \mathrm{~N} ., R_{1} 14 \mathrm{~W} ., \\
\text { near mouth } 8 \mathrm{mi} \text { west of Forks. }\end{array}$ & 44.4 & $\begin{array}{l}7-19-62 \\
8-28-62 \\
5-24-63 \\
7-26-63 \\
10-2-63 \\
8-11-64 \\
9-9-65 \\
8-17-66\end{array}$ & $\begin{array}{c}17.3 \\
31.8 \\
39.5 \\
92.3 \\
9.15 \\
62.3 \\
9.73 \\
12.5\end{array}$ \\
\hline 68 & Dickey River & Quillayute River & $\begin{array}{l}\text { Lat } 47057^{\prime} 53^{\prime \prime} \text {, long } 124032 \text { '53", } \\
\text { in NE INE } \frac{1}{4} \text { sec. } 6, T .28 \mathrm{~N} ., \text { R.14 W., } \\
\text { Clallam County, i.0 mi upstream from } \\
\text { Colby Creek, } 5.5 \mathrm{mi} \text { northeast of } \\
\text { La Push and at mile } 6.0 \text {. }\end{array}$ & 86.3 & $\begin{array}{l}11-19-75 \\
12-18-75 \\
4-8-76 \\
8-10-76\end{array}$ & $\begin{array}{r}683 \\
345 \\
256 \\
32\end{array}$ \\
\hline \multirow[t]{2}{*}{71} & Colby Creek & Dickey River & $\begin{array}{l}\text { NW 1 sec. } 8, T .28 \mathrm{~N} ., \mathrm{R} .14 \mathrm{~W} ., \text { at } \\
\text { logging road crossing } 1 \mathrm{mi} \text { above } \\
\text { mouth, and } 7 \mathrm{mi} \text { west of Forks. }\end{array}$ & 6.20 & $\begin{array}{l}7-19-62 \\
8-28-62 \\
5-24-63 \\
7-26-63 \\
10-2-63 \\
8-11-64 \\
9-9-65 \\
8-18-66\end{array}$ & $\begin{array}{l}2.97 \\
2.68 \\
7.56 \\
7.11 \\
1.16 \\
5.07 \\
1.25 \\
1.24\end{array}$ \\
\hline & \multicolumn{6}{|c|}{ Ozette River Basin } \\
\hline-- & Crooked Creek & Ozette Lake & 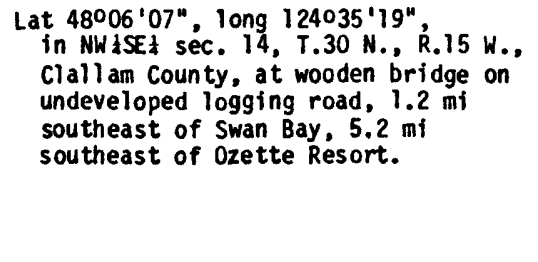 & 10.41 & $\begin{array}{l}1-14-76 \\
2-18-76 \\
3-30-76 \\
4-28-76 \\
5-26-75 \\
6-23-76 \\
7-19-76 \\
8-25-76 \\
9-30-76\end{array}$ & $\begin{array}{r}354 \\
155 \\
55.2 \\
12.2 \\
44.2 \\
7.6 \\
5.8 \\
12.1 \\
2.5\end{array}$ \\
\hline-- & Big River & ...Do & 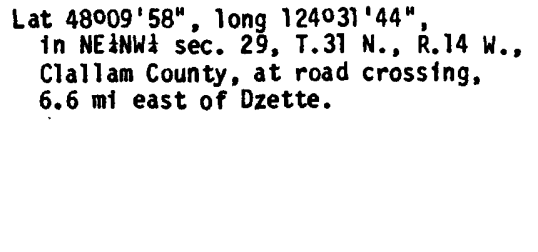 & 8.77 & $\begin{array}{l}7-20-62 \\
8-30-62 \\
5-23-63 \\
7-25-63 \\
10-1-63 \\
8-12-64 \\
9-9-65 \\
8-17-66 \\
8-28-73\end{array}$ & $\begin{array}{c}5.06 \\
10.7 \\
9.41 \\
22.7 \\
3.13 \\
13.7 \\
3.50 \\
6.22 \\
3.18\end{array}$ \\
\hline
\end{tabular}


TABLE 9.--Records of miscellaneous stream-flow measurement sites in $\mathrm{Clallam}$ County--continued

\begin{tabular}{|c|c|c|c|c|c|c|}
\hline $\begin{array}{l}\text { Si te } \\
\text { number }\end{array}$ & Stream & Tributary to & Location & $\begin{array}{l}\text { Orainage } \\
\text { area } \\
\text { (sq } \mathrm{mi} \text { ) }\end{array}$ & Meas & $\begin{array}{l}\frac{\text { rements }}{\text { Discharge }} \\
\text { (cubic feet } \\
\text { per second) }\end{array}$ \\
\hline-- & Trout Creek & Big River & $\begin{array}{l}\text { SWaNWi sec. 2, T.30 N., R.15 W., at } \\
\text { Ozette Road crossing and } 3 \text { miles } \\
\text { southeast of Ozette. }\end{array}$ & 3.59 & $\begin{array}{l}7-20-62 \\
8-30-62 \\
5-23-63 \\
7-25-63 \\
10-1-63 \\
8-12-64 \\
9-9-65 \\
8-17-66\end{array}$ & $\begin{array}{r}1.02 \\
1.99 \\
2.32 \\
6.51 \\
.35 \\
5.10 \\
.72 \\
2.19\end{array}$ \\
\hline-- & Big River & Ozette Lake & $\begin{array}{l}\text { Lat } 48007 \text { ' } 53^{\mathrm{N}}, \text { long } 124036^{\prime} 10^{\prime \prime}, \\
\text { in NE ISE i sec. } 3, T .30 \mathrm{~N} ., \mathrm{R} .15 \mathrm{~W} . \text {, } \\
\text { Cl al lam County, } 3.2 \mathrm{mi} \text { southeast of } \\
\text { of Ozette Resort, } 0.2 \text { mi upstream } \\
\text { from Dunham Creek. }\end{array}$ & 20.64 & $\begin{array}{l}7-19-76 \\
8-25-76 \\
9-29-76\end{array}$ & $\begin{array}{l}11.8 \\
24.6 \\
10.8\end{array}$ \\
\hline- & Dunham Creek & Big River & 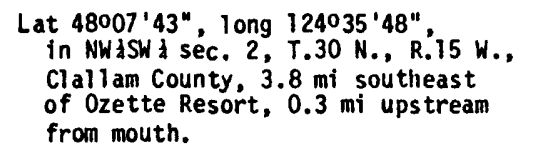 & 1.77 & $\begin{array}{l}7-19-76 \\
8-25-76 \\
9-29-76\end{array}$ & $\begin{array}{r}.63 \\
1.60 \\
.50\end{array}$ \\
\hline-- & Big River & Ozette Lake & $\begin{array}{l}\text { Lat } 48007^{\prime} 36 \text { ", long } 124036^{\prime} 16^{\prime \prime}, \\
\text { in SEtSEt sec. 3, T.30 N., R.15 W., } \\
\text { Clallam County, at old logging bridge } \\
\text { on road to Swan Bay, } 3.3 \text { mi southeast } \\
\text { of Ozette Resort. }\end{array}$ & 22.52 & $\begin{array}{l}1-14-76 \\
2-18-76 \\
3-29-76 \\
4-28-76 \\
5-26-76 \\
6-23-76\end{array}$ & $\begin{array}{c}5.78 \\
382 \\
174 \\
45.6 \\
140 \\
36.1\end{array}$ \\
\hline-- & Unbrella Creek & Ozette Lake & $\begin{array}{l}\text { Lat } 48008^{\prime} 07^{\prime \prime} \text {, long } 124037^{\prime} 18^{\prime \prime}, \\
\text { in NWiNW } 1 \text { sec. 3, T.30 N., R.15 W., } \\
\text { Clallam County, at concrete bridge } \\
\text { on Ozette Road, } 2.5 \text { mi southeast } \\
\text { of Ozette. }\end{array}$ & 11.4 & $\begin{array}{l}7-20-62 \\
8-30-62 \\
5-23-63 \\
7-25-63 \\
10-1-63 \\
8-12-64 \\
9-9-65 \\
8-17-66 \\
1-14-76 \\
2-17-76 \\
3-30-76 \\
4-26-76 \\
5-24-76 \\
6-21-76 \\
7-19-76 \\
8-25-76 \\
9-27-76\end{array}$ & $\begin{array}{c}5.13 \\
7.64 \\
8.16 \\
20.6 \\
2.64 \\
13.4 \\
2.80 \\
5.73 \\
219 \\
386 \\
73.2 \\
24.1 \\
7.5 \\
13.6 \\
6.93 \\
15.2 \\
6.31\end{array}$ \\
\hline- & Ozette River & Pacific Ocean & $\begin{array}{l}\text { Lat } 48009^{\prime} 14^{\prime \prime} \text {, long } 124040 \text { '08" } \\
\text { in SE } \mathrm{SE}_{1} \text { sec. 30, T.31 N., R.15 W., } \\
\text { Clallam County, Olympic National Park, } \\
\text { at Ozette and at outlet of lake. }\end{array}$ & 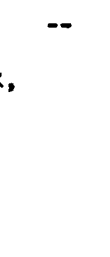 & $\begin{array}{l}7-20-62 \\
8-30-62 \\
5-23-63 \\
7-25-63 \\
10-1-63 \\
7-29-64 \\
9-9-65 \\
8-17-66 \\
8-28-73\end{array}$ & $\begin{array}{c}78.5 \\
81.3 \\
261 \\
145 \\
25.1 \\
216 \\
34.8 \\
44.7 \\
40.3\end{array}$ \\
\hline
\end{tabular}


TABLE 9.---Records of miscellaneous stream-flow measurement sites in Clallam County--continued

\begin{tabular}{|c|c|c|c|c|c|c|}
\hline $\begin{array}{l}\text { Site } \\
\text { number }\end{array}$ & Stream & Tributary to & Location & $\begin{array}{l}\text { Drainage } \\
\text { area } \\
\text { (sq mi) }\end{array}$ & Meas & $\begin{array}{l}\text { cements } \\
\text { Discharge } \\
\text { (cubic feet } \\
\text { per second) }\end{array}$ \\
\hline \multirow[t]{2}{*}{--} & Coal Creek & Ozette River & $\begin{array}{l}\text { SW } 1 \text { SW } \text { sec. } 29, \text { T. } 31 \mathrm{~N} ., \mathrm{R}_{.} 15 \mathrm{~W} . \\
\text { at private road crossing at Ozette, } \\
\text { I/4 mi above mouth. }\end{array}$ & 5.82 & $\begin{array}{l}7-20-62 \\
8-30-62 \\
5-23-63 \\
7-25-63 \\
10-1-63 \\
8-12-64 \\
9-9-65 \\
8-17-66\end{array}$ & $\begin{array}{r}4.22 \\
1.85 \\
1.57 \\
7.24 \\
.45 \\
4.25 \\
.59 \\
1.94\end{array}$ \\
\hline & \multicolumn{6}{|c|}{ Sooes River Basin } \\
\hline-- & $\begin{array}{l}\text { Thirty Cent } \\
\text { Creek }\end{array}$ & Sooes River & $\begin{array}{l}\text { NWaSEl sec. } 22, T .32 \mathrm{~N} ., \mathrm{R} .15 \mathrm{~W} \text {., } \\
\text { at private road crossing } 71 / 2 \mathrm{mi} \\
\text { south of Neah Bay. }\end{array}$ & 0.57 & $\begin{array}{l}7-20-62 \\
8-31-62 \\
5-23-63 \\
7-25-63 \\
10-2-63 \\
8-12-64 \\
9-9-65 \\
8-18-66\end{array}$ & $\begin{array}{r}.44 \\
.52 \\
.85 \\
1.51 \\
.21 \\
.84 \\
.14 \\
.14\end{array}$ \\
\hline-- & Miller Creek & $\ldots D_{0}$ & $\begin{array}{l}\text { SWINEt sec. } 22, T .32 \mathrm{~N} ., \mathrm{R} .15 \mathrm{~W} \text {, at } \\
\text { at private road crossing, } 7 \mathrm{mi} \\
\text { south of Neah Bay. }\end{array}$ & 1.11 & $\begin{array}{l}7-20-62 \\
8-31-62 \\
5-23-63 \\
7-25-63 \\
10-2-63 \\
8-12-64 \\
9-9-65 \\
8-18-66\end{array}$ & $\begin{array}{r}.63 \\
.87 \\
.81 \\
2.78 \\
.11 \\
1.03 \\
.33 \\
1.18\end{array}$ \\
\hline-- & Sooes River & Mukkaw Bay & 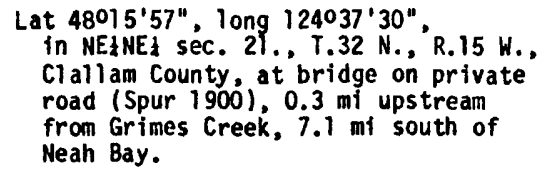 & 32.0 & $8-4-71$ & 27.0 \\
\hline-- & Grimes Creek & Sooes River & $\begin{array}{l}\text { NW\&SW s sec. } 15, T .32 \mathrm{~N} ., R_{.} 15 \mathrm{~W}, \text {, } \\
\text { at private road crossing } 61 / 2 \mathrm{mi} \\
\text { south of Neah Bay. }\end{array}$ & 1.38 & $\begin{array}{l}7-20-62 \\
8-31-62 \\
5-23-63 \\
7-25-63 \\
10-2-63 \\
8-12-64 \\
9-9-65 \\
8-18-66\end{array}$ & $\begin{array}{r}.73 \\
1.07 \\
.80 \\
3.05 \\
.12 \\
1.82 \\
.30 \\
1.24\end{array}$ \\
\hline-- & ...Do & $\ldots D 0$ & $\begin{array}{l}\text { Lat } 48017^{\prime} 32^{\prime \prime}, \text { long } 124038^{\prime} 56^{\prime \prime} \text {, } \\
\text { in NEiNE } 1 \text { sec. } 8, \text { T.32 N., R.15 W., } \\
\text { Clallam County, at bridge on private } \\
\text { road (Spur 7), } 5.5 \text { miles south of } \\
\text { Neah Bay. }\end{array}$ & -- & $\begin{array}{l}8-4-71 \\
9-3-71 \\
9-15-71\end{array}$ & $\begin{array}{l}27.3 \\
140 \\
59.4\end{array}$ \\
\hline
\end{tabular}


TABLE 9.--Records of miscellaneous stream-flow measurement sites in Clallam County--continued

\begin{tabular}{|c|c|c|c|c|c|c|}
\hline $\begin{array}{l}\text { Si te } \\
\text { number }\end{array}$ & Stream & Tributary to & Location & $\begin{array}{l}\text { Drainage } \\
\text { area } \\
\text { (sq } \mathrm{mi}) \\
\end{array}$ & Measu & $\begin{array}{l}\text { rements } \\
\text { Discharge } \\
\text { (cubic feet } \\
\text { per second) } \\
\end{array}$ \\
\hline & \multicolumn{6}{|c|}{ Waatch River Bas in } \\
\hline \multirow[t]{2}{*}{--} & Waatch River & $\begin{array}{l}\text { Mukkaw Bay } \\
\text { (Pacific Ocean) }\end{array}$ & $\begin{array}{l}\text { Lat } 48021^{\prime} 24 \text { ", long } 124037^{\prime} 42 \text { ", } \\
\text { in NE\&SW \& sec. i5, T.33 N., R. } 15 \mathrm{W.,} \\
\text { Clall am County, } 0.8 \mathrm{mi} \text { southwest of } \\
\text { of Neah Bay. }\end{array}$ & 10.7 & $\begin{array}{l}6-25-76 \\
9-9-76 \\
10-13-76 \\
12-15-76 \\
2-9-77 \\
4-17-77 \\
6-16-77 \\
8-4-77 \\
10-14-77 \\
12-16-77 \\
3-3-78\end{array}$ & $\begin{array}{c}11.9 \\
18.2 \\
13.5 \\
105 \\
21.3 \\
12.0 \\
7.88 \\
1.57 \\
9.98 \\
123 \\
12.3\end{array}$ \\
\hline & \multicolumn{6}{|c|}{ Waatch Creek Basin } \\
\hline \multirow[t]{2}{*}{--} & Waatch Creek & $\begin{array}{l}\text { Mukkaw Bay } \\
\text { (Pacific Ocean) }\end{array}$ & $\begin{array}{l}\text { NK \& sec. } 20, T .33 \mathrm{~N}_{\text {, }} \text { R.15 W., at } \\
\text { at road crossing i/4 mi above mouth } \\
\text { and } 3 \text { mi southwest of Neah Bay. }\end{array}$ & 0.55 & $\begin{array}{l}7-20-62 \\
8-30-62 \\
5-22-63 \\
7-24-63 \\
9-30-63 \\
8-11-64 \\
9-8-65 \\
8-16-66\end{array}$ & $\begin{array}{r}.58 \\
.94 \\
.66 \\
3.40 \\
.21 \\
1.44 \\
.28 \\
2.99\end{array}$ \\
\hline & \multicolumn{6}{|c|}{ Village Creek Basin } \\
\hline-- & Village Creek & Neah Bay & 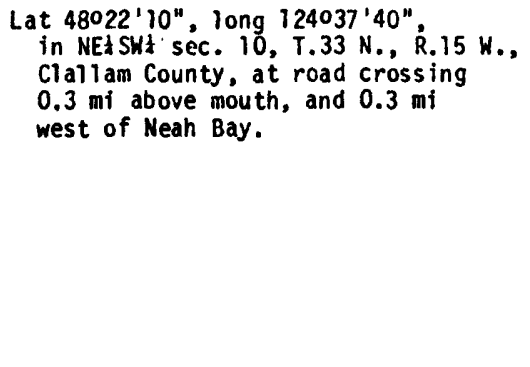 & 0.65 & $\begin{array}{l}4-14-76 \\
5-4-76 \\
6-18-76 \\
8-9-76 \\
10-13-76 \\
12-15-76 \\
2-9-77 \\
4-7-77 \\
6-29-77 \\
8-4-77 \\
10-14-77 \\
12-15-77 \\
3-3-78\end{array}$ & $\begin{array}{r}1.72 \\
.42 \\
2.79 \\
.21 \\
1.17 \\
8.96 \\
1.38 \\
.68 \\
.51 \\
.21 \\
.79 \\
21.2 \\
.75\end{array}$ \\
\hline- & ...Do & ...Do & $\begin{array}{l}\text { SW } \& \text { sec. } 10, T .33 \mathrm{~N} ., R_{.} 15 \mathrm{~W} \text {, at } \\
\text { road crossing at mouth at Neah Bay. }\end{array}$ & 0.68 & $\begin{array}{l}7-20-62 \\
8-30-62 \\
5-22-63 \\
7-24-63 \\
9-30-63 \\
8-11-64 \\
9-8-65 \\
8-16-66\end{array}$ & $\begin{array}{r}.31 \\
.72 \\
.46 \\
1.97 \\
.13 \\
.74 \\
.06 \\
1.55\end{array}$ \\
\hline
\end{tabular}


TABLE 9.--Records of miscellaneous stream-flow measurement sites in $\mathrm{Clallam} \mathrm{County--continued}$

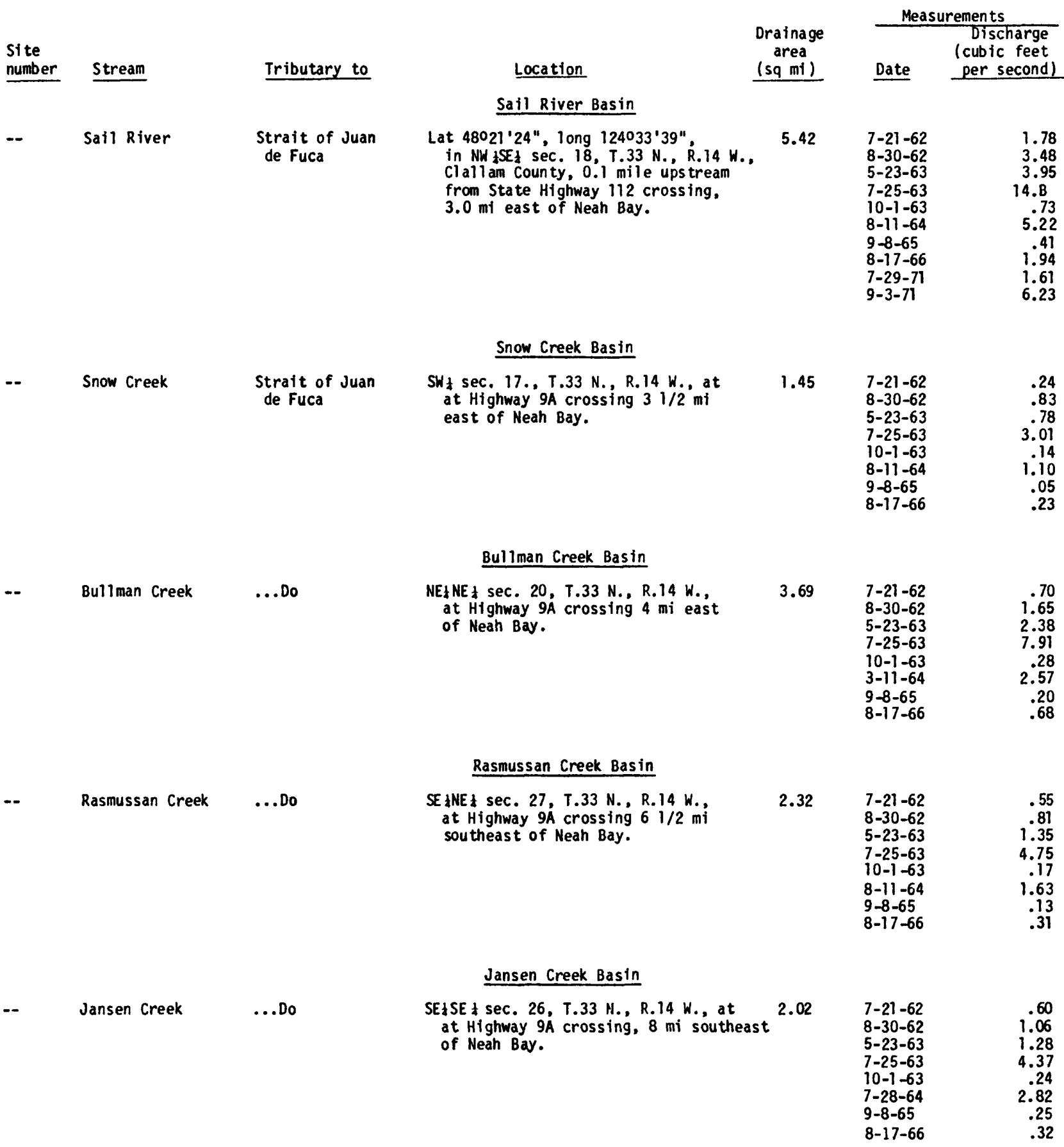


TABLE 9.--Records of miscellaneous stream-flow measurement sites in $\mathrm{Clallam}$ County--continued

\begin{tabular}{|c|c|c|c|c|c|c|}
\hline $\begin{array}{l}\text { Site } \\
\text { number }\end{array}$ & Stream & Tributary to & Location & $\begin{array}{l}\text { Drainage } \\
\text { area } \\
\text { (sq } \mathrm{mi} \text { ) }\end{array}$ & Meas & $\begin{array}{l}\text { rements } \\
\text { Discharge } \\
\text { (cubic feet } \\
\text { per second) }\end{array}$ \\
\hline & \multicolumn{6}{|c|}{ 01sen Creek Basin } \\
\hline-- & Dlsen Creek & ...Do & $\begin{array}{l}\text { NEł NE⿺ sec. 1, T.32 N., R. } 14 \text { W., at } \\
\text { State Highway } 112,7 \text { mi northwest } \\
\text { of Sekiu. }\end{array}$ & 1.14 & $\begin{array}{l}7-21-62 \\
8-30-62 \\
5-23-63 \\
7-25-63 \\
10-1-63 \\
7-28-64 \\
9-8-65 \\
8-17-66\end{array}$ & $\begin{array}{r}.41 \\
.83 \\
.96 \\
2.30 \\
.12 \\
1.68 \\
.15 \\
.20\end{array}$ \\
\hline & \multicolumn{6}{|c|}{ Sekiu River Basin } \\
\hline- & $\begin{array}{l}\text { North Fork } \\
\text { Sekiu River }\end{array}$ & Sekiu River & $\begin{array}{l}\text { NEt sec. } 15, T .32 \text { N., R. } 14 \text { W., at } \\
\text { confluence with South Fork, } 8 \mathrm{I} / 2 \mathrm{mi} \\
\text { west of Sekiu. }\end{array}$ & 11.1 & $\begin{array}{l}7-20-62 \\
8-31-62 \\
5-23-63 \\
7-25-63 \\
10-2-63 \\
8-12-64 \\
9-9-65 \\
8-18-66\end{array}$ & $\begin{array}{c}7.39 \\
11.5 \\
13.4 \\
33.4 \\
3.29 \\
13.8 \\
3.31 \\
6.76\end{array}$ \\
\hline-- & $\begin{array}{l}\text { South Fork } \\
\text { Sek iu River }\end{array}$ & $\cdots$ Do & $\begin{array}{l}\text { NE sec. } 15 ., \text { T.32 N., R.14 W., at } \\
\text { confluence with North Fork, } 8 \text { ' } 1 / 2 \mathrm{mi} \\
\text { west of Sekiu. }\end{array}$ & 8.86 & $\begin{array}{l}7-20-62 \\
8-31-62 \\
5-23-63 \\
7-25-63 \\
10-2-63 \\
8-12-64 \\
9-9-65 \\
8-18-66\end{array}$ & $\begin{array}{c}5.95 \\
8.66 \\
11.1 \\
21.3 \\
2.48 \\
10.1 \\
3.32 \\
4.41\end{array}$ \\
\hline 88 & Carpenters Creek & Sekiu River & $\begin{array}{l}\text { SW } 1 \text { SWt sec. } 8 ., \text { T. } 32 \text { N., R. } 13 \text { W., } \\
\text { at private road crossing } 500 \mathrm{ft} \\
\text { above mouth and } 5 \text { mf west of Sekfu. }\end{array}$ & 2.90 & $\begin{array}{l}7-20-62 \\
8-31-62 \\
5-23-63 \\
7-25-63 \\
10-2-63 \\
8-12-64 \\
9-9-65 \\
8-18-66\end{array}$ & $\begin{array}{l}.31 \\
.66 \\
2.06 \\
2.79 \\
0 \\
1.45 \\
0 \\
0\end{array}$ \\
\hline 89 & Sekiu River & $\begin{array}{l}\text { Strait of Juan } \\
\text { de Fuca }\end{array}$ & $\begin{array}{l}\text { Center sec. } 8, T .32 \mathrm{~N} ., R_{.} 13 \mathrm{~W} . \text {, } \\
0.6 \mathrm{mi} \text { above mouth and } 5 \mathrm{mi} \text { west } \\
\text { of Sekiu. }\end{array}$ & 31.6 & $\begin{array}{l}7-20-62 \\
8-31-62 \\
5-23-63 \\
7-25-63 \\
10-2-63 \\
8-12-64 \\
9-9-65 \\
8-18-66\end{array}$ & $\begin{array}{c}17.3 \\
26.5 \\
34.6 \\
84.9 \\
7.54 \\
33.5 \\
7.17 \\
14.5\end{array}$ \\
\hline
\end{tabular}


TABLE 9.--Records of miscellaneous stream-flow measurement sites in $\mathrm{Clallam}$ County--continued

\begin{tabular}{|c|c|c|c|c|c|c|}
\hline $\begin{array}{l}\text { Site } \\
\text { number } \\
\end{array}$ & Stream & Iributary to & Location & $\begin{array}{l}\text { Drainage } \\
\text { area } \\
\text { (sq mi) } \\
\end{array}$ & Measu & $\begin{array}{l}\text { rements } \\
\text { Oischarge } \\
\text { (cubic feet } \\
\text { per second) }\end{array}$ \\
\hline & \multicolumn{6}{|c|}{ Hoko River Basin } \\
\hline-- & $\begin{array}{l}\text { Hoko River } \\
\text { tributary }\end{array}$ & Hoko River & $\begin{array}{l}\text { NEINE1 sec. } 23, T .31 \text { N., R.14 W., at } \\
\text { Ozette Road crossing } 3 / 4 \text { mi south- } \\
\text { west of Hoko Camp. }\end{array}$ & 0.88 & $\begin{array}{l}7-20-62 \\
8-30-62 \\
5-23-63 \\
7-25-63 \\
10-1-63 \\
8-11-64 \\
9-8-65 \\
8-17-66\end{array}$ & $\begin{array}{l}.29 \\
.28 \\
.40 \\
.41 \\
.10 \\
.44 \\
.08 \\
.17\end{array}$ \\
\hline-- & $\begin{array}{l}\text { Hoko River } \\
\text { tributary No. } 2\end{array}$ & $\ldots D_{0}$ & $\begin{array}{l}\text { NWLWW sec. } 24, T .31 N ., \text { R.14 W., at } \\
\text { Ozette Road crossing } i / 2 \text { mi south- } \\
\text { west of Hoko Camp. }\end{array}$ & .94 & $\begin{array}{l}7-20-62 \\
8-30-62 \\
5-23-63 \\
7-25-63 \\
10-1-63 \\
8-11-64 \\
9-8-65 \\
8-17-66\end{array}$ & $\begin{array}{r}.46 \\
.63 \\
1.58 \\
.77 \\
.17 \\
1.23 \\
.22 \\
.27\end{array}$ \\
\hline \multirow[t]{2}{*}{91} & $\begin{array}{l}\text { Little Hoko } \\
\text { River }\end{array}$ & ...Do & 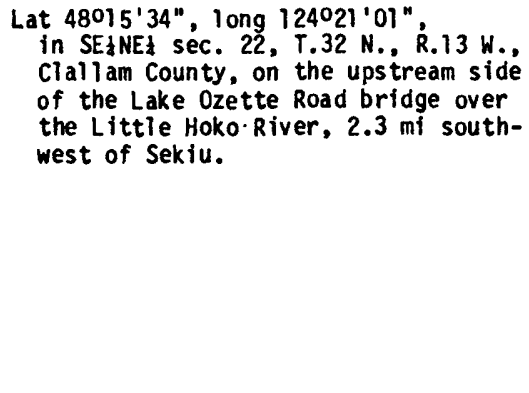 & 11.5 & $\begin{array}{l}7-20-62 \\
8-30-62 \\
5-23-63 \\
7-25-63 \\
10-1-63 \\
8-11-64 \\
9-8-65 \\
8-16-66 \\
12-15-71 \\
2-9-72 \\
4-12-72 \\
6-13-72 \\
8-8-72 \\
8-28-73\end{array}$ & $\begin{array}{c}4.25 \\
6.28 \\
10.2 \\
21.3 \\
3.79 \\
10.9 \\
2.24 \\
3.36 \\
122 \\
108 \\
119 \\
4.07 \\
3.64 \\
2.04\end{array}$ \\
\hline & \multicolumn{6}{|c|}{ Falls Creek Basin } \\
\hline \multirow[t]{2}{*}{92} & Falls Creek & Clallam Bay & $\begin{array}{l}\text { SEiNWI sec. } 19, \text { T.32 N., R.12 W., } \\
\text { at Highway } 9 A \text { crossing at Sekiu. }\end{array}$ & 1.43 & $\begin{array}{l}7-19-62 \\
8-30-62\end{array}$ & $\begin{array}{l}.16 \\
.10\end{array}$ \\
\hline & \multicolumn{6}{|c|}{ Clallam River Basin } \\
\hline 93 & Clallam River & ...Do & $\begin{array}{l}\text { NEISWI sec. 33, T.32 N., R. } 12 \text { W., } \\
\text { at U.S. Highway } 112, \text { i mi above } \\
\text { Charley Creek, and } 2 \text { mi south of } \\
\text { of Clall am Bay. }\end{array}$ & 137 & $\begin{array}{l}5-22-63 \\
7-24-62 \\
10-1-63 \\
7-28-64 \\
9-8-65 \\
8-16-66\end{array}$ & $\begin{array}{l}14.2 \\
40.4 \\
3.55 \\
17.3 \\
2.71 \\
6.09\end{array}$ \\
\hline
\end{tabular}


TABLE 9.--Records of miscellaneous stream-flow measurement sites in Clallam County--continued

\begin{tabular}{|c|c|c|c|c|c|c|}
\hline $\begin{array}{l}\text { Site } \\
\text { number }\end{array}$ & Stream & Tributary to & Location & $\begin{array}{l}\text { Drainage } \\
\text { area } \\
\text { (sq mi) }\end{array}$ & Meas & $\begin{array}{l}\text { ements } \\
\text { Discharge } \\
\text { (cubic feet } \\
\text { per second) }\end{array}$ \\
\hline 94 & Charley Creek & Clallam River & $\begin{array}{l}\text { SW 1SWH sec. 28, T.32 N., R.12 W., } \\
\text { at U.S. Highway } 112, i \text { mi south } \\
\text { of Ciallam County. }\end{array}$ & 5.23 & $\begin{array}{l}7-19-62 \\
8-30-62 \\
5-22-63 \\
7-24-63 \\
10-1-63 \\
7-28-64 \\
9-8-65 \\
8-16-66\end{array}$ & $\begin{array}{l}1.19 \\
1.15 \\
4.13 \\
7.48 \\
.94 \\
5.30 \\
.40 \\
.72\end{array}$ \\
\hline \multirow[t]{2}{*}{95} & Clallam River & Clallam Bay & Near East Clallam & -- & $\begin{array}{l}3-29-01 \\
6-21-01 \\
8-20-01\end{array}$ & $\begin{array}{r}100 \\
12 \\
6\end{array}$ \\
\hline & \multicolumn{6}{|c|}{ Pysht River Basin } \\
\hline 96 & Pysht River & $\begin{array}{l}\text { Strait of Juan } \\
\text { de Fuca }\end{array}$ & $\begin{array}{l}\text { SE iNW \& sec. } 23, \text { T.31 N., R.12 W., } \\
\text { at Burnt Mountain Road crossing, } \\
41 / 2 \mathrm{mi} \text { southwest of Pysht. }\end{array}$ & 10.2 & $\begin{array}{l}8-29-62 \\
5-22-63 \\
7-24-63 \\
10-1-63 \\
7-28-64 \\
9-9-65 \\
8-16-66\end{array}$ & $\begin{array}{c}7.21 \\
13.1 \\
33.5 \\
2.75 \\
14.6 \\
2.71 \\
5.13\end{array}$ \\
\hline 97 & Green Creek & Pysht River & $\begin{array}{l}\text { SW } W_{1} N W_{f} \text { Sec. } 14, T .31 \text { N., R.12 W., at } \\
\text { Sta te Highway } 112,1 \text { mi above mouth } \\
\text { and } 41 / 2 \text { mi southwest of Pysht. }\end{array}$ & 1.96 & $\begin{array}{l}8-29-62 \\
5-22-63 \\
7-24-63 \\
10-1-63 \\
7-28-64 \\
9-8-65 \\
8-16-66\end{array}$ & $\begin{array}{r}.58 \\
1.90 \\
4.29 \\
.26 \\
1.68 \\
.17 \\
.33\end{array}$ \\
\hline 98 & ...Do & $\ldots D 0$ & $\begin{array}{l}\text { SWl SEt sec. } 14, T .31 \text { N., R. } 12 \mathrm{~W} . \text {, } \\
\text { at Sta te Highway } 112 \text { at mouth, } \\
4 \text { mi southwest of Pysht. }\end{array}$ & 5.28 & $\begin{array}{l}7-19-62 \\
8-29-62 \\
5-22-63 \\
7-24-63 \\
10-1-63 \\
7-28-64 \\
9-8-65 \\
8-16-66\end{array}$ & $\begin{array}{r}1.17 \\
.92 \\
4.89 \\
8.67 \\
.58 \\
3.27 \\
.25 \\
.56\end{array}$ \\
\hline 99 & Pysht River & $\begin{array}{l}\text { Strait of Juan } \\
\text { de Fuca }\end{array}$ & Sec. 13, T.31 N., R.12 W., at the Forks & -- & $\begin{array}{l}5-29-01 \\
6-21-01 \\
9-20-01\end{array}$ & $\begin{array}{r}114 \\
29 \\
4\end{array}$ \\
\hline 100 & $\begin{array}{l}\text { South Fork } \\
\text { Pysht River }\end{array}$ & Pysht River & Sec. 13, T.31 N., R.12 W., at the Forks & -- & $6-27-01$ & 19 \\
\hline
\end{tabular}


TABLE 9.--Records of miscellaneous stream-flow measurenent sites in Clallam County--continued

\begin{tabular}{|c|c|c|c|c|c|c|}
\hline $\begin{array}{l}\text { Site } \\
\text { number }\end{array}$ & Stream & Tributary to & Location & $\begin{array}{l}\text { Drainage } \\
\text { area } \\
\text { (sq mi) }\end{array}$ & Meas & $\begin{array}{l}\text { rements } \\
\text { Discharge } \\
\text { (cubic feet } \\
\text { per second) }\end{array}$ \\
\hline \multirow[t]{2}{*}{101} & Pysht River & $\begin{array}{l}\text { Strait of Juan } \\
\text { de Fuca }\end{array}$ & 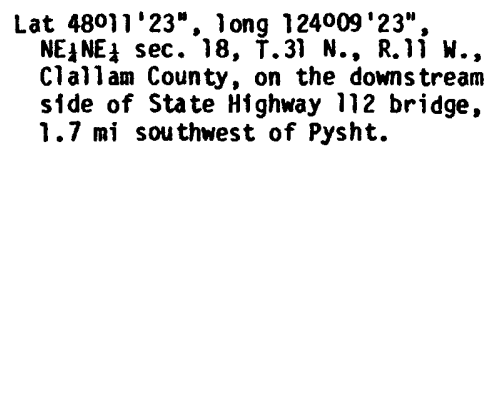 & 37.6 & $\begin{array}{l}7-19-62 \\
8-29-62 \\
5-22-63 \\
7-24-63 \\
9-30-63 \\
7-28-64 \\
9-8-65 \\
8-16-66 \\
12-15-71 \\
2-8-72 \\
4-11-72 \\
6-12-72 \\
8-7-72 \\
8-28-73\end{array}$ & $\begin{array}{c}9.74 \\
9.65 \\
38.0 \\
61.6 \\
4.20 \\
29.5 \\
3.31 \\
7.45 \\
366 \\
560 \\
285 \\
14.4 \\
8.14 \\
4.29\end{array}$ \\
\hline & \multicolumn{6}{|c|}{ Jim Creek Basin } \\
\hline \multirow[t]{2}{*}{--} & Jim Creek & $\begin{array}{l}\text { Strait of Juan } \\
\text { de Fuca }\end{array}$ & $\begin{array}{l}\text { SH \& sec. } 13, \text { T.31 N., R.11 W., } \\
\text { at State Highway } 112,3 \mathrm{mi} \\
\text { southeast of Pysht. }\end{array}$ & 3.70 & $\begin{array}{l}7-19-62 \\
8-29-62 \\
5-22-63 \\
7-24-63 \\
9-30-63 \\
7-28-64 \\
9-7-65 \\
8-16-66\end{array}$ & $\begin{array}{l}.16 \\
.09 \\
2.56 \\
.53 \\
.07 \\
.39 \\
.06 \\
.04\end{array}$ \\
\hline & \multicolumn{6}{|c|}{ - Joe Creek Basin } \\
\hline \multirow[t]{2}{*}{--} & Joe Creek & $\ldots D O$ & $\begin{array}{l}\text { NWINEt sec. } 24, T .31 \mathrm{~N} ., \mathrm{R} .11 \mathrm{H} \text {., } \\
\text { at State Highway } 112,1 / 2 \text { mi above } \\
\text { mouth, and } 31 / 2 \mathrm{mi} \text { southeast of } \\
\text { Pysht. }\end{array}$ & 1.51 & $\begin{array}{l}7-19-62 \\
8-29-62 \\
5-22-63 \\
7-24-63 \\
9-30-63 \\
7-28-64 \\
9-7-65 \\
8-16-66\end{array}$ & $\begin{array}{r}.17 \\
.08 \\
1.48 \\
.38 \\
.06 \\
.29 \\
.42 \\
.05\end{array}$ \\
\hline & \multicolumn{6}{|c|}{ Deep Creek Basin } \\
\hline-- & Deep Creek & $\ldots D O$ & $\begin{array}{l}N W_{1} N W_{1} \text { sec. } 20, T .31 \mathrm{~N} ., \text { R. } 10 \mathrm{~W} \text {., } \\
\text { at State } H i \text { ghway } 112,1 / 4 \mathrm{mi} \text { above } \\
\text { mouth and } 4 \text { mi west of Twin. }\end{array}$ & 17.3 & $\begin{array}{l}7-19-62 \\
8-29-62 \\
5-22-63 \\
7-24-63 \\
9-9-63 \\
7-28-64 \\
9-7-65 \\
8-16-66\end{array}$ & $\begin{array}{c}6.32 \\
4.36 \\
19.5 \\
20.3 \\
3.81 \\
11.9 \\
3.33 \\
4.65\end{array}$ \\
\hline
\end{tabular}


TABLE 9.--Records of miscellaneous stream-flow measurement sites in Clallam County--continued

\begin{tabular}{|c|c|c|c|c|c|c|}
\hline $\begin{array}{l}\text { Site } \\
\text { number } \\
\end{array}$ & Stream & Tributary to & Location & $\begin{array}{l}\text { Drainage } \\
\text { area } \\
\text { (sq mi) } \\
\end{array}$ & $\begin{array}{l}\text { Measu } \\
\text { Date }\end{array}$ & $\begin{array}{l}\text { rements } \\
\text { Discharge } \\
\text { (cubic feet } \\
\text { per second) }\end{array}$ \\
\hline \multirow[t]{2}{*}{101} & Pysht River & $\begin{array}{l}\text { Strait of Juan } \\
\text { de Fuca }\end{array}$ & $\begin{array}{l}\text { Lat } 480^{\circ} 11^{\prime} 23^{\prime \prime} \text {, long } 124009^{\prime} 23^{\prime \prime} \text {, } \\
\text { NEINEI sec. } 18 \text {, T.31 N., R. } 11 \text { W., } \\
\text { Clallam County, on the downstream } \\
\text { side of State Highway } 112 \text { bridge, } \\
1.7 \mathrm{mi} \text { southwest of Pysht. }\end{array}$ & 37.6 & $\begin{array}{l}7-19-62 \\
8-29-62 \\
5-22-63 \\
7-24-63 \\
9-30-63 \\
7-28-64 \\
9-8-65 \\
8-16-66 \\
12-15-71 \\
2-8-72 \\
4-11-72 \\
6-12-72 \\
8-7-72\end{array}$ & $\begin{array}{c}9.74 \\
9.65 \\
38.0 \\
61.6 \\
4.20 \\
29.5 \\
3.31 \\
7.45 \\
366 \\
560 \\
285 \\
14.4 \\
8.14\end{array}$ \\
\hline & \multicolumn{6}{|c|}{ Jim Creek Basin } \\
\hline \multirow[t]{2}{*}{-} & Jim Creek & $\begin{array}{l}\text { Strait of Juan } \\
\text { de Fuca }\end{array}$ & $\begin{array}{l}\text { SW } 1 \text { sec. } 13 \text { T. } 31 \mathrm{~N}_{\text {., R. R. } 11 \mathrm{~W} . \text {., }} \\
\text { at State Highway } 112,3 \mathrm{mi} \\
\text { southeast of Pysht. }\end{array}$ & 3.70 & $\begin{array}{l}7-19-62 \\
8-29-62 \\
5-22-63 \\
7-24-63 \\
9-30-63 \\
7-28-64 \\
9-7-65 \\
8-16-66\end{array}$ & $\begin{array}{l}.16 \\
.09 \\
2.56 \\
.53 \\
.07 \\
.39 \\
.06 \\
.04\end{array}$ \\
\hline & \multicolumn{6}{|c|}{ Joe Creek Basin } \\
\hline \multirow[t]{2}{*}{-} & Joe Creek & ...Do & $\begin{array}{l}\text { NWzNEX sec. } 24, T .31 \text { N., R.11 W., } \\
\text { at State Highway } 112,1 / 2 \mathrm{mi} \text { above } \\
\text { mouth, and } 31 / 2 \mathrm{mi} \text { southeast of } \\
\text { Pysht. }\end{array}$ & 1.51 & $\begin{array}{l}7-19-62 \\
8-29-62 \\
5-22-63 \\
7-24-63 \\
9-30-63 \\
7-28-64 \\
9-7-65 \\
8-16-66\end{array}$ & $\begin{array}{l}.17 \\
.08 \\
i .48 \\
.38 \\
.06 \\
.29 \\
.42 \\
.05\end{array}$ \\
\hline & \multicolumn{6}{|c|}{ Deep Creek Basín } \\
\hline-- & Deep Creek & ...Do & $\begin{array}{l}\text { NWt NWt sec. } 20, T .31 \text { N., R. } 10 \mathrm{~W} \text {., } \\
\text { at State Highway } 112,1 / 4 \mathrm{mi} \text { above } \\
\text { mouth and } 4 \text { mi west of Twin. }\end{array}$ & 17.3 & $\begin{array}{l}7-19-62 \\
8-29-62 \\
5-22-63 \\
7-24-63 \\
9-9-63 \\
7-28-64 \\
9-7-65 \\
8-16-66\end{array}$ & $\begin{array}{c}6.32 \\
4.36 \\
19.5 \\
20.3 \\
3.81 \\
11.9 \\
3.33 \\
4.65\end{array}$ \\
\hline
\end{tabular}


TABLE 9.--Records of miscellaneous stream-flow measurement sites in Clallam County--continued

\begin{tabular}{|c|c|c|c|c|c|c|}
\hline $\begin{array}{l}\text { Site } \\
\text { number }\end{array}$ & Stream & Tributary to & Location & $\begin{array}{l}\text { Drainage } \\
\text { area } \\
\text { (sq mi) } \\
\end{array}$ & Meas & $\begin{array}{l}\text { rements } \\
\text { Discharge } \\
\text { (cubic feet } \\
\text { per second) }\end{array}$ \\
\hline & \multicolumn{6}{|c|}{ West Twin River Basin } \\
\hline \multirow[t]{2}{*}{102} & West Twin River & ...Do & $\begin{array}{l}\text { NW } 4 \text { SE } 1 \text { sec. } 23, T .31 \text { N., R.10 W., } \\
\text { at State Highway } 112 \text { at Twin. }\end{array}$ & 12.8 & $\begin{array}{l}7-19-62 \\
8-29-62 \\
5-22-63 \\
7-24-63 \\
9-9-63 \\
7-28-64 \\
9-7-65 \\
8-16-66\end{array}$ & $\begin{array}{c}5.83 \\
5.53 \\
17.5 \\
16.1 \\
3.81 \\
9.86 \\
3.00 \\
5.07\end{array}$ \\
\hline & \multicolumn{6}{|c|}{ East Twin River Basin } \\
\hline \multirow[t]{2}{*}{103} & East Twin River & ...Do & 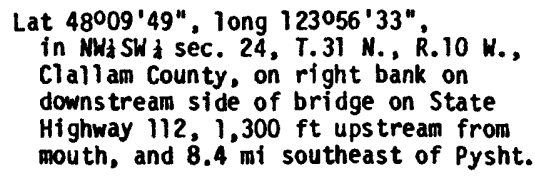 & 14.0 & $\begin{array}{l}8-28-73 \\
6-15-77\end{array}$ & $\begin{array}{l}3.86 \\
11.3\end{array}$ \\
\hline & \multicolumn{6}{|c|}{ Murdock Creek Basin } \\
\hline \multirow[t]{2}{*}{104} & Murdock Creek & ...Do & $\begin{array}{l}\text { SW } 1 \text { sec. } 29, \text { T.31 N., R. } 9 \text { W., at } \\
\text { private road crossing, } 1 / 2 \text { mi above } \\
\text { mouth, } 4 \text { mi east of Twin. }\end{array}$ & 2.26 & $\begin{array}{l}7-19-62 \\
8-30-62 \\
5-21-63 \\
7-23-63 \\
9-9-63 \\
7-27-64 \\
9-7-65 \\
8-16-66\end{array}$ & $\begin{array}{l}.08 \\
.06 \\
1.37 \\
.42 \\
.02 \\
.07 \\
.01 \\
.03\end{array}$ \\
\hline & \multicolumn{6}{|c|}{ Lyre River Basin } \\
\hline 105 & Fairholm Creek & Lake Crescent & $\begin{array}{l}\text { NEt sec. } 30, \text { T. } 30 \mathrm{~N} ., R_{.9} \mathrm{~W} \text {., } 50 \mathrm{ft} \\
\text { above mouth at Fairhoim. }\end{array}$ & 3.81 & $\begin{array}{l}7-17-62 \\
8-29-62 \\
5-22-63 \\
7-23-63 \\
9-9-63 \\
7-28-64 \\
9-8-65 \\
8-17-66\end{array}$ & $\begin{array}{r}.37 \\
.23 \\
1.10 \\
.37 \\
.23 \\
.41 \\
.20 \\
.27\end{array}$ \\
\hline 107 & Lapoel Creek & Lake Crescent & $\begin{array}{l}\text { Sut NWt sec. 32, T. } 30 \text { N., R.9 W., } \\
\text { at U.S. Highway } 101 \text { crossing } \\
21 / 2 \text { mi east of Fairholm. }\end{array}$ & 1.15 & $\begin{array}{l}7-17-62 \\
8-29-62 \\
5-22-63 \\
7-23-63 \\
9-9-63 \\
7-28-64 \\
9-8-65 \\
8-17-66\end{array}$ & $\begin{array}{r}2.92 \\
.76 \\
6.42 \\
2.61 \\
.72 \\
5.90 \\
.63 \\
1.11\end{array}$ \\
\hline 108 & Aurora Creek & ...Do & $\begin{array}{l}\text { SHA NE sec. } 33, T .30 \text { N., R.9 W. at } \\
\text { at U.S. Highway } 101 \text { crossing } 4 \text { mi } \\
\text { east of Fairholm. }\end{array}$ & .61 & $\begin{array}{l}7-17-62 \\
5-21-63 \\
7-23-63 \\
9-9-63 \\
7-28-64 \\
9-8-65 \\
8-17-66\end{array}$ & $\begin{array}{l}0 \\
0 \\
0 \\
0 \\
0 \\
0 \\
0\end{array}$ \\
\hline
\end{tabular}


TABLE 9.--Records of miscellaneous stream-flow measurement sites in $\mathrm{Clallam}$ County--continued

\begin{tabular}{|c|c|c|c|c|c|c|}
\hline $\begin{array}{l}\text { Site } \\
\text { number }\end{array}$ & Stream & Iributary to & Location & $\begin{array}{l}\text { Orainage } \\
\text { area } \\
(\mathrm{sq} \mathrm{mi}) \\
\end{array}$ & Meas & $\begin{array}{l}\text { rements } \\
\text { Oischarge } \\
\text { (cubic feet } \\
\text { per second) }\end{array}$ \\
\hline 109 & Smith Creek & ...Do & $\begin{array}{l}\text { SWl NWA sec. } 34, \text { T. } 30 \text { N., R.9 W., } \\
\text { at U.S. Highway } 101 \text { crossing } \\
5 \text { mi southeast of Fairholm. }\end{array}$ & 1.38 & $\begin{array}{l}7-16-62 \\
5-21-63 \\
7-23-63 \\
9-9-63 \\
9-8-65 \\
8-17-66\end{array}$ & $\begin{array}{l}0 \\
7.64 \\
0 \\
0 \\
0 \\
0\end{array}$ \\
\hline 111 & Falls Creek & Barnes Creek & $\begin{array}{l}\text { SE\&NEt sec. } 35, T .30 \mathrm{~N} . \text {. R.9 W., } \\
20 \mathrm{ft} \text { above mouth } 6 \mathrm{mi} \text { southeast } \\
\text { of Fairholm. }\end{array}$ & -- & $9-16-64$ & .45 \\
\hline 112 & Barnes Creek & Lake Crescent & 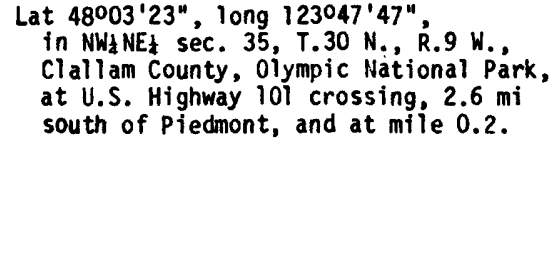 & 15.7 & $\begin{array}{l}7-16-62 \\
8-27-62 \\
5-21-63 \\
7-23-63 \\
9-9-63 \\
7-28-64 \\
9-7-65 \\
8-17-66 \\
8-27-73\end{array}$ & $\begin{array}{c}23.3 \\
8.96 \\
76.6 \\
27.5 \\
7.91 \\
38.6 \\
7.21 \\
13.3 \\
5.80\end{array}$ \\
\hline 115 & Lyre River & $\begin{array}{l}\text { Strait of Juan } \\
\text { de Fuca }\end{array}$ & $\begin{array}{l}\text { SWlNWL sec. } 14, \text { T. } 30 \mathrm{~N} . \text { R. R W. } \\
\text { at road crossing over outlet of } \\
\text { Lake Crescent, } 3 / 4 \mathrm{mi} \text { west of. Piedmont }\end{array}$ & 48.6 & $\begin{array}{l}7-16-62 \\
8-27-62 \\
5-21-63 \\
7-23-63 \\
9-9-63 \\
7-28-64 \\
9-7-65 \\
8-16-66\end{array}$ & $\begin{array}{c}96.6 \\
65.5 \\
186 \\
112 \\
48.9 \\
156 \\
41.3 \\
67.5\end{array}$ \\
\hline 116 & ...Do & $\ldots .00$ & $\begin{array}{l}\text { South line of NEt sec. } 15, \text { T. } 30 \mathrm{~N} . \text {, } \\
\text { R.9 W., at railroad bridge below } \\
\text { Lake Crescent at gaging station } \\
\text { "at Piedmont," (operated Oct. } 1917 \\
\text { to Sept. (927). }\end{array}$ & 48.5 & $\begin{array}{l}8-31-17 \\
9-26-58\end{array}$ & $\begin{array}{l}67.8 \\
39.4\end{array}$ \\
\hline \multirow[t]{2}{*}{117} & Susie Creek & Lyre River & $\begin{array}{l}\text { NW\&SE sec. } 28, T .31 \mathrm{~N}, \mathrm{R}_{\mathrm{S}} 9 \mathrm{~W} . \text {, } \\
\text { at State Highway } 112,1 / 4 \mathrm{mi} \text { above } \\
\text { mouth, and } 3 \text { mi west of Disque. }\end{array}$ & 3.56 & $\begin{array}{l}7-19-62 \\
8-30-62 \\
5-21-63 \\
7-23-63 \\
9-9-63 \\
7-27-64 \\
7-7-65 \\
8-16-66\end{array}$ & $\begin{array}{r}.54 \\
.30 \\
2.79 \\
2.70 \\
.33 \\
.81 \\
.22 \\
.40\end{array}$ \\
\hline & \multicolumn{6}{|c|}{ Field Creek Basin } \\
\hline 118 & Field Creek & $\begin{array}{l}\text { Strait of Juan } \\
\text { de Fuca }\end{array}$ & $\begin{array}{l}\text { Center of SE \& sec. } 26, T .31 \text { N., R.9 W., } \\
\text { at State Highway } 112, i \mathrm{mi} \text { northwest- } \\
\text { of Disque. }\end{array}$ & 3.90 & $\begin{array}{l}7-19-62 \\
8-30-62 \\
5-21-63 \\
7-23-63 \\
9-9-63 \\
7-27-64 \\
9-7-65 \\
8-16-66\end{array}$ & $\begin{array}{r}.56 \\
.13 \\
1.84 \\
1.04 \\
.12 \\
.26 \\
.08 \\
.14\end{array}$ \\
\hline
\end{tabular}


TABLE 9.--Records of miscellaneous stream-flow measurement sites in $\mathrm{Cla}$ allam County--continued

\begin{tabular}{|c|c|c|c|c|c|c|}
\hline $\begin{array}{l}\text { Site } \\
\text { number } \\
\end{array}$ & Stream & Tributary to & Location & $\begin{array}{l}\text { Drainage } \\
\text { area } \\
\text { (sq } \mathrm{mi}) \\
\end{array}$ & Meas & $\begin{array}{l}\text { rements } \\
\text { Discharge } \\
\text { (cubtc feet } \\
\text { per second) }\end{array}$ \\
\hline & \multicolumn{6}{|c|}{ Whiskey Creek Basin } \\
\hline 119 & Whiskey Creek & ...Do & $\begin{array}{l}\text { NE INE sec. } 36, \text { T.31 N., R.9 W. } \\
\text { at State Highway } 112,1 / 4 \mathrm{mi} \text { east } \\
\text { of Disque. }\end{array}$ & 1.63 & $\begin{array}{l}7-19-62 \\
8-29-62 \\
5-21-63 \\
7-23-63 \\
9-9-63 \\
7-27-64 \\
9-7-65 \\
8-16-66\end{array}$ & $\begin{array}{r}.47 \\
.18 \\
1.18 \\
.56 \\
.19 \\
.32 \\
.13 \\
.19\end{array}$ \\
\hline
\end{tabular}

\section{Salt Creek Basin}

120 Salt Creek $\quad$...Do

Lat $48007^{\prime} 08^{\prime \prime}$, long $123039^{\prime} 43^{\prime \prime}$,

in NW\& SE t sec. 2, T.30 N. R.8 W.

Clallam County, at State Highway 112 , and $3.5 \mathrm{mi}$ southeast of Joyce.

4.09

8-18-52

9-15-52

$7-16-62$

8-29-62

$5-21-63$

$7-23-63$

$9-9-63$

7-27-64

$9-7-65$

8-15-66

$8-27-73$

121 Salt Creek

Salt Creek

NEtSWl sec. 2, T.30 N., R.8 W., at at State Highway $112,1 / 2 \mathrm{mi}$ east

1.88

$7-16-62$

$8-29-62$

$5-21-63$

7-23-63

9-9-63

7-27-64

$9-7-65$

8-15-66

.40

.34

.80

1.68

.97

.43

.64

.43

.50
.28

tributary

122 Salt Creek

Crescent Bay

NWANWt sec. 2, T.30 N., R.8 W., $1 / 4 \mathrm{mi}$ above West Fork and $8 \mathrm{mt}$ west of Port Angeles.

7.07

4-25-61

6-15-61

$7-10-61$

$7-25-61$

$8-9-61$

$9-9-61$

.26

.16

1.03

.63

.11
.23

.09

.14

123 Salt Creek

Salt Creek

SWANEA SeC. 3, T.30 N., R.8 W.,

$9-15-52$

5.90

1.72

.96

.70

.49

at Piedmont Road crossing at Ramapo.

2.00

$8-9-61$

.09

NWłNWł sec. 3, T.30 N., R.8 W., at Piedmont Road crossing, $1 / 2 \mathrm{~m} 1$

1.49

6-18-52

$7-10-52$

$7-29-52$

8-13-52

$8-18-52$

$9-15-52$

$10-7-52$

$8-9-61$ 
TABLE 9.--Records of miscellaneous stream-flow measurement sites in Clallam County--continued

\begin{tabular}{|c|c|c|c|c|c|c|}
\hline $\begin{array}{l}\text { Site } \\
\text { number }\end{array}$ & Stream & Tributary to & Location & $\begin{array}{l}\text { Orainage } \\
\text { area } \\
\text { (sq } \mathrm{m} 1) \\
\end{array}$ & Measu & $\begin{array}{l}\text { rements } \\
\text { Discharge } \\
\text { (cubic feet } \\
\text { per second) }\end{array}$ \\
\hline \multirow[t]{2}{*}{125} & Salt Creek & $\begin{array}{l}\text { Strait of Juan } \\
\text { de Fuca }\end{array}$ & $\begin{array}{l}\text { SWINWA sec. } 27, T .31 \mathrm{~N} ., \mathrm{R.} 8 \mathrm{~W} . \\
1 \mathrm{mi} \text { above mouth and } 2 \mathrm{mi} \text { north } \\
\text { of Ramapo. }\end{array}$ & 15.9 & $\begin{array}{l}6-18-52 \\
7-10-52 \\
7-29-52 \\
8-13-52 \\
8-18-52 \\
9-15-52 \\
10-7-52 \\
8-9-61 \\
7-16-62 \\
8-30-62 \\
5-21-63 \\
7-23-63 \\
9-9-63 \\
7-27-64 \\
9-7-65 \\
8-15-66\end{array}$ & $\begin{array}{l}2.99 \\
1.10 \\
1.65 \\
.52 \\
.88 \\
.73 \\
.60 \\
1.02 \\
2.84 \\
1.03 \\
7.74 \\
3.86 \\
1.15 \\
1.77 \\
.93 \\
1.12\end{array}$ \\
\hline & \multicolumn{6}{|c|}{ Coville Creek Basin } \\
\hline \multirow[t]{2}{*}{126} & Coville Creek & Freshwater Bay & $\begin{array}{l}\text { SWISE sec. } 5, \text { T. } 30 \mathrm{~N} . \text {. R.7 W., } \\
\text { at State Highway } 9 A \text { (Piedmont Road). }\end{array}$ & 3.67 & $\begin{array}{l}8-18-52 \\
9-15-52\end{array}$ & $\begin{array}{l}0 \\
0\end{array}$ \\
\hline & \multicolumn{6}{|c|}{ Elwha River Basin } \\
\hline 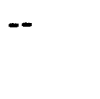 & Idaho Creek & Elwha River & $\begin{array}{l}\text { NEt sec. } 36, T .29 \mathrm{~N} ., \mathrm{R} .7 \mathrm{~W} . \mathrm{H} \\
200 \mathrm{ft} \text { east of road and } 2 \mathrm{mi} \\
\text { southeast of Hurricane } \mathrm{H} 111 \text { lookout. }\end{array}$ & - & $9-16-65$ & .01 \\
\hline 128 & Elwha River & Straft of Juan & $\begin{array}{l}\text { SEt sec. } 17, \text { T.29 N., R.7 W., } \\
\text { at powerpi ant. }\end{array}$ & 245 & $9-7-19$ & 53.2 \\
\hline 129 & ...Do & ...Do & $\begin{array}{l}\text { NEtNW \& sec. } 33 \mathrm{~T} .30 \mathrm{~N} ., \mathrm{R} .7 \mathrm{~W} \text {., } \\
\text { at gaging station "at McDonaid } \\
\text { bridge, near Port Angeles," } \\
\text { (operated Oct. } 1897 \text { to Dec. } 1902 \text {, } \\
\text { Oct. } 1918 \text { to } 1960 \text { ). }\end{array}$ & 269 & $\begin{array}{l}7-31-18 \\
8-12-18 \\
8-27-18 \\
9-16-18 \\
9-23-18 \\
9-24-18 \\
9-30-18\end{array}$ & $\begin{array}{l}832 \\
650 \\
526 \\
477 \\
346 \\
328 \\
475\end{array}$ \\
\hline 130 & Little River & Elwha River & $\begin{array}{l}\text { SEINEt sec. 28, T. } 30 \text { N., R.7 W., } \\
\text { OI ympic Hot Springs Road crossing, } \\
1,000 \mathrm{ft} \text { above mouth and } 7 \mathrm{mi} \\
\text { southwest of Port Angeles. }\end{array}$ & 23.0 & $\begin{array}{l}6-16-99 \\
3-15-00 \\
5-31-00 \\
6-27-00 \\
7-20-00 \\
8-22-00 \\
10-30-00 \\
2-28-01 \\
4-29-01 \\
9-26-01 \\
10-29-01 \\
11-27-01 \\
8-19-52 \\
9-17-52 \\
8-9-61\end{array}$ & $\begin{array}{r}84 \\
174 \\
68 \\
54 \\
28 \\
20 \\
52 \\
147 \\
55 \\
13 \\
20 \\
457 \\
13.6 \\
10.8 \\
14.8\end{array}$ \\
\hline
\end{tabular}


TABLE 9.--Records of miscellaneous stream-flow measurement sites in Clallam County--continued

\begin{tabular}{|c|c|c|c|c|c|c|}
\hline $\begin{array}{l}\text { Site } \\
\text { number }\end{array}$ & $\underline{\text { Stream }}$ & Tributary to & Location & $\begin{array}{l}\text { Drainage } \\
\text { area } \\
\text { (sq mi) }\end{array}$ & Measu & $\begin{array}{l}\text { rements } \\
\text { Discharge } \\
\text { (cubic feet } \\
\text { per second) }\end{array}$ \\
\hline 133 & Indian Creek & Lake Aldwell & 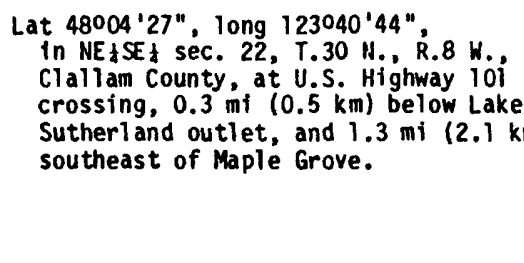 & $\begin{array}{l}8.33 \\
\mathrm{~km})\end{array}$ & $\begin{array}{l}7-16-62 \\
8-27-62 \\
5-21-63 \\
7-23-63 \\
9-9-63 \\
7-28-64 \\
9-7-65 \\
8-16-66 \\
8-27-73\end{array}$ & $\begin{array}{l}11.5 \\
9.98 \\
18.2 \\
13.6 \\
9.05 \\
19.6 \\
10.3 \\
10.4 \\
13.7\end{array}$ \\
\hline 134 & Indian Creek & $\ldots D 0$ & $\begin{array}{l}\text { SE d dNW } 1 \text { sec. } 28, \text { T. } 30 \text { N., R.7 W., } \\
\text { near mouth. }\end{array}$ & 20 & $6-16-99$ & 45 \\
\hline 135 & Elwha River & $\begin{array}{l}\text { Strait of Juan } \\
\text { de Fuca }\end{array}$ & $\begin{array}{l}\text { Nł sec. } 28, \text { T. } 30 \text { N., R.7 W., below } \\
\text { Indian Creek. }\end{array}$ & 312 & $10-25-98$ & 586 \\
\hline 137 & ...Do & $\ldots D 0$ & $\begin{array}{l}\text { SWINEl sec. 15, T.30 N., R.7 W., } \\
\text { below Olympic powerplant at gaging } \\
\text { station "near Port Angeles," } \\
\text { (operated May to Sept. 191i). }\end{array}$ & 315 & $\begin{array}{l}4-10-11 \\
10-24-11 \\
1-20-12 \\
6-10-12\end{array}$ & $\begin{array}{r}1,210 \\
445 \\
1,680 \\
2,340\end{array}$ \\
\hline 138 & $\begin{array}{l}\text { Port Angeles } \\
\text { Industrial } \\
\text { Canal }\end{array}$ & $\begin{array}{l}\text { Elwha River } \\
\text { (diverts from } \\
\text { right bank) }\end{array}$ & $\begin{array}{l}\text { NE } \text { sec. } 10, \text { T. } 30 \text { N., R. } 7 \mathrm{~W} \text {., at } \\
\text { road bridge and staff gage just } \\
\text { below rafliroad bridge. }\end{array}$ & -- & $\begin{array}{l}8-28-51 \\
10-18-51 \\
11-29-51 \\
12-5-51 \\
1-17-52 \\
2-28-52 \\
4-10-52 \\
5-15-52 \\
6-17-52 \\
8-7-52 \\
9-16-52 \\
10-30-52 \\
12-4-52 \\
1-14-53 \\
3-12-53 \\
4-16-53 \\
5-20-53 \\
7-15-53 \\
8-25-53 \\
9-24-53 \\
10-28-53 \\
12-5-53 \\
1-8-54 \\
2-19-54 \\
3-23-54 \\
5-14-54 \\
6-23-54 \\
7-26-54\end{array}$ & $\begin{array}{l}125 \\
116 \\
175 \\
161 \\
117 \\
133 \\
138 \\
158 \\
167 \\
142 \\
115 \\
130 \\
122 \\
189 \\
122 \\
111 \\
145 \\
151 \\
116 \\
103 \\
108 \\
152 \\
159 \\
190 \\
120 \\
141 \\
138 \\
145\end{array}$ \\
\hline
\end{tabular}


TABLE 9.--Records of miscellaneous stream-flow measurement sites in Clallam County--continued

\begin{tabular}{|c|c|c|c|c|c|c|}
\hline $\begin{array}{l}\text { Site } \\
\text { number } \\
\end{array}$ & Stream & Iributary to & Location & $\begin{array}{l}\text { Drainage } \\
\text { area } \\
\text { (sq } \mathrm{m} 1) \\
\end{array}$ & $\begin{array}{l}\text { Meas } \\
\text { Date }\end{array}$ & $\begin{array}{l}\text { rements } \\
\text { Discharge } \\
\text { (cubic feet } \\
\text { per second) }\end{array}$ \\
\hline 139 & Elwha River & $\begin{array}{l}\text { Strait of Juan } \\
\text { de Fuca }\end{array}$ & $\begin{array}{l}\text { Lat } 48^{\circ 006} \text { '52", long } 123033^{\prime} 08^{\prime \prime}, \\
\text { in NWtNE } 1 \text { sec. } 10, \text { T.30 N., R.7 W., } \\
\text { Clallam County, OIympic National } \\
\text { Forest, } 1.8 \mathrm{mi} \text { downstream from } \\
\text { powerplant, } 3.1 \text { mi ups tream from } \\
\text { mouth, } 5.7 \text { mi west of Port Angeles } \\
\text { ferry terminal. }\end{array}$ & 318 & $\begin{array}{l}12-5-51 \\
1-17-52 \\
2-28-52 \\
4-10-52 \\
4-10-52 \\
5-15-52 \\
6-17-52 \\
8-6-52 \\
9-16-52 \\
10-7-54 \\
3-18-58 \\
4-23-58 \\
6-3-58 \\
7-21-58 \\
8-27-58 \\
10-2-58 \\
12-14-61 \\
3-6-63 \\
6-4-64 \\
10-28-64 \\
8-5-65 \\
1-20-66 \\
7-6-66 \\
1-24-67 \\
9-7-67 \\
2-13-68 \\
9-6-68 \\
4-16-69 \\
12-4-69 \\
6-16-70\end{array}$ & $\begin{array}{r}1,780 \\
505 \\
983 \\
913 \\
1,150 \\
1,970 \\
2,080 \\
1,020 \\
384 \\
954 \\
861 \\
861 \\
2,720 \\
951 \\
597 \\
288 \\
965 \\
1,310 \\
3,440 \\
409 \\
676 \\
1,350 \\
1,920 \\
2,020 \\
412 \\
2,630 \\
618 \\
1,730 \\
816 \\
1,890\end{array}$ \\
\hline \multirow[t]{2}{*}{141} & Unnamed tributary & $\begin{array}{l}\text { Elwha West } \\
\text { Sough }\end{array}$ & $\begin{array}{l}\text { Lat } 48008^{\prime} 40^{\prime \prime} \text {, long } 123033^{\prime} 17^{\prime \prime} \text {, } \\
\text { on south line of SWI SEd sec. } 27 \text {, } \\
\text { T.31 N., R.7 W., Clall lam County, } \\
\text { at road crossing, } 2.1 \text { mi northwest } \\
\text { of Port Angeles city limits. }\end{array}$ & -- & $\begin{array}{l}3-18-76 \\
4-28-76 \\
6-1-76 \\
7-7-76 \\
9-2-76 \\
11-2-76 \\
1-6-77 \\
2-2-77 \\
3-23-77\end{array}$ & $\begin{array}{l}.94 \\
.74 \\
1.15 \\
1.61 \\
.61 \\
.43 \\
1.42 \\
2.27 \\
1.29\end{array}$ \\
\hline & \multicolumn{6}{|c|}{ Dry Creek Basin } \\
\hline \multirow[t]{2}{*}{144} & Dry Creek & ...Do & $\begin{array}{l}\text { SWdNE } \text { sec. } 12, T .30 \text { N., R.7 W., } \\
\text { at State Highway } 9 A^{2}\end{array}$ & 2.69 & $\begin{array}{l}9-18-52 \\
9-15-52\end{array}$ & $\begin{array}{l}0 \\
0\end{array}$ \\
\hline & \multicolumn{6}{|c|}{ Tumwater Creek Basin } \\
\hline 145 & Tumwater Creek & $\begin{array}{l}\text { Port Angeles } \\
\text { Harbor }\end{array}$ & $\begin{array}{l}\mathrm{SE}_{4} \mathrm{SE}_{1} \text { sec. } 4, \mathrm{~T} .30 \mathrm{~N} ., \mathrm{R} 6 \mathrm{~W} \text {, at } \\
\text { at small wooden bridge, i,000 ft } \\
\text { above mouth, in Port Angeles. }\end{array}$ & 5.54 & $2-16-49$ & 490 \\
\hline
\end{tabular}


TABLE 9.--Records of miscellaneous stream-flow measurement sites in Clallam County--continued

\begin{tabular}{|c|c|c|c|c|c|c|}
\hline $\begin{array}{l}\text { Site } \\
\text { number }\end{array}$ & Stream & Tributary to & Location & $\begin{array}{l}\text { Drainage } \\
\text { area } \\
\text { (sq mi) }\end{array}$ & Meas & $\begin{array}{l}\text { ements } \\
\text { Discharge } \\
\text { (cubic feet } \\
\text { per second) }\end{array}$ \\
\hline \multirow[t]{2}{*}{146} & $\ldots D 0$ & $\ldots 00$ & $\begin{array}{l}\text { SEzNE \& sec. 4, T. } 30 \mathrm{~N} \text {., R. } 6 \mathrm{~W} \text {., at } \\
\text { at culvert, } 500 \mathrm{ft} \text { above mouth, } \\
\text { in Port Angeles. }\end{array}$ & 5.59 & $\begin{array}{l}6-18-52 \\
7-10-52 \\
7-29-52 \\
8-13-52 \\
8-19-52 \\
9-17-52 \\
10-7-52 \\
4-25-61 \\
6-15-61 \\
7-10-61 \\
7-25-61 \\
8-9-61 \\
9-9-61\end{array}$ & $\begin{array}{l}2.15 \\
1.39 \\
1.21 \\
.93 \\
1.04 \\
.94 \\
.85 \\
4.15 \\
2.42 \\
1.48 \\
1.16 \\
1.07 \\
1.18\end{array}$ \\
\hline & \multicolumn{6}{|c|}{ Valley Creek Basin } \\
\hline 147 & $\begin{array}{l}\text { East Valley } \\
\text { Creek }\end{array}$ & Valley Creek & $\begin{array}{l}\text { SWINW sec. } 22, T .30 \mathrm{~N} ., \text { R. } 6 \mathrm{~W} . \text {, } \\
\text { above earth dam, } 1.7 \text { mi south' of } \\
\text { of Port Angeles city } 1 \text { imits. }\end{array}$ & .40 & $1-15-61$ & 15.6 \\
\hline 148 & ...Do & ...Do & $\begin{array}{l}\text { SWINW sec. } 22, \text { T. } 30 \text { N., R. } 6 \text { W., } \\
300 \mathrm{ft} \text { bel ow earth dam, } i .7 \text { mi } \\
\text { south of Port Angeles city limits. }\end{array}$ & -- & $1-15-61$ & 61 \\
\hline \multirow[t]{2}{*}{150} & Valley Creek & $\begin{array}{l}\text { Port Angeles } \\
\text { Harbor }\end{array}$ & $\begin{array}{l}\text { SW sec. } 3, T .30 \mathrm{~N} ., \mathrm{R} .6 \mathrm{~W} . \\
200 \mathrm{ft} \text { above culvert and } 500 \mathrm{ft} \\
\text { above mouth, in Port Angeles. }\end{array}$ & 4.20 & $\begin{array}{l}8-19-52 \\
9-17-52 \\
8-9-61\end{array}$ & $\begin{array}{l}.31 \\
.23 \\
.34\end{array}$ \\
\hline & \multicolumn{6}{|c|}{ Peabody Creek Basin } \\
\hline \multirow[t]{2}{*}{151} & Peabody Creek & $\begin{array}{l}\text { Port Angeles } \\
\text { Harbor }\end{array}$ & $\begin{array}{l}\text { NWINEA sec. } 10, \text { T. } 30 \text { N., R. } 6 \text { W., at } \\
\text { culvert } 2 \text { blocks east of Lincoln } \\
\text { Street, } 1 / 4 \text { mi above mouth, in Port } \\
\text { Angeles. }\end{array}$ & 2.55 & $\begin{array}{l}2-16-49 \\
8-20-52\end{array}$ & $\begin{array}{l}279 \\
9.93\end{array}$ \\
\hline & \multicolumn{6}{|c|}{ Ennis Creek Basin } \\
\hline 152 & Ennis Creek & ...Do & 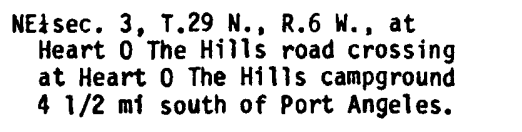 & - & $9-15-65$ & 2.44 \\
\hline 153 & Ennis Creek & $\ldots .00$ & 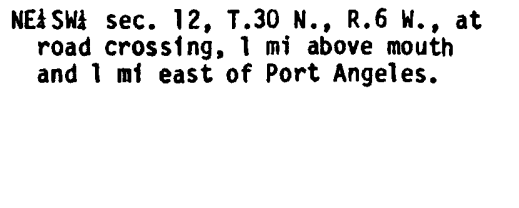 & 7.92 & $\begin{array}{l}11-6-52 \\
5-25-61 \\
6-15-61 \\
7-10-61 \\
7-25-61 \\
8-9-61 \\
9-9-61\end{array}$ & $\begin{array}{c}2.39 \\
18.0 \\
15.1 \\
8.95 \\
6.07 \\
4.98 \\
3.60\end{array}$ \\
\hline 154 & White Creek & Ennis Creek & $\begin{array}{l}\text { NEt SEt Sec. 11, T.30 N., R.6 W., } \\
\text { at U.S. Highway } 101 \text { crossing, at } \\
\text { east city I imits of Port Angeles. }\end{array}$ & 2.22 & $\begin{array}{l}8-19-52 \\
9-17-52 \\
9-9-6 !\end{array}$ & $\begin{array}{l}.08 \\
.11 \\
.05\end{array}$ \\
\hline
\end{tabular}


TABLE 9.--Records of miscellaneous stream-fl ow measurement sites in Clallam County--continued

\begin{tabular}{|c|c|c|c|c|c|c|}
\hline $\begin{array}{l}\text { Site } \\
\text { number }\end{array}$ & Stream & Tributary to & Location & $\begin{array}{l}\text { Drainage } \\
\text { area } \\
\text { (sq mi) }\end{array}$ & Meas & $\begin{array}{l}\text { ements } \\
\text { Discharge } \\
\text { (cubic feet } \\
\text { per second) }\end{array}$ \\
\hline \multirow[t]{2}{*}{155} & Ennis Creek & $\begin{array}{l}\text { Port Angeles } \\
\text { Harbor }\end{array}$ & $\begin{array}{l}\text { NEX NE } 1 \text { sec. } 11, \text { T.30 N., R. } 6 \mathrm{~W} \text {., } \\
\text { at rail road bridge at Rayonier } \\
\text { Company plant at Port Angeles. }\end{array}$ & 10.5 & $2-25-50$ & 1,980 \\
\hline & \multicolumn{6}{|c|}{ Lees Creek Basin } \\
\hline \multirow[t]{2}{*}{156} & Lees Creek & $\begin{array}{l}\text { Strait of Juan } \\
\text { de Fuca }\end{array}$ & $\begin{array}{l}\text { SEA SEt sec. } 12, T .30 \mathrm{~N} ., \text { R. } 6 \mathrm{~K} \text {., } \\
\text { U.S. Highway } 101 \text { crossing, } 2 \mathrm{mi} \\
\text { east of Port Angeles. }\end{array}$ & 4.77 & $8-9-61$ & .24 \\
\hline & \multicolumn{6}{|c|}{ Morse Creek Basin } \\
\hline 159 & Morse Creek & $\begin{array}{l}\text { Strait of Juan } \\
\text { de Fuca }\end{array}$ & $\begin{array}{l}\text { SWISWI sec. } 8, \text { T.30 N., R. } 5 \text { W., } \\
\text { near U.S. Highway } 10 i \text { crossing, } \\
4 \text { mi east of Port Angeles. }\end{array}$ & -- & $\begin{array}{l}6-3-99 \\
7-6-99 \\
9-15-99 \\
9-10-00 \\
3-8-01 \\
5-1-01 \\
8-1-01 \\
8-29-01 \\
9-28-01 \\
8-25-25 \\
5-25-61 \\
6-15-61 \\
7-10-61 \\
7-25-61 \\
8-9-61 \\
9-9-61\end{array}$ & $\begin{array}{c}242 \\
189 \\
62 \\
51 \\
173 \\
169 \\
122 \\
78 \\
50 \\
39.9 \\
201 \\
168 \\
90.1 \\
82.5 \\
39.7 \\
19.5\end{array}$ \\
\hline \multirow[t]{2}{*}{160} & ...Do & $\ldots D_{0}$ & $\begin{array}{l}\text { NEASWt sec. 8, T.30 N., R.5 W., } \\
1 / 4 \text { mi below U.S. Highway } 101 .\end{array}$ & 56.4 & $\begin{array}{l}7-10-52 \\
7-29-52 \\
8-13-52 \\
8-19-52 \\
9-17-52 \\
10-8-52\end{array}$ & $\begin{array}{c}104 \\
47.2 \\
30.0 \\
24.6 \\
10.5 \\
6.34\end{array}$ \\
\hline & \multicolumn{6}{|c|}{ Bagley Creek Basin } \\
\hline \multirow[t]{2}{*}{161} & Bagley Creek & $\ldots D_{0}$ & $\begin{array}{l}\text { NEANWA sec. } 16, \text { T.30 N., R.5 W., } \\
\text { U.S. Highway } 101 \text { crossing, } 5 \text { mi } \\
\text { east of Port Angeles. }\end{array}$ & 5.32 & $\begin{array}{l}9-10-47 \\
2-16-49 \\
8-19-52 \\
9-17-52 \\
8-9-61\end{array}$ & $\begin{array}{r}436.63 \\
.63 \\
.96 \\
.61\end{array}$ \\
\hline & \multicolumn{6}{|c|}{ Siebert Creek Basin } \\
\hline 162 & Siebert Creek & ...Do & $\begin{array}{l}\text { NWt SEt sec. } 14, \text { T.30 N., R. } 5 \text { W., } \\
\text { at gaging station "near Port } \\
\text { Angeles. }\end{array}$ & 16.1 & $2-16-49$ & 1,340 \\
\hline 164 & $\begin{array}{l}\text { Agnew Irrigation } \\
\text { Ditch diversion } \\
\text { from Dungeness }\end{array}$ & Siebert Creek & $\begin{array}{l}\text { NWtNWl sec. } 12, \text { T.29 N., R.4 W., } \\
4 \mathrm{mi} \text { southwest of Sequim. }\end{array}$ & -- & $\begin{array}{l}9-15-22 \\
9-13-45\end{array}$ & $\begin{array}{l}6.82 \\
9.05\end{array}$ \\
\hline
\end{tabular}


TABLE 9.--Records of miscellaneous stream-flow measurement sites in Clallam County--continued

\begin{tabular}{|c|c|c|c|c|c|c|}
\hline $\begin{array}{l}\text { Si te } \\
\text { number } \\
\end{array}$ & Stream & Iributary to & Location & $\begin{array}{l}\text { Drainage } \\
\text { area } \\
\text { (sq mi) }\end{array}$ & Measu & $\begin{array}{l}\text { rements } \\
\text { oischarge } \\
\text { (cubic feet } \\
\text { per second) }\end{array}$ \\
\hline 165 & Siebert Creek & $\begin{array}{l}\text { Strait of Juan } \\
\text { de Fuca }\end{array}$ & $\begin{array}{l}\text { SElSWt sec. 2, T. } 30 \text { N., R.5 W. } \\
0.1 \text { mi upstream of mouth, } 9.0 \mathrm{mi} \\
\text { northwest of Sequim. }\end{array}$ & 19.4 & $\begin{array}{l}9-11-78 \\
10-12-78 \\
11-13-78 \\
12-15-78 \\
1-15-79 \\
2-15-79 \\
3-16-79 \\
4-16-79 \\
5-14-79 \\
6-19-79 \\
7-16-79 \\
8-17-79 \\
9-18-79\end{array}$ & $\begin{array}{r}5.67 \\
2.87 \\
3.18 \\
10.7 \\
4.41 \\
32.4 \\
19.8 \\
19.9 \\
6.14 \\
3.83 \\
2.24 \\
1.96 \\
2.39\end{array}$ \\
\hline & & & McDonald Creek Basin & & & \\
\hline 166 & McDonald Creek & ...Do & $\begin{array}{l}\text { NE SW isec. } 30, T .30 \mathrm{~N} ., \text { R. } 4 \mathrm{~W} \text {., } \\
100 \mathrm{ft} \text { upstream of diversion from } \\
\text { Agnew Irrigation Ditch to strean, } \\
6.7 \mathrm{mi} \text { west of Sequim. }\end{array}$ & 13.0 & $\begin{array}{l}9-12-78 \\
10-12-78 \\
11-13-78 \\
12-14-78 \\
1-16-79 \\
2-16-79 \\
3-15-79 \\
4-17-79 \\
5-15-79 \\
6-19-79\end{array}$ & $\begin{array}{c}3.88 \\
1.88 \\
3.46 \\
10.1 \\
3.58 \\
16.4 \\
19.6 \\
27.0 \\
9.61 \\
3.43\end{array}$ \\
\hline 167 & $\begin{array}{l}\text { Agnew Irrigation } \\
\text { Ditch diversion } \\
\text { to McDonald Creek }\end{array}$ & McDonald Creek & $\begin{array}{l}\text { SE LNWI sec. } 30, \text { T. } 30 \text { N. , R.4 W., } \\
6.6 \text { mi west of Sequim. }\end{array}$ & -- & $\begin{array}{l}9-12-78 \\
10-12-78 \\
11-13-78 \\
12-14-78 \\
1-16-79 \\
2-16-79 \\
3-15-79 \\
4-17-79 \\
5-15-79 \\
6-19-79\end{array}$ & $\begin{array}{l}1.82 \\
3.62 \\
2.10 \\
3.11 \\
0 \\
1.81 \\
1.01 \\
0 \\
0 \\
5.25\end{array}$ \\
\hline 168 & McDonald Creek & $\begin{array}{l}\text { Strait of Juan } \\
\text { de Fuca }\end{array}$ & $\begin{array}{l}\text { SEt SE \& Sec. 18, T. } 30 \text { N., R.4 W., } \\
\text { above diversion fl ume above } \\
\text { U.S. Highway } 101 \text {. }\end{array}$ & 21.3 & $\begin{array}{l}7-11-52 \\
7-29-52 \\
9-11-52 \\
9-17-52 \\
10-8-52 \\
5-25-61 \\
6-15-61 \\
7-10-61 \\
7-25-61 \\
8-8-61 \\
9-9-61\end{array}$ & $\begin{array}{c}6.88 \\
5.93 \\
6.26 \\
6.04 \\
4.97 \\
24.7 \\
12.9 \\
10.3 \\
8.80 \\
9.10 \\
7.48\end{array}$ \\
\hline
\end{tabular}


TABLE 9.--Records of miscellaneous stream-flow measurement sites in $\mathrm{Clallam}$ County--continued

\begin{tabular}{|c|c|c|c|c|c|c|}
\hline $\begin{array}{l}\text { Site } \\
\text { number }\end{array}$ & Stream & Tributary to & Location & $\begin{array}{l}\text { Drainage } \\
\text { area } \\
\text { (sq } \mathrm{mi}) \\
\end{array}$ & Measu & $\begin{array}{l}\text { rements } \\
\text { Discharge } \\
\text { (cubic feet } \\
\text { per second) }\end{array}$ \\
\hline 169 & $\begin{array}{l}\text { Diversion from } \\
\text { McDonald Creek }\end{array}$ & -- & $\begin{array}{l}\text { SE ISE Sec. 18, T.30 N., R.4 K.. } \\
\text { at U.S. Highway } 101 .\end{array}$ & $\cdots$ & $\begin{array}{l}9-11-52 \\
9-17-52\end{array}$ & $\begin{array}{l}5.83 \\
5.43\end{array}$ \\
\hline 170 & McDonald Creek & $\begin{array}{l}\text { Strait of Juan } \\
\text { de Fuca }\end{array}$ & $\begin{array}{l}\text { SE \&SEt sec. } 18, \text { T.30 N. R. R.4 W., } \\
\text { below diversion flume at U.S. } \\
\text { Highway } 101 \text {. }\end{array}$ & 21.3 & $\begin{array}{l}9-10-47 \\
7-29-52\end{array}$ & $\begin{array}{r}1.10 \\
.62\end{array}$ \\
\hline 171 & McDonald Creek & ...DO & $\begin{array}{l}\text { SWINE sec. } 5, T .30 \mathrm{~N} ., R .4 \mathrm{~W} . \mathrm{m} \\
0.1 \mathrm{mi} \text { upstream of mouth, } 6.3 \mathrm{mi} \\
\text { northwest of Sequim. }\end{array}$ & 22.9 & $\begin{array}{l}9-11-78 \\
10-12-78 \\
11-13-78 \\
12-14-78 \\
1-15-79 \\
2-16-79 \\
3-15-79 \\
4-17-79 \\
5-15-79 \\
6-19-79 \\
7-16-79 \\
9-17-79 \\
9-17-79\end{array}$ & $\begin{array}{c}10.9 \\
7.70 \\
5.88 \\
15.1 \\
4.28 \\
22.5 \\
21.1 \\
27.6 \\
8.24 \\
2.90 \\
3.89 \\
1.79 \\
3.86\end{array}$ \\
\hline & & & New Dungeness Harbor Tributaries & & & \\
\hline 172 & $\begin{array}{l}\text { Unnamed tributary } \\
\text { to New Dungeness } \\
\text { Harbor }\end{array}$ & $\begin{array}{l}\text { New Dungeness } \\
\text { Harbor }\end{array}$ & $\begin{array}{l}\text { NWLNWt sec. } 34, T .31 \mathrm{~N} ., \mathrm{R} .4 \mathrm{~W} . \mathrm{M} \\
0.2 \mathrm{mi} \text { upstream of mouth, } 5.8 \mathrm{mi} \\
\text { northwest of Sequim. }\end{array}$ & .17 & $\begin{array}{l}9-11-78 \\
10-12-78 \\
11-13-78 \\
12-14-78 \\
1-15-79 \\
2-15-79 \\
3-16-79 \\
4-16-79 \\
5-14-79 \\
6-18-79\end{array}$ & $\begin{array}{r}2.84 \\
.31 \\
1.05 \\
.97 \\
.05 \\
.79 \\
.09 \\
2.43 \\
3.73 \\
.31\end{array}$ \\
\hline & & & Dungeness River Basin & & & \\
\hline$\cdots$ & Gold Creek & Dungeness River & $\begin{array}{l}\text { Lat } 47055^{\prime} 15^{\prime \prime} \text {. long } 123002^{\prime} 30^{\prime \prime}, \\
\text { near center of E } 1 / 2 \mathrm{sec} .15 \text {, } \\
\text { T.28 N. R. } 3 \mathrm{~W} . \mathrm{Cl}^{\circ} \mathrm{Cl} \text { am County } \\
\text { at } 10 \mathrm{gg} \text { ing road, } 7.5 \mathrm{mi} \text { southwest } \\
\text { of Blyn. }\end{array}$ & 2.28 & $\begin{array}{l}4-19-66 \\
2-24-67 \\
5-19-75\end{array}$ & $\begin{array}{l}5.52 \\
3.76 \\
5.62\end{array}$ \\
\hline 173 & Dungeness River & New Dungeness Bay & $\begin{array}{l}\text { Center of sec. } 12, \text { T.29 N., R. } 4 \text { W.. } \\
\text { at gaging station "near Sequim." }\end{array}$ & 156 & $\begin{array}{l}9-12-22 \\
10-18-30\end{array}$ & $\begin{array}{l}177 \\
197\end{array}$ \\
\hline
\end{tabular}


TABL 9.--Records of miscellaneous stream-flow measurement sites in Clallam County--continued

\begin{tabular}{|c|c|c|c|c|c|c|}
\hline $\begin{array}{l}\text { Site } \\
\text { number }\end{array}$ & Stream & Tributary to & Location & $\begin{array}{l}\text { Drainage } \\
\text { area } \\
\text { (sq mi) } \\
\end{array}$ & Measu & $\begin{array}{l}\text { rements } \\
\text { Díscharge } \\
\text { (cubic feet } \\
\text { per second) }\end{array}$ \\
\hline 174 & $\begin{array}{l}\text { Canyon Creek } \\
\text { near Sequim }\end{array}$ & Dungeness River & $\begin{array}{l}\text { SE INW } 1 \text { sec. } 12, T .29 \mathrm{~N} ., \text { R. } 4 \mathrm{~W} . \\
0.1 \mathrm{mi} \text { upstream of mouth, } 4.2 \mathrm{mi} \\
\text { southwest of Sequim. }\end{array}$ & 11.9 & $\begin{array}{l}6-19-52 \\
7-11-52 \\
8-7-52 \\
8-20-52 \\
9-16-52 \\
10-9-52 \\
5-25-61 \\
6-15-61 \\
7-10-61 \\
7-25-61 \\
8-8-61 \\
9-9-61 \\
9-12-78 \\
10-13-78 \\
11-13-78 \\
12-14-78 \\
1-16-79 \\
2-15-79 \\
3-15-79 \\
4-17-79 \\
5-15-79 \\
6-18-79 \\
7-16-79 \\
8-17-79 \\
9-17-79\end{array}$ & $\begin{array}{c}4.85 \\
3.77 \\
2.58 \\
1.87 \\
1.72 \\
1.49 \\
12.5 \\
6.28 \\
4.22 \\
2.61 \\
2.08 \\
1.76 \\
3.45 \\
1.77 \\
1.93 \\
11.7 \\
1.65 \\
22.5 \\
22.0 \\
25.7 \\
9.22 \\
4.31 \\
2.75 \\
1.74 \\
1.78\end{array}$ \\
\hline 175 & Dungeness River & $\begin{array}{l}\text { New Dungeness } \\
\text { Bay }\end{array}$ & $\begin{array}{l}\text { NE iNE sec. } 2, \text { T.29 N., R.4 W., } \\
\text { at gaging station "below Canyon } \\
\text { Creek". }\end{array}$ & 170 & $\begin{array}{l}7-28-98 \\
8-10-22 \\
11-8-22 \\
2-6-23\end{array}$ & $\begin{array}{l}336 \\
262 \\
125 \\
169\end{array}$ \\
\hline 176 & Bear Creek & Dungeness River & $\begin{array}{l}\text { NWtSWl sec. 35, T.30 N., R.4 W., } \\
\text { above Agnew Irrigation Ditch } \\
\text { diversion (leakage) to creek. }\end{array}$ & 1.09 & $\begin{array}{l}9-12-78 \\
10-13-78\end{array}$ & $\begin{array}{l}.08 \\
.03\end{array}$ \\
\hline 177 & ...Do & $\ldots D_{0}$ & $\begin{array}{l}\text { NWASWl sec. 35, T.30 N., R.4 W., } \\
\text { bel ow Agnew Irrigation Ditch } \\
\text { diversion (leakage) to creek. }\end{array}$ & 1.09 & $\begin{array}{l}9-12-78 \\
10-13-78\end{array}$ & $\begin{array}{l}.28 \\
.62\end{array}$ \\
\hline 178 & $\begin{array}{l}\text { Unnamed tributary } \\
\text { to 8ear Creek }\end{array}$ & Bear Creek & $\begin{array}{l}\text { SW1NWt sec. } 35, T .30 \text { N., R.4 W., } \\
\text { above Agnew Irrigation Ditch } \\
\text { diversion to creek. }\end{array}$ & 1.14 & $\begin{array}{l}9-12-78 \\
10-13-78\end{array}$ & $\begin{array}{l}\text { Trace } \\
\text { Trace }\end{array}$ \\
\hline 179 & $\begin{array}{l}\text { Diversion from } \\
\text { Agnew Irrigation } \\
\text { Ditch to unnamed } \\
\text { tributary to } \\
\text { Bear Creek. }\end{array}$ & $\begin{array}{l}\text { unnamed tributary } \\
\text { to Bear Creek }\end{array}$ & $\begin{array}{l}\text { SWINWt sec. } 35, T .30 \mathrm{~N} ., \text { R.4 W., } \\
\text { at point of diversion. }\end{array}$ & - & $\begin{array}{l}9-12-78 \\
10-13-78\end{array}$ & $\begin{array}{l}.54 \\
.09\end{array}$ \\
\hline
\end{tabular}


TABE 9.--Records of miscellaneous stream-flow measurement sites in Clallam County--continued

\begin{tabular}{|c|c|c|c|c|c|c|}
\hline $\begin{array}{l}\text { Si te } \\
\text { number }\end{array}$ & Stream & Tributary to & Location & $\begin{array}{l}\text { Drainage } \\
\text { area } \\
\text { (sq mi) }\end{array}$ & $\begin{array}{l}\text { Measu } \\
\text { Date }\end{array}$ & $\begin{array}{l}\text { rements } \\
\text { Discharge } \\
\text { (cubic feet } \\
\text { per second) }\end{array}$ \\
\hline 180 & Bear Creek & Dungeness River & $\begin{array}{l}\text { NWISE } 1 \text { sec. } 26, \text { T. } 30 \mathrm{~N} ., \text { R.4 W., } \\
0.1 \text { mi ups tream of mouth, } 2.7 \mathrm{mi} \\
\text { southwest of Sequim. }\end{array}$ & 3.75 & $\begin{array}{l}9-12-78 \\
10-13-78 \\
11-13-78 \\
12-14-78 \\
1-16-79 \\
2-15-79 \\
3-15-79 \\
4-17-79 \\
5-15-79 \\
6-18-79\end{array}$ & $\begin{array}{l}.66 \\
.21 \\
\text { Trace } \\
.70 \\
.39 \\
1.32 \\
1.52 \\
.72 \\
.02 \\
.34\end{array}$ \\
\hline 181 & Dungeness River & $\begin{array}{l}\text { New Dungeness } \\
\text { Bay }\end{array}$ & $\begin{array}{l}\text { SE \& SE \& Sec. } 23, \text { T. } 30 \text { N., R.4 W., } \\
\text { at U.S. Highway } 101 .\end{array}$ & 178 & $\begin{array}{l}12-14-71 \\
2-8-72 \\
4-10-72 \\
6-12-72 \\
8-7-72 \\
9-12-78 \\
10-12-78 \\
11-13-78 \\
12-13-78 \\
1-16-79 \\
2-15-79 \\
3-15-79 \\
4-16-79 \\
5-15-79 \\
6-18-79 \\
7-16-79 \\
8-14-79 \\
9-11-79\end{array}$ & $\begin{array}{l}227 \\
161 \\
421 \\
717 \\
306 \\
461 \\
104 \\
126 \\
129 \\
73.2 \\
203 \\
345 \\
209 \\
342 \\
186 \\
150 \\
52.1 \\
140\end{array}$ \\
\hline 182 & ...Do & ...Do & $\begin{array}{l}\text { SE } 1 \text { SE sec. 2, T.30 N, R.4 W, } \\
\text { at Woodcock Bridge. }\end{array}$ & 180 & $\begin{array}{l}8-5-52 \\
8-21-52 \\
9-12-78 \\
10-13-78 \\
11-13-78 \\
12-13-78 \\
1-16-79 \\
2-15-79 \\
3-15-79 \\
4-16-79 \\
5-15-79 \\
6-18-79 \\
7-16-79 \\
8-14-79 \\
9-11-79\end{array}$ & $\begin{array}{c}202 \\
64.3 \\
440 \\
98.3 \\
120 \\
120 \\
64.2 \\
203 \\
377 \\
216 \\
333 \\
193 \\
164 \\
48.8 \\
141\end{array}$ \\
\hline
\end{tabular}


TABLE 9.--Records of miscellaneous stream-flow measurement sites in Clallam County--continued

\begin{tabular}{|c|c|c|c|c|c|c|}
\hline $\begin{array}{l}\text { Si te } \\
\text { number }\end{array}$ & Stream & Tributary to & Location & $\begin{array}{l}\text { Drainage } \\
\text { area } \\
\text { (sq mi) } \\
\end{array}$ & $\begin{array}{l}\text { Measu } \\
\text { Date }\end{array}$ & $\begin{array}{l}\text { rements } \\
\text { Discharge } \\
\text { (cubtc feet } \\
\text { per second) }\end{array}$ \\
\hline 183 & Hurd Creek & Dungeness River & $\begin{array}{l}\text { SWlSWl sec. 1, T.30 N., R.4 W., } \\
\text { at Woodcock Road (mea surements } \\
\text { by Washington State Department } \\
\text { of Fisheries. }\end{array}$ & .88 & $\begin{array}{l}5-7-51 \\
5-10-51 \\
5-14-51 \\
5-18-51 \\
5-23-51 \\
5-28-51 \\
5-31-51 \\
6-4-51 \\
6-8-51 \\
6-12-51 \\
6-29-51 \\
7-3-51 \\
7-7-51 \\
7-10-51 \\
7-29-51 \\
8-3-51 \\
8-7-51 \\
8-12-51 \\
8-16-51 \\
8-22-51 \\
9-2-51 \\
9-6-51 \\
9-10-51 \\
9-30-51 \\
10-5-51 \\
10-11-5 \\
10-16-51 \\
10-21-51 \\
1-4-52 \\
3-11-52 \\
3-19-52 \\
4-18-52 \\
4-26-52 \\
4-30-52 \\
5-5-52\end{array}$ & $\begin{array}{l}2.6 \\
2.6 \\
2.1 \\
2.6 \\
3.1 \\
2.1 \\
2.1 \\
3.4 \\
3.6 \\
4.5 \\
4.5 \\
4.2 \\
4.2 \\
4.8 \\
4.8 \\
5.0 \\
5.4 \\
5.4 \\
5.0 \\
4.8 \\
4.8 \\
5.4 \\
6.3 \\
6.3 \\
6.6 \\
6.6 \\
6.3 \\
6.3 \\
3.6 \\
3.6 \\
3.1 \\
3.1 \\
2.6 \\
2.1 \\
2.1\end{array}$ \\
\hline 184 & ..Do & $\ldots D 0$ & $\begin{array}{l}\text { WW ASW } 1 \text { sec. } 1, T .30 \mathrm{~N} ., \text { R.4 W., } \\
0.1 \text { mi ups tream of mouth, } 3.5 \mathrm{mi} \\
\text { northwest of Sequim. }\end{array}$ & .95 & $\begin{array}{l}9-12-78 \\
10-13-78 \\
11-13-78 \\
12-14-78 \\
1-16-79 \\
2-15-79 \\
3-15-79 \\
4-17-79 \\
5-15-79 \\
6-18-79 \\
7-16-79 \\
8-17-79 \\
9-17-79\end{array}$ & $\begin{array}{l}7.90 \\
5.71 \\
6.00 \\
5.18 \\
5.26 \\
6.14 \\
6.74 \\
5.49 \\
6.20 \\
6.45 \\
8.45 \\
7.16 \\
6.69\end{array}$ \\
\hline
\end{tabular}


TAQE 9.--Records of miscellaneous stream-flow measurement st tes in Clallam County--continued

\begin{tabular}{|c|c|c|c|c|c|c|}
\hline $\begin{array}{l}\text { Site } \\
\text { number }\end{array}$ & Stream & Iributary to & Location & $\begin{array}{l}\text { Drainage } \\
\text { area } \\
\text { (sq mi) }\end{array}$ & Measu & $\begin{array}{l}\text { rements } \\
\text { Discharge } \\
\text { (cubic feet } \\
\text { per second) }\end{array}$ \\
\hline 185 & Matriotti Creek & ...Do & $\begin{array}{l}\text { SE ANE \& sec. } 28, \text { T. } 30 \text { N., R.4 W., } \\
\text { above Agnew Irrigation Ditch } \\
\text { diversion (leakage) to creek. }\end{array}$ & 1.96 & $\begin{array}{l}9-12-78 \\
10-13-78\end{array}$ & $\begin{array}{l}0 \\
0\end{array}$ \\
\hline 186 & ..Do & ...Do & $\begin{array}{l}\text { SEINEA se. } 28, \text { T.30 N., R.4 W., } \\
\text { below Agnew Irrigation Ditch } \\
\text { diversion (leakage) to creek. }\end{array}$ & 1.96 & $\begin{array}{l}9-12-78 \\
10-13-78\end{array}$ & 95 \\
\hline 187 & ..DO & ..DO & $\begin{array}{l}\text { NWhiNWl sec. } 27, T .30 \mathrm{~N}, \mathrm{R} .4 \mathrm{~W} ., \\
\text { at Keeting Road. }\end{array}$ & 2.38 & $8-8-61$ & .05 \\
\hline 188 & $\ldots D_{0}$ & ...Do & $\begin{array}{l}\text { SEt SWI sec. } 10, \text { T.30 N., R.4 W., } \\
\text { at old Oiympic Highway. }\end{array}$ & 5.31 & $9-9-47$ & 7.69 \\
\hline 189 & ...Do & ...DO & $\begin{array}{l}\text { NEISEA Sec. } 35, \text { T.31 N., R.4 W., } \\
\text { at Ward Road. }\end{array}$ & 13.6 & $\begin{array}{l}6-19-52 \\
7-11-52 \\
8-4-52 \\
8-21-52 \\
9-17-52 \\
10-8-52 \\
5-25-61 \\
6-25-61 \\
10-12-78 \\
11-13-78 \\
12-13-78 \\
1-16-79 \\
2-15-79 \\
3-15-79 \\
4-16-79 \\
5-15-79 \\
6-18-79 \\
7-16-79 \\
8-14-79 \\
9-11-79\end{array}$ & $\begin{array}{l}20.0 \\
19.6 \\
17.9 \\
14.4 \\
15.0 \\
11.9 \\
7.11 \\
15.9 \\
11.3 \\
11.2 \\
15.0 \\
11.5 \\
16.1 \\
12.8 \\
12.5 \\
17.0 \\
19.4 \\
20.4 \\
10.9 \\
15.4\end{array}$ \\
\hline 190 & Dungeness River & $\begin{array}{l}\text { New Dungeness } \\
\text { Bay }\end{array}$ & $\begin{array}{l}\text { NWJ NEA sec. } 36, T .31 \text { N., R.4 W., } \\
\text { at gaging station "at Dungeness." }\end{array}$ & 197 & $\begin{array}{l}7-29-98 \\
9-17-98\end{array}$ & $\begin{array}{l}327 \\
183\end{array}$ \\
\hline 191 & $\begin{array}{l}\text { Meadowbrook } \\
\text { Creek }\end{array}$ & Dungeness River & $\begin{array}{l}\text { On east line NEt NEA sec. } 36 \text {, } \\
\text { T.31 N., R. } 4 \mathrm{~W} ., \text { at road crossing } \\
\text { in Dungeness. }\end{array}$ & .53 & $\begin{array}{l}9-10-47 \\
8-20-52 \\
8-8-61 \\
9-12-78 \\
10-12-78 \\
11-14-78 \\
12-15-78 \\
1-15-79 \\
2-16-79 \\
3-16-79 \\
4-16-79 \\
5-14-79 \\
6-19-79 \\
7-17-79 \\
8-16-79 \\
9-18-79\end{array}$ & $\begin{array}{l}7.18 \\
4.23 \\
5.42 \\
4.31 \\
4.58 \\
5.74 \\
4.70 \\
4.95 \\
5.31 \\
5.16 \\
3.99 \\
5.56 \\
4.41 \\
4.16 \\
4.19 \\
4.28\end{array}$ \\
\hline
\end{tabular}


TABE 9.--Records of miscellaneous stream-flow measurement sites in Clallam County--continued

\begin{tabular}{|c|c|c|c|c|c|c|}
\hline $\begin{array}{l}\text { Site } \\
\text { number }\end{array}$ & Stream & Tributary to & Location & $\begin{array}{c}\text { Drainage } \\
\text { area } \\
\text { (sq mi) }\end{array}$ & Measu & $\begin{array}{l}\text { rements } \\
\text { Discharge } \\
\text { (cubic feet } \\
\text { per second) }\end{array}$ \\
\hline & \multicolumn{6}{|c|}{ Strait of Juan de Fuca } \\
\hline 199 & Cassalery Creek & $\begin{array}{l}\text { Strait of Juan } \\
\text { de Fuca }\end{array}$ & $\begin{array}{l}\text { NWASW sec. } 5, T .30 \text { N., R.3 W., } \\
\text { at State Highway } 9 \mathrm{~F} .\end{array}$ & 3.19 & $\begin{array}{l}8-21-52 \\
8-8-61\end{array}$ & $\begin{array}{l}16.5 \\
7.62\end{array}$ \\
\hline 200 & $\ldots D o$ & $\begin{array}{l}\text { Strait of Juan } \\
\text { de Fuca }\end{array}$ & $\begin{array}{l}\text { NWWWW sec. } 5, \text { T.30 N., R.3 W., } \\
\text { at Jamestown Road. }\end{array}$ & 3.39 & $\begin{array}{l}9-11-78 \\
10-13-78 \\
11-14-78 \\
12-15-78 \\
1-15-79 \\
2-16-79 \\
3-16-79 \\
4-16-79 \\
5-14-79 \\
6-19-79 \\
7-17-79 \\
8-16-79 \\
9-18-79\end{array}$ & $\begin{array}{l}5.18 \\
4.89 \\
5.04 \\
5.04 \\
4.80 \\
4.87 \\
5.28 \\
4.82 \\
3.71 \\
3.93 \\
5.40 \\
5.14 \\
6.18\end{array}$ \\
\hline 201 & Gierin Creek & $\ldots$ Do & $\begin{array}{l}\text { On south line SW } 2 \text { SW d sec. } 9 \text {, } \\
\text { T.30 N., R.3 W., at Hoiland Road }\end{array}$ & 3.13 & $\begin{array}{l}8-21-52 \\
8-8-61\end{array}$ & $\begin{array}{l}13.4 \\
8.30\end{array}$ \\
\hline 202 & $\begin{array}{l}\text { Unnamed tributary } \\
\text { to Gierin Creek }\end{array}$ & Gierin Creek & $\begin{array}{l}\text { On south line SW } 1 \text { SWt sec, } 9 \text {, } \\
\text { T.30 N., R.3 W., at Holl and Road. }\end{array}$ & .19 & $8-21-52$ & .76 \\
\hline \multirow[t]{2}{*}{203} & Gierin Creek & $\begin{array}{l}\text { Strait of Juan } \\
\text { de Fuca }\end{array}$ & $\begin{array}{l}\text { SWASWA sec. 9, T.30 N., R. } 3 \mathrm{~W},{ }^{\prime} \\
0.2 \mathrm{mi} \text { downstream of Holl and Road. }\end{array}$ & 3.49 & $\begin{array}{l}9-11-78 \\
10-13-78 \\
11-14-78 \\
12-15-78 \\
1-15-79 \\
2-16-79 \\
3-16-79 \\
4-16-79 \\
5-14-79 \\
6-19-79 \\
7-17-79 \\
8-16-79 \\
9-18-79\end{array}$ & $\begin{array}{l}6.14 \\
4.63 \\
3.97 \\
3.82 \\
2.94 \\
3.01 \\
2.54 \\
2.45 \\
4.70 \\
2.95 \\
4.62 \\
3.05 \\
3.88\end{array}$ \\
\hline & \multicolumn{6}{|c|}{ Sequim Bay Tributaries } \\
\hline 204 & $\begin{array}{l}\text { Highland } \\
\text { Irrigation Ditch }\end{array}$ & Bell Creek & $\begin{array}{l}\text { NWtNWt sec. } 1, \text { T.29 N., R.4 W., } \\
\text { at logging road crossing. }\end{array}$ & -- & $9-15-22$ & 6.8 \\
\hline 205 & Bell Creek & Sequim Bay & $\begin{array}{l}\text { NWdNWI sec. } 22, \mathrm{~T}^{\top} .30 \mathrm{~N} ., \text { R.3 W., } \\
2.1 \text { mi east of Sequim. }\end{array}$ & 8.86 & $\begin{array}{l}9-24-42 \\
8-21-52 \\
8-8-61 \\
9-11-78 \\
10-13-78 \\
11-14-78 \\
12-15-78 \\
1-15-79 \\
2-16-79 \\
3-16-79\end{array}$ & $\begin{array}{l}4.88 \\
3.78 \\
4.53 \\
4.84 \\
4.12 \\
4.70 \\
5.58 \\
4.10 \\
7.67 \\
8.53\end{array}$ \\
\hline
\end{tabular}


TABLE 9.--Records of miscellaneous stream-flow measurement sites in Clallam County--continued

\begin{tabular}{|c|c|c|c|c|c|c|}
\hline $\begin{array}{l}\text { Si te } \\
\text { number }\end{array}$ & Stream & Tributary to & Location & $\begin{array}{l}\text { Drainage } \\
\text { area } \\
\text { (sq } \mathrm{mi}) \\
\end{array}$ & Meas & $\begin{array}{l}\text { rements } \\
\text { Discharge } \\
\text { (cubic feet } \\
\text { per second) }\end{array}$ \\
\hline 205 & Bell Creek & Sequim Bay & $\begin{array}{l}\text { NWINWJ sec. } 22, \text { T. } 30 \text { N., R.3 W., } \\
2.1 \mathrm{mi} \text { east of Sequim. }\end{array}$ & 8.86 & $\begin{array}{l}4-16-79 \\
5-14-79 \\
6-19-79 \\
7-17-79 \\
8-16-79 \\
9-18-79\end{array}$ & $\begin{array}{l}9.24 \\
8.88 \\
7.10 \\
8.59 \\
3.08 \\
5.51\end{array}$ \\
\hline 206 & Johnson Creek & ...Do & $\begin{array}{l}\text { NW1 SWl sec. } 27, \text { T. } 30 \text { N., R. } 3 \text { W., } \\
200 \text { ft bel ow U.S. Highway } 101 \text {. }\end{array}$ & 4.72 & $\begin{array}{l}7-11-52 \\
8-7-52 \\
8-20-52 \\
9-10-52 \\
10-9-52 \\
5-26-61 \\
6-16-61 \\
7-9-61 \\
7-25-61 \\
8-8-61 \\
9-9-61\end{array}$ & $\begin{array}{l}5.77 \\
1.86 \\
.24 \\
1.08 \\
1.82 \\
4.90 \\
6.89 \\
4.06 \\
.28 \\
.92 \\
1.44\end{array}$ \\
\hline 207 & $\begin{array}{l}\text { Unnamed tributary } \\
\text { to Johnson Creek }\end{array}$ & Johnson Creek & $\begin{array}{l}\text { NEt SEA Sec. } 28, \text { T. } 30 \text { N., R.3 W., } \\
\text { at U.S. Highway 101. }\end{array}$ & .72 & $9-10-52$ & 0 \\
\hline 208 & $\begin{array}{l}\text { Sequim Bay } \\
\text { tributary }\end{array}$ & Sequim Bay & $\begin{array}{l}\text { SWl SEd sec. } 27, \text { T. } 30 \text { N., R. } 3 \text { W., } \\
\text { at road crossing at mouth. }\end{array}$ & .42 & $8-21-52$ & 1.12 \\
\hline 209 & $\begin{array}{l}\text { Sequim Bay } \\
\text { tributary No. } 2\end{array}$ & ...Do & $\begin{array}{l}\text { NEA SWA sec. } 35, \text { T.30 N., R.3 W., } \\
\text { at U.S. Highway 101. }\end{array}$ & 1.33 & $8-20-52$ & 0 \\
\hline \multirow[t]{2}{*}{210} & $\begin{array}{l}\text { Sequim Bay } \\
\text { tributary }\end{array}$ & $\ldots D o$ & $\begin{array}{l}\text { NWJ sec. } 2, T .29 \text { N. , R.3 W., at } \\
\text { U.S. Highway 101 crossing, at } \\
\text { Sequim Bay State Park. }\end{array}$ & - & $8-9-61$ & 0 \\
\hline & \multicolumn{6}{|c|}{ Dean Creek Basin } \\
\hline \multirow[t]{2}{*}{211} & Dean Creek & ...Do & $\begin{array}{l}\text { SWANWt sec. } 12, T .29 \mathrm{~N} ., \mathrm{R} .3 \mathrm{~W} ., \\
\text { at old highway, } 50 \mathrm{ft} \text { east of } \\
\text { U.S. Highway 101, at Blyn. }\end{array}$ & 2.96 & $\begin{array}{l}6-16-52 \\
8-20-52 \\
9-10-52 \\
8-10-61\end{array}$ & $\begin{array}{l}.17 \\
0^{.17} \\
.08\end{array}$ \\
\hline & \multicolumn{6}{|c|}{ Sequim Bay Tributaries } \\
\hline \multirow[t]{2}{*}{212} & $\begin{array}{l}\text { Sequim Bay } \\
\text { tributary no. } 3\end{array}$ & ...Do & $\begin{array}{l}\text { NEt SWI Sec. 12, T.29 N., R.3 W., } \\
\text { at U.S. HIghway } 101 \text { at Biyn. }\end{array}$ & .85 & $9-10-52$ & 0 \\
\hline & & & Jimnycomelately Creek Basin & & & \\
\hline-- & $\begin{array}{l}\text { East Fork } \\
\text { Jimnycomel ately } \\
\text { Creek }\end{array}$ & $\begin{array}{l}\text { Jimmycomelately } \\
\text { Creek }\end{array}$ & $\begin{array}{l}\text { SWt sec. } 24, T .29 \mathrm{~N} ., \mathrm{R}^{\mathrm{T}} 3 \mathrm{~W} \text {., at } \\
\text { road crossing } 1 / 2 \mathrm{mi} \text { above mouth. }\end{array}$ & 3.47 & $\begin{array}{l}8-20-52 \\
9-10-52\end{array}$ & .71 \\
\hline
\end{tabular}


TABLE 9.--Records of miscellaneous stream-flow measurement sites in $\mathrm{Clallam}$ County--continued

\begin{tabular}{|c|c|c|c|}
\hline $\begin{array}{l}\text { Site } \\
\text { number } \\
\end{array}$ & Stream & Tributary to & Location \\
\hline 213 & $\begin{array}{l}\text { Jimmycomelately } \\
\text { Creek }\end{array}$ & Sequim Bay & $\begin{array}{l}\text { SE iNE \& sec. 13, T.29 N., R. } 3 \text { W., } \\
i \mathrm{mi} \text { above mouth and } i \mathrm{mi} \text { south } \\
\text { of } 81 \mathrm{yn} \text {. }\end{array}$ \\
\hline & & & Salmon Creek Basin \\
\hline- & Saimon Creek & Discovery Bay & $\begin{array}{l}\text { NWLNW } 1 \text { sec. } 32, T .29 N_{.}, R .2 \text { W., } \\
\text { at crossing of Snow Creek Road, }\end{array}$ \\
\hline
\end{tabular}

at crossing of Snow Creek Road,
$41 / 2 \mathrm{mi}$ southwest of Maynard.

\begin{tabular}{ccc}
$\begin{array}{c}\text { Drainage } \\
\text { area } \\
\text { (sq mi) }\end{array}$ & Date & $\begin{array}{c}\text { Measurements } \\
\text { Discharge } \\
\text { (cubic feet } \\
\text { per second) }\end{array}$ \\
\cline { 3 - 4 } 14.6 & $5-24-61$ & 12.5 \\
& $6-16-61$ & 3.44 \\
& $6-16-61$ & 3.15 \\
& $6-16-61$ & 3.01 \\
& $6-16-61$ & 3.18 \\
& $7-9-61$ & 3.54 \\
& $7-9-61$ & 3.30 \\
& $7-26-61$ & 1.84 \\
& $8-10-61$ & 1.24 \\
& $9-10-61$ & 1.08
\end{tabular}

$4.69 \quad 8-20-52$

$9-10-52$

.31

.31 
TABLE 10.--Month-end reservoir contents of Lake Mills, 1927-78

ELHHA RIVER BASIN

12045000. Lake Mills at Glines Canyon, near Port Angeles, Wash. (Map number: 127)

LOCATION.--Lat $48^{\circ} 00^{\prime} 08^{\prime \prime}$, long $1233^{\prime} 55^{\prime \prime}$, in SW SEl $_{1}$ sec.17, T.29 N., R.7 W., Clallam County, Olympic National Park, at Glines Canyon Dam on Elwha River 2 miles upstream from Griff Creek, 4.1 miles south of Elwha, and 11 miles southwest of Port Angeles.

DRAINAGE AREA.--245 sq mi.

PERIOD OF RECORD.--April 1927 to current year.

GAGE. --Nonrecording gage. Datum of gage is $19.67 \mathrm{ft}$ below mean sea level.

EXTREMES FOR PERIOD OF RECORD.--Maximum contents observed, 39,940 acre-ft Dec. 22, 1936, gage height, 613.0 ft; minimum observed since reservoir first filled in May 1927, 24,290 acre-ft Nov. 14, 1929, gage height, $574.4 \mathrm{ft}$.

REMARKS. --Reservoir is formed by concrete dam, completed in 1927; storage began Apr. 1, 1927. Total capacity, 37,790 acre-ft at gage height $608 \mathrm{ft}$ (top of gates). Dead storage below gage height $579 \mathrm{ft}, 26,000 \mathrm{acre}-\mathrm{ft}$. Figures given herein represent total contents. Water is used for power by Crown Zellerbach Corp.

COOPERATION.--Gage-height record furnished by Crown Zellerbach Corp.

\begin{tabular}{|c|c|c|c|c|c|c|c|c|c|c|c|c|}
\hline $\begin{array}{l}\text { Water } \\
\text { year }\end{array}$ & Oct. & Nov. & Dec. & Jan. & Feb. & Mar. & Apr. & May & June & July & Aug. & Sept. \\
\hline $\begin{array}{l}1927 \\
1928 \\
1929 \\
1930\end{array}$ & $\begin{array}{l}37,780 \\
34,900 \\
27,800\end{array}$ & $\begin{array}{l}37,490 \\
37,120 \\
25,700\end{array}$ & $\begin{array}{l}36,230 \\
37,580 \\
38,220\end{array}$ & $\begin{array}{l}38,390 \\
32,360 \\
34,570\end{array}$ & $\begin{array}{l}36,740 \\
29,070 \\
38,690\end{array}$ & $\begin{array}{l}38,520 \\
37,450 \\
38,740\end{array}$ & $\begin{array}{l}20,060 \\
38,440 \\
36,610 \\
32,440\end{array}$ & $\begin{array}{l}35,260 \\
38,520 \\
38,050 \\
38,050\end{array}$ & $\begin{array}{l}35,520 \\
38,390 \\
38,090 \\
38,130\end{array}$ & $\begin{array}{l}10 \\
20 \\
50 \\
80\end{array}$ & $\begin{array}{l}70 \\
30 \\
50 \\
00\end{array}$ & $\begin{array}{l}36,560 \\
31,110 \\
34,290 \\
36,610\end{array}$ \\
\hline $\begin{array}{l}1931 \\
1932 \\
1933 \\
1934 \\
1935\end{array}$ & $\begin{array}{l}38,440 \\
38,330 \\
36,030 \\
38,760 \\
38,780\end{array}$ & $\begin{array}{l}38,690 \\
38,740 \\
38,130 \\
36,550 \\
35,210\end{array}$ & $\begin{array}{l}37,830 \\
37,600 \\
38,740 \\
38,350 \\
38,840\end{array}$ & $\begin{array}{l}38,310 \\
38,180 \\
38,560 \\
38,160 \\
36,190\end{array}$ & $\begin{array}{l}38,610 \\
38,590 \\
38,560 \\
38,480 \\
38,740\end{array}$ & $\begin{array}{l}38,560 \\
38,780 \\
35,520 \\
38,650 \\
34,530\end{array}$ & $\begin{array}{l}38,690 \\
37,200 \\
38,560 \\
38,610 \\
32,990\end{array}$ & $\begin{array}{l}38,780 \\
38,760 \\
38,390 \\
38,650 \\
38,740\end{array}$ & $\begin{array}{l}38,650 \\
38,610 \\
38,560 \\
36,680 \\
38,670\end{array}$ & & & $\begin{array}{l}37,240 \\
35,460 \\
38,650 \\
35,020 \\
35,840\end{array}$ \\
\hline $\begin{array}{l}1936 \\
1937 \\
1938 \\
1939 \\
1940\end{array}$ & $\begin{array}{l}35,520 \\
29,490 \\
37,920 \\
38,610 \\
34,700\end{array}$ & $\begin{array}{l}36,570 \\
32,910 \\
38,370 \\
37,120 \\
37,960\end{array}$ & $\begin{array}{l}38,970 \\
38,050 \\
35,460 \\
38,310 \\
38,560\end{array}$ & $\begin{array}{l}36,420 \\
31,850 \\
38,710 \\
38,560 \\
38,860\end{array}$ & $\begin{array}{l}37,940 \\
37,540 \\
38,540 \\
35,940 \\
38,910\end{array}$ & $\begin{array}{l}35,710 \\
35,960 \\
35,380 \\
37,880 \\
38,820\end{array}$ & $\begin{array}{l}38,890 \\
36,550 \\
37,850 \\
38,560 \\
38,560\end{array}$ & $\begin{array}{l}38,890 \\
38,760 \\
38,650 \\
38,910 \\
38,820\end{array}$ & $\begin{array}{l}38 \\
38 \\
38 \\
38 \\
37\end{array}$ & $\begin{array}{l}37,880 \\
36,970 \\
37,920 \\
38,820 \\
38,130\end{array}$ & $\begin{array}{l}37,390 \\
38,200 \\
33,140 \\
37,030 \\
35,350\end{array}$ & $\begin{array}{l}31,710 \\
35,400 \\
33,300 \\
34,660 \\
37,450\end{array}$ \\
\hline $\begin{array}{l}1946 \\
1947 \\
1948 \\
1949 \\
1950\end{array}$ & $\begin{array}{l}37,710 \\
34,330 \\
38,690 \\
37,540 \\
36,360\end{array}$ & $\begin{array}{l}38,440 \\
38,780 \\
37,290 \\
38,560 \\
38,740\end{array}$ & $\begin{array}{l}38,390 \\
38,310 \\
38,520 \\
37,710 \\
38,690\end{array}$ & $\begin{array}{l}38,480 \\
38,860 \\
37,830 \\
35,440 \\
37,920\end{array}$ & $\begin{array}{l}38,520 \\
38,820 \\
38,820 \\
38,860 \\
38,650\end{array}$ & $\begin{array}{l}37,960 \\
38,390 \\
37,370 \\
37,290 \\
36,320\end{array}$ & $\begin{array}{l}38,650 \\
38,820 \\
37,030 \\
38,260 \\
36,110\end{array}$ & $\begin{array}{l}38,740 \\
38,560 \\
38,480 \\
38,310 \\
38,740\end{array}$ & $\begin{array}{l}38,860 \\
38,820 \\
38,560 \\
38,740 \\
38,390\end{array}$ & $\begin{array}{l}38,820 \\
38,310 \\
38,910 \\
38,910 \\
38,740\end{array}$ & $\begin{array}{l}36,700 \\
37,790 \\
37,370 \\
36,910 \\
36,530\end{array}$ & $\begin{array}{l}35,940 \\
37,540 \\
37,960 \\
35,350 \\
37,240\end{array}$ \\
\hline $\begin{array}{l}1951 \\
1952 \\
1953 \\
1954 \\
1955\end{array}$ & $\begin{array}{l}38,520 \\
38,220 \\
32,930 \\
38,860 \\
37,450\end{array}$ & $\begin{array}{l}38,520 \\
38,740 \\
33,140 \\
38,350 \\
39,170\end{array}$ & $\begin{array}{l}38,560 \\
38,180 \\
38,310 \\
38,310 \\
38,130\end{array}$ & $\begin{array}{l}38,860 \\
38,990 \\
38,650 \\
37,330 \\
37,830\end{array}$ & $\begin{array}{l}38,310 \\
38,350 \\
38,130 \\
38,520 \\
37,240\end{array}$ & $\begin{array}{l}37,830 \\
37,750 \\
36,740 \\
36,780 \\
36,150\end{array}$ & $\begin{array}{l}38,000 \\
39,080 \\
38,440 \\
35,940 \\
34,200\end{array}$ & $\begin{array}{l}38,260 \\
38,350 \\
38,560 \\
38,180 \\
38,350\end{array}$ & $\begin{array}{l}38,690 \\
38,480 \\
38,610 \\
38,520 \\
38,130\end{array}$ & $\begin{array}{l}38,440 \\
38,860 \\
38,740 \\
39,080 \\
38,860\end{array}$ & $\begin{array}{l}37,160 \\
38,310 \\
38,350 \\
38,480 \\
37,750\end{array}$ & $\begin{array}{l}38,650 \\
35,110 \\
38,390 \\
36,990 \\
36,190\end{array}$ \\
\hline
\end{tabular}


TABLE 10.--Month-end reservoir contents of Lake Mills, 1927-78--Continued

ELWHA RIVER BASIN--Continued

\begin{tabular}{|c|c|c|c|c|c|c|c|c|c|c|c|c|}
\hline $\begin{array}{l}\text { Water } \\
\text { year }\end{array}$ & oct. & Nov. & Dec. & Jan. & Feb. & Mar. & Apr. & May & June & July & Aug. & Sept. \\
\hline $\begin{array}{l}1956 \\
1957 \\
1958 \\
1959 \\
1960\end{array}$ & $\begin{array}{l}38,610 \\
38,440 \\
38,650 \\
37,500 \\
37,880\end{array}$ & $\begin{array}{l}37,540 \\
36,450 \\
37,540 \\
38,820 \\
39,040\end{array}$ & $\begin{array}{l}36,320 \\
38,860 \\
38,480 \\
38,690 \\
38,820\end{array}$ & $\begin{array}{l}36,360 \\
37,580 \\
38,310 \\
38,520 \\
38,440\end{array}$ & $\begin{array}{l}36,700 \\
36,280 \\
39,040 \\
37,620 \\
38,310\end{array}$ & $\begin{array}{l}37,160 \\
35,860 \\
36,280 \\
37,370 \\
38,740\end{array}$ & $\begin{array}{l}38,910 \\
37,200 \\
35,190 \\
38,220 \\
37,830\end{array}$ & $\begin{array}{l}38,910 \\
38,220 \\
37,830 \\
38,690 \\
37,920\end{array}$ & $\begin{array}{l}38,390 \\
38,690 \\
38,690 \\
37,960 \\
38,000\end{array}$ & $\begin{array}{l}38,820 \\
37,710 \\
37,880 \\
38,000 \\
38,520\end{array}$ & $\begin{array}{l}37,540 \\
37,920 \\
37,660 \\
37,790 \\
37,120\end{array}$ & $\begin{array}{l}38,690 \\
37,540 \\
35,650 \\
37,160 \\
37,330\end{array}$ \\
\hline $\begin{array}{l}1961 \\
1962 \\
1963 \\
1964 \\
1965\end{array}$ & $\begin{array}{l}37,920 \\
36,780 \\
36,780 \\
38,520 \\
37,330\end{array}$ & $\begin{array}{l}37,750 \\
38,350 \\
38,350 \\
38,860 \\
37,790\end{array}$ & $\begin{array}{l}38,050 \\
38,860 \\
38,390 \\
38,820 \\
37,500\end{array}$ & $\begin{array}{l}38,350 \\
37,290 \\
37,330 \\
38,520 \\
38,480\end{array}$ & $\begin{array}{l}38,480 \\
35,560 \\
38,740 \\
37,160 \\
38,740\end{array}$ & $\begin{array}{l}38,480 \\
34,660 \\
38,350 \\
36,820 \\
36,320\end{array}$ & $\begin{array}{l}37,200 \\
37,790 \\
36,110 \\
34,370 \\
38,950\end{array}$ & $\begin{array}{l}38,820 \\
37,330 \\
38,520 \\
38,820 \\
38,520\end{array}$ & $\begin{array}{l}790 \\
090 \\
330 \\
690 \\
280\end{array}$ & $\begin{array}{l}38,130 \\
38,740 \\
37,660 \\
38,860 \\
37,450\end{array}$ & $\begin{array}{l}38,180 \\
37,580 \\
37,540 \\
38,260 \\
37,290\end{array}$ & $\begin{array}{l}36,610 \\
38,310 \\
37,290 \\
38,690 \\
36,530\end{array}$ \\
\hline $\begin{array}{l}1966 \\
1967 \\
1968 \\
1969 \\
1970\end{array}$ & $\begin{array}{l}37,710 \\
38,050 \\
36,740 \\
38,690 \\
36,870\end{array}$ & $\begin{array}{l}37,660 \\
37,960 \\
38,050 \\
38,260 \\
37,160\end{array}$ & $\begin{array}{l}37,290 \\
38,740 \\
38,480 \\
38,910 \\
37,540\end{array}$ & $\begin{array}{l}37,160 \\
38,560 \\
38,560 \\
38,090 \\
38,520\end{array}$ & $\begin{array}{l}36,950 \\
38,610 \\
38,390 \\
34,660 \\
38,130\end{array}$ & $\begin{array}{l}38,780 \\
38,990 \\
38,910 \\
38,440 \\
37,410\end{array}$ & $\begin{array}{l}36,910 \\
38,520 \\
34,000 \\
38,000 \\
37,030\end{array}$ & $\begin{array}{l}38,780 \\
38,000 \\
38,520 \\
38,390 \\
38,650\end{array}$ & $\begin{array}{l}38,520 \\
37,660 \\
37,790 \\
38,130 \\
38,260\end{array}$ & $\begin{array}{l}38,390 \\
38,740 \\
38,690 \\
38,220 \\
37,200\end{array}$ & $\begin{array}{l}37,620 \\
38,390 \\
37,240 \\
37,200 \\
37,410\end{array}$ & $\begin{array}{l}37,500 \\
38,390 \\
37,290 \\
38,050 \\
36,950\end{array}$ \\
\hline $\begin{array}{l}1976 \\
1977 \\
1978\end{array}$ & $\begin{array}{l}36,782 \\
38,134 \\
37,328\end{array}$ & $\begin{array}{l}38,736 \\
36,362 \\
37,833\end{array}$ & $\begin{array}{l}38,177 \\
38,650 \\
38,478\end{array}$ & $\begin{array}{l}38,478 \\
38,564 \\
36,866\end{array}$ & $\begin{array}{l}37,118 \\
37,664 \\
36,908\end{array}$ & $\begin{array}{l}35,438 \\
36,866 \\
38,005\end{array}$ & $\begin{array}{l}31,710 \\
38,349 \\
37,833\end{array}$ & $\begin{array}{l}38,478 \\
38,564 \\
37,202\end{array}$ & $\begin{array}{l}38,822 \\
38,177 \\
38,736\end{array}$ & $\begin{array}{l}38,865 \\
38,650 \\
38,220\end{array}$ & $\begin{array}{l}38,693 \\
38,263 \\
38,822\end{array}$ & $\begin{array}{l}38,607 \\
36,824 \\
37,328\end{array}$ \\
\hline
\end{tabular}




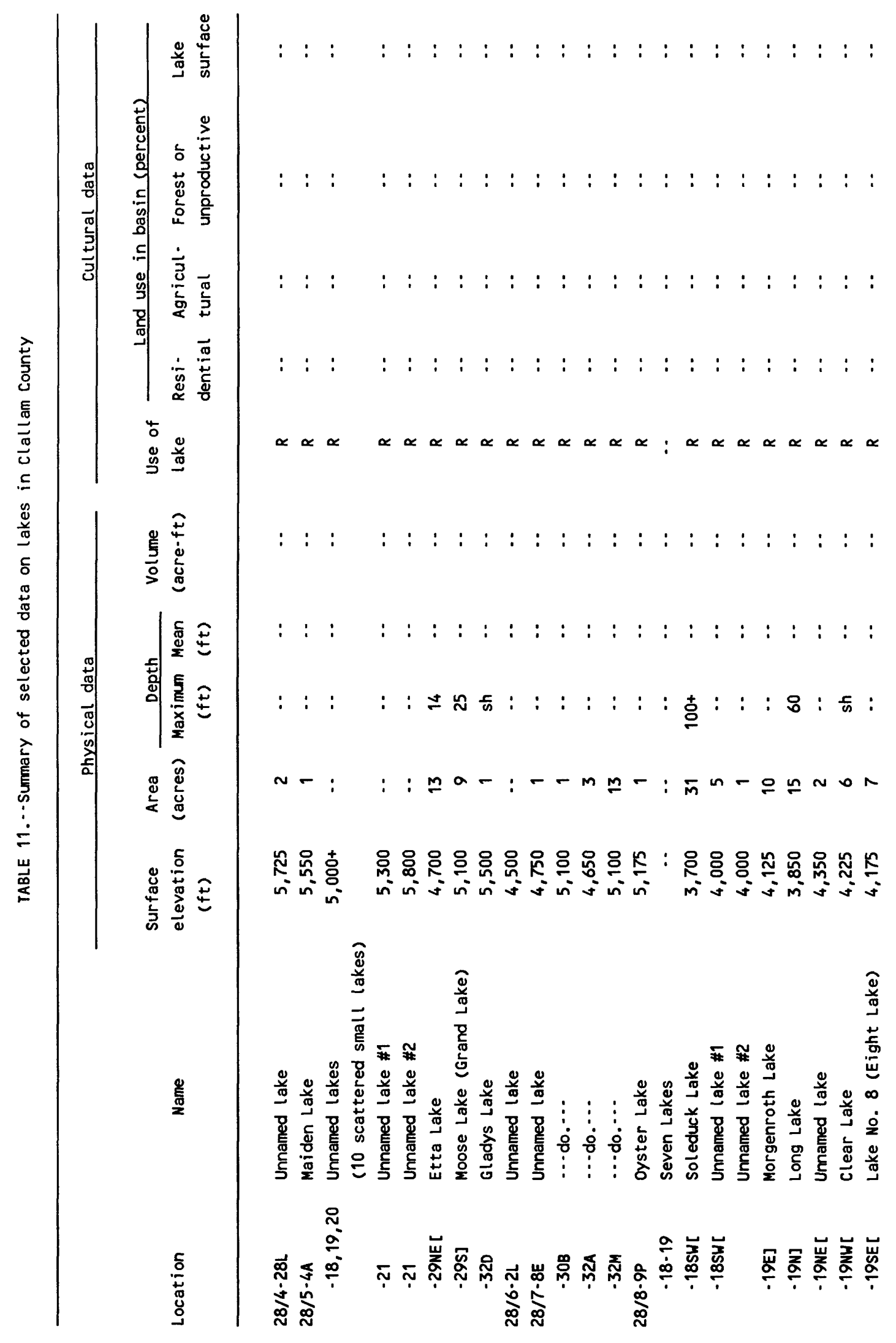




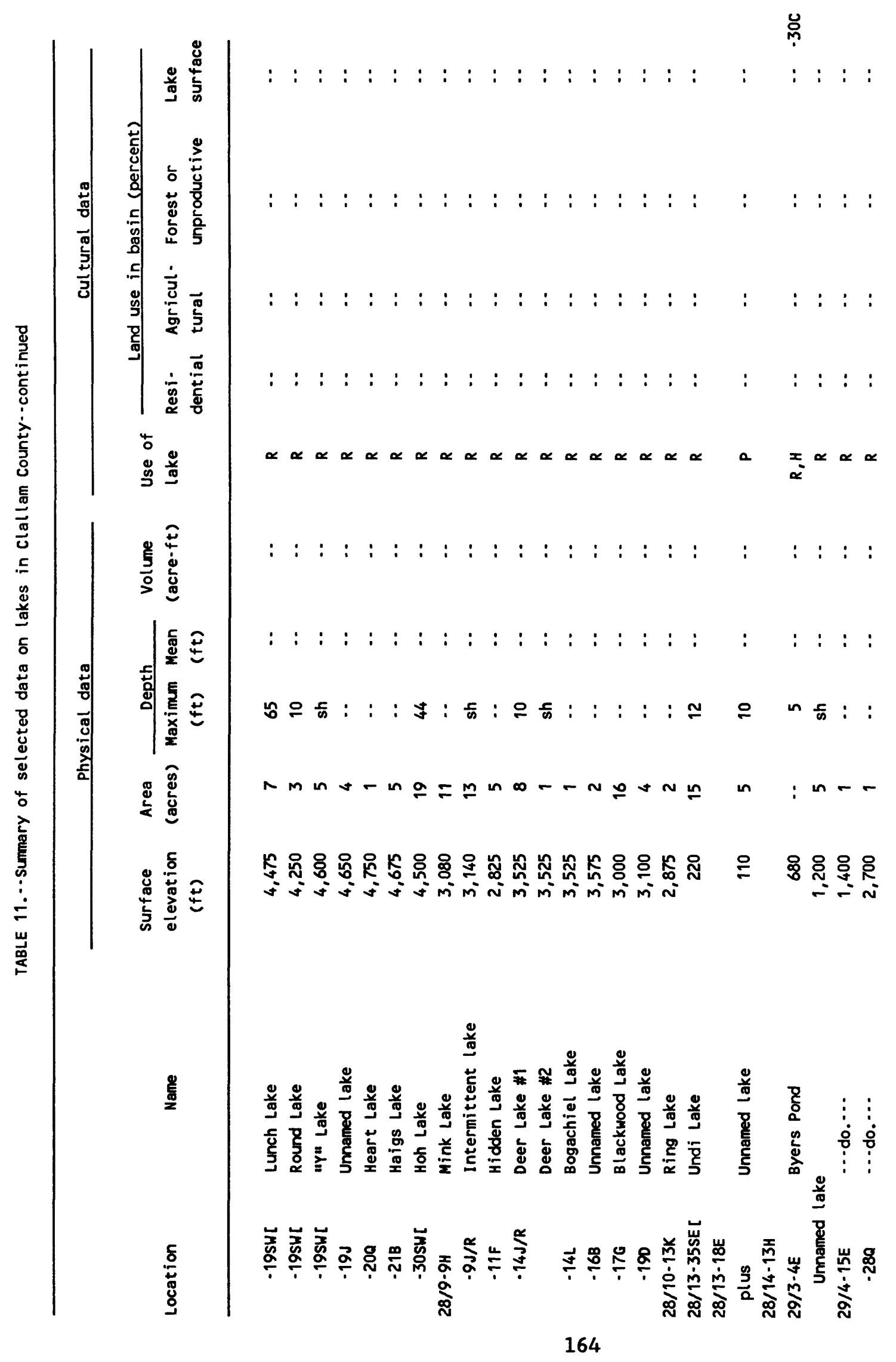




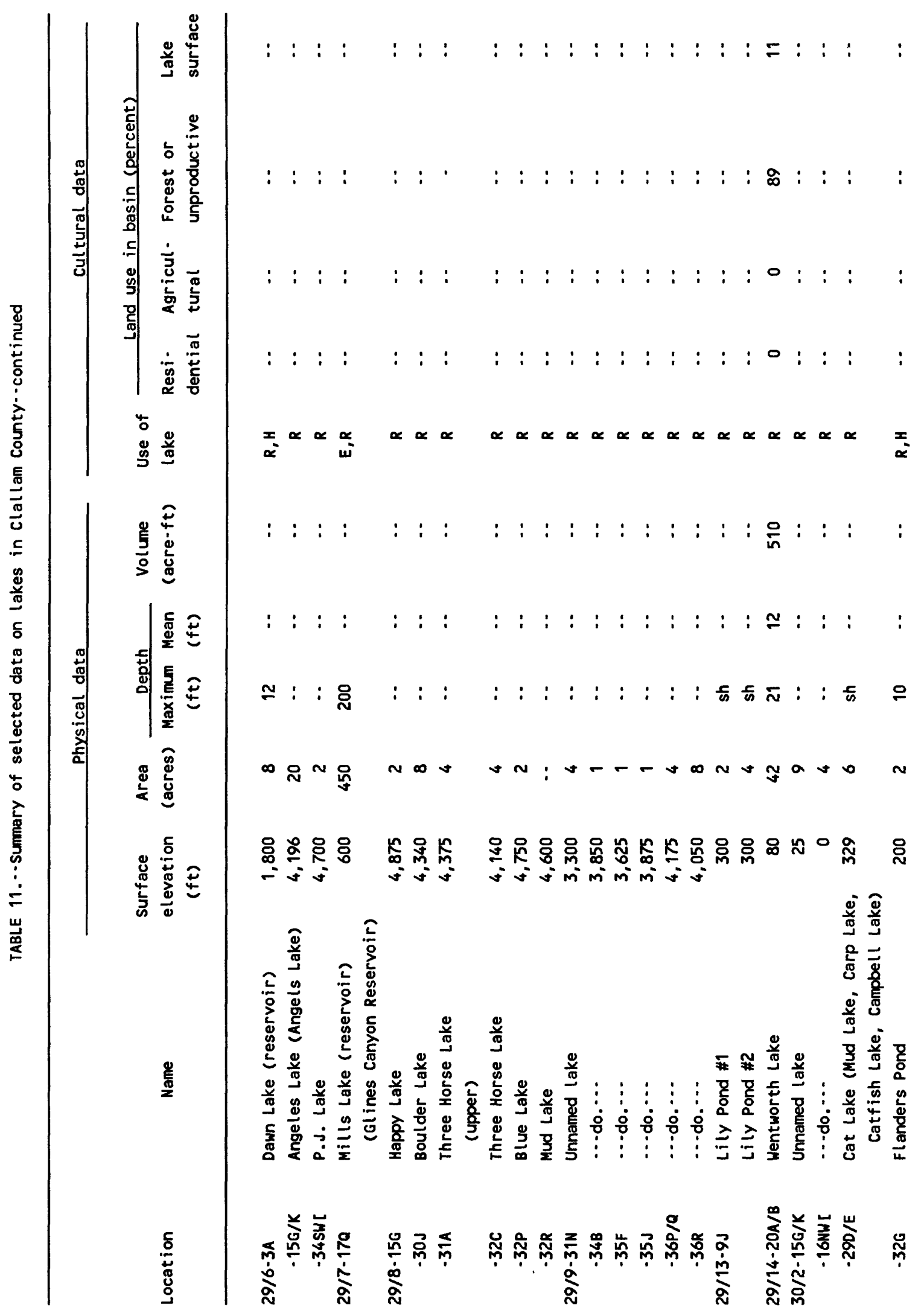




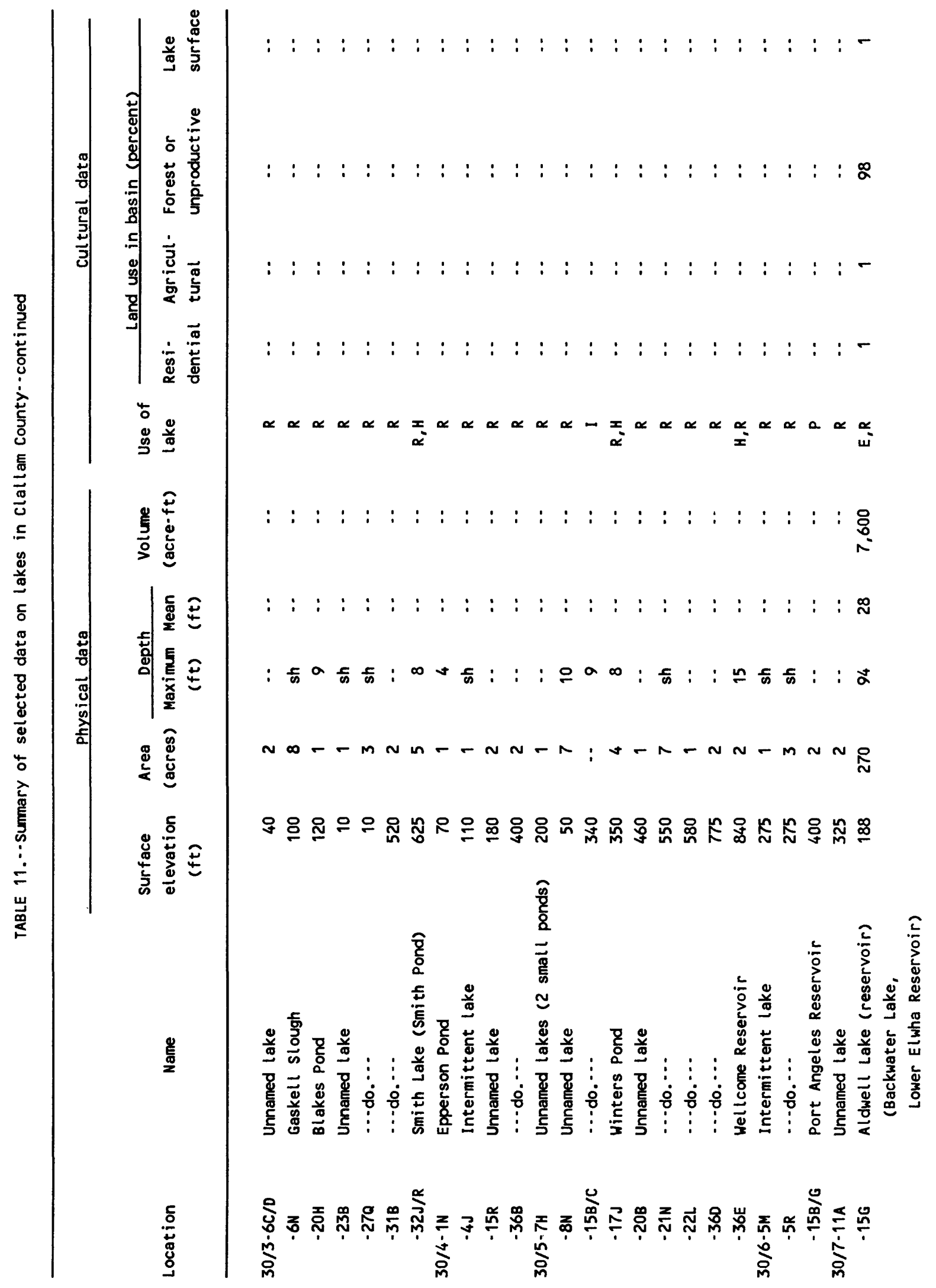




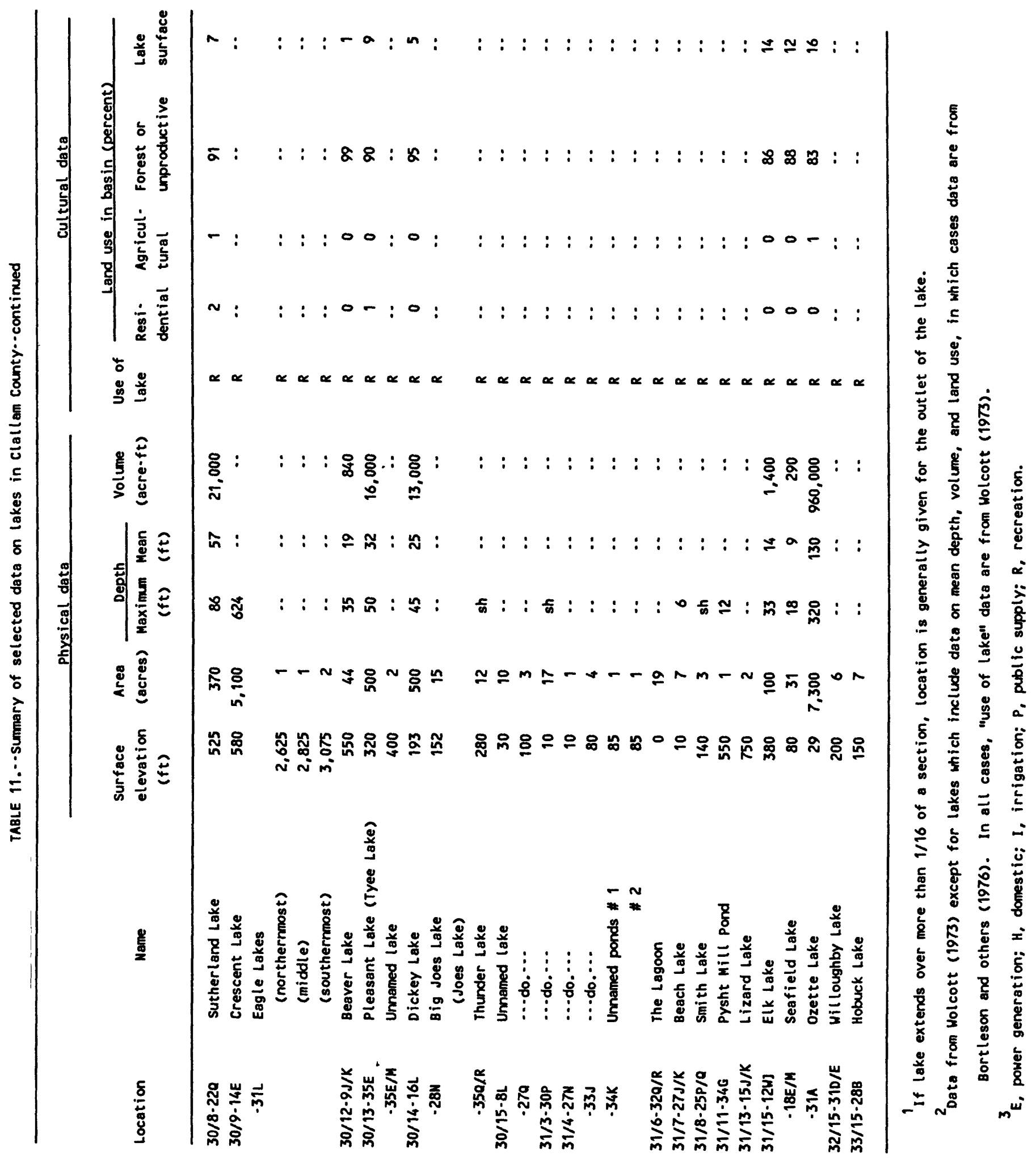


TABLE 12. - Chenical and physical quality of water from selected wells and springs in clallan touncy

", indicates value which exceeds maximm contaminant level or recommended level (toble 3); 1 , total concentration

\begin{tabular}{|c|c|c|c|c|c|c|c|c|c|c|c|c|c|}
\hline Site number & $\begin{array}{l}\text { Data } \\
\text { sempled }\end{array}$ & $\begin{array}{l}\text { Dis: } \\
\text { solved } \\
\text { silica } \\
(\mathrm{SiO}) \\
(\mathrm{mg} / \mathrm{L})\end{array}$ & $\begin{array}{l}\text { Dis: } \\
\text { solved } \\
\text { iron } \\
\text { (Fe) } \\
\text { (ug/L) }\end{array}$ & $\begin{array}{l}\text { Dis. } \\
\text { sol ved } \\
\text { mangan. } \\
\text { ese (Mn) } \\
\text { (ug/L) }\end{array}$ & $\begin{array}{l}\text { Dis. } \\
\text { solved } \\
\text { calcium } \\
\text { (Ca) } \\
(\mathrm{mg} / \mathrm{L})\end{array}$ & $\begin{array}{l}\text { nis. } \\
\text { solved } \\
\text { magnes. } \\
\text { iun }(\mathrm{Hg}) \\
(\mathrm{mg} / \mathrm{L})\end{array}$ & $\begin{array}{l}\text { Dis: } \\
\text { solved } \\
\text { sodium } \\
(\mathrm{Na}) \\
(\mathrm{mg} / \mathrm{L})\end{array}$ & $\begin{array}{l}\text { Disf } \\
\text { solved } \\
\text { potas. } \\
\text { sium (K) } \\
(\mathrm{mg} / \mathrm{h})\end{array}$ & $\begin{array}{l}\text { Bicar- } \\
\text { bonate } \\
\text { (HCO ) } \\
\text { (mg/L) }\end{array}$ & $\begin{array}{l}\text { Alka- } \\
\text { linity } \\
\text { as } \\
\text { Caco } \\
\text { (mg/L) }\end{array}$ & $\begin{array}{l}\text { Dis } \\
\text { solved } \\
\text { sulfate } \\
(\mathrm{so}) \\
(\mathrm{mg} / \mathrm{L})\end{array}$ & $\begin{array}{l}\text { Dis- } \\
\text { solvid } \\
\text { chloride } \\
(\mathrm{Cl}) \\
(\mathrm{mg} / \mathrm{L})\end{array}$ & $\begin{array}{l}\text { Dis: } \\
\text { solved } \\
\text { fluoride } \\
\text { (f) } \\
\text { (mg/L) }\end{array}$ \\
\hline \multirow[t]{2}{*}{$28 / 5 \mathrm{~W}-1 \mathrm{~J} 18$} & 8. .76 & $\cdots$ & 0.04 & 0.02 & $\cdots$ & $\cdots$ & 4 & $\cdot \cdot$ & .. & - & 4 & 1 & 0.1 \\
\hline & $7 \cdot 5 \cdot 78$ & $\cdots$ & .05 & .010 & $\cdots$ & $\cdot \cdot$ & $\cdots$ & $\cdots$ & $\cdots$ & $\cdots$ & $\cdots$ & $\cdots$ & .2 \\
\hline \multirow[t]{2}{*}{$28 / 124-601$} & $9 \cdot 10.64$ & 9.1 & .15 & .05 & 6.5 & 2.5 & 3.3 & 0.3 & 41 & 34 & 3.2 & 2.8 & .1 \\
\hline & $12-22-64$ & -. & .. & .. & 8.0 & 1.8 & $\cdot \cdot$ & $\cdots$ & 35 & 29 & $\cdots$ & $\cdots$ & $\cdots$ \\
\hline \multirow[t]{3}{*}{$28 / 134 \cdot 402$} & $3 \cdot 30 \cdot 71$ & 20 & .03 & .02 & 17 & 3.7 & 4.6 & .2 & 68 & 56 & 3.0 & 3.1 & .1 \\
\hline & $2 \cdot 29 \cdot 72$ & .. & .. & $\cdots$ & 15 & 4.3 & $\cdot \cdot$ & $\cdots$ & $\cdots$ & -. & $\cdot \cdot$ & 3.4 & $\cdot \cdot$ \\
\hline & $5 \cdot 15 \cdot 79$ & .. & $\cdot \cdot$ & .. & $\cdots$ & -. & .. & $\because$ & $\cdot \cdot$ & $\cdot \cdot$ & $\cdot \cdot$ & $\cdots$ & $\cdots$ \\
\hline $.402,3$ & $7 \cdot 9.64$ & 16 & .01 & .0 & 16 & 2.9 & 4.2 & .5 & 59 & $\cdots$ & 3.4 & 4.0 & 1.3 \\
\hline \multirow[t]{4}{*}{ E Ri } & $11 \cdot 27 \cdot 68$ & 14 & .17 & .011 & 16 & 6.6 & 5.8 & .8 & 85 & 70 & 7.8 & 1.5 & .1 \\
\hline & $9 \cdot 21 \cdot 70$ & 10 & .31 & .000 & 11 & 4.9 & 3.0 & .3 & 61 & 50 & 3.7 & 3.2 & .3 \\
\hline & $7 \cdot 6 \cdot 72$ & 4.6 & .16 & .009 & 18 & 9.2 & 4.4 & .3 & 56 & 46 & 11 & 7.0 & .1 \\
\hline & $11 \cdot 7 \cdot 75$ & $\cdots$ & .00 & .00 & -. & .. & .. & $\cdots$ & .. & $\cdots$ & 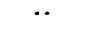 & .. & .1 \\
\hline .403 & $5 \cdot 15 \cdot 79$ & $\cdots$ & .. & .. & $\cdots$ & .. & $\cdot \cdot$ & $\cdots$ & $\cdots$ & $\cdot$ & .. & $\cdots$ & $\cdots$ \\
\hline$-4 R 1$ & 5. 2.61 & 15 & .07 & .. & 22 & 3.3 & 4.3 & .2 & 76 & 62 & 11 & 3.0 & .2 \\
\hline
\end{tabular}

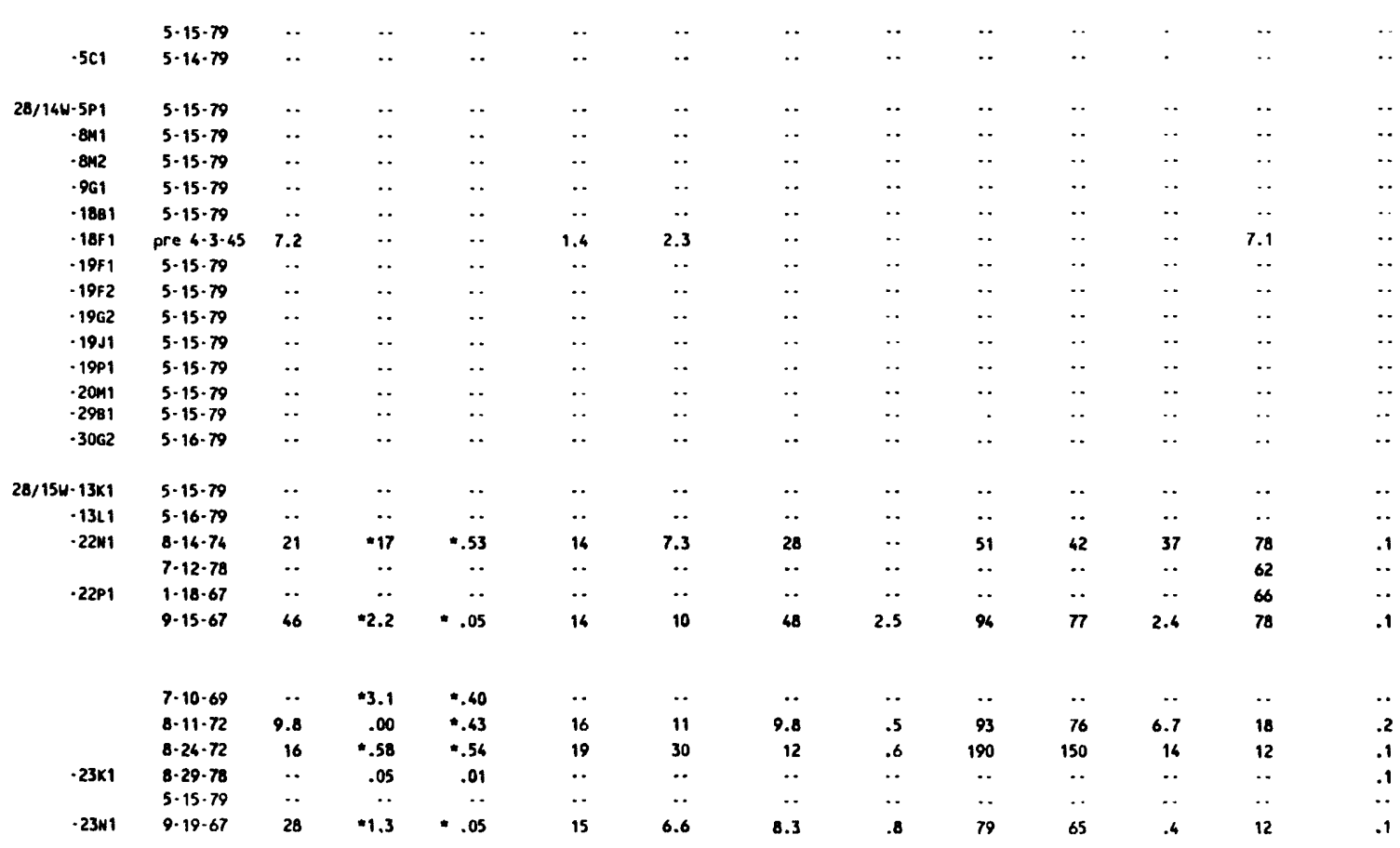

\begin{tabular}{|c|c|c|c|c|c|c|c|c|c|c|c|c|c|}
\hline$-23 M 2$ & $1 \cdot 17-67$ & $\cdot \cdot$ & .. & $* .05$ & $\cdots$ & $\cdots$ & $\cdots$ & .. & .. & $\cdots$ & $\cdots$ & 12 & .. \\
\hline & $9 \cdot 19 \cdot 67$ & 20 & .08 & .. & 8.2 & 3.5 & 7.5 & .8 & 38 & 31 & .4 & 13 & .1 \\
\hline$-23 k 3$ & 5.21 .68 & .. & .. & .. & $\cdots$ & $\cdots$ & .. & .. & .. & .. & $\cdots$ & 11 & .. \\
\hline .26141 & $5 \cdot 14 \cdot 79$ & .. & . & .. & .. & .. & .. & .. & .. & .. & .. & .. & . \\
\hline .2601 & $5 \cdot 25 \cdot 79$ & .. & $\cdots$ & .. & .. & -. & $\cdots$ & .. & .. & . & .. & .. & .. \\
\hline $29 / 2 W \cdot 581$ & $4 \cdot 25 \cdot 79$ & .. & .. & .. & .. & .. & .. & .. & .. & .. & .. & .. & . \\
\hline .511 & $4 \cdot 25 \cdot 79$ & -. & $\cdots$ & .. & .. & $\cdots$ & .. & -. & .. & .. & .. & .. & .. \\
\hline .512 & $4.26 \cdot 79$ & .. & .. & .. & .. & .. & .. & .. & .. & .. & .. & .. & .. \\
\hline .513 & $4 \cdot 25 \cdot 79$ & .. & .. & .. & .. & .. & .. & .. & .. & .. & $\ldots$ & .. & .. \\
\hline-681 & $4 \cdot 24 \cdot 79$ & .. & .. & .. & .. & $\cdots$ & $\cdots$ & .. & .. & .. & .. & .. & .. \\
\hline $.6 C 1$ & $4 \cdot 26 \cdot 79$ & .. & .. & .. & .. & $\cdots$ & .. & .. & .- & .. & .. & .. & .. \\
\hline $.6 C 2$ & $4 \cdot 26 \cdot 79$ & .. & .. & .. &.. & .. & .. & .. & .. & .. & $\cdots$ & .. & . \\
\hline $.6 F 1$ & $4-24 \cdot 79$ & .. & .. & .. & .. & .. & .. & .. & . & . & . & .. & .. \\
\hline .611 & $4 \cdot 26 \cdot 79$ & .. & .. & .. & .. & $\cdot \cdot$ & .. & .. & .. & .. & .. & .. & .. \\
\hline$\cdot 701$ & $4 \cdot 26 \cdot 79$ & .. & .. & .. & .. & .. & $\cdots$ & .. & .. & .. & .. & .. & .. \\
\hline
\end{tabular}




\begin{tabular}{|c|c|c|c|c|c|c|c|c|c|c|c|c|}
\hline $\begin{array}{l}\text { Total } \\
\text { nitrate } \\
\text { (W) } \\
\text { (mg/L) }\end{array}$ & $\begin{array}{l}\text { Iotal } \\
\text { nitrite } \\
\text { (W) } \\
(\mathrm{mg} / \mathrm{L})\end{array}$ & $\begin{array}{l}\text { Total } \\
\text { phos. } \\
\text { phorus } \\
\text { (P) } \\
\text { (mg/L) }\end{array}$ & $\begin{array}{l}\text { Dis. } \\
\text { solved } \\
\text { solids } \\
\text { (residue } \\
\text { ot }(80 \mathrm{C})\end{array}$ & $\begin{array}{l}\text { Mard- } \\
\text { ness } \\
(\mathrm{Ca}, \mathrm{Mg}) \\
(\mathrm{mg} / \mathrm{L})\end{array}$ & $\begin{array}{l}\text { Non- } \\
\text { carbon- } \\
\text { ate } \\
\text { hardness } \\
(\mathrm{mg} / \mathrm{L})\end{array}$ & $\begin{array}{l}\text { Specific } \\
\text { conduc. } \\
\text { tance } \\
\text { (micro- } \\
\text { mos) }\end{array}$ & $\begin{array}{l}\text { pH } \\
\text { (units) }\end{array}$ & $\begin{array}{l}\text { Mater } \\
\text { temper- } \\
\text { oture } \\
\text { (c) }\end{array}$ & $\begin{array}{l}\text { Color } \\
\text { (plat inum- } \\
\text { cobalt } \\
\text { units) }\end{array}$ & $\begin{array}{l}\text { Turbid- } \\
\text { ity } \\
\text { (JTU) }\end{array}$ & $\begin{array}{l}\text { Carbon } \\
\text { dioxide } \\
\text { (CO) } \\
(\mathrm{mg} / \mathrm{L})\end{array}$ & Remarks \\
\hline 0.8 & $\cdot \cdot$ & $\cdot \cdot$ & $\cdot \cdot$ & 56 & $\cdot \cdot$ & 126 & $\cdots$ & $\cdots$ & 0 & 0 & $\cdot \cdot$ & $\cdot \cdot$ \\
\hline .6 & $\cdots$ & .. & $\cdots$ & 48 & .. & 120 & .. & $\because$ & 5 & 0 & $\because$ & $\cdot \cdot$ \\
\hline .16 & 0.00 & $\cdots$ & 45 & 26 & 0 & 81 & 7.4 & $\cdot \cdot$ & 0 & $\cdots$ & 2.6 & $\begin{array}{l}\text { Dissolved or thophosphorus } \\
(P)=0.00 \mathrm{mg} / \mathrm{L}\end{array}$ \\
\hline$\cdots$ & $\cdot \cdot$ & -. & $\cdots$ & 28 & 0 & 73 & 6.9 & $\cdots$ & $\cdot \cdot$ & .. & 7.0 & .. \\
\hline .401 & -. & $\cdot \cdot$ & 80 & 58 & 2 & 128 & 7.7 & 8.4 & 0 & .. & 2.2 & Dissolved aluminm $=0.00 \mathrm{mg} / \mathrm{L}$. \\
\hline$\cdot \cdot$ & -. & .. & .. & 55 & .. & 129 & $\cdot \cdot$ & 9.2 & .. & $\cdots$ & *. & .. \\
\hline$\because$ & 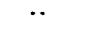 & $\cdots$ & $\because$ & $\because$ & $\because$ & 135 & . & 16.4 & .. & .. & .. & $\cdot \cdot$ \\
\hline .18 & $\cdot \cdot$ & $\cdots$ & 80 & 52 & 4 & 122 & 7.1 & .. & 0 & .. & -. & $\cdots$ \\
\hline .59 &.$\infty 0$ & .25 & 981 & 56 & -. & 120 & 7.3 & 10 & 1 & 2 & 8.5 & Sample chl or inated. \\
\hline .33 & .03 & .25 & 691 & 48 & $\cdot \cdot$ & 74 & 7.2 & 10 & 9 & 1 & 7.0 & -. \\
\hline .09 & .04 & .22 & 821 & 84 & 40 & 134 & 7.2 & $\cdots$ & 5 & 1 & 7.0 & $\cdot \cdot$ \\
\hline .5 & $\cdot \cdot$ & $\cdots$ & $\cdot$ & 64 & $\cdot$ & 150 & $\cdot \cdot$ & .. & .. & $\cdot \cdot$ & .. & $\cdot \cdot$ \\
\hline .. & $\cdot \cdot$ & .. & $\cdots$ & $\cdots$ & $\cdots$ & 160 & $\cdot \cdot$ & 11.4 & $\because$ & $\cdot \cdot$ & .. & -. \\
\hline .09 & .00 & $\cdots$ & 97 & 68 & 6 & 155 & 8.1 & 9.5 & 0 & $\cdots$ & 1.0 & $\begin{array}{l}\text { Dissolved orthophosphorus } \\
(P)=0.01 \mathrm{mg} / \mathrm{L} \text {. Dissolved } \\
\text { aluminum } 0.01 \mathrm{mg} / \mathrm{L} \text {. }\end{array}$ \\
\hline *. & $\cdots$ & $\cdots$ & -. & $\cdots$ & $\cdot-$ & 135 & $\cdots$ & 19.6 & $\cdots$ & .. & $\cdots$ & $\cdot \cdot$ \\
\hline -. & .. & -. & $\cdot \cdot$ & .. & 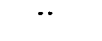 & 220 & .. & 7 & . & 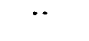 & $\because$ & $\cdot \cdot$ \\
\hline .. & *. & -. & .. & .. & .. & 120 & $\because$ & 11.6 & .. & .. & .. & .. \\
\hline .. & *. & $\cdot \cdot$ & $\cdot \cdot$ & $\cdots$ & 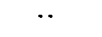 & 75 & *. & 20 & $\cdots$ & $\because$ & $\cdots$ & .. \\
\hline$\cdots$ & $\cdots$ & -. & -. & $\cdot \cdot$ & $\cdots$ & 120 & .. & 18.4 & $-\cdot$ & -. & -. & $\cdot \cdot$ \\
\hline *. & $\cdots$ & $\cdots$ & $\cdot \cdot$ & -. & $\cdots$ & 210 & *. & 5.6 & .. & $\cdots$ & -. & -. \\
\hline .. & $\cdots$ & $\cdots$ & .. & $\ddot{*}$ & $\cdots$ & 220 & $\because$ & 6.4 & $\cdots$ & .. & $\cdot \cdot$ & $\cdot \cdot$ \\
\hline . & $\cdots$ & $\cdot \cdot$ & $60 \mathrm{r}$ & 57 & $\because$ & .. & 6.5 & .. & .. & 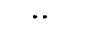 & 8.3 & Dissolved aluminum = $1.55 \mathrm{mg} / \mathrm{L}$. \\
\hline .. & $\cdots$ & $\cdots$ & .. & -. & $\cdot \cdot$ & 110 & -. & 13 & -. & - & .. & .. \\
\hline .. & -. & .. & .. & .. & - & 100 & .. & 15 & -. & .. &.. & $\cdot \cdot$ \\
\hline 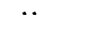 & $\cdots$ & $\cdot \cdot$ & ". & $\cdots$ & $\cdots$ & 100 &. & 12 & $\cdot \cdot$ & $\cdots$ & . & $\cdot$. \\
\hline$\cdot \cdot$ & 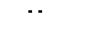 & $\cdot \cdot$ & .. & $\cdots$ & . & 100 & .. & 14 & -. & $\cdots$ & $\cdot$. & $\cdot \cdot$ \\
\hline$\cdot$. & $\cdots$ & $\cdots$ & $\cdots$ & -. & $\cdots$ & 110 & -. & 13 & .. & $\cdot \cdot$ & $\cdots$ & .. \\
\hline .. & .. & . &.. & .. & .. & 110 & -. & 13 & .. &.. & $\cdots$ & .. \\
\hline$\cdots$ & $\cdots$ & $\cdots$ & $\cdots$ & $\cdot \cdot$ & $\cdot \cdot$ & 250 & $\cdots "$ & 16 & -. & $\cdots$ & $\cdot \cdot$ & $\cdots$ \\
\hline$\cdot \cdot$ & $\cdots$ & $\cdot \cdot$ & - & $\cdot \cdot$ & $\cdots$ & 95 & $\cdots$ & 19 & $\cdot \cdot$ & $\cdot \cdot$ & $\cdot \cdot$ & $\cdots$ \\
\hline .. & .. & .. & .. & .. & .. & 90 & .. & 12 & .. &.. & .. & .. \\
\hline 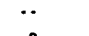 & $\cdots$ & $\ddot{*}$ & 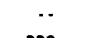 & .. &.. & 90 &.. & 11 & $\cdots$ & .. & $-\cdot$ & .. \\
\hline .8 & .01 & .15 & 229 & 60 & 26 & 320 & 7.1 & 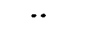 & $* 50$ & 4 & $\cdots$ & $\cdots$ \\
\hline$\cdots$ & $\cdot \cdot$ & * & $\because$ & $\cdots$ & .. & 376 & .. & .. & .. & .. & .. &.. \\
\hline .. & .. & .. & $\cdots$ & .. & .. & 372 &.. & $\cdots$ & .. & .. & -. & .. \\
\hline .02 & .. & .. & 260 & 76 & 0 & 420 & "6.3 & 9.5 & 10 & .. & 75 & $\begin{array}{l}\text { Cyanide, } 0.01 \mathrm{mg} / \mathrm{L} \text {. } \\
\text { Phenol, } 0.001 \mathrm{mg} / \mathrm{h} . \\
\text { ABS, } 0.01 \mathrm{mg} / \mathrm{L} .\end{array}$ \\
\hline 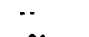 & $\ddot{*}$ & 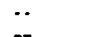 & $\because$ & $\ddot{*}$ & .. & 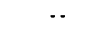 & .. & 10 & $\because$ & .. & $\cdots$ & .. \\
\hline .01 & .02 & .23 & 118 & 84 & 8 & 136 & 6.6 & 10 & 5 & $* 15$ & 55 & .. \\
\hline .08 & .06 & .39 & 198 & 120 & .. & 180 & 6.8 & 10 & $* 70$ & $* 65$ & 60 & Sulfide $=0.00 \mathrm{mg} / \mathrm{L}$. \\
\hline 2.0 & $\cdot \cdot$ & .. & 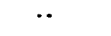 & 20 & $\cdots$ & 160 & .. & .. & 5 & 0 & $\cdots$ & $\because$ \\
\hline$\ddot{m}$ & $\cdots$ & $\cdots$ & 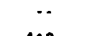 & $\ddot{*}$ & $\cdots$ & 120 & 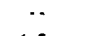 & 12.6 & 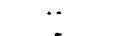 & $\cdot \cdot$ & $\because$ & - \\
\hline .00 & -・ & $\cdots$ & 118 & 65 & 0 & 167 & 6.5 & 9.0 & 5 & $\cdots$ & 40 & $\begin{array}{l}\text { Cyanide, } 0.01 \mathrm{mg} / \mathrm{h} \text {. } \\
\text { Phenol, } 0.001 \mathrm{mg} / \mathrm{L} \text {. } \\
\text { ABS, } 0.01 \mathrm{mg} / \mathrm{L} \text {. }\end{array}$ \\
\hline 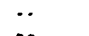 & *. & $\cdot \cdot$ & $\because$ & $\cdots$ & .• & 126 & $\cdots$ & -. & $\cdots$ & *. & $\because$ & $\cdots-D_{0} \cdot \cdots \cdots, \ldots, \ldots$ \\
\hline .20 & $\cdot \cdot$ & $\cdots$ & 83 & 35 & 4 & 113 & $" 6.2$ & 8.0 & 5 & $\cdots$ & 38 & $\cdots$ \\
\hline$\cdots$ & *. & .. & -. & $\cdots$ & .. & 182 & .. & 9.5 & $\cdots$ & *. & $\cdots$ & $\begin{array}{l}\text { Iron caste. Some } \mathrm{H}_{2} \mathrm{~S} \text {. } \\
\text { Becomes yellow and turbid } \\
\text {.. after standing several days. }\end{array}$ \\
\hline $\begin{array}{l}\cdots \\
\cdots\end{array}$ & $\ddot{*}$ & $\cdots$ & $\begin{array}{l}\ddot{ } \\
\cdots\end{array}$ & $\begin{array}{l}\ddot{ } \\
\cdots\end{array}$ & $\begin{array}{l}\ddot{ } \\
\cdots\end{array}$ & $\begin{array}{l}100 \\
100\end{array}$ & 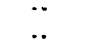 & $\begin{array}{l}14.8 \\
10.2\end{array}$ & $\begin{array}{l}\ddot{ } \\
\cdots\end{array}$ & $\ddot{*}$ & $\ddot{*}$ & . \\
\hline$\cdots$ & -. & .. & .. &.. & .. & 300 & $\cdots$ & 16 &. & .. & .. & .. \\
\hline .. & .. & .. & .. & .. & .. & 315 & .. & 11 & .. & $\cdots$ & .. & .. \\
\hline$\cdot \cdot$ & $\cdots$ & -. & .. & 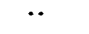 & $\cdot \cdot$ & 355 & 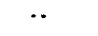 & 16 & .. & .. & 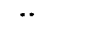 & .. \\
\hline$\cdot \cdot$ & .. & .. & .. & .. & . & 270 & $\because$ & 12 & .. & $\cdots$ & - & $\cdots$ \\
\hline$\cdot$ & $\cdots$ & .. & .. & $\cdots$ & .. & 242 & .. & 21 & .. & .. &.. & $\cdots$ \\
\hline . & $\cdots$ & - & .. & .. & .. & 260 & .. & 12 & .. & .. & .. & .. \\
\hline$\cdots$ & $\cdots$ & .. & -. & .. & .. & 234 & .. & 21 &.. & .. & .. & 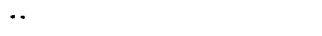 \\
\hline . &. & .. & .. & .. & -. & 207 & .. & 20.4 & $\cdots$ & .. & $\because$ & $\cdot \cdot$ \\
\hline$\cdot \cdot$ & $\cdots$ & $\cdot$ & $\cdot \cdot$ & $\cdots$ & $\cdots$ & 270 & $\because$ & 20 & $\because$ & $\cdot \cdot$ & $\cdot \cdot$ & $\because$ \\
\hline$\cdot \cdot$ & $\cdots$ & . & ・. & $\cdot \cdot$ & $\cdots$ & 315 & .. & 21 & $\cdot \cdot$ & . & 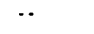 & .. \\
\hline
\end{tabular}




\begin{tabular}{|c|c|c|c|c|c|c|c|c|c|c|c|c|c|}
\hline si to number & $\begin{array}{l}\text { Date } \\
\text { sempled }\end{array}$ & $\begin{array}{l}\text { Dis. } \\
\text { solved } \\
\text { silica } \\
\text { (sio) } \\
\text { (mg/l) }\end{array}$ & $\begin{array}{l}\text { Dis- } \\
\text { solved } \\
\text { iron } \\
\text { (fe) } \\
(\mathrm{wg} / \mathrm{L})\end{array}$ & $\begin{array}{l}\text { Dis- } \\
\text { solved } \\
\text { mangan- } \\
\text { ese (in) } \\
\text { (ug/L) }\end{array}$ & $\begin{array}{l}\text { Dis- } \\
\text { solved } \\
\text { calcium } \\
\text { (Ca) } \\
\text { (mg/L) }\end{array}$ & $\begin{array}{l}\text { Dis- } \\
\text { solved } \\
\text { magnes- } \\
\text { inn (Mg) } \\
(\mathrm{mg} / \mathrm{L})\end{array}$ & $\begin{array}{l}\text { Dis: } \\
\text { solved } \\
\text { sodium } \\
\text { (Na) } \\
(\mathrm{mg} / \mathrm{L})\end{array}$ & $\begin{array}{l}\text { Dis. } \\
\text { solved } \\
\text { potes. } \\
\text { siun (K) } \\
\text { (mg/L) }\end{array}$ & $\begin{array}{l}\text { Bicar. } \\
\text { bonate } \\
\text { (HCO) } \\
\text { (mg/L) }\end{array}$ & $\begin{array}{l}\text { Alka- } \\
\text { linity } \\
\text { as } \\
\text { Caco } \\
\text { (mg/L) }\end{array}$ & $\begin{array}{l}\text { Dis- } \\
\text { solved } \\
\text { sulfate } \\
\text { (so) } \\
(\mathrm{mg} / \mathrm{L})\end{array}$ & $\begin{array}{l}\text { Dis- } \\
\text { solved } \\
\text { chloride } \\
(\mathrm{Cl}) \\
(\mathrm{mg} / \mathrm{l})\end{array}$ & $\begin{array}{l}\text { Dis: } \\
\text { solved } \\
\text { fluoride } \\
\text { (f) } \\
\text { (mg/L) }\end{array}$ \\
\hline $29 / 3 N-1 J 1$ & $7 \cdot 6 \cdot 78$ & .. & .. & .. & .. & -. & . & -. & .. & $\cdots$ & $\cdots$ & 7.3 & .. \\
\hline$\cdot 132$ & $4.26 \cdot 79$ & .. & .. & .. & .. & .. & .. & .. & .. & .. & $\cdots$ & .. & .. \\
\hline$\cdot 2 c 1$ & $12 \cdot 16 \cdot 59$ & 17 & $1 . \pi$ & $\because$ & 7.5 & 0.9 & 300 & 0.8 & 230 & .. & 19 & 320 & 1.1 \\
\hline$-2 \times 1$ & $7 \cdot 22 \cdot 68$ & -. & .. & $\cdot \cdot$ & .. & -. & .. & $\cdots$ & .. & .. & $\cdots$ & 150 & .. \\
\hline-201 & $7 \cdot 21-68$ & .. & -. & .. & -. & -. & .. & $\cdot \cdot$ & -. & .. & .. & $\cdot \cdot$ & $\cdots$ \\
\hline$-3 E 1$ & $4 \cdot 26 \cdot 79$ & $\cdots$ & -. & -. & $\cdots$ & .. & .. & .. & .. & 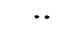 & $\cdot \cdot$ & .. & .. \\
\hline$-3 \mathbf{F 1}$ & $4 \cdot 26 \cdot 79$ & -. & -. & .. & $\cdot \cdot$ & -. & $\cdots$ & .. & .. & .. & .. & -. & . \\
\hline .302 & $4 \cdot 26 \cdot 79$ & .. & $-\cdot$ & $\cdots$ & $\cdots$ & $\cdots$ & .. & .. & *. & .. & $\cdots$ & $\cdots$ & .. \\
\hline$\cdot 3 R 2$ & $4 \cdot 26 \cdot 79$ & $\cdot \cdot$ & -. & $\cdot \cdot$ & $\cdot \cdot$ & .. & $\cdots$ & $\cdots$ & $\cdots$ & . & $\cdots$ & $\cdots$ & .. \\
\hline .581 & $4 \cdot 25 \cdot 79$ & .. & .. & .. & .. & 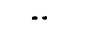 & *. & .. & .. & .. & .. & ・. & $\cdots$ \\
\hline .583 & $4 \cdot 25 \cdot 79$ & .. & .. & .. & .. & .. & .. & .. & -. & $\cdots$ & $\cdots$ & .. & .. \\
\hline .584 & $4-25 \cdot 79$ & .. & .. & .. & .. & .. & .. & .. & .. & .. & .. & .. & . \\
\hline $.5 C 1$ & $4 \cdot 25 \cdot 79$ & .. & .. & .. & .. & .. & .. & .. & .. & .. & .. & .. &.. \\
\hline $.5 c 2$ & $4-25 \cdot 79$ & .. & .. &.. & .. & .. & .. & .. & .. & .. & .. & .. & .. \\
\hline $.5 F 2$ & $4 \cdot 25 \cdot 79$ & $\cdots$ & $\cdots$ & .. & $\cdots$ & $\cdots$ & $\cdot \cdot$ & $\cdots$ & .. & .. & $\cdot \cdot$ & . & .. \\
\hline \multirow[t]{2}{*}{$-|2 A|$} & $10 \cdot 1.68$ & .. & .. & $\cdots$ & .. & .. & .. & .. & .. & $\cdot \cdot$ & $\cdot \cdot$ & 3.6 & .. \\
\hline & $7 \cdot 6 \cdot 78$ & $\cdots$ & .. & $\cdots$ & .. & .. & $\cdots$ & .. & .. & .. & .. & 6.0 & .. \\
\hline .1212 & $4 \cdot 27 \cdot 79$ & .. & .. & .. & .. & .. &.. & .. & .. & .. & .. & .. & .. \\
\hline \multirow[t]{2}{*}{.1201} & $7-23-68$ & .. & .. & .. & .. & .. & .. & .. & .. & .. & .. & 9.6 & .. \\
\hline & $7 \cdot 6 \cdot 78$ & .. & .. & .. & .. & .. & .. & .. & .. & .. & .. & 11 & .. \\
\hline \multirow{2}{*}{$-12 f 2$} & $7 \cdot 23.68$ & 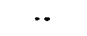 & .. & .. & .. & .. & .. & . & .. & -. & .. & 6.6 & .. \\
\hline & $7 \cdot 6.78$ & .. & -. & .. & $\cdots$ & .. & .. & $\cdots$ & .. & $\cdot \cdot$ & $\cdot \cdot$ & 6.5 & $\cdot \cdot$ \\
\hline$\cdot 12 \mathrm{H} 1$ & $4 \cdot 26 \cdot 79$ &.$\cdot$ & .. & .. & .. & $\cdot \cdot$ & .. & 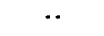 & .. & $\cdots$ & $\cdot \cdot$ & .. & .. \\
\hline$-12 \mathrm{H} 2$ & $4 \cdot 27 \cdot 79$ & -. & $\cdots$ & -. & $\cdots$ & $\cdots$ & .. & .. & .. & .. & .. & .. & .. \\
\hline -12M1 & $4 \cdot 26 \cdot 79$ & .. & .. & -. & .. & .. & .. & .. & .. & .. & .. & .. & .. \\
\hline$-15 E 1$ & $4 \cdot 24 \cdot 79$ & .. & .. & *. & $\cdots$ & .. & $\cdot \cdot$ & .. &.$\cdot$ & .. & .. & .. & .. \\
\hline$-31 R 1$ & $10 \cdot 11 \cdot 78$ & .. & .05 & 0.010 & $\cdot \cdot$ & .. & .. & .. & $\cdot \cdot$ & $\cdots$ & $\cdots$ & .. & .1 \\
\hline $29 / 4 W-1 M 1$ & .. & .. & .. & .. & .. & .. & .. & .. & .. & .. &.. & .. & .. \\
\hline$\cdot 2 \mathrm{C} 1$ & $\cdot \cdot$ & $\cdots$ & $\cdot \cdot$ & .. & $\cdots$ & $\cdot \cdot$ & $\cdots$ & $\cdots$ & $\cdot \cdot$ & $\cdots$ & $\cdot$. & -. & $\cdot \cdot$ \\
\hline $29 / 5 w-901$ & $6 \cdot 11 \cdot 79$ & $\cdots$ & $\cdots$ & .. & .. & $\cdots$ & .. & .. & $\cdots$ & $\cdots$ & .. & .. & $\cdots$ \\
\hline $29 / 7 \mathrm{~N}-901$ & $6 \cdot 26 \cdot 78$ & .. & .11 & .010 & .. & . & .. & . & .. & .. & .. & .. & .2 \\
\hline-911 & $6 \cdot 26 \cdot 78$ & .. & $* 1.2$ & .010 & .. & .. & .. & .. & .. & .. & .. & .. & .1 \\
\hline$-17 p / 8$ & $6 .-66$ & .. & $\cdots$ &. .06 & .. & .. & $\cdot \cdot$ & $\cdot \cdot$ & .. & $\cdot \cdot$ & .. & 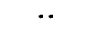 & .. \\
\hline \multirow[t]{3}{*}{$29 / 8 W \cdot 27 \mathrm{Kls}$} & $8-19-69$ & 31 & $\star 1.7$ & .01 & 6.6 & 1.0 & 30 & 2.0 & .. & $\cdots$ & 6 & 8.5 & 1.0 \\
\hline & $8 \cdot 19 \cdot 69$ & 29 & 2.4 & .01 & 4.8 & 1.5 & 38 & 2.3 & .. & .. & 10 & 6.0 & 1.0 \\
\hline & $8-19-69$ & 40 & $" 1.0$ & .00 & 4.0 & 1.5 & 31 & .2 & $\cdot \cdot$ & .. & 26 & 6.5 & 1.0 \\
\hline \multirow[t]{2}{*}{$29 / 9 \mathrm{w}-32 \mathrm{Cla}$} & $11 \cdot 30.54$ & 58 & .. & -. & 1.2 & .0 & 80 & 2.6 & $\cdots$ & .. & 34 & 17 & 1.6 \\
\hline & $10 \cdot 6.67$ & 58 & .02 & $\cdot \cdot$ & .. & .. & $\cdot \cdot$ & .. & $\cdots$ & .. & $\cdots$ & 4.0 & .. \\
\hline $.32 c 28$ & 10.4 .67 & 52 & .02 & .. & .. & .. & .. & .. & .. & $\cdots$ & .. & 3.5 & -. \\
\hline$\cdot 32 \mathrm{C3s}$ & $10 \cdot 4.67$ & 57 & .01 & .. & .. & $\cdots$ & .. & .. & .. & .. & .. & 4.2 & .. \\
\hline \multirow[t]{3}{*}{$.32 C 48$} & $10 \cdot 4.67$ & 47 & .07 & $\cdot \cdot$ & .. & .. & .. & $\cdots$ & .. & .. & .. & 19 & .. \\
\hline & $10-22.67$ & 38 & .10 & .. & 1.3 & .2 & $\cdot \cdot$ & $\cdots$ & .. & .. & .. & 11 & .. \\
\hline & 1.27 .68 & 49 & .01 & .. & 1.2 & .0 & 75 & 1.0 & .. & $*$ & 29 & 24 & 1.5 \\
\hline $.32 C 58$ & $10 \cdot 4.67$ & 43 & .02 & .. & -. & .. & $\cdot \cdot$ & .. & .. & .. & .. & 1.5 & .. \\
\hline \multirow[t]{2}{*}{$-32 c 1.58$} & 10.4 .67 & 48 & .20 & .. & .. & -. & .. & .. & .. & .. & .. & 16 & -. \\
\hline & $3-14-78$ & .. & .05 & .010 & .. & -. & .. & .. & .. & .. & .. & .. & 1.3 \\
\hline .32068 & $10 \cdot 4.67$ & .. & .02 & $\cdots$ & -. & .. & $\cdots$ & $\cdots$ & -. & .. & - & 4.0 & .. \\
\hline$-3201+F 1$ & $6 \cdot 26 \cdot 78$ & $\cdots$ & .05 & .010 & 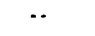 & $\cdots$ & $\cdot$. & .. &.. & $\cdots$ & .. & .. & .. \\
\hline $29 / 13 W-9 E 1$ & $5 \cdot 16 \cdot 79$ & .. & .. &.. & .. & $\cdots$ & .. & .. & $\cdots$ & .. & .. & .. & .. \\
\hline - 16N2 & $5 \cdot 14 \cdot 79$ & $\because$ & .. & $\because$ & $\because$ & .. & .. & $\cdots$ & .. &.$\cdot$ & .. & $\cdots$ & .. \\
\hline \multirow[t]{3}{*}{$30 / 2 W \cdot 15 L 1$} & $2 \cdot 4 \cdot 68$ & .. & .04 & .006 & -. & $\cdots$ & .. & $\cdots$ & . & .. & $\cdots$ & 170 & . \\
\hline & $10 \cdot 1-68$ & .. & .. & .. & .. & .. & .. & .. & .. & .. & .. & 140 & .. \\
\hline & $8 \cdot 9.74$ & 12 & .00 & .010 & 54 & 52 & 73 & .. & 310 & 260 & 34 & 170 & .4 \\
\hline \multirow[t]{4}{*}{$.15 L 3$} & $4 \cdot 26 \cdot 73$ & .. & .. & .. & .. & .. & 230 & .. &. &.. & .. & $\$ 880$ & .. \\
\hline & $1 \cdot 16 \cdot 75$ & 18 & .11 & .010 & 43 & 37 & 170 & .. & 100 & 80 & 75 & 330 & .1 \\
\hline & $2 \cdot 4 \cdot 75$ &.. & .. & . & .. & .. & 230 & $\cdots$ &. & .. & $\cdots$ & .650 & .. \\
\hline & $2 \cdot 4 \cdot 75$ & .. & .. & 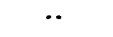 & .. & .. & 200 & $\cdot \cdot$ & .. & .. & .. & 1620 & $\cdot \cdot$ \\
\hline \multirow[t]{7}{*}{.1661} & $10 \cdot 1 \cdot 60$ & . & .. & .. & .. & .. & .. & - & . & - & .. & 4310 & .. \\
\hline & $6 \cdot 8.71$ & 2.5 & .04 & .003 & 120 & 70 & 66 & 2.7 & 180 & 140 & 52 & 390 & .1 \\
\hline & $11 \cdot 21 \cdot 71$ & 7.0 & .08 & .000 & 120 & 76 & 20 & 7.4 & 170 & 140 & 31 & $\$ 330$ & .1 \\
\hline & $2 \cdot 9 \cdot 72$ & $\cdot \cdot$ & -. & - & .. & . & $\cdots$ & .. & -. & .. & $\cdots$ & 32 & $\cdots$ \\
\hline & $4 \cdot 25 \cdot 72$ & 1.0 &. .52 & .006 & 52 & 27 & 23 & 5.0 & 270 & 220 & 32 & 26 & .1 \\
\hline & $1 \cdot 9 \cdot 73$ & 13 & .26 & .015 & 30 & 10 & 38 & 8.2 & .. & -. & 18 & 42 & .1 \\
\hline & $3 \cdot 6.75$ & 13 & .13 & .02 & 49 & 26 & 19 & - & 240 & 200 & 29 & 30 & .3 \\
\hline
\end{tabular}




\begin{tabular}{|c|c|c|c|c|c|c|c|c|c|c|c|c|}
\hline $\begin{array}{l}\text { Total } \\
\text { nitrate } \\
\text { (W) } \\
(\mathrm{mg} / \mathrm{L})\end{array}$ & $\begin{array}{l}\text { Total } \\
\text { nitrite } \\
\text { (M) } \\
\text { (ng/L) }\end{array}$ & $\begin{array}{l}\text { Total } \\
\text { phos. } \\
\text { phorus } \\
(P) \\
(\mathrm{mg} / \mathrm{L})\end{array}$ & $\begin{array}{l}\text { Dis. } \\
\text { solved } \\
\text { solids } \\
\text { (residue } \\
\text { ot }(80 \mathrm{C} \text { ) }\end{array}$ & $\begin{array}{l}\text { Mard. } \\
\text { ness } \\
(\mathrm{Ca}, \mathrm{Mg}) \\
(\mathrm{mg} / \mathrm{L})\end{array}$ & $\begin{array}{l}\text { Non. } \\
\text { carbon. } \\
\text { ate } \\
\text { harchess } \\
\text { (mg/L) }\end{array}$ & $\begin{array}{l}\text { Specific } \\
\text { conduc. } \\
\text { tence } \\
\text { (micro- } \\
\text { mos) }\end{array}$ & $\begin{array}{c}\text { PH } \\
\text { (units) }\end{array}$ & $\begin{array}{l}\text { Water } \\
\text { temper. } \\
\text { ature } \\
\text { ( c) }\end{array}$ & $\begin{array}{l}\text { Color } \\
\text { (platinum. } \\
\text { cobalt } \\
\text { units) }\end{array}$ & $\begin{array}{l}\text { Turbid- } \\
\text { ity } \\
\text { (JTU) }\end{array}$ & $\begin{array}{l}\text { Carbon } \\
\text { dioxide } \\
\text { (CO ) } \\
\text { (mg/L) }\end{array}$ & Remarks \\
\hline .. & .. & .. & .. & .. & .. & 390 & . & .. & .. & .. & . & .. \\
\hline .. & .. & .. & .. & .. & .. & 400 & .. & 14 & .. & .. & .. & .. \\
\hline 0.05 &.. & 0.22 & $* 778$ & 82 & 0 & 1,620 & $=6.0$ & 11 & 10 & .. & .. & $\begin{array}{l}\text { S! ight turbidity; probably due } \\
\text { to iron. }\end{array}$ \\
\hline .. & .. & .. & $\bullet$ & .. & . & 1,300 & .. & .. & .. & .. & $\cdots$ & -. \\
\hline .. & .. & .. & .. & .. & .. & .. & .. & .. & .. & .. & .. & Reportedly salty. \\
\hline .. & .. & $\cdots$ & .. & .. & $\cdots$ & 550 & .. & 13 & .. & .. & .. & $\therefore$ \\
\hline .. & .. & $\cdots$ & .. & .. & $*$ & 550 & .. & 13 & .. & .. & .. & .. \\
\hline .. & .. & .. & .. & .. & .. & 380 & .. & 10 & . & .. & .. & .. \\
\hline .. & .. & $\cdots$ & .. & .. & .. & 360 & .. & 12 & .. & .. & .. & . \\
\hline .. & .. & .. & .. & .. & .. & 355 & .. & 16.4 & .. & .. & .. & . \\
\hline .. & .. & . & .. & .. & .. & 325 & .. & 11.4 & .. & .. & .. & . \\
\hline .. & .. & .. & .. & .. & .. & 380 & . & 10 & $\cdots$ & .. & .. & .. \\
\hline .. & .. & $\cdots$ & .. & .. & .. & 370 & .. & 10 & .. & .. & .. & .. \\
\hline .. & .. & .. & .. & .. & .. & 278 & . & 13 & . & .. & .. & .. \\
\hline . & .. & .. & .. & .. & .. & 380 & .. & 10 & .. & .. & .. & .. \\
\hline .. &.. & .. & .. & .. & .. & 106 & .. & .. & .. & .. &.. & .. \\
\hline .. & .. & .. & .. & .. & .. & 193 & .. & .. & .. & .. & .. & . \\
\hline 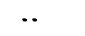 & .. & .. & .. & .. & .. & 294 & .. & 24 & .. & .. & .. & Bad taste and odor. \\
\hline .. & .. & .. & .. & .. & . & 339 & .. & .. & . & .. & .. & .. \\
\hline .. & .. & .. & .. & .. & .. & 331 & .. & .. & .. & .. & .. & .. \\
\hline$\cdots$ & .. & . & .. & .. & $\cdots$ & 307 & .. & .. & .. & .. & .. & .. \\
\hline .. & .. & .. & .. & .. & .. & 284 & .. & .. & .. & .. & .. & .. \\
\hline .. & .. & .. & .. & . & .. & 445 & .. & 14 & .. & -. & .. & .. \\
\hline .. & .. & .. & .. & .. & .. & 285 & .. & 22 & . & .. & .. & .. \\
\hline .. & .. & .. & .. & .. & -. & 310 & .. & 18 & .. & .. & .. & .. \\
\hline .. & .. & .. & .. & .. & .. & 320 & .. & 24 & -. & .. & .. & \\
\hline 1.3 & . & .. & .. & 120 & .. & 300 & .. & .. & 5 & 0 & $\cdots$ & .. \\
\hline .. & .. & .. & .. & .. & .. & .. & .. & .. & .. & .. & .. & Some "iron" problem. \\
\hline -. &.. & .. & -. & -. & $\cdots$ & .. & -. & .. & .. & .. & .. & Some "rust" problem. \\
\hline .. & .. & .. & .. & .. & $\cdots$ & 268 & .. & 17 & . & . &.. & .. \\
\hline .5 & .. & .. & .. & 50 & .. & 100 & .. & .. & 5 & 0 & .. & .. \\
\hline .2 & .. & .. & .. & 68 & .. & 135 & .. & .. & 5 & 0 & .. & .. \\
\hline .. & $\cdots$ & $\cdots$ & .. & 130 & .. & 250 & .. & .. & .. & .. & .. & -. \\
\hline .12 & 0.00 & 0.58 & 172 & 20 & .. & 284 & $* 9.4$ & 46.7 & 4 & 1 & 0 & Sample from drinking tap. \\
\hline .10 & .01 & .12 & 177 & 18 & .. & 296 & $\$ 9.6$ & 46.2 & 2 & 0 & 0 & Sample from reservolr. \\
\hline .20 & .06 & .19 & 155 & 16 & .. & 296 & $* 9.2$ & 43.3 & 2 & 3 & 0 & Sample from filter plant. \\
\hline .23 & .. & .. & 262 & 3 & 0 & 355 & $\$ 9.2$ & 55.5 & 0 & .. & .1 & .. \\
\hline .. & .. & .. & .. & 0 & .. & 357 & $\% 9.5$ & 48.0 & 5 & .. & $\therefore$ & .. \\
\hline -. & $\cdots$ & .. & $\cdots$ & 2 & .. & 355 & $\$ .5$ & 44.5 & 5 &.. & .. & .. \\
\hline -. & .. & .. & .. & 2 & .. & 354 & 9.5 & 50.0 & 5 & .. & .. & .. \\
\hline .. & .. & -. & . & 8 & .. & 330 & 9.3 & 42.0 & 5 & .. & .. & .. \\
\hline .00 & -. & .. & $\cdots$ & 4 & 0 & 234 & $\bullet 9.1$ & $\cdot$. & .. &.. & .1 & .. \\
\hline .00 & .. & .. & 252 & 3 & 0 & 342 & $\$ 9.4$ & 45 & 5 & .. & .1 & .. \\
\hline$\therefore$ & .. & .. &. & 5 & .. & 25 & $* 6.4$ & 11.7 & 5 & .. & 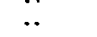 & .. \\
\hline -. & -. & .. & .. & 5 & .. & 330 & $* 9.4$ & 42.0 & $\therefore$ &.. & $\cdots$ & stight $H_{2}$ s odor. \\
\hline .8 & .. & .. & .. & 20 & .. & 348 & .. & .. & 0 & 0 & .. & .. 2 \\
\hline .. & -. & .. & -. & $\therefore$ & .. & 357 & -. & 48 & .. & .. & .. & .. \\
\hline .8 & -. & -. & .. & 65 & .. & 130 & .. & .. & 0 & 0 & .. & .. \\
\hline .. & .. & .. & .. & .. & .. & 189 & .. & 12.2 & .. & .. & .. & .. \\
\hline$\cdots$ & .. & $\cdots$ & .. & .. & .. & 119 & .. & 13.1 & .. & .. & .. & .. \\
\hline .. & .. & .. & .. & .. & .. & $\cdots$ & 7.8 & .. & .. & .. & .. & $\begin{array}{l}\text { Large amount of blue-green } \\
\text { algae (Anacystics). }\end{array}$ \\
\hline .. & $\cdots$ & .. & $\bullet$ & .. & .. & 1,300 & .. & .. & .. & .. & .. & .. \\
\hline .7 & .00 & .05 & H553 & 350 & 95 & 740 & 7.2 & .. & 6 & 0 & .. & .. \\
\hline.. & .. & .. & $\cdots$ & $\therefore$ & .. & .. & .. & .. & $\therefore$ & .. & .. & .. \\
\hline 3.5 & .03 & .38 & -726 & 260 & .. & .. & 7.4 & .. & 5 & 0 & .. & .. \\
\hline . & .. & $\cdots$ & 11,440 & . & .. & .. & .. & .. & .. & .. & .. & Sampled at 13:45. \\
\hline .. & .. & .. & 11,570 & .. & .. & .. & .. & .. & .. & .. & .. & sampled at 13:00. \\
\hline .. & .. & .. & 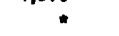 & .. & .. & 1,340 & .. & .. & .. & .. & .. & .. \\
\hline 2.8 &.$\infty 0$ & .04 & 793 & 590 & 440 & 1,400 & 7.7 & 10 & 15 & 0 & 7.1 & .. \\
\hline 2.6 & .05 & .37 & .675 & 600 & 450 & 1,400 & 7.8 & 10 & 4 & 1 & 5.8 & .. \\
\hline .. & .. &.. &.. & . & .. & .. &.. & 10 & . & $\therefore$ & . & $\begin{array}{l}\text { Well was } 421 \mathrm{ft} \text { deep; } \\
\text { changed to } 321 \mathrm{ft} \text {. }\end{array}$ \\
\hline .03 & .02 & .62 & 301 & 240 & 16 & 480 & 7.6 & .. & 5 & 3 & 14 & .. \\
\hline 1.7 & .00 & .9 & 286 & 120 & .. & 370 & 8.4 & .. & 5 & 0 & 1.2 & .. \\
\hline 1.3 & .00 & .35 & 289 & 230 & .. & 482 & 8.1 & .. & 7 & 0 & .. & .. \\
\hline
\end{tabular}




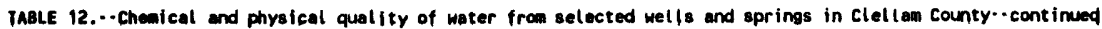

\begin{tabular}{|c|c|c|c|c|c|c|c|c|c|c|c|c|c|}
\hline site number & $\begin{array}{l}\text { Date } \\
\text { eampled }\end{array}$ & $\begin{array}{l}\text { Dis: } \\
\text { eolved } \\
\text { eillica } \\
\text { (sio) } \\
\text { (moll) }\end{array}$ & $\begin{array}{l}\text { Dis. } \\
\text { solved } \\
\text { iron } \\
\text { (Fe) } \\
\text { (ug/L) }\end{array}$ & $\begin{array}{l}\text { Dis. } \\
\text { solved } \\
\text { mangan" } \\
\text { ese (Mn) } \\
\text { (ug/L) }\end{array}$ & $\begin{array}{l}\text { Dis- } \\
\text { solved } \\
\text { calcilu } \\
\text { (ca) } \\
(\mathrm{moll})\end{array}$ & $\begin{array}{l}\text { Dis- } \\
\text { solved } \\
\text { magnes- } \\
\text { iun (Mg) } \\
(\mathrm{mg} / \mathrm{L})\end{array}$ & $\begin{array}{l}\text { Dis- } \\
\text { oolved } \\
\text { codicn } \\
\text { (Na) } \\
(\mathrm{ma} / \mathrm{L})\end{array}$ & $\begin{array}{l}\text { Dila- } \\
\text { solved } \\
\text { potas- } \\
\text { sium (K) } \\
(\operatorname{lng} / L)\end{array}$ & $\begin{array}{l}\text { Bicer. } \\
\text { bonate } \\
\text { (HCO) } \\
\text { (mo/L) }\end{array}$ & $\begin{array}{l}\text { Alka- } \\
\text { Iinity } \\
\text { as } \\
\text { Caco } \\
\text { (mo/L) }\end{array}$ & $\begin{array}{l}\text { Dis:- } \\
\text { solved } \\
\text { sul fata } \\
\text { (so) } \\
(\mathrm{mo} / \mathrm{L})\end{array}$ & $\begin{array}{l}\text { Dis: } \\
\text { oolved } \\
\text { chloride } \\
\text { (CI) } \\
(m / L)\end{array}$ & $\begin{array}{l}\text { Dis. } \\
\text { solved } \\
\text { fluoride } \\
\text { (f) } \\
(\mathrm{mo} / \mathrm{L})\end{array}$ \\
\hline \multirow[t]{3}{*}{$30 / 2 W-16 G 2$} & 1.9 .73 & 21 & $* 0.32$ & 0.000 & 20 & 23 & 39 & 8.2 & 160 & 130 & 22 & 38 & 0.2 \\
\hline & $11 \cdot 16 \cdot 74$ & 16 & .10 & .010 & 50 & 32 & 17 & -. & 270 & 220 & 27 & 29 & .2 \\
\hline & $3 \cdot 6 \cdot 75$ & 13 & .22 & .010 & 74 & 16 & 18 & .. & 230 & 190 & 24 & 28 & .3 \\
\hline \multirow[t]{2}{*}{-1761} & $10-19+20-77$ & $\quad$.. & . & .. & -. & .. & .. & .. & -. & .. & .. & .. & -. \\
\hline & $10-19+20-77$ & .. & .. & .. & .. & .. & .. & $\ldots$ & .. & $\cdots$ & .. & .. & $\cdots$ \\
\hline \multirow[t]{3}{*}{.2101} & $6 \cdot 6 \cdot 75$ & 4.5 & .19 & .01 & 24 & 15 & 5.6 & .. & 150 & 120 & 5.3 & 8.0 & .3 \\
\hline & $4-25 \cdot 79$ & .. & .. & .. & $\therefore$ & .. & .. & .. & .. & .. & .. & .. & - \\
\hline & $5 \cdot 6 \cdot 79$ & .. & .20 & .010 & .. & .. & .. & .. & *. & .. & .. & $\cdot \cdot$ & .. \\
\hline .3141 & $4 \cdot 25 \cdot 79$ & .. & .. & .. & .. & .. & .. & .. & .. & .. & .. & -. & $\cdots$ \\
\hline$\cdot 32 C 1$ & $4 \cdot 25 \cdot 79$ & .. & 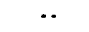 & .. & $\because$ & .. & .. & .. & $\cdots$ & .. & .. & .. & .. \\
\hline $.32 \times 1$ & $4 \cdot 26 \cdot 79$ & .. & .. & .. & -. & .. & .. & .. & .. & .. & .. & .. & -. \\
\hline-3212 & $4 \cdot 26 \cdot 79$ & *. & .. & .. & .. & .. & .. & $\cdots$ & .. & $\cdots$ & .. & $\cdots$ & .. \\
\hline$-32 L 3$ & 4.25 .79 & -. & $\cdots$ & .. & -. & .. & .. & .. & .. & 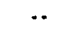 & .. & .. & $\cdots$ \\
\hline .3202 & $4 \cdot 26 \cdot 79$ & .. & .. & .. & .. & .. & .. & .. & ... & $\cdots$ & .. & .. & .. \\
\hline -33N1 & $4 \cdot 25 \cdot 79$ & .. & .. & .. & $\cdots$ & .. & -. & $\cdots$ & .. & .. & .. & .. & .. \\
\hline \multirow[t]{6}{*}{$30 / 3 N-5 B t$} & $7 \cdot 26 \cdot 50$ & .. & .. & .. & -. & .. & .. & .. & .. & .. & .. & 7 & -. \\
\hline & $7 \cdot 24 \cdot 66$ & .. & .. & .. & $\cdots$ & .. & .. & .. & .. & .. & .. & 2.2 & .. \\
\hline & $5 \cdot 24 \cdot 72$ & 27 & .12 & .02 & 26 & 11 & 13 & 2.8 & 149 & 122 & 20 & 2.2 & .1 \\
\hline & $11 \cdot 21 \cdot 72$ & 16 & .09 & .02 & 22 & 16 & 12 & 2.8 & 124 & 102 & 9.0 & 3.2 & .1 \\
\hline & $11 \cdot 30 \cdot 72$ & 13 & .28 &. .16 & 11 & 13 & 19 & 3.0 & 122 & 100 & 11 & 4.2 & .2 \\
\hline & $7 \cdot 7 \cdot 78$ & $\cdots$ & .. & -. & .. & .. & $\cdots$ & $\cdot \cdot$ & .. & $\cdot \cdot$ & $\cdots$ & 2.7 & -. \\
\hline \multirow[t]{2}{*}{.582} & $7 \cdot 24 \cdot 68$ & .. & .. & .. & .. & .. & .. & .. & .. & .. & .. & 5.0 & .. \\
\hline & $7 \cdot 7 \cdot 78$ & .. & .. & .. & .. & .. & .. & .. & .. & .. & .. & 6.5 & .. \\
\hline \multirow[t]{2}{*}{.586} & $11 \cdot 30 \cdot 72$ & 7.9 & .06 & .01 & 25 & 18 & 13 & 2.8 & 142 & 116 & 16 & 14 & .1 \\
\hline & $12 \cdot 5 \cdot 72$ & 22 & .14 & .01 & 26 & 12 & 13 & 3.1 & 137 & 112 & 13 & 6.5 & .1 \\
\hline \multirow[t]{2}{*}{$.5 \times 1$} & $7 \cdot 24 \cdot 68$ & .. & -. & .. & $\cdots$ & .. & .. & .. & .. & .. & .. & 4.3 & .. \\
\hline & $7 \cdot 7 \cdot 78$ & .. & .. & .. & .. & .. & .. & .. & .. & -. & .. & 4.0 & $\cdots$ \\
\hline$-6 C 1$ & . .60 &.. & .. & .. & .. & .. & .. & .. & 129 & 108 & 50 & 23 & .. \\
\hline .601 & - $\cdot 73$ & .. & .34 & .. & .. & .. & .. & .. & .. & .. & .. & .. & .. \\
\hline .661 & $\cdot \cdot \boldsymbol{B}$ & .. &. .5 & .. & .. & .. & .. & .. &.. & .. & .. & .. & .. \\
\hline .6114 & $8.25 \cdot 75$ & 19 & .02 & .00 & 44 & 13 & 8.2 & .. & 165 & 135 & 24 & 6.8 & .2 \\
\hline $.6 \mathrm{J3}$ & $12 \cdot 5 \cdot 74$ & 15 & .00 & .01 & 45 & 21 & 11 & .. & 211 & 173 & 24 & 10 & .1 \\
\hline -711 & -. & .. & .. & . & $\cdots$ & .. & .. & .. & .. & -. & .. & .. & $\cdots$ \\
\hline$-7 R I$ &.$\quad .60$ & .. & .. & .. & .. & .. &.. & .. & 149 & 122 & 50 & 4 & .. \\
\hline & - $\cdot 73$ & .. & .. & .. & .. & .. & .. & .. & .. & .. & .. & .. & .. \\
\hline -an & $12 \cdot 27-68$ & 15 & .16 & .02 & 46 & 8.7 & 2.6 & 2.3 & 198 & 162 & 6.8 & 3.0 & .0 \\
\hline -10н1 & $7-24.68$ & .. & .. & $\cdot-$ & $\because$ & .. & .. & .. & .. & -. & .. & 5.4 & .. \\
\hline & $7 \cdot 6 \cdot 78$ & .. & .. & .. & .. & .. & .. & .. & .. & .. & .. & 6.3 & .. \\
\hline .1561 & $11 \cdot 20-59$ & 25 & .01 & .. & 36 & 13 & 16 & 2.5 & 207 & 170 & 5.3 & 5.5 & .1 \\
\hline & $5 \cdot 24 \cdot 60$ & .. & .. & .. & $\cdots$ & .. & .. & .. & 206 & 167 & .. & .. & .. \\
\hline & 8. 2.68 & .. & .. & .. & .. & .. & .. & .. & .. & .. & .. & 3.8 & .. \\
\hline & $7 \cdot 6 \cdot 78$ & .. & .. & .. & .. & .. & .. & .. & .. & -. & .. & 4.8 & $\cdots$ \\
\hline .1682 & .. & .. & .. & .. & .. & .. & .. & .. & .. & -. & .. & .. & .. \\
\hline .1683 & $7 \cdot 7 \cdot 78$ & .. & .. & .. & .. & .. & .. & .. & .. & .. & .. & 5.3 & .. \\
\hline$-17 F 1$ & .60 & .. & .. & .. & -. & .. & .. &.. & 137 & 112 & 50 & 2 & .. \\
\hline$-18 E 1$ & - $\cdot 73$ & .. & .0 & .. & $\cdots$ & .. & .. & .. & -. & -. & $\because$ & .. & .. \\
\hline$-18 R 1$ & $11-28-69$ & -. & "1 & .. & $\cdots$ & .. & -. & .. & .. & .. & .. & .. & .. \\
\hline & - $\cdot 73$ & $\cdots$ & .2 & .. & $\cdots$ & .. & .. & .. & .. & .. & $\cdots$ & -. & .. \\
\hline - I9E1 & . .73 & .. & .2 & .. & $\cdots$ & .. & .. & $\cdot \cdot$ & .. & .. & .. & .. & .. \\
\hline $.19 \times 1$ & - $\cdot 73$ & .. & .2 & .. & .. & .. & .. & .. & .. & .. & .. & .. & .. \\
\hline-2081 & $7 \cdot 27 \cdot 50$ & .. & -. & -. & .. & .. & .. & .. & .. & .. & .. & 7 & $\cdots$ \\
\hline-2041 & - -73 & .. & .2 & .. & -. & .. & -. & .. & .. & .. & .. & -. & .. \\
\hline -20N1 & $7 \cdot 28 \cdot 50$ & .. & $\cdots$ & .. & .. & .. & .. & .. & .. & .. & .. & 9 & .. \\
\hline-2011 & $6 \cdot 9 \cdot 76$ & .. & 1 & -. & .. & .. & .. & .. & .. &.. & .. & .. & -. \\
\hline$\cdot 22 \times 1$ & $7 \cdot 24 \cdot 68$ & .. & .. & -. & .. & .. & .. &.$\cdot$ & -. & .. & .. & 6.0 & $\because$ \\
\hline & $7 \cdot 6 \cdot 68$ & .. & .. & .. & .. & .. & -. & .. & .. & .. & .. & 3.7 & $\cdots$ \\
\hline -2341 & $7 \cdot 23 \cdot 68$ & .. & .. & .. & .. & .. & .. & .. & .. & .. & -. & 8.0 & .. \\
\hline & $7 \cdot 6 \cdot 78$ & .. & $\cdot \cdot$ & -. & .. & .. & -. & .. & .. & -. & $-\cdot$ & 7.5 & $\cdots$ \\
\hline$-24 n 1$ & $4 \cdot 26 \cdot 79$ & .. & -. & .. & .. & .. &.. & .. &.. & .. & *. & .. & .. \\
\hline $.25 F 1$ & $7 \cdot 23 \cdot 68$ & .. & .. & .. & 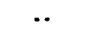 &.. & .. & .. & .. & .. & $\cdots$ & 10 & .. \\
\hline & $7 \cdot 6 \cdot 78$ & .. & .. & .. & .. & .. & .. & .. &.. &.. & .. & 16 & .. \\
\hline .2561 & $4 \cdot 26 \cdot 79$ & .. & .. & .. & .. & $\cdots$ & .. & .. & .. & .. & .. & .. & *. \\
\hline-2782 & $7 \cdot 26-68$ & .. & .. & .. & .. & .. & .. &.. & .. & .. & .. & 3.2 & .. \\
\hline & $7 \cdot 6 \cdot 78$ &.. & .. & .. & *. & .. & .. & .. & .. & .. & .. & 3.7 & .. \\
\hline-2701 & $7-23-68$ & .. & .. & .. & .. & .. & .. & .. & .. &.. & .. & 14 & .. \\
\hline -2841 & $7 \cdot 28 \cdot 50$ & .. & .. & .. & .. & .. & .. & .. & .. & .. & -. & 39 & $\cdots$ \\
\hline .3002 & $7 \cdot 28 \cdot 50$ & .. & .. & .. & .. & .. & -. & .. & .. & .. & .. & 10 & .. \\
\hline .3201 & $7 \cdot 28-50$ & .. & $\cdots$ & $\cdots$ & .. & .. & .. & .. & .. &.. & .. & 32 & .. \\
\hline $.34 a 1$ & $7 \cdot 6 \cdot 78$ & .. & .. & .. & .. & .. & .. & .. & .. & .. & .. & 260 & $\cdots$ \\
\hline
\end{tabular}




\begin{tabular}{|c|c|c|c|c|c|c|c|c|c|c|c|c|}
\hline $\begin{array}{l}\text { Total } \\
\text { nitrate } \\
(\mathrm{N}) \\
(m / L)\end{array}$ & $\begin{array}{l}\text { Total } \\
\text { ni trite } \\
\text { (H) } \\
(m / L)\end{array}$ & $\begin{array}{l}\text { Total } \\
\text { phos:- } \\
\text { phorus } \\
\text { (P) } \\
(\mathrm{mg} / \mathrm{L})\end{array}$ & $\begin{array}{l}\text { Dia- } \\
\text { solved } \\
\text { solids } \\
\text { (residus } \\
\text { et }(80 \mathrm{C})\end{array}$ & $\begin{array}{l}\text { Mard- } \\
\text { nease } \\
(\mathrm{Ca}, \mathrm{Mg}) \\
(\mathrm{mg} / \mathrm{L})\end{array}$ & $\begin{array}{l}\text { Mon. } \\
\text { carbon. } \\
\text { ate } \\
\text { hardhese } \\
\text { (ma/L) }\end{array}$ & $\begin{array}{l}\text { Specifle } \\
\text { concluc- } \\
\text { tence } \\
\text { (alcro- } \\
\text { whos) }\end{array}$ & $\begin{array}{c}\text { PNI } \\
\text { (units) }\end{array}$ & $\begin{array}{l}\text { Water } \\
\text { temper- } \\
\text { oture } \\
\text { ( C) }\end{array}$ & $\begin{array}{l}\text { Color } \\
\text { (platimu. } \\
\text { cobelt } \\
\text { units) }\end{array}$ & $\begin{array}{l}\text { Turbid- } \\
\text { (ty } \\
\text { (dTu) }\end{array}$ & $\begin{array}{l}\text { Carbon } \\
\text { díoxide } \\
\text { (co) } \\
(m g / L)\end{array}$ & Remarks \\
\hline 0.03 & 0.00 & .35 & 250 & 140 & 14 & 348 & 7.2 & -. & 2 & o & 19 & *. \\
\hline 1.6 & .07 & .57 & 308 & 260 & 32 & 534 & 7.6 & .. & 4 & $\mathbf{0}$ & -. & .. \\
\hline 1.1 & .00 & .11 & 288 & 250 & -. & 480 & 8.0 & *. & 5 & 1 & $\cdots$ & .. \\
\hline$\cdots$ & $\because$ & .. & * & -. & -. & 37,600 & 7.7 & $\cdot \cdot$ & $\because$ & $\cdot \cdot$ & $\cdots$ & Minien vatues of 16 semples. \\
\hline$\cdot \cdot$ & $\cdots$ & $\cdots$ & - & .. & -. & 44.000 & 8.7 & .. & -. & .. & .. & Maxiem vetues of 16 semples. \\
\hline .3 & .01 & .33 & 136 & 120 & .. & 260 & 8.0 & 9 & 4 & 0 & 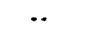 & .. \\
\hline 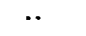 & .. & -. & -. & -. & -. & 278 & -. & 22 & -. & .. & -. & -. \\
\hline 1.0 & $\cdots$ & $\cdot \cdot$ & -. & 110 & $\cdots$ & 240 & $\cdot \cdot$ & $\cdot \cdot$ & 5 & 1 & $\cdot \cdot$ & $\cdot \cdot$ \\
\hline .. & .. & $\cdots$ & .. & -. & $\because$ & 390 & $\cdots$ & 18 & -. & -. & -. & -. \\
\hline$\cdots$ & $\cdots$ & $\cdot \cdot$ & • & $\cdot \cdot$ & $\cdots$ & 2.270 & $\cdots$ & 24 & .. & -. & $\cdots$ & .. \\
\hline .. & 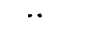 & .. & -. & .. & .. & 290 & -. & 15 & .. & .. & $\cdots$ & .. \\
\hline .. & .. & .. & .. & .. & .. & 96 & -. & 14 & -. & -. & .. & .. \\
\hline$\cdot \cdot$ & $\cdot \cdot$ & .. & .. & .. & -. & 206 & .. & 16 & $\cdots$ & .. & 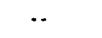 & .. \\
\hline .. & .. & .. & .. & $\because$ & $\cdots$ & 340 & $\cdots$ & 18 & .. & .. & $\cdots$ & $\cdots$ \\
\hline 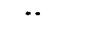 & $\because$ & -. & -. & - & -. & 392 & .. & 17 & .. & -. & - & .. \\
\hline$\cdots$ &.. & .. & .. & 112 & $\cdots$ & .. & -. & .. & .. & .. & .. & .. \\
\hline.. &.. & .. & .. & .. & .. & 250 & .. & 11 & $\cdots$ & .. & $\cdots$ & .. \\
\hline .23 & .02 & .23 & 175 & 110 & .. & 240 & 7.9 & -. & 5 & 0 & 4.0 & .. \\
\hline .01 & .00 & .16 & 162 & 118 & 16 & 240 & 7.4 & .. & 0 & 0 & 11.0 & .. \\
\hline .01 & .08 & .98 & 137 & 80 & .. & 220 & 7.3 & $\cdots$ & 5 & 4 & 10.5 & $\cdots$ \\
\hline .. & $\cdot \cdot$ & * & $\cdot \cdot$ & .. & -. & 241 & .. & .. & -. & $\cdots$ & .. & .. \\
\hline .. & .. & .. & $\cdots$ & $\cdot \cdot$ & -. & 366 & $\cdot \cdot$ & .. & .. & .. & .. & .. \\
\hline .. & $\cdots$ & .. & $\cdots$ & $\cdots$ & .. & 366 & $\therefore$ & .. & .. & .. & $\cdots$ & .. \\
\hline .01 & .01 & .05 & 166 & 138 & 22 & 220 & 7.4 & .. & 4 & 1 & 13 & Sulfide $=0.0 \mathrm{mg} / \mathrm{L}$. \\
\hline .01 & .02 & .43 & 206 & 116 & 4 & 230 & 8.4 & .. & 0 & 1 & 1.2 & . \\
\hline -. & .. & .. & .. & $\cdot \cdot$ & -. & 352 & $\cdots$ & -. & .. & $\cdots$ & .. & .. \\
\hline .. & 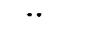 & -. & -. & $\cdot \cdot$ & $\cdot \cdot$ & 311 & $\cdot \cdot$ & $\cdots$ & -. & .. & .. & -. \\
\hline$\cdot \cdot$ & $\cdots$ & $\cdot \cdot$ & $\cdots$ & 169 & 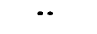 & $\cdot \cdot$ & $\cdot \cdot$ & -. & -. & -. & $\cdots$ & -. \\
\hline .26 & -. & .. & .. & 104 & -. & .. & 7.4 & .. & .. & .. & .. & .. \\
\hline -. & .. & .. & .. & 146 & -- & $\cdots$ & 7.0 & .. & .. & -. & .. & .. \\
\hline .8 & .01 & .02 & 197 & 164 & .. & 361 & 7.0 & .. & 3 & 0 & .. & * \\
\hline .9 & .. & .02 & 231 & 200 & -. & $3 n$ & 7.8 & 9.0 & 5 & 0 & .. & -. \\
\hline .. & -. & $\cdot \cdot$ & .. & $\cdot \cdot$ & .. & $\cdot \cdot$ & $\cdot \cdot$ & .. & $\cdots$ & $\cdots$ & $*$ & "Iron" problem; requires filter. \\
\hline *. & .. & .. & *. & 133 & $\cdots$ & $\cdot \cdot$ & 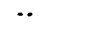 & -. & *. & .. & *. & $\cdot \cdot$ \\
\hline 4 & .. & $\cdot$ & $\cdots$ & .. & $\cdots$ & -. & 7.2 & .. & -. & .. & .. & .. \\
\hline .55 & .00 & .22 & 171 & 150 & -. & 288 & 7.1 & .. & 7 & 2 & 40 & $\cdot \cdot$ \\
\hline *. & .. & . & -. & .. & .. & 401 & .. & .. & .. & .. & -. & $\cdots$ \\
\hline$\ddot{~}$ & $\because$ & 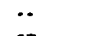 & 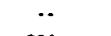 & 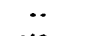 & -. & 391 & .. & .. & .. & -. & .. & -. \\
\hline .02 & .. & .07 & 206 & 142 & -. & 332 & 7.8 & $\cdots$ & 0 & .. & .. & .. \\
\hline$\because$ & $\cdot \cdot$ & -. & $\cdots$ & 146 & -. & 329 & 8.0 & -. & .. & .. & .. & .. \\
\hline .. & .. & .. & .. & .. & .. & 328 & -. & -. & .. & .. & -. & -. \\
\hline$\cdot \cdot$ & -• & -. & .. & .. & ・. & 325 & -. & -. & .. & .. & .. & .. \\
\hline$\cdots$ & .. & .. & .. & .. & .. & .. & $\cdots$ & -. & .. & -. & .. & "Pollutedr; unable to use. \\
\hline$\cdots$ & $\cdots$ & $\because$ & 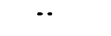 & $\because$ & 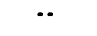 & 676 & $\cdots$ & -. & -. & *. & $\cdots$ & $\cdot \cdot$ \\
\hline 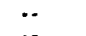 & $\cdots$ & $\cdot \cdot$ & *. & 118 & $\cdots$ & .. & $\cdots$ & -. & -. & .. & -. & $\because$ \\
\hline .12 & $\because$ & $\because$ & -. & 120 & $\cdots$ & -. & 7.6 & .. & -. & $\cdots$ & *. & $\because$ \\
\hline .. & $\cdots$ & $\cdots$ & 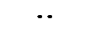 & 68 & -. & .. & 8 & .. & $\cdots$ & .. & .. & 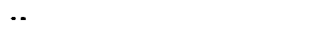 \\
\hline .2 & .. & -. & .. & 112 & -. & $\cdots$ & 7.2 & $\cdots$ & -. & -. & $\because$ & $\cdots$ \\
\hline .2 & .. & -. & -. & 140 & .. & .. & 6.9 & -. & .. & .. & -. & -. \\
\hline .2 & .. & .. & .. & 160 & .. & .. & 7.4 & .. & - & .. & .. & .. \\
\hline 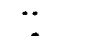 & $\because$ & $\cdots$ & .. & 146 & .. & .. & .. & .. & .. & .. & .. & .. \\
\hline .2 & $\because$ & $\because$ & $\cdots$ & 112 & $\cdots$ & .. & 7.0 & .. & -. & .. & -. & .. \\
\hline -. & $\cdots$ & 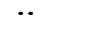 & $\cdot \cdot$ & 162 & $\cdots$ & .. & .. & $\cdots$ & .. & .. & .. & $\cdot \cdot$ \\
\hline 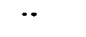 & $\cdots$ & $\cdot \cdot$ & .. & $\cdots$ & .. & .. & .. & .. & .. & .. & .. &.. \\
\hline .. & .. & $\because$ & .. & -. & .. & 358 & -. & -. & .. & .. & .. & .. \\
\hline -. & .. & .. & $\cdot$. & .. & .. & 375 & .. & $\cdots$ & .. & .• & .. & $\because$. \\
\hline$\cdots$ & .. & .. & .. & 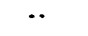 & -. & 490 & •. & .. & .. & .. & .. & .. \\
\hline$\cdot \cdot$ & $\because$ & $\cdots$ & $\cdots$ & .. & -- & 480 & .. & .. & .. & .. & -. & $\because$ \\
\hline$\cdot \cdot$ & $\cdots$ & $\because$ & 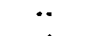 & $\cdots$ & -. & 956 & .. & 18 & -. & .. & $\cdots$ & .. \\
\hline$\cdot$. & $\cdot \cdot$ & .. & • & .. & -. & 1,050 & .. & .. & .. & .. & .. &.. \\
\hline .. & .. & $\cdots$ & • & .. & .. & 1,010 & .. & .. & .. & .. & -. & $\cdots$ \\
\hline .. & .. & -. & $\bullet$ & $\cdots$ & -. & 1,220 & .. & 15 & -. & $\cdot \cdot$ & .. & .. \\
\hline .. & $\because$ & .. & -. & .. & .. & 409 & -. & .. & .. & .. & .. & .. \\
\hline$\cdots$ & $\cdot \cdot$ & 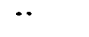 & .. & -. & -. & 370 & -. & .. & .. & .. & .. & $\cdot \cdot$ \\
\hline$\because$ & .. & $\cdots$ & -. & .. & $\cdots$ & 367 & .. & .. & .. & .. & .. & -. \\
\hline$\cdot$ & . & . & .. & & 18 & $\cdots$ &.. & .. & .. & .. & .. & -. \\
\hline -. & 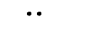 & 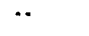 & .. & .. & 86 & $\cdots$ & .. & .. & .. & .. & -. & .. \\
\hline -. & .. & .. & $\because$ & 2.60 & $\cdots$ & .. & .. & .2 & -. & .. & .. & .. \\
\hline .. & .. & -. & $\bullet$ & .. & .. & 1,120 & -. & .. & .. & .. & -. & .. \\
\hline
\end{tabular}


TABLE 12.- Chealcal and physical quality of watar from selected walts and aprings in clallem county $\cdots$ contimued

\begin{tabular}{|c|c|c|c|c|c|c|c|c|c|c|c|c|c|}
\hline site number & $\begin{array}{l}\text { Date } \\
\text { sampled }\end{array}$ & $\begin{array}{l}\text { Dia. } \\
\text { solved } \\
\text { sillica } \\
\text { (sio) } \\
\text { (mg/L) }\end{array}$ & $\begin{array}{l}\text { Dis. } \\
\text { eolved } \\
\text { iron } \\
\text { (Fe) } \\
\text { (ug/L) }\end{array}$ & $\begin{array}{l}\text { Dis. } \\
\text { solved } \\
\text { mangan- } \\
\text { ese (Mn) } \\
\left(u_{g} / L\right)\end{array}$ & $\begin{array}{l}\text { Dis. } \\
\text { solved } \\
\text { calcium } \\
\text { (Cs) } \\
\text { (mg/L) }\end{array}$ & $\begin{array}{l}\text { Ois. } \\
\text { solved } \\
\text { magnes: } \\
\text { ium }(\mathrm{Mg}) \\
\text { (mg/L) }\end{array}$ & $\begin{array}{l}\text { Dis- } \\
\text { solved } \\
\text { eodium } \\
\text { (Ma) } \\
\text { (mg/L) }\end{array}$ & $\begin{array}{l}\text { Dis. } \\
\text { solved } \\
\text { potes. } \\
\text { silum }(K) \\
(\mathrm{mg} / \mathrm{L})\end{array}$ & $\begin{array}{l}\text { Bicar- } \\
\text { bonate } \\
\text { (HCO) } \\
\text { (mg/L) }\end{array}$ & $\begin{array}{l}\text { Alka- } \\
\text { linity } \\
\text { as } \\
\text { caco } \\
\text { (mg/L) }\end{array}$ & $\begin{array}{l}\text { Dis. } \\
\text { solved } \\
\text { sul fate } \\
\text { (so) } \\
\text { (mg/L) }\end{array}$ & $\begin{array}{l}\text { Dis. } \\
\text { colved } \\
\text { chtoride } \\
\text { (cl) } \\
(\mathrm{mg} / \mathrm{L})\end{array}$ & $\begin{array}{l}\text { Dia. } \\
\text { solved } \\
\text { fluoride } \\
\text { (F) } \\
\text { (mg/L) }\end{array}$ \\
\hline $30 / 34-3481$ & $10 \cdot 17 \cdot 79$ & .. & .. & .. & .. & .. & .. & .. & .. & .. & .. & .. & .. \\
\hline .3483 & $11 \cdot 5.79$ & .. & .. & .. & .. & .. & .. & .. & .. & .. & .. & 180 & .. \\
\hline$-34 L 1$ & $2 \cdot 13 \cdot 76$ & .. & $* 2$ & .. & .. & .. & .. & .. & .. & .. & .. & .. & .. \\
\hline \multirow[t]{2}{*}{$-35 E 1$} & $10 \cdot 7 \cdot 76$ & .. & .. & .. & .. & .. & .. & .. & .. & .. & .. & "380 & .. \\
\hline & 7.6 .78 & .. & .. & .. & .. & .. & .. & .. & .. & .. & .. & $* 400$ & .. \\
\hline $.35 \mathrm{M1}$ & 7.23 .68 & .. & .. & .. & .. & .. & .. &.. & .. & .. & .. & 20 & .. \\
\hline \multirow[t]{2}{*}{$.36 F 1$} & $7 \cdot 23.60$ & .. & .. & $\cdot \cdot$ & .. & .. & .. & .. & .. & .. & .. & 56 & .. \\
\hline & 7.6 .78 & .. & .. & .. & .. & .. & .. &.$\cdot$ & .. & .. & .. & 36 & .. \\
\hline$\cdot 36 f 2$ & 6.26.79 & .. & .. & .. & .. & .. & .. & .. & .. & & .. & .. & .. \\
\hline$\cdot 36 \times 1$ & $4 \cdot 24 \cdot 79$ & .. & .. & .. & .. & .. & .. & .. & $\cdot \cdot$ & .. & .. &.. & .. \\
\hline .3611 & $4 \cdot 24 \cdot 79$ & $\cdot \cdot$ & .. & .. & .. & .. & .. & .. & .. & .. & .. & .. & .. \\
\hline .3612 & $4 \cdot 24 \cdot 79$ & .. &.. & .. &.. &.. & .. & .. & .. & .. & .. & .. & .. \\
\hline $30 / 4 W-113$ & $5 \cdot 30 \cdot 73$ & 3.5 & 0.12 & 0.01 & 18 & 16 & 3.2 & 0.6 & 105 & 86 & 11 & 1.5 & 0.1 \\
\hline$\cdot 114$ & $4 \cdot 3 \cdot 74$ & 12 & .08 & .00 & 25 & 4.0 & 2.8 & .2 & 110 & 90 & 5.1 & 2.2 & .5 \\
\hline-301 & $5 \cdot 17 \cdot 73$ & 12 & .01 & .02 & 41 & 18 & 12 & 2.0 & 188 & 154 & 75 & 5.0 & .1 \\
\hline .531 & $7-10 \cdot 78$ & .. & .. & .. & .. & .. & .. & $\cdot \cdot$ & .. & .. & -. & 12 & .. \\
\hline \multirow[t]{2}{*}{$.5 L 1$} & $7 \cdot 26.68$ & .. & .. & .. & .. & -. & .. & .. & .. & .. & .. & 18 & .. \\
\hline & $7 \cdot 11 \cdot 78$ & .. & .. & .. & .. & .. & .. & .. & .. & .. & .. & 17 & .. \\
\hline .512 & $7 \cdot 26 \cdot 68$ & $\because$ & .. & .. & .. &.$\cdot$ & .. & .. & .. & .. & .. & 13 & .. \\
\hline $.5 L 3$ & $4 \cdot 20-76$ &.. & *3 & .. & .. & .. & .. & .. & .. & .. & .. & $\cdot \cdot$ & .. \\
\hline \multirow[t]{2}{*}{$.5 N 1$} & $8 \cdot 2.68$ & .. & .. & .. & .. & .. & .. & .. & .. &.. & .. & 7.9 & .. \\
\hline & $7 \cdot 11 \cdot 78$ & $\cdot \cdot$ & .. & $\cdots$ & .. & .. & .. & .. & .. & .. & .. & 13 & .. \\
\hline \multirow[t]{2}{*}{$-5 p 1$} & $1-24 \cdot 74$ & 9.0 & .03 & $\star .51$ & 64 & 2.3 & 16 & 3.4 & 300 & 246 & .0 & 18 & .2 \\
\hline & $3 \cdot 13 \cdot 74$ & .. & .. & $\star .55$ & $\cdot \cdot$ & .. & $\cdot \cdot$ & $\cdot \cdot$ & .. & .. &.$\cdot$ & .. & .. \\
\hline .781 & $3 \cdot 27 \cdot 79$ & .. & .11 & .00 & .. & .. & .. & .. & .. & .. & .. & .. & .. \\
\hline$\cdot 7 \times 1$ & .60 & .. & $\cdot \cdot$ & $\cdot \cdot$ & .. & .. & .. & .. & 112 & 92 & 50 & 6 & .. \\
\hline .861 & . .60 & .. & .. & .. & .. & .. & .. & .. & 212 & 174 & 50 & 8 & .. \\
\hline \multirow[t]{2}{*}{.912} & $2 \cdot 28 \cdot 74$ & $\cdot \cdot$ & .26 & $\star .22$ & .. & .. & .. & .. &.. &.. & .. & .. & .. \\
\hline & $3 \cdot 18 \cdot 74$ & .. & .05 & $\star .08$ & .. & $\cdot \cdot$ & .. & .. & .. & .. & .. & .. & .. \\
\hline$-12 R 2$ & $11 \cdot 14 \cdot 62$ & 12 & .07 & $\cdot \cdot$ & 29 & 6.5 & 4.4 & .6 & 118 & 97 & 8.4 & 1.5 & .1 \\
\hline-1301 & $7-28 \cdot 50$ & .. & .. & .. & $\cdot \cdot$ & .. & .. & $\cdot \cdot$ & .. & .. & .. & 7 & .. \\
\hline$-15 n 1$ & $\cdot \cdot$ & $\cdot \cdot$ & .. & .. & .. & .. & .. & .. & .. & .. & .. & .. & .. \\
\hline .1761 & .60 & .. & .. & .. & .. & .. & .. & .. & 115 & 94 & 50 & 3 & .. \\
\hline$-18 n 1$ &. .60 & .. & .. & .. & .. & .. & .. & .. & 127 & 104 & 50 & 6 & .. \\
\hline $.20 c 1$ & . . 60 & $\cdot \cdot$ & .. & $\cdot \cdot$ & .. & $\cdot \cdot$ & $\cdot \cdot$ & .. & 183 & 150 & 50 & 8 & .. \\
\hline-2162 & $\cdot \cdot$ & $\cdot \cdot$ & .. & .. & .. & .. & .. & $\cdot \cdot$ & $\cdot \cdot$ & $\cdot \cdot$ & .. & $\cdot \cdot$ & .. \\
\hline-2163 & .. & .. &.. & .. & .. & .. & .. & .. & .. & -. & .. & .. & .. \\
\hline .2164 & $12 \cdot 29 \cdot 75$ & .. & $* 1$ & .. & .. & .. & .. & .. & $\cdot \cdot$ & .. & $\cdot \cdot$ & .. & $\cdot \cdot$ \\
\hline \multirow[t]{3}{*}{$.22 \mathrm{HI}$} & $5.23 \cdot 68$ & 18 & .16 & .02 & 32 & 8.2 & 2.0 & 2.0 & 149 & 122 & 7.5 & 10 & .2 \\
\hline & $7 \cdot 21 \cdot 71$ & 6.0 & .00 & .00 & 42 & 18 & 13 & 1.5 & 237 & 194 & 7.0 & 7.5 & .1 \\
\hline & $6 \cdot 13 \cdot 75$ & 14 & .18 & .04 & 46 & 8.7 & 6.0 & .. & 176 & 144 & 16 & 7.0 & .5 \\
\hline$\cdot 23 \mathrm{J1}$ & . .60 & .. & $\cdot \cdot$ & $\cdot \cdot$ & .. & $\cdot \cdot$ & $\cdot \cdot$ & .. & 93 & 76 & 50 & 2 & .. \\
\hline .2305 & $\cdot \cdot$ & .. & .. & .. & .. & .. & .. & .. & .. & $\cdot \cdot$ & .. & .. & .. \\
\hline$-24 R 1$ & $\cdot \cdot \pi$ & .. & .2 & .. & .. & .. & .. & .. & $\cdot \cdot$ & .. & .. & .. & .. \\
\hline$-25 \wedge 1$ & $5-27 \cdot 73$ & 11 & .18 & .01 & 46 & 12 & 11 & 1.1 & .. & .. & 10 & 171 & .1 \\
\hline $.25 \wedge 2$ & $6 \cdot 3 \cdot 73$ & 4.0 & .10 & .00 & 34 & 20 & 8.9 & 1.4 & 146 & 120 & 17 & 3.0 & .1 \\
\hline $.25 F 1$ & $\cdot \cdot 3$ & .. & 2 & .. & .. & $\cdot \cdot$ & .. & .. & $\cdot \cdot$ & .. & .. & .. & .. \\
\hline$-26 \mathrm{J1}$ & $11 \cdot 11 \cdot 75$ & .. & .01 & .. & .. & $\cdot \cdot$ & $\cdot \cdot$ & .. & $\cdot \cdot$ & .. & .. & .. & .. \\
\hline $.26 R 1$ & $\cdot \cdot 73$ & .. & .2 & $\cdot \cdot$ & $\cdot \cdot$ & .. & .. & .. & .. & $\cdot \cdot$ & .. & $\cdot \cdot$ & .. \\
\hline 30/5W-2R1 & $7 \cdot 30.68$ & .. & .. & .. & .. & .. & .. & .. & $\cdot \cdot$ &.$\cdot$ & $\cdot \cdot$ & 7.7 & .. \\
\hline$-10 a 1$ & $6.15-79$ & .. & .. & $\cdot \cdot$ & $\cdot \cdot$ & .. & $\cdot \cdot$ & .. & $\cdot \cdot$ & .. & .. & .. & .. \\
\hline \multirow[t]{4}{*}{ - 10F1 } & $12-31.65$ & 21 & .15 & .001 & 38 & 15 & 6.0 & 2.2 & 170 &.$\cdot$ & 11 & 14 & .2 \\
\hline & $12-21 \cdot 71$ & 23 &. .52 & .000 & 84 & 37 & 37 & 1.4 & 240 & 200 & 27 & 140 & .1 \\
\hline & 3.6 .78 & $\cdot \cdot$ & .05 & .010 & .. & .. & 12 & .. & .. & $\cdot \cdot$ & .. & 11 & .1 \\
\hline & 3. $5-79$ & .. & .05 & .010 & .. & $\cdot \cdot$ & 20 & .. & .. & $\cdot \cdot$ & .. & 290 & .2 \\
\hline-1211 & $7 \cdot 30.60$ & .. & .. & .. & .. & $\cdot \cdot$ & .. & .. & .. & .. & .. & 21 & .. \\
\hline & $7-11 \cdot 78$ & .. & .. & .. & .. & .. & $\cdot \cdot$ & .. & .. & .. & .. & 6.8 & .. \\
\hline$-12 \mathrm{Cl}$ & $7-30.68$ & $\cdot \cdot$ & .. & .. & .. & .. & .. & .. & .. & .. & .. & 7.0 & .. \\
\hline & $7 \cdot 11 \cdot 78$ & $\cdot \cdot$ & .. & .. & .. & $\cdot \cdot$ & $\cdot \cdot$ & $\cdot \cdot$ & .. & .. & .. & 7.6 & $\cdot \cdot$ \\
\hline$-12 L 1$ & .60 & .. & .. & .. & $\cdot \cdot$ & $\cdot \cdot$ & .. & .. & 110 & 90 & 50 & 5 & .. \\
\hline -13E1 & . .60 & $\cdot \cdot$ & .. & $\cdot \cdot$ & $\cdot \cdot$ & .. & .. & $\cdot \cdot$ & 71 & 58 & 50 & 5 & .. \\
\hline$\cdot 13 K 1$ & - . .60 & .. & $\cdot \cdot$ & .. & .. & $\cdot \cdot$ & $\cdot \cdot$ & .. & 159 & 130 & 50 & 6 & .. \\
\hline$\cdot 18 \mathrm{~F} 1$ & $5 \cdot 18 \cdot 79$ & .. & .. & .. & .. & .. & .. & .. & .. & .. & .. & .. & .. \\
\hline$\cdot 1902$ & 6.12 .79 & $\cdot \cdot$ & $\cdot \cdot$ & $\cdot \cdot$ & $\cdot \cdot$ & .. & .. & $\cdot \cdot$ & $\cdot \cdot$ & $\cdots$ & $\cdot \cdot$ & $\cdot \cdot$ & .. \\
\hline$\cdot 1903$ & 6.12 .79 & .. & .. & .. & .. & .. & .. & .. & .. & .. & .. & .. & .. \\
\hline-2081 & 6.11 .79 & $\cdot \cdot$ & $\cdot \cdot$ & .. & $\cdot \cdot$ & .. & $\cdot \cdot$ & $\cdot \cdot$ & .. & .. & .. & .. & .. \\
\hline-2181 & $6.12-79$ & .. & .. & .. & .. & .. & .. & .. & .. & .. & .. & .. & $\cdot \cdot$ \\
\hline $.23 c 1$ & 6.11 .79 & .. & .. & .. & .. & .. & .. & .. & .. & .. & .. & $\cdot \cdot$ & $\cdot \cdot$ \\
\hline
\end{tabular}




\begin{tabular}{|c|c|c|c|c|c|c|c|c|c|c|c|c|}
\hline $\begin{array}{l}\text { Total } \\
\text { nitrate } \\
\text { (M) } \\
\text { (mg/L) }\end{array}$ & $\begin{array}{l}\text { Total } \\
\text { nitrita } \\
\text { (W) } \\
\text { (mg/L) }\end{array}$ & $\begin{array}{l}\text { Total } \\
\text { phos: } \\
\text { phorus } \\
(P) \\
(\mathrm{mg} / \mathrm{L})\end{array}$ & $\begin{array}{l}\text { Dis. } \\
\text { solved } \\
\text { sol ids } \\
\text { (residus } \\
\text { at } 180 \mathrm{C} \text { ) }\end{array}$ & $\begin{array}{l}\text { Hard- } \\
\text { nese } \\
(\mathrm{Ca}, \mathrm{Mg}) \\
(\mathrm{mg} / \mathrm{L})\end{array}$ & $\begin{array}{l}\text { Mon- } \\
\text { carbon- } \\
\text { ato } \\
\text { harchese } \\
\text { (mg/L) }\end{array}$ & $\begin{array}{l}\text { Specific } \\
\text { conduc- } \\
\text { tanco } \\
\text { (micro- } \\
\text { moso) }\end{array}$ & $\begin{array}{c}\text { PN1 } \\
\text { (units) }\end{array}$ & $\begin{array}{l}\text { Vater } \\
\text { temper- } \\
\text { aturo } \\
\text { ( C) }\end{array}$ & $\begin{array}{l}\text { Color } \\
\text { (platinum- } \\
\text { cobalt } \\
\text { enits) }\end{array}$ & $\begin{array}{l}\text { Turbld- } \\
\text { ity } \\
\text { (JTU) }\end{array}$ & $\begin{array}{l}\text { Carbon } \\
\text { dioxide } \\
(c o) \\
(m a / L)\end{array}$ & Remorke \\
\hline .. & .. & .. & .. & .. & .. & $\cdots$ & .. & .. & .. & .. & .. & salty water at $320.340 \mathrm{ft}$. \\
\hline$\cdots$ & .. & .. & .. & .. & .. & .. & .. & .. & .. & .. & .. & .. \\
\hline .. & .. & .. & .. & 34 & .. & .. & $\leftrightarrow$ & .. & - & .. & .. & .. \\
\hline .. & .. & .. & .. & -. & .. & $\cdot \cdot$ & $\because$ & .. & .. & $\because$ & .. & .. \\
\hline .. & .. & .. & * & .. & .. & 1,580 & .. & $\cdots$ & . & .. & 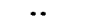 & .. \\
\hline .. & .. & .. & .. & .. & .. & 607 & .. & .. & .. & .. & .. & .. \\
\hline *. & $\cdots$ & $\because$ & $\cdots$ & .. & .. & 601 & .. & .. & $\because$ & .. & .. & .. \\
\hline .. & -. & $\cdots$ & .. & .. & .. & 520 & .. & $\because$ & .. & .. & .. &.. \\
\hline.. & .. & .. & .. & .. & .. & 655 & .. & 14 & .. &.. & .. & .. \\
\hline.. & .. & .. & .. & .. & .. & 280 & .. & 24 & .. & .. & .. & .. \\
\hline .. & .. & .. & .. & .. &.. & 260 & .. & 26 & .. & .. & .. & .. \\
\hline 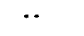 & .. & .. & • & -. & .. & 2,900 & $\cdots$ & 16 & 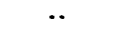 & .. & .. & .. \\
\hline 0.09 & 0.01 & 0.07 & 106 & 110 & 24 & 130 & 7.2 & .. & 7 & 0 & .. & .. \\
\hline 1.7 & .00 & .1 & 109 & 80 & .. & 160 & 7.6 & $\cdots$ & 5 & 0 & .. & .. \\
\hline .16 & .04 & .01 & 319 & 176 & 22 & 312 & 8.6 &.. & 0 & 1 & 1.1 & .. \\
\hline .. & $\cdots$ & .. & .. & .. & .. & 359 & .. & 10.1 & .. &.. & .. &.. \\
\hline$\cdots$ & .. & .. & .. & .. & .. & 484 & .. & $\cdots$ & .. & .. & .. & .. \\
\hline .. & .. & .. & .. & .. & .. & 482 & .. & .. & .. & .. & .. & .. \\
\hline.. & .. & .. & $\cdots$ & .. & .. & 430 & .. & .. & .. &.. & $\cdots$ & .. \\
\hline.. &.. &.. & .. & 154 & .. & .. & 7.5 & $\cdots$ & .. & .. & .. & .. \\
\hline$\cdots$ & .. & .. &.. & .. & .. & 325 & .. & .. &.. & .. & .. & .. \\
\hline .. &.$\cdot$ & .. & .. & .. & $\because$ & 429 & .. & 10.2 & .. & .. & .. & .. \\
\hline 1.5 & .01 & 1.2 & 263 & 169 & $\therefore$ & 390 & 8.2 & .. & 7 & 0 & 3.5 & .. \\
\hline 2.0 & .01 & 1.4 & .. &.. & .. & . & .. & .. & .. & .. & .. & .. \\
\hline .. & .. & .. & .. & .. & .. & 200 & .. &.. & 10 & $\star 10$ & .. &.. \\
\hline.. & .. &.. & .. & 93 & .. & .. &.. & .. & .. & . & .. & .. \\
\hline .. & .. & . & .. & 166 & .. & .. & .. & .. & .. & $\ldots$ & .. & .. \\
\hline .. & .. & .. & .. & . & .. & .. & .. & .. & .. & .. & .. & .. \\
\hline .. &.. & .. & .. &.. & .. & .. & .. & .. & . & .. & .. & .. \\
\hline .28 & .. & .02 & 122 & 100 & 3 & 205 & 6.9 & 9.5 & .. & .. & .. & .. \\
\hline .. & .. & .. & .. & 122 & .. & .. & .. & .. & .. & .. & .. & .. \\
\hline .. & $\cdots$ & .. & .. & .. & .. & .. & .. & .. & .. & .. & .. & "Rust and iron" problens. \\
\hline 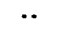 & .. & .. & .. & 65 & -. & $\cdots$ & .. & .. & $\cdots$ & $\cdots$ & -. & $\cdots$ \\
\hline -. & -. & •. & $\because$ & 111 & -. & .. & $\cdots$ & -. & .. & .. & *. & .. \\
\hline$\cdots$ & -. & .. & .. & 144 & *. & .. & .. & .. & .. & .. & .. & .. \\
\hline -. & .. & -. & .. & .. & $\cdots$ & -. & .. & .. & .. & .. & .. & "Ruat" problem. \\
\hline .. & .. & -. & .. & .. & $\cdots$ & .. & .. & .. & .. & .. & .. & $\cdots D 0 . \cdots$ \\
\hline .. & .. & .. & .. & 120 & -. & .. & 7.5 & .. & .. & .. & .. & .. \\
\hline .32 & .01 & .05 & 154 & 122 & $\cdots$ & 2,40 & 7.2 & .. & 4 & 2 & 19.0 & .. \\
\hline 2.3 & .01 & .19 & 110 & 182 & .. & 292 & 7.4 & 10 & 1 & 1 & 21.0 & .. \\
\hline .5 & .01 & .08 & 186 & 152 & .. & 316 & 7.1 & .. & 5 & 0 & .. & .. \\
\hline$\cdots$ & .. & .. & -. & 85 & $\cdots$ & .. & .. & $\cdots$ & .. & . & .. & -. \\
\hline$\cdots$ & 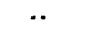 & .. & .. & .. & $\because$ & .. & .. & -. & .. & .. & .. & "Rust" problem. \\
\hline 1 & .. & .. & .. & 140 & .. & .. & 7.5 & $\cdots$ & .. & .. & .. & .. \\
\hline 1.1 & .03 & .02 & 263 & 164 & .. & 820 & .. & .. & 5 & 1 & .. & .. \\
\hline .27 & .01 & .18 & 161 & 168 & 48 & 216 & 7.3 & 10 & 5 & 3 & 15 & .. \\
\hline 1 & .. & $\because$ & $\cdots$ & 120 & $\because$ & $\cdots$ & 7.2 &. & .. & .. & .. & .. \\
\hline$\cdot \cdot$ & -. & .. & .. & 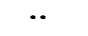 & .. & .. & .. & .. & .. & .. & .. & .. \\
\hline$\cdots$ & .. & .. & .. & 94 & -. & .. & 7.9 & .. & .. & .. & .. & .. \\
\hline .. & .. & .. & .. & -. & .. & 321 & .. & .. & .. & .. & .. & .. \\
\hline .. & .. & .. & .. & .. & .. & 350 & .. & 12 & .. & .. & $\ldots$ & .. \\
\hline .61 & .00 & .31 & 150 & 160 & 20 & 255 & 8.3 & .. & 4 & 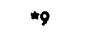 & 1.6 & .. \\
\hline 2.3 & .06 .26 & 4681 & 360 & 160 & 260 & 7.9 &.. & 5 & 0 & 6.7 & .. & \\
\hline 2.4 & .. & .. & .. & 160 &. & 339 & .. & .. & 0 & 0 & .. & . \\
\hline 3.0 & $\cdots$ & .. & $\bullet$ & 490 & .. & 1,200 & .. & .. & 5 & 0 & .. & .. \\
\hline .. & $\because$ & $\cdots$ & .. & .. & .. & 321 & .. & 11 & .. &.. & .. & .. \\
\hline .. & .. & .. & .. & $\cdots$ & .. & 283 & . & 9.5 & .. & .. & .. & $*$ \\
\hline .. & $\cdots$ & .. & .. & .. & .. & 315 & .. &. & .. & .. & .. & .. \\
\hline .. & 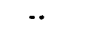 & $\because$ & .. & -. & .. & 310 & .. & 10.3 & .. &.. & $\cdots$ & -. \\
\hline .. & $\cdots$ & .. & .. & 89 & .. & .. & .. & . & $\ldots$ & .. & .. &.. \\
\hline .. & .. & .. & .. & 75 & .. & .. & .. & .. & .. & .. & .. & .. \\
\hline .. & $\cdots$ & .. & .. & 129 & .. & .. & .. &.. &.. & .. & $\cdots$ & .. \\
\hline .. & .. & .. & .. & .. & $\ldots$ & 290 & .. & 13 & .. & .. & .. & .. \\
\hline.. & .. & .. & .. & .. & .. & 220 & .. & 10 & .. & .. & .. & .. \\
\hline .. & .. & .. & .. &.. & .. & 220 & .. & 14 & .. &.. & .. & .. \\
\hline$\cdots$ & .. & . & . & .. & .. & 355 & .. & 16 & .. & .. & .. & .. \\
\hline .. & .. & .. & .. & .. & .. & 100 & .. & 15 & .. &.. & .. & .. \\
\hline$\cdot \cdot$ & .. & .. & .. & .. & .. & 205 & .. & 16 & .. & .. &.. & .. \\
\hline
\end{tabular}




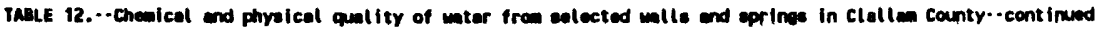

\begin{tabular}{|c|c|c|c|c|c|c|c|c|c|c|c|c|c|}
\hline 1 & $\begin{array}{l}\text { Date } \\
\text { eempled }\end{array}$ & $\begin{array}{l}\text { Dis- } \\
\text { solved } \\
\text { silica } \\
\text { (sio) } \\
\text { (Dio) }\end{array}$ & $\begin{array}{l}\text { Dis:- } \\
\text { colved } \\
\text { iron } \\
\text { (fo) } \\
\text { (uo/L) }\end{array}$ & $\begin{array}{l}\text { Dis- } \\
\text { eol red } \\
\text { mengan- } \\
\text { ase (In) } \\
\text { (ug/L) }\end{array}$ & $\begin{array}{l}\text { Dise } \\
\text { solved } \\
\text { celctur } \\
\text { (Co) }\end{array}$ & 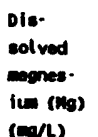 & $\begin{array}{l}\text { Dis: } \\
\text { solved } \\
\text { sodiun } \\
\text { (Wa) } \\
(0,1 / 2)\end{array}$ & $\begin{array}{l}\text { Dis. } \\
\text { solved } \\
\text { potes- } \\
\text { giv (K) } \\
\text { (n) }\end{array}$ & $\begin{array}{l}\text { Bicar. } \\
\text { benate } \\
\text { (nco ) } \\
\text { (mg/L) }\end{array}$ & $\begin{array}{l}\text { Alka- } \\
\text { linity } \\
\infty \\
\text { Coco } \\
(m g / L)\end{array}$ & $\begin{array}{l}\text { Dis. } \\
\text { solved } \\
\text { sulfate } \\
\text { (so) } \\
(n / L)\end{array}$ & $\begin{array}{l}\text { Dis: } \\
\text { eolved } \\
\text { chloride } \\
\text { (cl) } \\
(\mathrm{mg} / \mathrm{l})\end{array}$ & $\begin{array}{l}\text { Dle- } \\
\text { eolved } \\
\text { fluoride } \\
\text { (f) } \\
(n g / L)\end{array}$ \\
\hline
\end{tabular}

\begin{tabular}{|c|c|c|c|c|c|c|c|c|c|c|c|c|c|}
\hline $30 / 5 W-2761$ & $6-11-79$ & $\cdots$ & $-\cdot$ & .. & $\ldots$ & $\cdots$ & .. & $\cdots$ & $\cdots$ & -. & -. & $\cdots$ & $\cdots$ \\
\hline$-27 \mathrm{ni1}$ & $6-11-79$ & $\cdots$ & $\cdot \cdot$ & 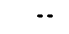 & $\cdots$ & $\cdots$ & $\cdots$ & $\cdots$ & $\cdots$ & .. & $\cdot \cdot$ & $\cdots$ & $\cdots$ \\
\hline-292 & $6-12-79$ & $\cdots$ & .. & -- & .. & $\cdots$ & -. & $-\cdot$ & $\cdots$ & .. & .. & $\cdots$ & $\cdots$ \\
\hline-2914 & $6-12-79$ & .. & -. & $\cdots$ & $\cdots$ & $\cdots$ & -. & $\cdots$ & $\cdots$ & .. & $\cdots$ & $\cdots$ & .. \\
\hline-29015 & $6-12-79$ & -. & -. & $\cdots$ & $\cdots$ & -. & .. & .. & $\cdots$ & .. & 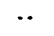 & $\cdots$ & .. \\
\hline-2901 & $6-12-79$ & -. & $\cdots$ & $\cdots$ & $\cdots$ & $\cdots$ & $\cdots$ & $\cdots$ & $\cdots$ & $\cdots$ & $\cdots$ & $\cdots$ & $\cdots$ \\
\hline$-29 P 1$ & $6-12-79$ & $\cdots$ & $\cdots$ & $\cdots$ & $\cdots$ & $\cdots$ & $\cdot \cdot$ & $\cdot-$ & .. & .. & $\cdots$ & .. & $\cdots$ \\
\hline$-30 \mathrm{c2}$ & $6-14-79$ & $\cdot \cdot$ & $\cdots$ & $\cdots$ & $\cdots$ & -. & .. & $\cdots$ & $\cdots$ & $\cdots$ & $\cdot \cdot$ & $\cdots$ & $\cdots$ \\
\hline .3003 & $6-14-79$ & .. & -. & $\cdots$ & $\cdots$ & -. & -. & $-\cdot$ & $\cdots$ & -. & .. & $\cdots$ & $\cdots$ \\
\hline$-30 F 1$ & $6-14-79$ & -. & -. & $-\cdot$ & $\cdots$ & $\cdots$ & -. & -- & $\cdots$ & .. & -. & $\cdots$ & -. \\
\hline$-30 f 2$ & $6-14-79$ & -. & $-\cdot$ & .. & .. & $\cdots$ & -. & $\cdots$ & $\cdots$ & -. & .. & $\cdots$ & .. \\
\hline$-30 F 4$ & $6-14-79$ & .. & $\cdots$ & .. & $\cdots$ & -. & -. & $\cdot-$ & $\cdots$ & .. & .. & $\cdots$ & 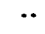 \\
\hline .3041 & $6 \cdot 16-79$ & $\cdot \cdot$ & $\cdots$ & $\cdots$ & .. & .. & $\cdot \cdot$ & $\cdots$ & .. & $\cdots$ & $\cdot \cdot$ & $\cdots$ & $\cdots$ \\
\hline-3012 & $6-14-79$ & $\cdot$. & $\because$ & $\cdots$ & $\cdots$ & $\cdots$ & $\cdots$ & $\cdots$ & $*$ & $\cdots$ & $\cdot \cdot$ & $\cdots$ & $\cdots$ \\
\hline-3013 & $6-16-79$ & .. & $\cdots$ & $\cdots$ & $\cdots$ & $\cdots$ & $\cdots$ & $\cdots$ & $\cdots$ & -. & .. & $\cdots$ & $\cdots$ \\
\hline$\cdot 30 \mathrm{R1}$ & $6-14 \cdot 79$ & .. & $\cdots$ & -. & .. & $\cdots$ & .. & $\cdots$ & $\cdots$ &.- & .. & $\cdots$ & $\cdots$ \\
\hline-30122 & $6 \cdot 12-79$ & -. & $-\cdot$ & $\cdots$ & $\cdots$ & $\cdots$ & 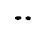 & -. & $\cdots$ & .. & $\cdots$ & $\cdots$ & $\cdots$ \\
\hline-3013 & $6 \cdot 12 \cdot 79$ & $\cdot \cdot$ & .. & $\cdots$ & $\cdots$ & -. & $\cdots$ & $\cdots$ & $\cdots$ & .. & $\cdots$ & $\cdots$ & *. \\
\hline$-31 a 1$ & $6-14-79$ & .. & $\cdot \cdot$ & $\cdots$ & $\cdots$ & $\cdots$ & $\cdot \cdot$ & $\cdots$ & $\cdots$ & $\cdots$ & 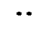 & $\cdots$ & $\cdots$ \\
\hline-3101 & $6-12-79$ & $\cdot \cdot$ & $\cdots$ & $\cdots$ & $\cdots$ & $\cdots$ & $\cdots$ & $\cdots$ & $\cdots$ & $\cdot \cdot$ & .. & $\cdots$ & -. \\
\hline-3102 & $6 \cdot 12 \cdot 79$ & .. & -. & $\cdots$ & $\cdots$ & .. & $\cdot \cdot$ & $\cdot \cdot$ & $\cdots$ & -. & .. & $\cdots$ & $-\cdot$ \\
\hline-3162 & $6-12-79$ & .. & .. & $\cdots$ & $\cdots$ & $\cdots$ & .. & 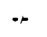 &.- & .. & .. & $\cdots$ & .. \\
\hline-3201 & $6-12 \cdot 79$ & .. & -. & $\cdots$ & $\cdots$ & $\cdots$ & -. & .. & -. & -. & .. & $\cdots$ & .. \\
\hline-3221 & $6 \cdot 15-79$ & $\cdot \cdot$ & $\cdots$ & .. & $\cdots$ & $\cdots$ & .. & $\cdots$ & $\cdots$ & .. & $\cdot \cdot$ & $*$ & $\cdots$ \\
\hline-3401 & $6-15 \cdot 79$ & -. & $\cdots$ & $\cdots$ & $\cdots$ & $\cdots$ & $\cdots$ & $\cdots$ & $\cdots$ & $\cdots$ & $\cdot \cdot$ & $\cdots$ & -. \\
\hline $30 / 6 \mathrm{~N}-7 \mathrm{B1}$ & 2. $2-54$ & $\cdots$ & 0.1 & $\cdots$ & $\cdots$ & $\cdots$ & -. & $\cdots$ & .. & .. & .. & 6 & .. \\
\hline-761 & 2. 4.56 & $\cdots$ & $\cdots$ & $\cdots$ & $\cdot \cdot$ &.. & .. & .. & .. & 43 &. & 8 & .. \\
\hline$\cdot 7 \mathrm{n}$ & $5-17-79$ & $\cdots$ & .. & $\cdots$ & $\cdots$ & $\cdots$ & -. & $\cdot-$ & $\cdots$ & .. & $\cdots$ & 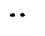 & .. \\
\hline -9P1 & $5-17-79$ & .. & -. & $\cdots$ & $\cdots$ & $\cdots$ & -. & $\cdot$. & $\cdots$ & .. & $\cdot \cdot$ & $\ddot{*}$ & $\cdots$ \\
\hline \multirow{2}{*}{$-12 m 1$} & $7-11 \cdot 73$ & $\cdots$ & $\cdot \cdot$ & $\cdots$ & $\cdots$ & $\cdots$ & $\cdot \cdot$ & $\cdots$ & $\cdots$ & $\cdots$ & $\cdot \cdot$ & 22 & *. \\
\hline & $5-17-79$ & $\cdots$ & $\cdots$ & $\cdots$ & $\cdots$ & $\cdots$ & $\cdots$ & $\cdots$ & $\cdots$ & $\cdots$ & 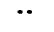 & $\cdots$ & $\cdots$ \\
\hline-1601 & $5 \cdot 17 \cdot 79$ & $\cdots$ & $\cdots$ & $\cdots$ & $\cdots$ & $\cdots$ & $\cdot \cdot$ & $\cdots$ & $\cdots$ & -. & $\cdots$ & $\cdots$ & $\cdots$ \\
\hline$-16 m 1$ & $5-17-79$ & $-\cdot$ & $\cdots$ & $\cdots$ & $\cdots$ & $\cdots$ & -. & .. & $\cdots$ & .. & -. & $\cdots$ & .. \\
\hline-1702 & $5-17-79$ & $\cdots$ & .. & .. & $\cdots$ & $\cdots$ & $-\cdot$ & $\cdots$ & $\cdots$ & -. & $\cdot \cdot$ & $\cdots$ & 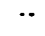 \\
\hline - 1aA1 & $5-17 \cdot 79$ & $\cdots$ & $\cdots$ & -. & -. & -. & $\cdots$ & -. & .. & .. & $\cdots$ & $\cdot$ & $\cdots$ \\
\hline$-22 c 1$ & $6-13 \cdot 79$ & $\cdots$ & $\cdots$ & $\cdots$ & -. & $\cdots$ & .. & $\cdots$ & $\cdots$ & .. & $\because$ & $\cdots$ & $\cdots$ \\
\hline -22E1 & $6-13 \cdot 79$ & $\cdots$ & $\cdots$ & $\cdots$ & $\cdots$ & $-\cdot$ & $\cdots$ & $\cdot-$ & $\cdots$ & $\cdot \cdot$ & $\cdot \cdot$ & $\cdots$ & $\because$ \\
\hline$-22 F 2$ & $6 \cdot 13 \cdot 79$ & $\cdots$ & $\cdots$ & $\cdots$ & $\cdots$ & $\cdots$ & -. & $\cdot-$ & $\cdots$ & .. & $\cdots$ & $\cdots$ & $\cdot \cdot$ \\
\hline-2361 & $6-13-79$ & $\cdots$ & $\cdots$ & $\cdots$ & $\cdots$ & $\cdots$ & $-\cdot$ & $\cdots$ & $\cdot$. & -. & $\cdots$ & $\cdots$ & $\cdots$ \\
\hline-2312 & $6 \cdot 13 \cdot 79$ & $\cdots$ & $\cdots$ & $\cdot \cdot$ & -. & $\cdots$ & -. & $\cdots$ & $\cdots$ & .. & $\cdots$ & $\cdots$ & $\cdots$ \\
\hline$-23 \times 1$ & $6-13 \cdot 79$ & -. & 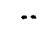 & $\cdots$ & $\cdots$ & -. & $\cdots$ & -- & $\cdots$ & .. & $\cdots$ & $\cdots$ & $\cdots$ \\
\hline-2641 & $6-13-79$ & $\cdot \cdot$ & $\cdots$ & •• & $\cdots$ & $\cdots$ & $\cdots$ & $\cdots$ & $\cdots$ & -. & $\cdots$ & $\cdots$ & 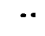 \\
\hline$-24 \times 1$ & $6 \cdot 13-79$ & .. & $\cdot$. & .. & .. & $\cdots$ & $\cdots$ & $\cdots$ & $\cdots$ & $*$ & $\cdots$ & $\cdots$ & $\cdots$ \\
\hline$-24 k 2$ & $6-13 \cdot 79$ & $\cdots$ & $\cdots$ & .. & $\cdots$ & $\cdots$ & $\cdots$ & $\cdots$ & $\bullet$ & $\cdot \cdot$ & $\because$ & $\cdots$ & $\cdots$ \\
\hline$-24 k 6$ & $6-13 \cdot 79$ & $\cdots$ & $\cdots$ & $\cdots$ & $\cdots$ & $\cdots$ & $\cdots$ & $\cdot \cdot$ & .. & .. & $\cdots$ & $\cdots$ & $\cdots$ \\
\hline$-24 k 5$ & $6 \cdot 13-79$ & .. & $\cdots$ & $\cdots$ & $\cdots$ & $\cdots$ & $\cdots$ & $\cdot-$ & $\cdots$ & .. & $\because$ & $\cdots$ & .. \\
\hline$-24 P 1$ & $6-11-79$ & $\cdots$ & $\cdots$ & -. & $\cdots$ & $\cdots$ & $\cdots$ & $\cdots$ & $\cdots$ & -. & $\because$ & $\cdots$ & $\because$ \\
\hline$-26 \mathrm{p3}$ & $6-11 \cdot 79$ & $\cdots$ & $\because$ & -. & $\cdots$ & $\cdots$ & $\cdots$ & $\cdot \cdot$ & $\cdots$ & -. & $\because$ & $\cdots$ & $\cdots$ \\
\hline$-24 n 1$ & $6-11-79$ & $\cdots$ & 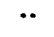 & .. & $*$ & $\cdot$. & $\bullet$ & 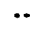 & $\cdots$ & $\cdots$ & $\because$ & $\cdots$ & 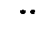 \\
\hline-24183 & $5 \cdot 18 \cdot 79$ & .. & $\cdots$ &.. & $\cdots$ & $\cdots$ & $*$ & .. & -. & .. & 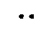 & $\cdot \cdot$ & .. \\
\hline-2581 & $6-12 \cdot 79$ & -. & $\cdots$ & .. & -. & $\cdots$ & $\cdots$ & .. & $\cdots$ & .. & $\cdots$ & .. & .. \\
\hline-2582 & $6 \cdot 11 \cdot 79$ & $\cdots$ & $\cdots$ & $\cdot \cdot$ & 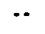 & .. & $\cdots$ & $\cdot \cdot$ & .. & .• & $\because$ & $\cdot \cdot$ & $\cdots$ \\
\hline$-25 c 1$ & $6-12.79$ & .. & $\cdots$ & .. & $*$ & $\cdots$ & $\cdots$ & .. & 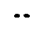 & $\cdot \cdot$ & $\because$ & $\cdot \cdot$ & $\cdots$ \\
\hline .2502 & $6-12 \cdot 79$ & $\cdots$ & $\cdots$ & •• & $\cdots$ & $\cdots$ & $\because$ & •• & $\cdots$ & $\cdot$ & $\cdots$ & $\cdots$ & $\cdots$ \\
\hline .2512 & $6-12-79$ & $\cdots$ & $\cdots$ & $\cdots$ & -. & $\cdots$ & $\cdots$ & .. & .. & .. & $\cdots$ & $\cdot \cdot$ & $\because$ \\
\hline$-25 F 1$ & $6-12 \cdot 79$ & $\cdot \cdot$ & $\cdots$ & $\cdot \cdot$ & -. & $\cdot \cdot$ & $\cdot \cdot$ & -• & .• & . & $\cdots$ & $\cdots$ & 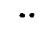 \\
\hline $.25 F 2$ & $6-12-79$ & $\cdots$ & $\cdots$ & •. & $\cdots$ & .. & •. & .. & .. & .. & -. & $\cdots$ & •. \\
\hline \multirow[t]{2}{*}{-2501} & $5 \cdot 2-66$ & 18 & .18 & 0.046 & 30 & 12 & 5.1 & D.6 & 350 & .. & 9.0 & 1.0 & 0.1 \\
\hline & $6 \cdot 12-79$ & $\cdots$ & $\cdots$ & -. & $\cdots$ & -. & $\cdots$ & -. & -. & -. & $\cdots$ & $\cdot$. & .. \\
\hline .2564 & $6-12 \cdot 79$ & .. & -. & .. & $\cdots$ & -. & $\cdots$ & $\cdot \cdot$ & $\cdots$ & $\cdots$ & $\because$ & $\cdots$ & $\cdots$ \\
\hline -25m1 & $6 \cdot 12-79$ & $-\cdot$ & $\cdots$ & .. & .. & $\cdots$ & $\cdots$ & .. & -. & -. & $\cdots$ & $\cdot \cdot$ & $\cdots$ \\
\hline-2511 & $6 \cdot 12 \cdot 79$ & $\cdots$ & $\cdots$ & .. & .. & -. & $\cdots$ & .. & $\cdots$ & .. & •. & $\cdots$ & $\cdots$ \\
\hline-2602 & $6-13-79$ & $\cdots$ & $\cdots$ & .. & 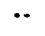 & .. & $\cdots$ & $\cdot \cdot$ & $\cdots$ & .. & 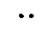 & 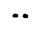 & -. \\
\hline -26E1 & $6-13 \cdot 79$ & $\cdots$ & $\cdots$ & .. & $\cdots$ & .. & $\cdots$ & $\cdot \cdot$ & $\cdots$ & .. & •. & $\cdot \cdot$ & $\because$ \\
\hline-2652 & $6 \cdot 12 \cdot 79$ & .. & $\cdots$ & -・ & .. & $\cdots$ & $\cdots$ & $\cdot \cdot$ & .• & $\cdot \cdot$ & $\cdots$ & $\cdots$ & $\because$ \\
\hline$-26 m 2$ & $6-13.79$ & $\cdots$ & $\cdots$ & $\cdots$ & -. & $\cdots$ & $\cdots$ & $\cdot \cdot$ & -. & .. & $\cdots$ & $\cdots$ & $\because$ \\
\hline$-27 a 1$ & $6-13 \cdot 79$ & .. & $\cdots$ & $\cdots$ & $\cdots$ & .. & $\cdots$ & $\cdot \cdot$ & .. & $\cdots$ & $\cdots$ & $\cdots$ & $\because$ \\
\hline$-27 n 2$ & $6-13-79$ & .. & $\cdots$ & .. & $\cdots$ & -. & -. & $*$ & -. & -. & $\cdots$ & $\cdots$ & $\cdots$ \\
\hline \multirow[t]{2}{*}{-2701} & 4. - 72 & 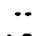 & $\cdots$ & $\ddot{*}$ & 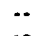 & $\ddot{*}$ & 6.5 & 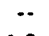 & $\ddot{*}$ & 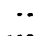 & $\cdots$ & $\because$ & $\because$ \\
\hline & $3 \cdot 18-74$ & 6.5 & .1 & .010 & 19 & 19 & 42 & 1.8 & 140 & 110 & 30 & 15 & .4 \\
\hline
\end{tabular}




\begin{tabular}{|c|c|c|c|c|c|c|c|c|c|c|c|c|}
\hline $\begin{array}{l}\text { Total } \\
\text { nitrate } \\
(\mathrm{W}) \\
(\mathrm{mg} / \mathrm{L})\end{array}$ & $\begin{array}{l}\text { Total } \\
\text { nitrite } \\
(\mathrm{N}) \\
(\mathrm{ng} / \mathrm{L})\end{array}$ & $\begin{array}{l}\text { Totel } \\
\text { phos: } \\
\text { phorus } \\
(P) \\
(m / L)\end{array}$ & $\begin{array}{l}\text { ols- } \\
\text { solved } \\
\text { solide } \\
\text { (residun } \\
\text { at } 180 \mathrm{c} \text { ) }\end{array}$ & $\begin{array}{l}\text { Mard- } \\
\text { ness } \\
(\mathrm{Ca}, \mathrm{Hg}) \\
(\mathrm{mg} / \mathrm{L})\end{array}$ & $\begin{array}{l}\text { Won- } \\
\text { carbon- } \\
\text { ate } \\
\text { hardhese } \\
(m g / L)\end{array}$ & $\begin{array}{l}\text { Specifle } \\
\text { conduc. } \\
\text { tenca } \\
\text { (nicro. } \\
\text { whos) }\end{array}$ & $\begin{array}{c}\text { PH } \\
\text { (uni te) }\end{array}$ & $\begin{array}{l}\text { Water } \\
\text { temper: } \\
\text { ature } \\
\text { (C) }\end{array}$ & $\begin{array}{l}\text { Color } \\
\text { (platinum. } \\
\text { cobalt } \\
\text { units) }\end{array}$ & $\begin{array}{l}\text { Turbid- } \\
\text { Ity } \\
\text { (JTU) }\end{array}$ & $\begin{array}{l}\text { Carbon } \\
\text { dioxide } \\
(\infty \infty) \\
(m / L)\end{array}$ & Remarks \\
\hline
\end{tabular}

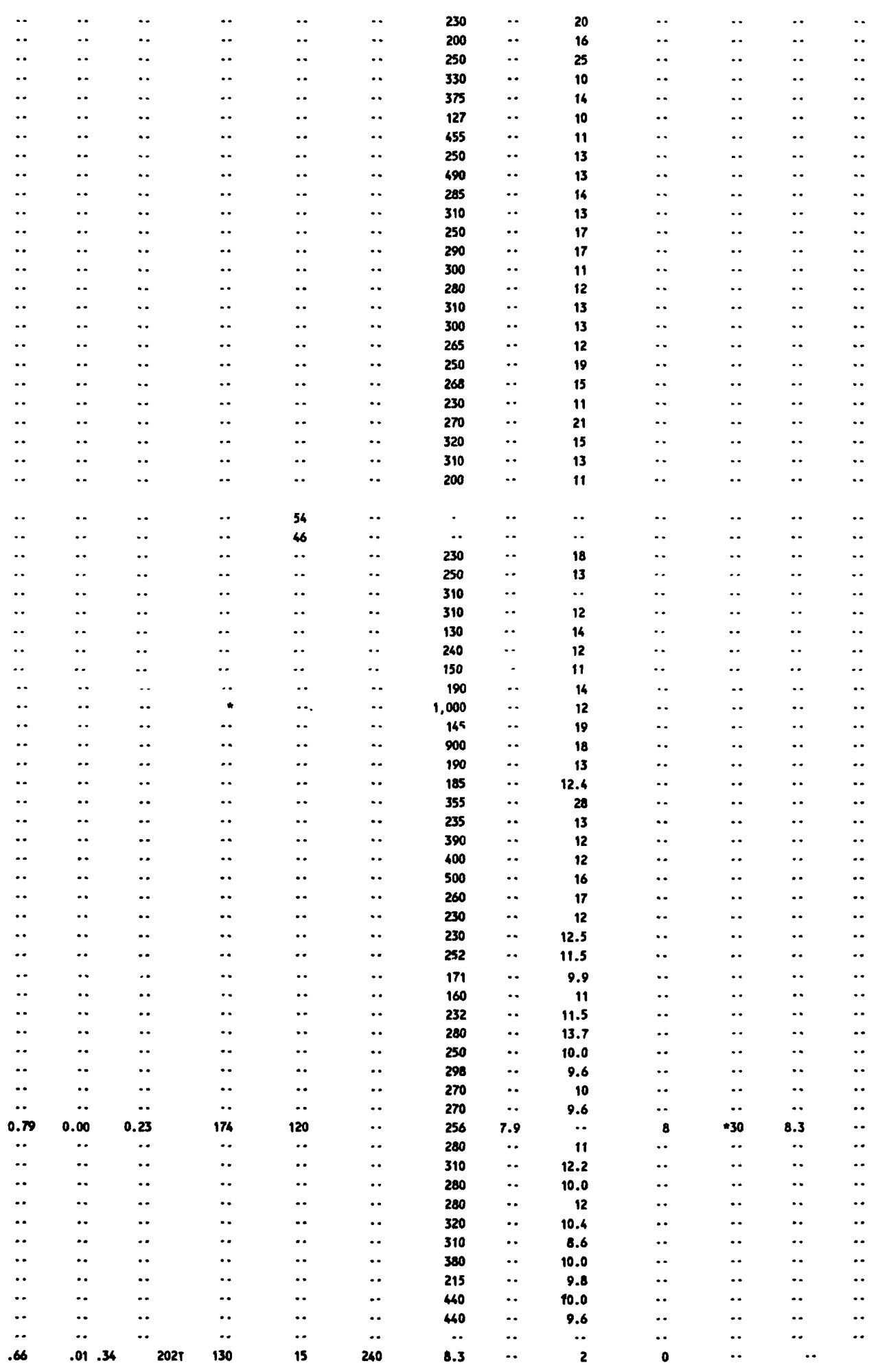




\begin{tabular}{|c|c|c|c|c|c|c|c|c|c|c|c|c|c|}
\hline site number & $\begin{array}{l}\text { Dote } \\
\text { sempled }\end{array}$ & $\begin{array}{l}\text { Dis. } \\
\text { solved } \\
\text { silica } \\
\text { (sio) } \\
\text { (mg/L) }\end{array}$ & $\begin{array}{l}\text { Dis. } \\
\text { solved } \\
\text { iron } \\
\text { (Fe) } \\
\text { (ug/L) }\end{array}$ & $\begin{array}{l}\text { Dis- } \\
\text { solved } \\
\text { mangen- } \\
\text { esa (Mn) } \\
\text { (ug/L) }\end{array}$ & $\begin{array}{l}\text { Dif- } \\
\text { solved } \\
\text { colcium } \\
\text { (ca) } \\
(\mathrm{mg} / \mathrm{L})\end{array}$ & $\begin{array}{l}\text { Dis. } \\
\text { solved } \\
\text { megnes. } \\
\text { lum (Mg) } \\
\text { (mg/L) }\end{array}$ & $\begin{array}{l}\text { Dis. } \\
\text { solved } \\
\text { sodi im } \\
\text { (Ma) } \\
(\mathrm{mo} / L)\end{array}$ & $\begin{array}{l}\text { Dis. } \\
\text { solved } \\
\text { potos. } \\
\text { sium }(K) \\
\text { (mg/L) }\end{array}$ & $\begin{array}{l}\text { eicar } \\
\text { bonate } \\
\text { (HCO) } \\
\text { (mg/L) }\end{array}$ & $\begin{array}{l}\text { Alka. } \\
\text { linity } \\
\text { os } \\
\text { coco } \\
\text { (mg/L) }\end{array}$ & $\begin{array}{l}\text { Dis. } \\
\text { solved } \\
\text { sul fote } \\
\text { (so) } \\
\text { (mg/L) }\end{array}$ & $\begin{array}{l}\text { Dis. } \\
\text { solved } \\
\text { chloride } \\
\text { (Cl) } \\
\text { (mg/L) }\end{array}$ & $\begin{array}{l}\text { Dis. } \\
\text { solvod } \\
\text { fluoride } \\
\text { (F) } \\
\text { (mg/h) }\end{array}$ \\
\hline $30 / 7 N \cdot 1 M 1$ & $2 \cdot 2.54$ & .. & 0.1 & .. & .. & .. & .. & .. & .. & .. & .. & 10 & .. \\
\hline .101 & 2. 2.54 &.. &.. & .. & .. & .. & .. & .. & .. & 130 & .. & 16 & .. \\
\hline$-2 \mathrm{H1}$ & $2 \cdot 2 \cdot 54$ & .. & .1 & .. & .. & .. & .. & .. & .. & .. & .. & 6 & .. \\
\hline$-3 R 1$ & $6 \cdot 13 \cdot 79$ & .. & .. & .. & .. & .. & .. & .. & .. & .. & .. & .. & .. \\
\hline$-3 R 2$ & pra 12.6 .78 & .. & .07 & 0.010 & .. & .. & .. & .. & .. & .. & .. & .. & 0.1 \\
\hline$\cdot 7 \mathrm{~s} 2$ & $5 \cdot 17-79$ & .. & .. & .. & .. & .. & .. & .. & .. & .. & .. & .. & .. \\
\hline .911 & $5 \cdot 17 \cdot 79$ & .. & .. & .. & .. & .. & .. & .. & .. & .. & .. & .. & .. \\
\hline $.9 \times 1$ & $5 \cdot 17 \cdot 79$ & .. & .. & .. & .. & .. & .. & .. & .. &.. & .. & .. & .. \\
\hline$\cdot 11 \mathrm{s1}$ & $2 \cdot 2.54$ & .. & .. & .. & .. & .. & .. & .. & .. & 120 & .. & 30 & .. \\
\hline$\cdot 1261$ & $2 \cdot 2.54$ & .. & .. & .. & .. & .. & .. & .. & .. & 31 & .. & 10 & .. \\
\hline$\cdot 12 \mathrm{H1}$ & $2 \cdot 2.54$ & .. & .. & .. & .. & .. & .. & .. & .. & 79 & .. & 6 & .. \\
\hline$-14 C 1$ & 2.3 .54 & .. & .. & .. & .. & .. & .. & .. & .. & 31 & .. & 14 & .. \\
\hline$\cdot 1401$ & 6.14 .79 & .. & .. & .. & .. & .. & .. & .. & .. & .. & .. & .. & .. \\
\hline$\cdot 14 E 1$ & $6 \cdot 14 \cdot 79$ & .. & .. & .. & .. & .. & .. & .. & .. & .. & .. & .. & .. $\quad-14 F 1$ \\
\hline $5 \cdot 17 \cdot 79$ &. & .. & .. & .. & .. & .. & .. & .. & .. & .. & .. & .. & \\
\hline$-15 \wedge 1$ & $6 \cdot 14 \cdot 79$ & .. & .. & .. & .. & .. & .. & .. & .. & .. & .. & .. & .. \\
\hline-1501 & $5 \cdot 17 \cdot 79$ & .. & .. & .. & .. & .. & .. & .. & .. & .. & .. & .. & .. \\
\hline$\cdot 1601$ & $6 \cdot 14 \cdot 79$ & .. & .. & .. & .. & .. & .. & .. & .. & .. & .. & .. & ... \\
\hline$\cdot 18 E 1$ & 6.14 .79 & .. & .. & .. & .. & .. & .. & .. & .. & .. & .. & .. & .. \\
\hline$\cdot 18 F 1$ & 6.14 .79 & .. & .. & .. & .. & .. & .. & .. & .. & .. & .. & .. & .. \\
\hline$\cdot 27 \mathrm{s1}$ & 0.14 .79 & .. & .. & .. & .. & .. & .. & .. & .. & .. & .. & .. & .. \\
\hline$\cdot 27\lrcorner 2$ & $6 \cdot 14.79$ & .. & .. & .. & .. & .. & .. & .. & .. & .. & .. & .. & .. \\
\hline$\cdot 28 R 2$ & 6.14 .79 & .. & .. & .. & .. & .. & .. & .. & .. & .. & .. & .. & .. \\
\hline $.29 F 1$ & $6 \cdot 14.79$ & .. & .. & .. & .. & .. & .. & -. & .. & .. & .. & .. & .. \\
\hline $30 / 94-26 R 1$ & 9.20 .67 & 11 & .00 & .. & 19 & 4.0 & 2.7 & 0.4 & 78 & 64 & 5.8 & 1.0 & .0 \\
\hline \multirow[t]{2}{*}{$-30 F 1$} & $1 \cdot 10.67$ &.. & .07 & .. &.. & .. & $\ldots$ & .. &.. & .. &.. & 2.8 &.. \\
\hline & 9.20 .67 & 16 & .01 & .. & 22 & 4.0 & 5.2 & .5 & 92 & $\pi$ & 5.6 & 3.0 & .1 \\
\hline$\cdot 3581$ & 1.20 .78 & $\cdot \cdot$ & .10 & .010 & .. & .. & .. & .. & $\cdot \cdot$ & .. & .. & .. & .2 \\
\hline $30 / 10 \mathrm{~W}-25 \mathrm{G} 1 \mathrm{~s}$ & $6 \cdot 12 \cdot 78$ & .. & .05 & .010 &.. & .. & .. &.. & .. &.. & .. & .. & .1 \\
\hline $30 / 11 W-28 G 1$ & 10.5 .78 & .. & .23 & .010 & .. & .. & .. & .. & .. & .. & .. & .. & .2 \\
\hline \multirow{2}{*}{$.28 \mathrm{H1}$} & $7 \cdot 8.64$ & 8.7 & .22 & .05 & 10 & 2.1 & 2.7 & .4 & 40 & 33 & 4.0 & 2.5 & .1 \\
\hline & $10 \cdot 3 \cdot 77$ & .. & .25 & .010 &.. & .. &.. &.. &.. & .. &.. &.. & $\therefore$ \\
\hline$-28 \mathrm{~Hz}$ & 7.24 .64 & 16 & .05 & .05 & 5.0 & .5 & 22 & .2 & 55 & 52 & 5.2 & 4.0 & .1 \\
\hline $30 / 124-2501$ & 5.16 .79 & .. & .. & .. & .. & .. & .. & .. & .. & .. & .. & .. & .. \\
\hline$\cdot 2601$ & 5.15 .79 & .. & .. & .. & .. & .. & .. & .. & .. &.. & .. & .. & .. \\
\hline $.27 m 1$ & 5.15 .79 & .. & .. & .. & .. & .. & .. & ... & .. & .. & .. & .. & .. \\
\hline$\cdot 27 N 1$ & $5 \cdot 15.79$ & .. & .. & .. & .. & .. & .. & .. & .. & .. & .. & .. & .. \\
\hline .2841 & $5.16-79$ & .. & .. & .. & .. & .. & .. & .. & .. & .. & .. & .. & .. \\
\hline$\cdot 3041$ & $5 \cdot 16 \cdot 79$ & .. & .. & .. & .. & .. & .. & .. & .. & .. & .. & .. & .. \\
\hline $30 / 13 w-34 \times 2$ & $5 \cdot 16 \cdot 79$ & .. & .. & .. & .. & .. & .. & .. & .. & .. & .. & .. & .. \\
\hline$-34 \mathrm{P} 1$ & $5 \cdot 15 \cdot 79$ & .. & .. & .. & .. & .. & .. & .. & .. & .. & .. & .. & .. \\
\hline-3501 & $5 \cdot 15 \cdot 79$ & .. & .. & .. & .. & .. & .. & .. & .. & .. & .. & .. & .. \\
\hline .3641 & 5.15 .79 & .. & .. & .. & .. & .. & .. & .. & .. & .. & .. & .. & .. \\
\hline \multirow[t]{3}{*}{ 31/3W-1861 } & 2.21 .66 & 11 & .13 &. .07 & 24 & 10 & 12 & 5.2 & 117 & 96 & 9.0 & 4.5 & .1 \\
\hline & 3.11 .66 & $\cdots$ & .. &.. & $\because$. &.. &.$\cdot$ & $\cdot$. & $\because \cdot$ & .. &.. & $\therefore$ &.. \\
\hline & $7 \cdot 26.68$ & .. & .. & .. & .. & .. & .. & .. & .. & .. & .. & 3.6 & .. \\
\hline \multirow[t]{2}{*}{-3041} & 7.25 .68 & .. & .. & .. & .. & .. & .. & .. & .. & .. & .. & 4.3 & .. \\
\hline & $7 \cdot 7 \cdot 78$ & .. & .. & .. & .. & .. & .. & .. & .. & .. & .. & 5.0 & .. \\
\hline .3001 & 7.25 .68 & .. & .. & .. & .. & .. & .. & .. & .. & .. &.. & 2.5 & .. \\
\hline-3004 & $7 \cdot 24 \cdot 75$ & .. & .. & .. & $\cdot \cdot$ & $\because$ & $\cdot \cdot$ & .. &.$\cdot$ & .. & $\cdot \cdot$ & $\cdot \cdot$ & $\cdots$ \\
\hline \multirow[t]{2}{*}{.3181} & $1 \cdot 16 \cdot 75$ & 16 & .12 &. .10 & 33 & 3.4 & 8.4 & .. & 111 & 91 & 11 & 1.2 & .1 \\
\hline & $7 \cdot 7 \cdot 78$ &.. & .. & .. &.. & .. & .. & .. & .. &.. &.. & 2.7 &.. \\
\hline$\cdot 3182$ & $\cdot \cdot \pi$ & .. & .. & .. & .. & .. & .. & .. & .. & .. & .. & 5.0 & .. \\
\hline \multirow[t]{3}{*}{.3141} & $11-28-67$ & .. &. .31 & .. & .. & .. & .. & .. & 178 & .. & 7.0 & 3.3 & .. \\
\hline & 7.25 .68 & .. & .. & .. & .. & .. & .. & .. & .. & .. &.. & 3.6 & .. \\
\hline & $1.31 \cdot 74$ & 19 & .00 & .03 & 36 & 9.4 & 8.4 & 1.3 & 146 & 120 & .0 & 0.4 & .2 \\
\hline \multirow[t]{2}{*}{$31 / 4 \mathrm{~W}-25 \mathrm{M1}$} & 7.25 .68 & .. & .. & .. & .. & .. & .. & .. & .. & .. & .. & 5.1 & .. \\
\hline & $7 \cdot 7 \cdot 78$ & .. & .. & .. & .. & .. & .. & .. & .. & .. & .. & 5.0 & .. \\
\hline $.25 \mathrm{P} 3$ & $7 \cdot 7 \cdot 78$ & .. & .. & .. & .. & .. & .. & .. & .. & .. & .. & 4.8 & .. \\
\hline \multirow[t]{2}{*}{.2661} & 7.25 .68 & .. & .. & .. & .. & .. & .. & .. & .. & .. & .. & 7.3 & .. \\
\hline & $7 \cdot 10 \cdot 78$ & .. & .. & .. & .. & .. & .. & .. & .. & .. &.. & 7.3 & .. \\
\hline $.26 \mathrm{~J} 2$ & $\cdot .78$ & .. & .. & -. &.. & .. & .. &.. & .. & $\cdots$ &.$\cdot$ & .. & $\because$ \\
\hline$-26 m 1$ & - .60 & .. & .. & .. & .. & .. &.. & .. & 193 & 158 & 50 & 12 & .. \\
\hline .2602 & $7 \cdot 10-78$ & .. & .. & .. & .. & .. & .. & .. & .. & .. &.. & 10 & .. \\
\hline
\end{tabular}




\begin{tabular}{|c|c|c|c|c|c|c|c|c|c|c|c|c|}
\hline $\begin{array}{l}\text { Total } \\
\text { nitrata } \\
\text { (W) } \\
(\mathrm{mg} / \mathrm{L})\end{array}$ & $\begin{array}{l}\text { Totol } \\
\text { nitrite } \\
(\mathrm{W}) \\
(\mathrm{mg} / \mathrm{L})\end{array}$ & $\begin{array}{l}\text { Potal } \\
\text { phos: } \\
\text { phorus } \\
\text { (P) } \\
\text { (ng/L) }\end{array}$ & $\begin{array}{l}\text { ole. } \\
\text { oolved } \\
\text { eol Ids } \\
\text { (rasidue } \\
\text { ot } 180 \mathrm{C} \text { ) }\end{array}$ & $\begin{array}{l}\text { Mord- } \\
\text { noese } \\
(\mathrm{Co}, \mathrm{Hg}) \\
(\mathrm{mg} / \mathrm{L})\end{array}$ & $\begin{array}{l}\text { Mon- } \\
\text { carbon- } \\
\text { ete } \\
\text { harchess } \\
\text { (mg/L) }\end{array}$ & $\begin{array}{l}\text { specific } \\
\text { conduc- } \\
\text { tance } \\
\text { (micro- } \\
\text { mos) }\end{array}$ & $\begin{array}{c}\text { PII } \\
\text { (units) }\end{array}$ & $\begin{array}{l}\text { Wotar } \\
\text { temper- } \\
\text { ature } \\
\text { (C) }\end{array}$ & $\begin{array}{l}\text { Color } \\
\text { (plot imum. } \\
\text { cobslt } \\
\text { unlts) }\end{array}$ & $\begin{array}{l}\text { Turbld- } \\
\text { lty } \\
\text { (JTU) }\end{array}$ & $\begin{array}{l}\text { Carbon } \\
\text { dioxide } \\
\text { (CO) } \\
\text { (ng/L) }\end{array}$ & Remarke \\
\hline
\end{tabular}

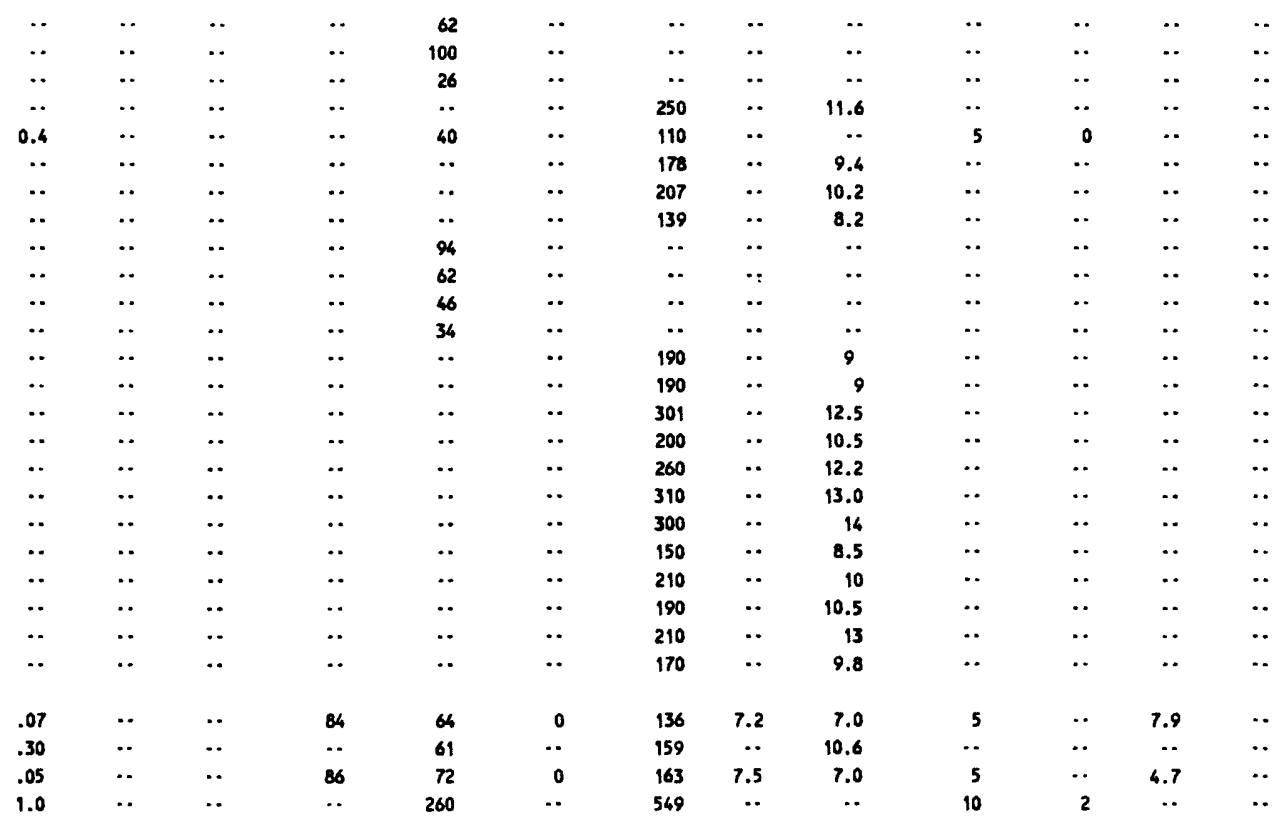

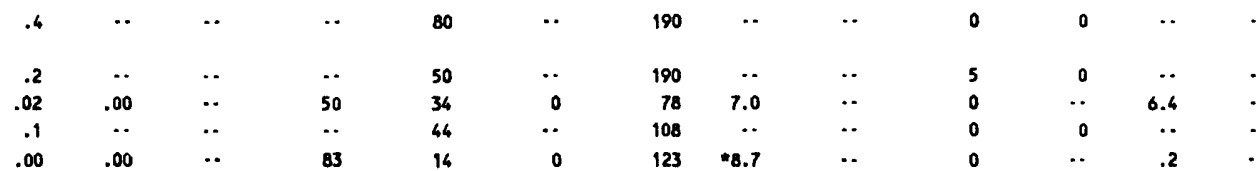

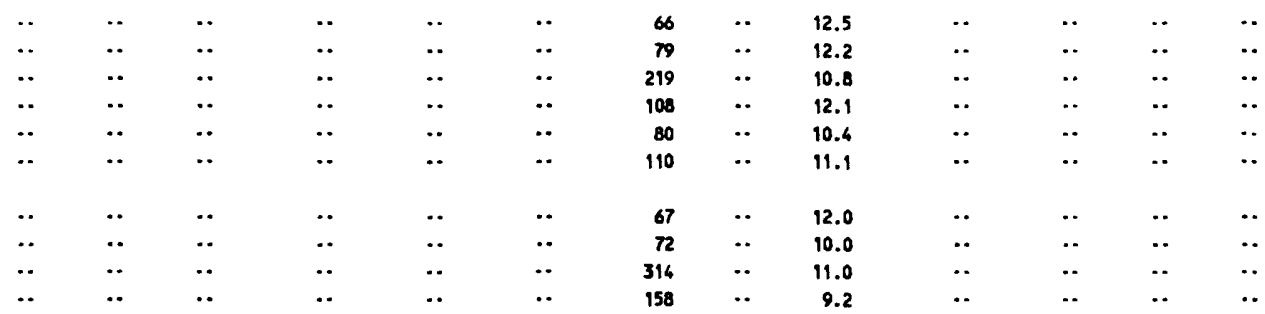

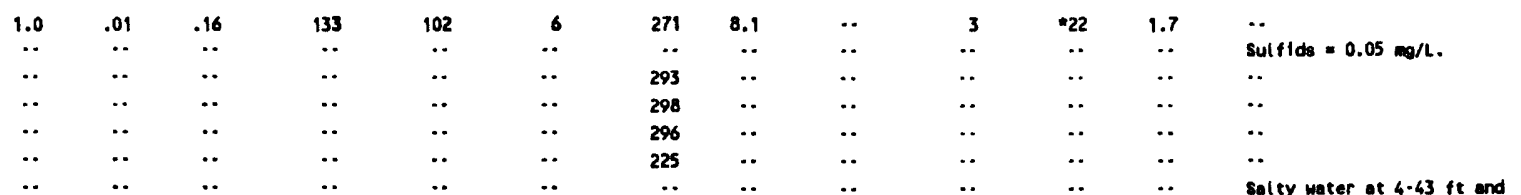

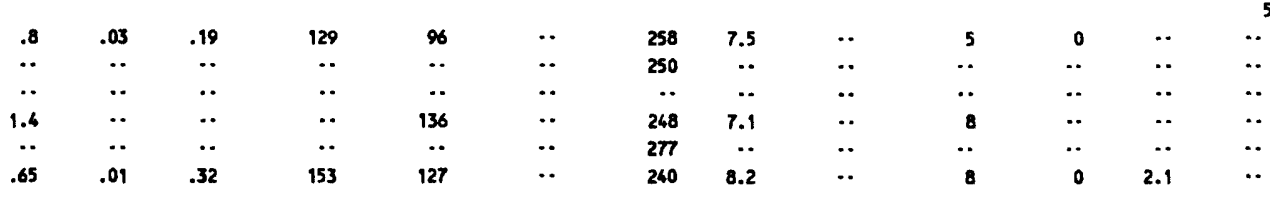

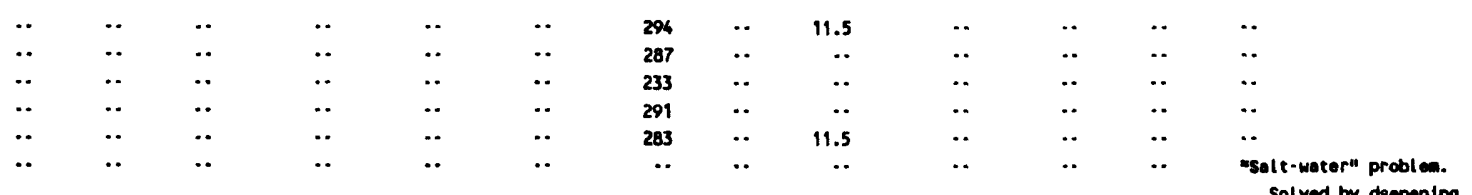

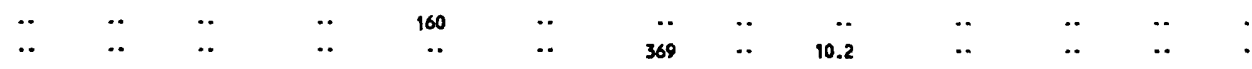

Solved by dsepening. 
TABLE 12. - cheaical and physical quality of water frem eelected wella and epringe in clallem county-continued

\begin{tabular}{|c|c|c|c|c|c|c|c|c|c|c|c|c|c|}
\hline site nuber & $\begin{array}{l}\text { Date } \\
\text { empled }\end{array}$ & $\begin{array}{l}\text { Dis- } \\
\text { Eolved } \\
\text { sllice } \\
\text { (s10) } \\
(\mathrm{mo} / \mathrm{L})\end{array}$ & $\begin{array}{l}\text { Dle. } \\
\text { golved } \\
\text { Iron } \\
\text { (fos) } \\
\text { (uo/L) }\end{array}$ & $\begin{array}{l}\text { Dis. } \\
\text { solvod } \\
\text { mangen. } \\
\text { eee (Mn) } \\
\text { (Lu/L) }\end{array}$ & $\begin{array}{l}\text { Dis. } \\
\text { solved } \\
\text { calcilum } \\
\text { (Co) } \\
(m / L)\end{array}$ & $\begin{array}{l}\text { Dis. } \\
\text { solved } \\
\text { mognes. } \\
\text { im (Mo) } \\
\text { (mo/L) }\end{array}$ & $\begin{array}{l}\text { Dis. } \\
\text { solved } \\
\text { sodium } \\
\text { (Ma) } \\
\left(\mathrm{mo}_{\mathrm{g}} / \mathrm{L}\right)\end{array}$ & $\begin{array}{l}\text { Dig. } \\
\text { solved } \\
\text { potose } \\
\text { olum (K) } \\
(\operatorname{mog} / L)\end{array}$ & $\begin{array}{l}\text { Bicar- } \\
\text { bonate } \\
\text { (HCO) } \\
\text { (mg/L) }\end{array}$ & $\begin{array}{l}\text { Alke- } \\
\text { linity } \\
\text { as } \\
\text { coco } \\
\text { (mo/L) }\end{array}$ & $\begin{array}{l}\text { Dis. } \\
\text { oolved } \\
\text { culfote } \\
\text { (so) } \\
(\mathrm{mg} / \mathrm{L})\end{array}$ & $\begin{array}{l}\text { Dis. } \\
\text { solved } \\
\text { chloride } \\
\text { (Cl) } \\
\text { (mo/L) }\end{array}$ & $\begin{array}{l}\text { Die: } \\
\text { oolved } \\
\text { fluoride } \\
\text { (f) } \\
\text { (mg/L) }\end{array}$ \\
\hline \multirow{2}{*}{$31 / 4 \mathrm{H}-27 \mathrm{MI}$} & $7 \cdot 25 \cdot 68$ & .. & .. & .. & .. & .. & .. & .. & .. &.$\cdot$ & .. & 11 & .. \\
\hline & $7 \cdot 10 \cdot 78$ & .. & .. & .. & .. & .. & .. & .. & .. & .. & .. & 12 & .. \\
\hline \multirow[t]{4}{*}{$\cdot 27 R 1$} & $7 \cdot 25.68$ & .. & .. & .. & .. & .. & .. & $\cdot \cdot$ & .. &.. & .. & 13 & $\cdot \cdot$ \\
\hline & $3 \cdot 30-71$ & 24 & 0.06 & 0.02 & 31 & 24 & 2.1 & 18 & 208 & 102 & 15 & 13 & 0.1 \\
\hline & $2-29 \cdot 72$ & .. & .. & .. & 30 & 23 & .. & .. & .. & .. & .. & 14 & .. \\
\hline & $7 \cdot 10 \cdot 78$ & .. & .. & .. & .. & .. & .. & .. & .. & .. & $\cdot \cdot$ & 10 & $\cdot \cdot$ \\
\hline .3501 & $7 \cdot 10 \cdot 78$ & .. & .. & .. & .. &.. & .. & .. & .. &.$\cdot$ & .. & 5.8 & .. \\
\hline .3514 & $2 \cdot 8 \cdot 72$ & .. & .05 & $\cdot \cdot$ & .. & $\cdot \cdot$ & .. & $\cdot \cdot$ & .. & .. & .. & $\cdot \cdot$ & $\cdot \cdot$ \\
\hline -35P1 & 7.27 .50 & -. & $\cdot \cdot$ & .. & .. & .. & $\cdot \cdot$ & .. & .. & $\cdot \cdot$ & .. & 14 & $\cdot \cdot$ \\
\hline \multirow[t]{2}{*}{$31 / 7 N-26 N 1$} & $8 \cdot 2 \cdot 68$ & .. & $\therefore$ &.. & .. & .. & .. & .. & .. & .. & .. & 3.8 & .. \\
\hline & $7-11 \cdot 78$ & .. & .. & .. & .. & .. & .. & .. & $\cdot \cdot$ & .. & $\cdot \cdot$ & 1.7 & $\cdot \cdot$ \\
\hline $.27 \mathrm{s1}$ & 7.31 .68 & .. & .. & $\cdot \cdot$ & .. & .. & .. & .. & .. & .. & .. & .7 & $\cdot \cdot$ \\
\hline $.27 \mathrm{~s} 2$ & 7.31 .68 & .. & .. & .. & .. &.. & .. & .. & .. & .. & .. & 1.8 & $\cdot \cdot$ \\
\hline$-27 \mathrm{J3}$ & $5 \cdot 11 \cdot \pi$ & 11 & .02 & .00 & 16 & 2.6 & 2.8 & .3 & 55 & 45 & 10 & 1.3 & .1 \\
\hline$\cdot 32 \times 1$ & 6.15 .79 & .. & .. & .. & .. & .. & .. & .. &.. & .. & .. & $\cdot \cdot$ & .. \\
\hline \multirow[t]{2}{*}{-33n1 } & $7 \cdot 31.68$ & .. & .. & $\cdot \cdot$ & .. & .. & .. & .. & .. & .. & .. & 12 & .. \\
\hline & $7 \cdot 11 \cdot 78$ & .. & .. & .. & .. & .. & .. &.. &.. & .. &.. & 13 & .. \\
\hline$\cdot 33 \wedge 2$ & 6.15 .79 & -. & .. & .. & .. &.. & .. & .. &.. & .. & .. & $\cdot \cdot$ & $\cdot$. \\
\hline \multirow{2}{*}{$-33 F 1$} & 7.31 .68 & .. & .. & .. & .. & .. & .. & .. & .. &.. &.. & 27 & .. \\
\hline & $7 \cdot 11 \cdot 78$ & .. & .. & .. & .. &.. & .. & .. & .. & .. & .. & 39 & .. \\
\hline \multirow[t]{3}{*}{$\cdot 34 A 2$} & $7 \cdot 13 \cdot 76$ & 8.3 & $\cdot \cdot$ & .. & 14 & 1.7 & 2.0 & .2 & 49 & 40 & 7.8 & 1.5 & .1 \\
\hline & $2 \cdot 2 \cdot \pi$ & $\cdot \cdot$ &.$\cdot$ & $\cdot \cdot$ & .. & $\cdot \cdot$ &.. & .. &.. & $\cdot \cdot$ & .. & $\cdot \cdot$ & $\cdot \cdot$ \\
\hline & $2 \cdot 2 \cdot \pi$ & .. &.. & .. & .. & .. & $\ldots$ & .. & .. &.. &.. &.. & .. \\
\hline .3483 & $5 \cdot 11 \cdot \pi$ & 6.9 & .16 & .01 & 14 & 1.7 & 2.1 & .1 & 42 & 34 & 13 & 1.3 & .1 \\
\hline-3401 & $7 \cdot 11 \cdot 78$ & .. & .. & $\cdot \cdot$ & .. & .. & .. & .. & .. & .. & .. & .9 & .. \\
\hline .3402 & 6.15 .79 & .. & .. & .. & .. & .. & .. & .. & .. & .. & .. & .. & $\cdot \cdot$ \\
\hline \multirow[t]{2}{*}{-35E1 } & 3.15 .69 & 10 & .16 & .021 & 17 & 3.6 & 3.1 & .8 & 66 & 54 & 5.0 & 3.0 & .0 \\
\hline & $5-11 \cdot \pi 7$ & 13 & .02 & .01 & 17 & 2.7 & 4.1 & .3 & 57 & 47 & 11 & 3.0 & .1 \\
\hline -35E2 & 6.1 .76 & .. & .1 & .05 & .. & .. & .. & .. & .. & .. & 6 & 3 & .1 \\
\hline$-35 E 3$ & 6.1 .76 & .. & .1 & .05 & .. & .. & .. & .. & .. & .. & 6 & 3 & .1 \\
\hline$-35 k 1$ & 2. 3.54 & .. & .1 &.. & .. & .. &.. & .. &.. & .. & .. & 6 & .. \\
\hline \multirow[t]{2}{*}{$.35 \mathrm{N1}$} & $3-15.69$ & 18 & .16 & .011 & 16 & 4.9 & 1.8 & .2 & 66 & 54 & 7.3 & .0 & .0 \\
\hline & $5 \cdot 11 \cdot \pi$ & 8.9 & .02 & .00 & 15 & 2.0 & 2.8 & .2 & 46 & 38 & 8.6 & 1.8 & .1 \\
\hline \multirow{3}{*}{$31 / 94-31610$} & $9 \cdot 7 \cdot 71$ & 6.2 & $\bullet .58$ & .01 & 21 & 4.9 & 5.0 & .2 & 81 & 66 & 7.9 & 3.5 & .1 \\
\hline & $4 \cdot 10-73$ & 7.6 & .01 & .00 & 11 & 18 & 4.6 & .0 & 130 & 100 & 8.6 & 3.0 & .1 \\
\hline & $11 \cdot 16 \cdot 77$ & $\cdot \cdot$ & -1.4 & -.09 &.. & $\because$ & .. &. & .. & $\cdot \cdot$ &.. & .. & .1 \\
\hline 31/19w-9191 & 8.1 .68 & .. & .. & .. & .. & .. & .. & .. & .. & .. & .. & .. & .. \\
\hline$\cdot 10 E 1$ & 8.1 .68 & .. & .. & $\cdot \cdot$ & .. & $\cdot \cdot$ & .. & $\cdot \cdot$ & .. & .. & .. & 11 & .. \\
\hline $32 / 124-21 M 1$ & 11.58 & .. & $\cdot 6.0$ & .. & .. & .. & .. & .. & .. & .. & .. & .640 & .. \\
\hline $.28 \times 2$ & $1 \cdot 12 \cdot \pi$ &.. & .14 & .. & .. & .. & .. & .. & .. & .. & .. &.. & .. \\
\hline .3301 & $5 \cdot 16 \cdot 79$ & .. & .. & .. & .. & .. & .. & .. & .. & $\cdot \cdot$ & .. & .. & $\cdot \cdot$ \\
\hline \multirow[t]{7}{*}{$32 / 134-2261$} & $8 \cdot 12.58$ & 2.5 & .. & .. & 64 & 42 & .. & .. & .. & 72 & 10 & $\cdot 360$ & .. \\
\hline & $1 \cdot 14.65$ & 11 & .2 &. .12 & 8.8 & 1.5 & 3.1 & .5 & 35 & 29 & 4.4 & 4.2 & .4 \\
\hline & 1.14 .65 & 12 & .06 & .003 & 10 & 1.3 & 2.8 & .6 & 35 & 29 & 3.0 & 4.0 & .3 \\
\hline & 3-28-67 & 62 & .19 & .028 & 5.6 & 3.4 & 13 & .6 & 34 & 28 & 7.5 & 3.5 & .2 \\
\hline & 3.28 .67 & 7.8 & .25 & .017 & 12 & 2.9 & 14 & .6 & 51 & 42 & 4.0 & 3.3 & .6 \\
\hline & $12 \cdot 27 \cdot 68$ & 12 & .26 & .023 & 9.6 & 4.9 & 2.7 & .4 & 49 & 40 & 3.4 & 4.0 & .0 \\
\hline & $5 \cdot 30 \cdot 78$ & 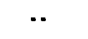 & .22 & .022 & & & $\cdot$ & .. & .. & .. & $\cdot \cdot$ & $\cdot \cdot$ & .1 \\
\hline \multirow[t]{2}{*}{$32 / 14 W \cdot 1 A 1$} & $10 \cdot 2.60$ & .. & .. & .. & .. & .. & .. & .. & .. & .. & .. & 2.7 & .. \\
\hline & $7 \cdot 12 \cdot 78$ & $\cdot \cdot$ & .. & .. & .. & $\cdot \cdot$ & $\cdot \cdot$ & $\cdot \cdot$ & .. & .. & $\cdot \cdot$ & 5.0 & .. \\
\hline $32 / 15 N \cdot 5 \times 1$ & $11 \cdot 30 \cdot \pi$ &.. & .05 & .01 &.. & .. & 49 & .. & .. & .. & .. & 27 & .3 \\
\hline .512 & $11 \cdot 30 \cdot 7$ & .. & .12 & .02 & .. & .. & 56 & $\cdot$. & $\because$ & .. & $\cdot \cdot$ & 16 & .5 \\
\hline \multirow[t]{2}{*}{ 33/15H-10R1 } & $5 \cdot 16 \cdot 52$ &.. & .09 & .. & .. & .. & .. & .. & 270 & .. & 15 & 26 & .4 \\
\hline & $2-27-53$ & .. & .11 & .. & 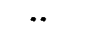 & .. & .. & .. & .. & .. & .. & .. & .. \\
\hline$\cdot 1111$ & $5 \cdot 16.52$ & .. & .10 & .. & .. & .. & .. & .. & 120 & .. & 13 & 4 & .4 \\
\hline .121 .1 & $10 \cdot 2.68$ & 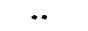 & .. & .. & .. & .. & .. &.. &.. & .. &.. & 28 & .. \\
\hline $.14 C 1$ & $2-27.53$ & 6.7 & .. & .. & 49 & 6.7 & 16 & 1.0 & 210 & .. & 20 & 7 & .. \\
\hline$-15 A 1$ & $3 \cdot 20 \cdot 62$ & 17 & $\cdot .76$ & .05 & 65 & 3.9 & 4.2 & 1.4 & 210 & 170 & 13 & 11 & .2 \\
\hline & 1.14 .65 & .. & .01 & .02 & .• & .. & .. & $\cdot \cdot$ & .. & .. & .8 & .. & .1 \\
\hline & $10 \cdot 2 \cdot 68$ & .. & $\cdot \cdot$ & .. & .. & .. & $\cdot \cdot$ & .. & .. & .. & .. & 17 & .. \\
\hline
\end{tabular}




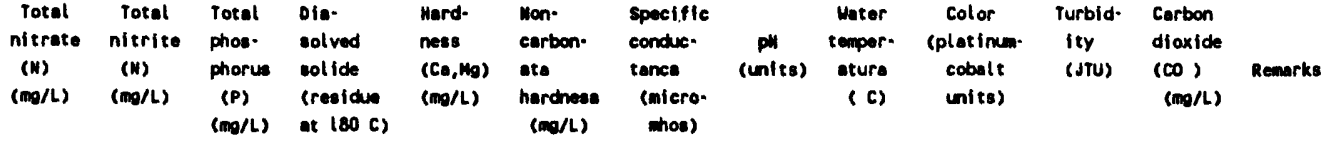

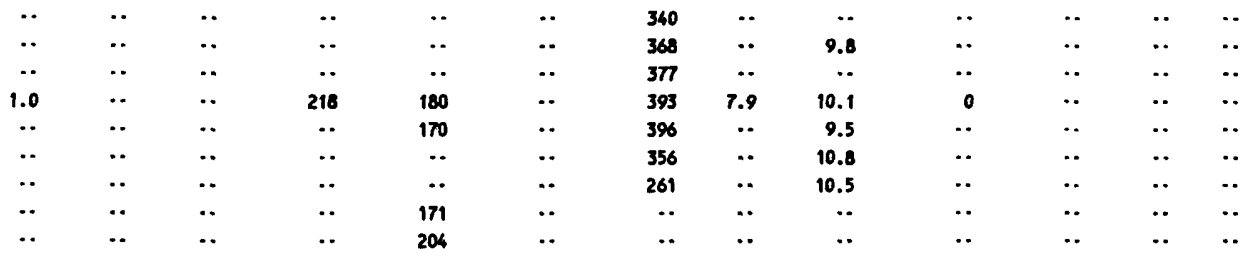

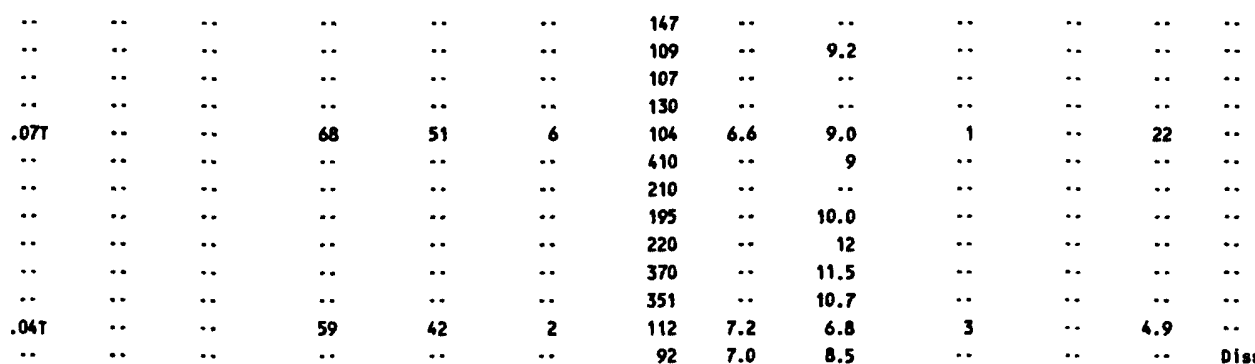

$$
\begin{aligned}
& \begin{array}{lllllllll}
. .04 & \cdots & . . & . & . . & . . & 92 & 7.0 & 8.5 \\
. . & . . & . . & . & \ldots & \ldots & 92 & 7.0 & 8.5
\end{array}
\end{aligned}
$$

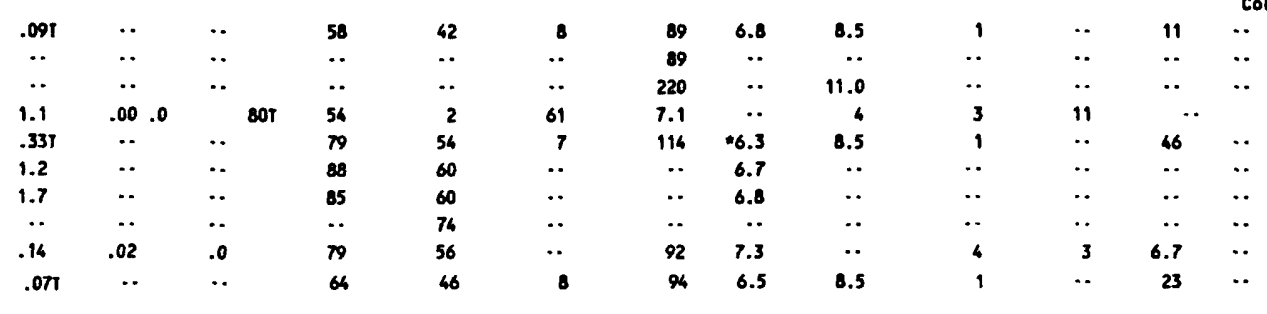

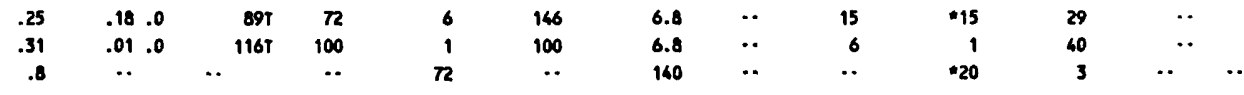

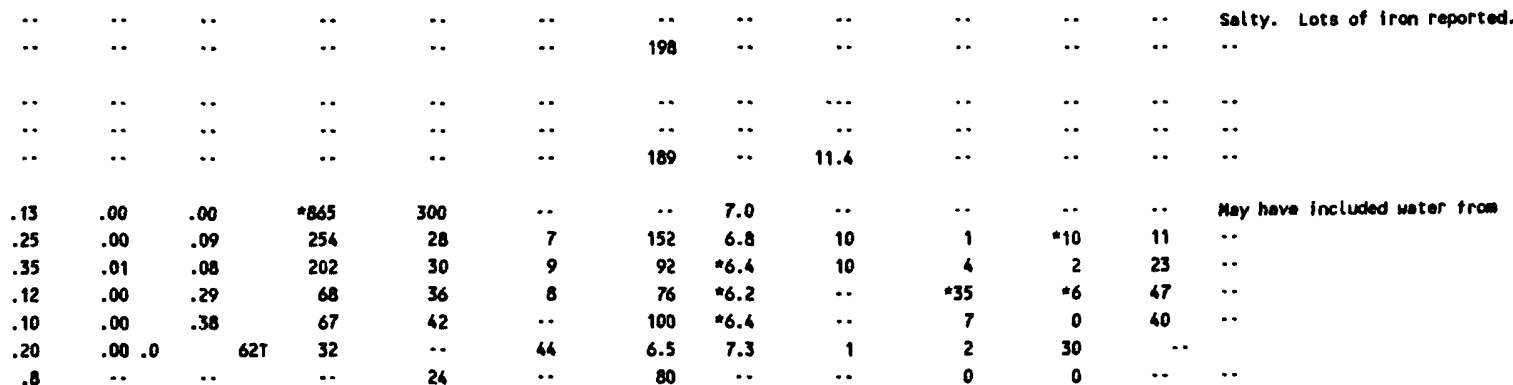

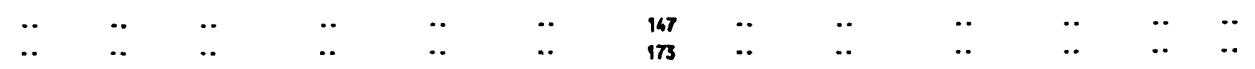

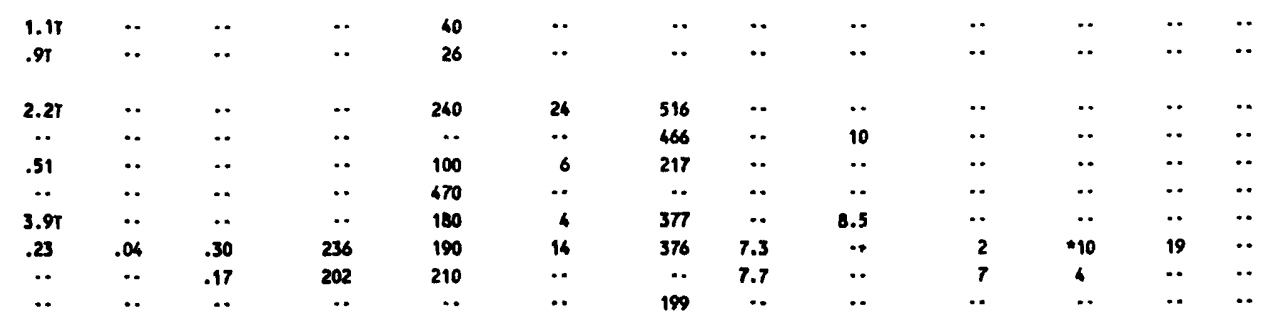




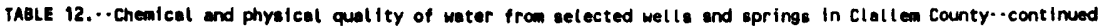

\begin{tabular}{|c|c|c|c|c|c|c|c|c|c|c|c|c|c|}
\hline site number & $\begin{array}{l}\text { Data } \\
\text { sompled }\end{array}$ & $\begin{array}{l}\text { Dia. } \\
\text { solved } \\
\text { sillica } \\
\text { (sio) } \\
(\mathrm{mg} / \mathrm{L})\end{array}$ & $\begin{array}{l}\text { Dife } \\
\text { eolved } \\
\text { Iron } \\
\text { (Fe) } \\
\text { (ug/L) }\end{array}$ & $\begin{array}{l}\text { Difo- } \\
\text { solved } \\
\text { mangan- } \\
\text { ese (Mn) } \\
\text { (ug/L) }\end{array}$ & $\begin{array}{l}\text { Dis. } \\
\text { solved } \\
\text { calcium } \\
\text { (Ca) } \\
(\mathrm{mg} / \mathrm{h})\end{array}$ & $\begin{array}{l}\text { Dis- } \\
\text { oolved } \\
\text { mognes. } \\
\text { ium (Mg) } \\
\text { (mo/L) }\end{array}$ & $\begin{array}{l}\text { Dite- } \\
\text { sol ved } \\
\text { sodium } \\
\text { (No) } \\
\text { (mo/L) }\end{array}$ & $\begin{array}{l}\text { Dis. } \\
\text { solved } \\
\text { potas. } \\
\text { eilum }(K) \\
(\mathrm{mg} / \mathrm{L})\end{array}$ & $\begin{array}{l}\text { Bicar. } \\
\text { bonate } \\
\text { (HCO) } \\
\text { (Ma/L) }\end{array}$ & $\begin{array}{l}\text { Alka. } \\
\text { linity } \\
\text { as } \\
\text { Caco } \\
\text { (mg/L) }\end{array}$ & $\begin{array}{l}\text { Dis. } \\
\text { solved } \\
\text { sulfate } \\
\text { (so) } \\
(\mathrm{mg} / \mathrm{L})\end{array}$ & $\begin{array}{l}\text { Dis. } \\
\text { solved } \\
\text { chloride } \\
\text { (Cl) } \\
\text { (mg/L) }\end{array}$ & $\begin{array}{l}\text { Dig- } \\
\text { solved } \\
\text { fluoride } \\
\text { (f) } \\
\text { (mo/L) }\end{array}$ \\
\hline \multirow[t]{3}{*}{$33 / 15 W \cdot 15 A 1,2$} & $12-21.66$ & 11 & 0.13 & 0.01 & 71 & 4.4 & 2.8 & 2.6 & 280 & 230 & 28 & 10 & 0.2 \\
\hline & 5.18 .68 & 55 & .16 &. .10 & 65 & 50 & 6.5 & 1.6 & 190 & 160 & 39 & 12 & .3 \\
\hline & $12 \cdot 12 \cdot 68$ & 7 & $=2.9$ &. .10 & 38 & 14 & 20 & 7.8 & 190 & 160 & 81 & 17 & .1 \\
\hline \multirow[t]{3}{*}{$-15 \wedge 2$} & 3.20 .62 & 18 & .07 &. .13 & 67 & 5.9 & 5.7 & 1.3 & 210 & 180 & 26 & $1 n$ &.$v$ \\
\hline & 1.14 .65 & .. & .00 &. .07 & .. & .. & .. & .. & .. & .. & .0 &.. & .1 \\
\hline & $10 \cdot 2 \cdot 68$ & .. & .. & $\cdot \cdot$ & .. & .. & .. & .. & .. & .. & .. & 6.5 & .. \\
\hline .1581 & $4 \cdot 7.67$ & .. & .. & .. & .. & .. & .. & .. & .. & .. & .. & $* 3,300$ & .. \\
\hline .1561 & $11 \cdot 3 \cdot 77$ & .. & .. & .. & .. & .. & .. & .. & .. & $\cdot \cdot$ & .. & -. & .. \\
\hline .1562 & $11 \cdot 3 \cdot 7$ & .. & .. &.. & .. & .. & .. & .. & .. & .. & .. & .. & .. \\
\hline .1563 & $11 \cdot 3 \cdot 77$ & .. & .. &.$\cdot$ & .. &.. & .. & .. & .. & .. &.. & .. & $\cdot \cdot$ \\
\hline .1564 & $11 \cdot 3 \cdot 77$ & .. & .. & .. & .. & .. & .. & .. & .. & .. & .. & .. & .. \\
\hline$-16 R 1$ & $9 \cdot 23 \cdot 76$ & .. & .. & .. & $\cdot$ & $\cdot$ & $\cdot$ & .. & .. & .. & .. & \$550 & .. \\
\hline
\end{tabular}




\begin{tabular}{|c|c|c|c|c|c|c|c|c|c|c|c|c|}
\hline $\begin{array}{l}\text { Total } \\
\text { nițrate } \\
\text { (N) } \\
\text { (ng/L) }\end{array}$ & $\begin{array}{l}\text { Total } \\
\text { nitrite } \\
\text { (H) } \\
\text { (mg/L) }\end{array}$ & $\begin{array}{l}\text { Total } \\
\text { phos- } \\
\text { phorus } \\
\text { (P) } \\
\text { (ms/L) }\end{array}$ & $\begin{array}{l}\text { Dis: } \\
\text { solved } \\
\text { solids } \\
\text { (residue } \\
\text { ot }(80 \mathrm{C})\end{array}$ & $\begin{array}{l}\text { Hard- } \\
\text { ness } \\
\text { (Co, Mg) } \\
(\mathrm{mg} / \mathrm{L})\end{array}$ & $\begin{array}{l}\text { Non- } \\
\text { carbon- } \\
\text { ate } \\
\text { hardhess } \\
\text { (mg/L) }\end{array}$ & $\begin{array}{l}\text { Speclfic } \\
\text { conduc- } \\
\text { tence } \\
\text { (micro- } \\
\text { mos) }\end{array}$ & $\begin{array}{c}\text { PH } \\
\text { (unites) }\end{array}$ & $\begin{array}{l}\text { Water } \\
\text { temper- } \\
\text { ature } \\
\text { (C) }\end{array}$ & $\begin{array}{l}\text { Color } \\
\text { (platinum. } \\
\text { cobalt } \\
\text { units) }\end{array}$ & $\begin{array}{l}\text { Turbid- } \\
\text { Ity } \\
\text { (JTu) }\end{array}$ & $\begin{array}{l}\text { Carbon } \\
\text { dioxide } \\
\text { (CO) } \\
(\mathrm{mg} / \mathrm{L})\end{array}$ & Remarks \\
\hline 0.16 & 0.00 & 0.38 & 253 & 200 & .. & 362 & 7.9 & .. & 10 & 1 & 5.2 & $\begin{array}{l}\text { suspect iron bacterit on well } \\
\text { screen. }\end{array}$ \\
\hline .46 & .00 & .42 & 252 & 370 & 210 & 370 & 7.8 & .. & 4 & 20 & 5.6 & $\cdots$ \\
\hline .63 & . 19.00 & $298 T$ & 200 & 46 & 466 & 7.6 & 10 & $=90$ & "8 & 9.6 & $\because$ & \\
\hline .22 & .10 & .46 & 254 & 200 & 26 & 404 & 7.8 & .. & 3 & 0 & 5.4 & . \\
\hline$\cdot \cdot$ & .. & .26 & 254 & 200 & .. & .. & 8.1 & .. & 7 & 16 & $\cdot \cdot$ & .. \\
\hline$\cdots$ & $\cdots$ & $\cdot \cdot$ & $\cdot \cdot$ & *. & -. & 560 & .. & $\cdots$ & $\cdots$ & $\cdots$ & 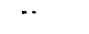 & $\cdots$ \\
\hline .. & .. & .. & " & .. & -. & 11,500 & -. & $\cdots$ & 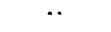 & . & $\cdots$ & $\cdot \cdot$ \\
\hline $.01 \mathrm{~T}$ & $\cdots$ & -. & $\cdot \cdot$ & .. & .. & -. & .. & 10.0 & .. & 3 & .. & $\begin{array}{l}\text { Ammonis }=0.79 \mathrm{mg} / \mathrm{L} \\
\text { Total nitrogen }-1.3 \mathrm{mg} / \mathrm{L} \text {. }\end{array}$ \\
\hline .01T & .. & .. & .. & .. & .. & .. & .. & 10.0 & .. & 2 & .. & $\begin{array}{l}\text { Amonis }=0.02 \mathrm{mg} / \mathrm{L} . \\
\text { Total nitrogen }=0.11 \mathrm{mg} / \mathrm{L} \text {. }\end{array}$ \\
\hline ז01ז & .. & .. & .. & . & $\cdots$ & $\cdots$ & .. & 10.0 & $\cdots$ & $* 20$ & .. & $\begin{array}{l}\text { Amonia }=0.25 \mathrm{mg} / \mathrm{L} . \\
\text { Total } \mathrm{n} \text { itrogen }=0.63 \mathrm{mg} / \mathrm{L} \text {. }\end{array}$ \\
\hline T1T. & .. & .. & .. & .. & .. & .. & .. & 10.0 & $*$ & $" 15$ & .. & $\begin{array}{l}\text { Armonia }=0.27 \mathrm{mg} / \mathrm{h} . \\
\text { Total nitrogen }=0.59 \mathrm{mg} / \mathrm{h} \text {. }\end{array}$ \\
\hline
\end{tabular}



TABLE 13. - Trace constituents in watar from selected wells and springs in clallan county

\begin{tabular}{|c|c|c|c|c|c|c|c|c|c|c|c|c|c|c|}
\hline \multirow[b]{2}{*}{$\begin{array}{c}\text { sita } \\
\text { number }\end{array}$} & \multirow[b]{2}{*}{$\begin{array}{l}\text { Date } \\
\text { sampled }\end{array}$} & \multirow[b]{2}{*}{$\begin{array}{c}\text { Arsenic } \\
(\text { As }) \\
\end{array}$} & \multirow[b]{2}{*}{$\begin{array}{c}\text { Barium } \\
\text { (Ba) }\end{array}$} & \multirow[b]{2}{*}{$\begin{array}{l}\text { Boron } \\
\text { (B) }\end{array}$} & \multicolumn{7}{|c|}{ Dissolved constituents (ug/L) } & \multirow[b]{2}{*}{$\begin{array}{l}\text { stron- } \\
\text { tium } \\
\text { (Sr) }\end{array}$} & \multirow[b]{2}{*}{$\begin{array}{l}\text { zine } \\
(Z n)\end{array}$} & \multirow[b]{2}{*}{ Remarks } \\
\hline & & & & & $\begin{array}{l}\text { Cad- } \\
\text { aiun } \\
\text { (Cr) }\end{array}$ & $\begin{array}{l}\text { Chro: } \\
\text { micm } \\
\text { (Cr) }\end{array}$ & $\begin{array}{c}\text { Copper } \\
\text { (CU) }\end{array}$ & $\begin{array}{l}\text { Lead } \\
(P b)\end{array}$ & $\begin{array}{l}\text { Mer. } \\
\text { cury } \\
\text { (Ho) }\end{array}$ & $\begin{array}{l}\text { selen- } \\
\text { iun } \\
\text { (Sa) }\end{array}$ & $\begin{array}{l}\text { Silvar } \\
(A g)\end{array}$ & & & \\
\hline \multirow[t]{2}{*}{$28 / 5 u-1 \mathrm{Jls}$} & 8. .76 & 5 & 100 & -. & 1 & 5 & .. & 5 & 1 & 2 & 0.5 & -. & $\cdots$ & -. \\
\hline & $7 \cdot 5 \cdot 78$ & 10 & 250 & $\cdots$ & 2 & 10 & $\cdots$ & 10 & 5 & 3 & 10 & $\cdots$ & $\cdots$ & $\cdots$ \\
\hline $28 / 124-601$ & $9-10-64$ & $\cdots$ & $\because$ & -. & $\cdots$ & 5 & 20 & $\cdots$ & $\cdots$ & $\cdots$ & $\cdots$ & $\cdots$ & $\cdots$ & Hexavalent chronium $5 \mathrm{ug} / \mathrm{L}$. \\
\hline $28 / 13 w-491-3$ & $11 \cdot 7 \cdot 75$ & 10 & 120 & -. & 5 & 5 & $\cdots$ & 5 & .5 & 6 & 5 & $\cdots$ & $\cdots$ & 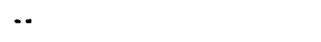 \\
\hline-402 & $3-30-71$ & $\cdots$ & $\cdots$ & $\because$ & $\because$ & 30 & 50 & 100 & .. & $\cdots$ & -. & 50 & 10 & $\begin{array}{l}\text { Aluminum } 5 \mathrm{ug} / \mathrm{L} \text {. } \\
\text { Lithium } 20 \mathrm{ug} / \mathrm{L} \text {. }\end{array}$ \\
\hline$-4 R 1$ & $5 \cdot 2 \cdot 61$ & $\cdot \cdot$ & -. & $\cdots$ & $\cdots$ & 30 & 50 & 100 & -. & $\cdots$ & -. & 50 & 10 & $\begin{array}{l}\text { Aluminum } 10 \mathrm{ug} / \mathrm{L} \text {. } \\
\text { Lithium } 20 \mathrm{ug} / \mathrm{L} \text {. }\end{array}$ \\
\hline $28 / 154-22 \mathrm{PI}$ & $9 \cdot 15 \cdot 67$ & 10 & 1,000 & $\cdots$ & 5 & 50 & 400 & 40 & -. & 10 & 40 & $\cdots$ & 500 & -. \\
\hline$-23 \times 1$ & $8-29-78$ & 10 & 250 & $\cdots$ & 2 & 10 & - & 10 & 1 & 3 & 10 & $\because$ & -. & -. \\
\hline$-23 N 1$ & $9-19-67$ & 10 & 1,000 & -. & 5 & 50 & 400 & 60 & $\cdots$ & 10 & 40 & $\cdots$ & 500 & -. \\
\hline$-23 N 2$ & $1-17-67$ & 10 & 1,000 & $\cdots$ & 5 & 50 & 400 & 40 & $\cdots$ & 10 & 40 & $\cdots$ & 500 & $\cdots$ \\
\hline $29 / 3 U-31 R 1$ & $10-11-78$ & 10 & 250 & $\cdots$ & 2 & 10 & $\cdots$ & 10 & 1 & 3 & 10 & $\cdots$ & $\cdots$ & $\cdots$ \\
\hline $29 / 7 \mathrm{~N}-901$ & $6-26-78$ & 10 & 250 & .. & 2 & 10 & $\cdots$ & 19 & 1 & 3 & 10 & $\cdots$ & $\cdots$ & $\cdots$ \\
\hline -9N1 & $6 \cdot 26 \cdot 78$ & 10 & 250 & $\cdot \cdot$ & 2 & 10 & $\cdots$ & 35 & 1 & 3 & 10 & $\cdots$ & $\cdots$ & 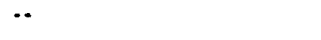 \\
\hline \multirow[t]{3}{*}{$29 / 8 \mathrm{u}-27 \mathrm{~K}$ Is } & $8 \cdot 19 \cdot 69$ & $\cdots$ & $\cdots$ & 800 & $\cdots$ & $\cdots$ & $\cdots$ & $\cdots$ & $\cdots$ & -. & $\cdots$ & - & $\cdots$ & orinking tap. \\
\hline & $8-19-69$ & -. & .. & 500 & $\cdots$ & $\cdots$ & $\cdots$ & $\cdots$ & $\cdots$ & .. & -. & .. & .. & Resarvoir. \\
\hline & $8 \cdot 19 \cdot 69$ & $\cdots$ & $\cdots$ & 500 & $\cdots$ & $\cdots$ & $\cdots$ & $\cdots$ & $\cdots$ & $\cdots$ & $\cdots$ & $\cdots$ & $\cdots$ & filtar plant. \\
\hline $29 / 9 \mathrm{~d}-32 \mathrm{Cl}-5 \mathrm{~s}$ & $3-14-78$ & 5 & 300 & $\cdots$ & 2 & 10 & $\cdots$ & 10 & .6 & 4 & 10 & $\cdots$ & $\cdots$ & $\cdots$ \\
\hline-3201 & $6 \cdot 26-78$ & 10 & 250 & $\cdots$ & 2 & 10 & $\cdots$ & 11 & 2.3 & 3 & 10 & .. & $\cdots$ & $\cdots$ \\
\hline F1 & $6 \cdot 26 \cdot 78$ & 10 & 250 & $\cdots$ & 2 & 10 & 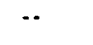 & 10 & 1 & 3 & 10 & $\cdots$ & $\cdots$ & $\cdots$ \\
\hline $30 / 21-2101$ & $5 \cdot 6 \cdot 79$ & 10 & 250 & .. & 2 & 10 & $\cdots$ & 10 & 1 & 5 & 10 & $\cdots$ & $\cdots$ & $\cdots$ \\
\hline $30 / 44-781$ & $3 \cdot 27-79$ & 5 & 50 & $\cdots$ & 2 & 2 & $\cdots$ & 10 & 2 & 5 & 2 & $\cdots$ & $\cdots$ & $\cdots$ \\
\hline$-12 R 2$ & $11-16-62$ & $\cdots$ & 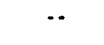 & 10 & $\cdots$ & $\cdots$ & $\cdots$ & $\cdots$ & -. & $\cdots$ & $-\cdot$ & $\cdots$ & $\because$ & $\because$ \\
\hline \multirow[t]{3}{*}{$30 / 5 u-10 F 1$} & $3 \cdot 6 \cdot 78$ & 10 & 250 & $\cdots$ & 2 & 12 & $\cdots$ & 10 & 1 & 3 & 10 & $\cdots$ & $\cdots$ & $\cdots$ \\
\hline & $3 \cdot 5 \cdot 79$ & 10 & 250 & -. & 2 & 10 & $\cdots$ & 10 & 1 & 5 & 10 & $\cdots$ & $\cdots$ & $\cdots$ \\
\hline & $3-20-79$ & $\cdots$ & $\cdots$ & $\cdots$ & $\cdots$ & $\cdots$ & $\cdots$ & $\cdots$ & 1 & $\cdots$ & $-\cdot$ & $\cdots$ & $\cdots$ & $\cdots$ \\
\hline $30 / 7 \mathrm{U}-3 \mathrm{R2}$ pre & $12-6-78$ & 10 & 250 & $\cdots$ & 2 & 10 & $\cdots$ & 10 & 1 & 3 & 10 & $\cdots$ & $\cdots$ & $\cdots$ \\
\hline $30 / 94-3581$ & $1-20-78$ & 10 & 100 & -. & 5 & 10 & $\cdots$ & 10 & 1 & 5 & 10 & $\cdots$ & $\cdots$ & $\cdots$ \\
\hline $30 / 10 \mathrm{~d}-25 \mathrm{G} 1 \mathrm{~s}$ & $6-12-78$ & 10 & 250 & -. & 2 & 10 & $\cdots$ & 25 & 1.3 & 3 & 10 & $\cdots$ & .. & $\cdots$ \\
\hline $30 / 114-2801$ & $10 \cdot 5 \cdot 78$ & 10 & 250 & -- & 2 & 10 & $\cdots$ & 10 & 1 & 3 & 10 & $\cdots$ & $\cdots$ & $\cdots$ \\
\hline
\end{tabular}


Quilleyute River (river mile 1.5) (Site 60)

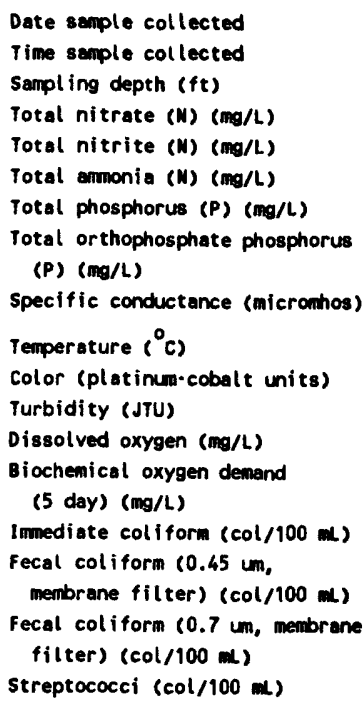

\begin{tabular}{|c|c|c|c|c|c|c|c|c|c|c|c|c|}
\hline \multirow{2}{*}{$\frac{8 / 2 / 76}{1600}$} & \multicolumn{2}{|c|}{$9 / 10 / 76$} & \multicolumn{9}{|c|}{$9 / 8 / 77$} & \multirow{2}{*}{$\frac{10 / 5 / 77}{1655}$} \\
\hline & 0755 & 1345 & 0710 & 0850 & 1030 & 1145 & 1325 & 1435 & 1605 & 1725 & 1855 & \\
\hline$\because$ & .8 & 1.6 & $\cdots$ & $\because$ & $\because$ & $\cdots$ & $\cdots$ & $\cdots$ & $\cdots$ & -. & .. & -. \\
\hline .01 & .. & .. & .. & .. & $\cdots$ & .. & .. & .. & $\cdots$ & .. & $\cdots$ & .04 \\
\hline .00 & $\cdots$ & $\cdots$ & $\cdots$ & $\cdots$ & $\cdots$ & .. & $\cdots$ & $\cdots$ & $\cdots$ & $\cdots$ & $\cdots$ & .00 \\
\hline .02 & .03 & .02 & $\cdots$ & $\cdots$ & $\cdots$ & $\cdots$ & $\cdots$ & $\cdots$ & $\cdots$ & $\cdots$ & $\cdots$ & .03 \\
\hline .00 & $\cdots$ & $\cdots$ & $\cdots$ & $\cdots$ & $\cdots$ & $\cdots$ & $\cdots$ & $\because$ & $\cdots$ & $\because$ & $\cdots$ & .00 \\
\hline .00 & $\cdots$ & -. & $\cdots$ & $\cdots$ & $\cdots$ & .. & $\cdots$ & .. & $\cdots$ & $\cdots$ & $\cdots$ & .00 \\
\hline 82 & $\cdots$ & .. & 85 & 79 & 80 & 85 & 79 & 81 & 85 & 81 & 82 & 78 \\
\hline 15.2 & 12.4 & 15.5 & $\cdots$ & -. & $\because$ & $\cdots$ & $\cdots$ & $\cdots$ & $\cdots$ & $\cdots$ & -. & 10.8 \\
\hline 0 & $\cdots$ & $\cdots$ & $\because$ & $\cdots$ & $\cdots$ & $\cdots$ & $\cdots$ & $\cdots$ & $\cdots$ & .. & .. & 0 \\
\hline 0 & $\because$ & $\because$ & $\because$ & $\cdots$ & $\because$ & $\cdots$ & $\cdots$ & $\because$ & $\because$ & $\cdots$ & $\cdots$ & 0 \\
\hline 10.7 & 9.5 & 11.3 & -. & 10.2 & $\cdots$ & $\cdots$ & $\cdots$ & 11.2 & $\cdots$ & . & $\cdots$ & 12.8 \\
\hline$\cdots$ & 1.0 & -. & . & $\cdots$ & $\cdots$ & $\cdots$ & $\cdots$ & $\cdots$ & $\cdots$ & .. & - & - \\
\hline 20 & 65 & 42 & 78 & 2900 & 420 & 8 & 2 & 54 & 2 & 8 & 40 & 930 \\
\hline$\cdots$ & 15 & 11 & $\cdots$ & $\cdots$ & $\cdots$ & $\cdots$ & -. & $\cdots$ & $\cdots$ & .. & .. & .. \\
\hline$\cdots$ & .. & $\cdots$ & .. & .. & -. & $\cdots$ & -. & . & .. & .. & .. & 5 \\
\hline .. & 10 & 1 & .. & $\cdots$ & $\cdots$ & $\cdots$ & $\cdots$ & $\cdots$ & $\cdots$ & $\cdots$ & $\cdots$ & $\because$ \\
\hline
\end{tabular}

Quillayute Estuary, at Dickey River Mile 0.1 (river mile 1.3) (Site 62)

\begin{tabular}{|c|c|c|c|}
\hline \multirow{2}{*}{$\begin{array}{l}\text { Date sample collected } \\
\text { Time ample collected }\end{array}$} & \multicolumn{3}{|c|}{$9 / 10 / 76$} \\
\hline & 0810 & 1030 & 1400 \\
\hline Sampl ing depth (ft) & .5 & 1.6 & 1.6 \\
\hline Total ammonia (N) (mg/L) & .03 & .03 & .02 \\
\hline Temperature $\left(^{\circ} \mathrm{C}\right)$ & 11.8 & 12.7 & 15.0 \\
\hline Dissolved oxygen (mg/t) & 8.9 & 9.3 & 10.6 \\
\hline Biochemical oxygen demand & & & \\
\hline (5 day) (mg/L) & 1.4 & $\cdots$ & $\cdots$ \\
\hline $\begin{array}{l}\text { Immediate coliform }(\mathrm{col} / 100 \mathrm{~mL}) \\
\text { Fecal coliform }(0.45 \mathrm{~mm}\end{array}$ & 42 & 88 & 800 \\
\hline menbrane filter) col/100 m.) & 48 & $\cdots$ & 22 \\
\hline Streptococci (col/100 m) & 24 & .. & 2 \\
\hline
\end{tabular}

Quillayute Estuary (river mile 1.4) (Site 61)

Dote sample collected Time sample collected Sampl ing depth ( $f t$ ) Total nitrate (N) (mg/t)

Total nitrite (W) (mg/L) Total amonie $(N)(m g / L)$

Temperature $\left({ }^{\circ} \mathrm{C}\right)$

Turbidity (JTU)

Dissolved oxygen (mg/L)

Biochemical oxygen demand

(5 day) (mg/L)

Imediate coliform (col/100 m)

Fecal coliform $(0.45 \mathrm{um}$,

membrane filter) (col/100 wh)

\begin{tabular}{|c|c|c|c|c|c|c|c|c|}
\hline \multicolumn{2}{|c|}{1976} & \multirow{2}{*}{\multicolumn{7}{|c|}{$8 / 19 / 77$}} \\
\hline $8 / 11$ & $8 / 12$ & & & & & & & \\
\hline$\overline{1930}$ & 0905 & 0840 & 1030 & 1250 & 1300 & 1440 & 1720 & 2015 \\
\hline 1.6 & 1.6 & 4.9 & 8.2 & 8.2 & 8.2 & 8.2 & 8.2 & 8.2 \\
\hline .. & $\cdots$ & .02 & .02 & .02 & .02 & .02 & .02 & .02 \\
\hline$\cdots$ & $\cdots$ & .00 & .00 & .00 & .00 & .00 & .00 & .00 \\
\hline$\cdots$ & $\cdots$ & .01 & .02 & .01 & .01 & .00 & .02 & .09 \\
\hline 17.0 & 15.2 & 14.0 & - & -. & -. & 14.0 & 14.0 & 14.0 \\
\hline$\cdots$ & .. & D & 0 & 0 & 0 & 0 & 0 & 1 \\
\hline .. & 9.6 & 10.4 & 9.6 & 10.1 & 10.2 & 8.8 & 10.5 & 10.0 \\
\hline$\cdots$ & .. & .7 & $\cdots$ & $\cdots$ & .9 & 1.3 & -. & 1.0 \\
\hline$\cdots$ & 76 & $\cdots$ & 720 & 290 & 140 & 120 & 150 & 480 \\
\hline • & .. & .. & .. & .. & 58 & .. & $\because$ & .. \\
\hline
\end{tabular}


Pacific Ocean Tributary No. 5 (Site 1)

\begin{tabular}{|c|c|c|c|c|}
\hline Date sample collected & $5 / 20 / 65$ & $4 / 27 / 67$ & $4 / 27 / 67^{\circ}$ & $8 / 4 / 70$ \\
\hline Dissolved sillica $\left(\mathrm{siO}_{2}\right)(\mathrm{mg} / \mathrm{L})$ & 31 & 2.5 & 9.5 & 21 \\
\hline Dissolved iron (Fe) (ug/L) & 30 & 220 & 490 & 270 \\
\hline Dissolve manganese $(\mathrm{Mn})(\mathrm{ug} / \mathrm{L})$ & 16 & 39 & 4 & 3 \\
\hline Dissolved calcium (Ca) (mg/L) & 4.8 & 4.0 & 3.2 & 4.0 \\
\hline Dissolved magnesium (Mg) (mg/L) & 2.9 & 2.6 & 1.4 & 5.3 \\
\hline Dissolved sodium (Na) (mg/L) & 10 & 15 & 4.1 & 18 \\
\hline Dissolved potassiun (K) (mg/L) & 1.3 & 1.1 & .8 & .7 \\
\hline Bicarbonate $\left(\mathrm{HCO}_{3}\right)(\mathrm{mg} / \mathrm{L})$ & 39 & 17 & 10 & 22 \\
\hline Alkal inity $\left(\mathrm{CaCO}_{3}\right)(\mathrm{mg} / \mathrm{L})$ & 32 & 14 & 8 & 18 \\
\hline Dissolved sulfata $\left(\mathrm{SO}_{4}\right)(\mathrm{mg} / \mathrm{L})$ & 8.5 & 2.7 & 2.5 & 1.9 \\
\hline Dissolved chloride (Cl) (mg/L) & 28 & 22 & 15 & 27 \\
\hline Dissolved fluoride (F) (mg/L) & .3 & .2 & .6 & .0 \\
\hline Dissolved nitrate $(N)(m g / L)$ & .40 & .31 & .22 & .21 \\
\hline Dissolved nitrite $(N)(\mathrm{mg} / \mathrm{L})$ & .00 & .00 & .01 & .02 \\
\hline Dissolved phosphorus (P) (mg/L) & .11 & .13 & .16 & .04 \\
\hline \multicolumn{5}{|l|}{ Dissolved solids, residue at } \\
\hline $180^{\circ} \mathrm{C}(\mathrm{mg} / \mathrm{L})$ & 122 & 70 & 50 & $\cdots$ \\
\hline Suspended solids, at $105^{\circ} \mathrm{C}(\mathrm{mg} / \mathrm{L})$ & 1 & 2 & 3 & $\cdots$ \\
\hline Hardness $\left(\mathrm{CaCO}_{3}\right)(\mathrm{mg} / \mathrm{L})$ & 12 & 10 & 8 & 10 \\
\hline Noncarbonate hardness (mg/L) & $\cdot \cdot$ & 8 & 10 & 14 \\
\hline Specific conductance (micromhos) & 123 & 114 & 71 & 149 \\
\hline pH (units) & 7.1 & 7.5 & 7.2 & 7.1 \\
\hline Color (platimu-cobalt units) & 7 & 25 & 50 & 25 \\
\hline Turbidity (JTU) & 3 & 2 & 1 & 2 \\
\hline Dissolved carbon dioxide (mg/L) & 6.2 & 1.2 & 1.5 & 3.8 \\
\hline
\end{tabular}

the stream.

12041500 Soleduck River near Fairholm, Wash. (Site 4)

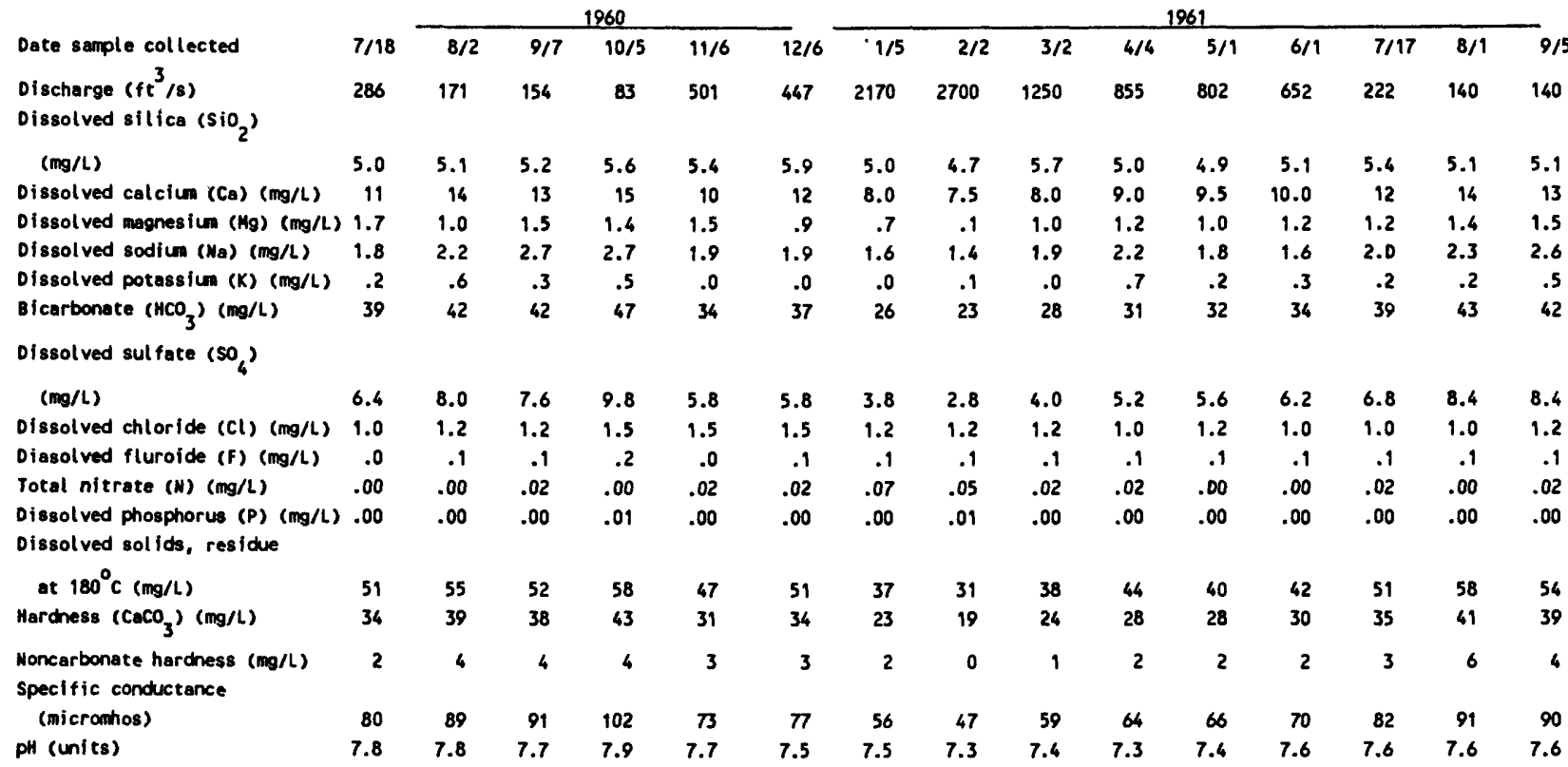


12042000 Solectuck River near Beaver, Wash. (sitte 6)

Date sample collected

Discharge $\left(\mathrm{ft}^{3} / \mathrm{s}\right)$

Dissolved siltica $\left(\mathrm{SiO}_{2}\right)(\mathrm{mg} / \mathrm{L})$

Total iron (fe) (ug/L)

Dissolved celciun (Ca) (mo/L)

Dissolved manesiu $(\mathrm{Mg})(\mathrm{mg} / \mathrm{L})$

Dissolved sodiun ( $\mathrm{Na})(\mathrm{ma} / \mathrm{L})$

Dissolved potassium (K) $(\mathrm{m} / \mathrm{L} / \mathrm{L})$

sicarbonate $\left(\mathrm{BCO}_{3}\right)(\mathrm{m} / \mathrm{L})$

Alkal inity $\left(\mathrm{CaCO}_{3}\right)(\mathrm{mg} / \mathrm{L})$

Dissolved sulfate $\left(\mathrm{SO}_{4}\right)(\mathrm{mg} / \mathrm{L})$

Dissolved chloride (Ct) (m/L)

Dissolved fluoride (F) (mg/L)

Total nitrate (W) $(\mathrm{mg} / \mathrm{L})$

Diseolved or thophosphate

phosphorws (P) $(\mathrm{mo/L})$

Diseolved solids, residus at

$180^{\circ} \mathrm{C}(\mathrm{mg} / \mathrm{L})$

Msrchess $\left(\mathrm{CaCO}_{3}\right)(\mathrm{mg} / \mathrm{L})$

Moncarbonata harchess $(\mathrm{mg} / \mathrm{L})$

Specific conductance (micromos)

pil (units)

Temperature ( $\left.{ }^{\circ} \mathrm{C}\right)$

Color (plstinu-cobalt units)

Turbidity (JTU)

Dissolved oxygen (ma/L)

Diseolved cerbon dioxide

$\left(\mathrm{CO}_{2}\right)(\mathrm{ma} / \mathrm{L})$

Completa coliform (MPN)

Oissolved arsenic (As) (ug/L)

Dissolved boron (B) (ug/L)

Dissolved chromiun (Cr) (ug/L)

Dissolved hexavalent chromiun

(Cr) (ug/L)

Dissolved copper (Cu) (ug/L)

Dissolved zine ( $2 n)(\mathrm{ug} / L)$

\begin{tabular}{|c|c|c|c|c|c|c|c|c|c|c|c|c|c|c|}
\hline \multicolumn{6}{|c|}{1960} & \multicolumn{9}{|c|}{1961} \\
\hline $7 / 18$ & $8 / 2$ & $9 / 7$ & $10 / 5$ & $11 / 4$ & $12 / 6$ & $1 / 5$ & $2 / 2$ & $3 / 2$ & $4 / 4$ & $5 / 1$ & $6 / 1$ & $7 / 17$ & $8 / 1$ & $9 / 5$ \\
\hline 286 & 171 & 154 & 83 & 501 & 447 & 2170 & 2700 & 1250 & 855 & 802 & 652 & 222 & 140 & 140 \\
\hline 5.0 & 5.1 & 5.2 & 5.6 & 5.4 & 5.9 & 5.0 & 4.7 & 5.7 & 5.0 & 4.9 & 5.1 & 5.4 & 5.1 & 5.1 \\
\hline 10 & 10 & 40 & 20 & 30 & 30 & 140 & 450 & 60 & 10 & 60 & 20 & 10 & 20 & 0 \\
\hline 11 & 14 & 13 & 15 & 10 & 12 & 8.0 & 7.5 & 8.0 & 9.0 & 8.5 & 10 & 12 & 14 & 13 \\
\hline 1.7 & 1.0 & 1.5 & -1.4 & 1.5 & .9 & .7 & .1 & 1.0 & 1.2 & 1.0 & 1.2 & 1.2 & 1.4 & 1.5 \\
\hline 1.8 & 2.2 & 2.7 & 2.7 & 1.9 & 1.9 & 1.6 & .14 & 1.9 & 2.2 & 1.8 & 1.6 & 2.0 & 2.3 & 2.6 \\
\hline .2 & .6 & .3 & .5 & .0 & .0 & .0 & .1 & .0 & .7 & .2 & .3 & .2 & .2 & .5 \\
\hline 39 & 42 & 42 & 47 & 34 & 37 & 26 & 23 & 28 & 31 & 32 & 34 & 39 & 43 & 42 \\
\hline 32 & 34 & 34 & 39 & 28 & 30 & 21 & 19 & 23 & 25 & 26 & 28 & 32 & 35 & 34 \\
\hline 6.4 & 8.0 & 7.6 & 9.8 & 5.8 & 5.8 & 3.8 & 2.8 & 4.0 & 5.2 & 5.6 & 6.2 & 6.8 & 8.4 & 8.4 \\
\hline 1.0 & 1.2 & 1.2 & 1.5 & 1.5 & 1.5 & 1.2 & 1.2 & 1.2 & 1.0 & 1.2 & 1.0 & 1.0 & 1.0 & 1.2 \\
\hline .0 & .1 & .1 & .2 & .0 & .1 & .1 & .1 & .1 & .1 & .1 & .1 & .1 & .1 & .1 \\
\hline .00 & .00 & .02 & .00 & .02 & .02 & .07 & .05 & .02 & .02 & .00 & .00 & .02 & .00 & .02 \\
\hline .00 & .00 & .00 & .01 & .00 & .00 & .00 & .01 & .00 & .00 & .00 & .00 & .00 & .00 & .00 \\
\hline 51 & 55 & 52 & 58 & 47 & 51 & 37 & 31 & 39 & 44 & 40 & 42 & 51 & 58 & 54 \\
\hline 34 & 39 & 38 & 43 & 321 & 34 & 23 & 19 & 24 & 28 & 28 & 30 & 35 & 41 & 39 \\
\hline 2 & 5 & 4 & 4 & 3 & 4 & 2 & 0 & 1 & 3 & 2 & 2 & 3 & 6 & 5 \\
\hline 80 & 90 & 91 & 102 & 73 & $\pi$ & 56 & 47 & 59 & 64 & 66 & 70 & 82 & 91 & 90 \\
\hline 7.8 & 7.8 & 7.7 & 7.9 & 7.7 & 7.5 & 7.5 & 7.3 & 7.4 & 7.3 & 7.4 & 7.6 & 7.6 & 7.6 & 7.6 \\
\hline 16.5 & -. & 10.5 & 12.0 & 6.1 & 3.4 & 4.8 & 5.1 & 3.7 & 6.0 & 8.0 & 11.0 & 18.8 & 18.6 & 15.0 \\
\hline 0 & 5 & 5 & 5 & 5 & 5 & 10 & 10 & 5 & 5 & 5 & 5 & 0 & 0 & 5 \\
\hline .. & -. & -. & -. & -. & -. & $\cdots$ & .. & .. & .. & .. & $\cdots$ & $\cdots$ & $\cdots$ & - \\
\hline 9.4 & 9.8 & 10.8 & 10.6 & 12.3 & 13.2 & $\cdots$ & 11.7 & 12.9 & . & 11.4 & 10.9 & 9.2 & 9.7 & 10.2 \\
\hline 1.0 & 1.1 & 1.3 & .9 & 1.1 & 1.9 & 1.3 & 1.8 & 1.8 & 2.5 & 2.0 & 1.4 & 1.6 & 1.7 & 1.7 \\
\hline 0 & 36 & 0 & 0 & 0 & 0 & 0 & 0 & 0 & 0 & 0 & 0 & 36 & 0 & 91 \\
\hline$\cdots$ & .. & 0 & .. & $\cdots$ & $\cdots$ & 0 & $\cdots$ & $\cdots$ & $\cdots$ & $\cdots$ & $\cdots$ & $\cdots$ & $\cdot \cdot$ & .. \\
\hline .. & -. & 0 & -. & $\cdots$ & $\cdots$ & 0 & $\cdots$ & - & $\cdots$ & $\because$ & $\cdots$ & $\cdots$ & $\cdots$ & - \\
\hline$\cdot \cdot$ & .. & 10 & $\cdots$ & $\cdots$ & $\cdots$ & 40 & $\cdots$ & $\because$ & -. & $\cdots$ & $\cdots$ & $\because$ & $\cdots$ & - \\
\hline .. & - & -. & $\cdots$ & .. & $\cdots$ & .. & .. & .. & -. & .. & .. & .. & .. & . \\
\hline$\cdots$ & .. & 0 & $\cdots$ & -. & $\cdots$ & 90 & $\cdots$ & .. & $\cdots$ & $\cdots$ & $\cdots$ & $\cdot$. & $\cdots$ & - \\
\hline -. & $\cdots$ & $\cdots$ & 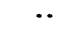 & .. & $\because$ & $\because$ & 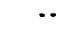 & $\because$ & $\cdots$ & 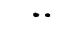 & .. & $\because$ & 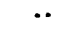 & . \\
\hline
\end{tabular}


TABLE 14.--Chemical and physical quality of water from selected surface-water sites in clallam County--Continued

12042000 Solectuck River near Beaver, Wash. (Site 6)--Continued

\begin{tabular}{|c|c|c|c|c|c|c|c|c|c|c|c|c|c|c|c|c|c|}
\hline \multirow[b]{2}{*}{$11 / 1 / 61$} & \multicolumn{4}{|c|}{1962} & \multicolumn{2}{|c|}{1963} & \multicolumn{2}{|c|}{1964} & \multirow[b]{2}{*}{$6 / 2 / 65$} & \multicolumn{2}{|c|}{1966} & \multicolumn{2}{|c|}{1967} & \multicolumn{2}{|c|}{1968} & \multirow[b]{2}{*}{$9 / 9 / 69$} & \multirow[b]{2}{*}{$6 / 9 / 70$} \\
\hline & $2 / 14$ & $5 / 14$ & $8 / 14$ & $12 / 13$ & $6 / 17$ & $12 / 23$ & $6 / 15$ & $12 / 14$ & & $2 / 7$ & $8 / 2$ & $5 / 18$ & $10 / 18$ & $4 / 48$ & $11 / 13$ & & \\
\hline 630 & 420 & 928 & 140 & 1000 & 382 & 5080 & 456 & .. & .. & .. & .. & .. & .. & .. & .. & .. & .. \\
\hline 5.4 & 5.8 & 5.9 & 5.8 & 5.3 & 4.2 & 3.5 & 4.3 & 5.4 & 5.4 & 5.3 & 4.7 & 8.0 & 7.3 & 7.7 & 6.6 & 9.5 & 4.5 \\
\hline 0 & 10 & 30 & 0 & 40 & 0 & 630 & 40 & 30 & 20 & .. & .. & .. & .. & .. & -. & $\cdots$ & $\cdots$ \\
\hline 10 & 11 & 11 & 15 & 10 & 11 & 5.0 & 9.0 & 11 & 10 & 9.2 & 12 & 8.8 & 8.8 & 7.9 & 7.9 & 12 & 10 \\
\hline .9 & 1.2 & 1.6 & .8 & 1.0 & 1.3 & .8 & 1.0 & 1.2 & 1.4 & 1.2 & 1.5 & 1.3 & 1.4 & 1.5 & 1.6 & 2.1 & 1.2 \\
\hline 1.9 & 2.3 & 2.2 & 2.4 & 2.2 & 1.6 & 1.8 & 1.7 & 2.3 & 2.0 & 2.0 & 2.0 & 2.0 & 2.8 & 3.0 & 2.8 & 3.6 & 1.9 \\
\hline .3 & .3 & .4 & .3 & .2 & .2 & .3 & .3 & .3 & .4 & .2 & .3 & .3 & .2 & .1 & .3 & .4 & .3 \\
\hline 32 & 36 & 38 & 44 & 32 & 36 & 18 & 30 & 36 & 35 & 32 & 39 & 33 & 33 & 33 & 32 & 46 & 35 \\
\hline 26 & 30 & 31 & 36 & 26 & 30 & 15 & 25 & 30 & 29 & 26 & 32 & 27 & 27 & 27 & 26 & 38 & 29 \\
\hline 5.2 & 6.0 & 6.8 & 8.2 & 6.4 & 5.4 & 3.2 & 4.8 & 6.4 & 6.2 & 5.2 & 6.4 & 4.4 & 5.0 & 3.8 & 4.0 & 5.2 & 5.8 \\
\hline 1.0 & 1.5 & 1.0 & 1.2 & 1.8 & .8 & 1.0 & 1.2 & 1.2 & 1.0 & 1.5 & 1.5 & 1.5 & 1.5 & 1.5 & 1.4 & 1.8 & .7 \\
\hline .1 & .1 & .0 & .1 & .1 & .1 & .1 & .1 & .1 & .1 & .1 & .1 & .1 & .1 & .1 & .1 & .1 & .1 \\
\hline .00 & .00 & .02 & .00 & .02 & .00 & .07 & .05 & .05 & .07 & .02 & .02 & .02 & .11 & .05 & .14 & .07 & .02 \\
\hline .00 & .01 & .00 & .00 & .00 & .00 & .00 & .01 & $\cdots$ & $\cdots$ & $\cdots$ & . & $\cdots$ & $\cdots$ & $\cdots$ & $\cdots$ & $\cdots$ & $\cdots$ \\
\hline 46 & 51 & 53 & 56 & 45 & 46 & 27 & 39 & 47 & 43 & 41 & 44 & 41 & 43 & 42 & 44 & 58 & 41 \\
\hline 29 & 32 & 34 & 41 & 29 & 33 & 16 & 27 & 32 & 30 & 28 & 36 & 28 & 28 & 26 & 26 & 39 & 30 \\
\hline 3 & .3 & 3 & 5 & 3 & 6 & 0 & 2 & 3 & 2 & 2 & 4 & 0 & 1 & 0 & 0 & 1 & 2 \\
\hline 69 & $\pi$ & 82 & 94 & 69 & 75 & 38 & 60 & 76 & 72 & 70 & 82 & 68 & 69 & 67 & 62 & 95 & 73 \\
\hline 7.1 & 7.4 & 7.6 & 7.5 & 7.0 & 7.5 & 7.0 & 7.5 & 7.5 & 7.5 & 7.4 & 7.0 & 7.7 & 7.5 & 7.5 & 7.1 & 7.2 & 7.2 \\
\hline 6.1 & 6.0 & 6.7 & 15.0 & 6.1 & 16.7 & 6.7 & 8.9 & 2.8 & 12.6 & 5.0 & 12.4 & 7.2 & 11.0 & 8.0 & .. & $\cdots$ & -8.5 \\
\hline 5 & 5 & 5 & 5 & 5 & 0 & 15 & 5 & 5 & 0 & 5 & 0 & 5 & 5 & 5 & 5 & 0 & 0 \\
\hline 0 & 0 & 0 & 0 & 0 & 0 & 35 & 0 & 0 & 0 & .. & .. & -. & .. & -. & -. & $\cdots$ & .. \\
\hline 12.1 & 12.0 & 11.7 & 10.2 & 13.7 & 10.7 & 12.0 & 11.6 & 11.7 & 11.3 & 11.8 & 10.8 & $\cdots$ & $\cdots$ & $\cdots$ & -. & $\cdots$ & -. \\
\hline 4.1 & 2.3 & 1.5 & 2.2 & 5.1 & 1.8 & 2.9 & 1.5 & 1.8 & 1.8 & 2.0 & 6.2 & 1.1 & 1.7 & 1.7 & 4.1 & 4.6 & 3.5 \\
\hline 36 & 23 & 360 & 36 & 73 & 23 & 23 & 23 & 30 & 23 & 30 & 36 & .. & $\cdots$ & .. & .. & .. & .. \\
\hline 0 & $\cdots$ & 0 & $\cdot \cdot$ & 0 & 0 & 0 & 0 & 0 & 0 & 0 & 0 & 0 & 0 & $\cdots$ & .. & $\cdots$ & $\cdot \cdot$ \\
\hline 0 & $\cdots$ & 0 & .. & 0 & 0 & 0 & 10 & 20 & 10 & 30 & 10 & 40 & 0 & $\cdots$ & 20 & $\cdots$ & .. \\
\hline 0 & $\cdots$ & 0 & $\because$ & 0 & 0 & 1. & 0 & 0 & 0 & 0 & 0 & 0 & 0 & -. & 0 & 0 & .. \\
\hline 0 & .. & 0 & .. & 0 & 0 & 10 & 0 & 0 & 0 & 0 & 0 & 0 & 0 & $\cdots$ & 0 & 0 & .. \\
\hline 40 & $\cdots$ & 0 & $\cdots$ & 0 & 10 & 90 & 0 & 10 & 20 & 10 & 20 & 0 & 0 & $\cdots$ & 0 & 0 & $\cdots$ \\
\hline 50 & $\cdots$ & 50 & $\cdots$ & 50 & 50 & 50 & 50 & 50 & 50 & 50 & 0 & 20 & 0 & $\cdots$ & 0 & 0 & $\cdots$ \\
\hline
\end{tabular}


Beaver Laka, naar Sappho, Wash. (Site 9)

Data sample collacted

Sample site number

Depth sampla collected ( $f t)$

Total nitrate (W) (mg/L)

Total nitrite (N) (mg/L)

Total emonis (N) (mg/L)

Total organic nitrogen (W) (mg/L)

Total phosphorus (P) (mg/L)

Total or thophosphate phosphorus

(P) $(\mathrm{mg} / \mathrm{L})$

Specific conductanca (micromhos)

Water temperatura $\left({ }^{\circ} \mathrm{C}\right)$

Color (plotinum-cobalt units)

Secchi-disc visibility ( $f t$ )

Dissolved oxygen (mg/L)

Lake shoreline covered by

emersed plants (pct)

Lake surface covered by

emersed plants (pct)

Number of fecal coliform samples

Fecal coliform, minimu (col/100m)

facal coliform, maximum

Fecal collform, mean (col/100m)

\begin{tabular}{ccc}
\multicolumn{3}{c}{$8 / 12 / 74$} \\
\hline 3 & 1 & 30 \\
.00 & & .03 \\
.00 & & .00 \\
.04 & & .37 \\
.29 & & .15 \\
.01 & & .02 \\
.00 & & .02 \\
76 & & 80 \\
17.3 & & 9.0 \\
20 & & 45 \\
& 9 & \\
9.3 & & 0.1 \\
& & \\
& $76-100$ & \\
& & \\
& $1-10$ & \\
& 3 & \\
& 1 & \\
& 1 &
\end{tabular}

Pleasant Lake, near Sappho, Wash. (Site 15)

Data sampla collected

Sampla site number

Depth semple collected ( $f t)$

Total nitrata (W) (mg/L)

Total nitrita (N) (mg/L)

Total emonia (N) (mg/L)

total organic nitrogen (N) (mg/L)

Total phosphorus (P) (mg/L)

Total or thophosphate phosphorus

$$
\text { (P) (mg/L) }
$$

Specific conductance (micromhos)

watar temperaturs $\left({ }^{\circ} \mathrm{C}\right)$

Color (platinum-cobalt units)

Secchi-disc visibility (ft)

Dissolved oxygen ( $m g / L$ )

Lake shoreline covered by

emersed plants (pct)

Laka surface covered by

enersed plants (pct)

Number of facal coliform semples

Facal coliform, minimum (col/100m)

fecal coliform, maximum

Fecal coliform, mean (col/100m)

\begin{tabular}{rrr}
\multicolumn{3}{c}{$8 / 12 / 74$} \\
\hline 3 & 1 & \\
.00 & & .03 \\
.00 & & .00 \\
.02 & & .16 \\
.16 & & .06 \\
.01 & & .03 \\
.00 & & .03 \\
40 & & 50 \\
20.7 & & 10.9 \\
0 & & 30 \\
9.2 & 13 & \\
& & 1.0
\end{tabular}

Littla or none

None or 1

1

12042300 Soleduck River near Forks, Wash. (Site 17)

\begin{tabular}{|c|c|c|c|c|c|c|c|c|c|c|c|c|c|c|c|c|c|c|}
\hline \multirow[b]{2}{*}{ Dote sample collected } & \multicolumn{2}{|c|}{1971} & \multicolumn{5}{|c|}{1972.} & \multicolumn{6}{|c|}{1973} & \multicolumn{5}{|c|}{1974} \\
\hline & $10 / 27$ & $12 / 14$ & $2 / 8$ & $4 / 11$ & $6 / 13$ & $8 / 8$ & $11 / 29$ & $1 / 16$ & $3 / 13$ & $5 / 30$ & $7 / 17$ & $9 / 25$ & $11 / 13$ & $1 / 23$ & $3 / 19$ & $5 / 15$ & $7 / 23$ & $9 / 17$ \\
\hline Diacharge $\left(\mathrm{ft}^{3} / \mathrm{s}\right)$ & $\cdots$ & 2150 & 1720 & 1500 & 863 & 401 & $\cdot \cdot$ & -. & $\cdots$ & $\cdots$ & $\because$ & $\cdots$ & -. & $\cdots$ & $\cdots$ & .. & $\cdots$ & $\cdots$ \\
\hline Dissolved calciun (Ca) (mg/L) & 9.0 & 7.5 & 7.9 & 8.4 & 10 & 11 & 9.7 & 5.1 & 8.8 & 10 & 12 & 12 & $\cdots$ & $\cdots$ & .. & .. & -. & .. \\
\hline Dissolved megnesiun $(\mathrm{Mg})(\mathrm{mg} / \mathrm{L})$ & .8 & 1.4 & 1.3 & 1.3 & 1.4 & 1.7 & 1.6 & .9 & 1.5 & 1.5 & 1.7 & 1.8 & .. & $\cdots$ & $\cdots$ & .. & .. & -. \\
\hline Dissolved sodiun (Na) (mg/L) & 1.8 & 3.0 & 2.9 & 3.2 & 2.3 & 2.4 & 3.2 & 2.3 & 3.0 & 2.4 & 3.0 & 3.6 & -. & $\cdots$ & $\cdots$ & 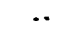 & $\cdots$ & $\cdots$ \\
\hline Dissolved potassium (K) $(\mathrm{mg} / \mathrm{L})$ & .4 & .3 & .3 & .4 & .2 & .2 & .5 & .6 & 1.9 & .3 & .4 & .3 & .. & $\cdots$ & $\cdots$ & $\cdots$ & $\cdots$ & .. \\
\hline Bicarbonate $\left(\mathrm{HCO}_{3}\right)(\mathrm{mg} / \mathrm{L})$ & 33 & 30 & 30 & 32 & 26 & 42 & 26 & 24 & 30 & 38 & 47 & 48 & .. & .. & $\cdots$ & $\cdots$ & $\cdots$ & -. \\
\hline $\begin{array}{l}\text { Alkalinity }\left(\mathrm{CaCO}_{3}\right)(\mathrm{mg} / \mathrm{L}) \\
\text { Dissolved aulfate }\left(\mathrm{SO}_{4}\right)\end{array}$ & 27 & 25 & 25 & 26 & 30 & 34 & 30 & 20 & 25 & 31 & 39 & 39 & $\cdots$ & $\cdots$ & $\cdots$ & $\cdot \cdot$ & $\cdots$ & .. \\
\hline $\begin{array}{l}(\mathrm{mg} / \mathrm{L}) \\
\text { Dissolved chloride (Cl) }\end{array}$ & $\cdots$ & $\cdots$ & $\cdots$ & $\cdots$ & $\cdots$ & $\cdots$ & 5.9 & 3.1 & 1.2 & 5.6 & 5.9 & 5.2 & $\cdots$ & $\cdots$ & $\cdots$ & $\cdot \cdot$ & 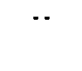 & $\cdots$ \\
\hline$(\mathrm{mg} / \mathrm{L})$ & 1.3 & 2.7 & 2.4 & 2.2 & 2.6 & 1.5 & 2.4 & 2.2 & 2.7 & 1.7 & 2.1 & 2.3 & .. & $\cdots$ & $\cdots$ & $\cdots$ & $\cdots$ & $\cdots$ \\
\hline Total nitrata (W) $(\mathrm{mg} / \mathrm{L})$ & $\cdots$ & .26 & .06 & .01 & .04 & .02 & .22 & .17 & .15 & .06 & .05 & .08 & $\cdots$ & $\because$ & $\because$ & $\cdots$ & 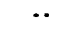 & $\cdots$ \\
\hline $\begin{array}{l}\text { Total nitrite (N) (mg/L) } \\
\text { Total nitrita plus nitrate }\end{array}$ & $\because$ & .00 & .00 & .01 & .00 & .00 & .00 & .01 & .00 & .00 & .01 & .00 & $\cdots$ & $\cdots$ & -. & $\cdot \cdot$ & 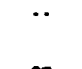 & $\cdots$ \\
\hline (N) $(m g / L)$ & $\cdots$ & .26 & .06 & .02 & .04 & .02 & .22 & .18 & .15 & .06 & .06 & .08 & .23 & .15 & .20 & .04 & .03 & .06 \\
\hline $\begin{array}{l}\text { Total ammonia (N) (mg/L) } \\
\text { Total organic nitrogen }\end{array}$ & $\cdots$ & .01 & .04 & .16 & .03 & .02 & .09 & .18 & .11 & .03 & .02 & .01 & .07 & .05 & .07 & .11 & .07 & .05 \\
\hline (N) $(\mathrm{mg} / \mathrm{L})$ & $\cdots$ & .03 & .09 & .06 & .11 & .06 & -. & $\cdots$ & $-\cdot$ & $\cdots$ & $\cdots$ & $\cdots$ & .. & .. & $\cdots$ & $-\cdot$ & -. & .. \\
\hline $\begin{array}{l}\text { Total phosphorus }(P)(\mathrm{mg} / \mathrm{L}) \\
\text { Dissolved or thophosphate }\end{array}$ & $\cdots$ & .01 & .01 & .05 & .01 & .00 & .01 & .11 & .00 & .00 & .00 & .00 & .02 & .02 & .02 & .01 & .01 & .01 \\
\hline phosphorus (P) (mg/L) & $\because$ & .00 & .01 & .05 & .00 & .00 & .00 & .00 & .00 & .00 & .00 & .00 & .00 & .00 & .00 & .00 & .00 & .00 \\
\hline Hordness $\left(\mathrm{CaCO}_{3}\right)(\mathrm{mg} / \mathrm{L})$ & 26 & 24 & 25 & 26 & 31 & 34 & 31 & 16 & 28 & 31 & 37 & 37 & $\cdots$ & $\cdot \cdot$ & -. & $\cdots$ & .. & $\cdots$ \\
\hline $\begin{array}{l}\text { Noncarbonate hardness (mg/t) } \\
\text { Specific conductance }\end{array}$ & 0 & 0 & 0 & 0 & 1 & 0 & 1 & 0 & 4 & 0 & 0 & 0 & 0 & $\cdots$ & .. & .. & $\cdots$ & .. \\
\hline (micromhos) & 66 & 67 & 64 & 67 & 86 & 98 & 67 & 48 & 94 & 78 & $\varphi$ & 100 & 61 & 66 & 67 & $n$ & 76 & 94 \\
\hline pH (units) & 7.2 & 7.1 & 7.5 & 7.5 & 7.4 & 7.8 & 7.6 & 7.1 & 7.0 & 7.6 & 7.8 & 7.8 & 7.4 & 7.4 & 7.4 & 7.5 & 7.7 & 7.5 \\
\hline Temperature $\left({ }^{\circ} \mathrm{C}\right)$ & 4.4 & 5.3 & 5.3 & 5.4 & 9.6 & 16.8 & 4.7 & 5.5 & 6.6 & 10.6 & 15.7 & 12.0 & 6.0 & 6.0 & 5.8 & 7.0 & 11.6 & 12.7 \\
\hline Color (platinum-cobalt units) & $\cdots$ & 40 & 26 & 9 & 6 & 5 & 0 & 55 & 19 & 23 & 5 & 18 & 29 & 17 & 10 & 16 & 11 & 14 \\
\hline Turbidity (JTU) & $\because$ & 2 & 4 & 2 & 1 & 1 & 3 & 45 & 3 & 1 & 1 & 1 & 8 & 15 & 7 & 5 & 2 & 2 \\
\hline $\begin{array}{l}\text { Dissolved oxygen (mg/L) } \\
\text { Dissolved carbon dioxide }\end{array}$ & 12.6 & 12.5 & 12.9 & 12.6 & 11.7 & 9.8 & 12.9 & 12.4 & 12.9 & 11.1 & 9.7 & 11.1 & 12.1 & 12.8 & 13.0 & $\$ 3.0$ & 11.4 & 11.1 \\
\hline $\begin{array}{l}\left(\mathrm{CO}_{2}\right)(\mathrm{mg} / \mathrm{L}) \\
\text { Total immediate coli }\end{array}$ & 3.3 & 3.8 & 1.5 & 1.6 & 2.3 & 1.1 & 1.4 & 3.1 & 4.8 & 1.5 & 1.2 & 1.2 & .. & .. & -. & .. & $\cdots$ & $\therefore$ \\
\hline$(\mathrm{col} / 100 \mathrm{~mL})$ & 130 & 350 & 100 & 75 & 225 & 120 & 80 & 160 & 100 & 75 & 100 & 540 & 440 & 270 & 120 & 260 & 650 & 660 \\
\hline
\end{tabular}


12043003 Calawah River at mouth near Forks, Wash. (sita 47)

\begin{tabular}{|c|c|c|}
\hline Dota sample collected & $8 / 24 / 76$ & $10 / 6 / 77$ \\
\hline Oischerga $\left(\mathrm{ft}^{3}\right)$ & 170 & 185 \\
\hline Oissolved silica $\left(\mathrm{SiO}_{2}\right)(\mathrm{mg} / \mathrm{L})$ & 8.0 & -. \\
\hline Dlssol ved calcium (Ca) (mg/L) & 7.9 & $\because$ \\
\hline Dissolved magnes/um ( $\mathrm{Hg})(\mathrm{mg} / \mathrm{L})$ & 1.8 & $\because$ \\
\hline Dissolved sodium (Na) (mg/L) & 3.1 & $\because$ \\
\hline Oissolved potassium (K) (mg/L) & .3 & -. \\
\hline Bicarbonate $\left(\mathrm{HCO}_{3}\right)(\mathrm{mg} / \mathrm{L})$ & 34 & $\cdots$ \\
\hline Alkal inity $\left(\mathrm{CaCO}_{3}\right)(\mathrm{mg} / \mathrm{L})$ & 28 & $\cdots$ \\
\hline Oissolved sulfata $\left(\mathrm{SO}_{4}\right)(\mathrm{mog} / \mathrm{L})$ & 5.7 & -. \\
\hline oissolved chloride (Cl) (mg/L) & 2.3 & $\because$ \\
\hline Dissolved fluoride $(F)(m g / L)$ & .1 & $\because$ \\
\hline Total nitrata (N) (mg/L) & .03 & .09 \\
\hline Total nitrite (M) (mg/L) & .00 & .00 \\
\hline Total ammonis (N) (mg/L) & .02 & .04 \\
\hline $\begin{array}{l}\text { Total phosphorus (P) }(\mathrm{mag} / \mathrm{L}) \\
\text { Total orthophosphate phosphorus }\end{array}$ & .00 & .00 \\
\hline (P) $(\mathrm{mg} / \mathrm{L})$ & .00 & .00 \\
\hline Harchess $\left(\mathrm{CaCO}_{3}\right)(\mathrm{mg} / \mathrm{L})$ & 27 & .. \\
\hline Noncarbonata hardness $(\mathrm{mg} / \mathrm{L})$ & 0 & $\because$ \\
\hline Specific conductanco (micromhos) & 73 & 68 \\
\hline Temperaturs $\left({ }^{\circ} \mathrm{C}\right)$ & 14.0 & 8.6 \\
\hline Color (platimum-cobalt units) & 0 & 0 \\
\hline Turbidity (JTU) & 0 & 0 \\
\hline Dissolved oxygen (mo/L) & .. & 12.1 \\
\hline Total immediata coliform (col/100 m $)$ & 3 & 290 \\
\hline $\begin{array}{l}\text { Fecal coliform ( } 0.7 \mathrm{um} \text {, menbrana } \\
\text { filter) (col/100 m. }\end{array}$ & . & 11 \\
\hline
\end{tabular}

Bogachial Rivar at mouth, at Soleduck (rivar mila 6.5) (Sita 56)

Oata sampla collected

Dissolved silica $\left(\mathrm{SiO}_{2}\right)(\mathrm{mg} / \mathrm{L})$

Dissolved calcium (Ca) (mg/ $\mathrm{L})$

Dissolved magnasium ( $/ \mathrm{Hg})(\mathrm{mg} / \mathrm{L})$

Dissolved sodium ( $\mathrm{Ha}$ ) (mg/L)

Dissolved potassium (K) (mg/L)

Bicarbonate $\left(\mathrm{HCO}_{3}\right)(\mathrm{mg} / \mathrm{L})$

Alkalinity $\left(\mathrm{CaCO}_{3}\right)(\mathrm{mg} / \mathrm{L})$

0issolved sulfata $\left(\mathrm{SO}_{4}\right)(\mathrm{mg} / \mathrm{L})$

Dissolved chloride (Cl) (mg/L)

Dissolved fluoride (F) (mg/L)

Total nitrata (N) (mg/L)

Total nitrite (N) (mg/L)

Total ammonis (N) (mg/L)

Total phosphorus (P) (mg/L)

Total or thophosphata phosphorus (P) (mg/L)

Dissolved solids, sum of constituents ( $m g / L$ )

Hardness (Ca, Mg) (mg/L)

Moncarbonate harchess ( $\mathrm{mg} / \mathrm{L}$ )

Specific conductance (micromhos)

Temperatura $\left({ }^{\circ} \mathrm{C}\right)$

Color (platinum-cobalt units)

Turbidity (JTU)

0 issolved oxygen (mg/L)

Biochemical oxygen demand (5 day) (mg/L)

Immediate coliform ( $\mathrm{col} / 100 \mathrm{~mL}$ )

fecal colifore $\$ 0.7 \mathrm{um}$, membrane filter) (col/100 mL)

\begin{tabular}{|c|c|c|}
\hline $8 / 12 / 76$ & $8 / 25 / 76$ & $10 / 5 / 77$ \\
\hline$\cdots$ & 6.5 & $\cdots$ \\
\hline -. & 9.8 & $\cdots$ \\
\hline -. & 1.5 & .. \\
\hline -. & 3.1 & $\cdots$ \\
\hline$\cdot \cdot$ & .3 & $\cdots$ \\
\hline$\cdots$ & 32 & $\cdots$ \\
\hline$\cdots$ & 26 & $\cdots$ \\
\hline 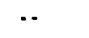 & 6.2 & $\cdots$ \\
\hline$\cdots$ & 3.1 & -. \\
\hline -. & .1 & -. \\
\hline$\cdots$ & .00 & .03 \\
\hline$\cdots$ & .00 & .00 \\
\hline .. & .03 & .02 \\
\hline$\because$ & .01 & .00 \\
\hline -. & .00 & .00 \\
\hline .. & .00 & .00 \\
\hline$\cdots$ & 31 & $\cdots$ \\
\hline$\cdots$ & 4 & $\cdots$ \\
\hline$\cdots$ & 79 & 80 \\
\hline 9.8 & 15.1 & 9.4 \\
\hline$\cdots$ & 0 & 5 \\
\hline .. & 0 & 1 \\
\hline$\cdots$ & 10.2 & 12.3 \\
\hline 1.8 & $\cdots$ & $\cdots$ \\
\hline 116 & 44 & 64 \\
\hline .. & $\cdots$ & 14 \\
\hline
\end{tabular}

Dickey River at Mora Bridga, at mouth (river mile 0.2 ) (Site 78)

Data sampla collacted

Time sampla collacted

Sampling depth (ft)

Dissolved silica $\left(\mathrm{SiO}_{2}\right)(\mathrm{mg} / \mathrm{L})$

Oissolved calcium (Ca) (mg/L)

oissolved megnes ium ( $\mathrm{Mg})(\mathrm{mg} / \mathrm{L})$

Dissolved sodium ( $\mathrm{Na})(\mathrm{mg} / \mathrm{L})$

Dissoled potassiun (K) (mg/L)

Bicarbonate $\left(\mathrm{HCO}_{3}\right)(\mathrm{Mg} / \mathrm{L})$

Alkalinity $\left(\mathrm{CaCO}_{3}\right)(\mathrm{mg} / \mathrm{L})$

Dissolved sul fata $\left(\mathrm{SO}_{4}\right)(\mathrm{mg} / \mathrm{h})$

Dissolved chloride (Cl) (mg/L)

oissolved fluoride (f) (mg/L)

Total nitrate (W) (mg/t)

Total nitrite (N) (mg/l)

Total ammonia (N) (mg/L)

Total phosphorus (P) (mg/L)

Total orthophosphate phosphorus

(P) (mg/L)

Dissolved solids (sum of constituents)

(mg/t)

Mardness ( $\mathrm{Ca}, \mathrm{Mg}$ ) (mg/L)

Moncarbonato hardness (mg/L)

Specific conductanco (micromhos)

Temperatura $\left({ }^{\circ} \mathrm{C}\right)$

color (platinum-cobalt units)

Turbidity (JTU)

oisaolved oxygen (mg/L)

Immediata colliform (col/100 mu)

fecal coliform $(0.7 \mathrm{um}$, membrane

filter) (col/100 mh)

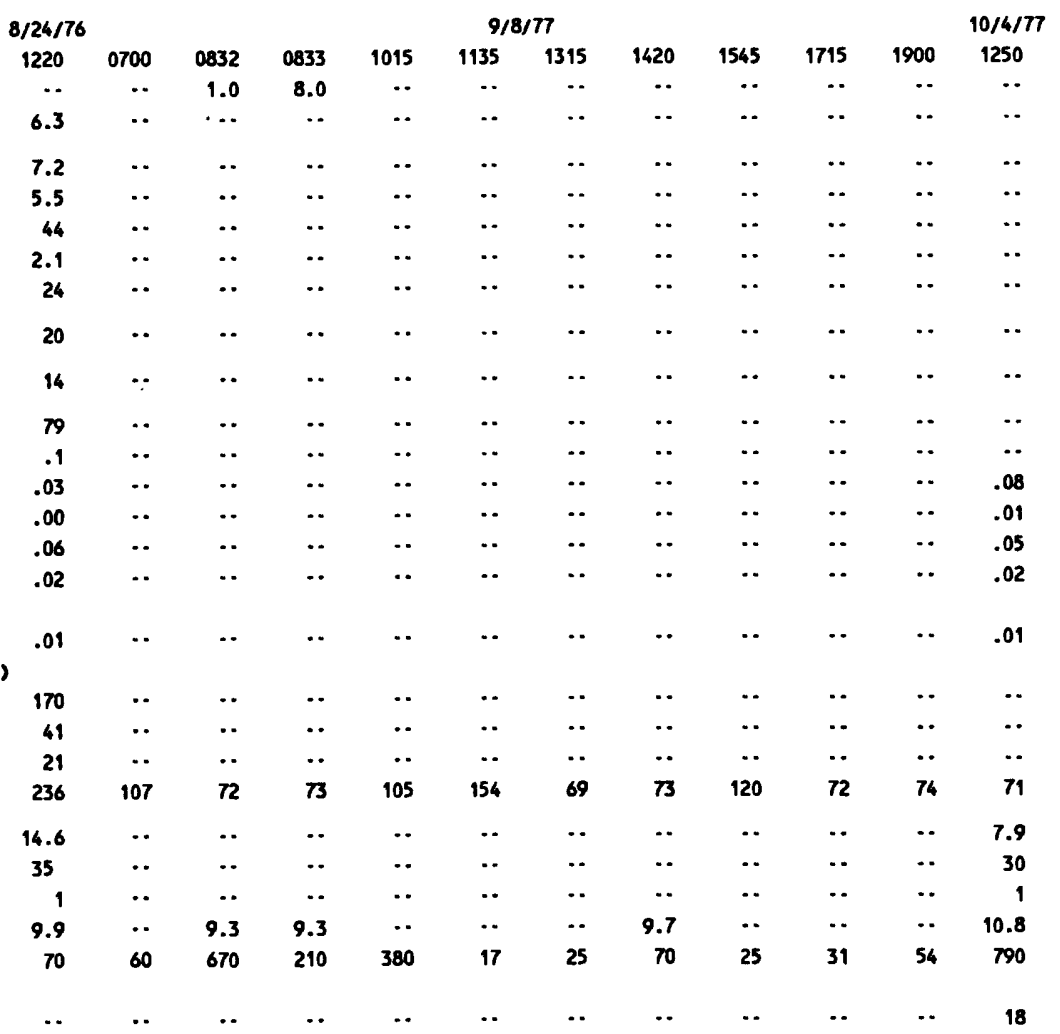


Dickay Laka, near Forks, Wash.

\begin{tabular}{|c|c|c|}
\hline Dota sample & & $8 / 12 / 74$ \\
\hline Sample site number & & 1 \\
\hline Depth semple collected & 3 & \\
\hline Total nitrata $(N)(n g / L)$ & .00 & \\
\hline Total nitrate $(N)(\mathrm{mg} / \mathrm{L})$ & .00 & \\
\hline Total anonle (N) $(\mathrm{ng} / \mathrm{L})$ & .05 & \\
\hline Total orgenic nitrogen (N) (ng/L) & .32 & \\
\hline Total phosphorus $(P)(\mathrm{mg} / \mathrm{L})$ & .02 & \\
\hline Total or thophosphate phosphorus (P) & /L) .01 & \\
\hline Specific conductance (nicromhos) & 32 & \\
\hline Uater temperature $\left({ }^{\circ} \mathrm{C}\right)$ & 20.2 & \\
\hline Color (pletinum-cobalt units) & 45 & \\
\hline secchi-disc visibility (ft) & & 9 \\
\hline Diseolved oxygen $(\mathrm{mg} / \mathrm{L})$ & 8.7 & \\
\hline $\begin{array}{l}\text { Lake shoreline covered by emersed } \\
\text { plents (pct) }\end{array}$ & & $76 \cdot 100$ \\
\hline $\begin{array}{l}\text { Laka surfece covered by emersed } \\
\text { plents (pct) }\end{array}$ & & $1-10$ \\
\hline Nuber of fecal colliform saples & & 3 \\
\hline Fecel collform minimu (col/100 m) & & 1 \\
\hline Facal coliform, maximm (col/100 m) & & 2 \\
\hline Facal coliform, wean (col/100 m) & & 1 \\
\hline
\end{tabular}

Elk Lake, near Ozetta, Wesh.

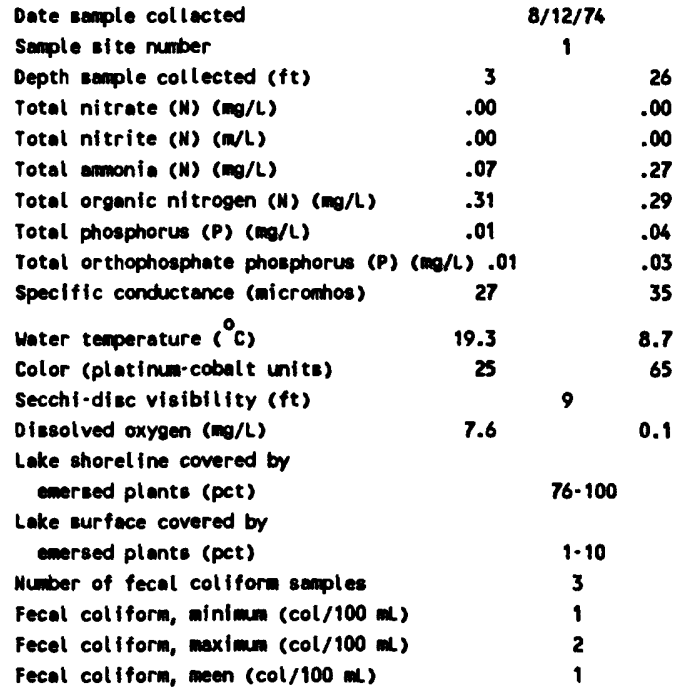

Wentworth Laka, near Forks, Wash.

\begin{tabular}{|c|c|c|}
\hline \multicolumn{2}{|l|}{ Doto sample collected } & $8 / 12 / 74$ \\
\hline & & 1 \\
\hline Depth semple collected $(f t)$ & 3 & \\
\hline Total nitrate $(K)(\mathrm{ma} / L)$ & .00 & \\
\hline Total nitrite (N) $(n / L)$ & .00 & \\
\hline Total amonile $(W)(m a / L)$ & .04 & \\
\hline Total orgenic nitrogen (W) (mo/L) & .31 & \\
\hline Total phosphorus (P) $(\mathrm{mg} / \mathrm{L})$ & .01 & \\
\hline \multicolumn{3}{|c|}{ Total or thophosphate phosphorus (P) $(\mathrm{mg} / \mathrm{L}) . D 0$} \\
\hline Specific conductence (n/cromhos) & 30 & \\
\hline Water temperature $\left({ }^{\circ} \mathrm{C}\right)$ & 21.2 & \\
\hline Color (plotínum cobalt units) & 40 & \\
\hline Secchi-disc visibility (ft) & & 8 \\
\hline Dissolved oxygen $(m g / L)$ & 8.7 & \\
\hline \multicolumn{3}{|l|}{ Laka shoraline covered by } \\
\hline enersed plants (pct) & & $76-100$ \\
\hline \multicolumn{3}{|l|}{ Lake surface covered by } \\
\hline emersed plants (pct) & & $11-25$ \\
\hline Number of fecal colliform semplas & & 3 \\
\hline Fecal coliform, winimum $(\mathrm{col} / 100 \mathrm{~m})$ & & 1 \\
\hline Fecal collform, maximan (col/100 m) & & 5 \\
\hline Fecal colliform, maen (col/100 m) & & 2 \\
\hline
\end{tabular}

Ozette Laka, near Ozette, Wash.

Semple site numer

Depth sample collected ( $f t$ )

Total nitrate (W) $(\mathrm{mg} / \mathrm{L})$

rotal nitrite (N) $(\mathrm{mg} / \mathrm{L})$

Total amonie (W) $(\mathrm{mg} / \mathrm{L})$

Total orgenic nitrogen (K) $(\mathrm{mg} / \mathrm{L})$

Total phosphorus (P) $(\mathrm{mg} / \mathrm{L})$

Total or thoephosphate phosphorus

(P) $(\mathrm{ng} / \mathrm{L})$

Specific conductance (micromhos)

Water temperature $\left({ }^{\circ} \mathrm{C}\right)$

Color (platinum-cobalt unite)

Secchi-disc viafbility (ft)

Diseolved oxygen $(\mathrm{mg} / \mathrm{L})$

Lake shoraline covered by

emersed plents (pet)

Lake surface covered by

emersed plants (pct)

Nuber of fecal collform samples

Fecal colliform, ninimu ( $\mathrm{col} / 100 \mathrm{~m}$ )

Fecal colliform, maximm (col/100 m)

fecal colliform, mean (col/100 m)

$\begin{array}{rrrr}3 & 89 & 3 & 164 \\ .01 & .01 & .01 & .10 \\ .00 & .00 & .00 & .00 \\ .08 & .06 & .06 & .04 \\ .26 & .32 & .10 & .03 \\ .00 & .00 & .01 & .01\end{array}$

$\begin{array}{llll}.00 & .00 & .00 & .00\end{array}$

$39 \quad 41 \quad 40 \quad 40$

$\begin{array}{llll}19.6 & 7.4 & 19.6 & 7.3\end{array}$

$\begin{array}{llll}15 & 20 & 35 & 40\end{array}$

13

10.2 
12043150 Ozatte River at Ozetta, Wash.

\begin{tabular}{|c|c|c|c|c|c|c|c|c|c|c|}
\hline \multirow[b]{2}{*}{ Date semple collected } & \multicolumn{5}{|c|}{1976} & \multicolumn{5}{|c|}{1977} \\
\hline & $4 / 27$ & $5 / 27$ & $6 / 21$ & $7 / 21$ & $11 / 11$ & $2 / 8$ & $3 / 9$ & $5 / 17$ & $7 / 12$ & $9 / 20$ \\
\hline Discharge $\left(\mathrm{ft}^{3} / \mathrm{s}\right)$ & $\cdots$ & -. & -. & $\cdots$ & 173 & 420 & 1010 & 230 & 101 & 110 \\
\hline Total nitrate $(W)(\mathrm{mg} / \mathrm{L})$ & .05 & $\cdots$ & .. & $\cdots$ & $\cdots$ & $\cdot \cdot$ & $\cdots$ & $\cdots$ & $\cdots$ & $\cdots$ \\
\hline Total nitrite (W) (ng/L) & .00 & $\cdots$ & .. & $\cdots$ & -. & .. & $\cdots$ & $\cdots$ & $\cdots$ & $\because$ \\
\hline Total ammonia (N) $(\mathrm{mg} / \mathrm{L})$ & .05 & $\cdots$ & .. & $\cdots$ & $\cdots$ & .. & $\cdots$ & $\cdots$ & $\cdots$ & $\cdots$ \\
\hline Total organic nitrogen (N) (mo/L) & .12 & $\cdots$ & .. & $\cdots$ & $\cdots$ & -. & $\cdots$ & $\cdots$ & $\cdots$ & 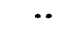 \\
\hline Total Kjeldahl nitrogen (N) (mg/L) & .17 & $\cdots$ & $\cdots$ & $\cdots$ & *. & $\cdot \cdot$ & $\cdots$ & $\cdots$ & $\because$ & $\cdots$ \\
\hline $\begin{array}{l}\text { Total phosphorus }(P)(\mathrm{mg} / \mathrm{L}) \\
\text { Total or thosphosphate phosphorus }\end{array}$ & .01 & $\cdots$ & $\cdots$ & $\cdots$ & $\cdots$ & $\cdots$ & $\cdots$ & $\cdots$ & $\cdots$ & $\cdots$ \\
\hline (P) $(\mathrm{mg} / \mathrm{L})$ & .00 & $\cdots$ & $\cdots$ & .. & $\cdots$ & $\cdots$ & $\cdots$ & $\cdots$ & $\cdots$ & 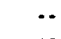 \\
\hline Specific conductance (aicromhos) & 37 & 37 & 36 & .. & $\cdots$ & 39 & 43 & 42 & 40 & 42 \\
\hline PH (units) & $\cdots$ & 7.0 & 6.3 & $\cdots$ & $\cdots$ & $\cdots$ & $\cdots$ & $\cdots$ & $\cdots$ & $\cdots$ \\
\hline Temperatura $\left({ }^{\circ} \mathrm{C}\right)$ & 10.0 & 13.0 & 14.0 & 17.8 & 12.5 & 8.2 & 6.9 & 13.3 & 18.3 & 16.0 \\
\hline Turbidity (JTu) & 0 & 0 & 1 & $\cdots$ & 0 & 0 & 1 & 0 & 0 & 0 \\
\hline Dissolved oxygen (mg/L) & 11.0 & 9.8 & $\cdots$ & 9.3 & 9.6 & 11.7 & 11.6 & 10.0 & 9.2 & 9.2 \\
\hline $\begin{array}{l}\text { Totsl inmediste coll form (col/100 mL) } \\
\text { fecal colliform }(0.7 \mathrm{w} \text {, membrane }\end{array}$ & $\cdots$ & 7 & 4 & 12 & 90 & $\cdots$ & 14 & 26 & 73 & 160 \\
\hline filter $)(\mathrm{col} / 100 \mathrm{~m})$ & .. & 4 & 1 & 9 & 94 & $\cdots$ & 3 & 1 & 1 & 17 \\
\hline
\end{tabular}

Seafield Lake near Ozatte, Wash.

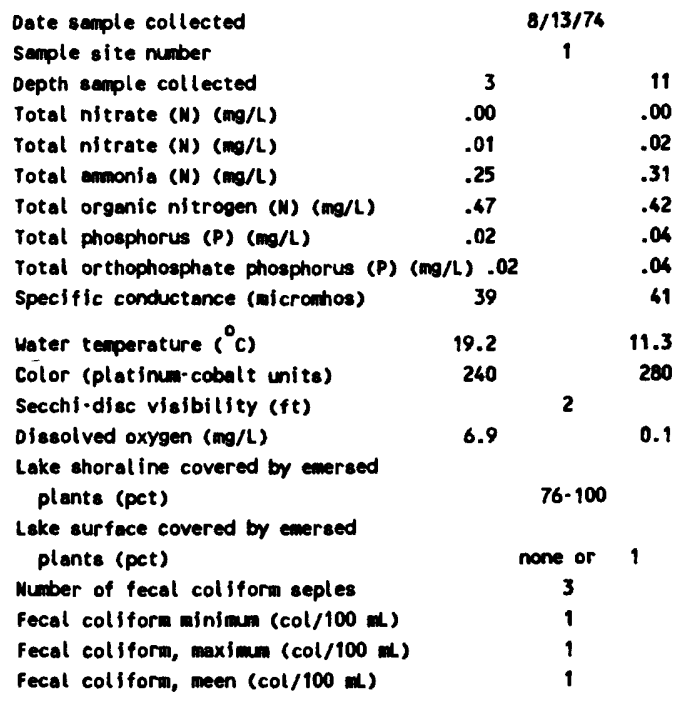


12043156 Sooes River above Pilchuck Creek, Ozette Lake, near Ozette, Wash.

Date sample collected

Dissolved calcium (Ca) (mg/L)

Dissolved magnesium $(\mathrm{Hg})(\mathrm{mg} / \mathrm{L})$

Dissolved sodium ( $\mathrm{Na}$ ) (mg/L)

Dissolved potassium (K) (mg/L)

Bicarbonate $\left(\mathrm{HCO}_{3}\right)(\mathrm{mg} / \mathrm{L})$

Alkalinity $\left(\mathrm{CaCO}_{3}\right)(\mathrm{mg} / \mathrm{L})$

Dissolved sulfate $\left(\mathrm{SO}_{4}\right)(\mathrm{mg} / \mathrm{L})$

dissolved chloride (Cl) (mg/L)

Total nitrate (N) (mg/L)

Total nitrite (N) $(\mathrm{mg} / \mathrm{L})$

Total annonia (N) (mg/L)

Total Kjeldahl nitrogen (N) (mg/L)

Total phosphorus (P) (mg/L)

Dissolved orthophosphate

phosphorus (P) $(\mathrm{mg} / \mathrm{L})$

Harchass $\left(\mathrm{CaCO}_{3}\right)(\mathrm{mg} / \mathrm{L})$

Noncarbonte harchess $(\mathrm{mg} / \mathrm{L}$ )

Specific conductance (micromhos)

pH (units)

Temperatue $\left({ }^{\circ} \mathrm{C}\right)$

Turbidity (JTU)

Dissolved oxygen (mg/L)

Total immediata coliform (col/100 ms) 30

Fecal coliform (0.7 um, membrane

filter) (col/100 mL)

0 issolved chromium (Cr) (ug/L)

Dissolved copper (Cu) (ug/L)

Total mercury (Hg) (ug/L)

Dissolved zine $(2 n)(u g / L)$

12043159 pilchuck Creek near Ozette, Wash.

Date sample collected

Dissolved calcium (Ca) (mg/L)

Dissolved magnesium ( $\mathrm{Mg})(\mathrm{mg} / \mathrm{L})$

Dissolved sodium (Na) (mg/L)

Dissolved potaasium $(K)(\mathrm{mg} / \mathrm{L})$

Bicarbonate $\left(\mathrm{HCO}_{3}\right)(\mathrm{mg} / \mathrm{L})$

Alkal inity $\left(\mathrm{CaCO}_{3}\right)(\mathrm{mg} / \mathrm{L})$

Dissolved aulfate $\left(\mathrm{SO}_{4}\right)(\mathrm{mg} / \mathrm{L})$

Dissolved chloride $(\mathrm{Cl})(\mathrm{mg} / \mathrm{h})$

Total nitrate (W) $(\mathrm{mg} / \mathrm{L})$

Total nitrite (N) $(\mathrm{mg} / \mathrm{L})$

Total nitrite plus nitrate $(W)$ ( $\mathrm{mg} / \mathrm{L}$ )

Total anmonia (N) (mg/L)

Total Kjaldahl nitrogen (N) (mg/L)

Total phosphorus (P) (mg/L)

Dissolved orthophosphate phosphorus

(P) $(\mathrm{mg} / \mathrm{L})$

Harcheas $\left(\mathrm{CaCO}_{3}\right)(\mathrm{mg} / \mathrm{L})$

Noncarbonate harchess (mg/ $\mathrm{L})$

Specific conductence (micromhos)

pH (units)

Temperature $\left({ }^{\circ} \mathrm{C}\right)$

Turbidity

Dissolved oxgyen (mg/L)

Total imediate coliform (col/100 mu

Fecal coliform (0.7 un, membrane

filtar) (col/100 $\mathrm{m}$ )

Dissolved chromium (Cr) (ug/L)

Dissolved copper (CU) (ug/L)

Dissolved lead $(\mathrm{Pb})(\mathrm{ug} / \mathrm{L})$

Total mercury ( $\mathrm{Hg})(\mathrm{ug} / \mathrm{L}$ )

Dissolved zinc $(2 n)(\mathrm{ug} / L)$

\begin{tabular}{|c|c|c|c|c|c|}
\hline \multicolumn{4}{|c|}{1976} & \multicolumn{2}{|c|}{1977} \\
\hline $4 / 21$ & $6 / 2$ & $8 / 25$ & $11 / 17$ & $1 / 18$ & $3 / 23$ \\
\hline 4.7 & $\cdots$ & 6.6 & . & . & .. \\
\hline 1.6 & .. & 2.0 & $\cdots$ & .. & $\cdots$ \\
\hline 4.0 & .. & 4.7 & .. & $\cdots$ & $\cdots$ \\
\hline .2 & .. & .4 & $\cdots$ & .. & $\cdots$ \\
\hline 19 & 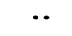 & 28 & .. & .. & $\cdots$ \\
\hline 61 & $\cdots$ & 23 & .. & .. & $\cdots$ \\
\hline 2.7 & .. & 56 & .. & .. & $\cdots$ \\
\hline 4.6 & .. & 4.9 & $\cdots$ & .. & $\cdots$ \\
\hline .02 & .. & .01 & .. & $\cdots$ & .. \\
\hline .00 & .. & .00 & .. & .. & $\cdots$ \\
\hline .07 & .. & .03 & .. & .. & $\cdots$ \\
\hline .14 & .. & .10 & $\cdots$ & .. & $\cdots$ \\
\hline .01 & $\cdots$ & .01 & .. & .. & .. \\
\hline .01 & . & .01 & . & .. & $\cdots$ \\
\hline 18 & .. & 25 & $\cdots$ & $\cdots$ & $\cdots$ \\
\hline 3 &.. & 2 & $\cdots$ & $\cdots$ & $\cdots$ \\
\hline 46 & 52 & 68 & 40 & 38 & 53 \\
\hline 6.7 & 7.1 & 7.6 & 6.9 & 7.2 & 6.9 \\
\hline 6.5 & 8.5 & 14.6 & 10.2 & 7.8 & 6.2 \\
\hline 2 & 1 & .. & 90 & 55 & 10 \\
\hline 11.8 & 11.5 & 10.2 & 10.6 & 11.3 & 10.9 \\
\hline 30 & $\cdots$ & 800 & 2700 & 910 & $\cdots$ \\
\hline 8 & .. & 140 & 1 & 1 & $\cdots$ \\
\hline .. & .. & 0 & .. & -. & $\cdots$ \\
\hline .. &.. & 1 & .. & .. & $\cdots$ \\
\hline -. & $\cdots$ & .0 & .. & .. & $\cdots$ \\
\hline -. & .. & 0 & - & $\cdots$ & $\cdots$ \\
\hline
\end{tabular}

\begin{tabular}{|c|c|c|c|c|c|c|}
\hline \multicolumn{3}{|c|}{1976} & \multicolumn{4}{|c|}{1977} \\
\hline $4 / 20$ & $6 / 2$ & $8 / 25$ & $11 / 17$ & $1 / 18$ & $3 / 23$ & $11 / 3$ \\
\hline 4.7 & .. & 5.5 & .. & .. & .. & .. \\
\hline 1.5 & .. & 1.8 & .. & .. & .. & .. \\
\hline 3.9 & .. & 4.9 & .. & . & .. & .. \\
\hline .6 & .. & .6 & .. & .. & .. & .. \\
\hline 13 & .. & 23 & .. & $\cdots$ & $\cdots$ & $\cdots$ \\
\hline 11 & .. & 19 & .. & .. & .. & $\cdots$ \\
\hline 4.6 & .. & 5.7 & . & .. & . & .. \\
\hline 5.4 & .. & 5.7 & .. & .. & .. & .. \\
\hline .00 & .. & .01 & .. & . & .. & .. \\
\hline .01 & -. & .00 & .. & -. & $\cdots$ & $\cdots$ \\
\hline .01 & .. & .01 & .. & . & .. & .24 \\
\hline .12 & .. & .06 & .. & .. & $\cdots$ & .06 \\
\hline .22 & .. & .21 & .. & .. & .. & .27 \\
\hline .02 & .. & .06 & .. & .. & .. & .. \\
\hline .01 & $\cdots$ & .01 & .. & .. & . & .. \\
\hline 18 & $\cdots$ & 21 & .. & -. & $\cdots$ & .. \\
\hline 7 & .. & 2 & .. & $\cdots$ & .. & .. \\
\hline 40 & 48 & 65 & 46 & 38 & 68 & 55 \\
\hline 6.5 & 6.7 & 7.2 & 6.5 & 6.9 & 6.8 & 6.5 \\
\hline 8.5 & 9.0 & 14.5 & 10.2 & 7.8 & 6.4 & 7.0 \\
\hline 4 & 2 & . & 35 & 10 & 10 & 2 \\
\hline 11.5 & 10.4 & 9.0 & 95. & 10.8 & 10.5 & 10.5 \\
\hline 35 & .. & B70 & 3700 & 1500 & .. & 230 \\
\hline 12 & .. & 36 & 130 & 6 & $\cdots$ & 44 \\
\hline . & .. & 0 & .. & .. & $\cdots$ & .. \\
\hline .. & .. & 1 & -. & .. & $\cdots$ & .. \\
\hline .. & .. & 2 & .. & $\cdots$ & $\cdots$ & .. \\
\hline .. & $\cdots$ & .0 & .. & .. & .. & .. \\
\hline -. & . & 0 & .. & $\cdots$ & -. & \\
\hline
\end{tabular}


TABLE 14, - Chemical and physical quality of water from selected surface-water sites in clallam County--Continued

12043163 Sooes River below Miller Creek near Ozette, Wesh.

\begin{tabular}{|c|c|c|c|c|c|c|c|}
\hline \multirow[b]{2}{*}{ Date aample collected } & \multirow[b]{2}{*}{$6 / 30 / 71$} & \multicolumn{4}{|c|}{1976} & \multicolumn{2}{|c|}{1977} \\
\hline & & $4 / 20$ & $6 / 2$ & $8 / 25$ & $11 / 17$ & $1 / 18$ & $3 / 23$ \\
\hline Discharge $\left(\mathrm{ft}^{3} / 8\right)$ & $\cdot \cdot$ & 230 & 144 & 49 & 870 & 1430 & 230 \\
\hline Dissolved calciun (Ca) (mg/L) & 6.2 & 4.0 & *. & 6.7 & $\cdots$ & -. & $\cdots$ \\
\hline Dissolved magnesium (Mg) (mg/) & 1.7 & 1.3 & -. & 2.0 & .. & .. & $\because$ \\
\hline Dissolved sodium (Ne) (mg/L) & $\cdot \cdot$ & 3.8 & $\cdots$ & 4.9 & -. & $\cdots$ & $\cdots$ \\
\hline Dissolved potessium $(K)(m g / L)$ & -. & .5 & -. & .5 & -. & $\cdots$ & $\cdots$ \\
\hline Bicarbonate $\left(\mathrm{HCO}_{3}\right)(\mathrm{mg} / \mathrm{h})$ & 33 & 15 & $\cdots$ & 29 & 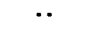 & $\cdots$ & $\cdots$ \\
\hline Alkal inity $\left(\mathrm{CaCO}_{3}\right)(\mathrm{mg} / \mathrm{L})$ & -. & 12 & $\cdots$ & 24 & $\cdots$ & $\cdots$ & $\cdots$ \\
\hline Dissolved aulfate $\left(\mathrm{SO}_{4}\right)(\mathrm{mg} / \mathrm{L})$ & 4.0 & 3.1 & 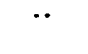 & 5.8 & -. & $\cdots$ & $\cdots$ \\
\hline Dissolved chlorde (Cl) (ng/L) & *. & 5.1 & $\cdots$ & 5.3 & $\because$ & -. & $\because$ \\
\hline Total nitrate $(N)(n g / L)$ & .04 & .01 & $\cdots$ & .01 & ・. & $\cdots$ & $\cdots$ \\
\hline Total nitrite $(N)(n g / L)$ & $\cdots$ & .01 & .. & .00 & -. & $\cdots$ & $\because$ \\
\hline Total Eamonia (N) (ng/L) & $\cdots$ & .12 & $\cdots$ & .04 & -・ & $\cdots$ & $\cdots$ \\
\hline Total KJeldahl nitrogen (W) $(\mathrm{mg} / \mathrm{L})$ & -. & .26 & $\cdots$ & .19 & -. & $\cdot-$ & $\cdots$ \\
\hline $\begin{array}{l}\text { Total phosphorus (P) (mg/L) } \\
\text { Dissolved or thophosphate }\end{array}$ & 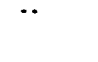 & .03 & $\cdots$ & .01 & *. & $\cdot \cdot$ & $\cdots$ \\
\hline -phosphorus (P) (mg/L) & $\because$ & .01 & $\because$ & .00 & $\cdots$ & $\cdots$ & $\because$ \\
\hline Hardness $\left(\mathrm{CaCO}_{3}\right)(\mathrm{mg} / \mathrm{L})$ & 22 & 15 & $\cdot \cdot$ & 25 & -. & -. & $\cdots$ \\
\hline Moncarbonate hardness $(\mathrm{mg} / \mathrm{L})$ & 0 & 3 & 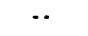 & 1 & -. & -. & $\cdots$ \\
\hline specific concuctance (micromhos) & 72 & 41 & 53 & 75 & 45 & 39 & 55 \\
\hline pH (units) & 7.2 & 6.5 & 7.2 & 7.3 & 7.1 & 6.9 & 7.1 \\
\hline Temperature $\left({ }^{\circ} \mathrm{C}\right)$ & -・ & 7.0 & 9.2 & 15.3 & 10.6 & 8.0 & 6.8 \\
\hline Turbidity (JTU) & 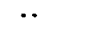 & 7 & 2 & -. & 22 & 45 & 15 \\
\hline Dissolvad oxygen (mg/L) & -. & 12.0 & 11.2 & 9.7 & 10.3 & 10.1 & 11.1 \\
\hline Total immediate coliform (col/100 m $)$ & $\cdots$ & 43 & $\cdots$ & 670 & 5400 & 1400 & $\cdots$ \\
\hline $\begin{array}{l}\text { Fecal colliform (0.7 un, menbrane } \\
\text { filter) (col/100 m) }\end{array}$ & $\cdots$ & 7 & $\cdots$ & 92 & 200 & 9 & $\cdots$ \\
\hline Dissolved chromiun (Cr) (ug/L) & -. & -. & -. & 0 & .. & -. & $\cdots$ \\
\hline Dissolved copper (Cu) (ug/L) & 6 & $\cdots$ & - & 1 & .. & $\because$ & $\cdots$ \\
\hline Dissolved lead $(\mathrm{Pb})(\mathrm{ug} / \mathrm{L})$ & *. & $\because$ & - & 6 & •. & -・ & $\because$ \\
\hline Total mercury (Hg) (ug/L) & $\cdots$ & $\cdots$ & $\cdots$ & 0 & $\because$ & $\cdots$ & $\cdots$ \\
\hline Dissolved zinc $(2 n)(u g / L)$ & 20 & $\cdot \cdot$ & $\cdots$ & 0 & $\cdot \cdot$ & *. & $\cdots$ \\
\hline
\end{tabular}


12043173 Waatch Rivar below Educkat Creek at Weah Bay, Mash.

Data sanpla collected

Discharge $\left(\mathrm{ft}^{3} / \mathrm{s}\right)$

Dissolved siltice $\left(\mathrm{SiO}_{2}\right)(\mathrm{mg} / \mathrm{L})$

Dissolved iron (fe) (ug/t)

Dissolved manganese $(\mathrm{Mn})(\mathrm{ug} / \mathrm{L})$

Dissolved calciun (Ca) (mo/L)

Dissolved magnesiun (Ha) (mo/L)

Dissolved sodiun ( $\mathrm{Ha})(\mathrm{mo} / \mathrm{L})$

Dissolved potassiun (K) (mo/L)

Bicarbonate $\left(\mathrm{HCO}_{3}\right)(\mathrm{mo} / \mathrm{L})$

Alkalinity $\left(\mathrm{CaCO}_{3}\right)(\mathrm{mg} / \mathrm{L})$

Dissolved sulfata $\left(\mathrm{SO}_{4}\right)(\mathrm{mg} / \mathrm{L})$

Dissolved chloride (Cl) (ng/L)

Dissolved fluoride (F) (mo/L)

rotal nitrate (W) (mg/L)

rotal nitrita (N) $(\mathrm{mol} / \mathrm{L})$

Total nitrite plus nitrata (W) (mo/L)

total amonia (W) (ma/L)

Total Kjeldahl nitrosen (W) (mg/L)

total phosphorus (P) (mo/L)

Dissolved or thophosphate phosphorus

(P.) $(\mathrm{mo} / \mathrm{L})$

Narchess $\left(\mathrm{CaCO}_{3}\right)(\mathrm{mg} / \mathrm{L})$

Noncarbonate harchess ( $\mathrm{mg} / \mathrm{L}$ )

specific conductence (nicronhos)

pH (unts)

Temperature $\left({ }^{\circ} \mathrm{C}\right)$

color (platinu-cobalt units)

Turbidity (JTU)

Dissolved oxygen ( $\mathrm{mg} / \mathrm{L}$ )

Dissolved carbon dioxide

$$
\left(\mathrm{CO}_{2}\right)(\mathrm{m} / \mathrm{L})
$$

Total immediate coliform (col/100 m)

fecal coliform $(0.7 \mathrm{um}$, menbrane

filter) (col/100 $m$ )

\begin{tabular}{|c|c|c|c|c|c|c|c|c|c|}
\hline \multirow{2}{*}{$\frac{1971}{4 / 29^{\circ}}$} & \multicolumn{2}{|c|}{1972} & \multicolumn{4}{|c|}{1976} & \multicolumn{3}{|c|}{1977} \\
\hline & $1 / 19$ & $4 / 7$ & $4 / 20$ & $6 / 2$ & $8 / 25$ & $11 / 16$ & $1 / 18$ & $3 / 22$ & $11 / 2$ \\
\hline .. & .. & $\ldots$ & .. & 37 & 13 & 156 & 450 & 70 & 165 \\
\hline 6.0 & 3.5 & 2.8 & $\cdots$ & $\cdot \cdot$ & $\cdots$ & $\cdots$ & $\cdots$ & $\cdots$ & $\cdots$ \\
\hline 120 & 120 & 180 & .. & $\cdots$ & .. & .. & .. & .. & .. \\
\hline 6 & 9 & 3 & .. & .. & .. & $\cdots$ & $\cdots$ & $\cdots$ & $\cdots$ \\
\hline 4.4 & 4.0 & .. & 5.7 & $\cdots$ & $\cdots$ & .. & $\cdots$ & $\cdots$ & $\cdots$ \\
\hline 6.3 & 5.3 & 5.3 & 1.1 & -. & $\cdots$ & $\cdots$ & $\cdots$ & $\cdots$ & $\cdots$ \\
\hline 11 & 8.0 & 6.2 & 3.8 & .. & 5.1 & $\cdots$ & $\cdots$ & $\cdots$ & $\cdots$ \\
\hline .7 & .5 & .6 & .4 & .. & .4 & .. & $\because$ & $\cdots$ & $\cdots$ \\
\hline 26 & 51 & 24 & 12 & $\cdot \cdot$ & 20 & $\cdots$ & $\cdots$ & $\because$ & $\cdots$ \\
\hline 21 & 42 & 20 & 10 & 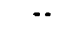 & 16 & $\cdots$ & $\cdots$ & $\cdots$ & $\cdots$ \\
\hline 16 & 13 & 7.8 & 4.3 & .. & 6.3 & $\cdots$ & $\cdots$ & .. & *. \\
\hline 9.5 & 7.2 & 5.5 & 6.6 & .. & 5.9 & $\cdots$ & .. & $\because \cdot$ & $\cdots$ \\
\hline .1 & .1 & .1 & .. & .. & .. & $\cdots$ & $\cdots$ & .. & .. \\
\hline .11 & .05 & .02 & .15 & .. & .15 & $\cdots$ & $\cdots$ & .. & 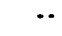 \\
\hline .01 & .01 & .01 & .01 & .. & .00 & $\cdots$ & $\cdots$ & $\cdots$ & $*$ \\
\hline -. & $\cdots$ & .. & .16 & .. & .15 & -. & .. & .. & .69 \\
\hline$\cdots$ & .. & .. & .07 & .. & .03 & -. & .. & .. & .07 \\
\hline$\cdots$ & $\cdots$ & -. & .17 & $\cdots$ & .11 & $\cdots$ & $\cdots$ & $\cdots$ & .20 \\
\hline .03 & .04 & .02 & .02 & $\cdots$ & .01 & $\cdots$ & $\cdots$ & $\cdots$ & $\cdots$ \\
\hline .. & .. & .. & .00 & .. & .01 & .. & $\cdots$ & .. & $\cdots$ \\
\hline 18 & 10 & $\cdots$ & 19 & . & $\cdots$ & $\cdots$ & $\cdots$ & .. & -. \\
\hline 23 & .. & 2 & 9 & $\cdots$ & $\cdots$ & $\cdots$ & $\cdots$ & $\cdot \cdot$ & $\because$ \\
\hline 75 & 80 & 52 & 37 & 47 & 60 & 51 & 44 & 38 & 50 \\
\hline 7.4 & 7.0 & 6.5 & 6.6 & 6.9 & 7.1 & 7.0 & 7.3 & 6.9 & 6.3 \\
\hline .. & .. & .. & 5.5 & 7.2 & 11.1 & 9.9 & 7.8 & 7.0 & 8.0 \\
\hline 18 & 12 & 35 & $\cdots$ & -. & $\cdots$ & .. & .. & .. & .. \\
\hline 1 & 2 & 2 & 4 & 1 & $\cdot$. & 2 & 8 & 1 & 1 \\
\hline$\cdots$ & $\cdots$ & $\cdots$ & 11.9 & 11.8 & 10.0 & 10.7 & 11.5 & 10.3 & 11.3 \\
\hline 3.8 & 11 & 17 & $\cdots$ & $\cdots$ & $\cdots$ & $\cdots$ & $\cdots$ & $\cdots$ & $\cdots$ \\
\hline$\cdots$ & $\cdots$ & $\cdot \cdot$ & 4 & 280 & 560 & 1000 & 400 & $\cdots$ & 100 \\
\hline .. & .. & .. & 1 & 24 & 25 & 31 & 18 & $\cdots$ & 6 \\
\hline
\end{tabular}

Avarage of two semples. 
12043176 Waatch River at Meah Bay, Uash.

Data sample collected

Dissolved calcium (Ca) (mo/L)

Dissolved magnesium (Mg) (mg/L)

Dissolved sodium (Ma) (mg/L)

Dissolved potassium (K) (mo/L)

Bicarbonate $\left(\mathrm{HCO}_{3}\right)(\mathrm{mg} / \mathrm{L})$

Alkalinity $\left(\mathrm{CaCO}_{3}\right)(\mathrm{mg} / \mathrm{L})$

Dissolved sulfate $\left(\mathrm{SO}_{4}\right)(\mathrm{mo} / \mathrm{L})$

Dissolved chloride (Cl) (mg/L)

Total nitrate (N) (no/L)

Total nitrite (M) (mg/L)
Total nitrite plust nitrate (N) (mg/L)

Total amonila (N) (mg/L)

Total Kjeldaht nitrogen (M) (mo/L)

Total phosphorus (P) (mg/L)

Dissolved or thophosphate phosphorus

(P) $(\mathrm{mg} / \mathrm{L})$

Hardness $\left(\mathrm{CaCO}_{3}\right)(\mathrm{mg} / \mathrm{L})$

Noncerbonate hardness (mg/L)

Specific conductance (micromhos)

pH (units)

Temperature $\left({ }^{\circ} \mathrm{C}\right)$

Turbldity

Dissolved oxygen (ng/L)

Total imediate coliform (col/100 m)

Facal coliform ( 0.7 um, membrane

filter) (col/100 m. $)$

\begin{tabular}{|c|c|c|c|}
\hline \multicolumn{4}{|c|}{1976} \\
\hline $4 / 20$ & $6 / 2$ & $8 / 25^{\circ}$ & $11 / 17$ \\
\hline 3.2 & $\cdots$ & 7.4 & .. \\
\hline 1.8 & $\cdots$ & 7.3 & .. \\
\hline 4.4 & .. & 56 & .. \\
\hline .4 & $\cdots$ & 2.6 & $\cdots$ \\
\hline 11 & .. & 23 & . \\
\hline 9 & .. & 19 & .. \\
\hline 4.2 & $\because$ & 18 & .. \\
\hline 6.5 & .. & 97 & .. \\
\hline .14 & .. & .15 & .. \\
\hline .01 & .. & .00 & $\cdots$ \\
\hline .15 & .. & .15 & .. \\
\hline .08 & .. & .05 & $\cdots$ \\
\hline .22 & .. & .16 & $\because$. \\
\hline .02 & $\cdots$ & .02 & $\cdots$ \\
\hline .01 & .. & .02 & .. \\
\hline 15 & $\cdots$ & 49 & .. \\
\hline 6 & .. & 30 & .. \\
\hline 41 & 81 & -. & 61 \\
\hline 6.2 & 7.1 & 7.3 & 6.6 \\
\hline 5.5 & 8.8 & 12.0 & 9.4 \\
\hline 3 & 1 & $\because$ & 4 \\
\hline 11.6 & 11.8 & 8.0 & 10.6 \\
\hline 50 & 1400 & 660 & 900 \\
\hline
\end{tabular}

64

$\frac{1977}{1 / 18 \quad 3 / 22 \quad 11 / 2}$

$\begin{array}{lll} & \cdots & 3 / 22\end{array}$

$\cdots \quad \cdots \quad$.

$\begin{array}{lll}\cdots & \cdots & \\ \cdots & . & \end{array}$

. $\quad$.. $\quad$..

.. $\quad$.. $\quad$..

.. $\quad .$.

. $\quad$. 10

.. $\quad$.. $\quad$.

$\begin{array}{lll}. & & \\ & & .54\end{array}$

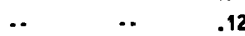

$\begin{array}{lll}. . & . . & .26\end{array}$

-

..

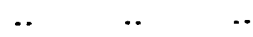

$51 \quad 72 \quad 65$

$\begin{array}{lll}6.7 & 6.8 & 6.1\end{array}$

$\begin{array}{rrr}8.2 & 7.0 & 8.3 \\ 3 & 1 & 2 \\ 11.2 & 10.9 & 10.5 \\ 580 & . . & 160\end{array}$

$19 \quad \ldots \quad 3$

12043186 Village Creek at Weah Bay, Wash.

Date sample collected

Dissolved calcium (Ca) (mg/L)

Dissolved magnesium $(\mathrm{Mg})(\mathrm{mg} / \mathrm{L})$

Dissolved sodiun (Ma) (mg/L)

Diasolved potassium (K) (mg/L)

Bicarbonate $\left(\mathrm{HCO}_{3}\right)(\mathrm{mg} / \mathrm{L})$

Alkallity $\left(\mathrm{CaCO}_{3}\right)(\mathrm{mg} / \mathrm{L})$

Disaolved aulfate $\left(\mathrm{SO}_{4}\right)(\mathrm{mg} / \mathrm{L})$

Dissolved chloride (Cl) (mg/L)

Total nitrate $(M)(\mathrm{N} / \mathrm{g} / \mathrm{L})$

Total nitrite (W) (mg/L)

Total nitrite plus nitrate $(N)(\mathrm{mg} / \mathrm{L}) .02$

Total ammonia (W) (mg/L)

Total Kjeldehl nitrogen (W) (mg/L)

Total phosphorus (P) (mo/L)

Dissolved or thophosphete phosphorus

(P) $(m / L)$

Nardnesa $\left(\mathrm{CacO}_{3}\right)(\mathrm{ma} / \mathrm{L})$

Moncarbonata hardness $(\mathrm{mg} / L)$

Specific conductance (micromhos)

pH (units)

Temperature $\left({ }^{\circ} \mathrm{C}\right)$

Turbidity (JTU)

Dianol ved oxygen $(\mathrm{mg} / \mathrm{L})$

Total inmediate colliform

( col/100 mo/L)

Fecal collform $(0.7$ um, nembrane

filtar) (col/100 ma)

\begin{tabular}{cccc}
\hline $4 / 21$ & $6 / 2$ & $8 / 25$ & $11 / 17$ \\
4.6 &.. &.. &.. \\
1.5 &.. &.. &.. \\
4.2 &.. & 5.1 &.. \\
.3 &. & .4 &.. \\
11 &.. & 15 &.. \\
9 &.. & 12 &.. \\
3.8 &.. & 6.7 &.. \\
6.6 &.. & 6.5 &.. \\
.02 &.. & .03 &.. \\
.00 &.. & .00 &.. \\
.02 &.. & .03 &.. \\
.05 &.. & .03 &.. \\
.18 &.. & .17 &.. \\
.01 &.. & .01 &.. \\
.00 &.. & .01 &.. \\
18 &.. &.. &.. \\
9 &.. &.. &.. \\
40 & 51 & 65 & 46 \\
6.7 & 6.8 & 7.3 & 6.4 \\
6.5 & 7.5 & 10.8 & 10.0 \\
1 & 1 &.. & 5 \\
.. & 11.4 & 9.1 & 11.4 \\
& & & \\
40 & 370 & 340 & 180
\end{tabular}

\begin{tabular}{|c|c|c|}
\hline & 1977 & \\
\hline $1 / 17$ & $3 / 22$ & $11 / 2$ \\
\hline .. & .. & .. \\
\hline .. & .. & .. \\
\hline .. & .. & .. \\
\hline .. & .. & $\ldots$ \\
\hline .. & .. & .. \\
\hline . & $\cdots$ & -. \\
\hline .. & .. & $\cdots$ \\
\hline .. & . & .. \\
\hline . & .. & .. \\
\hline .. & .. & .. \\
\hline .. & .. & .22 \\
\hline .. & .. & .03 \\
\hline .. & .. & .10 \\
\hline .. & .. & .. \\
\hline
\end{tabular}

2 
12043190 Sail River near Nech Bay, Wash.

\begin{tabular}{|c|c|c|c|c|c|c|c|c|}
\hline \multirow[b]{2}{*}{ Date sample collected } & \multirow[b]{2}{*}{$6 / 30 / 71$} & \multicolumn{4}{|c|}{1976} & \multicolumn{3}{|c|}{1977} \\
\hline & & $4 / 19$ & $6 / 2$ & $8 / 25$ & $11 / 17$ & $1 / 17$ & $3 / 22$ & $11 / 2$ \\
\hline Discharge $\left(\mathrm{ft}^{3} / \mathrm{s}\right)$ & $\cdots$ & -. & 17 & 5.2 & 70 & 186 & 39 & 153 \\
\hline Dissolved calcium (Ca) (mo/L) & 4.9 & 5.1 & $\cdots$ & -. & .. & -. & $\cdots$ & 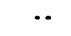 \\
\hline Dissolved magnesium (Mg) (mg/L) & 1.5 & .9 & $\cdots$ & .. & .. & .. & .. & .. \\
\hline Dissolved sodium (Na) $(m g / L)$ & $\cdots$ & 4.2 & $\cdots$ & 6.1 & $\cdots$ & $\cdots$ & .. & $\cdots$ \\
\hline Dissolved potassium $(K)(\mathrm{mg} / \mathrm{L})$ & .. & .4 & $\cdots$ & .4 & .. & .. & .. & -. \\
\hline Bicarbonate $\left(\mathrm{HCO}_{3}\right)(\mathrm{mg} / \mathrm{L})$ & 24 & 15 & $\cdots$ & 21 & $\cdots$ & .. & $\cdot \cdot$ & $\cdots$ \\
\hline Alkal inity $\left(\mathrm{CaCO}_{3}\right)(\mathrm{mg} / \mathrm{L})$ & 20 & 12 & $\cdots$ & 17 & $\because$ & $\cdots$ & .. & $\cdots$ \\
\hline Dissolved sulfate $\left.\left(\mathrm{SO}_{4}\right) 9 \mathrm{mg} / \mathrm{L}\right)$ & 7.0 & 4.0 & $\cdots$ & 8.7 & $\cdots$ & $\because$ & $\cdots$ & $\cdots$ \\
\hline Dissolved chloride $(\mathrm{Cl})(\mathrm{mg} / \mathrm{L})$ & $\cdots$ & 4.0 & $\cdots$ & 4.5 & $\cdots$ & $\cdots$ & $\cdots$ & $\cdots$ \\
\hline Total nitrate $(N)(\mathrm{mg} / \mathrm{L})$ & .26 & .07 & $\cdots$ & .26 & 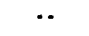 & .. & -. & $\cdots$ \\
\hline Total nitrite (N) $(\mathrm{mg} / \mathrm{L})$ & $\cdots$ & .01 & $\cdots$ & .00 & $\cdots$ & .. & $\cdots$ & $\cdots$ \\
\hline \multicolumn{9}{|l|}{ Total nitrite plus nitrate } \\
\hline (N) $(m g / L)$ & $\cdots$ & .08 & $\cdots$ & .26 & $\cdots$ & $\cdots$ & $\cdots$ & .63 \\
\hline Total ammonia (N) (mg/L) & $\cdots$ & .06 & $\cdots$ & .03 & $\cdots$ & 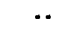 & -. & .05 \\
\hline Total Kjeldahl nitrogen (N) ( $\mathrm{mg} / \mathrm{L}$ ) & -. & .12 & $\cdots$ & .20 & .. & -. & $\cdots$ & .20 \\
\hline Total phosphorus $(P)(m g / L)$ & $\cdots$ & .02 & $\cdots$ & .01 & -. & $\cdots$ & $\cdots$ & $\cdots$ \\
\hline \multicolumn{9}{|l|}{ Dissolved or thophosphate phosphorus } \\
\hline (P) $(\mathrm{mg} / \mathrm{t})$ & -. & .00 & $\cdots$ & .01 & 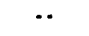 & $\cdots$ & .. & $\cdots$ \\
\hline Hardness $\left(\mathrm{CaCO}_{3}\right)(\mathrm{mg} / \mathrm{L})$ & 18 & 16 & $\cdots$ & .. & $\because$ & -. & .. & $\cdots$ \\
\hline Noncarbonate hardness (mg/L) & 0 & 4 & $\cdots$ & $\cdots$ & $\cdots$ & 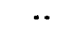 & $\cdots$ & $\cdots$ \\
\hline Specific conductance (micromhos) & 74 & 42 & 45 & 72 & 56 & 48 & 47 & 50 \\
\hline pH (units) & 8.4 & 6.6 & 6.6 & 7.6 & 6.8 & 7.7 & 7.0 & 7.5 \\
\hline Temperature $\left({ }^{\circ} \mathrm{C}\right)$ & -. & 7.4 & 8.9 & 10.8 & 12.2 & 8.0 & 7.2 & 8.0 \\
\hline Turbidity (JTU) & $\cdots$ & 6 & 5 & -. & 2 & 6 & 1 & 1 \\
\hline Oissolved oxygen (mg/L) & $\cdots$ & 12.0 & 11.6 & .. & 10.8 & 19.9 & 11.5 & 10.3 \\
\hline Total immediate coliform (col/100 mL) & 00 & 8 & 220 & 800 & 00 & 150 & -. & 120 \\
\hline \multicolumn{9}{|l|}{ Fecal coliform 10.7 un, menbrane } \\
\hline filter $)(\mathrm{col} / 100 \mathrm{~mL})$ & $\cdots$ & 1 & 79 & 8 & 00 & 1 & 00 & 3 \\
\hline Dissolved copper (Cu) (ug/L) & 11 & $\cdots$ & $\cdots$ & 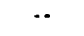 & $\cdot \cdot$ & $\cdots$ & $\cdots$ & $\cdots$ \\
\hline Dissolved zinc $(2 n)(u g / L)$ & 40 & -. & $\cdots$ & $\cdots$ & -. & $\cdots$ & -. & $\cdots$ \\
\hline
\end{tabular}

Clallam River, near East Clellem, Wash. (Site 95)

Date sample collected

Dissolved iron (Fe) (ug/L)

Dissolved manganese (Mn) (ug/L)

Dissolved fluoride (F) (mg/L)

Dissolved nitrate (N) (mg/L)

Mardness $\left(\mathrm{CaCO}_{3}\right)$ ( $\left.\mathrm{mg} / \mathrm{L}\right)$

Specific conductance (micromos)

color (platínm-cobalt units)

Turbidity (JTU)

Oissolved arsenic (As) (ug/L)

Dissolved barium (Ba) (ug/L)

Oiasolved cadnium (Cd) (ug/L)

Dissolved chromiun (Cr) (ug/L)

Dissolved lead (Pb) (ug/L)

Dissolved mercury ( $\mathrm{Hg})(\mathrm{ug} / \mathrm{h})$

Dissolved seleniun (se) (ug/L)

Dissolved silver (Ag) (ug/L)

\begin{tabular}{c}
$6 / 28 / 77$ \\
\hline 50 \\
31 \\
.0 \\
.5 \\
32 \\
80 \\
14 \\
1 \\
10 \\
250 \\
2 \\
10 \\
10 \\
1 \\
3 \\
10
\end{tabular}

12043365 Pysht River near Sappho, Wash. (Site 96)

\begin{tabular}{|c|c|c|}
\hline Date sample collected & $2 / 12 / 68$ & $6 / 25 / 6$ \\
\hline Dissolved silice $\left(\mathrm{sio}^{2}\right)(\mathrm{mg} / \mathrm{L})$ & 9.3 & 8.8 \\
\hline Dissolved calcium (Ca) ( $\mathrm{mg} / \mathrm{L})$ & 7.0 & 8.8 \\
\hline Dissolved magnesium (Mg) (mg/L) & 1.8 & 2.0 \\
\hline Dissolved sodium (Ma) (mg/L) & 4.3 & 5.2 \\
\hline Dissolved potassium (K) (mg/L) & .3 & .5 \\
\hline Bicarbonate $\left(\mathrm{HCO}_{3}\right)(\mathrm{ma} / \mathrm{L})$ & 28 & 29 \\
\hline Alkal inity $\left(\mathrm{CaCO}_{3}\right)(\mathrm{mg} / \mathrm{L})$ & 23 & 32 \\
\hline Dissolved sulfate $\left(\mathrm{SO}_{4}\right)(\mathrm{mg} / \mathrm{L})$ & 6.4 & 7.8 \\
\hline Diasolved chloride (cl) (mo/l) & 4.5 & .7 \\
\hline Dissolved fluoride $(F)(\mathrm{mg} / \mathrm{t})$ & .1 & .1 \\
\hline $\begin{array}{l}\text { Total nitrate (N) }(\mathrm{mg} / \mathrm{L}) \\
\text { Dissolved solids, residue at }\end{array}$ & .50 & .10 \\
\hline $180^{\circ} \mathrm{C}(\mathrm{mg} / \mathrm{L})$ & 52 & 57 \\
\hline Hardness $\left(\mathrm{CaCO}_{3}\right)(\mathrm{mg} / \mathrm{L})$ & 25 & 30 \\
\hline Noncarbonata hardhess (mg/L) & 2 & 0 \\
\hline Specific conductene (micronhos) & 73 & 90 \\
\hline pH (units) & 7.7 & 7.2 \\
\hline Temperature $\left({ }^{\circ} \mathrm{C}\right)$ & -. & 14.5 \\
\hline Color (platinum-cobalt units) & 5 & 5 \\
\hline Turbidity (JTU) & 0 & $\cdots$ \\
\hline Diseolved (ithium (Li) (ug/L) & 0 & 0 \\
\hline Diesolved stroutiun (Sr) (ug/L) & 20 & 0 \\
\hline
\end{tabular}


12043400 Pysht River near Pysht, Wash. (Site 6)

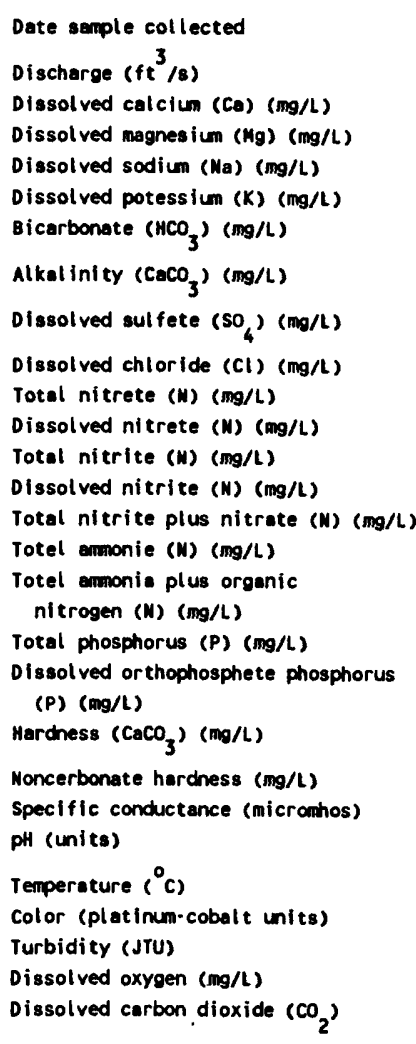

Total immediate coliform (col/100 mL)

\begin{tabular}{|c|c|c|c|c|c|c|c|c|c|c|c|}
\hline \multirow{2}{*}{$\begin{array}{l}1971 \\
12 / 14\end{array}$} & \multicolumn{5}{|c|}{1972} & \multicolumn{6}{|c|}{1973} \\
\hline & $2 / 8$ & $4 / 11$ & $6 / 13$ & $8 / 8$ & $11 / 29$ & $1 / 16$ & $3 / 13$ & $5 / 30$ & $7 / 17$ & $9 / 25$ & $11 / 13$ \\
\hline 620 & 575 & 315 & 16 & 7.8 & . & .. & .. & $\ldots$ & .. & .. & .. \\
\hline 4.5 & 4.6 & 4.8 & 9.1 & 8.7 & 7.9 & 4.1 & 5.6 & 7.9 & 93 & 11 & .. \\
\hline 1.1 & 1.0 & 1.2 & 1.9 & 1.8 & 1.6 & .9 & 1.2 & 1.4 & 2.0 & 2.1 & .. \\
\hline 3.9 & 3.9 & 4.3 & 5.5 & 5.4 & 5.4 & 3.6 & 4.1 & 4.9 & 5.7 & 6.7 & $\cdots$ \\
\hline .1 & .8 & .6 & .6 & .5 & .7 & 2.4 & 2.0 & .4 & .5 & .5 & $\cdots$ \\
\hline 17 & 16 & 20 & 38 & 36 & 25 & 18 & 16 & 29 & 44 & 42 & .. \\
\hline 14 & 13 & 165 & 31 & 30 & 21 & 15 & 13 & 24 & 36 & 34 & $\cdots$ \\
\hline .. & .. & $\cdots$ & $\cdots$ & .. & 10 & 6.1 & 13 & 9.4 & 10 & 14 & . \\
\hline 3.6 & 2.6 & 2.9 & 3.7 & 3.2 & 3.5 & 3.5 & 3.4 & 3.5 & 4.1 & 4.5 & .. \\
\hline .. & -. & .. & .. & .. & 1.1 & .76 & .38 & .23 & .08 & .15 & . \\
\hline .80 & .11 & .42 & .07 & .05 & .. & . & -. & .. & -. & .. & -. \\
\hline -. & .. & -. & .. & .. & .00 & .02 & .00 & .00 & -. & .. & .. \\
\hline .00 & .00 & .01 & .01 & .00 & -. & -. & -. & .. & $\cdots$ & .. & .. \\
\hline -. & -. & . & .. & -. & 1.1 & .78 & .38 & .23 & .. & -. & 1.3 \\
\hline .03 & .11 & .04 & .03 & .02 & .07 & .27 & .10 & .05 & .05 & .02 & .18 \\
\hline .14 & .25 & .05 & .19 & .14 & .. & .. & .. & .. & - & .. & .. \\
\hline .04 & .03 & .04 & .00 & .02 & .01 & .15 & .00 & $.0 D$ & .02 & .00 & .06 \\
\hline .00 & .01 & .00 & .00 & .01 & .00 & .00 & .00 & .00 & .00 & .00 & .00 \\
\hline 16 & 16 & 17 & 31 & 29 & 26 & 14 & 19 & 26 & 33 & 36 & -. \\
\hline 2 & 2 & 1 & 0 & 0 & 6 & D & 6 & 2 & 0 & 2 & $\cdots$ \\
\hline 59 & 57 & 18 & 104 & 106 & 67 & 62 & 86 & 83 & 110 & 120 & 71 \\
\hline 7.2 & 7.7 & 7.3 & 7.2 & 7.1 & 7.3 & 7.2 & 6.8 & 7.6 & 7.4 & 7.4 & 6.9 \\
\hline 5.2 & 4.7 & 51 & 13.0 & 18.6 & 4.4 & 5.0 & 6.8 & 10.4 & 15.2 & 11.4 & 6.6 \\
\hline 55 & 35 & 18 & 18 & 15 & 2 & 70 & 30 & 25 & 23 & 47 & 55 \\
\hline 8 & 15 & 3 & 1 & 2 & 2 & 70 & 3 & 1 & 2 & 1 & 30 \\
\hline 12.1 & 12.8 & 12.4 & 9.8 & 7.0 & 12.6 & 11.5 & 12.3 & 10.5 & 8.8 & 9.9 & 11.6 \\
\hline$\cdots$ & $\cdots$ & 1.6 & 3.8 & 4.6 & 2.0 & 1.8 & 4.1 & 1.2 & 2.8 & 2.7 & .. \\
\hline 500 & 150 & 200 & 900 & 500 & 350 & 400 & 100 & 325 & 550 & 1500 & 660 \\
\hline
\end{tabular}


TABLE 14.-.Chemical and physical quality of wotar from alected surface-watar sites in clallam County--Continued

\begin{tabular}{|c|c|c|c|c|c|}
\hline \multirow[b]{2}{*}{ Date semple collected } & \\
\hline & $1 / 23$ & $3 / 19$ & $5 / 15$ & $7 / 23$ & $9 / 17$ \\
\hline Discharge $\left(\mathrm{ft}^{3} / \mathrm{s}\right)$ & .• & .. & .. & .. & .. \\
\hline Dissolved calcium (Ca) $(\mathrm{mg} / \mathrm{L})$ & $\cdot \cdot$ & $\cdot \cdot$ & .. & .. & .. \\
\hline Disaolved magnesium $(\mathrm{Mg})(\mathrm{mg} / \mathrm{L})$ & .. & .. & $\cdot \cdot$ & $\cdot \cdot$ & .. \\
\hline Disẹolved sodiun (Ma) $(\mathrm{mo} / \mathrm{L})$ & $\cdots$ & .. & .. & .. & .. \\
\hline Dinsolved potensium $(K)(m / L)$ & .. & .. & .. & .. & .. \\
\hline Bicarbonata $\left(\mathrm{HCO}_{3}\right)(\mathrm{mg} / \mathrm{L})$ & $\because$ & $\cdot \cdot$ & .. & $\cdot \cdot$ & $\cdot \cdot$ \\
\hline Alkal inity $\left(\mathrm{CaCO}_{3}\right)(\mathrm{mg} / \mathrm{L})$ & 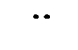 & 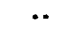 & .. & 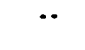 & $\cdot \cdot$ \\
\hline Dissolved aul fate $\left(\mathrm{SO}_{4}\right)(\mathrm{ma} / \mathrm{L})$ & $\cdots$ & -. & -. & -. & .. \\
\hline Dissolved chloride (Cl) (ma/l) & $\cdots$ & -. & $\cdots$ & $\cdots$ & $\cdot \cdot$ \\
\hline Total nitrate $(\mathrm{N})(\mathrm{m} / \mathrm{L})$ & .. & .. & .. & .. & .. \\
\hline Dissolved nitrate $(N)(\mathrm{mg} / \mathrm{L})$ & .. & .. & .. & -. & .. \\
\hline Totel nitrite $(W)(m / L)$ & $\cdots$ & ・. & $\cdots$ & -. & .. \\
\hline Dissolved nitrita (N) $(\mathrm{mg} / \mathrm{L})$ & .. & .. & .. & .. & .. \\
\hline Total nitrite plus nitrata (W) (mas & .46 & .47 & .26 & .10 & .11 \\
\hline $\begin{array}{l}\text { Total monie (U) (mg/L) } \\
\text { Total ammonis plus orgenic }\end{array}$ & .06 & .07 & .15 & .13 & .13 \\
\hline nitrogen $(U)(\mathrm{m} / \mathrm{L})$ & .. & .. & .. & .. & .. \\
\hline Total phosphorus $(P)(m / L)$ & .03 & .03 & .02 & .02 & .02 \\
\hline Dissolved or thophosphate phosphoru: & & & & & \\
\hline (P) $(m / L)$ & .00 & .00 & .01 & .00 & .01 \\
\hline Mardness $\left(\mathrm{CaCO}_{3}\right)(\mathrm{mg} / \mathrm{L})$ & $\cdot \cdot$ & $\cdot \cdot$ & .. & $\cdot \cdot$ & $\cdot \cdot$ \\
\hline Noncarbonate herchess $(\mathrm{mg} / \mathrm{L})$ & .. & .. & .. & .. & .. \\
\hline Specific conductanca (micromhos) & 60 & 43 & 99 & 90 & 110 \\
\hline pH (units) & 7.2 & 7.2 & 7.3 & 7.5 & 7.2 \\
\hline Temperatura $\left({ }^{\circ} \mathrm{C}\right)$ & 6.5 & 5.8 & 6.7 & 13.5 & 12.3 \\
\hline Color (platinum-cobalt units & 25 & 17 & 29 & 32 & 34 \\
\hline Turbidity (JTU) & 10 & 7 & 6 & 4 & 2 \\
\hline Dissol ved oxygen (mo/L) & 12.3 & 12.3 & 12.5 & 10.3 & 9.6 \\
\hline Dissol ved carbon dioxide $\left(\mathrm{CO}_{2}\right)$ & 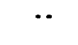 & $\cdot \cdot$ & $\cdots$ & 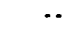 & *. \\
\hline Total immediate coliform (col/100 & 390 & 280 & 450 & 1600 & 2100 \\
\hline
\end{tabular}


TABLE 14. - Chemical and physical quality of water from selected surface-water sites in Clallam county-.-Continued

Lyre River at railroad bridge below Lake Crescent, et Piectnont, Wash.

Date sample collected

Dissolved siltica $\left(\mathrm{SiO}_{2}\right)(\mathrm{mg} / \mathrm{L})$

Dissolved iron (Fe) (ug/L)

Dissolved manganese ( $\mathrm{Hn}$ ) ( $\mathrm{ug} / \mathrm{L}$ )

Dissolved calcium ( $\mathrm{CaO}(\mathrm{mg} / \mathrm{L})$

Dissolved magnesium ( $\mathrm{Mg})(\mathrm{mg} / \mathrm{L})$

Dissolved sodium (Na) (mg/L)

Dissolved potessium (K) (ng/L)

Bicarbonate $\left(\mathrm{HCO}_{3}\right)(\mathrm{mg} / \mathrm{L})$

Alkal inity $\left(\mathrm{CaCO}_{3}\right)(\mathrm{mg} / \mathrm{L})$

Dissolved sulfete $\left(\mathrm{SO}_{4}\right)(\mathrm{mg} / \mathrm{L})$

Dissolved chloride (Cl) (mg/L)

Dissolved fluoride (F) (mg/L)

Total nitrete (N) (ng/L)

Total nitrite (N) (no/L)

Totel phosphorus (P) (mg/L)

Harchess $\left(\mathrm{CeCO}_{3}\right)(\mathrm{mg} / \mathrm{L})$

Noncarbonate harchess ( $\mathrm{mg} / \mathrm{L}$ )

Specific conductence (micromos)

pH (units)

Color (pletinum-cobalt units)

Turbidity (JTU)

Dissolved carbon dioxide $\left(\mathrm{CO}_{2}\right)(\mathrm{mg} / \mathrm{L})$
Crescent Leke (site 113)

Dete sample collected
Dissolved chloride $(\mathrm{Cl})(\mathrm{mg} / \mathrm{L})$
Total nitrete $(\mathrm{H})(\mathrm{mg} / \mathrm{L})$
total nitrite $(\mathrm{H})(\mathrm{mg} / \mathrm{L})$
Totel phosphorus $(\mathrm{P})(\mathrm{mg} / .00$
pH (units)
Meximan value of 18 semples.
bedian value of 18 samples.
CMinimu value of 18 samples.

$\begin{array}{lll}7 / 27 / 71^{\mathrm{a}} & 7 / 27 / 71^{\mathrm{b}} & 7 / 27 / 71^{\mathrm{c}} \\ 8.5 & 2.2 & .2 \\ .62 & .26 & .15 \\ .05 & .01 & .00 \\ .29 & .14 & .00 \\ 8.1 & 7.8 & 7.2\end{array}$


TABLE 14. - Chemical and physical quality of water from selected surface-water sites in clallam county-Cont inued

12045500 Elwha River at McDonald Bridge near Port Angeles, Wash (Site 129)

Date sample collected

Discharge $\left(\mathrm{ft}^{3} / \mathrm{s}\right)$

Dissolved silica (SiD $)(\mathrm{mg} / \mathrm{L})$

Total iron (fe) (ug/L)

Dissolved iron (Fe) (ug/L)

Dissolved calcium (Ca) (mg/L)

Dissolved magnesium (Mg) (mg/L)

Dissolved sodium (Ma) ( $\mathrm{mg} / \mathrm{L})$

Dissolved potassium (K) (mg/L)

Bicarbonete $\left(\mathrm{HCO}_{3}\right)(\mathrm{mg} / \mathrm{L})$

Alkalinity $\left(\mathrm{CaCO}_{3}\right)(\mathrm{mg} / \mathrm{L})$

Dissolved sulfate $\left(\mathrm{SO}_{4}\right)(\mathrm{mg} / \mathrm{L})$

Dissolved chloride (Cl) (mg/h)

Dissolved fluoride (F) (mg/L)

Dissolved nitrate (W) (mg/L)

Total phosphorus (P) (mg/h)

Dissolved or thophosphate phosphorus

(P) $(\mathrm{mg} / \mathrm{L})$

Dissolved sol ids, residue at

$180^{\circ} \mathrm{C}$ (mg/L)

Herdness $\left(\mathrm{CaCO}_{3}\right)(\mathrm{mg} / \mathrm{L})$

Noncarbonate hardness (mg/L)

Specific conductance (micromhos)

pH (units)

Temperature $\left({ }^{\circ} \mathrm{C}\right)$

Color (plat inum-cobalt units)

Dissolved oxygen (mg/L)

Complete coliform (MPN)

Dissolved arsenic (As) (ug/L)

Dissolved boron (B) (ug/L)

Dissolved chromium (Cr) (ug/L)

Dissolved copper (Cu) (ug $/ L$ )

Dissolved zinc $(Z n)(u g / L)$

\begin{tabular}{|c|c|c|c|c|c|c|c|c|c|c|c|}
\hline \multicolumn{3}{|c|}{1959} & \multicolumn{9}{|c|}{1960} \\
\hline $10 / 19$ & $11 / 20$ & $12 / 16$ & $1 / 21$ & $2 / 18$ & $3 / 17$ & $4 / 11$ & $5 / 9$ & $6 / 9$ & $7 / 18$ & $9 / 7$ & $10 / 5$ \\
\hline 616 & 6500 & 4910 & 627 & 1940 & 1060 & 1900 & 1770 & 2330 & 1510 & -. & 348 \\
\hline 5.9 & 5.4 & 6.0 & 7.4 & 7.7 & 7.8 & 6.7 & 6.9 & 5.6 & 4.0 & -. & 5.9 \\
\hline 50 & 200 & 620 & 170 & 280 & 170 & 80 & 70 & 90 & 40 & $\cdots$ & $\cdots$ \\
\hline -. & $\cdots$ & -. & $\cdots$ & $\cdots$ & $\cdots$ & -. & -. & $\cdot \cdot$ & $\cdots$ & $\cdots$ & 0 \\
\hline 15 & 13 & 10 & 16 & 13 & 16 & 14 & 14 & 11 & 12 & -. & 16 \\
\hline .5 & 1.0 & .9 & 1.6 & 1.5 & 1.2 & .9 & 1.2 & 1.0 & .8 & -. & 1.2 \\
\hline 1.9 & 1.5 & 1.4 & 2.1 & 1.7 & 2.2 & 1.7 & 1.9 & 1.6 & 1.6 & -. & 2.2 \\
\hline .6 & .4 & .4 & .4 & .1 & .2 & .2 & .1 & .1 & .2 & .. & .0 \\
\hline 42 & 42 & 33 & 60 & 45 & 51 & 43 & 44 & 35 & 36 & -. & 49 \\
\hline 34 & 34 & 27 & 41 & 37 & 42 & 35 & 36 & 29 & 30 & $\cdots$ & 40 \\
\hline 8.0 & 4.9 & 6.4 & 9.0 & 6.8 & 8.7 & 7.0 & 1.0 & 5.8 & 6.4 & .. & 89.8 \\
\hline .8 & .0 & .0 & 1.0 & .6 & 1.0 & .2 & 1.0 & .5 & .2 & -. & 1.0 \\
\hline .0 & .3 & .1 & .1 & .1 & .1 & .1 & .1 & .1 & .0 & $\because$ & .0 \\
\hline .00 & .02 & .06 & .00 & .07 & .02 & .00 & .00 & .00 & .00 & $\cdots$ & .00 \\
\hline - & -. & -. & -. & -. & $\cdots$ & $\cdots$ & 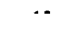 & -. & $\cdots$ & $\cdots$ & $\cdots$ \\
\hline .01 & .02 & .04 & .00 & .00 & .01 & .00 & .00 & .00 & .00 & .. & .00 \\
\hline 55 & 55 & 46 & 66 & 54 & 65 & 52 & 54 & 42 & 47 & -. & 67 \\
\hline 40 & 36 & 30 & 46 & 39 & 45 & 38 & 40 & 32 & 33 & -. & 45 \\
\hline 5 & 2 & 2 & 6 & 2 & 3 & 4 & 4 & 3 & 4 & $\because$ & 5 \\
\hline 90 & 80 & 71 & 106 & 90 & 103 & 89 & 91 & 70 & 76 & .. & 100 \\
\hline 7.4 & 6.4 & 7.3 & $7 . .7$ & 8.0 & 7.8 & 7.6 & 7.8 & 7.8 & 7.6 & $\cdots$ & 7.6 \\
\hline 11.3 & 5.9 & 5.4 & 3.2 & 5.8 & 5.0 & 7.0 & 10.5 & 10.5 & 13.9 & 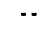 & 13.3 \\
\hline 5 & 5 & 10 & 6 & 10 & 5 & 5 & 5 & 5 & 5 & $\cdots$ & 5 \\
\hline 10.9 & 12.2 & 12.6 & 13.1 & 12.4 & $\cdots$ & 12.1 & 10.6 & 10.4 & 10.4 & $\cdots$ & 10.6 \\
\hline 30 & 0 & 0 & 0 & 0 & 0 & 0 & 0 & 0 & 30 & $\cdots$ & 0 \\
\hline$\cdots$ & 0 & $\cdots$ & .. & $\cdots$ & $\cdots$ & 0 & $\cdots$ & $\cdots$ & $\cdot \cdot$ & 0 & - \\
\hline$\cdots$ & $\cdots$ & $\because$ & $\cdots$ & $\cdots$ & $\cdots$ & 10 & $\cdots$ & $\cdots$ & $\cdot-$ & $\cdots$ & - \\
\hline -. & 0 & .. & $\cdots$ & $\cdots$ & .. & 0 & $\cdots$ & $\cdot$ & $\cdots$ & 40 & $\cdots$ \\
\hline$\because$ & 0 & .. & $\cdots$ & $\cdots$ & $\cdots$ & - & $\cdots$ & $\cdots$ & $\cdots$ & 0 & $\cdots$ \\
\hline$\cdots$ & $\cdots$ & $\cdots$ & -. & $\cdots$ & $\cdots$ & $\cdots$ & $\cdots$ & $\cdot-$ & $\cdot \cdot$ & $\cdots$ & $\cdots$ \\
\hline
\end{tabular}


TABLE 14.--Chemical and physical quality of water from selected surface-water sites in clallam County--Continued

12045500 El wha River at McDonald Bridge neor Port Angeles, Wash (site 129) -. Continued

\begin{tabular}{|c|c|c|c|c|c|c|c|c|c|c|c|c|c|c|}
\hline \multicolumn{3}{|c|}{1961} & \multicolumn{5}{|c|}{1962} & \multicolumn{2}{|c|}{1963} & \multicolumn{2}{|c|}{1964} & \multirow{2}{*}{$\frac{1965}{6 / 2}$} & \multicolumn{2}{|c|}{1966} \\
\hline $1 / 5$ & $4 / 4$ & $7 / 17$ & $11 / 1$ & $2 / 14$ & $5 / 14$ & $8 / 14$ & $12 / 13$ & $6 / 17$ & $12 / 23$ & $6 / 15$ & $12 / 14$ & & $2 / 7$ & $7 / 9$ \\
\hline 2510 & 1940 & 1660 & 952 & 1190 & 1440 & 761 & 2970 & 1560 & 1230 & 3170 & 1240 & 2110 & $\cdots$ & $\cdots$ \\
\hline 6.9 & 7.0 & 4.8 & 5.9 & 6.6 & 6.8 & 5.6 & 6.6 & 5.5 & 6.0 & 4.3 & 5.7 & 5.7 & $\cdots$ & -. \\
\hline 60 & 80 & 30 & 60 & 708 & 80 & 50 & 360 & 20 & 580 & 550 & 130 & 40 & . & .. \\
\hline .. & $\cdots$ & -. & .. & $\cdots$ & $\cdots$ & .. & $\cdots$ & .. & . & $\ldots$ & .. & .. & $\cdots$ & $\cdots$ \\
\hline 14 & 16 & 11 & 15 & 14 & 14 & 13 & 14 & 12 & 14 & 10 & 12 & 12 & .. & .. \\
\hline 1.2 & .9 & .7 & .6 & 1.1 & 1.3 & .8 & .5 & 1.0 & .8 & 1.0 & 1.5 & 1.1 & $\cdots$ & .. \\
\hline 1.9 & 1.9 & 1.5 & 2.0 & 1.9 & 2.1 & 1.8 & 2.1 & 1.4 & 2.3 & 1.5 & 2.0 & 1.9 & .. & $\cdots$ \\
\hline .0 & .1 & .0 & .3 & .3 & .2 & .4 & .0 & .2 & .3 & .1 & .2 & .4 & .. & $\cdots$ \\
\hline 44 & 44 & 34 & 44 & 43 & 44 & 39 & 40 & 37 & 41 & 32 & 41 & 38 & . & 36 \\
\hline 36 & 36 & 28 & 36 & 35 & 36 & 32 & 33 & 30 & 34 & 26 & 34 & 31 & .. & 30 \\
\hline 7.4 & 8.6 & 6.6 & 8.6 & 8.2 & 8.2 & 8.0 & 8.2 & 7.2 & 7.4 & 5.8 & 7.2 & 7.2 & .. & .. \\
\hline .5 & .8 & .8 & 1.0 & .8 & .5 & .5 & 1.0 & .5 & .8 & .5 & .5 & 1.0 & .. & $\cdots$ \\
\hline .1 & .1 & .1 & .1 & .1 & .1 & .1 & .1 & .0 & .1 & .1 & .0 & .1 & .. & $\cdots$ \\
\hline .02 & .02 & .02 & .05 & .07 & .00 & .02 & .02 & .00 & .05 & .02 & .05 & .07 & $\cdots$ & $\cdots$ \\
\hline$\cdots$ & $\cdots$ & $\cdots$ & $\cdots$ & -. & $\cdots$ & $\cdots$ & - & $\cdots$ & $\cdots$ & .. & .00 & .00 & $\cdots$ & $\cdots$ \\
\hline .01 & .00 & .00 & .00 & .00 & .00 & .00 & .01 & .00 & .00 & .01 & $\cdots$ & $\cdots$ & .. & . \\
\hline 57 & 62 & 44 & 58 & 58 & 58 & so & 57 & 48 & 54 & 43 & 52 & 49 & .. & - \\
\hline 40 & 41 & 30 & 40 & 40 & 40 & 36 & 37 & 34 & 38 & -. & 36 & 34 & $\cdots$ & -. \\
\hline 4 & 5 & 2 & 4 & 4 & 4 & 4 & 4 & 4 & 5 & 3 & 2 & 4 & -. & .. \\
\hline 93 & 92 & 69 & 93 & 90 & 94 & 82 & 83 & 76 & 88 & 63 & 82 & 79 & .. & .. \\
\hline 7.6 & 7.6 & 7.7 & 7.5 & 7.7 & 7.3 & 7.7 & 7.5 & 7.5 & 7.4 & 7.2 & 7.7 & 7.6 & $\cdots$ & - \\
\hline 4.8 & 7.5 & 15.5 & 7.8 & 5.6 & 6.7 & 15.5 & 5.5 & 14.5 & 6.5 & 10.1 & 4.4 & 12.0 & 5.3 & . \\
\hline 5 & 5 & 5 & 5 & 5 & 5 & 5 & 5 & 0 & 5 & 0 & 5 & 0 & $\cdots$ & .. \\
\hline .. & 12.6 & 10.0 & 19.0 & 12.5 & 11.2 & 10.0 & 14.1 & 10.8 & 12.5 & 11.5 & 12.6 & 11.9 & 12.2 & . \\
\hline 0 & 0 & 0 & 0 & 0 & 0 & 0 & 430 & 0 & 30 & 0 & 30 & 0 & 36 & .. \\
\hline 0 & $\cdots$ & .. & 0 & $\because$ & 0 & $\cdots$ & 0 & 0 & 0 & $\cdots$ & 0 & 0 & 0 & 0 \\
\hline 0 & $\cdots$ & .. & 0 & .. & 30 & $\cdots$ & 10 & 0 & 0 & 10 & 0 & 0 & 0 & 30 \\
\hline 10 & $\cdots$ & $\cdots$ & 20 & .. & 0 & $\cdots$ & 0 & 0 & 10 & 0 & 0 & 0 & 10 & - \\
\hline 0 & .. &.. & 10 & .. & 0 & .. & 0 & 0 & 40 & 0 & 20 & 10 & 10 & -. \\
\hline . & .. & $\cdots$ & 0 & $\cdot \cdot$ & 0 & $\cdots$ & 0 & 0 & 0 & 0 & 0 & 0 & 0 & $\cdots$ \\
\hline
\end{tabular}


TABLE 14.--Chenical and physical quality of watar from selected surface-water sites in clallan County--Continued

12045500 Elwhe River at McDonald Bridge near Port Angeles, Wash. (Site 129) (continued)

Date sample collected

Diacharge $\left(\mathrm{ft}^{3} / \mathrm{s}\right)$

Dissolved sillica $\left(\mathrm{SiO}_{2}\right)(\mathrm{mg} / \mathrm{L})$

Total iron (fa) (ug/L)

Dissolved iron ( $F a)(u g / L)$

Total manganesa $(\mathrm{H} n)(\mathrm{ug} / \mathrm{L})$

Suspended manganesa (Mn) (Ug/L)

Dissolved manganesa (Mn) (Ug/L)

Dissolved calciun (Ca) (mo/L)

Dissolved magnesiun (Mg) (mo/L)

Dissolved sodiun (Ha) (mo/L)

Dissolved potassiun (K) (mo/L)

Bicarbonate $\left(\mathrm{HCO}_{3}\right)(\mathrm{mo} / \mathrm{L})$

Alkalinity $\left(\mathrm{CaCO}_{3}\right)(\mathrm{ma} / \mathrm{L})$

Diasolved sulfata $\left(\mathrm{SO}_{4}\right)(\mathrm{mo} / \mathrm{L})$

Dissolved chloride (Cl) (mo/L)

Dissolved fluoride (F) (mo/L)

Total nitrata (N) (mo/L)

Dissolved nitrata (W) $(\mathrm{mg} / \mathrm{L})$

Total nitrite (N) (mo/L)

Total nitrite plus nitrate (W) $(\mathrm{mo} / \mathrm{L})$

Total amonia (H) (mo/L)

Total organic nitrogen (N) $(\mathrm{mo} / \mathrm{L})$

Total amonila plus organic

nitrooen (N) $(m / L)$

Total nitrogen (N) $(m / L)$

Total phosphorus (P) (mo/L:

Dissolved orthophosphata

phosphorus (P) (mo/L)

Dissolved sol ids, residue at

$180^{\circ} \mathrm{C}(\mathrm{mo} / \mathrm{L})$

Hardness $\left(\mathrm{CaCO}_{3}\right)(\mathrm{mo} / \mathrm{L})$

Noncarbonate hardness (mo/L)

Specific conductance (micromhos)

PH (units)

chlorophyll B periphyton,

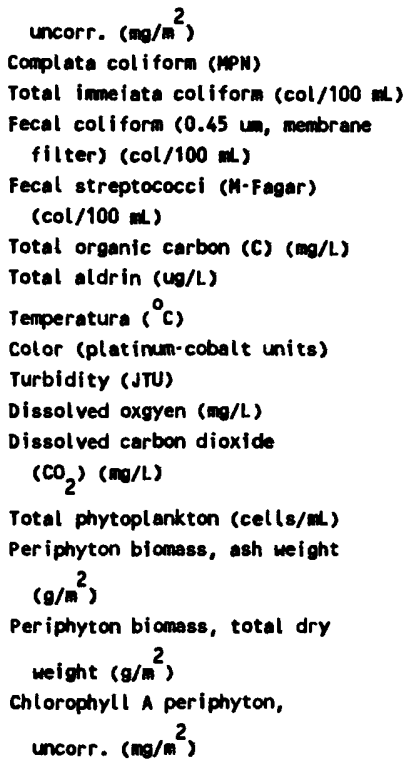

\begin{tabular}{|c|c|c|c|c|c|c|c|c|c|c|c|}
\hline \multirow{2}{*}{$\frac{1966}{8 / 1}$} & \multicolumn{2}{|c|}{1967} & \multicolumn{2}{|c|}{1968} & \multicolumn{5}{|c|}{1974} & \multirow[b]{2}{*}{$1 / 21$} & \multirow[b]{2}{*}{$2 / 19$} \\
\hline & $5 / 17$ & $10 / 17$ & $4 / 17$ & $11 / 12$ & $9 / 3$ & $6 / 12$ & $10 / 25$ & $11 / 21$ & $12 / 18$ & & \\
\hline -. & 2680 & 1440 & 1040 & 1010 & 786 & 1460 & 446 & 2710 & 2010 & 1620 & 1070 \\
\hline 5.7 & 6.3 & 4.5 & 6.7 & 5.5 & 5.6 & 5.0 & 5.8 & 5.4 & 5.7 & 6.2 & 6.4 \\
\hline -. & $\cdots$ & $\cdots$ & .. & $\cdots$ & .. & .. & 50 & .. & .. & 840 & $\cdots$ \\
\hline$\cdots$ & $\cdots$ & $\because$ & $\because$ & $\cdots$ & $\cdots$ & .. & 30 & $\cdots$ & $\cdots$ & 10 & $\cdots$ \\
\hline .. & .. & .. & .. & $\cdots$ & .. & -. & 20 & .. & .. & 10 & $\cdots$ \\
\hline -. & $\cdots$ & $\cdots$ & $\because \cdot$ & $\cdots$ & .. & $\cdots$ & 0 & $\cdots$ & .. & 10 & $\cdots$ \\
\hline .. & $\cdots$ & . & .. & .. & .. & .. & 20 & .. & .. & 0 & $\cdots$ \\
\hline 12 & 15 & 12 & 15 & 11 & 14 & 11 & 15 & 17 & 14 & 14 & 15 \\
\hline 1.1 & 1.5 & 1.0 & 1.7 & 2.2 & 1.1 & .9 & .5 & 1.1 & 1.1 & 1.6 & 1.5 \\
\hline .19 & 2.1 & 1.6 & 2.5 & 1.8 & 2.1 & 1.4 & 2.2 & 2.6 & 2.2 & 2.4 & 2.0 \\
\hline .4 & .4 & .1 & .7 & .2 & .2 & .2 & .1 & .5 & .1 & .4 & .3 \\
\hline 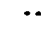 & 47 & 37 & 52 & 40 & 42 & 34 & 46 & 48 & 38 & 40 & 46 \\
\hline .. & 39 & 30 & 43 & 33 & 34 & 28 & 38 & 39 & 31 & 33 & 38 \\
\hline 7.2 & 8.2 & 5.8 & 7.0 & 7.2 & 7.4 & 6.2 & 8.5 & 7.6 & 6.9 & 6.2 & 10 \\
\hline 1.0 & .5 & .6 & .7 & .5 & 1.2 & .3 & 1.0 & .3 & .2 & .8 & 1.5 \\
\hline .1 & .1 & .1 & .1 & .1 & .1 & .1 & .0 & .0 & .0 & .0 & .1 \\
\hline .07 & 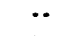 & $\because$ & $\cdots$ & $\cdots$ & $\cdots$ & $\cdots$ & 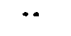 & $\cdots$ & $\cdots$ & $\because$ & $\cdots$ \\
\hline .05 & .02 & .00 & .00 & .00 & .05 & .07 & $\cdots$ & -. & .. & .. & $\cdots$ \\
\hline$\cdots$ & $\cdots$ & $\because$ & $\cdots$ & . & .. & $\cdots$ & $\cdots$ & $\cdots$ & $\cdots$ & .. & $\cdots$ \\
\hline$\cdots$ & $\cdots$ & $\because$ & $\cdots$ & $\cdots$ & $\cdots$ & 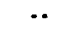 & .02 & .03 & .05 & .02 & .03 \\
\hline$\cdots$ & $\because$ & $\cdots$ & 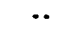 & -. & -. & $\cdots$ & $\cdots$ & .. & -. & . & $\cdots$ \\
\hline$\cdots$ & $\cdots$ & $\cdots$ & $\cdots$ & 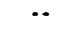 & $\cdots$ & $\cdots$ & $\cdots$ & $\cdots$ & $\cdots$ & $\cdots$ & $\cdots$ \\
\hline .. & .. & .. & .. & .. & $\cdots$ & $\cdots$ & .05 & .20 & .11 & .12 & .10 \\
\hline -. & $\cdots$ & $\cdots$ & .. & .. & .. & .. & .07 & .23 & .15 & .14 & .13 \\
\hline$\cdots$ & $\cdots$ & $\cdots$ & $\cdots$ & . & $\cdots$ & $\cdots$ & .02 & .04 & .02 & .08 & .02 \\
\hline$\cdots$ & $\cdots$ & $\cdots$ & $\cdots$ & $\cdots$ & $\cdots$ & $\cdots$ & .. & . & $\cdots$ & $\cdots$ & $\cdots$ \\
\hline 49 & 60 & 47 & 62 & 51 & 56 & 41 & 57 & 54 & 55 & 55 & 55 \\
\hline$\cdots$ & 44 & 34 & 45 & 37 & 40 & 31 & 40 & 47 & 40 & 42 & 44 \\
\hline .. & 5 & 4 & 2 & 4 & 5 & 3 & 2 & 8 & 8 & 9 & 6 \\
\hline$\cdots$ & 96 & 72 & 100 & 83 & 90 & 71 & 76 & 99 & 89 & 95 & 100 \\
\hline •. & 8.1 & 7.7 & 7.7 & 7.5 & 7.3 & 7.2 & 7.2 & 7.2 & 7.4 & 6.9 & 7.4 \\
\hline
\end{tabular}

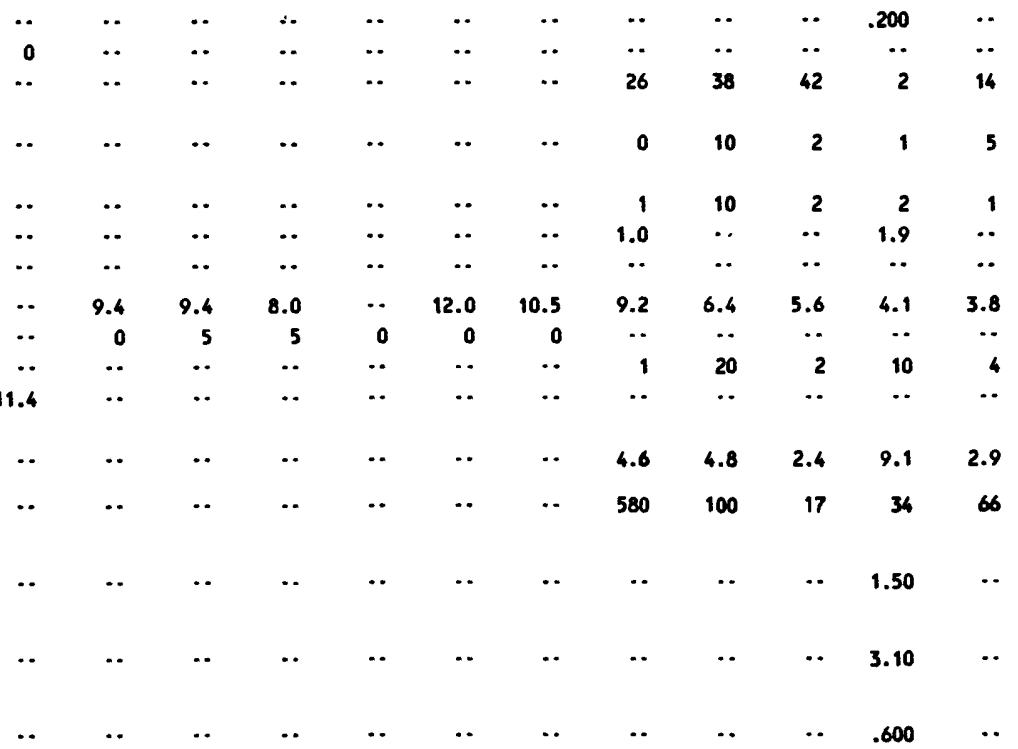




\begin{tabular}{|c|c|c|c|c|c|c|c|c|c|c|c|c|c|}
\hline \multicolumn{10}{|c|}{1975} & \multicolumn{4}{|c|}{1976} \\
\hline $3 / 11$ & $4 / 14$ & $5 / 20$ & $6 / 17$ & $7 / 14$ & $8 / 12$ & $9 / 16$ & $10 / 16$ & $11 / 21$ & $12 / 22$ & $1 / 21$ & $2 / 19$ & $3 / 22$ & $4 / 21$ \\
\hline 1270 & 877 & 1800 & $2450^{\circ}$ & 2170 & 912 & 730 & 1240 & 2130 & 1340 & 2100 & 1530 & 1100 & 1010 \\
\hline 6.0 & 6.3 & 5.3 & 4.6 & 4.2 & 4.5 & 5.3 & 4.7 & 5.9 & 5.9 & 5.4 & 6.1 & 6.6 & 6.3 \\
\hline .. & 120 & .. & .. & 320 & 00 & 00 & 90 & .. & $\cdots$ & 1100 & .. & $\cdots$ & 60 \\
\hline .. & 10 & .. & $\cdots$ & 20 & .. & -. & 30 & .. & .. & 60 & .. & .. & 30 \\
\hline .. & 210 & $\cdots$ & .. & 10 & .. & $\cdots$ & 20 & $\cdots$ & $\cdots$ & 20 & -. & $\cdots$ & 20 \\
\hline .. & 210 & .. & .. & 10 & .. & $\cdots$ & 10 & $\cdots$ & $\cdots$ & 20 & $\cdots$ & .. & 10 \\
\hline .. & 0 & .. & -. & 0 & .. & .. & 10 & .. & $\cdots$ & 0 & .. & .. & 10 \\
\hline 15 & 17 & 13 & 11 & 11 & 12 & 12 & 13 & 12 & 14 & 12 & 15 & 16 & 15 \\
\hline 1.7 & 1.7 & .9 & .6 & 1.1 & .8 & .3 & 1.0 & 1.2 & 1.3 & 1.8 & 1.8 & 3.0 & 2.1 \\
\hline 2.2 & 2.6 & 2.1 & 1.7 & 1.8 & 2.2 & 3.0 & 2.1 & 1.8 & 2.1 & 2.0 & 2.1 & 3.0 & 2.7 \\
\hline .3 & .8 & .3 & .1 & .1 & .1 & .1 & .2 & .2 & .5 & .4 & .3 & .3 & .2 \\
\hline 46 & 52 & 40 & 31 & 29 & 36 & 32 & 39 & 38 & 41 & 40 & 67 & 56 & 52 \\
\hline 38 & 43 & 33 & 25 & 24 & 30 & 26 & 32 & 31 & 34 & 33 & 39 & 46 & 43 \\
\hline 9.6 & 8.9 & 7.1 & 5.6 & 5.7 & 6.3 & 7.5 & 7.6 & 5.2 & 7.2 & 6.2 & 8.0 & 10 & 11 \\
\hline 1.6 & 1.1 & .5 & 1.3 & .8 & 1.7 & 1.6 & 1.9 & 1.4 & 1.6 & 1.3 & 1.1 & 1.3 & .8 \\
\hline .1 & .1 & .1 & .1 & .0 & .1 & .0 & .1 & .1 & .1 & .1 & .1 & .1 & .1 \\
\hline .. & -. & .. & .02 & .00 & .00 & .00 & .02 & .02 & .00 & .04 & .02 & .01 & .00 \\
\hline$\cdots$ & .. & .. & .. & -. & -. & $\cdots$ & $\cdots$ & -. & -. & -. & -. & -. & -. \\
\hline$\cdots$ & -. & .. & .00 & .00 & .00 & .00 & .00 & .01 & .01 & .00 & .00 & .00 & .00 \\
\hline .02 & .01 & .01 & .02 & .00 & .00 & .00 & .02 & .03 & .01 & .04 & .02 & .01 & .00 \\
\hline .. & -. & .. & .04 & .05 & .03 & .03 & .04 & .13 & .10 & .10 & .04 & .02 & .04 \\
\hline .. & $\because$ & .. & .04 & .03 & .11 & .12 & .08 & .00 & .03 & .02 & .02 & .06 & .06 \\
\hline .10 & .07 & .09 & .08 & .08 & $: 14$ & .15 & .12 & .13 & .13 & .12 & .06 & .08 & .10 \\
\hline .12 & .08 & .10 & .10 & .08 & .16 & .15 & .14 & .16 & .14 & .16 & .8 & .009 & .10 \\
\hline .01 & .00 & .01 & .03 & .01 & .01 & .01 & .01 & .05 & .03 & 03 & .01 & .01 & .01 \\
\hline$\cdots$ & .. & $\cdot \cdot$ & .03 & .00 & .00 & .00 & .00 & .00 & .00 & .02 & .01 & .01 & .00 \\
\hline 66 & 62 & 44 & 40 & 35 & 48 & 54 & 52 & 56 & 68 & 46 & 54 & 65 & 66 \\
\hline 44 & 49 & 36 & 30 & 32 & 33 & 31 & 37 & 35 & 40 & 37 & 45 & 52 & 46 \\
\hline 7 & 7 & 3 & 5 & 8 & 4 & 5 & 5 & 4 & 7 & 4 & 5 & 5 & 3 \\
\hline 112 & 100 & $\pi$ & 60 & 60 & 87 & 84 & 82 & 76 & 82 & 79 & 97 & 100 & 87 \\
\hline 6.8 & 7.3 & 7.5 & 6.9 & 7.0 & 7.0 & 7.8 & $7: 7$ & 7.2 & 7.2 & 7.1 & 7.0 & 7.1 & 7.0 \\
\hline .. & .100 & 6.8 & 6.6 & 10.6 & 16.4 & 10.6 & 9.4 & 4.0 & 4.4 & 4.0 & 4.6 & 4.6 & 5.7 \\
\hline$\cdots$ & $\cdots$ & $\cdots$ & .. & .. & .. & $\cdots$ & -. & .. & - & .. & .. & $\cdots$ & - \\
\hline 1 & 4 & 2 & 4 & 3 & 7 & 1 & 2 & 25 & 10 & 10 & 3 & 2 & 1 \\
\hline 3 & 1 & $\therefore$ & .. & $\cdots$ & $\cdots$ & .. & -. & $\cdots$ & $\cdots$ & $\cdots$ & .. & $\cdots$ & .. \\
\hline 2 & 1 & 2.0 & 6.2 & 4.6 & 5.8 & .8 & 1.2 & 3.8 & 4.1 & 5.1 & 7.4 & 7.1 & 8.3 \\
\hline .. & .4 & 190 & 120 & 12 & 38 & 100 & 11 & 0 & 10 & 2 & 140 & 120 & 160 \\
\hline .. & $\cdots$ & $\cdot \cdot$ & $\cdot \cdot$ & .200 & -. & $\cdots$ & .000 & -. & -. & .. & -. & -. & -. \\
\hline 3.5 & 5.5 & .. & .. & .300 & $\cdots$ & $\cdots$ & .000 & .. & -. & $-\cdot$ & $\cdots$ & .. & .. \\
\hline .. & -. & .. & -. & .000 & $\cdots$ & $\cdots$ & .000 & -. & .. & $\cdots$ & .. & -. & $\cdots$ \\
\hline 1 & 1 & .. & .. & .000 & $\cdots$ & .. & .000 & .. & -. & $\cdots$ & .. & $\cdots$ & $\cdots$ \\
\hline$\cdot \cdot$ & -. & .. & $\cdots$ & $\because$ & $\cdots$ & $\cdot \cdot$ & $\cdots$ & .. & $\because$ & $\cdots$ & $\cdots$ & $\cdots$ & -. \\
\hline 12 & 4.2 & 11 & 18 & 2 & 5 & 10 & 95 & 27 & 11 & 4 & 7 & 2 & 16 \\
\hline 170 & 260 & 2 & 1 & 1 & 1 & 1 & 1 & 1 & 1 & 3 & 1 & 2 & 3 \\
\hline$\cdots$ & 1.00 & 1 & 1 & 3 & 3 & 1 & 1 & 2 & 1 & 1 & 1 & 1 & 1 \\
\hline . & 2.10 & $\cdots$ & -. & 2.7 & $\cdots$ & $\cdots$ & -. & -. & . & .8 & .. & .. & 1.4 \\
\hline$\cdots$ & .700 & $\mathbf{N D}$ & $\cdots$ & $\cdots$ & $\cdots$ & ND & $\cdots$ & $\cdots$ & $m$ & $\cdots$ & .. & ND & $\cdots$ \\
\hline
\end{tabular}


12045500 El wha River at MCDonald Bridge near Port Angeles, Wash. (Site 129) (continued)

Date sample collected

Discharge $\left(\mathrm{ft}^{3} / \mathrm{s}\right)$

Dissolved silice $\left(\mathrm{SiO}_{2}\right)$

Total iron (fe) (ug/L)

Dissolved iron (fe) (ug/L)

Total manganese (Mn) (ug/L)

Suspended manganese (Mn) (ug/L)

Dissolved manganese (Mn) (ug/L)

Dissolved calcium (Ca) (mg/L)

Dissolved magnesium ( $\mathrm{Mg})(\mathrm{mg} / \mathrm{L}$ )

Dissolved sodium (Na) ( $\mathrm{mg} / \mathrm{L}$ )

Dissoved potassium (K) (mg/L)

Bicarbonate $\left(\mathrm{HCO}_{3}\right)(\mathrm{mg} / \mathrm{L})$

Alkalinity $\left(\mathrm{CaCO}_{3}\right)(\mathrm{mg} / \mathrm{L})$

Dissolved sulfste $\left(\mathrm{SO}_{4}\right)(\mathrm{mg} / \mathrm{L})$

Dissolved chloride (Cl) (mg/L)

Dissolved fluoride (f) $(\mathrm{mg} / \mathrm{L})$

Total nitrate $(N)(\mathrm{mg} / \mathrm{L})$

Total nitrite (N) (mg/L)

Total nitrite plus nitrata (W) (mg/L)

Total amonia (N) $(\mathrm{mg} / \mathrm{L})$

Total organic nitrogen (N) (mg/L)

Suspended amonia plus organic nitrogen (N) (mg/l)

Dissolved amonia plus organic nitrogen

(N) $(\mathrm{mg} / \mathrm{L})$

Total nitrogen (W) $(\mathrm{mg} / \mathrm{L})$

Total phosphorus (P) (mg/L)

0 issolved phosphorus (P) (mg/L)

Dissolved orthophosphate phosphorus (P) (ng/L)

Dissolved solids, residue at $180^{\circ} \mathrm{C}(\mathrm{mg} / \mathrm{L})$

suspended solids, at $105^{\circ} \mathrm{C}(\mathrm{mg} / \mathrm{L})$

suspended solids, at $110^{\circ} \mathrm{C}(\mathrm{mg} / \mathrm{L})$

Hardness $\left(\mathrm{CaCO}_{3}\right)(\mathrm{mg} / \mathrm{L})$

Moncarbonate hardness (mg/L)

Specific conductance (micromhos)

pH (units)

Temperature $\left({ }^{\circ} \mathrm{C}\right)$

Turbidity (JTU)

Dissolved oxygen ( $m g / L$ )

Dissolved carbon dioxide $\left(\mathrm{CO}_{2}\right)(\mathrm{mg} / \mathrm{t})$

Total phytoplankton (cells/m)

Periphyton biamass, ash weight $\left(9 / \mathrm{m}^{2}\right)$

Periphyton biomass, total dry weight $\left(9 / \mathrm{m}^{2}\right)$

Chlorophyll A periphyton, uncorr. (mg/m $\mathrm{m}^{2}$ )

Chlorophyll A periphyton, chromographic

fluorom. $\left(\mathrm{mg} / \mathrm{m}^{2}\right)$

Chlorophyll B periphyton, uncorr. (mg/m ${ }^{2}$ ) chlorophyli B periphyton, chromographic

fluorom. $\left(\mathrm{mg} / \mathrm{m}^{2}\right)$

Total inmediste coliform (col/100 m)

fecal coliform ( $0.45 \mathrm{um}$, membrane filter)

(col/100 m)

\begin{tabular}{|c|c|c|c|c|c|c|c|c|c|c|c|}
\hline \multicolumn{8}{|c|}{1976} & \multicolumn{4}{|c|}{1977} \\
\hline $5 / 19$ & $6 / 30$ & $7 / 22$ & $8 / 25$ & $9 / 27$ & $10 / 26$ & $12 / 1$ & $12 / 27$ & $1 / 26$ & $3 / 1$ & $3 / 28$ & $4 / 22$ \\
\hline 2080 & 2590 & 1730 & 1000 & 698 & 572 & 435 & 1260 & 650 & 1030 & 704 & 872 \\
\hline 4.9 & 4.3 & 4.3 & 4.4 & 4.6 & 5.1 & 4.5 & 5.7 & 5.4 & 5.0 & 6.3 & 6.3 \\
\hline .. & .. & 170 & .. & .. & 60 & .. & .. & 240 & $\cdots$ & $\cdots$ & 80 \\
\hline .. & $\cdots$ & 10 & $\ldots$ & .. & 30 & -. & .. & 10 & .. & $\cdots$ & 10 \\
\hline .. & -. & 10 & .. & - & 10 & -. & .. & 0 & -. & .. & 10 \\
\hline .. & .. & 10 & .. & $\cdots$ & 10 & -. & -. & 0 & -. & -. & 0 \\
\hline .. & -. & 0 & .. & $\cdots$ & 0 & .. & $\cdots$ & 0 & $-\cdot$ & -. & 10 \\
\hline 12 & 10 & 11 & 13 & 14 & 15 & 14 & 15 & 14 & 13 & 15 & 16 \\
\hline .9 & .9 & .7 & 1.0 & 1.1 & 1.2 & 1.3 & 1.8 & 1.2 & 1.1 & 1.7 & 1.3 \\
\hline 1.4 & 1.3 & 1.3 & 1.8 & 1.8 & 2.1 & 1.9 & 2.1 & 1.8 & 2.2 & 2.6 & 2.5 \\
\hline .1 & .1 & .1 & .1 & .1 & .1 & .1 & .1 & .1 & .2 & .2 & .2 \\
\hline 35 & 33 & 31 & 37 & 41 & 43 & 45 & 45 & 40 & 41 & 44 & 45 \\
\hline 29 & 27 & 25 & 30 & 34 & 35 & 37 & 37 & 33 & 34 & 36 & 37 \\
\hline 6.9 & 7.0 & 6.0 & 7.5 & 8.3 & 7.9 & 8.7 & 8.4 & 8.2 & 8.4 & 9.7 & 7.5 \\
\hline .9 & 1.0 & .8 & 1.1 & 2.2 & .8 & .8 & 1.1 & 2.1 & .8 & 1.3 & 1.5 \\
\hline .1 & .1 & .2 & .1 & .2 & .0 & .1 & .1 & .1 & .0 & .1 & .0 \\
\hline .02 & .00 & .00 & .00 & .00 & .00 & .01 & .04 & .03 & .03 & .00 & .00 \\
\hline .00 & .00 & .00 & .00 & .00 & .00 & $.0 !$ & .04 & .03 & .03 & .00 & .00 \\
\hline .02 & .00 & .00 & .00 & .00 & .00 & .01 & .04 & .04 & .03 & .01 & .00 \\
\hline .04 & .02 & .02 & .02 & .01 & .02 & .01 & .02 & .04 & .02 & .04 & .03 \\
\hline .05 & .02 & .00 & .00 & .01 & .07 & .07 & .11 & .05 & .08 & .10 & .04 \\
\hline
\end{tabular}

$\begin{array}{llllllllllll}. & . & . . & . . & . . & . . & . . & . . & . . & . . & . . & . . \\ .11 & .04 & .02 & .02 & .02 & .09 & .10 & .17 & .13 & .13 & .15 & .07 \\ .02 & .01 & .01 & .01 & .01 & .01 & .01 & .01 & .01 & .01 & .01 & .01\end{array}$

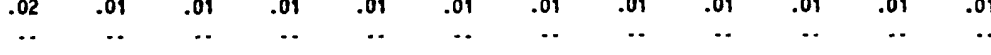

$\begin{array}{llllllllllll}.00 & .00 & .00 & .00 & .00 & .01 & .00 & .00 & .00 & .00 & .00 & .01\end{array}$

$\begin{array}{llllllllllll}50 & 45 & 30 & 45 & 56 & 63 & 51 & 68 & 59 & 50 & 50 & 57\end{array}$

$\begin{array}{lllllllllll}. . & \ldots & \ldots & 0 & 5 & 13 & 5 & 0 & 0 & 0 & 4\end{array}$

$\begin{array}{rrrrrrrrrrrr}. . & . . & 0 & . & 5 & . . & \ldots & \ldots & \ldots & . . & . . & . . \\ 34 & 29 & 30 & 37 & 40 & 42 & 40 & 45 & 40 & 37 & 44 & 45\end{array}$

$\begin{array}{rrrrrrrrrrrr}5 & 2 & 5 & 6 & 6 & 7 & 3 & 9 & 7 & 3 & 8 & 8 \\ 69 & 62 & 63 & 62 & 65 & 88 & 88 & 86 & 70 & 82 & 90 & 100\end{array}$

$\begin{array}{llllllllllll}7.1 & 7.3 & 7.1 & 7.2 & 7.2 & 6.6 & 7.1 & 7.0 & 7.0 & 7.0 & 7.1 & 7.0\end{array}$

$\begin{array}{llllllllllll}7.2 & 8.9 & 9.4 & 10.8 & 11.6 & 9.1 & 4.9 & 4.6 & 2.6 & 4.4 & 5.4 & 8.5\end{array}$

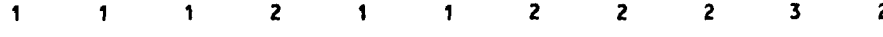

$\begin{array}{llllllllllll}4.6 & 2.6 & 3.9 & 3.7 & 4.1 & 17 & 5.7 & 7.2 & 6.4 & 5.5 & 5.6 & 7.2\end{array}$

$\begin{array}{llllllllllll}75 & 160 & 60 & 70 & 300 & 210 & 8 & 90 & 58 & \ldots & \ldots & \end{array}$

$\begin{array}{lllllllllllll}\ldots & \ldots & \ldots & \ldots & .07 & .462 & .538 & \ldots & \ldots & \ldots & \ldots & \ldots & \ldots\end{array}$

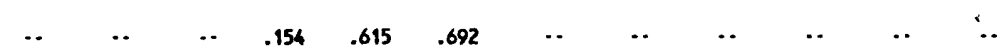

$\begin{array}{lllllllllllll}\cdots & \ldots & \ldots & .000 & \ldots & \ldots & \ldots & \ldots & \ldots & \ldots & \ldots & \ldots\end{array}$ 
TABLE 14.-.Chemical and physical quality of water from selected surface-water sites in Clallam County - Cont inued

12045500 Elwha River at McDonald Bridge near Port Angeles, Wash. (Site 129) (cont inued)

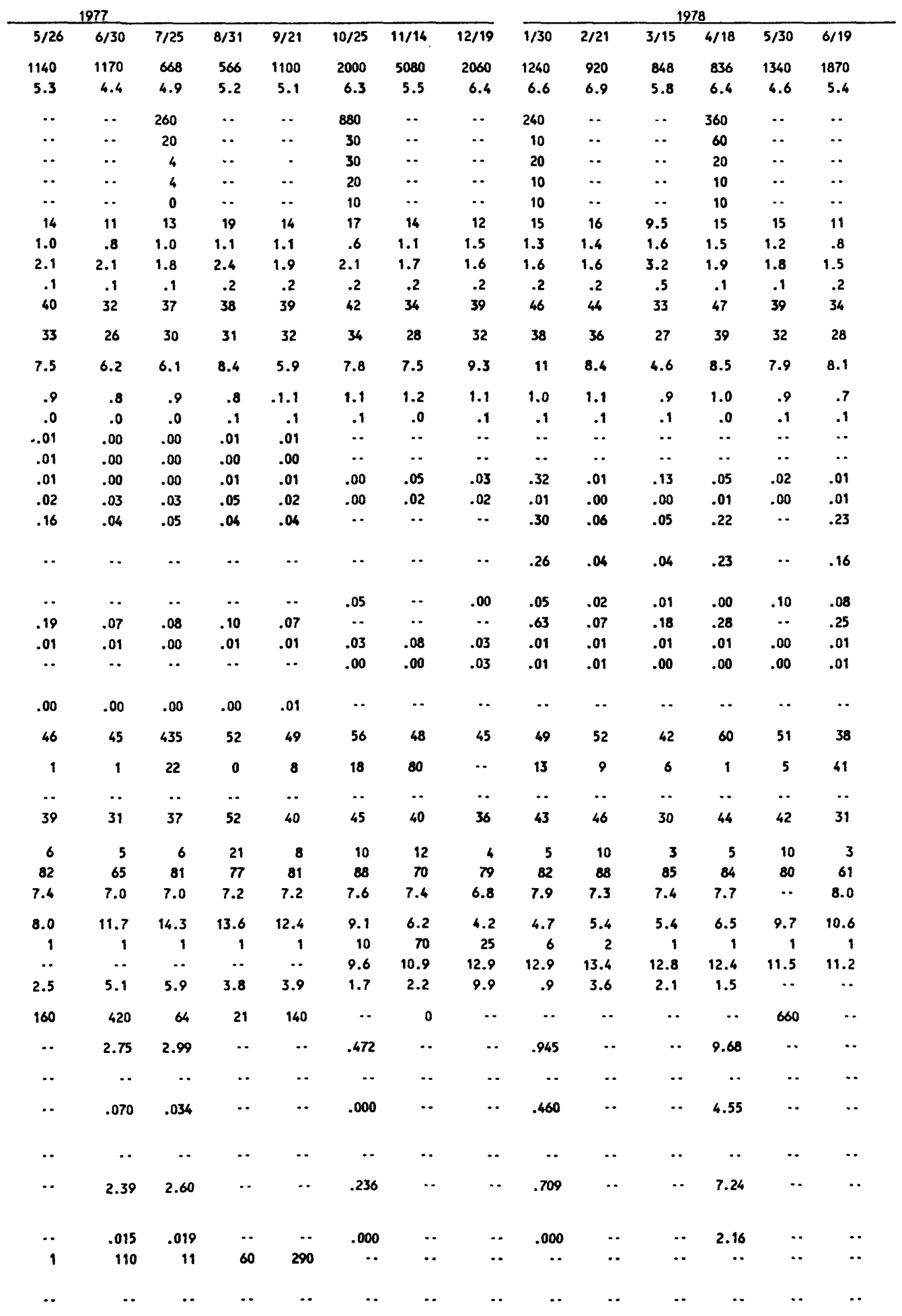


12045500 Elwhe River at McDonald Bridge near Port Angeles, Mash. (Site 129) (continued)

Date aample collected

Total otrazine (ug/L)

Total chloridane (ug/L)

Total DoD (Lo/L)

Total DOE ( $\mathrm{Lg} / \mathrm{L})$

Total DOT ( $u / / L$ )

Total diazinon (La/l)

Total dieldrin (ua/L)

Total endrin (La/L)

Total ethion (uo/L)

Total heptochlor (ug/L)

Total ehptechlor epoxide (ug/L)

Total I indane ( $\mathrm{Lg} / \mathrm{L})$

Total matathion ( $u g / L)$

Total wethoxychlor (ug/L)

Total methyl parathion (ug/L)

Total wethyl trittion ( $u=/ L$ )

Total parathion (ug/L)

Total trithion (ua/L)

Total toxaphene $(\mathrm{ug} / L)$

Total 2, 4-D (ug/L)

Total 2, 4, 5.T (ug/L)

Total allvex $\left(\omega_{0} / L\right)$

Total arsenic (As) (ua/L)

Dissolved aresenic (As) ( $u g / L)$

Dissolved boron (B) (us/L)

Total cadhiun (Cd) (ug/L)

Diseolved cadmiun (Cd) ( $\mathrm{Ug} / \mathrm{L}$ )

Total chromiun (Cr) (ug/L)

Diseolved chromiun (Cr) ( $\mathrm{U} / \mathrm{h} / \mathrm{s})$

Total cobalt (co) (uon)

Dissolved cobelt (Co) ( ug/L)

Total copper (Cu) (ug/L)

Dissolved copper (Cu) (ug/h)

Total lead (Pb) (ug/L)

Dissolved lead $(\mathrm{Pb})(\mathrm{co} / \mathrm{L})$

Total mercury (Mg) (La/L)

Dissolved mercury (Hg) (ug/L)

Total seleniun (Se) (ug/L)

Dissolved seleniun (Se) (ug/L)

Total zinc ( $2 n)$ (ug/L)

Dissolved zinc (2n) (ug/L)

\begin{tabular}{|c|c|c|c|c|c|c|c|c|c|c|c|c|c|}
\hline \multirow{2}{*}{$\begin{array}{r}1966 \\
8 / 1\end{array}$} & \multicolumn{2}{|c|}{1967} & \multicolumn{2}{|c|}{1968} & \multicolumn{5}{|c|}{1974} & \multirow[b]{2}{*}{$1 / 21$} & \multirow[b]{2}{*}{$2 / 19$} & \multicolumn{2}{|r|}{1975} \\
\hline & $5 / 17$ & $10 / 17$ & $4 / 17$ & $11 / 12$ & $9 / 3$ & $6 / 12$ & $10 / 25$ & $11 / 21$ & $12 / 18$ & & & $3 / 11$ & $4 / 14$ \\
\hline$\cdots$ & -. & -. & -. & .. & .. & .. & -. & .. & $\cdots$ & .. & $\cdots$ & $\cdots$ & $\therefore$ \\
\hline$\cdots$ & $\cdots$ & -. & $\cdots$ & .. & -. & $\cdots$ & -. & -. & .. & $\cdots$ & .. & -. & .. \\
\hline$\cdots$ & .. & .. & .. & .. & .. & .. & .. & .. & .. & .. & $\cdots$ & .. & $\cdots$ \\
\hline$\cdots$ & -. & -. & -. & -. & .. & $\because$ & .. & .. & .. & $\cdots$ & .. & -. & . \\
\hline$\cdots$ & -. & .. & $\cdots$ & -. & .. & $\cdots$ & $\cdots$ & $\cdots$ & $\cdots$ & $\cdots$ & .. & $\because$ & . \\
\hline$\cdots$ & 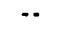 & -. & .. & -. & -. & $\cdots$ & .. & $\cdots$ & -. & .. & .. & -. & $\therefore$ \\
\hline$\cdots$ & .. & .. & $\cdots$ & $\cdots$ & -. & .. & $\cdots$ & .. & -. & .. & $\cdots$ & -. & .. \\
\hline$\cdots$ & $\cdots$ & $\cdots$ & $\cdots$ & .. & .. & $\cdots$ & .. & $\ldots$ & -. & .. & $\cdots$ & $\cdot$. & $\cdots$ \\
\hline$\cdots$ & $\cdots$ & .. & $\because$. & .. & .. & .. & $\cdots$ & -. & .. & $\cdots$ & $\cdots$ & $\cdots$ & $\cdots$ \\
\hline$\cdots$ & $\because$ & -. & $\cdots$ & -. & -. & -. & .. & .. & -. & $\cdots$ & $\cdots$ & -. & - \\
\hline .. & 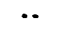 & .. & .. & $\cdots$ & -. & -. & $\cdots$ & $\cdots$ & 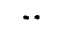 & $\cdots$ & $\cdots$ & .. & . \\
\hline$\cdots$ & $\cdots$ & $\cdots$ & $\cdots$ & $\cdots$ & -. & $\cdots$ & .. & .. & $\cdots$ & $\cdots$ & $\cdots$ & $\cdots$ & $\cdots$ \\
\hline$\cdots$ & $\cdots$ & .. & $\cdots$ & $\cdots$ & .. & $\cdots$ & .. & $\cdots$ & $\cdots$ & $\cdots$ & $\cdots$ & $\because$ & .. \\
\hline$\cdots$ & $\cdots$ & $\cdots$ & $\cdots$ & $\cdots$ & -. & -. & .. & $\cdots$ & .. & $\cdots$ & $\cdots$ & .. & $\cdots$ \\
\hline$\cdots$ & 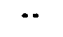 & 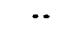 & $\cdots$ & $\cdots$ & -. & $\cdots$ & -. & .. & $\cdots$ & .. & .. & .. & $\because$ \\
\hline$\cdots$ & $\cdots$ & $\cdots$ & $\cdots$ & $\cdots$ & .. & - & $\cdots$ & $\cdots$ & $\cdots$ & $\cdots$ & $\cdots$ & .. & $\cdots$ \\
\hline$\cdots$ & $\cdots$ & $\cdots$ & $\cdots$ & $\cdots$ & -. & $\cdots$ & .. & 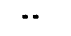 & .. & -. & 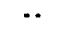 & .. & .. \\
\hline$\cdots$ & $\cdots$ & $\because$ & $\cdots$ & -. & -. & .. & $\cdots$ & $\cdots$ & -. & .. & $\cdots$ & $\cdots$ & $\cdots$ \\
\hline$\cdots$ & $\cdots$ & $\because$ & 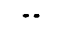 & 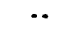 & $\cdots$ & $\cdots$ & $\cdots$ & $\cdots$ & $\cdots$ & -. & .. & $\because$ & $\because$ \\
\hline$\cdots$ & $\because$ & $\because$ & $\cdots$ & $\cdots$ & .. & -. & $\cdots$ & .. & 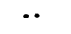 & $\cdots$ & .. & .. & - \\
\hline$\cdots$ & $\because$ & $\cdots$ & $\cdots$ & $\cdots$ & -. & $\cdots$ & . & .. & .. & $\cdots$ & $\cdots$ & 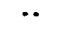 & - \\
\hline$\cdots$ & .. & $\cdots$ & $\cdots$ & $\cdots$ & -. & $\cdots$ & .. & .. & .. & .. & $\cdots$ & -. & $\cdots$ \\
\hline$\cdots$ & -. & $\cdots$ & .. & $\cdots$ & .. & .. & 0 & $\cdots$ & -. & 0 & $\cdots$ & $\cdots$ & 0 \\
\hline$\cdots$ & 0 & 0 & $\cdots$ & $\cdots$ & .. & $\cdots$ & 0 & $\cdots$ & $\cdots$ & 0 & .. & $\cdots$ & 0 \\
\hline$\cdots$ & 40 & 10 & -. & 20 & -. & $\cdots$ & $\cdots$ & $\cdots$ & $\cdots$ & $\because$ & $\cdots$ & .. & . \\
\hline$\cdots$ & .. & -. & $\cdots$ & $\cdots$ & .. & .. & 10 & .. & $\cdots$ & 10 & .. & .. & 20 \\
\hline$\cdots$ & 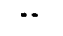 & $\because$. & 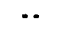 & $\cdots$ & .. & . & 0 & $\cdots$ & $\cdots$ & 1 & $\cdots$ & .. & 0 \\
\hline$\cdots$ & -. & 0 & $\cdots$ & 0 & 0 & .. & 0 & $\cdots$ & .. & 0 & .. & $\cdots$ & 0 \\
\hline 10 & 0 & $\cdots$ & $\because$ & .. & .. & .. & 0 & -. & $\cdots$ & 0 & .. & .. & 0 \\
\hline$\cdots$ & $\cdots$ & $\cdots$ & $\cdots$ & -. & .. & $\cdots$ & 50 & .. & .. & 50 & $\cdots$ & $\cdots$ & 50 \\
\hline$\cdots$ & 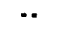 & .. & $\cdots$ & $\cdots$ & .. & $\cdots$ & 0 & $\cdots$ & 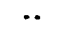 & 0 & .. & $\because$. & 0 \\
\hline .. & .. & .. & -. & .. & .. & $\cdots$ & 10 & -. & .. & 30 & .. & 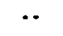 & 10 \\
\hline 20 & 0 & 0 & $\cdots$ & 0 & 0 & $\cdots$ & 5 & $\cdots$ & $\cdots$ & 7 & 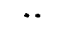 & $\cdot-$ & 1 \\
\hline$\cdots$ & -. & .. & $\cdots$ & .. & .. & $\cdots$ & 100 & $\cdots$ & .. & 100 & .. & $\cdots$ & 100 \\
\hline$\cdots$ & $\cdots$ & -. & -. & -. & -. & $\cdots$ & 5 & .. & .. & 0 & $\cdots$ & $\cdots$ & 3 \\
\hline$\because$ & -. & $\cdots$ & $\cdots$ & $\cdots$ & $\cdots$ & .. & .0 & $\cdots$ & .. & .0 & $\cdots$ & *. & .1 \\
\hline$\cdots$ & 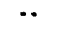 & $\because$ & $\cdots$ & -. & -. & $\cdots$ & .0 & $\mathbf{0 0}$ & $\infty$ & .0 & $\cdots$ & $\cdots$ & .1 \\
\hline$\cdots$ & $\cdots$ & $\because$ & $\cdots$ & $\cdots$ & -. & $\cdots$ & 0 & $\cdots$ & $\cdots$ & 1 & $\cdots$ & $\cdots$ & 0 \\
\hline$\cdots$ & $\cdots$ & 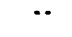 & $\because$ & $\cdots$ & -. & $\cdots$ & 0 & $\cdots$ & $\cdots$ & 0 & .. & .. & 0 \\
\hline -. & $\because$ & .. & $\cdots$ & $\cdots$ & .. & $\cdots$ & 120 & $\cdots$ & $\cdots$ & 30 & $\cdots$ & $\cdots$ & 50 \\
\hline$\cdots$ & $\cdots$ & -. & .. & .. & .. & .. & 50 & $\cdots$ & $\cdots$ & 6 & .. & $\cdots$ & 10 \\
\hline
\end{tabular}


TABLE 14.--Chealcal and physical quality of watar from salected surfaca-water sites in Clallam County--Continued

12045500 Etwha Rivar at McDonald Bridge near Port Angales, Uash. (Sita 129) (continued)

\begin{tabular}{|c|c|c|c|c|c|c|c|c|c|c|c|}
\hline \multicolumn{8}{|c|}{1975} & \multicolumn{4}{|c|}{1976} \\
\hline $5 / 20$ & $6 / 17$ & $7 / 14$ & $8 / 12$ & $9 / 16$ & $10 / 16$ & $11 / 21$ & $12 / 22$ & $1 / 21$ & $2 / 19$ & $3 / 22$ & $4 / 21$ \\
\hline - & .. & $\cdots$ & $\cdots$ & $\cdots$ & $\cdots$ & -. & ND & .. & $\cdots$ & No & -. \\
\hline NO & $\cdots$ & $\cdots$ & $\cdots$ & NO & $\cdots$ & . & NO & - & .. & ND & - \\
\hline ND & .. & $\cdots$ & $\cdots$ & No & .. & $\cdots$ & MD & .. & .. & ND & .. \\
\hline ND & .. & .. & .. & No & .. & .. & ND & $\cdots$ & $*$ & ND & -. \\
\hline ND & .. & .. & .. & No & .. & .. & ND & .. & .. & MD & .. \\
\hline No & .. & .. & $\cdots$ & No & .. & .. & ND & $\cdots$ & $\cdots$ & MD & •. \\
\hline$\omega$ & .. & .. & .. & ND & .. & $\cdots$ & MD & .. & $\cdots$ & MD & .. \\
\hline MD & .. & .. & .. & ND & $\cdots$ & .. & ND & .. & -. & No & . \\
\hline MD & .. & .. & .. & ND & .. & .. & MD & .. & .. & No & .. \\
\hline ND & .. & .. & $\cdots$ & ND & . & . & MD & $\cdots$ & $\cdots$ & MD & . \\
\hline MD & .. & .. & .. & ND & .. & .. & No & .. & $\cdots$ & MD & -. \\
\hline MD & .. & .. & -. & MD & .. & .. & MD & .. & $\cdot \cdot$ & MD & $\cdots$ \\
\hline ND & -. & .. & $\cdots$ & No & .. & $\cdots$ & No & .. & .. & MD & - \\
\hline ND & $\cdots$ & $\cdots$ & $\cdots$ & No & .. & .. & MD & $\cdots$ & $\cdots$ & No & $\cdots$ \\
\hline MD & .. & .. & $\cdots$ & No & .. & $\cdots$ & ND & . & $\cdots$ & MD & . \\
\hline ND & .. & $\cdots$ & $\cdots$ & No & .. & .. & ND & $\cdots$ & $\cdots$ & ND & - \\
\hline ND & $\cdots$ & .. & $\cdots$ & No & .. & .. & MD & $\cdots$ & .. & MD & . \\
\hline $\mathbf{M D}$ & $\cdots$ & .. & $\cdots$ & 10 & .. & .. & ND & $\cdots$ & $\cdots$ & MD & $\cdots$ \\
\hline ND & .. & .. & .. & ND & $\cdots$ & .. & MO & $\cdots$ & $\cdots$ & ND & . \\
\hline .. & $\cdots$ & .. & $\cdots$ & .. & .. & .. & ND & $\cdots$ & 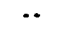 & MD & .. \\
\hline$\cdots$ & .. & $\cdots$ & $\cdots$ & $\cdots$ & .. & $\cdots$ & No & $\cdots$ & $\cdots$ & ND & $\cdots$ \\
\hline .. & -. & $\cdots$ & .. & -. & .. & $\cdots$ & ND & $\cdots$ & .. & WD & .. \\
\hline .. & .. & 0 & .. & .. & 1 & .. & .. & 0 & .. & $\cdots$ & 1 \\
\hline .. & .. & 0 & .. & .. & 1 & .. & $\cdots$ & 0 & $\cdots$ & $\cdots$ & 1 \\
\hline .. & .. & .. & $\cdots$ & $\cdots$ & .. & .. & .. & .. & .. & $\cdots$ & .. \\
\hline .. & .. & 10 & .. & .. & 0 & .. & .. & 10 & .. & .. & 10 \\
\hline . & -. & 0 & $\cdots$ & .. & 0 & $\cdots$ & $\cdots$ & 0 & $\cdots$ & .. & 1 \\
\hline - & .. & 0 & $\cdots$ & .. & 50 & . & $\cdots$ & 0 & $\cdots$ & $*$ & 0 \\
\hline - & .. & 0 & .. & $\cdots$ & 0 & .. & .. & 0 & .. & $\cdots$ & 0 \\
\hline -. & .. & 50 & .. & .. & 60 & $\cdots$ & $\cdots$ & 50 & $\cdots$ & $\cdots$ & 50 \\
\hline .. & $\cdots$ & 1 & $\cdots$ & .. & 3 & .. & $\cdots$ & 0 & $\cdots$ & $\cdots$ & 1 \\
\hline$\cdots$ & $\cdots$ & 10 & .. & .. & 0 & .. & .. & 20 & $\because$ & $\cdots$ & 10 \\
\hline .. & .. & 6 & .. & .. & 2 & .. & .. & 2 & $\cdots$ & $\cdots$ & 2 \\
\hline .. & .. & 100 & .. & .. & 100 & .. & .. & 100 & $\cdots$ & $\cdots$ & 100 \\
\hline .. & .. & 5 & .. & .. & 3 & $\cdots$ & .. & 3 & $\cdots$ & .. & 10 \\
\hline .. & $\cdots$ & .0 & $\cdots$ & $\cdots$ & .0 & $\cdots$ & -. & .1 & $\cdots$ & $\cdots$ & .1 \\
\hline .. & .. & .0 & .. & $\cdots$ & .0 & . & .. & .0 & $\cdots$ & .. & .1 \\
\hline .. & $\ldots$ & 0 & .. & .. & 0 & .. & .. & 0 & . & .. & 0 \\
\hline .. & $\cdots$ & 0 & .. & .. & 0 & .. & .. & D & 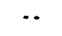 & $\cdots$ & 0 \\
\hline .. & $\cdots$ & 30 & $\cdots$ & .. & 20 & $\cdots$ & .. & 20 & $*$ & $\cdots$ & 0 \\
\hline .. & .. & 30 & .. & .. & 10 & .. & .. & 10 & .. & $\cdots$ & 0 \\
\hline
\end{tabular}


JABLE 14.--Chemical and physical quality of water from selected surface-water sites in Clallam County-Continued

12045500 Elwha River at McDonald Bridgo near Port Angeles, Wash. (Site 129) (continued)

Dote sample collected

Fecal coliform $(0.7 \mathrm{um}$, membrane

filter) $(\mathrm{col} / 100 \mathrm{~mL})$

Fecal streptococcl ( $H-F$ agar) (col/100 ml)

fecal streptococcl (KF agar) (col/100 mL)

Total organic carbon (C) (mg/L) Dissolved organic carbon (C) $(\mathrm{mg} / \mathrm{L})$ Total aldrin (ug/L)

Total atrazine (ug/L)

Total chlordane (ug/L)

Total DDD (ug/L)

Total DDE (ug/L)

Total DOT (ug/L)

Total diazinon (ug/L)

Total dieldrin (ug/L)

Total endrin (ug/L)

Total ethion (ug/L)

Total heptachlor (ug/L)

Total heptachlor expoxide (ug/L)

Total lindane (ug/L)

Total malathion ( $u g / L$ )

Total methoxychlor (ug/L)

rotal methyl parathion (ug/L)

rotal methyl trithion (ug/L)

Total parathion (ug/L)

Total PCB (ug/L)

rotal coulson cond. simazine (ug/L)

Total toxaphene (ug/L)

rotal trithion (ug/L)

Total 2,4-D (ug/L)

Total 2,4,5-1 (ug/L)

Total silvex (ug/L)

Total arsenic (AS) (ug/L)

Dissolved arsenic (As) (Ug/L)

Total barium (Ba) (ug/L)

Dissolved barium (Ba) (ug/L)

Total cadmium (Cd) (ug/L)

Dissolved cadmium (Cd) (ug/L)

Total chromium (Cr) (ug/L)

Dissolved chromium (Cr) (ug/L)

Total cobalt (Co) ( $\mathrm{ug} / \mathrm{L}$ )

Dissolved cobalt ( $\mathrm{CO}$ ) (ug/L)

Total copper (Cu) (ug/L)

Dissolved copper (CU) (ug/LO

Total lead (Pb) (ug/L)

Dissolved leed $(\mathrm{Pb})(\mathrm{ug} / \mathrm{L})$

Total mercury (Hg) (ug/L)

Dissolved mercury ( $\mathrm{Hg}$ ) (ug/L)

total selenium (Se) (ug/L)

Dissolved selenium (Se) (ug/L)

Total silver (Ag) (ug/L)

Dissolved silver (ag) (ug/L)

Total zinc $(2 n)$ (ug/L)

Dissolved zinc $(\mathrm{Zn})(\mathrm{ug} / \mathrm{L})$

\begin{tabular}{|c|c|c|c|c|c|c|c|c|}
\hline \multicolumn{8}{|c|}{1976} & 77 \\
\hline & & 2 & $8 / 25$ & $9 / 27$ & $10 / 26$ & $12 / 1$ & $12 / 27$ & $5 / 26$ \\
\hline
\end{tabular}

\begin{tabular}{|c|c|c|c|c|c|c|c|c|c|c|c|}
\hline$\cdots$ & $\cdots$ & $\cdots$ & .. & $\cdots$ & $\cdots$ & 1 & 2 & 1 & 60 & 1 & 1 \\
\hline 1 & 1 & 3 & 15 & 4 & 8 & 1 & 8 & 3 & 1 & 1 & 1 \\
\hline
\end{tabular}

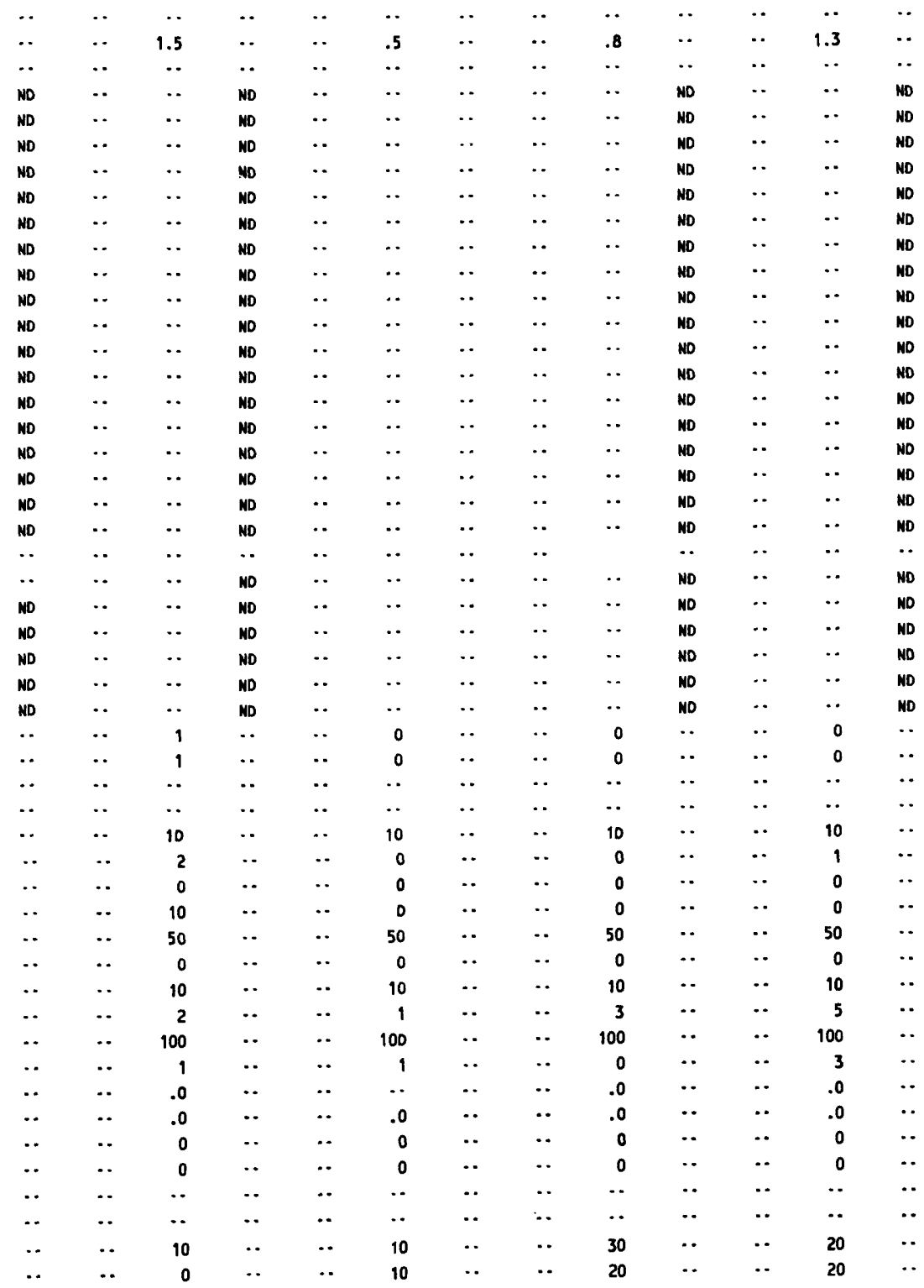


TABLE 14.--Chemical and physical quality of water from selected surface-water sites in clallam County--Continued

12045500 Elwha River at McDonald Bridge near Port Angeles, Wash. (Site 129) (continued)

\begin{tabular}{|c|c|c|c|c|c|c|c|c|c|c|c|c|}
\hline \multicolumn{2}{|c|}{1977} & \multirow[b]{2}{*}{$8 / 31$} & \multirow[b]{2}{*}{$9 / 21$} & \multirow[b]{2}{*}{$10 / 25$} & \multirow[b]{2}{*}{$19 / 14$} & \multicolumn{7}{|c|}{1978} \\
\hline $6 / 30$ & $7 / 25$ & & & & & $12 / 19$ & $1 / 30$ & $2 / 21$ & $3 / 14$ & $4 / 18$ & $5 / 30$ & $6 / 19$ \\
\hline 1 & 1 & 2 & 6 & 3 & 1 & 1 & 1 & 180 & 1 & 1 & 1 & 6 \\
\hline 1 & 10 & .. & 12 & 19 & 30 & 2 & 1 & 1 & 1 & 2 & .. & .. \\
\hline .. & .. & .. & .. & .. & .. & $\cdots$ & .. & .. & .. & .. & 2 & 3 \\
\hline .. & 1.5 & .. & .. & .. & 1.9 & .6 & .. & .6 & 1.1 & .. & 1.1 & 7.9 \\
\hline .. & .. & .. & .. & 1.4 & .. & .. & 3.6 & .. & .. & 5.0 & .. & .. \\
\hline .. & $\cdots$ & MD & .. & .. & ND & .. & .. & HD & .. & .. & ND & .. \\
\hline .. & $\cdots$ & HD & .. & .. & ND & .. & .. & MD & .. & .. & ND & .. \\
\hline .. & .. & HD & .. & .. & ND & .. & . & ND & .. & .. & HD & $\cdots$ \\
\hline .. & .. & ND & .. & .. & ND & .. & .. & ND & .. & .. & ND & .. \\
\hline .. & .. & MD & .. & .. & ND & .. & .. & ND & .. & .. & ND & .. \\
\hline .. & $\cdots$ & ND & .. & .. & ND & $\cdots$ & $\cdots$ & ND & .. & .. & MD & $\cdots$ \\
\hline .. & $\cdots$ & MD & .. & .. & ND & .. & $\cdots$ & ND & .. & .. & NO & .. \\
\hline - & .. & ND & $\cdots$ & .. & ND & $\cdots$ & .. & MD & .. & .. & NO & .. \\
\hline .. & .. & ND & .. & .. & ND & .. & .. & MD & .. & .. & MD & .. \\
\hline .. & .. & MD & .. & .. & MD & .. & .. & MD & .. & .. & ND & .. \\
\hline .. & .. & ND & .. & .. & ND & .. & .. & MD & .. & .. & ND & .. \\
\hline .. & .. & MD & .. & .. & MD & .. & .. & MD & .. & .. & ND & .. \\
\hline .. & .. & ND & .. & .. & ND & .. & .. & ND & .. & .. & NO & .. \\
\hline .. & .. & ND & $\cdots$ & . & ND & .. & .. & ND & .. & .. & ND & .. \\
\hline .. & .. & ND & .. & .. & ND & .. & .. & ND & $\cdots$ &.. & ND & $\cdots$ \\
\hline .. & .. & MD & .. & .. & ND & .. & .. & MD & .. & .. & ND & .. \\
\hline .. & .. & ND & .. & .. & ND & .. &.. & ND & .. & .. & ND & .. \\
\hline . & .. & MD & .. & .. & ND & .. &.. & MD & .. & .. & ND & .. \\
\hline .. & $\cdots$ & ND & .. & $\cdots$ & ND & .. & .. & ND & .. & .. & ND & .. \\
\hline .. & .. & MD & .. & .. & MD & .. & .. & MD & .. & .. & ND & .. \\
\hline . & .. & ND & .. & .. & ND & .. & .. & MD & .. & .. & ND & .. \\
\hline .. & .. & MD & .. & .. & ND & .. & .. & MD & .. & $\cdots$ & ND & .. \\
\hline . & .. & MD & .. & .. & ND & .. & .. & MD & .. & $\cdots$ & ND & .. \\
\hline -. & .. & MD & .. & .. & MD & .. & .. & MD & .. & .. & ND & .. \\
\hline . & .. & ND & .. & .. & ND & .. & .. & MD & .. & .. & ND & .. \\
\hline .. & 0 & .. & .. & 1 & -. & .. & 1 & . & .. & 4 & .. & .. \\
\hline .. & 0 & .. & .. & 0 & .. & .. & 0 & .. & .. & 3 & .. & .. \\
\hline . & $\ldots$ & -. & .. & 0 & .. & .. & 0 & .. & .. & 0 & .. & .. \\
\hline .. & .. & .. & .. & 0 & .. & .. & 0 & .. & .. & 0 & .. & .. \\
\hline .. & 10 & .. & .. & 10 & .. & .. & 2 & . & .. & 2 & .. & .. \\
\hline .. & 1 & .. & .. & 2 & .. & .. & 1 & .. & .. & 1 & .. & .. \\
\hline .. & 0 & .. & .. & 4 & .. & .. & 10 & . & .. & 10 & $\cdots$ & .. \\
\hline 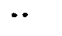 & 0 & .. & .. & D & .. & .. & 0 & . & .. & 0 & $\cdots$ & .. \\
\hline$\cdots$ & 5D & .. & .. & 50 & .. & . & 1 & . & .. & 2 & .. & .. \\
\hline .. & 0 & .. & .. & D & .. & .. & 0 & .. & .. & 2 & .. & .. \\
\hline .. & 10 & .. & .. & 10 & .. & .. & 19 & .. & .. & 5 & .. & .. \\
\hline .. & 3 & .. & .. & 3 & $\cdots$ & .. & 2 & .. & .. & 1 & .. & .. \\
\hline .. & 100 & .. & .. & 100 & .. & .. & 22 & .. &.. & 8 & .. & .. \\
\hline .. & 9 & .. & .. & 5 & .. & .. & 11 & .. & .. & 7 & .. & .. \\
\hline .. & .0 & .. & .. & .0 & $\cdots$ & .. & 13 & .. & .. & .0 & .. & .. \\
\hline .. & .0 & .. & .. & .0 & . & .. & .3 & .. & .. & .0 & .. & .. \\
\hline .. & 0 & .. & .. & 0 & $\cdots$ & .. & 0 & .. & .. & 0 & .. & .. \\
\hline .. & 0 & .. & .. & 0 & .. & .. & 0 & .. & .. & 0 & .. & .. \\
\hline .. & .. & .. & .. & 10 & .. & $\cdots$ & 2 & .. & .. & 0 & .. & .. \\
\hline .. & .. & .. & .. & 0 & . & .. & 0 & .. & .. & 0 & .. & .. \\
\hline .. & 10 & .. & .. & 40 & $\cdots$ & .. & 100 & .. & .. & 10 & .. & $\cdots$ \\
\hline .. & 4 & .. & .. & 10 & .. & .. & 10 & .. & .. & 10 & .. & .. \\
\hline
\end{tabular}


12045500 Elwho River at McDonald Bridge near Port Angeles, Wash. (Sito 129) (continued)

\begin{tabular}{|c|c|c|c|c|c|c|c|c|c|c|c|}
\hline \multirow[b]{2}{*}{ Data emple collected } & \multicolumn{6}{|c|}{1978} & \multicolumn{5}{|c|}{1979} \\
\hline & $7 / 26$ & $8 / 29$ & $9 / 13$ & $10 / 25$ & $11 / 28$ & $12 / 15$ & $1 / 18$ & $2 / 13$ & $3 / 21$ & $4 / 18$ & $5 / 15$ \\
\hline Discharge $\left(\mathrm{ft}^{3} / \mathrm{s}\right)$ & 968 & 686 & 992 & .. & $\cdots$ & .. & .. & .. & .. & $\cdots$ & $\cdots$ \\
\hline Dissolved sillica $\left(\mathrm{SiO}_{2}\right)(\mathrm{mg} / \mathrm{L})$ & 5.0 & 4.9 & 5.2 & 5.2 & 5.4 & 6.0 & 5.3 & 5.7 & 6.5 & 6.6 & 5.0 \\
\hline Total Iron (Fe) (ug/L) & 160 & -. & .. & 520 & -. & $\cdots$ & 300 & $\cdots$ & . & 140 & $\cdots$ \\
\hline Suspended Iron (Fa) (ug/L) & 110 & $\cdots$ & -. & 430 & .. & $\cdots$ & 150 & .. & .. & 130 & .. \\
\hline Diseolved Iron (Fa) (ug/LO & 50 & -. & $\cdots$ & 90 & $\cdots$ & $\cdots$ & 150 & -. & $\cdots$ & 10 & -. \\
\hline Total manganesa $(\mathrm{Mn})(\mathrm{ug} / \mathrm{L})$ & 10 & -. & $\cdots$ & 30 & .. & $\cdots$ & 60 & .. & -. & 20 & -. \\
\hline Suspended anganese (Mn) (ug/L) & 10 & $\cdots$ & $\cdots$ & 20 & $\because$ & $\cdots$ & 0 & -. & $\cdots$ & 10 & -. \\
\hline Dissolved manganess (Mn) (ug/l) & $\mathbf{0}$ & -. & -. & 10 & -. & -. & 60 & .. & $\cdots$ & 9 & $\cdots$ \\
\hline Diasolved calciun $\left(C_{a}\right)(\mathrm{mo} / \mathrm{L})$ & 12 & 13 & 13 & 9.0 & 14 & 15 & 16 & 15 & 14 & 15 & 14 \\
\hline Diseolved magnesiun $(\mathrm{Mg})(\mathrm{mg} / \mathrm{l})$ & 1.0 & 1.0 & 1.2 & 1.0 & 1.1 & 1.3 & 1.5 & 1.4 & 4.9 & 1.4 & 1.2 \\
\hline Diseolved sodiun (Na) (mo/L) & 2.0 & 1.8 & 2.9 & 2.8 & 1.9 & 2.1 & 2.4 & 2.3 & 2.1 & 2.2 & 2.2 \\
\hline Diegsolved potesssiun (K) (ma/l) & .1 & .1 & .2 & .3 & .2 & .3 & .2 & .1 & .2 & .1 & .0 \\
\hline Elcarbonata $\left(\mathrm{HCO}_{3}\right)(\mathrm{mg} / \mathrm{L})$ & 35 & 38 & 37 & 26 & 43 & 43 & 44 & 38 & 41 & 40 & 31 \\
\hline Alkalinity $\left(\mathrm{CacO}_{3}\right)(\mathrm{mg} / \mathrm{t})$ & 29 & 31 & 30 & 21 & 35 & 35 & 36 & 31 & 34 & 33 & 25 \\
\hline Dissolved sulfate $\left(\mathrm{SO}_{4}\right)(\mathrm{mg} / \mathrm{L})$ & 7.9 & 11 & 11 & 7.0 & 11 & 9.3 & 10 & 9.5 & 15 & 12 & 7.0 \\
\hline Dissolved chloride $(\mathrm{Cl})(\mathrm{mg} / \mathrm{L})$ & .7 & .9 & 1.0 & 2.7 & 1.1 & 1.0 & 1.2 & 1.6 & 1.2 & .9 & 1.5 \\
\hline Dissolved fluoride (F) (mo/L) & .0 & .0 & .1 & .0 & .0 & .0 & .1 & .1 & .1 & .1 & .1 \\
\hline Total nitrita plus nitrate $(W)(m / L)$ & .00 & .01 & .02 & .02 & .03 & .04 & .10 & .05 & .02 & .02 & .01 \\
\hline Dissolved nitrite plus nitrate $(W)(m g / L)$ & $\cdots$ & .. & $\cdots$ & 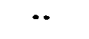 & $\cdots$ & $\cdots$ & $\cdots$ & $\cdots$ & $\cdots$ & 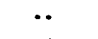 & $\because$ \\
\hline Total amonia $(H)(\mathrm{mg} / \mathrm{L})$ & .00 & .01 & .00 & .01 & .01 & .00 & .00 & .02 & .00 & .01 & .00 \\
\hline Dissolved emonia (W) (ma/L) & $\because$ & -. & $\cdots$ & $\cdots$ & $\cdots$ & $\cdots$ & $\cdots$ & $\cdots$ & $\cdots$ & $\cdots$ & $\cdots$ \\
\hline Total organic nitrogen (W) $(\mathrm{mg} / \mathrm{L})$ & .15 & .20 & .24 & .18 & .09 & .05 & .04 & .01 & .02 & .12 & .06 \\
\hline $\begin{array}{l}\text { Dissolved orgenic nitrogen }(W)(m a / L) \\
\text { Total sumonie plus organic nitrogen }\end{array}$ & .. & .. & .. & $\cdots$ & $\cdots$ & $\cdots$ & $\cdots$ & $\cdots$ & .. & $\cdots$ & $\cdots$ \\
\hline (N) $(m g / L)$ & .15 & .21 & .24 & .19 & .13 & .05 & .06 & .05 & .04 & .13 & .06 \\
\hline Suspended amonia plus organic & & & & & & & & & & & \\
\hline nitrogen ( $(W)(\mathrm{mg} / \mathrm{t})$ & .05 & .10 & .03 & .04 & .00 & .02 & .00 & .00 & .00 & .07 & .04 \\
\hline Dissolved amonie plus organic & & & & & & & & & & & \\
\hline nitrogen $(W)(m g / L)$ & .11 & .11 & .21 & 15 & .13 & .03 & .06 & .05 & .04 & .05 & .02 \\
\hline Total nitrogen (W) $(\mathrm{mo} / \mathrm{L})$ & .15 & .22 & .26 & .21 & .13 & .09 & .14 & .08 & .04 & .15 & .07 \\
\hline Dissolved nitrogen (W) (mg/ll) & $\ddot{*}$ & 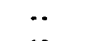 & $\because$ & $\cdots$ & $\cdots$ & $\cdots$ & $\because$ & $\cdots$ & $\cdots$ & $\because$ & 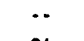 \\
\hline Total phosphorus (P) (mo/L) & .00 & .02 & .01 & .01 & .02 & .01 & .01 & .04 & 01 & .01 & .04 \\
\hline Dissolved phosphorus $(P)(m \theta / L)$ & .00 & .01 & .00 & .00 & .01 & .00 & .01 & .02 & .01 & .00 & .00 \\
\hline Diseolved sollids, residue at $180^{\circ} \mathrm{C}(\mathrm{mg} / \mathrm{l})$ & 42 & 51 & 52 & 45 & 54 & 52 & 67 & 60 & 52 & 54 & 58 \\
\hline Suspended solids, residue at $180^{\circ} \mathrm{C}(\mathrm{mg} / \mathrm{h})$ & 12 & 11 & 6 & $\cdots$ & $\cdots$ & -. & *. & $\because$ & $\cdots$ & $\cdots$ & .. \\
\hline Hercheses $\left(\mathrm{CaCO}_{3}\right)(\mathrm{mg} / \mathrm{L})$ & 34 & 37 & 37 & 27 & 40 & 43 & 46 & 43 & 55 & 43 & 40 \\
\hline Noncarbonata harchess (ma/L) & 5 & 6 & 7 & 6 & 5 & 8 & 10 & 12 & 21 & 10 & 15 \\
\hline Specific conductence (micromhos) & 79 & 80 & 78 & 73 & 91 & 102 & 101 & 92 & 80 & 99 & 90 \\
\hline pH (units) & 7.6 & 7.4 & 7.2 & 7.6 & 7.7 & 7.7 & 7.7 & 7.8 & 7.4 & 7.9 & 7.5 \\
\hline Texperatura $\left({ }^{\circ} \mathrm{C}\right)$ & 16.3 & 15.3 & 10.9 & 6.6 & 4.6 & 3.3 & 1.2 & 3.0 & 6.0 & 6.0 & 9.5 \\
\hline Turbidity (JTU) & 1 & 1 & 4 & 2 & 7 & 2 & 2 & 3 & 5 & 1 & 2 \\
\hline Dissolved oxygen (mo/LO & 10.6 & 10.2 & 10.8 & 11.3 & 13.1 & 13.2 & 13.4 & 13.2 & 12.4 & 12.7 & 11.3 \\
\hline Totel phytoplankton (cells/mL) & 160 & 5 & 14 & $\cdots$ & 56 & $\cdots$ & $\cdots$ & .. & 25 & $\cdots$ & 1800 \\
\hline $\begin{array}{l}\text { Pariphyton bicases, wh wight }\left(g / m^{2}\right) \\
\text { Pariphyton bicasess, total dry wight }\end{array}$ & 2.13 & $\cdots$ & $\cdots$ & .472 & $\because$ & $\because$ & 4.49 & $\because$ & $\cdots$ & $\cdots$ & $\cdots$ \\
\hline $\begin{array}{l}\left(g / m^{2}\right) \\
\text { Chlorophyll A periphyton, chrowographic }\end{array}$ & 3.23 & .. & $\cdots$ & .630 & $\because$ & - & 4.80 & -• & $\cdots$ & $\because$ & $\cdots$ \\
\hline $\begin{array}{l}\text { fluorom. }\left(m g / m^{2}\right) \\
\text { Chl orophyll periphyton, chromographic }\end{array}$ & .510 & $\cdots$ & $\cdots$ & 1.00 & $\cdots$ & $\cdots$ & 1.63 & $\cdots$ & -. & -• & -. \\
\hline $\begin{array}{l}\text { fluoram. }\left(\mathrm{mg} / \mathrm{m}^{2}\right) \\
\text { Fecal coliform }(0.7 \mathrm{~m} \text {, membrane }\end{array}$ & .420 & $\cdots$ & $\cdots$ & .310 & $\cdots$ & $\cdots$ & .900 & $\cdots$ & 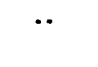 & .. & -. \\
\hline filter $)(\mathrm{col} / 100 \mathrm{~mL})$ & 2 & 2 & 7 & 1 & 5 & 1 & 1 & 1 & 1 & 1 & 1 \\
\hline Fecal atreptococci (KF ogar) (col/100 mL) & 5 & 2 & 11 & 1 & 2 & 1 & 1 & 2 & 1 & 1 & 1 \\
\hline Total organic carbon (C) (mo/L) & $\cdots$ & .8 & 1.0 & $\cdots$ & .9 & .7 & .. & .8 & 1.6 & .. & 1.8 \\
\hline
\end{tabular}


TABLE 14.*-Chemical and physical quality of water from selected surface-water sites in clatlan County--Cont inued

12045500 Etwhe River at MCDonald Bridge near Port Angeles, Wash. (site 129) (continued)

\begin{tabular}{|c|c|c|c|c|c|c|c|}
\hline \multicolumn{7}{|c|}{1979} & \multirow{2}{*}{$\frac{1980}{1 / 16}$} \\
\hline $6 / 21$ & $7 / 17$ & $8 / 15$ & $9 / 12$ & $10 / 17$ & $11 / 14$ & $12 / 12$ & \\
\hline .. & .. & .. & $\cdots$ & . & .. & $\cdots$ & $\cdots$ \\
\hline 3.8 & 4.7 & 5.6 & 5.2 & 5.4 & 5.6 & 5.7 & 6.7 \\
\hline -. & 170 & .. & .. & 80 & .. & .. & 2200 \\
\hline .. & 160 & .. & .. & 50 & .. & .. & 2100 \\
\hline .. & 10 & .. & 100 & 30 & $\cdots$ & .. & 60 \\
\hline .. & 20 & .. & . & 20 & .. & .. & 20 \\
\hline .. & 20 & .. & .. & 0 & .. & .. & 20 \\
\hline .. & 1 & .. & 5 & 20 & .. & .. & 4 \\
\hline 13 & 11 & 14 & 13 & 15 & 13 & 12 & 13 \\
\hline .8 & .9 & 1.1 & 1.1 & 1.3 & 1.2 & 1.0 & 1.1 \\
\hline 1.8 & 1.4 & 2.3 & 2.0 & 3.2 & 1.9 & 2.0 & 1.8 \\
\hline .2 & .3 & .1 & .1 & .1 & .2 & .2 & .0 \\
\hline 28 & 27 & 32 & 32 & 38 & 39 & 35 & 39 \\
\hline 23 & 22 & 26 & 26 & 31 & 32 & 29 & 32 \\
\hline 7.6 & 13 & 11 & 11 & 14 & 12 & 9.4 & 6.7 \\
\hline .8 & .9 & .9 & 3.1 & 1.0 & .9 & 1.0 & 1.6 \\
\hline .0 & .1 & .1 & .1 & .1 & .1 & .1 & .1 \\
\hline .02 & .01 & .05 & .02 & .03 & .08 & .08 & .04 \\
\hline .. & .. & .. & .04 & .02 & .05 & .05 & .03 \\
\hline .02 & .02 & .00 & .01 & .00 & .01 & .03 & .00 \\
\hline .. & -. & .. & - & .00 & .. & .02 & .00 \\
\hline .04 & .06 & .11 & .22 & 1.2 & .38 & .37 & .80 \\
\hline -. & .. & .. & $\cdots$ & 1.2 & -. & .30 & .76 \\
\hline .06 & .17 & .18 & .23 & 1.2 & .38 & .40 & .80 \\
\hline .06 & .00 & .00 & .01 & .00 & .16 & .08 & .04 \\
\hline .00 & .17 & .18 & .22 & 1.2 & .23 & .32 & .76 \\
\hline .08 & .09 & .16 & .25 & 1.2 & .47 & .48 & .84 \\
\hline .. & .. & .. & .26 & 1.2 & .28 & .37 & .79 \\
\hline .00 & .01 & .02 & .03 & .01 & .01 & .09 & .03 \\
\hline .00 & .00 & .00 & .01 & .01 & .01 & .00 & .02 \\
\hline 48 & 42 & 50 & 90 & 59 & 72 & 57 & 56 \\
\hline .. & .. & .. & . & .. & .. & -. & .. \\
\hline 36 & 31 & 40 & 37 & 43 & 37 & 34 & 37 \\
\hline 13 & 9 & 14 & 11 & 12 & 5 & 5 & 5 \\
\hline 72 & 78 & 86 & 82 & 102 & 90 & 77 & 84 \\
\hline 8.0 & 7.2 & 7.5 & 7.1 & 5.9 & 7.3 & 7.3 & 8.1 \\
\hline 9.5 & 12.9 & 14.0 & 1.17 & 10.2 & 4.8 & 4.6 & 4.0 \\
\hline 1 & 1 & 2 & 5 & 1 & 5 & 75 & 33 \\
\hline 11.1 & 11.0 & 10.1 & 10.6 & 11.1 & 12.3 & 12.2 & .. \\
\hline 610 & .. & .. & $\cdots$ & .. & .. & .. & .. \\
\hline 1.26 & 2.60 & .. & .. & 100 & .. & .. & .. \\
\hline 1.42 & 2.68 & . & - & .620 & - & -. & -. \\
\hline .810 & .640 & - & $\cdots$ & .450 & -. & .. & $\because$ \\
\hline .310 & .090 & 5 & 5 & 2 & 1 & 11 & . \\
\hline 1 & 1 & 32 & 28 & 2 & 5 & 3 & .. \\
\hline 2 & 44 & 1.0 & .. & . & 5.5 & 2.2 & .. \\
\hline .4 & $\cdots$ & & & & & & \\
\hline
\end{tabular}


Date sample collected

Dissolved organic carbon (c) $(\mathrm{mg} / \mathrm{L})$

Total aldrin (ug/L)

Total atrazine (ug/L)

Total chlordane (ug/L)

Total DOD (ug/L)

Total DDE (ug/L)

Total DDT (ug/L)

Total diazinon (ug/L)

Total dieldrin (ug/L)

Total endrin (ug/L)

Total ethion (ug/L)

Total heptachlor (ug/h)

Total heptechlor epoxide (ug/L)

Total lindane (ug/L)

Total malation aldrin (ug/L)

Total mathoxychlor (ug/L)

Total mathyl parathion (ug/L)

rotal mathyl trithion (ug/L)

Total parathion (ug/ $L$ )

Total PCB (ug/L)

Total coulson cond. simazine (ug/L)

Total toxaphene (ug/L)

Total trithion ( $\mathrm{Ug} / \mathrm{L})$

Total 2, 4-D (ug/L)

Total 2,4,5-T (ug/L)

Total silvex (ug/L)

Total arsenic (As) ( $\mathrm{ug} / \mathrm{L}$ )

Dissolved arsenic (As) (ug/L)

Total barium (Ba) (ug/L)

Dissolved beriuom (Ba) (ug/LO

Total cadmium (Cd) ( $u g / L$ )

Dissolved cadmium (Cd) (ug/L)

Total chromium (Cr) (ug/L)

Dissolved chromium (Cr) (ug/L)

Total cobale (Co) (ug/L)

Dissolved cobalt (CO) (ug/L)

Total copper (Cu) (ug/L)

Dissolved copper (Cu) (ug/L)

Total lead (Pb) (ug/h)

Dissolved leed (Pb) (ug/L)

Total marcury (Hg) (ug/L)

Dissolved mercury (Hg) (ug/L)

Total nickel (Ni) ( $\mathrm{ug} / \mathrm{L}$ )

Dissolved nickel (Ni) (ug/L)

Total selenium (Se) (ug/L)

Dissolved selenium (Se) (ug/L)

Total silver (Ag) (ug/L)

Dissolved silver (Ag) (ug/L)

Totel zinc $(2 n)(u g / L)$

Dissol ved zinc $(2 n)$

\begin{tabular}{|c|c|c|c|c|c|c|c|c|c|c|}
\hline \multicolumn{6}{|c|}{1978} & \multirow[b]{2}{*}{$1 / 18$} & \multirow[b]{2}{*}{$2 / 13$} & \multirow[b]{2}{*}{$3 / 21$} & \multirow[b]{2}{*}{$4 / 18$} & \multirow[b]{2}{*}{$5 / 15$} \\
\hline $7 / 26$ & $8 / 29$ & $9 / 13$ & $10 / 25$ & $11 / 28$ & $12 / 15$ & & & & & \\
\hline 1.1 & .. & .. & 3.6 & .. & .. & .8 & .. & .. & 1.3 & .. \\
\hline .. & ND & .. & .. & ND & .. & .. & .. & ND & .. & ND \\
\hline .. & ND & .. & .. & ND & $\cdots$ & $\cdots$ & .. & ND & .. & ND \\
\hline$\cdots$ & ND & .. & $\cdots$ & MD & .. & .. & .. & ND & .. & ND \\
\hline .. & ND & . & .. & ND & .. & .. & .. & ND & .. & ND \\
\hline .. & ND & .. & .. & ND & .. & .. & .. & MD & .. & MD \\
\hline .. & ND & -. & .. & ND & .. & .. & . & ND & .. & ND \\
\hline .. & MD & .. & .. & ND & .. & .. & . & ND & .. & ND \\
\hline .. & ND & .. & .. & MD & .. & .. & .. & ND & .. & ND \\
\hline .. & ND & .. & .. & ND & .. & .. & .. & ND & .. & MD \\
\hline .. & ND & - & .. & ND & .. & .. & . & ND & .. & ND \\
\hline .. & ND & $\cdots$ & .. & ND & .. & .. & . & ND & .. & ND \\
\hline . & MD & - & .. & ND & .. & .. & - & ND & .. & ND \\
\hline .. & ND & . & .. & ND & $\cdots$ & .. & .. & ND & .. & ND \\
\hline .. & ND & .. & .. & ND & $\cdot \cdot$ & .. & $\ldots$ & ND & .. & ND \\
\hline -. & ND & .. & .. & ND & .. & .. & - & ND & .. & ND \\
\hline -. & ND & .. & .. & ND & .. & .. & . & ND & .. & ND \\
\hline .. & ND & .. & .. & ND & .. & .. & .. & ND & .. & NO \\
\hline . & ND & $\cdots$ & -. & ND & .. & .. & $\cdots$ & ND & .. & ND \\
\hline -. & ND & $\cdots$ & -. & ND & .. & .. & .. & ND & .. & ND \\
\hline$\cdots$ & ND & . & .. & -. & $\cdot \cdot$ & .. & . & - & .. & $\cdots$ \\
\hline - & ND & . & -. & ND & .. & .. & . & ND & . & ND \\
\hline -. & ND & . & $\cdots$ & ND & .. & .. & .. & ND & .. & ND \\
\hline -. & MD & . & .. & $\cdots$ & .. & .. & .. & .. & .. & .. \\
\hline .. & ND & .. & .. & .. & .. & .. & .. & .. & .. & .. \\
\hline -. & ND & . & -. & . & .. & .. & .. & .. & .. & .. \\
\hline .. & .. & $\cdots$ & 0 & .. & .. & 1 & . & .. & 0 & $\cdots$ \\
\hline 1 & .. & -. & 0 & .. & .. & 0 & . & . & 0 & .. \\
\hline 300 & -. & . & 0 & $\cdots$ & -. & 10 & . & -. & 4 & -. \\
\hline 100 & .. & .. & 0 & .. & .. & 0 & .. & . & 0 & .. \\
\hline 2 & .. & .. & 2 & .. & .. & 2 & .. & .. & 2 & .. \\
\hline 1 & .. & $\cdots$ & 1 & .. & $\cdots$ & 1 & -. & .. & 1 & .. \\
\hline 10 & .. & $\cdots$ & 0 & .. & .. & 0 & .. & . & 0 & .. \\
\hline D & .. & .. & 0 & .. & .. & 0 & -. & . & 0 & .. \\
\hline 2 & .. & .. & 0 & .. & .. & 3 & .. & . & 3 & .. \\
\hline 1 & .. & .. & 0 & .. & .. & 2 & .. & .. & 0 & $\cdots$ \\
\hline 9 & .. & $\cdots$ & 7 & -. & .. & 11 & .. & .. & 8 & .. \\
\hline 4 & .. & .. & 4 & .. & .. & 1 & .. & .. & 0 & .. \\
\hline 17 & .. & .. & 10 & . & .. & 5 & .. &.. & 66 & .. \\
\hline 6 & .. & .. & 0 & .. & .. & 3 & .. & .. & 0 & .. \\
\hline .0 & .. & -. & .0 & .. & .. & .0 & $\because$. &.. & .0 & .. \\
\hline .0 & .. & $\cdots$ & .0 & .. & .. & .0 & .. & .. & .0 & -. \\
\hline - & -. & $\cdots$ & .. & .. & $\because$ & .. & .. & .. & .. & -. \\
\hline - & .. & .. & .. & .. & $\therefore$ & .. & .. & .. & .. & .. \\
\hline -. & .. & .. & 0 & .. & .. & 0 & .. &.. & 0 & .. \\
\hline 0 & .. & .. & 0 & .. & .. & 0 & .. & .. & 0 & .. \\
\hline 1 & -. & .. & 0 & .. & .. & 0 & .. & .. & 3 & .. \\
\hline 0 & .. & .. & D & .. & .. & 0 & .. & .. & 0 & .. \\
\hline 20 & .. & .. & 20 & .. & .. & 30 & .. & .. & 10 & .. \\
\hline 20 & -. & .. & 10 & .. & .. & 6 & .. & .. & 3 & .. \\
\hline
\end{tabular}


TABLE 14. - Chemical and physical qualtty of watar from selacted surface-water sites in Clallan County- Continued

12045500 Elwha River at McDonald Bridge near Port Angeles, Wash. (Site 129) (continued)

\begin{tabular}{|c|c|c|c|c|c|c|c|}
\hline$\frac{1979}{6 / 21}$ & $7 / 17$ & $8 / 15$ & $9 / 12$ & $10 / 17$ & $11 / 14$ & $12 / 12$ & $\frac{1980}{1 / 16}$ \\
\hline$\cdots$ & .4 & $\cdots$ & $\cdots$ & 2.0 & $\cdots$ & $\cdots$ & .. \\
\hline .. & $\cdots$ & $\cdots$ & $\cdots$ & -. & $\cdots$ & $\cdots$ & $\cdots$ \\
\hline .. & .. & . & .. & .. & -. & .. & .. \\
\hline -. & - & $\cdots$ & $\cdots$ & $\cdots$ & $\cdots$ & $\cdots$ & $\cdots$ \\
\hline .. & .. & $\cdots$ & $\cdots$ & $\cdots$ & $\cdots$ & $\cdots$ & $\cdots$ \\
\hline -. & .. & .. & $\cdots$ & .. & $\cdots$ & $\cdots$ & .. \\
\hline$\cdots$ & -. & .. & $\cdot \cdot$ & .. & $\cdots$ & $*$ & $\cdots$ \\
\hline$\cdots$ & -. & $\cdots$ & $\cdot \cdot$ & $\cdots$ & $\cdots$ & $\cdots$ & $\cdots$ \\
\hline .. & .. & .. & .. & $\cdots$ & $\cdots$ & $\cdot \cdot$ & $\cdots$ \\
\hline .. & .. & .. & $\cdot \cdot$ & $\cdots$ & -. & $\cdots$ & $\cdots$ \\
\hline$\cdots$ & .. & $\because$ & $\because$ & $\because$ & 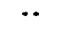 & $\because$ & $\because \cdot$ \\
\hline -. & -. & $\cdots$ & $\cdot$. & - & 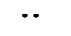 & $\cdots$ & $\cdots$ \\
\hline$\because$ & .. & $\cdots$ & $\cdots$ & .. & .. & $\cdots$ & $\cdots$ \\
\hline$\cdots$ & .. & $\cdots$ & $\cdots$ & . & .. & $\cdots$ & $\because$ \\
\hline$\cdots$ & $\cdots$ & $\cdots$ & $\cdots$ & $\cdots$ & .. & $\cdots$ & $\cdots$ \\
\hline$\cdot-$ & .. & $\cdots$ & 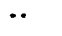 & -. & $\cdots$ & $*$ & .. \\
\hline$\cdot \cdot$ & - & $\cdots$ & $\because$ & $\cdots$ & $\cdots$ & $\cdots$ & $\cdots$ \\
\hline$\cdots$ & .. & .. & $\cdots$ & .. & .. & $\cdots$ & 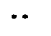 \\
\hline$\cdots$ & -. & $\cdots$ & $\because$ & - & $\cdots$ & $\cdots$ & $\cdots$ \\
\hline$\cdots$ & -. & $\cdots$ & $\because \cdot$ & -. & $\cdots$ & $\cdots$ & $\cdots$ \\
\hline$\cdots$ & . & $\cdots$ & 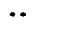 & . & $\cdots$ & $\cdots$ & 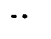 \\
\hline .. & -. & $\cdots$ & .. & . & $\cdots$ & $\cdots$ & .. \\
\hline$\cdots$ & - & $\cdots$ & $\cdots$ & $\because$ & $\because$ & $\cdots$ & $\cdots$ \\
\hline$\because \cdot$ & - & $\cdots$ & $\cdot \cdot$ & $\cdots$ & $\cdots$ & $\cdots$ & $\cdots$ \\
\hline$\cdots$ & - & $\cdots$ & $\because \cdot$ & $\cdots$ & $\because$ & $\cdots$ & $\cdots$ \\
\hline -. & . & $\cdots$ & $\because \cdot$ & - & $\because$ & $\cdots$ & $\cdots$ \\
\hline$\cdots$ & 1 & $\because \cdot$ & $\because \cdot$ & 1 & 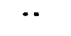 & $\because \cdot$ & 2 \\
\hline$\cdots$ & 0 & $\cdot$. & 2 & 1 & $\because$ & -. & 1 \\
\hline$\cdots$ & 2 & $\cdots$ & $\because$ & 200 & $\cdots$ & $\cdots$ & 10 \\
\hline$\cdots$ & 0 & .. & 20 & 6 & $\cdots$ & $\cdots$ & 0 \\
\hline$\cdots$ & 3 & $\cdots$ & $\cdots$ & 1 & $\cdots$ & $\cdots$ & 2 \\
\hline$\cdots$ & 1 & $\cdots$ & 1 & 0 & $\cdots$ & 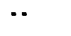 & 0 \\
\hline .. & 10 & .. & $\because$ & 0 & $\cdots$ & 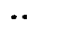 & 0 \\
\hline .. & 0 & $\cdots$ & 10 & 0 & $\because$ & $\cdots$ & 0 \\
\hline -. & 3 & .. & 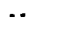 & 3 & .. & 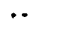 & 3 \\
\hline .. & 0 & .. & 3 & 0 & .. & $\cdots$ & 2 \\
\hline$\cdots$ & 21 & .. & 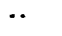 & 16 & .. & $\cdots$ & 10 \\
\hline$\cdots$ & 0 & .. & 2 & 0 & $\cdots$ & $\cdots$ & 1 \\
\hline . & 5 & -. & $\cdots$ & 5 & . & 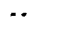 & 12 \\
\hline - & 0 & $\because$ & 0 & 0 & .. & .. & 0 \\
\hline •• & .0 & -. & -. & .0 & . & 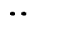 & .1 \\
\hline -. & .0 & $\cdots$ & .0 & .0 & .. & $\cdots$ & .0 \\
\hline$\cdots$ & .. & $\cdots$ & $\cdots$ & 8 & .. & .. & 4 \\
\hline$\cdots$ & -. & .. & $\cdots$ & 0 & $\cdots$ & $\cdots$ & 0 \\
\hline -. & 0 & -. & -. & 0 & -. & $\cdots$ & 0 \\
\hline$\cdots$ & 0 & $\cdots$ & 0 & 0 & $\cdots$ & -. & 0 \\
\hline .. & 0 & .. & .. & 0 & $\cdots$ & 0 & 0 \\
\hline$\cdots$ & 0 & 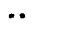 & 0 & 0 & .. & 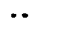 & 0 \\
\hline .. & 30 & $\cdots$ & .. & 20 & . & .. & 20 \\
\hline . & 6 & $\cdots$ & 9 & 0 & . & $\cdots$ & 6 \\
\hline
\end{tabular}




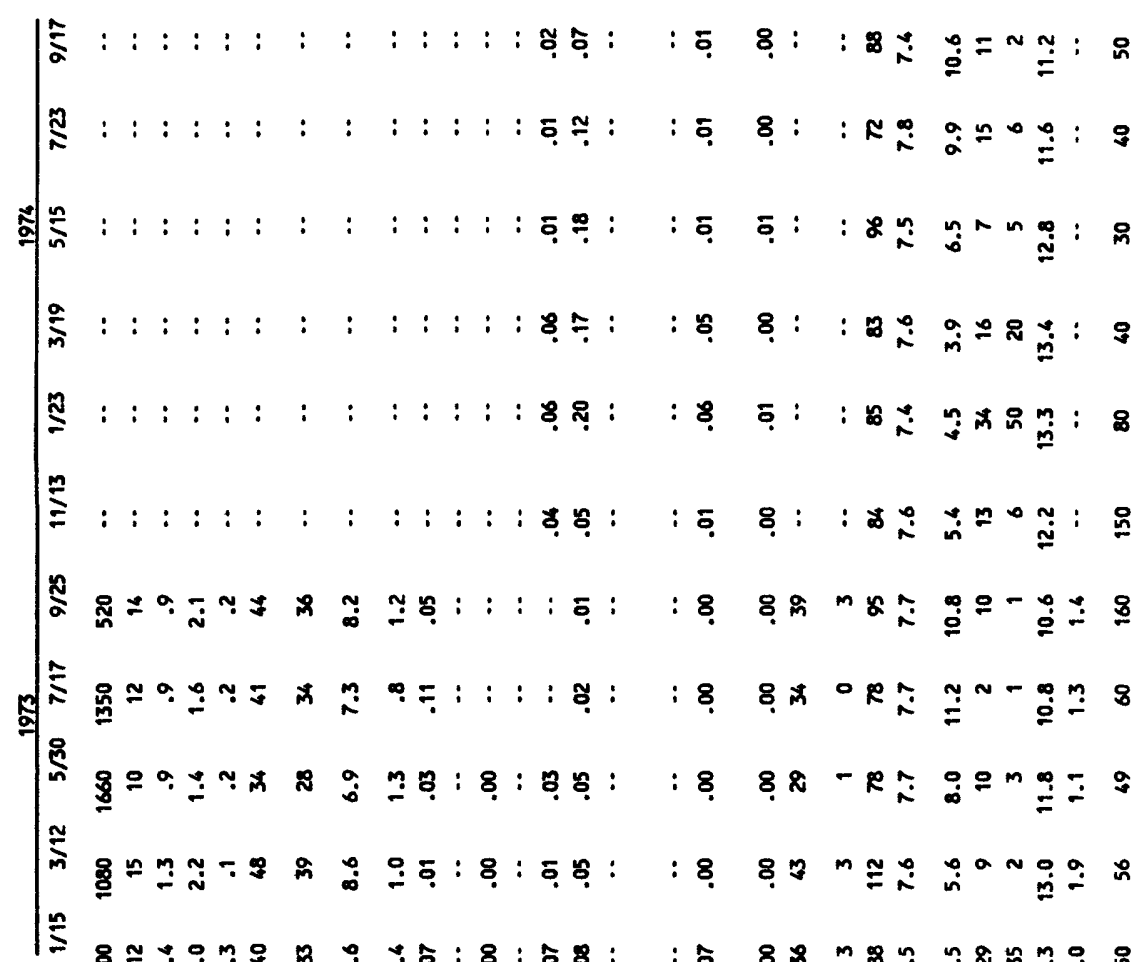

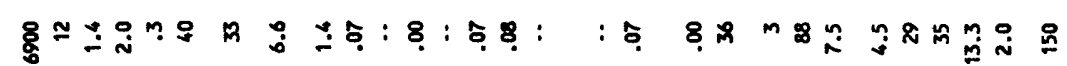
|

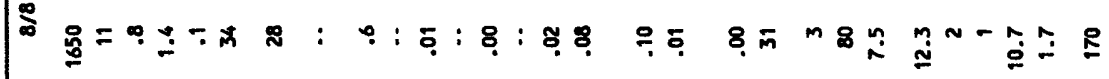

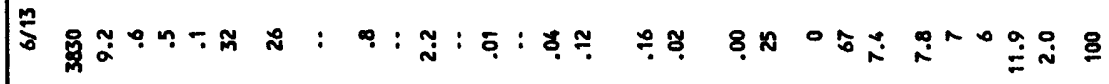

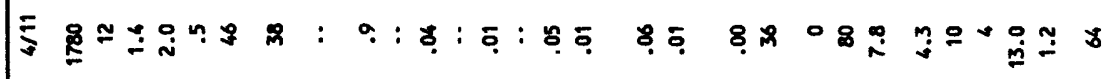

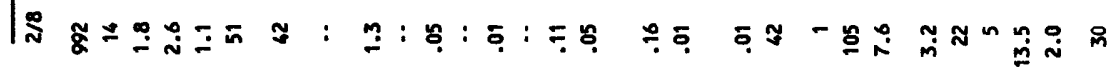

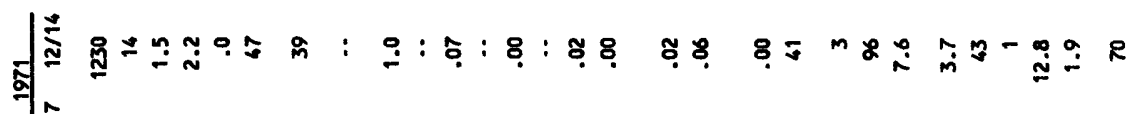

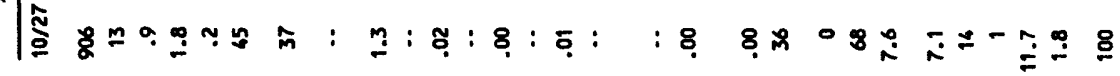

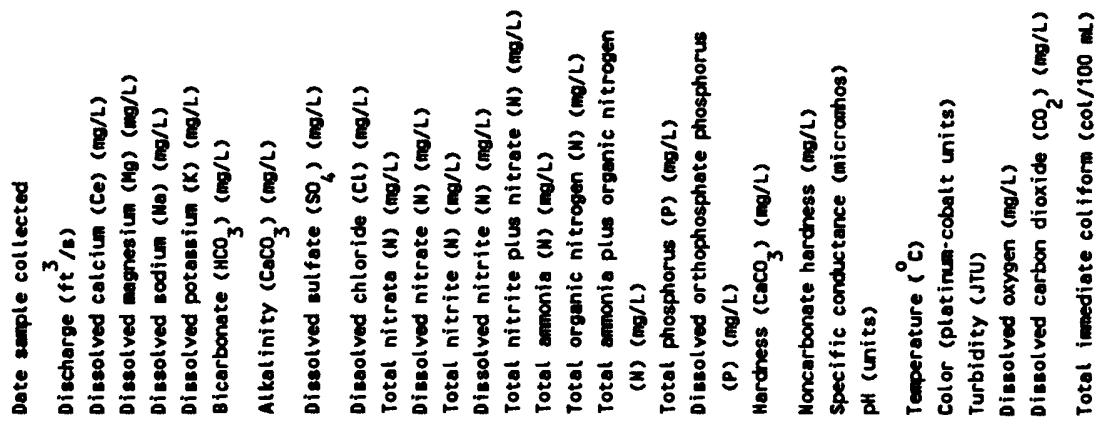


Sutherlend Lake, near Port Angeles, Wash. (site 132)

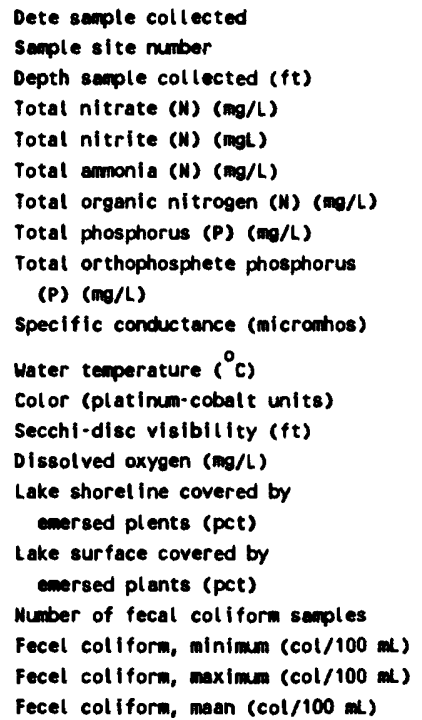

\begin{tabular}{ccc}
\multicolumn{3}{c}{$8 / 12 / 74$} \\
\hline 3 & 1 & \\
.00 & & .00 \\
.00 & & .00 \\
.02 & & .02 \\
.02 & & .11 \\
.01 & & .01 \\
.01 & & .00 \\
140 & & 130 \\
20.1 & & 7.4 \\
0 & & 0 \\
8.8 & & 6.6
\end{tabular}

Little or none

None or $\begin{array}{r}1 \\ 3 \\ 1 \\ 1 \\ 1\end{array}$

Aldwell Leke, near Port Angeles, MA. (site 136)

Date ample collected

Semple site number

Depth sample collected $(f t)$

Total nitrate (W) (ng/t)

Total nitrite (K) (ng/L)

Total amonic (N) (mg/L)

Total organic nitrogen $(N)(m g / L)$

Total phoaphorus $(P)(\mathrm{mo} / L)$

Total orthophosphate phosphorus

(P) $(\mathrm{mo} / L)$

Specific coductance (microhos)

water temperature $\left({ }^{\circ} \mathrm{c}\right)$

Color (pletinum-cobalt units)

secchi-disc visibility (ft)

Dissolved oxygen (ang/L)

Lake shorel ine covered by

emersed plants (pct)

Lake surface covered by

emersed plents (pct)

Number of fecal collform semples

fecal coliform, minimam (col/100 m)

fecal collform, maximan $(\mathrm{col} / 100 \mathrm{~m})$

fecal collform, maan (col/100 mL)

\section{Mone or 1}

12046510 Elwha River near mouth, near Port Angeles, WA. (Site 140)

Date samples collected

Total alunimu (ug/L)

Total manganese (ug/t)

Dissolved calcilun (Ca) (mo/L)

Dissolved magnesiun $(\mathrm{Mg})(\mathrm{mg} / \mathrm{L})$

Dissolved aodiun (Na) (mo/L)

Dissolved potassium (K) (mg/L)

Bicarbonate $\left(\mathrm{HCO}_{3}\right)(\mathrm{ma} / \mathrm{L})$

Alkel inity $\left(\mathrm{CaCO}_{3}\right)(\mathrm{mo} / \mathrm{L})$

Dissolved sulfete $\left(\mathrm{SO}_{4}\right)(\mathrm{mo} / \mathrm{L})$

Dissolved chloride (Cl) (ma/L)

Total nitrate (N) (mo/l )

Total nitrite (W) $(\mathrm{ma} / \mathrm{L})$

Total amonia (W) (mo/L)

Total organic nitrogen $(N)(m g / L)$

fotal phosphorus (P) ( $\mathrm{mg} / \mathrm{L})$

Dissolved orthophosphate

phosphorus (P) (mo/L)

Mardness $\left(\mathrm{CaCO}_{3}\right)(\mathrm{ma} / \mathrm{L})$

Moncarbonate hardness (mg/L)

Specific conductance (miconhos)

pH (units)

Temperature $\left({ }^{\circ} \mathrm{C}\right)$

Turbidity (JTU)

Diasolved oxygen (mo/LO

Diasolved carbon dioxide

$$
\left(\mathrm{CO}_{2}\right)(\mathrm{mg} / \mathrm{L})
$$

Total dissolved gas pressure

$$
\text { (percent saturation) }
$$

Total immediate colifrm ( $\mathrm{col} / 100 \mathrm{ml}$ )

Fecal coliform $(0.45 \mathrm{um}$, membrane filter) (col/100 ml)

Nitrogen plus argon (percent saturation )

Total chromium (Cr) (ug/L)

Dissolved chromium (Cr) (ug/L)

Total copper (Cu) (ug/L)

Dissolved copper (Cu) (ug/L)

Total leed (Pb) (ug/L)

Dissolved leed $(\mathrm{Pb})(\mathrm{ug} / \mathrm{L})$

Total mercury (Hg) (ug/L)

Total molybdenum (Mo) (Ug/L)

Total zine (2n) (ug/L)

Dissolved zine (2n) (ug/h)

103
Total fron (ug/L)

Total coctnium (Cd) (ug/L)

$\begin{array}{rccc} & 1976 & & \frac{1971}{2 / 18} \\ 0 & 4 / 28 & 6 / 1 & . . \\ 0 & . . & . . & . . \\ 0 & . . & . . & . . \\ 15 & . . & . . & . . \\ 2.2 & . . & . . & . . \\ 5.1 & . . & . . & . . \\ .3 & . . & . . & . . \\ 55 & . . & . . & . . \\ 45 & . . & . . & . . \\ 9.3 & . . & . . & . . \\ 4.3 & . . & . . & . . \\ .01 & . . & . . & . . \\ .00 & . . & . . & . . \\ .05 & . . & . . & . . \\ .17 & . . & . . & . . \\ .01 & . . & . . & . . \\ & & & \\ .01 & . . & . . & . . \\ 47 & . . & . . & . . \\ 2 & . . & . . & . . \\ 115 & 111 & 94 & . . \\ 6.4 & 7.4 & 6.7 & 7.2 \\ 5.0 & 9.5 & 7.5 & 3.6 \\ 1 & 0 & 1 & . \\ 12.9 & . . & 11.8 & 13.2 \\ & & & \\ 35 & . . & . . & .\end{array}$

$\because 101 \quad 101$

$37 \quad$.. $\quad$..

$1 \quad \cdots \quad$

$\begin{array}{lll}\cdot . & 102 & 101\end{array}$

$\begin{array}{lll} & 102 & 101\end{array}$

$\begin{array}{lll}-. & \\ & & \\ & & \end{array}$

. 1 ..

$\begin{array}{lll}\cdots & \cdots & \\ \cdots & -\end{array}$

.

$\begin{array}{lll}- & & \\ \cdots & & \end{array}$

.

- 2 -

$\begin{array}{lll}\text {. } & \ldots & \\ & & \end{array}$ 


\begin{tabular}{|c|c|c|c|c|c|c|c|c|c|c|c|c|c|c|}
\hline \multirow[b]{2}{*}{ Date sample collacted } & \multicolumn{9}{|c|}{1976} & \multicolumn{5}{|c|}{1977} \\
\hline & $\overline{3 / 18}$ & $4 / 28$ & $6 / 1$ & $6 / 3$ & $7 / 8$ & $7 / 14$ & $9 / 2$ & $11 / 2$ & $11 / 18$ & $\overline{1 / 6}$ & $2 / 2$ & $3 / 23$ & $5 / 10$ & $5 / 11$ \\
\hline Discharge $\left(\mathrm{ft}^{3} / \mathrm{a}\right)$ & .94 & .74 & 1.2 & .. & 1.6 & $\because$ & .61 & .63 & $\cdots$ & 1.4 & 2.3 & 1.3 & 1.6 & - \\
\hline Total aluminum $(A L)(U g / L)$ & 0 & .. & .. & $\cdots$ & .. & .. & .. & .. & $\cdots$ & $\cdots$ & -. & -. & $\cdots$ & .. \\
\hline Total iron $\left(F_{0}\right)(u g / L)$ & 0 & .. & .. & -. & -. & .. & .. & .. & 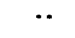 & $\cdots$ & .. & $\cdots$ & $\cdots$ & *. \\
\hline Total manganese $\left(\mathrm{H}_{n}\right)(\mathrm{ug} / \mathrm{L})$ & 0 & .. & .. & .. & .. & .. & -. & .. & $\cdots$ & .. & .. & .. & $\cdots$ & $\cdots$ \\
\hline Dissolved calcium (Ca) $(\mathrm{mg} / \mathrm{L})$ & 14 & .. & $\cdots$ & $\cdots$ & .. & -. & 15 & $\cdots$ & $\cdots$ & $\cdots$ & -. & *. & $\cdots$ & $\cdots$ \\
\hline Dissolved mggnesium (mg/L) & 2.1 & .. & .. & $\cdots$ & .. & .. & 1.5 & $\cdots$ & .. & -. & -. & $\cdots$ & $*$ & -. \\
\hline Dissolved sodium (Na) (mg/L) & 2.3 & .. & .. & $\cdots$ & -. & $\cdots$ & 2.1 & -. & $\cdots$ & .. & $\cdots$ & $\cdots$ & $\cdots$ & $\cdots$ \\
\hline Dissolved potassium (K) (mg/L) & .1 & -. & .. & $\cdots$ & .. & .. & .2 & * & -. & $\cdots$ & .. & $\cdots$ & $\cdots$ & -. \\
\hline Bicarbonate $\left(\mathrm{HCO}_{3}\right)(\mathrm{mg} / \mathrm{L})$ & 49 & $\cdots$ & -. & $\cdots$ & $\cdots$ & $\cdots$ & 47 & .. & $\cdots$ & $\cdots$ & $\cdots$ & $\cdots$ & $\cdots$ & $\cdots$ \\
\hline Alkal inity $\left(\mathrm{CaCO}_{3}\right)(\mathrm{mg} / \mathrm{L})$ & 40 & .. & -. & $\cdots$ & .. & $\cdots$ & 39 & .. & $\cdots$ & $\cdots$ & .. & $\cdots$ & $\cdots$ & -. \\
\hline Dissolved sulfate $\left(\mathrm{SO}_{4}\right)(\mathrm{mg} / \mathrm{L})$ & 7.5 & .. & .. & $\cdots$ & .. & -. & 8.7 & - & $\cdots$ & $\cdots$ & $\cdots$ & $\cdots$ & -. & -. \\
\hline Dissolved chloride (Cl) (mg/L) & 1.4 & .. & .. & .. & .. & .. & .6 & .. & .. & -. & $\cdots$ & $\because$ & . & .. \\
\hline Total nitrate $(N)(m g / L)$ & .12 & -. & .. & $\cdots$ & $\cdots$ & $\cdots$ & .02 & .. & $\cdots$ & $\cdots$ & .• & $\cdots$ & $\cdots$ & $\cdots$ \\
\hline Total nitrite (N) (mg/L) & .00 & .. & .. & $\cdots$ & $\cdots$ & .. & .00 & .. & $\cdots$ & .. & -. & $\cdots$ & .. & .. \\
\hline Total ammonia (N) $(\mathrm{mg} / \mathrm{L})$ & .05 & .. & .. & $\cdots$ & -. & -. & .05 & - & $\cdots$ & -. & $\cdots$ & $\cdots$ & $\cdots$ & -. \\
\hline Total organic nitrogen ( $N$ ) (mg/L) & .01 & .. & .. & .. & .. & $\cdots$ & .04 & -. & -. & $\cdots$ & .. & $\cdots$ & .. & .. \\
\hline Total phosphorua $(P)(m g / L)$ & .00 & .. & .. & $\cdots$ & -. & -. & .01 & -. & $\cdots$ & $\cdots$ & $\cdots$ & $\cdots$ & $\cdots$ & $\cdots$ \\
\hline Dissolved or thophosphate phosphorus & & & & & & & & & & & & & & \\
\hline (P) $(m g / L)$ & .00 & $\cdots$ & .. & .. & .. & -. & .00 & 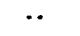 & $\cdots$ & .. & $\cdots$ & $\cdots$ & $\cdots$ & $\cdots$ \\
\hline Harchese $\left(\mathrm{CaCO}_{3}\right)(\mathrm{mg} / \mathrm{L})$ & 44 & $\cdots$ & $\cdots$ & $\cdots$ & -. & $\because$ & 44 & .. & -. & .. & .. & $\cdots$ & -. & $\cdots$ \\
\hline Moncarbonate harchesa (mg/L) & 3 & .. & -. & $\cdots$ & $\cdots$ & $\cdots$ & 5 & *. & $\cdots$ & $\cdots$ & -. & .. & -. & .. \\
\hline Specific conductance (micromhos) & 73 & 220 & 99 & .. & 103 & .. & 96 & 77 & .. & 90 & 148 & 67 & - & .. \\
\hline PH (units) & 6.3 & 6.8 & 6.6 & $\cdots$ & 6.8 & .. & 6.4 & 7.1 & $\cdots$ & 6.9 & 6.8 & 7.2 & 6.5 & .. \\
\hline Temperatura $\left({ }^{\circ} \mathrm{C}\right)$ & 7.6 & 9.6 & 7.4 & $\cdots$ & 7.5 & 7.4 & 7.8 & 8.8 & 7.9 & 7.6 & 5.8 & 8.5 & 8.5 & 8.5 \\
\hline Turbidity (JTU) & 0 & 0 & 0 & .. & .. & 0 & 0 & .. & 1 & $\cdots$ & $\cdots$ & 1 & $\cdots$ & .. \\
\hline Dissolved oxygen (mg/L) & 8.8 & .. & 10.0 & .. & .. & 8.7 & 7.6 & 7.8 & $\cdots$ & 8.8 & 11.5 & 10.9 & 10.71 & *. \\
\hline Dissolved carbon dioxide $\left(\mathrm{CO}_{2}\right)(\mathrm{mg} / \mathrm{L})$ & 39 & .. & .. & .. & $\cdots$ & $\cdots$ & 30 & .. & $\because$ & $\cdots$ & $\cdots$ & $\cdots$ & $\cdots$ & - \\
\hline Mitrogen plus argon (percant & & & & & & & & & & & & & & \\
\hline saturation) & 112 & .. & 106 & $\cdots$ & 105 & $\cdots$ & 105 & 101 & $\cdots$ & 103 & 102 & 107 & 105 & $\cdots$ \\
\hline Total dissolved gas pressure & & & & & & & & & & & & & & \\
\hline (percent saturation) & 104 & .. & 101 & $\cdots$ & 98 & $\cdots$ & 96 & 94 & $\cdots$ & 97 & 100 & 105 & 102 & $\cdots$ \\
\hline Total immediate coliform (col/100 mL) & 10 & 1 & .. & 36 & .. & 110 & .. & $\cdots$ & 160 & $\cdots$ & -. & .. & -. & 6 \\
\hline Fecal coliform $(0.45 \mathrm{um}$ & & & & & & & & & & & & & & \\
\hline membrane filter) $(\mathrm{col} / 100 \mathrm{~m})$ & 1 & 1 & .. & 1 & .. & 13 & 170 & $\cdots$ & .. & $\because$ & $\cdots$ & $\cdots$ & $\cdots$ & $\cdots$ \\
\hline facal colliform $(0.7 \mathrm{um}$, menbrane & & & & & & & & & & & & & & \\
\hline filtar) (col/100 mL) & .. & .. & .. & $\cdots$ & .. & $\cdots$ & -. & .. & 1 & -. & $\cdots$ & $\cdots$ & $\because$ & 1 \\
\hline Total cadmium (cd) (ug/L) & 0 & . & $\cdots$ & $\cdots$ & .. & $\cdots$ & -. & $\cdots$ & 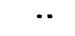 & 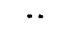 & $\cdots$ & $\because$ & $\because$ & $\cdots$ \\
\hline Total chromium (Cr) (ug/L) & 0 & .. & $\cdots$ & $\cdots$ & .. & $\cdots$ & -. & .. & 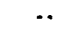 & $\cdots$ & $\cdots$ & $\cdots$ & $\cdots$ & $\cdots$ \\
\hline Dissolved chromiun (Cr) (ug/L) & 0 & - & .. & .. & .. & $\cdots$ & 10 & -. & $\cdots$ & $\cdots$ & -. & $\cdots$ & $\cdots$ & $\cdots$ \\
\hline Total copper (Cu) (ug/L) & 0 & .. & -. & -. & *. & $\cdots$ & -. & $\cdots$ & $\cdots$ & $\cdots$ & $\cdots$ & $\cdots$ & 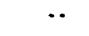 & $\cdots$ \\
\hline Dissolved copper (Cu) (ug/L) & 0 & .. & $\because$ & $\cdots$ & -. & $\cdots$ & 1 & -. & $\cdots$ & $\cdots$ & $\cdots$ & $\cdots$ & $\cdots$ & $\cdots$ \\
\hline Total loed $(P b)(u g / L)$ & 0 & . & .. & $\cdots$ & .. & $\cdots$ & .. & - & $\cdots$ & .. & $\cdots$ & $\cdots$ & - & . \\
\hline Dissolved lead (Pb) (ug/h) & 0 & . & -. & $\cdots$ & $\cdots$ & $\cdots$ & 1 & .. & $\cdots$ & $\cdots$ & $\cdots$ & $\cdots$ & $\cdots$ & - \\
\hline Total mercury $(\mathrm{Hg})(\mathrm{ug} / \mathrm{L})$ & 0 & .. & .. & $\cdots$ & $\cdots$ & $\cdots$ & -. & $\cdots$ & $\cdots$ & $\cdots$ & $\cdots$ & $\cdots$ & $\cdots$ & $\cdots$ \\
\hline Total molytodenum (Mo) $(u g / L)$ & 0 & .. & .. & $\cdots$ & $\cdot \cdot$ & $\cdots$ & $\cdots$ & $\cdots$ & $\cdots$ & $\cdots$ & $\cdots$ & $\cdots$ & $\cdots$ & $\cdots$ \\
\hline Total zinc $(2 n)(4 g / L)$ & 0 & .. & -. & $\cdots$ & $\because \cdot$ & 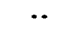 & $\cdots$ & *. & $\cdot \cdot$ & $\cdots$ & $\cdots$ & $\cdot \cdot$ & $\cdots$ & $\cdots$ \\
\hline Dissolved zinc $(2 n)(u g / L)$ & 0 & .. & -. & -. & .. & .. & 10 & - & .. & $\cdots$ & $\cdots$ & -. & .. & .. \\
\hline
\end{tabular}




\begin{tabular}{|c|c|c|c|c|c|c|c|c|c|c|c|c|c|c|}
\hline \multirow[b]{2}{*}{ Date sample collected } & \multicolumn{9}{|c|}{1976} & \multicolumn{5}{|c|}{1977} \\
\hline & $3 / 18$ & $4 / 28$ & $6 / 1$ & $6 / 3$ & $7 / 7$ & $7 / 14$ & $9 / 2$ & $11 / 2$ & $11 / 18$ & $1 / 6$ & $2 / 2$ & $3 / 23$ & $5 / 10$ & $5 / 11$ \\
\hline Dissolved calcium (Ca) (mg/L) & 13 & .. & $\cdots$ & $\cdots$ & $\cdots$ & $\cdots$ & 14 & -. & $\cdots$ & $\cdots$ & $\cdots$ & $\cdots$ & $\cdots$ & $\cdots$ \\
\hline Dissolved magnesium $(\mathrm{Mg})(\mathrm{mg} / \mathrm{L}) 5$ & 1.8 & $\cdots$ & $\cdots$ & $\cdots$ & $\cdots$ & 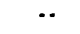 & 1.6 & 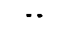 & $\cdots$ & $\cdots$ & $\cdots$ & $\cdots$ & $\because$ & $\cdots$ \\
\hline Dissolved sodium ( $\left.\mathrm{Ha}_{\mathrm{a}}\right)(\mathrm{mg} / \mathrm{L})$ & 2.8 & -. & -. & $\cdots$ & $\cdots$ & $\cdot \cdot$ & 2.2 & $-\cdot$ & $\cdots$ & $\cdots$ & $\because$ & $\cdots$ & $\cdots$ & $\cdots$ \\
\hline Dissolved potassium (K) (mg/L) & .2 &.- & $\cdots$ & $\cdots$ & $\cdots$ & $\cdot \cdot$ & .3 & $\cdots$ & $\cdots$ & $\cdots$ & $\cdots$ & $\cdots$ & $\because$ & $\cdots$ \\
\hline Bicarbonate $\left(\mathrm{HCO}_{3}\right)(\mathrm{mg} / \mathrm{L})$ & 48 & $\cdots$ & $\cdots$ & $\cdot \cdot$ & $\cdots$ & $\cdots$ & 46 & -. & $\cdots$ & $\cdots$ & $\cdots$ & $\cdots$ & $\because$ & $\cdots$ \\
\hline Alkalinity $\left(\mathrm{CaCO}_{3}\right)(\mathrm{mg} / \mathrm{L})$ & 39 & $\cdots$ & $\cdots$ & $\cdots$ & $\cdots$ & $\cdots$ & 38 & -. & $\cdots$ & $\cdots$ & $\cdots$ & $\cdots$ & $\cdots$ & $\cdots$ \\
\hline Dissolved sulfate $\left(\mathrm{SO}_{4}\right)(\mathrm{mg} / \mathrm{L})$ & 7.4 & -. & $\cdots$ & $\cdots$ & $\cdots$ & $\cdots$ & 7.6 & $\cdots$ & $\cdots$ & $\cdots$ & $\cdots$ & $\cdots$ & $\cdots$ & $\cdots$ \\
\hline Dissolved chloride (cl) (mg/L) & 1.8 & - & 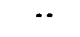 & $\cdots$ & $\cdots$ & $\because$ & .7 & $\cdots$ & $\cdots$ & $\cdots$ & $\cdots$ & $\cdots$ & $\cdots$ & $\cdots$ \\
\hline Total nitrate $(N)(\mathrm{mg} / \mathrm{h})$ & .02 & $\cdots$ & .. & $\cdots$ & $\cdots$ & $\because$ & .01 & $\cdots$ & $\cdots$ & $\cdots$ & $\cdots$ & $\cdots$ & $\cdots$ & $\cdots$ \\
\hline Total nitrite (N) $(\mathrm{mg} / \mathrm{L})$ & .00 & -. & $\cdots$ & $\cdots$ & $\cdots$ & $\because$ & .00 & 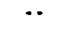 & $\cdots$ & $\cdots$ & $\because$ & $\cdots$ & $\cdots$ & $\cdots$ \\
\hline Total emmonia (N) (mg/L) & .05 & -. & -. & $\cdots$ & $\cdots$ & $\cdots$ & .05 & $\cdots$ & $\cdots$ & $\because$ & $\cdots$ & $\cdots$ & $\cdots$ & $\cdots$ \\
\hline Total organic nitrogen (H) (mg/L) & .16 & - & $\cdots$ & $\cdots$ & $\cdots$ & $\cdots$ & .11 & 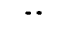 & $\cdots$ & $\cdots$ & $\cdots$ & $\cdots$ & $\cdots$ & $\cdots$ \\
\hline Total phosphorus (P) (mg/L) & .02 & $\cdots$ & $\cdots$ & $\cdots$ & $\cdots$ & 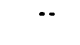 & .01 & $\cdots$ & 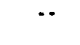 & $\cdots$ & $\because$ & $\cdots$ & $\cdots$ & $\cdots$ \\
\hline \multicolumn{15}{|l|}{ Dissolved or thophosphate } \\
\hline phosphorus (p) (mg/h) & .01 & $\cdot \cdot$ & -. & $\cdots$ & $\cdots$ & $\cdots$ & .01 & $\cdots$ & $\cdots$ & $\cdots$ & $\cdots$ & $\cdots$ & -. & $\cdots$ \\
\hline Hardhess $\left(\mathrm{CaCO}_{3}\right)(\mathrm{mg} / \mathrm{L})$ & 40 & -• & 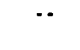 & $\cdots$ & $\cdots$ & $\cdots$ & 42 & $\cdots$ & $\cdots$ & $\cdots$ & $\cdots$ & $\cdots$ & -・ & $\cdots$ \\
\hline Noncarbonate hardness (mg/t) & 1 & 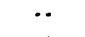 & 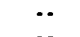 & $\cdots$ & $\because$ & $\cdots$ & 4 & $\cdots$ & $\ddot{*}$ & 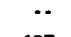 & 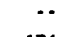 & 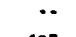 & 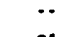 & $\cdots$ \\
\hline Specific conductance (micromhos) & 81 & 380 & 89 & $\cdots$ & 98 & $\cdots$ & 93 & 280 & 160 & 127 & 136 & 105 & 91 & $\cdots$ \\
\hline pH (units) & 7.2 & 7.6 & 6.6 & $\cdots$ & 7.1 & $\cdots$ & 6.7 & 7.2 & $\cdot \cdot$ & 7.2 & 6.9 & 7.5 & 6.8 & $\cdots$ \\
\hline Temperature $\left({ }^{\circ} \mathrm{C}\right)$ & 8.5 & 12.8 & 11.8 & $\cdots$ & 15.0 & 12.2 & 15.2 & 7.8 & 6.8 & 2.6 & 5.4 & 9.0 & 12.0 & $\cdots$ \\
\hline Turbidity (JTU) & 1 & D & 0 & $\cdots$ & $\cdots$ & o & 0 & $\cdots$ & 5 & -. & -. & 1 & -. & $\cdots$ \\
\hline Dissolved oxygen (mg/L) & 13.2 & -. & 11.5 & $\cdots$ & $\cdots$ & 10.2 & 9.8 & 9.2 & $\cdots$ & 11.3 & 11.8 & 13.0 & 11.0 & $\cdots$ \\
\hline \multicolumn{15}{|l|}{ Dissolved carbon dioxide } \\
\hline$\left(\mathrm{CO}_{2}\right)(\mathrm{mg} / \mathrm{L})$ & 4.8 & $\cdots$ & -• & $\cdots$ & $\cdots$ & $\cdots$ & 15 & -. & $\cdot \cdot$ & $\cdots$ & $\cdots$ & $\cdots$ & $\cdots$ & $\cdots$ \\
\hline \multicolumn{15}{|l|}{ Mitrogen plus argon (percent } \\
\hline saturation) & 103 & - & 104 & $\cdots$ & 104 & $\cdots$ & 101 & 102 & $\cdots$ & 99 & 101 & 102 & 104 & -. \\
\hline \multicolumn{15}{|l|}{ Total dissolved gas pressure } \\
\hline (percent saturation) & 106 & $\cdots$ & 104 & $\cdots$ & 102 & $\cdot-$ & 100 & 97 & $\cdots$ & 96 & 99 & 104 & 103 & $\cdots$ \\
\hline Total immediate coliform $(\mathrm{col} / 100 \mathrm{~mL})$ & 2 & 1 & -. & 200 & $\cdots$ & 100 & 100 & $\cdots$ & $\cdots$ & 450 & $\cdots$ & -・ & $\cdots$ & 3 \\
\hline \multicolumn{15}{|l|}{ Fecal coliform $0.45 \mathrm{~mm}$, membrane } \\
\hline filter) $(\mathrm{col} / 100 \mathrm{~mL})$ & 1 & 1 & ‥ & 1 & $\cdots$ & 1 & 10 & $\cdots$ & $\cdots$ & $\cdots$ & $\cdots$ & $\cdots$ & $\cdots$ & $\cdots$ \\
\hline \multicolumn{15}{|l|}{ Fecal col iform 0.7 um, membrane } \\
\hline filter) $(\mathrm{col} / 100 \mathrm{~mL})$ & $\cdots$ & $\cdots$ & $\cdots$ & $\cdots$ & 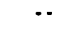 & $\because$ & $\because$ & $\cdots$ & $\cdots$ & 3 & $\cdots$ & $\because$ & $\cdots$ & 1 \\
\hline Dissolved chromium (Cr) (ug/t) & -. & $\cdots$ & $\cdots$ & $\cdot \cdot$ & $\cdots$ & $\cdots$ & $\mathbf{0}$ & $\cdots$ & $\cdots$ & $\cdots$ & $\cdots$ & $\because$ & $\because$ & $\cdots$ \\
\hline Dissoled copper (Cu) (ug/h) & $\cdots$ & $\cdots$ & $\cdots$ & $\cdots$ & $\because$ & $\because$ & 1 & $\cdots$ & $\cdots$ & $\cdots$ & $\cdots$ & $\cdots$ & $\cdots$ & $\cdots$ \\
\hline Dissolved lead $(\mathrm{Pb})(\mathrm{ug} / \mathrm{L})$ & $\because$ & $\cdots$ & $\cdots$ & $\cdots$ & $\cdots$ & $\because$ & 0 & $\cdots$ & $\cdots$ & $\cdots$ & $\because$ & $\cdots$ & $\cdots$ & $\cdots$ \\
\hline Dissolved zinc $(2 n)(\mathrm{ug} / \mathrm{L})$ & $\cdots$ & $\cdots$ & -. & $\cdots$ & $\cdots$ & $\cdots$ & 20 & $\cdots$ & $\cdots$ & $\cdots$ & $\cdots$ & $\cdots$ & $\cdots$ & $\cdots$ \\
\hline
\end{tabular}


TABLE 14, - Chemical and physical qulity of water from selected surface-water sites in clellam County--Continued

12046526 Fast slowph ot Angeles Point, neer Port Angeles, wa (site 143)

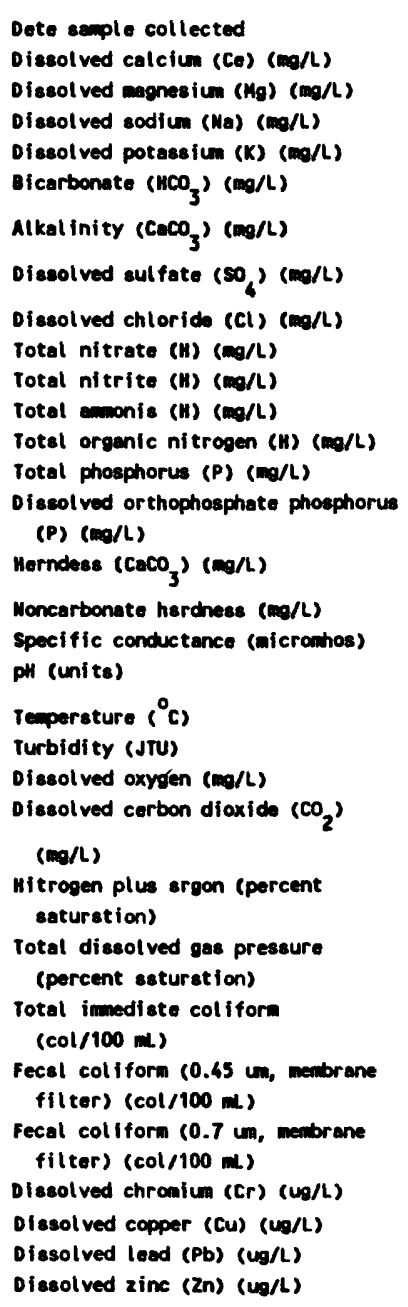

\begin{tabular}{|c|c|c|c|c|c|c|c|c|c|c|c|c|}
\hline & & & & 1976 & & & & & & & & \\
\hline $3 / 18$ & $4 / 20$ & $6 / 1$ & $6 / 3$ & $7 / 8$ & $7 / 16$ & $9 / 2$ & $11 / 2$ & $11 / 18$ & $1 / 6$ & $2 / 2$ & $3 / 23$ & $5 / 11$ \\
\hline 15 & $\cdots$ & •. & $\cdots$ & $\cdots$ & .. & 16 & -. & $\cdots$ & $\cdots$ & $\because$ & $\cdots$ & $\cdots$ \\
\hline 3.4 & -・ & $\cdots$ & $\cdots$ & .. & .. & 2.6 & .. & -. & $\cdots$ & $\cdots$ & $\cdots$ & -. \\
\hline 8.6 & .. & $\cdots$ & $\cdots$ & -. & .. & 3.1 & .. & -. & -. & $\cdots$ & $\cdots$ & $\cdots$ \\
\hline .5 & -. & $\cdots$ & .. & .. & .. & .3 & -. & -. & $\cdots$ & $\cdots$ & $\cdots$ & -. \\
\hline 59 & .. & $\cdots$ & -. & .. & .. & 51 & .. & - & .. & $\cdots$ & $\cdots$ & $\cdots$ \\
\hline 48 & .. & .. & $\cdots$ & -. & .. & 42 & -. & $\cdots$ & $\cdots$ & $\cdots$ & -. & $\cdot$ \\
\hline B.7 & .. & .. & .. & .. & .. & 8.0 & -. & -. & .. & -. & .. & *. \\
\hline 11 & .. & $\cdots$ & $\cdots$ & .. & -. & 1.5 & .. & -. & .. & .. & -. & $\cdots$ \\
\hline .04 & .. & $\cdots$ & -. & .. & -. & .00 & $\cdots$ & -. & $\cdots$ & $\cdots$ & $\cdots$ & $\because$ \\
\hline .00 & .. & -. & $\cdots$ & -. & $\cdots$ & .00 & -. & -. & $\cdot \cdot$ & $\cdot \cdot$ & $\cdots$ & $\because$ \\
\hline .03 & -. & $\cdots$ & -. & -. & $\cdots$ & .06 & -. & -• & -. & -. & $\cdots$ & $\cdots$ \\
\hline .13 & .. & $\cdots$ & $\cdots$ & $\cdots$ & $\cdots$ & .15 & -. & $\cdots$ & -. & $\cdots$ & $\cdots$ & $\cdots$ \\
\hline .01 & $\cdots$ & $\cdots$ & $\cdots$ & $\cdots$ & $\cdots$ & .01 & -. & -. & -. & -. & $\cdots$ & $\cdots$ \\
\hline .00 & -• & $\cdots$ & $\because$ & -. & .. & .00 & -. & .. & .. & $\cdots$ & .. & .. \\
\hline 51 & $\cdots$ & $\cdots$ & $\cdots$ & $\cdots$ & $\cdots$ & 51 & $\cdots$ & .. & .. & $\cdots$ & $\cdots$ & $\because$ \\
\hline 3 & .. & .. & $\cdots$ & .. & $\cdots$ & 9 & .. & .. & .. & $\cdots$ & $\cdots$ & $\cdots$ \\
\hline 136 & 537 & 196 & $\cdots$ & 118 & $\cdots$ & 107 & 90 & 116 & 368 & 265 & 560 & $\cdots$ \\
\hline 7.2 & 7.3 & 6.8 & $\cdots$ & 7.2 & $\cdots$ & 7.2 & 7.5 & -. & 7.1 & 7.2 & 7.3 & 7.1 \\
\hline 9.0 & 12.5 & 12.5 & - & 16.4 & 15.2 & 16.0 & 8.8 & 7.2 & 2.0 & 5.2 & 10.6 & 12.5 \\
\hline 0 & 0 & 0 & $\cdots$ & $\cdots$ & D & 0 & -. & D & $\cdot \cdot$ & $\cdots$ & 0 & • \\
\hline 13.0 & $\cdots$ & 13.2 & $\cdots$ & -. & 9.4 & 10.0 & 9.7 & -. & 9.1 & 10.6 & 11.0 & 10.6 \\
\hline 6.0 & $\because$ & -. & $\cdots$ & $\cdots$ & $\cdots$ & 5.1 & .. & - & -. & -. & - & 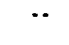 \\
\hline 105 & $\because$ & 99 &.. & 104 & -. & 104 & 100 & -. & 101 & 102 & 102 & 101 \\
\hline 107 & . & 104 & $\cdots$ & 102 & $\cdots$ & 103 & 97 & -. & 94 & 98 & 102 & 101 \\
\hline 1 & 8 & -. & 490 & $\cdots$ & 1800 & - & .. & 460 & $\cdots$ & $\cdots$ & -. & 5 \\
\hline 1 & 4 & -. & 1 & $\cdots$ & 4 & 1 & -. & -. & . & $\cdots$ & . & $\cdots$ \\
\hline .. & .. & .. & $\cdots$ & -. & $\cdots$ & .. & .. & 1 & -. & .. & $\cdot \cdot$ & 1 \\
\hline$\cdots$ & $\cdots$ & - & . & -. & -. & 0 & -. & $\cdots$ & •. & . & $\cdots$ & $\cdots$ \\
\hline$\cdots$ & -. & .. & $\cdots$ & $\cdots$ & .. & 5 & .. & .. & $\cdots$ & -• & -. & 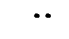 \\
\hline$\cdots$ & $\cdots$ & - & $\cdots$ & $\cdots$ & $\cdots$ & 0 & $\cdots$ & -. & $\cdots$ & $\cdots$ & $\cdots$ & $\cdots$ \\
\hline$\cdots$ & $\cdots$ & $\cdots$ & $\cdots$ & -. & $\cdots$ & 0 & -- & -. & $\because \cdot$ & $\cdots$ & $\because$ & $\cdots$ \\
\hline
\end{tabular}


12048600 Dungeness River at Highway 101 bridge (Site 181)

Date sample collected

Diecharge $\left(\mathrm{ft}^{3} / \mathrm{a}\right)^{1}$

Dissolved silica $\left(\mathrm{sio}_{2}\right)(\mathrm{ma} / \mathrm{L})$

Dissolved iron (fe) (ug/L)

Dissolved calciun (Ca) (mo/L)

Diesolved magnesium $(\mathrm{Mg})(\mathrm{mg} / \mathrm{L})$

Dissolved sodiun ( $\mathrm{Na})(\mathrm{mg} / \mathrm{L})$

Dissolved potassium (K) (mo/L)

Bicarbonate $\left(\mathrm{NCO}_{3}\right)(\mathrm{ma} / \mathrm{L})$

Alkalinity $\left(\mathrm{CaCO}_{3}\right)(\mathrm{ma} / \mathrm{L})$

Diseolved sulfate $\left(\mathrm{SO}_{4}\right)(\mathrm{mg} / \mathrm{L})$

Dissolved chloride (Cl) $(\mathrm{mg} / \mathrm{L})$

Dissolved flworide (F) (mg/L)

Diseolved nitrate (W) (mo/L)

Diseolved nitrite (mo/L)

Iotal amaonia (N) (mg/L)

Total Kjeldahl nitrogen (W) (mo/L)

Total phosphorus (P) (mo/L)

Dissolved orthophosphate phosphorus

$$
\text { (P) ( }(n / L)
$$

Diseolved solids, residue at

$$
\begin{gathered}
180^{\circ} \mathrm{C} \mathrm{(mg/L)} \\
\text { Harchess ( } \left.\mathrm{CaCO}_{3}\right)(\mathrm{mo} / \mathrm{L})
\end{gathered}
$$

Moncarbonate harchess (mo/L)

Specific conductence (micromos)

pH (units)

Temperature $\left({ }^{\circ} \mathrm{C}\right)$

Color (platinum-cobalt unite)

Turbidity

Dissolved oxygen ( $\mathrm{mg} / \mathrm{L}$ )

Diesolved carbon dioxide

$$
\left(\mathrm{CO}_{2}\right)(\mathrm{mo} / \mathrm{L})
$$

Total colliform (col/100 mL)

Dissolved arsenic (As) (ug/L)

Dissolved boron (B) (ug/L)

Total chromium (Cr) (ug/L)

Hexavalent chromiun (Cr) (ug/L)

oissolved copper (Cu) (ug/L)

\begin{tabular}{|c|c|c|c|c|c|c|c|c|c|c|c|c|c|}
\hline \multicolumn{6}{|c|}{1959} & \multicolumn{7}{|c|}{1960} & 1961 \\
\hline $7 / 9$ & $8 / 11$ & $9 / 21$ & $10 / 49$ & $11 / 20$ & $12 / 15$ & $1 / 21$ & $2 / 18$ & $3 / 17$ & $4 / 11$ & $5 / 9$ & $6 / 9$ & $7 / 18$ & $11 / 1$ \\
\hline 504 & 257 & 303 & 150 & 825 & 1300 & 150 & 440 & 205 & 445 & 532 & 732 & 420 & 157 \\
\hline 3.0 & 6.7 & 6.3 & 6.8 & 5.5 & 7.1 & 7.0 & 8.4 & 8.7 & 7.2 & 6.8 & 5.3 & 4.9 & 7.2 \\
\hline . & .. & .. & .. & $\cdots$ & .. & .. & $\cdot \cdot$ & -. & $\cdot \cdot$ & .. & .. & .. & .06 \\
\hline 16 & 18 & 15 & 20 & 13 & 12 & 21 & 19 & 23 & 18 & 16 & 14 & 14 & 21 \\
\hline 2.0 & 2.1 & 2.1 & 2.5 & 1.6 & 1.7 & 3.6 & 2.8 & 2.7 & 1.7 & 2.7 & 1.6 & 1.6 & 2.5 \\
\hline 1.9 & 2.8 & 2.5 & 3.2 & 1.8 & 1.9 & 3.5 & 3.2 & 4.1 & 2.5 & 2.5 & 1.9 & 1.9 & 3.3 \\
\hline .1 & .6 & .4 & .5 & .3 & .3 & .4 & .3 & .3 & .2 & .1 & .3 & .3 & .4 \\
\hline 54 & 65 & 58 & $n$ & 44 & 45 & 76 & 68 & 79 & 62 & 59 & 50 & 48 & 72 \\
\hline 45 & 54 & 48 & 59 & 36 & 37 & 63 & 56 & 65 & 51 & 48 & 41 & 39 & 59 \\
\hline 5.7 & 7.0 & 6.6 & 9.0 & 5.6 & 6.1 & 9.6 & 8.0 & 10 & 7.4 & 7.0 & 4.4 & 5.2 & 9.4 \\
\hline .5 & .8 & 1.0 & 1.5 & .8 & .5 & 1.8 & 1.2 & 2.2 & .8 & 1.0 & 1.0 & 1.0 & 2.0 \\
\hline .0 & .1 & .1 & .1 & .1 & .1 & .0 & .0 & .1 & .1 & .0 & .1 & .0 & .1 \\
\hline .02 & .09 & .05 & .05 & .14 & .07 & .00 & .05 & .02 & .00 & .00 & .00 & .00 & .07 \\
\hline .. & .. & . & .. & $\cdots$ & .. & - & $\cdots$ & - & - & .. & .. & -. & 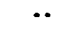 \\
\hline$\cdots$ & $\cdot \cdot$ & .. & -. & -. & $\cdots$ & .. & .. & .. & .. & .. & $\cdots$ & -. & \\
\hline •. & $\cdot \cdot$ & . & $\cdots$ & . & $\cdots$ & - & . & . & . & $\because$ & $\cdots$ & -. & \\
\hline .00 & .00 & .00 &.$\infty 0$ & .00 & .02 & .00 & .02 & .01 & .00 & .00 & .00 & .00 & .01 \\
\hline$\cdots$ & .. & $\cdots$ & 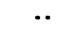 & $\cdots$ & $\cdots$ & $\cdots$ & $\cdots$ & $\cdots$ & $\cdots$ & $\cdots$ & $\cdots$ & $\cdots$ & \\
\hline 59 & 72 & 67 & 84 & 55 & 53 & 92 & 77 & 94 & 69 & 63 & 54 & 54 & 86 \\
\hline 48 & 54 & 49 & 50 & 39 & 38 & 68 & 59 & 68 & 52 & 51 & 42 & 42 & 62 \\
\hline 4 & 0 & 2 & 1 & 3 & 1 & 5 & 4 & 4 & 1 & 2 & 0 & 2 & 4 \\
\hline 101 & 125 & 113 & 137 & 89 & 94 & 150 & 130 & 152 & 117 & 112 & 93 & 92 & 141 \\
\hline 7.6 & 7.5 & 7.6 & 7.9 & 6.3 & 7.2 & 7.9 & 7.9 & 7.9 & 7.9 & 7.7 & 7.89 & 7.7 & 7.9 \\
\hline .. & $\ldots$ & .. & .. & $\cdots$ & $\cdots$ & .. & .. & -. & $\cdots$ & .. & .. & $\cdots$ & \\
\hline$\cdots$ & $\cdots$ & .. & $*$ & $\cdots$ & $\because$ & $\cdots$ & $\cdot \cdot$ & $\cdots$ & $\cdots$ & $\cdots$ & $\cdots$ & $\cdots$ & 5 \\
\hline -. & $\cdots$ & -. & .. & $\because$ & $\cdot \cdot$ & $\cdots$ & .. & . & $\cdots$ & $\because$ & -. & $\cdots$ & . \\
\hline -. & $\cdot$ & $\cdot \cdot$ & $\cdot \cdot$ & $\because$ & $\cdots$ & $\cdot \cdot$ & $\cdot \cdot$ & $\cdots$ & $\cdots$ & $\cdots$ & $\cdot \cdot$ & $\cdot \cdot$ & 11.9 \\
\hline . & $\cdots$ & $\cdots$ & *. & .. & . & . & $\cdots$ & $\cdots$ & $\cdots$ & .. & . & .. & \\
\hline .. & -. & .. & .. & .. & .. & 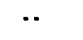 & .. & .. & .. & .. & .. & .. & 36 \\
\hline .. & $\cdots$ & 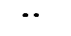 & $\cdots$ & $\cdots$ & $\cdots$ & $\cdots$ & $\cdot \cdot$ & $\cdots$ & .. & $\cdots$ & $\cdot \cdot$ & .. & .00 \\
\hline . & $\cdots$ & . & $\cdots$ & 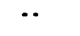 & $\cdots$ & $\because$ & -. & $\cdots$ & $\cdots$ & $\cdot \cdot$ & ・. & -. & .00 \\
\hline 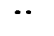 & $\cdots$ & -. & 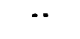 & 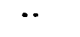 & $\cdots$ & $\cdots$ & .. & $\because$ & $\because$ & . & -. & -. & .00 \\
\hline -. & $\cdots$ & -. & $\because$ & -. & $\cdots$ & $\cdots$ & .. & $\cdots$ & -. & 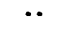 & -• & -・ & .00 \\
\hline$\cdots$ & $\cdots$ & $\cdots$ & 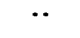 & $\because$ & 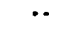 & . & $\cdots$ & $\cdots$ & $\cdots$ & .. & .. & - & .01 \\
\hline - & $\cdot \cdot$ & $\cdots$ & -・ & $\because$ & $\cdots$ & $\cdots$ & $\cdot \cdot$ & $\cdots$ & $\cdots$ & .. & $\cdot \cdot$ & -. & .05 \\
\hline
\end{tabular}

Dissolved zinc (2n) (ug/l)

'Discharges are as computed at site 173.

2 Witrate plus nitrite.

$\frac{1961}{11 / 1}$

157

.04

2.5

3.3

2.0

.1

-.

.01

86

4 
12048600 Dungeness River at Highway 101 bridge (Site 181)

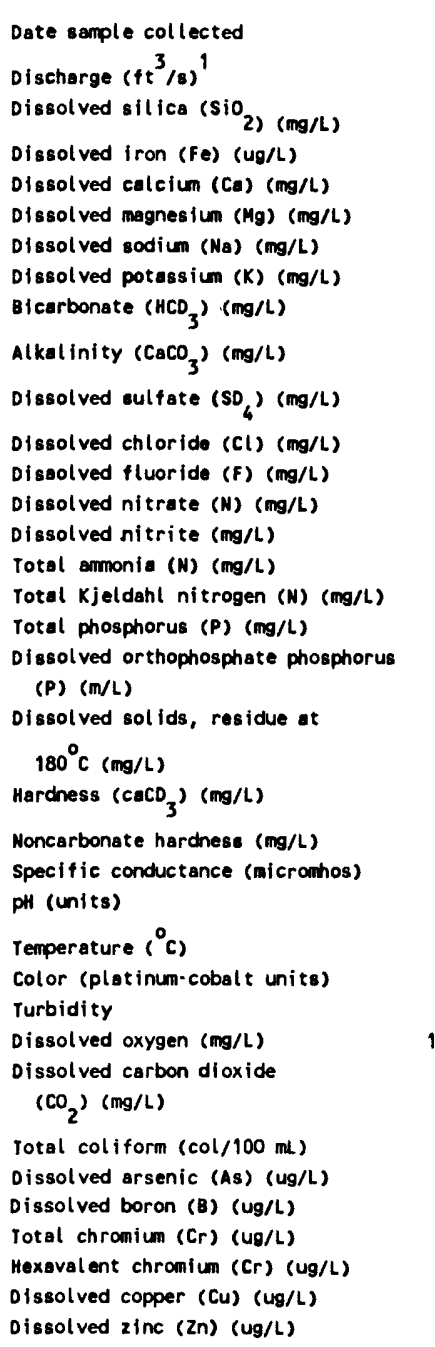

\begin{tabular}{|c|c|c|c|c|c|c|c|c|c|c|c|c|c|}
\hline & & 962 & & 19 & 63 & & 64 & 1965 & & 1966 & & 67 & 1968 \\
\hline $2 / 14$ & $5 / 14$ & $8 / 14$ & $12 / 13$ & $6 / 17$ & $12 / 23$ & $6 / 15$ & $12 / 14$ & $6 / 2$ & $2 / 17$ & $8 / 1$ & $5 / 17$ & $10 / 16$ & $4 / 16$ \\
\hline 225 & 311 & 228 & 560 & 776 & 1340 & 898 & 187 & 626 & 220 & 458 & 918 & 363 & 214 \\
\hline 7.1 & 7.7 & 6.3 & 7.2 & 4.4 & 5.6 & 4.2 & 6.3 & 5.5 & 14 & 4.6 & 5.9 & 5.2 & 6.8 \\
\hline .08 & .11 & .05 & $\cdots$ & . & .. & .. & .04 & $\cdots$ & .. & .. & $\cdots$ & -. & - \\
\hline 19 & 18 & 18 & 18 & 12 & 14 & 12 & 20 & 14 & 19 & 14 & 14 & 17 & 21 \\
\hline 2.7 & 2.4 & 1.6 & 2.0 & 1.7 & 2.0 & 2.3 & 2.8 & 1.9 & 3.9 & 1.9 & 2.2 & 2.1 & 3.2 \\
\hline 3.3 & 3.2 & 2.6 & 2.7 & 1.6 & 2.5 & 2.1 & 3.6 & 2.5 & 3.9 & 2.1 & 2.3 & 2.5 & 3.7 \\
\hline .5 & .4 & .6 & .1 & .2 & .3 & .2 & .4 & .4 & .3 & .3 & .6 & .2 & .1 \\
\hline 67 & 64 & 60 & 62 & 44 & 50 & 45 & 70 & 51 & 74 & 50 & 52 & 59 & 76 \\
\hline 55 & 53 & 49 & 51 & 36 & 41 & 37 & 58 & 42 & 61 & 41 & 43 & 48 & 63 \\
\hline 8.8 & B. 4 & 7.8 & 7.8 & 4.4 & 6.0 & 5.2 & 10 & 5.8 & 9.0 & 6.0 & 6.0 & 6.6 & 8.0 \\
\hline 1.2 & 1.0 & 1.0 & 1.2 & .2 & .5 & .5 & 1.4 & 1.0 & 2.0 & .5 & .0 & .7 & 1.0 \\
\hline .1 & .1 & .1 & .1 & .0 & .1 & .1 & .2 & .1 & .1 & .1 & .1 & .1 & .2 \\
\hline .07 & .00 & .05 & .05 & .02 & .09 & .02 & .02 & .07 & .05 & .07 & .02 & .05 & .02 \\
\hline .. & $\cdots$ & $\cdots$ & .. & .. & .. & $\cdots$ & .. & .. & .. & $\cdots$ & .. & . & . \\
\hline .. & .. & .. & .. & $\cdots$ & .. & $\cdots$ & .. & .. & .. & .. & .. & .. & 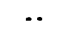 \\
\hline .. & .. & .. & .. & .. & -. & $\cdots$ &.. & $\cdots$ & $\cdots$ & .. & .. & .. & $\cdots$ \\
\hline .01 & .01 & .01 & .01 & .00 & .00 & .01 & .01 & .00 & .. & .. & .10 & .. & .. \\
\hline$\cdots$ & . & $\cdots$ & -. & - & - & $\cdots$ & $\cdots$ & $\cdots$ & . & $\cdots$ & $\cdots$ & .. & - \\
\hline 80 & 78 & 68 & 74 & 50 & 57 & 53 & 84 & 58 & 88 & 55 & 57 & 64 & 80 \\
\hline 58 & 55 & 52 & 53 & 37 & 43 & 40 & 62 & 43 & 64 & 43 & 44 & 51 & 66 \\
\hline 4 & 2 & 3 & 2 & 1 & 2 & 2 & 4 & 1 & 3 & 2 & 2 & 3 & 3 \\
\hline 130 & 123 & 115 & 116 & 80 & 94 & 83 & 135 & 97 & 142 & 95 & 99 & 109 & 147 \\
\hline 7.9 & 7.4 & 7.9 & 7.7 & 7.5 & 7.2 & 7.5 & 7.7 & 7.7 & 7.9 & 7.4 & 7.6 & 7.9 & 8.0 \\
\hline .. & .. & .. & . & .. & .. & .. & . & .. & .. & . & .. & 9 & 7 \\
\hline 5 & 10 & 5 & 5 & 0 & 10 & 5 & 0 & 0 & 5 & 5 & 5 & 5 & 5 \\
\hline .. & .. & .. & .. & $\cdots$ & .. & .. & -. & $\cdots$ & $\cdots$ & $\cdots$ & .. & -. & .. \\
\hline 12.2 & 11.7 & 10.1 & 13.4 & 10.6 & 12.1 & 10.9 & 13.1 & 10.4 & 11.9 & 11.2 & -. & .. & .. \\
\hline .. & .. & .. & .. & $\cdots$ & .. & . & .. & - & . & $\cdots$ & .. & .. & . \\
\hline 91 & 23 & 36 & 230 & 23 & 91 & 23 & 230 & 36 & 91 & 23 & 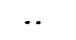 & .. & .. \\
\hline .. & .00 & .. & .01 & .00 & .00 & .00 & .00 & .00 & .00 & .00 & .. & .. & $\cdots$ \\
\hline .. & .04 & .. & .00 & .00 & .00 & .01 & .01 & .00 & .04 & .02 & $\cdots$ & .00 & $\cdots$ \\
\hline .. & .00 & .. & .00 & .00 & .01 & .00 & .00 & .00 & .00 & .00 & .. & .00 & $\ldots$ \\
\hline .. & .0 & .. & .00 & .00 & .01 & .00 & .00 & .00 & .00 & .02 & .00 & .. & .. \\
\hline .. & .03 & .. & .00 & .02 & .07 & .00 & .01 & .01 & .03 & .04 & .00 & .00 & $\cdots$ \\
\hline .. & .05 & 00 & .05 & .05 & .05 & .05 & .05 & .05 & .05 & .00 & .01 & .00 & .. \\
\hline
\end{tabular}

'Dischargee are as computed at ite 173.

2 Nitrate plus nitrite. 
TABLE 14. - Chemical and physical quality of water from selected surface-water sites in clollom County-. Continued

12048600 Dungeness River Highwoy 101 bridge (Site 181)... Continued

\begin{tabular}{|c|c|c|c|c|c|c|c|c|c|c|c|c|c|c|c|c|c|}
\hline \multicolumn{2}{|c|}{1971} & \multicolumn{5}{|c|}{1972} & \multicolumn{6}{|c|}{1973} & \multicolumn{5}{|c|}{1974} \\
\hline $10 / 26$ & $12 / 13$ & $2 / 17$ & $4 / 10$ & $6 / 12$ & $8 / 7$ & $11 / 28$ & $1 / 15$ & $3 / 12$ & $5 / 29$ & $7 / 16$ & $9 / 24$ & $11 / 12$ & $1 / 22$ & $3 / 18$ & $5 / 14$ & $7 / 22$ & $9 / 16$ \\
\hline .. & 240 & 161 & 421 & 717 & 306 & .. & .. & .. & .. & .. & .. & .. & .. & $\cdots$ & -. & -. & -. \\
\hline .. & .. & .. & .. & . & .. & .. & .. & $\cdots$ & .. & .. & .. & .. & .. & .. & .. & .. & .. \\
\hline .. & .. & $\cdots$ & .. & . & .. & .. & .. & .. & .. & .. & .. & .. & .. & $\cdots$ & .. & .. & $\cdots$ \\
\hline 19 & 18 & 22 & 19 & 12 & 13 & 21 & 13 & 21 & 15 & 13 & 19 & $\cdots$ & .. & .. & .. & .. & $\cdots$ \\
\hline 2.3 & 2.8 & 3.7 & 2.7 & 1.5 & 1.5 & 3.0 & 2.1 & 3.3 & 1.8 & 1.6 & 2.6 & .. & .. & .. & .. & .. & -. \\
\hline 3.2 & 3.6 & 4.4 & 3.3 & 1.3 & 1.8 & 3.9 & 2.6 & 3.7 & 3.0 & 2.1 & 3.6 & .. & -. & -. & .. & .. & $\cdots$ \\
\hline .4 & .4 & .8 & .6 & .2 & .2 & .6 & .7 & .3 & .3 & .3 & .5 & $\cdots$ & .. & .. & .. & .. & .. \\
\hline 69 & 70 & 83 & 75 & 47 & 46 & 74 & 48 & 79 & 59 & 53 & 75 & -. & .. & $\cdots$ & .. & 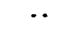 & .. \\
\hline 57 & 57 & 68 & 62 & 39 & 38 & 61 & 39 & 65 & 48 & 43 & 62 & .. & . & .. & $\cdots$ & $\cdots$ & $\cdots$ \\
\hline .. & . & . & . & $\cdots$ & . & 9.8 & 7.0 & 9.5 & 7.3 & 5.9 & 7.1 & $\cdots$ & $\cdots$ & -. & $\cdots$ & .. & . \\
\hline 1.9 & 1.6 & 3.2 & 1.2 & .9 & .3 & 2.5 & 1.9 & 1.5 & 1.2 & .9 & 1.9 & .. & $\cdots$ & $\cdot \cdot$ & -. & $\cdots$ & $\cdot$ \\
\hline .. & .. & .. & .. & .. & .. & .. & .. & .. & . & .. & .. & $\ddot{z}$ & $\cdots$ & .. & $\cdot \cdot$ & $\cdots$ & $\cdots$ \\
\hline .01 & .10 & .06 & .06 & .03 & .02 & .02 & .13 & .02 & .03 & .02 & .15 & $.10^{2}$ & .09 & .06 & .01 & .01 & .04 \\
\hline .01 & .00 & .00 & .00 & .00 & .00 & .00 & .01 & .00 & .00 & $\cdots$ & 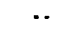 & $\cdots$ & . & .. & $\cdots$ & .. & $\because$ \\
\hline .02 & .02 & .07 & .13 & .06 & .02 & .05 & .17 & .06 & .04 & .02 & .08 & .07 & .07 & .10 & .11 & .08 & .09 \\
\hline .. & .11 & .15 & .13 & .30 & .10 & $\cdot \cdot$ & $\cdots$ & $\cdots$ & .. & $\cdots$ & $\cdots$ & .. & .. & . & .. & .. & .. \\
\hline .01 & .01 & .02 & .04 & .03 & .01 & .01 & .07 & .00 & .00 & .00 & .01 & .03 & .04 & .04 & .02 & .01 & .01 \\
\hline .00 & .00 & .02 & .00 & .00 & .00 & .00 & .00 & .00 & .00 & .00 & .00 & .00 & .01 & $\begin{array}{l}.00 \\
.\end{array}$ & .01 & .00 & .00 \\
\hline .. & .. & $\cdots$ & $\cdots$ & .. & .. & .. & .. & .. & .. & .. & $\cdots$ &.. & $\cdots$ & .. & .. & .. & .. \\
\hline 57 & 56 & 70 & 59 & 26 & 39 & 65 & 41 & 66 & 45 & 39 & 58 & $\cdot$. & $\cdots$ & $\because$ & $\because$ & . & $\because$ \\
\hline 0 & 0 & 2 & 0 & 0 & 1 & 4 & 2 & 1 & 0 & 0 & 0 & .. & $\cdots$ & .. & $\cdots$ & $\cdot$ & -. \\
\hline 91 & 135 & 160 & 128 & 97 & 89 & 150 & 105 & 170 & 110 & 109 & 130 & 101 & 130 & 40 & 130 & 95 & 130 \\
\hline 7.7 & 7.7 & 7.8 & 7.9 & 7.6 & 7.7 & 7.8 & 7.4 & 7.9 & 7.9 & 7.9 & 8.0 & 7.8 & 7.8 & 7.8 & 7.8 & 7.9 & 7.8 \\
\hline 7.1 & 3.4 & 2.7 & 4.6 & 8.2 & 17.4 & 4.1 & 5.6 & 7.0 & 12.0 & 15.5 & 12.6 & 6.3 & 4.2 & 7.0 & 8.8 & 12.7 & 14.6 \\
\hline 12 & 16 & 13 & 11 & 9 & 5 & 0 & 45 & 9 & 11 & 7 & 11 & 22 & 29 & 20 & 7 & 6 & 12 \\
\hline 1 & 1 & 2 & 16 & 16 & 2 & 2 & 4 & 2 & 3 & 2 & 1 & 15 & 20 & 15 & 8 & 8 & 2 \\
\hline 11.7 & 13.1 & 13.1 & 12.5 & 11.7 & 9.7 & 13.0 & 12.3 & 12.6 & 11.0 & 9.8 & 11.0 & 11.3 & 13.2 & 12.9 & 12.6 & 11.2 & 11.6 \\
\hline .. & .. & .. & . & $\cdots$ & -. & 1.9 & 3.1 & 2.0 & 1.2 & 1.1 & 1.2 & .. & .. & .. & -. & $\cdots$ & $\cdots$ \\
\hline 100 & 100 & 21 & 100 & 150 & .. & 50 & 150 & 120 & 41 & .. & 600 & 200 & 60 & 50 & 60 & 70 & 80 \\
\hline .. & -. & .. & $\cdots$ & 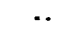 & $\cdots$ & .. & .. & 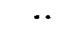 & .. & .. & .. & .. & .. & .. & $\cdots$ & $\because$ & $\cdots$ \\
\hline$\cdots$ & -. & .. & $\because$ & $\cdots$ & $\cdots$ & $\cdot \cdot$ & 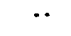 & $\cdots$ & $\cdots$ & .. & $\because$ & $\cdots$ & $\cdots$ & $\cdot \cdot$ & $\cdots$ & $\cdots$ & $\cdots$ \\
\hline .. & .. & .. & $\cdots$ & $\cdots$ & $\cdots$ & .. & $\cdots$ & $\cdots$ & .. & .. & $\cdots$ & .. & $\cdot \cdot$ & .. & $\cdots$ & $\cdots$ & $\cdots$ \\
\hline .. & $\cdots$ & .. & $\cdots$ & $\cdots$ & $\cdots$ & .. & -. & $\cdots$ & .. & $\cdots$ & $\cdots$ & -. & .. & . & .. & $\cdots$ & $\because$ \\
\hline.. & .. & $\ldots$ & $\cdots$ & $\because$ & $\cdots$ & -. & $\cdots$ & $\cdots$ & .. & $\cdots$ & $\cdots$ & .. & .. & . & $\cdots$ & $\cdots$ & $\cdots$ \\
\hline .. & .. & .. & .. & .. & $\cdots$ & .. & $\cdots$ & $\cdots$ & . & .. & $\cdots$ & .. & .. & -. & .. & $\cdots$ & $\cdots$ \\
\hline
\end{tabular}


Morse Creek et City of Port Angeles diversion near Port Angeles, un (Site 157)

Date sumple collected

Dissolved sillice $\left(\mathrm{SiO}_{2}\right)(\mathrm{mo} / \mathrm{L})$

Dissolved iron (Fe) (ug/L)

Dissolved anganese (Hn) (ug/h)

Dissolved calcium (CA) (mg/L)

Dissolved magnesiun $(\mathrm{Mg})(\mathrm{mg} / \mathrm{L})$

Diesolved sodium (Ma) (mg/L)

Dissolved potassium $(K)(\mathrm{mg} / \mathrm{L})$

Bicerbonate $\left(\mathrm{HCO}_{3}\right)(\mathrm{mg} / \mathrm{L})$

Alkalinity $\left(\mathrm{CaCO}_{3}\right)(\mathrm{mg} / \mathrm{L})$

Dissolved sulfate $\left(\mathrm{SO}_{4}\right)(\mathrm{mol} / \mathrm{L})$

Dissolved chloride (Cl) (ma/L)

Diseolved fluoride (F) $(\mathrm{mg} / \mathrm{L})$

Diseolved nitrate (N) $(\mathrm{mg} / \mathrm{L})$

Diseolved nitrite (N) $(\mathrm{m} / \mathrm{h})$

Diseolved phosphorus (P) (mg/L)

Dissolved solids, residue nt

$180^{\circ} \mathrm{C}(\mathrm{mg} / \mathrm{L})$

Suspended solids, at $105^{\circ} \mathrm{C}$ ( $m g / L$ )

Harchess $\left(\mathrm{CaCO}_{3}\right)(\mathrm{mg} / \mathrm{L})$

Noncarbonte harchess (mg/L) Specific conductence $(m / L)$ pH (units)

Color (platimum-cobalt units)

Turbidity (JTU)

Dissolved carbon dioxide (mg/L)

Dissolved arsenic (As) (ug/L)

Dissolved barium (Ba) (ug/L)

Dissolved cadnium (Cd) (ug/L)

Dissolved chromium (Cr) (ug/L)

Dissolved leed (Pb) (ug/L)

Dissolved mercury (Hg) (ug/L)

Dissovled selenium (Se) (ug/L)

Dissolved silver (Ag) (ug/L)

\begin{tabular}{|c|c|c|c|c|c|c|c|c|c|c|c|c|c|}
\hline 1988 & 1960 & & & & 1968 & & & & & 72. & 1973 & 1975 & 1978 \\
\hline $8 / 6$ & $8 / 9$ & $2 / 23$ & $12 / 20$ & 91. & $11 / 25$ & $12 / 30$ & $1 / 6$ & $9 / 8$ & $7 / 26$ & $8 /$. & $2 / 1$ & $1 / 20$ & $5 / 30$ \\
\hline 7.4 & 7.4 & 26 & 6.1 & 6.8 & 3.8 & 7.4 & 2.5 & 5.0 & 4.6 & 4.0 & 29 & 8.2 & $\cdots$ \\
\hline .. & 40 & 500 & 20 & 160 & 60 & 120 & 180 & 80 & 10 & 220 & 0 & 90 & 0 \\
\hline$\therefore$ & 22 & 80 & 8 & 14 & 10 & 43 & 20 & 0 & 0 & 12 & 0 & 10 & 10 \\
\hline 21 & 14 & 25 & 20 & 19 & 13 & 14 & 14 & 22 & 20 & 17 & 10 & 14 & $\cdots$ \\
\hline 8.1 & 1.9 & 22 & 3.4 & 7.8 & 7.4 & 2.8 & 6.3 & 5.8 & 7.3 & 1.5 & 8.4 & 15 & .. \\
\hline -. & 3.1 & $\cdots$ & 1.8 & 1.4 & .0 & 1.6 & .. & 2.9 & 2.4 & 2.2 & 3.0 & 4.8 & .. \\
\hline - & .5 & $\cdots$ & .4 & .6 & .7 & .6 & 1.0 & .2 & .3 & .3 & .0 & .. & .. \\
\hline 75 & 90 & .. & 55 & 88 & 73 & 54 & 51 & 82 & 73 & 63 & 56 & 95 & 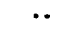 \\
\hline 62 & 74 & .. & 45 & 72 & 60 & 44 & 42 & 57 & 50 & 52 & 46 & 78 & $\cdot$ \\
\hline .0 & 6.3 & .0 & 7.8 & 6.0 & .0 & 6.9 & 5.5 & 12 & 12 & 16 & 1.0 & 9.6 & 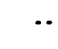 \\
\hline .. & .0 & 9.2 & 1.0 & .5 & .0 & 2.0 & 2.5 & 2.0 & 4. & 5.0 & 1.6 & 3.7 & $\cdots$ \\
\hline .. & .1 & .4 & .1 & .0 & .0 & .0 & .2 & .1 & .1 & .1 & .0 & .1 & .2 \\
\hline .03 & .01 & .18 & .05 & .12 & .10 & .20 & .30 & .16 & .08 & .09 & .00 & 1.7 & .30 \\
\hline .00 & .00 & .04 & .00 & .04 & .00 & .00 & .02 & .03 & .01 & .01 & .00 & .01 & $\cdots$ \\
\hline .00 & .13 & .12 & .02 & .05 & .08 & .00 & .00 & .04 & .15 & .05 & .00 & .02 & \\
\hline
\end{tabular}

Soquin Bay Tributary at Sequin Bay Park, near Soquim, WA. (Site 210)

Date sample collected

Dissolved ailice $\left(\mathrm{SiO}_{2}\right)(\mathrm{ma} / \mathrm{L})$

Disuolved iron (Fe) (ug/L)

Diseolved eangenese ( $(\mathrm{H} n)$ (ug/L)

Disaolved calcium (Ce) (mg/L)

Dissolved magnes iun (Mg) (mo/L)

Dissolved sodiun ( $\mathrm{Wa})(\mathrm{mg} / \mathrm{L})$

Dissolved potessium $(K)(m g / L)$

Bicerbonate $\left(\mathrm{HCO}_{3}\right)(\mathrm{mo} / \mathrm{L})$

Alkal inity $\left(\mathrm{CaCO}_{3}\right)(\mathrm{mg} / \mathrm{L})$

Dissolved sulfete $\left(\mathrm{SO}_{4}\right)(\mathrm{ma} / \mathrm{L})$

Dissolved chloride (Cl) (ma/L)

Diseolved fluoride (F) (mo/L)

Diseolved nitrate (N) (m/L)

Dissolved nitrite (W) (mo/L)

Dissolved phosphorue (P) (mo/L)

Diseolved solide, residue at

$180^{\circ} \mathrm{C}(\mathrm{mo} / \mathrm{L})$

$\begin{array}{ccc}10 / 12 / 65 & 4 / 11 / 73 & 11 / 16 / 7 \\ 12 & 9.3 & . . \\ 210 & 90 & 50 \\ 40 & 6 & 10 \\ 21 & 14 & . . \\ 13 & 15 & . . \\ .7 & 12 & . . \\ 1.9 & .7 & . . \\ 129 & 117 & . . \\ 106 & 96 & . . \\ 15 & 12 & . . \\ 7.8 & 5.0 & . . \\ .2 & .1 & .1 \\ .68 & .16 & .70 \\ .01 & .00 & . . \\ .11 & .00 & . .\end{array}$

151
Dete sample collected

Suspended sol is, ot $105^{\circ} \mathrm{C}(\mathrm{mg} / \mathrm{L})$ Herdhess $\left(\mathrm{CaCO}_{3}\right)(\mathrm{ma} / \mathrm{L})$

Specific conductance (micromhos) pH (units)

Color (pletinum-cobalt units)

Turbidity (JTU)

Dissolved cerbon dioxida (mg/L)

Dissolved arsenic (As) (La/L)

Dissolved bariun (Ba) (ug/L)

Dissolved cadiniun (Cd) (ug/L)

Dissolved chromiun (Cr) (ug/L)

Dissolved leed (Pb) (ug/L)

Dissolved mercury (Hg) (ug/L)

Dissolved selenium (Se) (ug/L)

Dissolved silver (Ag) (ug/L)
$10 / 12 / 65 \quad 4 / 11 / 73 \quad 11 / 16 / 7$

$\begin{array}{rrr}3 & . . & \ldots \\ 104 & 96 & 88 \\ 222 & 144 & 200 \\ 8.0 & 7.0 & \ldots \\ 28 & 25 & 35 \\ 19 & 1 & 1 \\ 2.1 & 26 & \ldots \\ . . & . . & 10 \\ \ldots & \ldots & 250 \\ . . & \ldots & 2 \\ . . & \ldots & 11 \\ . . & \ldots & 10 \\ . . & \ldots & 1 \\ . . & . . & 3 \\ . . & . . & 10\end{array}$


12042400 Solectuck River at Mighwey 101 et Forke, Wash. (river mile 19.0) (site 18)

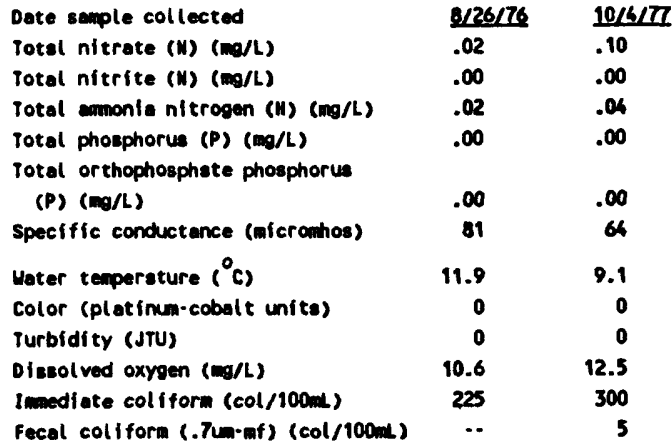

Soleduck River (river wile 17.4) (site 19)

Dats sample collected

Total nitrate (II) (mg/L)

Total nitrite (ii) (mo/L)

Total amonia nitrogen (H) (mo/L)

Totsl phosphorus (P) (n/L)

Total orthophosphate phosphorus

(P) $(\mathrm{mg} / \mathrm{L})$

Specifle conductance (micromhos)

$\begin{array}{cc}\frac{8226 / 76}{.02} & \frac{10 / 6 / 77}{.10} \\ .00 & .00 \\ .02 & .03 \\ .01 & .00 \\ .00 & .00 \\ 82 & 85 \\ 12.0 & 9.0 \\ 0 & 0 \\ 0 & 0 \\ 10.8 & 12.5 \\ 94 & .200 \\ . . & 4\end{array}$

Water temperature $\left({ }^{\circ} \mathrm{c}\right)$

Color (platinum-cobalt unite)

Turbidity (JTu)

Dissolved oxygen (mg/L)

Inmediate colliform (col/100m)

fecal collform (.7m-nf) (col/100-1)
Soleduck River (river mile 16.1) (Site 21)

\begin{tabular}{|c|c|c|}
\hline Date sample collected & $8 / 26 / 76$ & $10 / 4 / 77$ \\
\hline Total nitrate $(M)(\mathrm{mg} / \mathrm{L})$ & .02 & .12 \\
\hline Total nitrite (H) $(\mathrm{mg} / \mathrm{L})$ & .00 & .00 \\
\hline Total amonfe nitrogen (H) (mg/L) & .02 & .08 \\
\hline Total phosphorus $(P)(\mathrm{mg} / L)$ & .00 & .00 \\
\hline \multicolumn{3}{|l|}{ Total or thophosphats phosphorus } \\
\hline (P) $(m / L)$ & .00 & .00 \\
\hline Specific conductanca (micronhos) & 82 & 63 \\
\hline Water temperature $\left({ }^{\circ} \mathrm{C}\right)$ & 12.3 & 9.0 \\
\hline Color (platinum-cobalt units) & o & 0 \\
\hline Turbidity (JTU) & 0 & 0 \\
\hline Diseolved oxyoen (mg/L) & 10.8 & 12.6 \\
\hline Imsediate colsform (col/100m) & 54 & 300 \\
\hline Fecal colfform (.7un-mf) (col/100m) & $\cdots$ & 5 \\
\hline
\end{tabular}

Solectuck River (river mila 15.2) (Site 22)

Date sample collected

Total nitrate (II) (mo/L)

Total nitrite (M) $(\mathrm{mg} / \mathrm{L})$

rotal amanía nitrogen (N) $(\mathrm{mol} / \mathrm{L})$

Total phosphorus (P) (mo/L)

Total or thophosphate phosphorus

(P) $(n g / L)$

Specific conductance (micromhos)

Water temperature $\left({ }^{\circ} \mathrm{c}\right)$

color (platinu-cobalt units)

Turbidity (JTu)

Dissolved oxysen (m/L)

Immedista colliform (col/100m)

Fecal collform (.7um-mf) (col/100m)

$\begin{array}{rr}8 / 26 / 76 & 10 / 4 / 77 \\ .03 & .08 \\ .00 & .00 \\ .05 & .04 \\ .01 & .00 \\ .00 & .00 \\ 83 & 63 \\ 12.2 & 9.0 \\ 0 & 0 \\ 0 & 0 \\ 10.8 & 12.6 \\ 116 & 330 \\ \ldots & 6\end{array}$

12042500 Solectuck River near oullloyute, Wash. (river mila 13.9) (SIta 23)

\begin{tabular}{|c|c|c|}
\hline Dats semple collected & $2 / 26 / 76$ & $10 / 4 / 77$ \\
\hline Total nitrate (H) (mg/L) & .03 & .08 \\
\hline Total nitrite (H) (mg/L) & .00 & .00 \\
\hline Total ammonie nitrogen (II) (mg/L) & .04 & .04 \\
\hline $\begin{array}{l}\text { Total phosphorus }(P)(m / L) \\
\text { Total orthophosphate phosphorus }\end{array}$ & .01 & .00 \\
\hline (P) $(\mathrm{mg} / \mathrm{L})$ & .01 & .00 \\
\hline Specific conductance (micramhos) & 83 & 83 \\
\hline Water temperature $\left({ }^{\circ} \mathrm{C}\right)$ & 12.6 & 9.1 \\
\hline Color (platínu-cobelt unite) & $\mathbf{0}$ & o \\
\hline Turbidity (JTU) & 0 & $\mathbf{0}$ \\
\hline Dissol ved oxygen (mg/t) & 11.0 & 12.7 \\
\hline Inmediate collform (col/100m) & 118 & 200 \\
\hline Fecsl collform (.7nm-nf) (col/100m) & $\cdots$ & 6 \\
\hline
\end{tabular}


Solectuck River (river mile 12.6) (Site 24)

Date sempla collected

Total nitrata (W) $(\mathrm{mg} / \mathrm{L})$

Total nitrita (U) (mg/L)

Total monia nitrogen (U) (ma/L)

Total phosphorue (P) (mg/L)

Total orthophosphate phosphorus

(P) $(\mathrm{mg} / \mathrm{L})$

Specific conductence (micromhos)

Watar temperature $i^{\circ} \mathrm{c}$ )

color (platinu-cobalt units)

Turbidity (JTU)

Dissolved oxygen $(m g / L)$

Imediata coliform (col/100m)

fecal coliform (.7um-nf) (col/100m)

$\begin{array}{cc}8 / 26 / 76 & 10 / 4 / 77 \\ .02 & .08 \\ .00 & .00 \\ .05 & .04 \\ .01 & .00 \\ & \\ .00 & .00 \\ 83 & 81 \\ 12.6 & 8.9 \\ 0 & 0 \\ 0 & 0 \\ 10.9 & 12.7 \\ 74 & 180 \\ . . & 2\end{array}$

Soleduck River (river mila 11.0) (sita 25)

Date aemple callected

Total nitrate (W) $(\mathrm{mg} / \mathrm{L})$

Total nitrita (U) $(\mathrm{mg} / \mathrm{L})$

Total monia nitrogen $(U)(m a / L)$

Total phosphorue (P) (mg/L)

Total orthophosphate phosphorus

$$
\text { (P) }(\mathrm{mg} / \mathrm{L})
$$

Specific conductence (micromhos)

(sita 25$)$

Water temperatura $\left({ }^{\circ} \mathrm{C}\right)$

color (platinum-cobalt units)

Turbidity (JTU)

Dissolved oxygen (mo/l)

Impediate colliform (col/100m)

fecal coliform (.7m-mf) (col/100m)

\begin{tabular}{|c|c|}
\hline $8 / 26 / 76$ & $10 / 4 / \pi$ \\
\hline .02 & .08 \\
\hline .00 & .00 \\
\hline .04 & .07 \\
\hline .01 & .00 \\
\hline .00 & .00 \\
\hline 83 & 64 \\
\hline 13.0 & 9.2 \\
\hline 0 & 0 \\
\hline 0 & 0 \\
\hline .. & 12.6 \\
\hline 88 & 150 \\
\hline .. & 4 \\
\hline
\end{tabular}

Soleduck River (river mila 9.4) (sita 26)

\begin{tabular}{|c|c|c|}
\hline Date smple collected & $8 / 26 / 76$ & $10 / 4 / 7 \pi$ \\
\hline Total nitrate (II) (mo/L) & .02 & .07 \\
\hline Total nitrita (II) (mo/l) & .00 &.$\infty$ \\
\hline Total cmonia nitrogen $(\mathrm{K})(\mathrm{mg} / \mathrm{L})$ & .03 & .04 \\
\hline $\begin{array}{l}\text { Total phosphorus (P) (mg/L) } \\
\text { Total or thophosphata phosphorus }\end{array}$ & .01 & .00 \\
\hline (P) $(\mathrm{mg} / \mathrm{L})$ &.$\infty$ & .00 \\
\hline Specific conductance (microathos) & 82 & 83 \\
\hline Watar temperature $\left({ }^{\circ} \mathrm{C}\right)$ & 13.4 & 9.2 \\
\hline color (platínu-cobalt units) & $\mathbf{0}$ & 0 \\
\hline Turbidity (JTU) & $\mathbf{0}$ & $\mathbf{0}$ \\
\hline Dissolved oxygen (mg/L) & 10.9 & 12.1 \\
\hline Imnediate collform & 38 & 170 \\
\hline fecal coliform (.7m-mf) (col/1 & .. & 4 \\
\hline
\end{tabular}

Soleduck River (river mila 8.3) (site 27)

\begin{tabular}{|c|c|c|}
\hline Data sample collected & $8 / 26 / 76$ & $10 / 4 / 77$ \\
\hline rotal nitrata $(W)(\mathrm{mg} / \mathrm{L})$ & .01 & .07 \\
\hline rotal nitrita (N) (mo/L) & .01 & .00 \\
\hline rotal mmonia nitrogen (W) (mg/L) & .05 & .04 \\
\hline $\begin{array}{l}\text { rotal phosphorus }(P)(\mathrm{mg} / \mathrm{L}) \\
\text { rotal or thophosphata phosphorus }\end{array}$ & .01 & .00 \\
\hline (P) $(m g / L)$ & .00 & .00 \\
\hline Specific conductanca (micromos) & 83 & 62 \\
\hline Water temperatura $\left({ }^{\circ} \mathrm{C}\right)$ & *. & 9.1 \\
\hline Color (platinum-cobalt units) & 0 & 0 \\
\hline Turbidity (JTU) & 0 & 0 \\
\hline Dissolved oxygen (mg/L) & 10.8 & 12.2 \\
\hline Imnediate coliform & 45 & 160 \\
\hline Fecal coliform (.7m-mf) (col/100m & -. & 3 \\
\hline
\end{tabular}

12042503 Soleduck Rivar at mouth near ta Push, Wash. (river mile 6.5) (Site 28)

\begin{tabular}{|c|c|}
\hline Data sample collected & $10 / 4 / 77$ \\
\hline Total nitrate $(N)(m g / L)$ & .06 \\
\hline Total nitrite (N) (mg/L) & .00 \\
\hline Total monia nitrogen ( $(K)(\mathrm{mg} / \mathrm{t})$ & .04 \\
\hline Total phosphorus $(P)(\mathrm{mg} / \mathrm{l})$ & .00 \\
\hline Total or thophosphate phosphorus & \\
\hline (P) $(\mathrm{mg} / \mathrm{l})$ & .00 \\
\hline Specific conductance (micromhos) & 69 \\
\hline Watar teaperature $\left({ }^{\circ} \mathrm{c}\right)$ & 9.5 \\
\hline color (platinum-cobalt units) & 0 \\
\hline Turbidity (JTU) & $\mathbf{0}$ \\
\hline Dissolved oxygen (mg/L) & 11.9 \\
\hline Immediate coliform (col/100m) & 2400 \\
\hline ecal coliform (.7um-mf) (col/10 & 7 \\
\hline
\end{tabular}

East Fork Dickay Rivar at mouth, at Dickey River mila 7.9 (sita 64)

\begin{tabular}{|c|c|c|}
\hline Date sample collected & $8 / 24 / 76$ & $10 / 4 / 77$ \\
\hline total nitrata $(N)(\mathrm{mg} / \mathrm{L})$ & .05 & .12 \\
\hline Total nitrita (N) $(\mathrm{mg} / \mathrm{L})$ & .00 & .00 \\
\hline Total ammia nitrogen (W) (mg/L) & .05 & .04 \\
\hline Total phosphorus (P) (mg/L) & .02 & .02 \\
\hline \multicolumn{3}{|l|}{ Total or thophosphata phosphorus } \\
\hline (P) $(m g / L)$ & .01 & .00 \\
\hline Specific conductence (micromos) & 74 & 65 \\
\hline Water temperature $\left({ }^{\circ} \mathrm{c}\right)$ & 12.6 & 7.3 \\
\hline Color (platinum-cobalt units) & 25 & 20 \\
\hline Turbidity (JTU) & 1 & 1 \\
\hline 0issolved oxygen (mg/L) & 10.0 & 11.5 \\
\hline Imediate colliform (col/100m ) & 62 & 260 \\
\hline fecal coliform $(.7 \mathrm{~m}-\mathrm{mf})(\mathrm{col} / 100 \mathrm{~m})$ & - & 25 \\
\hline
\end{tabular}


West fork Dickey River at mouth (site 65)

\begin{tabular}{|c|c|c|}
\hline Data sample collected & $8 / 24 / 76$ & $10 / 4 / 77$ \\
\hline Total nitrate $(N)(n g / L)$ & .02 & .02 \\
\hline Total nitrite $(N)\left(\mathrm{mg}_{\mathrm{g}} / \mathrm{L}\right)$ & .00 & .01 \\
\hline Total anmonia nitrogen (N) (mg/L) & .10 & .03 \\
\hline Total phosphorus (P) $(\mathrm{mg} / \mathrm{L})$ & .02 & .02 \\
\hline \multicolumn{3}{|l|}{ Total orthophosphate phosphorus } \\
\hline (P) $(m g / L)$ & .01 & .01 \\
\hline Specific conductance (micromhos) & 44 & 48 \\
\hline Water temperature $\left({ }^{\circ} \mathrm{C}\right)$ & 13.7 & 8.9 \\
\hline Color (platinum-cobalt units) & 40 & 40 \\
\hline Turbidity (JTU) & 1 & 1 \\
\hline Dissolved oxygen (mg/L) & 9.4 & 10.5 \\
\hline Immediate coliform (col/100mL) & 90 & 240 \\
\hline fecal coliform $(.7 \mathrm{~mm}-\mathrm{mf})(\mathrm{col} / 10 \mathrm{~mL})$ & $\cdots$ & 44 \\
\hline
\end{tabular}

Dickey River (river mile 7.7 ) (site 66)

\begin{tabular}{|c|c|c|}
\hline Data sample collected & $8 / 24 / 76$ & $10 / 4 / \pi$ \\
\hline Total nitrate (N) (ma/L) & .03 & .08 \\
\hline Total nitrite (N) $(\mathrm{mg} / \mathrm{L})$ & .00 & .00 \\
\hline Total amonia nitrogen (N) (mg/L) & .05 & .04 \\
\hline Total phosphorus $(P)(\mathrm{mg} / \mathrm{L})$ & .02 & .02 \\
\hline \multicolumn{3}{|l|}{ Total orthophosphate phosphorus } \\
\hline (P) $(m g / L)$ & .01 & .00 \\
\hline Specific conductance (micromhos) & 56 & 56 \\
\hline Water temperature $\left({ }^{\circ} \mathrm{c}\right)$ & 13.5 & 8.0 \\
\hline Color (platinum-cobatt units) & 35 & 30 \\
\hline Turbidity (JTU) & 1 & 1 \\
\hline Dissolved oxygen (mg/L) & 9.8 & 11.1 \\
\hline Immediate coliform (col/100mL) & 84 & 300 \\
\hline fecal coliform $(.7 \mathrm{um} \cdot \mathrm{mf})(\mathrm{col} / 100 \mathrm{~mL}$ & $\cdots$ & 30 \\
\hline
\end{tabular}

Oickey River (river mile 6.8) (site 67)

\begin{tabular}{|c|c|c|}
\hline Date sample collected & $8 / 24 / 76$ & $10 / 4 / 77$ \\
\hline Total nitrate (N) (mg/L) & .03 & .08 \\
\hline Total nitrite (N) (mg/L) & .00 & .00 \\
\hline Total amonia nitrogen (N) (mg/L) & .10 & .03 \\
\hline $\begin{array}{l}\text { Total phosphorus (P) (mg/L) } \\
\text { Total orthophosphate phosphorus }\end{array}$ & .04 & .02 \\
\hline (P) $(m g / L)$ & .04 & .01 \\
\hline Specific conductance (nicromhos) & 59 & 57 \\
\hline Weter temperature $\left({ }^{\circ} \mathrm{C}\right)$ & 13.4 & 8.0 \\
\hline Color (platinum-cobalt units) & 35 & 30 \\
\hline Turbidity (JTU) & 1 & 1 \\
\hline Dissolved oxygen (mg/L) & 9.8 & 10.8 \\
\hline Immediate coliform (col/100m) & 78 & 130 \\
\hline Fecel coliform (.7um-nf) (col/100mL) & $\cdots$ & 48 \\
\hline
\end{tabular}

12043100 Dickey River near La Push, Wash. (river mile 6.D) (Site 68)

\begin{tabular}{|c|c|c|}
\hline Date sample collacted & $8 / 24 / 76$ & $10 / 4 / 7 \pi$ \\
\hline Total nitrate $(N)(\mathrm{mg} / \mathrm{L})$ & .02 & .07 \\
\hline Total nitrite (N) $(\mathrm{mg} / \mathrm{L})$ & .01 & .00 \\
\hline Total ammonia nitrogen $(N)(\mathrm{mg} / \mathrm{L})$ & .07 & .04 \\
\hline Total phosphorus (P) (mg/L) & .02 & .01 \\
\hline \multicolumn{3}{|l|}{ Total or thophosphata phosphorus } \\
\hline (P) $(m g / t)$ & .01 & .00 \\
\hline Specific conductance (micromhos) & 57 & 58 \\
\hline Watar temperature $\left({ }^{\circ} \mathrm{C}\right)$ & 13.2 & 7.9 \\
\hline Color (platimu-cobalt units) & 35 & 30 \\
\hline Turbidity (JTU) & 1 & 1 \\
\hline Dissolved oxygen (mg/L) & 9.8 & 11.2 \\
\hline Immediate coliform (col/ $100 \mathrm{~mL}$ ) & 90 & 280 \\
\hline Fecal coliform (.7um-mf) (col/100m) & .. & 30 \\
\hline
\end{tabular}

Dickey River (river mile 5.1) (site 70)

\begin{tabular}{|c|c|c|}
\hline Date sample collected & $8 / 24 / 76$ & $10 / 4 / 77$ \\
\hline Total nitrate $(N)(\mathrm{mg} / \mathrm{L})$ & .03 & .09 \\
\hline Total nitrite $(N)(\mathrm{mg} / \mathrm{L})$ & .00 & .00 \\
\hline Total ammonia nitrogen (N) (mg/L) & .06 & .06 \\
\hline Total phosphorus (P) (mg/L) & .02 & .02 \\
\hline \multicolumn{3}{|l|}{ Total orthophosphate phosphorus } \\
\hline (P) $(m g / L)$ & .01 & .01 \\
\hline Specific conductance (micromhos) & 58 & 58 \\
\hline Water temperature $\left({ }^{\circ} \mathrm{C}\right)$ & 13.0 & 7.9 \\
\hline Color (platinum-cobalt units) & 35 & 30 \\
\hline Turbidity (JTU) & 1 & 1 \\
\hline Dissolved oxygan (mg/L) & 9.7 & 11.2 \\
\hline Imediate coliform (col/ $100 \mathrm{~mL}$ ) & 156 & 82 \\
\hline Fecal coliform (.7um-nf) (col/100m $)$ & $\because$ & 37 \\
\hline
\end{tabular}

Colby Creek at mouth at Dickey River mile 5.0 (Site 72)

\begin{tabular}{|c|c|c|}
\hline Date sample collected & $8 / 24 / 76$ & $10 / 4 / 77$ \\
\hline Total nitrate $(N)(\mathrm{mg} / \mathrm{L})$ & .07 & .14 \\
\hline Total nitrite (N) (mg/L) & .01 & .00 \\
\hline Total ammonia nitrogen $(H)(\mathrm{mg} / \mathrm{L})$ & .11 & .04 \\
\hline Total phosphorus (P) (mg/L) & .02 & .01 \\
\hline $\begin{array}{l}\text { Total or thophosphate phosphorus } \\
\text { (P) }(m g / L)\end{array}$ & .02 & .00 \\
\hline Specific conductance (micromos) & 83 & 80 \\
\hline Water temperature $\left({ }^{\circ} \mathrm{C}\right)$ & 11.4 & 6.9 \\
\hline Color (platinum-cobalt units) & 40 & 30 \\
\hline Turbidity (JTU) & 2 & 1 \\
\hline Dissolved oxygen (mg/L) & 10.0 & 11.3 \\
\hline Immediate coliform (col/100m) & 76 & 200 \\
\hline fecal colliform (.7um-at) (col/1 & $\cdots$ & 22 \\
\hline
\end{tabular}




\begin{tabular}{|c|c|c|}
\hline Date eample collected & $2 / 26 / 76$ & $10 / 4 / 77$ \\
\hline Total nitrate (W) $(\mathrm{mg} / \mathrm{L})$ & .02 & .08 \\
\hline Totel nitrite (W) $(\mathrm{mg} / \mathrm{L})$ & .01 & .01 \\
\hline Total monis nitrogen $(M)(\mathrm{mo} / L)$ & .12 & .04 \\
\hline $\begin{array}{l}\text { Total phosphorus }(P)(\mathrm{mg} / \mathrm{L}) \\
\text { rotal or thophosphete phosphorus }\end{array}$ & .04 & .01 \\
\hline (P) $(\min / L)$ & .01 & .00 \\
\hline Specific conductance (micranhos) & 62 & 60 \\
\hline Water teapereture $\left({ }^{\circ} \mathrm{C}\right)$ & 13.2 & 7.9 \\
\hline Color (plat inum-cobalt unite) & 30 & 30 \\
\hline Purbldity (JTU) & 1 & 1 \\
\hline Diecolved axygen $(\mathrm{mig} / \mathrm{L})$ & 9.7 & 11.3 \\
\hline Immediete colliform (col/100m) & 155 & 160 \\
\hline Fecal colliform (.7um-mf) (col/100ml) & $\cdots$ & 27 \\
\hline
\end{tabular}

Coal Creek at mouth et Dickey River mile 3.9 (site 74)

\begin{tabular}{|c|c|c|}
\hline Date sample collected & $8 / 24 / 76$ & $10 / 4 / 77$ \\
\hline Total nitrate $(K)(\mathrm{mg} / \mathrm{L})$ & .01 & .05 \\
\hline Total nitrite $(H)(m g / L)$ & .01 & .00 \\
\hline rotel amonia nitrogen (W) $(\mathrm{mg} / \mathrm{L})$ & .09 & .04 \\
\hline $\begin{array}{l}\text { Total phosphorus }(P)(\mathrm{mg} / \mathrm{L}) \\
\text { Total or thophosphate phosphorus }\end{array}$ & .03 & .02 \\
\hline (P) $(m g / L)$ & .02 & .01 \\
\hline Specific conductance (micranhos) & 52 & 54 \\
\hline Water tenperature $\left({ }^{\circ} \mathrm{C}\right)$ & 12.1 & 7.3 \\
\hline Color (platimu-cobalt unita) & 35 & 20 \\
\hline Turbidity (JTU) & 6 & 2 \\
\hline Diecolved axyoen (mg/L) & 10.4 & 12.0 \\
\hline mediate colliform (col/100m & 180 & 430 \\
\hline
\end{tabular}

Dete sample collected

Total nitrate (W) (m/L)

Potel nitrite (W) (m/L)

Total amonia nitrogen (W) (m/L)

Total phosphorus (P) (m/L)

Total orthophosphete phosphorus

(P) $(\mathrm{m} / \mathrm{L})$

Specific conductance (nicranhos)

Water temperature $\left({ }^{\circ} \mathrm{C}\right.$ )

Color (plotimu-cobalt unite)

Turbidi ty (JTU)

Diseolved axygen (ng/L)

Imediate colliform (col/1900m)

Fecal colliform (.7nmenf) (col/100m)

$\begin{array}{cc}8 / 24 / 76 & 10 / 4 / 77 \\ .03 & .08 \\ .00 & .01 \\ .06 & .05 \\ .02 & .01 \\ & \\ .01 & .00 \\ 59 & 61 \\ 13.1 & 8.3 \\ 35 & 30 \\ 1 & 11 \\ 9.6 & 11.1 \\ 126 & 380 \\ . . & 46\end{array}$

Dickey River (river mile 0.3 ) (site 7 )

Dete sample collected

Potal nitrate (W) (mg/L)

Total nitrite (W) (mo/L)

Total amonia nitrogen (W) (mg/L)

Total phoephorus (P) $(\mathrm{m} / \mathrm{h} / \mathrm{h})$

Totel or thophosphate phosphorus

$$
\text { (P) }(\mathrm{mg} / \mathrm{L})
$$

specific conductance (nicromhos)

Weter temperature $\left({ }^{\circ} \mathrm{C}\right)$

Color (platimn-cobalt unite)

Turbidity (JTU)

Diesolved axyoen $(\mathrm{mg} / \mathrm{L})$

Immediate colliform (col/100m

Fecal colliform (.7m-nf) (col/100m)

$\begin{array}{cc}8 / 24 / 76 & 10 / 4 / 77 \\ .02 & \cdots \\ .00 & \ldots \\ .04 & \ldots \\ .01 & \ldots \\ .01 & \ldots \\ 61 & 79 \\ 14.8 & \ldots \\ 30 & \ldots \\ 1 & \ldots \\ 9.6 & 9.4 \\ 158 & 700 \\ . . & \ldots\end{array}$

Dickey River (river mile 2.8) (Site 75)

\begin{tabular}{|c|c|c|}
\hline Date semple collected & $8 / 24 / 76$ & $10 / 4 / 77$ \\
\hline Total nitrate (W) (mo/L) & .02 & .09 \\
\hline Total nitrite (K) (mo/L) & .01 & .00 \\
\hline Total manonle nitrogen $(K)(\mathrm{mg} / \mathrm{L})$ & .06 & .06 \\
\hline $\begin{array}{l}\text { Total phosphorus (P) }(\mathrm{mg} / \mathrm{L}) \\
\text { Total orthophosphate phosphorus }\end{array}$ & .02 & .01 \\
\hline (P) $(\mathrm{mg} / \mathrm{L})$ & .01 & .01 \\
\hline Specifle conductance (micranhos) & 59 & 61 \\
\hline Water temperature $\left({ }^{\circ} \mathrm{C}\right)$ & 13.4 & 8.0 \\
\hline Color (platinum-cobalt unite) & 35 & 30 \\
\hline Turbidity (JTU) & 1 & 1 \\
\hline Diacolved oxygen (mg/L) & 9.8 & 11.2 \\
\hline Imsediate colliform (col/100m) & 96 & 320 \\
\hline fecal collform (.7um-nf) (col/10 & - & 30 \\
\hline
\end{tabular}


TABLE 15.--Suspended sediment and related parameters in water from selected surface-water sites in the study area.

12042503 Soleduck River at mouth near La Push, Wash. (Site 28)

$\begin{array}{llcccc} & \begin{array}{l}\text { Streamflow, } \\ \text { instantaneous, }\end{array} & \begin{array}{c}\text { Tempera- } \\ \text { ture }\end{array} & \text { Turbidity } & \text { Sediment, } & \text { Sediment } \\ \text { suspended discharge, } & \text { (cubic feet } & \left({ }^{\circ} \mathrm{C}\right) & \text { (JT) } & \text { (milligrams suspended } \\ \text { Date } & & & \text { per liter) (tons per day) }\end{array}$

1975

December

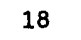

1130

$--$

4.4

--

1245

$-$

5.5

1115

6.0

1030

$--$

8.0

1500

$--$

$-$

7.0

1015

$-$

7.2

1005

1610

$-$

5.7

31

$--$

$--$

1976

January

1100

1130

1330

1400

1530

1340

1150

1230

1100

1230

1130

1115

1155

1200

--
--
1170
--
1140
1070
1160
1040
1130
1160
--
1200
1190
1230

.5

5.0

--

6.1

5.0

6.0

4.0

6.0

6.0

6.0

5.0

7.0

5.0

4.0

$--$

--

$-$

$-$

$--$

$-$

per liter)

(tons per day)

25

1230

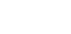

-- 10

$--18$

-- 16

-- 32

-- 20

-- 34

-- 15

-- 16

-- 62

-- 18

-- 30

$--13$

$-$

$--$

$-$ 
TABLE 15.--Suspended sediment and related parameters in water from selected surface-water sites in the study area.

12042503 Soleduck River at mouth near La Push, Wash. (Site 28)--cont.

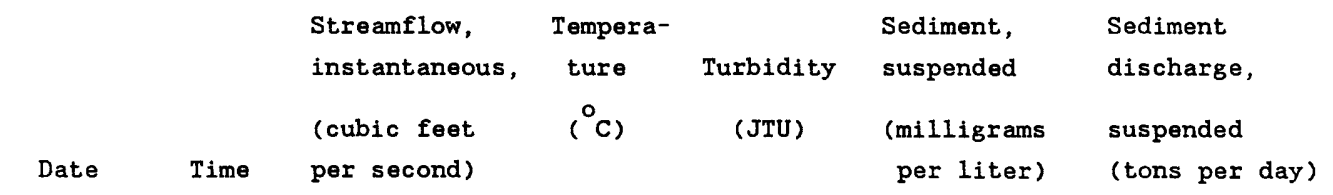

1976

January

$26 \quad 1200$

$28 \quad 1245$

1245

0950

0940

1250

5.0

$-$

10

-- 6.5

6.0

6.0

$-$

$-$

$--$

30

$-$

February

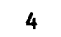

1420

1330

4. 3

5.5

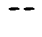

3

11

$11 \quad 1435$

2120

2970

3910

5.5

$-$

30

172

1215

5.0

$-$

217

17

1130

2900

5.0

$-$

306

141

March

12

$1330 \quad 1220$

2510

7.0

$-$

13

1120

8.0

$-$

237

1130

2770

8.0

-

142

1200

4270

7.0

$-$

1420

1100

3870

7.0

$-$

481

1020

3150

7.0

$--$

170

April

1

1230

1860

7.5

$-$

90

15

1500

1430

9.0

--

19 
TABLE 15.--Suspended sediment and related parameters in water from selected surface-water sites in the study area.

12042503 Soleduck River at mouth near La Push, Wash. (Site 28)--cont.

$\begin{array}{llcccc} & \text { Streamflow, } & \text { Tempera- } & \text { Sediment } \\ \text { instantaneous, } & \text { ture } & \text { Turbidity } & \begin{array}{l}\text { Sediment, } \\ \text { suspended discharge, }\end{array} \\ \text { (cubic feet } & \left({ }^{\circ} \mathrm{C}\right) & \text { (JTU) } & \text { (milligrams suspended } \\ \text { Date } & & & \text { per liter) (tons per day) }\end{array}$

1976

June

8

1025

1080

$-$

$-$

3

8. 7

17
24

1215

1045

July

2

8

14

23

1125

1720

1030

--

$-$

$-$

$-$

26

$-$

562
1140
766
626

11.5

$-$

$-$

--

13.5

1

1

18

19

1.7

August

$4 \quad 1255$

1300

502

14.5

1

1

1.4

20

$--$

$--$

$-\quad 17.0$

1700

1300

September

29

October

6

20

27

$\because$

$\because$

$\cdots$

12.0 
TABLE 15.--Suspended sediment and related parameters in water from selected surface-water sites in the study area.

12042503 Soleduck River at mouth near La Push, Wash. (Site 28)--cont.

Date Time per second)

\begin{tabular}{|c|c|c|c|c|}
\hline $\begin{array}{l}\text { Streamflow, } \\
\text { instantaneous, }\end{array}$ & $\begin{array}{l}\text { Tempera- } \\
\text { ture }\end{array}$ & Turbidity & $\begin{array}{l}\text { Sediment, } \\
\text { suspended }\end{array}$ & $\begin{array}{l}\text { Sediment } \\
\text { discharge, }\end{array}$ \\
\hline $\begin{array}{l}\text { (cublc feet } \\
\text { per second) }\end{array}$ & $\left({ }^{\circ} \mathrm{C}\right)$ & (JTU) & $\begin{array}{l}\text { (milligrams } \\
\text { per liter) }\end{array}$ & $\begin{array}{l}\text { suspended } \\
\text { (tons per day) }\end{array}$ \\
\hline
\end{tabular}

1976

November

1

4

17

$-$

100

1430

$--$

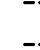

$--$

9.0

8.0

December

1

8

1130

--

1530

960

1130

1130

$--$

--

$--$

1510

$-$

1977

January

$$
5
$$

4.0

1645

$-$

$$
4.0
$$$$
-
$$

$--$

17.0

1530

$--$

1540

$--$

7.0

6.0

6.0

9.0

6.0

$-$

6.0

--
--
--
--

--

--
-
--
--
--
-
-
-

$-$

$-$

18

$--$

$--$

$--$

$--$

$-$

13

$-$

$-$

$-$

$-$

$-$

$--$

$\begin{aligned} 1 & -- \\ 4 & -- \\ 20 & -- \\ 547 & -- \\ 63 & -- \\ 2 & --\end{aligned}$


TABLE 15.--Suspended sediment and related parameters in water from selected surface-water sites in the study area.

12042503 Soleduck River at mouth near La Push, Wash. (Site 28)--cont.

\begin{tabular}{|c|c|c|c|c|c|c|}
\hline Date & Time & $\begin{array}{l}\text { Streamflow, } \\
\text { instantaneous, } \\
\text { (cubic feet } \\
\text { per second) }\end{array}$ & $\begin{array}{l}\text { Tempera- } \\
\text { ture } \\
\left.{ }^{\circ} \mathrm{C}\right)\end{array}$ & $\begin{array}{l}\text { Turbidity } \\
\text { (JTU) }\end{array}$ & $\begin{array}{l}\text { Sediment, } \\
\text { suspended } \\
\text { (milligrams } \\
\text { per liter) }\end{array}$ & $\begin{array}{l}\text { Sediment } \\
\text { discharge, } \\
\text { suspended } \\
\text { (tons per day) }\end{array}$ \\
\hline \multicolumn{7}{|l|}{1977} \\
\hline \multicolumn{7}{|c|}{ February } \\
\hline 4 & 1245 & -- & 6.5 & -- & 2 & -- \\
\hline 9 & 1035 & -- & 8.0 & -- & 2 & -- \\
\hline 10 & -- & -- & 4.5 & -- & 3 & -- \\
\hline 11 & 1500 & -- & 7.1 & -- & 8 & -- \\
\hline 13 & 1200 & 2970 & 5.6 & -- & 27 & 217 \\
\hline 15 & 1515 & -- & 5.5 & -- & 1 & -- \\
\hline 16 & - & -- & -- & -- & 1 & -- \\
\hline 17 & 1340 & -- & 9.0 & -- & 4 & -- \\
\hline 18 & 1445 & 3790 & 5.0 & -- & 35 & 358 \\
\hline 22 & -- & -- & 9.0 & -- & 140 & -- \\
\hline 23 & 1130 & 2630 & 7.5 & -- & 26 & 185 \\
\hline 25 & 1230 & 2450 & 3.9 & -- & 12 & 79 \\
\hline 25 & 1445 & -- & 6.5 & -- & 11 & -- \\
\hline 27 & 1215 & 2130 & 4.4 & -- & 15 & 86 \\
\hline 28 & 1505 & -- & 7.0 & -- & 4 & -- \\
\hline \multicolumn{7}{|l|}{ March } \\
\hline 1 & 1500 & 1060 & 6.2 & -- & 4 & 11 \\
\hline 2 & 1010 & 1660 & 6.0 & -- & 3 & 13 \\
\hline 8 & -- & -- & 5.5 & -- & 93 & -- \\
\hline 9 & 1500 & -- & 5.5 & -- & 66 & -- \\
\hline 10 & 1200 & 1660 & 7.0 & -- & 30 & 134 \\
\hline 16 & 1500 & --- & 7.0 & -- & 12 & -- \\
\hline 31 & 1530 & 1180 & 6.0 & -- & 1 & 3.2 \\
\hline
\end{tabular}


TABLE 15.--Suspended sediment and related parameters in water from selected surface-water sites in the study area.

12042503 Soleduck River at mouth near La Push, Wash. (Site 28)--cont.

$\begin{array}{llcccc} & \begin{array}{l}\text { Streamflow, } \\ \text { instantaneous, }\end{array} & \begin{array}{c}\text { Tempera- } \\ \text { ture }\end{array} & \text { Turbidity } & \begin{array}{l}\text { Sediment, } \\ \text { suspended }\end{array} & \text { discharge, } \\ \text { (cubic feet } & \left({ }^{\circ} \mathrm{C}\right) & \text { (JTU) } & \text { (milligrams suspended } & \text { sent } \\ \text { Date } & \text { Time second) } & & \text { per liter) (tons per day) }\end{array}$

1977

April

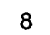

1510

1460

9.0

--

12

14

1240

1150

7.0

$--$

8.5

-.

1320

10.0

$-$

3.1

--

$1430 \quad 1400$

9.0

$-$

11

$1530 \quad 1220$

9.5

-

13

$1450 \quad 910$

10.5

-

54

$1610 \quad 800$

11.0

$-$

1055

10.5

-

4.3

775

11.0

-

4.2

$1750 \quad 700$

11.5

$-$

3. 8

1545

$-$

9.0

$--$

--

16

1400

775

10.5

$--$

11.5

12.5

13.0

12.0

14.0

$\begin{array}{lr}1650 & 1280 \\ 1230 & 1360 \\ 1145 & -- \\ 1200 & 710 \\ 1635 & 530\end{array}$

1280

--

1

1

1

1

27

510

1 
TABLE 15.--Suspended sediment and related parameters in water from selected surface-water sites in the study area.

12042503 Soleduck River at mouth near La Push, Wash. (Site 28)--cont.

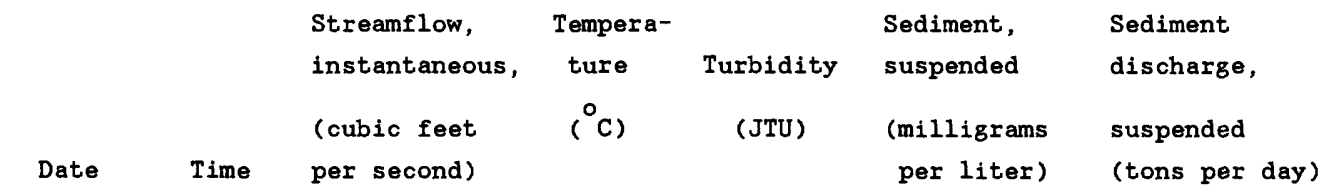

1977

July

4

$18-0950$

0950

$--$

6.0

1240

$-$

14.5

21

$-$

13.7

$--$

80

178

August

11

1050

$\begin{array}{ll}- & 7.0\end{array}$

7.0
23.0

$--23.0$

1615

$\begin{array}{ll}- & 16.0 \\ -- & 14.0\end{array}$

1200

1710

-14.0
$--\quad 15.5$

24

September

2

1305

-- $\quad 14.0$

1315

$\begin{array}{ll}-- & 15.5\end{array}$

1645

$--$

16.0

14

1135

--

14.0

20

1000

755

11.0

0930

898

11.0

22

1035

382

11.0

2
2
--
1
1

$\begin{aligned} 8 & -- \\ 12 & -- \\ 8 & -- \\ 10 & -- \\ 35 & --\end{aligned}$

October

24

1200

964

1870

11.0

9.0

2

13

34

25

1000

1870

2

45

227 
TABLE 15.--Suspended sediment and related parameters in water from selected surface-water sites in the study area.

12042503 Soleduck River at mouth near La Push, Wash. (Site 28)--cont.

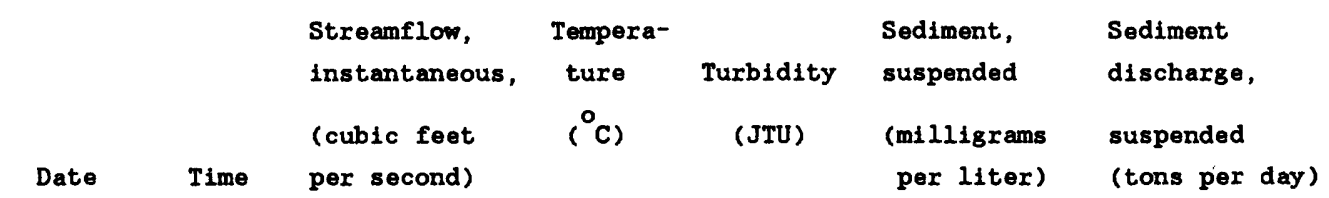

1977

October

1600
1250

1570

9.6

9.0

9

38

31

1400

2640

8.0

28

24

200

\section{November}

1

1300

3290

5580

7.0

1545

1315

2240

4430

1115

1150

3200

1230

1520

4640

1590

3790

1040

1000

6410

10.0

8.0

8.0

8.0

8.0

3.0

7.0

7.0

$\begin{array}{rr}60 & 533 \\ 204 & 3070 \\ 90 & 544 \\ 108 & 1290 \\ 42 & 363 \\ 80 & 1000 \\ 9 & 39 \\ 43 & 440 \\ 312 & 5400\end{array}$

December

$\begin{array}{rrr}1 & 1200 & 3910 \\ 4 & -- & 4950 \\ 4 & 1050 & 5070 \\ 6 & 1100 & 3560 \\ 9 & 1430 & 2610 \\ 12 & 1345 & 7230 \\ 19 & 1300 & 2370 \\ 30 & 1200 & 1280\end{array}$

--
7.2
6.1
4.0
--
7.0
5.0

5
--
--
2
13
1
1

111

1170

2180

2330

452

113

3030

90

24 
TABLE 15.--Suspended sediment and related parameters in water from selected surface-water sites in the study area.

12042503 Soleduck River at mouth near La Push, Wash. (Site 28)--cont.

$\begin{array}{llcccc} & \begin{array}{l}\text { Streamflow, } \\ \text { instantaneous, }\end{array} & \begin{array}{c}\text { Tempera- } \\ \text { ture }\end{array} & \text { Turbidity } & \text { Sediment, } & \text { Sediment } \\ \text { suspended } & \text { discharge, } \\ \text { (cubic feet } & \left.{ }^{\circ} \mathrm{C}\right) & \text { (JTU) } & \text { (milligrams suspended } \\ \text { Date } & & & \text { per liter) (tons per day) }\end{array}$

1978

January

$\begin{array}{rrrrrrr}3 & 1120 & 1060 & 4.1 & 1 & 14 & 40 \\ 6 & 1530 & 2090 & 5.0 & 4 & 11 & 62 \\ 10 & 1020 & 2550 & 6.0 & 5 & 20 & 138\end{array}$

May

\section{8}

10

15

17

23

25

26

1020

2550

6.0

12.0

11.0

$-$

11.0

10.0

11.0

1415

795

680

670

$-$

646

14.8

17.9

13.0

12.5

--

16.0

1100

780

514

14.0

546

1120

-. 1180

-.

1

4

5.9

12

$-$

11

.00
1.8
.00
1.8
21
5.6

1.4

3.1

90

5.0

2.1

11

1.8

$\begin{array}{ll}1 & 1.8\end{array}$


TABLE 15.--Suspended sediment and related parameters in water from selected surface-water sites in the study area.

12042503 Soleduck River at mouth near La Push, Wash. (Site 28)--cont.

\begin{tabular}{|c|c|c|c|c|c|c|}
\hline Date & Time & $\begin{array}{l}\text { Streamflow, } \\
\text { instantaneous, } \\
\text { (cubic feet } \\
\text { per second) }\end{array}$ & $\begin{array}{l}\text { Tempera- } \\
\text { ture } \\
\left.\text { ( }^{\circ} \mathrm{C}\right)\end{array}$ & $\begin{array}{c}\text { Turbidi ty } \\
\text { (JTU) }\end{array}$ & $\begin{array}{l}\text { Sediment, } \\
\text { suspended } \\
\text { (milligrams } \\
\text { per liter) }\end{array}$ & $\begin{array}{l}\text { Sediment } \\
\text { discharge, } \\
\text { suspended } \\
\text { (tons per day) }\end{array}$ \\
\hline \multicolumn{7}{|l|}{1978} \\
\hline \multicolumn{7}{|c|}{ September } \\
\hline 13 & 1100 & 970 & -- & 2 & 8 & 21 \\
\hline 18 & 1455 & 660 & 13.0 & 1 & 3 & 5.3 \\
\hline 20 & 0955 & 566 & 14.0 & 1 & 3 & $4.5 \quad 221050$ \\
\hline 976 & 12.5 & 1 & 4 & 11 & & \\
\hline 25 & 1050 & 1020 & 14.0 & 2 & 6 & 17 \\
\hline 28 & 0930 & 690 & 13.0 & -- & 5 & 9.3 \\
\hline 29 & 0750 & 646 & 12.0 & -- & 4 & 7.0 \\
\hline
\end{tabular}


TABLE 15.--Suspended sediment and related parameters in water from selected surface-water sites in the study area.

12043000 Calawah River near Forks, Wash. (Site 42)

\begin{tabular}{|c|c|c|c|c|c|c|}
\hline & & $\begin{array}{l}\text { Streamflow, } \\
\text { instantaneous, }\end{array}$ & $\begin{array}{l}\text { Tempera- } \\
\text { ture }\end{array}$ & Turbidity & $\begin{array}{l}\text { Sediment, } \\
\text { suspended }\end{array}$ & $\begin{array}{l}\text { Sediment } \\
\text { discharge, }\end{array}$ \\
\hline Date & Time & $\begin{array}{l}\text { (cubic feet } \\
\text { per second) }\end{array}$ & C) & (JTU) & $\begin{array}{l}\text { (milligrams } \\
\text { per liter) }\end{array}$ & $\begin{array}{l}\text { suspended } \\
\text { (tons per day) }\end{array}$ \\
\hline
\end{tabular}

1975

December

$1330 \quad 5050$

$1200 \quad 1930$

6.0

$--$

210

2860

$25 \quad 1200$

5.5

$--$

68

1860

7.5

$-$

231

1130

2790

6.0

$--$

271

1976

January

21020

1020

1000

$$
2250
$$

$1120 \quad 1720$

$1030 \quad 2440$

1045

1890

$$
1890
$$

$$
1600
$$

$$
3430
$$

7080

2640

1990

1520

1380

814

942

750

695

\begin{tabular}{|c|c|}
\hline 1.0 & -- \\
\hline 5.0 & -- \\
\hline-- & -- \\
\hline 6.0 & -- \\
\hline 5.0 & -- \\
\hline 6.0 & -- \\
\hline 6.0 & - \\
\hline 6.0 & -- \\
\hline 7.0 & -- \\
\hline 7.0 & -- \\
\hline 8.0 & -- \\
\hline 6.0 & -- \\
\hline 6.0 & -- \\
\hline 7.0 & -- \\
\hline 6.0 & -- \\
\hline 4.0 & -- \\
\hline 6.0 & -- \\
\hline 7.5 & -- \\
\hline 7.0 & -- \\
\hline 6.0 & - \\
\hline
\end{tabular}

2100

$\begin{array}{ll}1500 & 1410 \\ 1020 & 1230\end{array}$

$\begin{array}{ll}1500 & 1410 \\ 1020 & 1230\end{array}$

$\because$
$\because$
$\because$
$\because$
$\because$
$\because$
$\because$
$\because$
$\because$
-

12

32

170

28

422

122

240

60

2290

5390

185

365

53

45

70

61

12

7.5

130

15

6.0

45

149 
TABLE 15.--Suspended sediment and related parameters in water from selected surface-water sites in the study area.

12043000 Calawah River near Forks, Wash. (Site 42)--cont.

\begin{tabular}{|c|c|c|c|c|c|c|}
\hline Date & Time & $\begin{array}{l}\text { Streamflow, } \\
\text { instantaneous, } \\
\text { (cubic feet } \\
\text { per second) }\end{array}$ & $\begin{array}{l}\text { Tempera- } \\
\text { ture } \\
\left.\text { ( }{ }^{\circ} \mathrm{C}\right)\end{array}$ & $\begin{array}{l}\text { Turbidity } \\
\text { (JTU) }\end{array}$ & $\begin{array}{l}\text { Sediment, } \\
\text { suspended } \\
\text { (milligrams } \\
\text { per liter) }\end{array}$ & $\begin{array}{l}\text { Sediment } \\
\text { discharge, } \\
\text { suspended } \\
\text { (tons per day) }\end{array}$ \\
\hline \multicolumn{7}{|l|}{1976} \\
\hline \multicolumn{7}{|c|}{ February } \\
\hline 4 & 1100 & 600 & 3.0 & -- & 3 & 4.9 \\
\hline 11 & 1230 & 2060 & 5.5 & -- & 64 & 356 \\
\hline 13 & 1330 & 2710 & 5.0 & -- & 40 & 293 \\
\hline 17 & 1030 & 4280 & 5.0 & -- & 50 & 578 \\
\hline 18 & 1400 & 3940 & 5.0 & -- & 49 & 521 \\
\hline 19 & 1000 & 2840 & 4.5 & -- & 16 & 123 \\
\hline \multicolumn{7}{|l|}{ March } \\
\hline 12 & 1530 & 1030 & 7.0 & -- & 9 & 25 \\
\hline 25 & 1020 & 3900 & 7.0 & -- & 39 & 411 \\
\hline 26 & 1045 & 2940 & 7.5 & -- & 18 & 143 \\
\hline \multicolumn{7}{|l|}{ April } \\
\hline 1 & 1100 & 1710 & 7.0 & -- & 17 & 78 \\
\hline 15 & 1400 & 966 & -- & -- & 1 & 2.6 \\
\hline \multicolumn{7}{|l|}{ July } \\
\hline 8 & 1100 & 514 & 13.0 & -- & 13 & 18 \\
\hline \multicolumn{7}{|l|}{ October } \\
\hline 27 & -- & 480 & -- & -- & 1 & 1.3 \\
\hline
\end{tabular}


TABLE 15.--Suspended sediment and related parameters in water from selected surface-water sites in the study area.

12043000 Calawah River near Forks, Wash. (Site 42)--cont.

\begin{tabular}{|c|c|c|c|c|c|c|}
\hline Date & Time & $\begin{array}{l}\text { Streamflow, } \\
\text { instantaneous, } \\
\text { (cubic feet } \\
\text { per second) }\end{array}$ & $\begin{array}{l}\text { Tempera- } \\
\text { ture } \\
\left({ }^{\circ} \mathrm{C}\right)\end{array}$ & $\begin{array}{c}\text { Turbidity } \\
\text { (JTU) }\end{array}$ & $\begin{array}{l}\text { Sediment, } \\
\text { suspended } \\
\text { (milligrams } \\
\text { per liter) }\end{array}$ & $\begin{array}{l}\text { Sediment } \\
\text { discharge, } \\
\text { suspended } \\
\text { (tons per day) }\end{array}$ \\
\hline \multicolumn{7}{|l|}{1976} \\
\hline \multicolumn{7}{|c|}{ December } \\
\hline 8 & 1100 & 2320 & -- & -- & 61 & 382 \\
\hline 9 & 1100 & 1410 & 7.0 & -- & 4 & 15 \\
\hline 14 & 0730 & 690 & 7.2 & -- & 1 & 1.9 \\
\hline 16 & 1330 & 916 & 9.0 & -- & 1 & 2.5 \\
\hline 20 & 1100 & 832 & -- & -- & 1 & 2.2 \\
\hline 22 & 1400 & 600 & 7.0 & -- & 1 & 1.6 \\
\hline 28 & 1030 & 1798 & 7.0 & -- & 2 & 9.7 \\
\hline 30 & 1430 & 946 & 6.0 & -- & 4 & 10 \\
\hline \multicolumn{7}{|l|}{1977} \\
\hline \multicolumn{7}{|l|}{ January } \\
\hline 5 & 1130 & 452 & 4.0 & - & 3 & 3.7 \\
\hline 17 & 1600 & 1410 & 6.0 & - & 18 & 69 \\
\hline 18 & 1400 & 6040 & 7.0 & -- & 158 & 2580 \\
\hline 20 & 1500 & 1307 & 6.5 & -- & 1 & 3.5 \\
\hline 31 & 1455 & 1006 & 6.0 & -- & 14 & 38 \\
\hline \multicolumn{7}{|c|}{ February } \\
\hline 4 & 1200 & 464 & 6.0 & $\cdots$ & 1 & 1.3 \\
\hline 9 & 1125 & 416 & 8.0 & -- & 1 & 1.1 \\
\hline 10 & -- & 1610 & 6.0 & -- & 29 & 126 \\
\hline 11 & 1345 & 1405 & 6.5 & -- & 5 & 19 \\
\hline
\end{tabular}


TABLE 15.--Suspended sediment and related parameters in water from selected surface-water sites in the study area.

12043000 Calawah River near Forks, Wash. (Site 42)--cont.

$\begin{array}{llcccc} & \begin{array}{l}\text { Streamflow, } \\ \text { instantaneous, }\end{array} & \begin{array}{c}\text { Tempera- } \\ \text { ture }\end{array} & \text { Turbidity } & \begin{array}{l}\text { Sediment, } \\ \text { suspended }\end{array} & \begin{array}{l}\text { Sediment } \\ \text { discharge, }\end{array} \\ \text { (cubic feet } & \left({ }^{\circ} \mathrm{C}\right) & \text { (JTU) } & \text { (milligrams suspended } \\ \text { Date } & \text { Time second) } & & \text { per liter) (tons per day) }\end{array}$

1977

February

$1400 \quad 856$

6.5

$-$

$1405 \quad 850$

$-$

$-$

8.0

$-$

1150

$1330 \quad 5610$

1030

2920

-.

$-$

7.0

$--$

$3.3--$

1145

1360

1690

$\begin{array}{ll}1540 & 1690 \\ 1116 & 2090\end{array}$

6.5

$-$

$4.4 \quad--$

per liter)

(tons per day)

1600

1055

1310

7.0

$-$

6.5

$-$

--

$-$

7.0

$-$

856
874

6.5

$--$

8.5

$-$

22

53

$\begin{array}{ll}1430 & 886 \\ 1120 & 868\end{array}$

6.0

$--$

1515

8.5

-

$-$

6

9.7

590

9.0

4

6.4

May

8.5

$-$

10

8.0

2

5.1 
TABLE 15.--Suspended sediment and related parameters in water from selected surface-water sites in the study area.

12043000 Calawah River near Forks, Wash. (Site 42)--cont.

\begin{tabular}{|c|c|c|c|c|c|c|}
\hline Date & Time & $\begin{array}{l}\text { Streamflow, } \\
\text { instantaneous, } \\
\text { (cubic feet } \\
\text { per second) }\end{array}$ & $\begin{array}{l}\text { Tempera- } \\
\text { ture } \\
\left(^{\circ} \mathrm{C}\right)\end{array}$ & $\begin{array}{l}\text { Turbidity } \\
\text { (JTU) }\end{array}$ & $\begin{array}{l}\text { Sediment, } \\
\text { suspended } \\
\text { (milligrams } \\
\text { per liter) }\end{array}$ & $\begin{array}{l}\text { Sediment } \\
\text { discharge, } \\
\text { suspended } \\
\text { (tons per day) }\end{array}$ \\
\hline \multicolumn{7}{|l|}{1977} \\
\hline \multicolumn{7}{|l|}{ May } \\
\hline 10 & 1326 & 460 & 10.5 & -- & 5 & 6.2 \\
\hline 13 & 1540 & 505 & 10.0 & -- & 16 & 22 \\
\hline 16 & 1000 & 448 & 10.0 & -- & 7 & 8.5 \\
\hline 26 & 1600 & 600 & 10.0 & -- & 3 & 4.9 \\
\hline 27 & 1115 & 525 & 7.0 & -- & 4 & 5.7 \\
\hline 27 & 1530 & 747 & 8.0 & -- & 5 & 10 \\
\hline 31 & 1320 & 690 & 10.0 & -- & 4 & 7.5 \\
\hline \multicolumn{7}{|l|}{ June } \\
\hline 1 & 1610 & 880 & 11.0 & -- & 4 & 9.5 \\
\hline 7 & 1145 & 505 & 10.5 & 1 & 4 & 5.5 \\
\hline 8 & 1230 & 472 & 14.0 & 1 & 5 & 6.4 \\
\hline \multicolumn{7}{|l|}{ July } \\
\hline 8 & 1405 & 1555 & 13.5 & 1 & 2 & 8.4 \\
\hline \multicolumn{7}{|c|}{ September } \\
\hline 20 & 1115 & 1120 & 12.0 & 5 & 15 & 45 \\
\hline 20 & 1430 & 1060 & 13.0 & 3 & 10 & 28 \\
\hline 20 & 1845 & 1020 & 13.0 & 2 & 6 & 17 \\
\hline 21 & 0935 & 802 & 10.0 & 2 & 6 & 13 \\
\hline
\end{tabular}


TABLE 15.--Suspended sediment and related parameters in water from selected surface-water sites in the study area.

12043000 Calawah River near Forks, Wash. (Site 42)--cont.

\begin{tabular}{|c|c|c|c|c|c|c|}
\hline & & $\begin{array}{l}\text { Streanflow, } \\
\text { instantaneous, } \\
\text { (cublc feet }\end{array}$ & $\begin{array}{l}\text { Tempera- } \\
\text { ture } \\
\left(^{\circ} \mathrm{C}\right)\end{array}$ & $\begin{array}{l}\text { Turbidity } \\
\text { (JTU) }\end{array}$ & $\begin{array}{l}\text { Sediment, } \\
\text { suspended } \\
\text { (milligrams }\end{array}$ & $\begin{array}{l}\text { Sediment } \\
\text { discharge, } \\
\text { suspended }\end{array}$ \\
\hline Date & Time & per second) & & & per liter) & (tons per day) \\
\hline \multicolumn{7}{|l|}{1977} \\
\hline \multicolumn{7}{|c|}{ September } \\
\hline 22 & 0950 & 490 & 10.0 & 1 & 7 & 9.3 \\
\hline 22 & 1810 & 452 & 11.0 & 1 & 5 & 6.1 \\
\hline 23 & 0900 & 416 & 10.0 & 8 & 2 & 2.2 \\
\hline \multicolumn{7}{|l|}{ October } \\
\hline 24 & 1345 & 1270 & 10.0 & 1 & 6 & 21 \\
\hline 25 & 1045 & 1610 & 9.0 & 2 & 11 & 48 \\
\hline 25 & 1155 & 1580 & 9.0 & 2 & 11 & 47 \\
\hline 26 & 1520 & 1310 & 10.0 & 1 & 13 & 46 \\
\hline 28 & 1250 & 1380 & 9.0 & 1 & 9 & 34 \\
\hline 31 & 1145 & 2180 & 8.0 & 2 & 12 & 71 \\
\hline \multicolumn{7}{|c|}{ November } \\
\hline 1 & 1330 & 12500 & 9.0 & 5 & 1130 & 38100 \\
\hline 4 & 1115 & 1570 & 8.0 & -- & 2 & 8.5 \\
\hline 7 & 1200 & 1340 & 8.0 & 3 & 15 & 54 \\
\hline 10 & 0950 & 3490 & 9.0 & 2 & 107 & 1010 \\
\hline 10 & 1330 & 3570 & 9.0 & 1 & 100 & 964 \\
\hline 11 & 1115 & 2290 & 8.0 & 4 & 33 & 204 \\
\hline 14 & 1135 & 8250 & -- & 2 & 1080 & 24100 \\
\hline 15 & 1030 & 3120 & 8.0 & 4 & 192 & 1620 \\
\hline 21 & 1420 & 832 & 4.0 & 4 & 5 & 11 \\
\hline 28 & 1010 & 3020 & 7.0 & 3 & 163 & 1330 \\
\hline 29 & 0920 & 4820 & 8.0 & 1 & 30 & 390 \\
\hline
\end{tabular}


TABLE 15.--Suspended sediment and related parameters in water from selected surface-water sites in the study area.

12043000 Calawah River near Forks, Wash, (Site 42)--cont.

$\begin{array}{llllll} & \begin{array}{l}\text { Streamflow, } \\ \text { instantaneous, }\end{array} & \text { Tempera- } & \text { Sediment, } & \text { Sediment } \\ & & \text { ture } & \text { Turbidity } & \text { suspended } & \text { discharge, } \\ \text { (cubic feet } & \left({ }^{\circ} \mathrm{C}\right) & \text { (JTU) } & \text { (milligrams suspended } \\ \text { Date } & \text { Time second) } & & \text { per liter) (tons per day) }\end{array}$

1977

December

9.0

9

693

10100

$40735 \quad 3440$

--

156

1450

$1030 \quad 2480$

$--$

2

66

442

2560

2.2

42

290

$1345 \quad 1420$

5.0

19

73

5010

7.0

179

2420

$\begin{array}{rr}1230 & 1260 \\ 1630 & 555\end{array}$

7.0

14

48

7.0

1

12

18

1978

January

$3 \quad 1050$

$61250 \quad 1550$

650
550

4.1

5.0

1

5

8. 8

1550
1630

8.0

2

5

18

75

48

May

12

$\begin{array}{lr}1030 & 420 \\ 1140 & 1890 \\ 0945 & 404 \\ 1215 & 420 \\ 1115 & 660 \\ 1315 & 476\end{array}$

9.0
--
8.5
10.0
8.0
--

9.0

\section{1}

3

3

8.5

3

3
--

1

2.3

51

2.2

12

23

2.6 
TABLE 15.--Suspended sediment and related parameters in water from selected surface-water sites in the study area.

12043000 Calawah River near Forks, Wash. (Site 42)--cont.

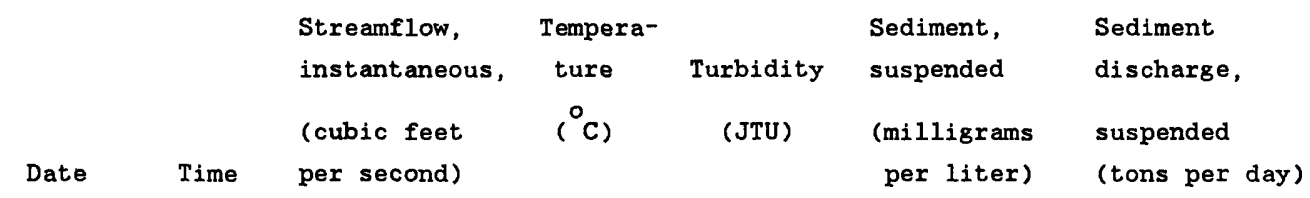

1977

June

$13 \quad 1040$

1010

408

11.0

2

9.0

1

2

2. 2

.00

September

6

11

13

18

20

22

25

26

28

28

$$
1045
$$

1000

1400

0910

1000

1000

--

0830

1615

464
1500
856
490
412
934
820
655
510
495

464

856

490

412

934

820

655

495
12.0

14.0

12.0

11.5

14.0

10.5

11.0

11.0

12.0

11.9
0

0

0

0

0

1

3

2

2

2

1
.00

.00

.00

.00

1.1

7.6

4.4

3.5

2.8

1.3 
TABLE 15.--Suspended sediment and related parameters in water from selected surface-water sites in the study area.

12043015 Bogachiel River near LaPush, Wash. (Site 55)

\begin{tabular}{|c|c|c|c|c|c|c|}
\hline Date & Time & $\begin{array}{l}\text { Streamflow, } \\
\text { instantaneous, } \\
\text { (cubic feet } \\
\text { per second) }\end{array}$ & $\begin{array}{l}\text { Tempera- } \\
\text { ture } \\
\left.\text { ( }{ }^{\circ} \mathrm{C}\right)\end{array}$ & $\begin{array}{c}\text { Turbidity } \\
\text { (JTU) }\end{array}$ & $\begin{array}{l}\text { Sediment, } \\
\text { suspended } \\
\text { (milligrams } \\
\text { per liter) }\end{array}$ & $\begin{array}{l}\text { Sediment } \\
\text { discharge, } \\
\text { suspended } \\
\text { (tons per day) }\end{array}$ \\
\hline \multicolumn{7}{|l|}{1975} \\
\hline \multicolumn{7}{|c|}{ December } \\
\hline 24 & 1230 & 11800 & 5.5 & -- & 776 & 24700 \\
\hline 25 & 1100 & 4580 & 6.0 & -- & 24 & 297 \\
\hline 26 & 1010 & 38300 & 8.0 & -- & 2150 & 222000 \\
\hline 28 & 1430 & 5120 & 7.0 & -- & 86 & 1190 \\
\hline 29 & 0955 & 4420 & 7.0 & -- & 64 & 764 \\
\hline 30 & 0950 & 6570 & 6.0 & -- & 98 & 1740 \\
\hline 31 & 1630 & 3780 & -- & -- & 52 & 531 \\
\hline \multicolumn{7}{|l|}{1976} \\
\hline \multicolumn{7}{|l|}{ January } \\
\hline 2 & 1130 & 2410 & 1.5 & -- & 25 & 163 \\
\hline 5 & 1150 & 5320 & 4.0 & -- & 42 & 603 \\
\hline 6 & 1350 & 4090 & -- & -- & 37 & 409 \\
\hline 8 & 1415 & 5780 & 5.9 & -- & 64 & 999 \\
\hline 9 & 1555 & 4490 & 5.0 & -- & 26 & 315 \\
\hline 10 & 1400 & 7500 & 6.0 & -- & 240 & 4860 \\
\hline 12 & 1230 & 4490 & 5.0 & -- & 38 & 461 \\
\hline 13 & 1200 & 3800 & 4.0 & -- & 22 & 226 \\
\hline 14 & 1300 & 9350 & 5.0 & -- & 274 & 6920 \\
\hline 15 & 1230 & 17700 & 6.5 & -- & 692 & 33100 \\
\hline 17 & 1100 & 6060 & 7.0 & -- & 86 & 1410 \\
\hline 18 & 1120 & 4760 & 7.0 & -- & 32 & 411 \\
\hline
\end{tabular}


TABLE 15.--Suspended sediment and related parameters in water from selected surface-water sites in the study area.

12043015 Bogachiel River near LaPush, Wash. (Site 55)--cont.

\begin{tabular}{|c|c|c|c|c|c|c|}
\hline & & $\begin{array}{l}\text { Streamflow, } \\
\text { instantaneous, } \\
\text { (cubic feet }\end{array}$ & $\begin{array}{l}\text { Tempera- } \\
\text { ture } \\
\left({ }^{\circ} \mathrm{C}\right)\end{array}$ & $\begin{array}{c}\text { Turbidity } \\
\text { (JTU) }\end{array}$ & $\begin{array}{l}\text { Sediment, } \\
\text { suspended } \\
\text { (milligrams }\end{array}$ & $\begin{array}{l}\text { Sediment } \\
\text { discharge, } \\
\text { suspended }\end{array}$ \\
\hline Date & Time & per second) & & & per liter) & (tons per day) \\
\hline \multicolumn{7}{|l|}{1976} \\
\hline \multicolumn{7}{|c|}{ January } \\
\hline 19 & 1400 & 3270 & 6.0 & -- & 12 & 106 \\
\hline 20 & 1115 & 3010 & 4.0 & - & 34 & 276 \\
\hline 22 & 1130 & 2970 & 7.0 & -- & 22 & 176 \\
\hline 23 & 1100 & 2260 & 5.0 & -- & 12 & 73 \\
\hline 27 & 1300 & 11300 & 6.0 & $\cdots$ & 572 & 17500 \\
\hline 28 & 1300 & 4720 & 7.0 & -- & 52 & 663 \\
\hline 29 & 1030 & 3550 & 6.0 & -- & 30 & 288 \\
\hline 30 & 0925 & 2860 & 6.0 & -- & 26 & 201 \\
\hline \multicolumn{7}{|c|}{ February } \\
\hline 11 & 1445 & 7180 & 5.5 & - & 66 & 1280 \\
\hline 12 & 1215 & 11400 & 5.5 & -- & 755 & 23200 \\
\hline 17 & 1230 & 9380 & 5.0 & -- & 114 & 2890 \\
\hline 18 & 1510 & 7760 & 5.0 & $\cdots$ & 68 & 1430 \\
\hline 19 & 0930 & 5640 & 4.5 & -- & 50 & 761 \\
\hline \multicolumn{7}{|l|}{ March } \\
\hline 18 & 1145 & 9140 & 7.0 & -- & 110 & 2720 \\
\hline 22 & 1100 & 7300 & 7.0 & -- & 56 & 1100 \\
\hline 24 & 1030 & 12700 & 7.5 & $\cdots$ & 709 & 24300 \\
\hline 25 & 1110 & 7790 & 7.0 & -- & 58 & 1220 \\
\hline 26 & 1030 & 6270 & 7.0 & -- & 15 & 254 \\
\hline
\end{tabular}


TABLE 15.--Suspended sediment and related parameters in water from selected surface-water sites in the study area.

12043015 Bogachiel River near LaPush, Wash. (Site 55)--cont.

$\begin{array}{llllll} & \begin{array}{l}\text { Streamflow, } \\ \text { instantaneous, }\end{array} & \begin{array}{c}\text { Tempera- } \\ \text { ture }\end{array} & \text { Turbidity } & \text { Sediment, } & \text { Sediment } \\ \text { (cubic feet } & \left({ }^{\circ} \mathrm{C}\right) & \text { (JTU) } & \text { (milligrams } & \text { suspended } \\ \text { Date } & \text { Time second) } & & \text { per liter) } & \text { (tons per day) }\end{array}$

1976

April

$1 \quad 1015 \quad 3250$

15002250

6.5

$-$

4

35

15

9.0

$-$

3

18

November

1

17

1500

2530

$-$

$-$

10

68

6130

$-$

$-$

705

11700

December

9

1400

2940

7.5

11.0

$-$

33

262

16

1200

2380

7.0

$-$

$\begin{array}{ll}1145 & 4170 \\ 1530 & 2250\end{array}$

6.0

$-$

$-$

$--$

51

32

6.0

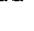

1977

January

17

$1500 \quad 3270$

3270
12500

7.0

$--$

82

724

18

1600

7.0

$--$

323

10900

$1600 \quad 3230$

6.0

$--$

10

87 
TABLE 15.--Suspended sediment and related parameters in water from selected surface-water sites in the study area.

12043015 Bogachiel River near LaPush, Wash. (Site 55)--cont.

$\begin{array}{llllll} & \begin{array}{l}\text { Streamflow, } \\ \text { instantaneous, }\end{array} & \begin{array}{l}\text { Tempera- } \\ \text { ture }\end{array} & \text { Turbidity } & \begin{array}{l}\text { Sediment, } \\ \text { suspended }\end{array} & \begin{array}{l}\text { Sediment } \\ \text { discharge, }\end{array} \\ \text { (cubic feet } & \left.{ }^{\circ} \mathrm{C}\right) & \text { (JTU) } & \text { (milligrams } & \text { suspended } \\ \text { Date } & & & \text { per liter) } & \text { (tons per day) }\end{array}$

1977

February

10

11

16

17

22

23

25

25

27

28

March

1

2

8

9

10

April

8

May

4

5
1440

1130

3190

2560

10200

9680

5850

2330

9.0

--
--
--
--
--
--
--
--
--
--

6.1

$--$

$\because$

-

$--$

$-$

5.5

6.5

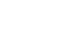

1525

1445

2500

2290 $\begin{array}{rr}9.0 & -- \\ -- & --\end{array}$

$\begin{array}{rr}62 & 688 \\ 21 & 195 \\ 9 & 51 \\ 20 & 177 \\ 357 & 13400 \\ 85 & 1340 \\ 20 & 158 \\ 80 & 860 \\ 6 & 80 \\ 10 & 106\end{array}$

138

35

5010

1570

521

25

47

49 
TABLE 15.--Suspended sediment and related parameters in water from selected surface-water sites in the study area.

12043015 Bogachiel River near LaPush, Wash. (Site 55)--cont.

\begin{tabular}{|c|c|c|c|c|c|c|}
\hline Date & Time & $\begin{array}{l}\text { Streamflow, } \\
\text { instantaneous, } \\
\text { (cubic feet } \\
\text { per second) }\end{array}$ & $\begin{array}{l}\text { Tempera- } \\
\text { ture } \\
\left({ }^{\circ} \mathrm{C}\right)\end{array}$ & $\begin{array}{c}\text { Turbidity } \\
\text { (JTU) }\end{array}$ & $\begin{array}{l}\text { Sediment, } \\
\text { suspended } \\
\text { (milligrams } \\
\text { per liter) }\end{array}$ & $\begin{array}{l}\text { Sediment } \\
\text { discharge, } \\
\text { suspended } \\
\text { (tons per day) }\end{array}$ \\
\hline \multicolumn{7}{|l|}{1977} \\
\hline \multicolumn{7}{|l|}{ June } \\
\hline 1 & 1700 & 2270 & 11.5 & -- & 9 & 55 \\
\hline \multicolumn{7}{|c|}{ September } \\
\hline 20 & 0950 & 3310 & 11.5 & 7 & 69 & 617 \\
\hline 20 & 1505 & 2960 & 14.0 & 11 & 28 & 224 \\
\hline 20 & 1725 & 2930 & 14.0 & 5 & 30 & 237 \\
\hline 21 & 0930 & 2010 & 11.0 & 3 & 9 & 49 \\
\hline \multicolumn{7}{|l|}{ October } \\
\hline 25 & 0945 & 3820 & 10.0 & 3 & 24 & 248 \\
\hline 25 & 1255 & 3690 & 11.0 & 5 & 21 & 209 \\
\hline 26 & 1655 & 3220 & 12.0 & 2 & 8 & 70 \\
\hline 28 & 1300 & 2220 & 9.0 & 5 & 29 & 174 \\
\hline 31 & 1415 & 5300 & 9.0 & 4 & 32 & 458 \\
\hline \multicolumn{7}{|c|}{ November } \\
\hline 10 & 1125 & 9290 & 9.0 & 3 & 287 & 7200 \\
\hline 11 & -- & 6220 & -- & 2 & 63 & 1050 \\
\hline 14 & 1120 & 21500 & -- & 2 & 1240 & 72000 \\
\hline 14 & 1450 & 16600 & -- & 3 & 792 & 35500 \\
\hline 15 & 1245 & 6900 & 9.0 & 6 & 139 & 2590 \\
\hline 28 & 0945 & 7300 & 8.0 & 5 & 124 & 2440 \\
\hline 29 & 1000 & 11800 & 7.0 & 2 & 521 & 16600 \\
\hline
\end{tabular}


TABLE 15.--Suspended sediment and related parameters in water from selected surface-water sites in the study area.

12043015 Bogachiel River near LaPush, Wash. (Site 55)--cont.

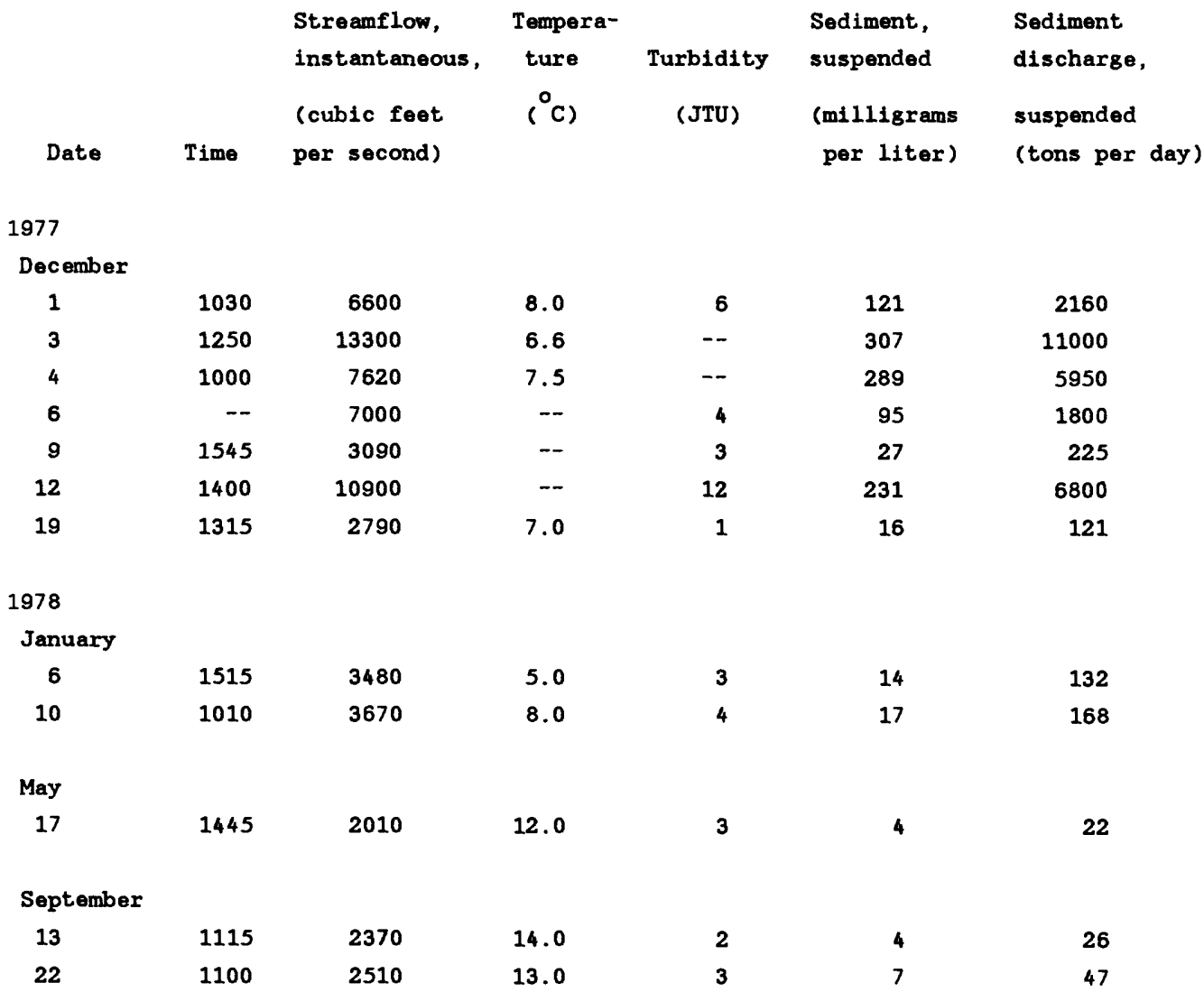


TABLE 15.--Suspended sediment and related parameters in water from selected surface-water sites in the study area.

12043101 Dickey River above Colby Creek near La Push, Wash. (Site 69)

\begin{tabular}{|c|c|c|c|c|c|c|}
\hline Date & Time & $\begin{array}{l}\text { Streamflow, } \\
\text { instantaneous, } \\
\text { (cubic feet } \\
\text { per second) }\end{array}$ & $\begin{array}{l}\text { Tempera- } \\
\text { ture } \\
\left({ }^{\circ} \mathrm{C}\right)\end{array}$ & $\begin{array}{l}\text { Turbidity } \\
\text { (JTU) }\end{array}$ & $\begin{array}{l}\text { Sediment, } \\
\text { suspended } \\
\text { (milligrams } \\
\text { per liter) }\end{array}$ & $\begin{array}{l}\text { Sediment } \\
\text { discharge, } \\
\text { suspended } \\
\text { (tons per day) }\end{array}$ \\
\hline \multicolumn{7}{|l|}{1975} \\
\hline \multicolumn{7}{|c|}{ December } \\
\hline 24 & 1030 & 3560 & 5.5 & - & 559 & 5370 \\
\hline 25 & 1015 & 1090 & 5.5 & $\cdots$ & 22 & 65 \\
\hline 29 & 1045 & 1230 & 7.5 & -- & 197 & 654 \\
\hline 30 & 1030 & 1710 & 6.0 & -- & 106 & 489 \\
\hline 31 & 1330 & 880 & -- & - & 14 & 33 \\
\hline \multicolumn{7}{|l|}{1976} \\
\hline \multicolumn{7}{|l|}{ January } \\
\hline 2 & 1340 & 536 & 2.0 & -- & 10 & 14 \\
\hline 5 & 1420 & 1210 & 5.0 & - & 14 & 46 \\
\hline 6 & 1300 & 1100 & - & - & 14 & 42 \\
\hline 8 & 1315 & 1170 & 6.0 & - & 50 & 158 \\
\hline 10 & 1430 & 3020 & 6.0 & - & 220 & 1790 \\
\hline 12 & 1430 & 1190 & 5.0 & - & 46 & 148 \\
\hline 13 & 1500 & 828 & 4.0 & -- & 8 & 18 \\
\hline 14 & 1500 & 1710 & 7.0 & - & 40 & 185 \\
\hline 15 & 1430 & 4750 & 7.0 & -- & 172 & 2210 \\
\hline 19 & 1330 & 704 & 6.0 & $\cdots$ & 11 & 21 \\
\hline 20 & 1045 & 578 & 4.0 & - & 10 & 16 \\
\hline 22 & 1330 & 672 & 6.5 & -- & 20 & 36 \\
\hline 23 & 1345 & 536 & 6.0 & $\cdots$ & 9 & 13 \\
\hline 27 & 1430 & 2280 & - & -- & 128 & 788 \\
\hline 28 & 1600 & 935 & 7.0 & - & 16 & 40 \\
\hline 30 & 1230 & 628 & 6.0 & - & 4 & 6.8 \\
\hline
\end{tabular}


TABLE 15.--Suspended sediment and related parameters in water from selected surface-water sites in the study area.

12043101 Dickey River above Colby Creek near La Push, Wash. (Site 69)--cont.

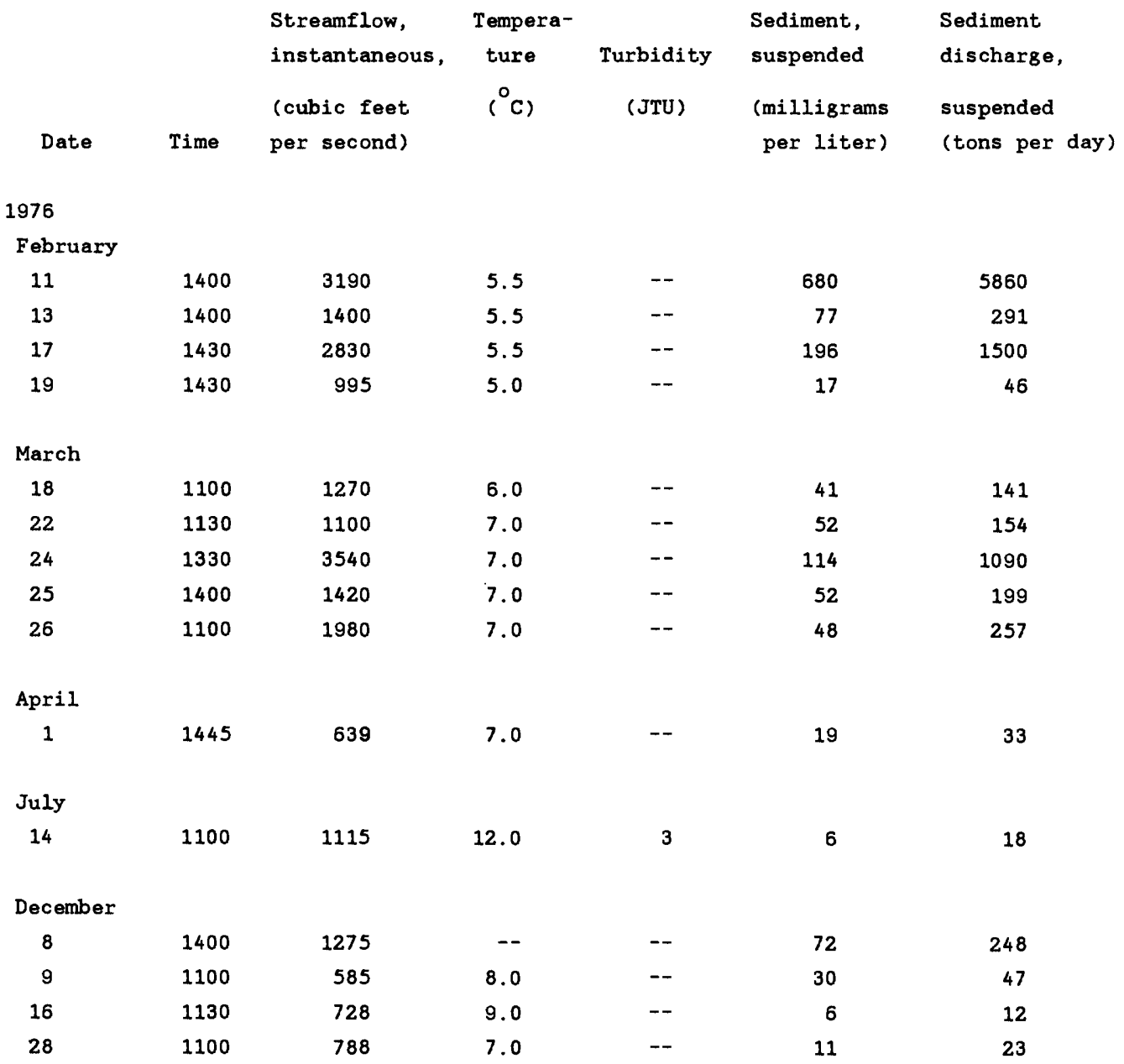


TABLE 15.--Suspended sediment and related parameters in water from selected surface-water sites in the study area.

12043101 Dickey River above Colby Creek near La Push, Wash. (Site 69)- cont.

\begin{tabular}{|c|c|c|c|c|c|c|}
\hline Date & Time & $\begin{array}{l}\text { Streamflow, } \\
\text { instantaneous, } \\
\text { (cubic feet } \\
\text { per second) }\end{array}$ & $\begin{array}{c}\text { Tempera- } \\
\text { ture } \\
\left.{ }^{\circ} \mathrm{C}\right)\end{array}$ & $\begin{array}{l}\text { Turbidity } \\
\text { (JTU) }\end{array}$ & $\begin{array}{l}\text { Sediment, } \\
\text { suspended } \\
\text { (milligrams } \\
\text { per liter) }\end{array}$ & $\begin{array}{l}\text { Sediment } \\
\text { discharge, } \\
\text { suspended } \\
\text { (tons per day) }\end{array}$ \\
\hline \multicolumn{7}{|l|}{1977} \\
\hline \multicolumn{7}{|l|}{ January } \\
\hline 17 & 1630 & 2870 & -- & -- & 69 & 535 \\
\hline 18 & 1430 & 2110 & 8.0 & -- & 270 & 1540 \\
\hline 20 & 1500 & 654 & 6.0 & -- & 14 & 25 \\
\hline 31 & 1520 & 1050 & 6.0 & -- & 163 & 462 \\
\hline \multicolumn{7}{|c|}{ February } \\
\hline 10 & -- & 813 & 4.5 & -- & 68 & 149 \\
\hline 11 & 1410 & 756 & 6.5 & -- & 23 & 47 \\
\hline 12 & 1330 & 2250 & 6.1 & -- & 222 & 1350 \\
\hline 17 & 1400 & 880 & 8.0 & -- & 72 & 167 \\
\hline 22 & -- & 2140 & 8.0 & -- & 108 & 624 \\
\hline 25 & 1430 & 945 & 3.9 & -- & 42 & 107 \\
\hline 27 & 1430 & 1170 & 3.3 & -- & 9 & 28 \\
\hline \multicolumn{7}{|l|}{ March } \\
\hline 1 & 1530 & 724 & 7.2 & -- & 10 & 20 \\
\hline 9 & 1445 & 2320 & 6.0 & - & 127 & 796 \\
\hline \multicolumn{7}{|l|}{ May } \\
\hline 27 & 1600 & 529 & 9.5 & - & 42 & 60 \\
\hline
\end{tabular}


TABLE 15.--Suspended sediment and related parameters in water from selected surface-water sites in the study area.

12043101 Dickey River above Colby Creek near La Push, Wash. (Site 69)--cont.

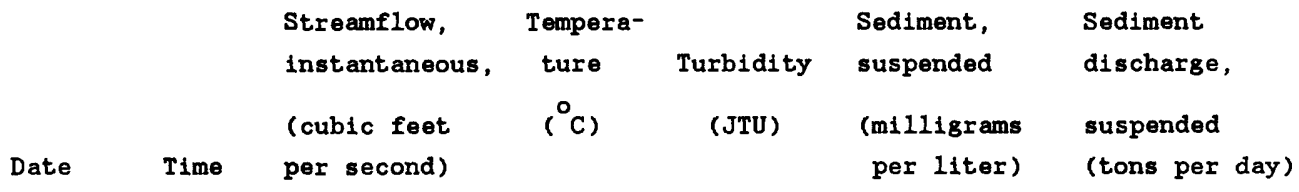

1977

October

24

1210

660
985
1010
816
596
996

13.0

11.0

11.0

1230

12.0

10.0

1330

1330

8.0

5
5
3
4
--
2

22

17

18

16

14

11

39

45

49

35

23

31

1345

3020

10.0

10.0

3340

1330

990

2850

1100

8.0

10.0

9.0

4900

--

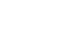

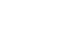

30

1530

1620

1220

1530

9.0

8.0

220

1790

$466 \quad 4200$

$55 \quad 147$

117900

$76 \quad 277$

$222 \quad 2940$

$70 \quad 306$

$29 \quad 120$

December

$\begin{array}{rrr}1 & 1350 & 4830 \\ 4 & 1120 & 1570 \\ 6 & 1100 & 1410 \\ 7 & 1615 & 1070 \\ 9 & 1410 & 603\end{array}$

29

1430

668

--
7.2
6.7
2.8
4.0
--

1

361

4710

119

504

316

95

41

52 
TABLE 15.--Suspended sediment and related parameters in water from selected surface-water sites in the study area.

12043101 Dickey River above Colby Creek near La Push, Wash. (Site 69)--cont.

\begin{tabular}{|c|c|c|c|c|c|c|}
\hline Date & Time & $\begin{array}{l}\text { Streamflow, } \\
\text { instantaneous, } \\
\text { (cubic feet } \\
\text { per second) }\end{array}$ & $\begin{array}{c}\text { Tempera- } \\
\text { ture } \\
\left(^{\circ} \mathrm{C}\right)\end{array}$ & $\begin{array}{c}\text { Turbidity } \\
\text { (JTU) }\end{array}$ & $\begin{array}{l}\text { Sediment, } \\
\text { suspended } \\
\text { (milligrams } \\
\text { per liter) }\end{array}$ & $\begin{array}{l}\text { Sediment } \\
\text { discharge, } \\
\text { suspended } \\
\text { (tons per day) }\end{array}$ \\
\hline \multicolumn{7}{|l|}{1978} \\
\hline \multicolumn{7}{|l|}{ January } \\
\hline 6 & 1200 & 596 & 5.0 & 5 & 14 & 23 \\
\hline 10 & 1300 & 684 & 7.5 & 5 & 15 & 28 \\
\hline \multicolumn{7}{|l|}{ May } \\
\hline 15 & 1145 & 564 & -- & 4 & 10 & 15 \\
\hline \multicolumn{7}{|c|}{ September } \\
\hline 12 & 1030 & 960 & 15.0 & -- & 71 & 184 \\
\hline 13 & 1030 & 664 & -- & 3 & 15 & 27 \\
\hline
\end{tabular}


TABLE 15.--Suspended sediment and related parameters in water from selected surface-water sites in the study area.

12045500 Elwha River at McDonald Bridge near Port Angeles, Wash. (Site 129)

\begin{tabular}{|c|c|c|c|c|c|c|}
\hline Date & Time & $\begin{array}{l}\text { Streamflow, } \\
\text { instantaneous, } \\
\text { (cubic feet } \\
\text { per second) }\end{array}$ & $\begin{array}{l}\text { Tempera- } \\
\text { ture } \\
\left({ }^{\circ} \mathrm{C}\right)\end{array}$ & $\begin{array}{c}\text { Turbidity } \\
\text { (JTU) }\end{array}$ & $\begin{array}{l}\text { Sediment, } \\
\text { suspended } \\
\text { (milligrams } \\
\text { per liter) }\end{array}$ & $\begin{array}{l}\text { Sediment } \\
\text { discharge, } \\
\text { suspended } \\
\text { (tons per day) }\end{array}$ \\
\hline \multicolumn{7}{|l|}{1974} \\
\hline \multicolumn{7}{|l|}{ October } \\
\hline 25 & 1215 & 444 & 9.2 & 1 & 1 & 1.2 \\
\hline \multicolumn{7}{|c|}{ November } \\
\hline 21 & 1030 & 2710 & 6.4 & 20 & 27 & 198 \\
\hline \multicolumn{7}{|c|}{ December } \\
\hline 18 & 0945 & 2010 & 5.6 & 2 & 5 & 27 \\
\hline \multicolumn{7}{|l|}{1975} \\
\hline \multicolumn{7}{|l|}{ January } \\
\hline 21 & 1100 & 1620 & 4.1 & 10 & 11 & 48 \\
\hline \multicolumn{7}{|c|}{ February } \\
\hline 19 & 0930 & 1070 & 3.8 & 4 & 6 & 17 \\
\hline \multicolumn{7}{|l|}{ March } \\
\hline 11 & 0900 & 1270 & 3.6 & 1 & 4 & 14 \\
\hline \multicolumn{7}{|l|}{ April } \\
\hline 14 & 1030 & 877 & 5.5 & 1 & 3 & 7.1 \\
\hline \multicolumn{7}{|l|}{ May } \\
\hline 20 & 0830 & 1800 & 6.8 & 2 & 5 & 24 \\
\hline \multicolumn{7}{|l|}{ June } \\
\hline 17 & 1100 & 2460 & 6.6 & 4 & 6 & 40 \\
\hline \multicolumn{7}{|l|}{ July } \\
\hline 14 & 1200 & 2170 & 10.6 & 3 & 5 & 29 \\
\hline \multicolumn{7}{|l|}{ August } \\
\hline 12 & 1400 & 912 & 14.4 & 7 & 2 & 4.9 \\
\hline \multicolumn{7}{|c|}{ September } \\
\hline 16 & 0905 & 730 & 10.6 & 1 & 2 & 3.9 \\
\hline \multicolumn{7}{|l|}{ October } \\
\hline 16 & 1010 & 1240 & 9.4 & 2 & 3 & 10 \\
\hline
\end{tabular}


TABLE 15.--Suspended sediment and related parameters in water from selected surface-water sites in the study area.

12045500 Elwha River at McDonald Bridge near Port Angeles, Wash. (Site 129)--cont.

\begin{tabular}{|c|c|c|c|c|c|c|}
\hline Date & Time & $\begin{array}{l}\text { Streamflow, } \\
\text { instantaneous, } \\
\text { (cubic feet } \\
\text { per second) }\end{array}$ & $\begin{array}{c}\text { Tempera- } \\
\text { ture } \\
\left({ }^{\circ} \mathrm{C}\right)\end{array}$ & $\begin{array}{c}\text { Turbidity } \\
\text { (JTU) }\end{array}$ & $\begin{array}{l}\text { Sediment, } \\
\text { suspended } \\
\text { (milligrams } \\
\text { per liter) }\end{array}$ & $\begin{array}{l}\text { Sediment } \\
\text { discharge, } \\
\text { suspended } \\
\text { (tons per day) }\end{array}$ \\
\hline \multicolumn{7}{|l|}{1975} \\
\hline \multicolumn{7}{|c|}{ November } \\
\hline 21 & 0830 & 2130 & 4.0 & 25 & 35 & 201 \\
\hline \multicolumn{7}{|c|}{ December } \\
\hline 22 & 1300 & 1340 & 4.4 & 10 & 13 & 47 \\
\hline \multicolumn{7}{|l|}{1976} \\
\hline \multicolumn{7}{|l|}{ January } \\
\hline 21 & 0930 & 2100 & 4.0 & 10 & 19 & 108 \\
\hline \multicolumn{7}{|c|}{ February } \\
\hline 19 & 1200 & 1530 & 4.4 & 3 & 5 & 21 \\
\hline \multicolumn{7}{|l|}{ March } \\
\hline 22 & 1045 & 1100 & 4.6 & 2 & 3 & 8.9 \\
\hline \multicolumn{7}{|l|}{ April } \\
\hline 21 & 0830 & 1010 & 5.7 & 1 & 1 & 2.7 \\
\hline \multicolumn{7}{|l|}{ May } \\
\hline 19 & 1230 & 2080 & 7.2 & 2 & 20 & 112 \\
\hline \multicolumn{7}{|l|}{ June } \\
\hline 30 & 1230 & 2590 & 8.9 & 1 & 3 & 21 \\
\hline \multicolumn{7}{|l|}{ July } \\
\hline 22 & 0930 & 1730 & 9.4 & 1 & 2 & 9.3 \\
\hline \multicolumn{7}{|l|}{ August } \\
\hline 25 & 1245 & 1000 & 10.8 & 1 & 1 & 2.7 \\
\hline \multicolumn{7}{|c|}{ September } \\
\hline 27 & 1230 & 698 & 11.6 & 2 & 1 & 1.9 \\
\hline
\end{tabular}


TABLE 15.--Suspended sediment and related parameters in water from selected surface-water sites in the study area.

12045500 Elwha River at McDonald Bridge near Port Angeles, Wash. (Site 129)--cont.

\begin{tabular}{|c|c|c|c|c|c|c|}
\hline Date & Time & $\begin{array}{l}\text { Streamflow, } \\
\text { instantaneous, } \\
\text { (cubic feet } \\
\text { per second) }\end{array}$ & $\begin{array}{c}\text { Tempera- } \\
\text { ture } \\
\left({ }^{\circ} \mathrm{C}\right)\end{array}$ & $\begin{array}{c}\text { Turbidity } \\
\text { (JTU) }\end{array}$ & $\begin{array}{l}\text { Sediment, } \\
\text { suspended } \\
\text { (milligrams } \\
\text { per liter) }\end{array}$ & $\begin{array}{l}\text { Sediment } \\
\text { discharge, } \\
\text { suspended } \\
\text { (tons per day) }\end{array}$ \\
\hline \multicolumn{7}{|l|}{1976} \\
\hline \multicolumn{7}{|l|}{ October } \\
\hline 26 & 1145 & 572 & 9.1 & 1 & 1 & 1.5 \\
\hline \multicolumn{7}{|c|}{ December } \\
\hline 01 & 0900 & 435 & 4.9 & 1 & 1 & 1.2 \\
\hline 27 & 0945 & 1260 & 4.6 & 2 & 2 & 6.8 \\
\hline \multicolumn{7}{|l|}{1977} \\
\hline \multicolumn{7}{|l|}{ January } \\
\hline 26 & 1015 & 650 & 2.6 & 2 & 4 & 7.0 \\
\hline \multicolumn{7}{|l|}{ March } \\
\hline 01 & 1000 & 1030 & 4.4 & 2 & 1 & 2.8 \\
\hline 28 & 1030 & 704 & 5.4 & 3 & 2 & 3.8 \\
\hline \multicolumn{7}{|l|}{ April } \\
\hline 22 & 1330 & 872 & 8.5 & 2 & 2 & 4.7 \\
\hline \multicolumn{7}{|l|}{ May } \\
\hline 26 & 0930 & 1140 & 8.0 & 1 & 2 & 6.2 \\
\hline \multicolumn{7}{|l|}{ June } \\
\hline 30 & 0930 & 1170 & 11.7 & 1 & 1 & 3.2 \\
\hline \multicolumn{7}{|l|}{ July } \\
\hline 25 & 1200 & 668 & 14.3 & 1 & 3 & 5.4 \\
\hline \multicolumn{7}{|l|}{ August } \\
\hline 31 & 1200 & 566 & 13.6 & 1 & 3 & 4.6 \\
\hline \multicolumn{7}{|c|}{ September } \\
\hline 21 & 1030 & 1100 & 12.4 & 1 & 2 & 5.9 \\
\hline
\end{tabular}


TABLE 15.--Suspended sediment and related parameters in water from selected surface-water sites in the study area.

12045500 Elwha River at McDonald Bridge near Port Angeles, Wash. (Site 129)--cont.

$\begin{array}{llcccc} & \begin{array}{l}\text { Streamflow, } \\ \text { instantaneous, }\end{array} & \text { Tempera- } & \text { Sediment, } & \text { Sediment } \\ & & \text { ture } & \text { Turbidity } & \text { suspended } & \text { discharge, } \\ \text { (cubic feet } & \left({ }^{\circ} \mathrm{C}\right) & \text { (JTU) } & \text { (milligrams suspended } \\ \text { Date } & \text { Time second) } & & \text { per liter) (tons per day) }\end{array}$

1977

October

0930

2000

9.1

10

14

76

November

14

1230

5080

6.2

70

94

1290

December

19

1430

2060

4.2

25

42

234

1978

January

30

1230

1240

4.7

6

5

17

February

21

1130

920

5.4

2

41

102

March

15

1000

848

5.4

1

6.9

April

18

0930

836

5.4

1

9.0

May

30

1215

1340

9.7

$--$

14

June

19

1400

1870

10.6

$--$

25

26

0930

968

14.3

$-$

4

10

August

29

1600

686

15.3

2

3.7 
TABLE 15.--Suspended sediment and related parameters in water from selected surface-water sites in the study area.

12045500 Elwha River at McDonald Bridge near Port Angeles, Wash. (Site 129)--cont.

\begin{tabular}{|c|c|c|c|c|c|c|}
\hline Date & Time & $\begin{array}{l}\text { Streamflow, } \\
\text { instantaneous, } \\
\text { (cubic feet } \\
\text { per second) }\end{array}$ & $\begin{array}{c}\text { Tempera- } \\
\text { ture } \\
\left({ }^{\circ} \mathrm{C}\right)\end{array}$ & $\begin{array}{l}\text { Turbidity } \\
\text { (JTU) }\end{array}$ & $\begin{array}{l}\text { Sediment, } \\
\text { suspended } \\
\text { (milligrams } \\
\text { per liter) }\end{array}$ & $\begin{array}{l}\text { Sediment } \\
\text { discharge, } \\
\text { suspended } \\
\text { (tons per day) }\end{array}$ \\
\hline \multicolumn{7}{|l|}{1978} \\
\hline \multicolumn{7}{|c|}{ September } \\
\hline 13 & 0830 & 992 & 10.9 & -- & 8 & 21 \\
\hline \multicolumn{7}{|l|}{ October } \\
\hline 25 & 1030 & 450 & 6.6 & --- & 5 & 6.1 \\
\hline \multicolumn{7}{|c|}{ November } \\
\hline 28 & 1245 & 860 & 4.6 & -- & 9 & 21 \\
\hline \multicolumn{7}{|c|}{ December } \\
\hline 15 & 1055 & 1090 & 3.3 & -- & 3 & 8.8 \\
\hline \multicolumn{7}{|l|}{1979} \\
\hline \multicolumn{7}{|l|}{ January } \\
\hline 18 & 0930 & 380 & 1.2 & -- & 2 & 2.1 \\
\hline \multicolumn{7}{|c|}{ February } \\
\hline 13 & 1600 & 2880 & 3.0 & -- & 9 & 70 \\
\hline \multicolumn{7}{|l|}{ March } \\
\hline 21 & 1100 & 1240 & 6.0 & -- & 7 & 23 \\
\hline \multicolumn{7}{|l|}{ April } \\
\hline 18 & 0930 & 812 & 6.0 & -- & 2 & 4.4 \\
\hline \multicolumn{7}{|l|}{ May } \\
\hline 15 & 1230 & 1660 & 9.5 & -- & 3 & 13 \\
\hline \multicolumn{7}{|l|}{ June } \\
\hline 21 & 1000 & 1110 & 9.5 & -- & 2 & 6.0 \\
\hline \multicolumn{7}{|l|}{ July } \\
\hline 17 & 0730 & 1020 & 12.9 & -- & 4 & 11 \\
\hline
\end{tabular}


TABLE 15.--Suspended sediment and related parameters in water from selected surface-water sites in the study area.

12045500 Elwha River at McDonald Bridge near Port Angeles; Wash. (Site 129)--cont.

\begin{tabular}{|c|c|c|c|c|c|c|}
\hline Date & Time & $\begin{array}{l}\text { Streamflow, } \\
\text { instantaneous, } \\
\text { (cubic feet } \\
\text { per second) }\end{array}$ & $\begin{array}{c}\text { Tempera- } \\
\text { ture } \\
\left({ }^{\circ} \mathrm{C}\right)\end{array}$ & $\begin{array}{l}\text { Turbidity } \\
\text { (JTU) }\end{array}$ & $\begin{array}{l}\text { Sediment, } \\
\text { suspended } \\
\text { (milligrams } \\
\text { per liter) }\end{array}$ & $\begin{array}{l}\text { Sediment } \\
\text { discharse, } \\
\text { suspended } \\
\text { (tons per day) }\end{array}$ \\
\hline \multicolumn{7}{|l|}{1979} \\
\hline \multicolumn{7}{|l|}{ August } \\
\hline 15 & 0745 & 596 & 14.0 & -- & 3 & 4.8 \\
\hline \multicolumn{7}{|c|}{ September } \\
\hline 12 & 0900 & 576 & 7.1 & -- & 12 & 19 \\
\hline \multicolumn{7}{|l|}{ October } \\
\hline 17 & 0930 & -- & 10.2 & -- & 3 & -- \\
\hline \multicolumn{7}{|c|}{ November } \\
\hline 14 & 0945 & -- & 4.8 & -- & 9 & -- \\
\hline \multicolumn{7}{|c|}{ December } \\
\hline 12 & 0900 & -- & 4.6 & -- & 81 & -- \\
\hline 1980 & & & + & & & \\
\hline \multicolumn{7}{|l|}{ January } \\
\hline 16 & 1000 & -- & 4.0 & -- & -- & -- \\
\hline \multicolumn{7}{|c|}{ February } \\
\hline 13 & 0745 & -- & 3.7 & -- & 21 & -- \\
\hline
\end{tabular}

